\title{
The Borrego Mountain Earthquake of April 9, 1968
}

GE O L O G I A L S U R VEY PR OFE S S I O N A L P A P E R 787

Contributions from:

California Institute of Technology

Lamont-Doherty Geological Observatory of Columbia University

Seismological Field Survey, National Oceanic and Atmospheric Administration

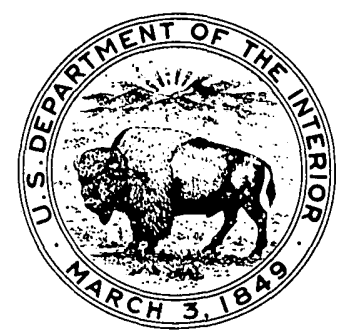

UNITED STATES GOVERNMENT PRINTING OFFICE, WASHINGTON : 1972 


\section{UNITED STATES DEPARTMENT OF THE INTERIOR}

ROGERS C. B. MORTON, Secretary

\section{GEOLOGICAL SURVEY}

V. E. McKelvey, Director

Library of Congress catalog-card No. 72-600201

For sale by the Superintendent of Documents, U.S. Government Printing Office

Washington, D.C. 20402 - Price $\$ 6.25$

Stock Number 2401-00218 


\section{BORREGO MOUNTAIN EARTHQUAKE, APRIL 9, 1968}




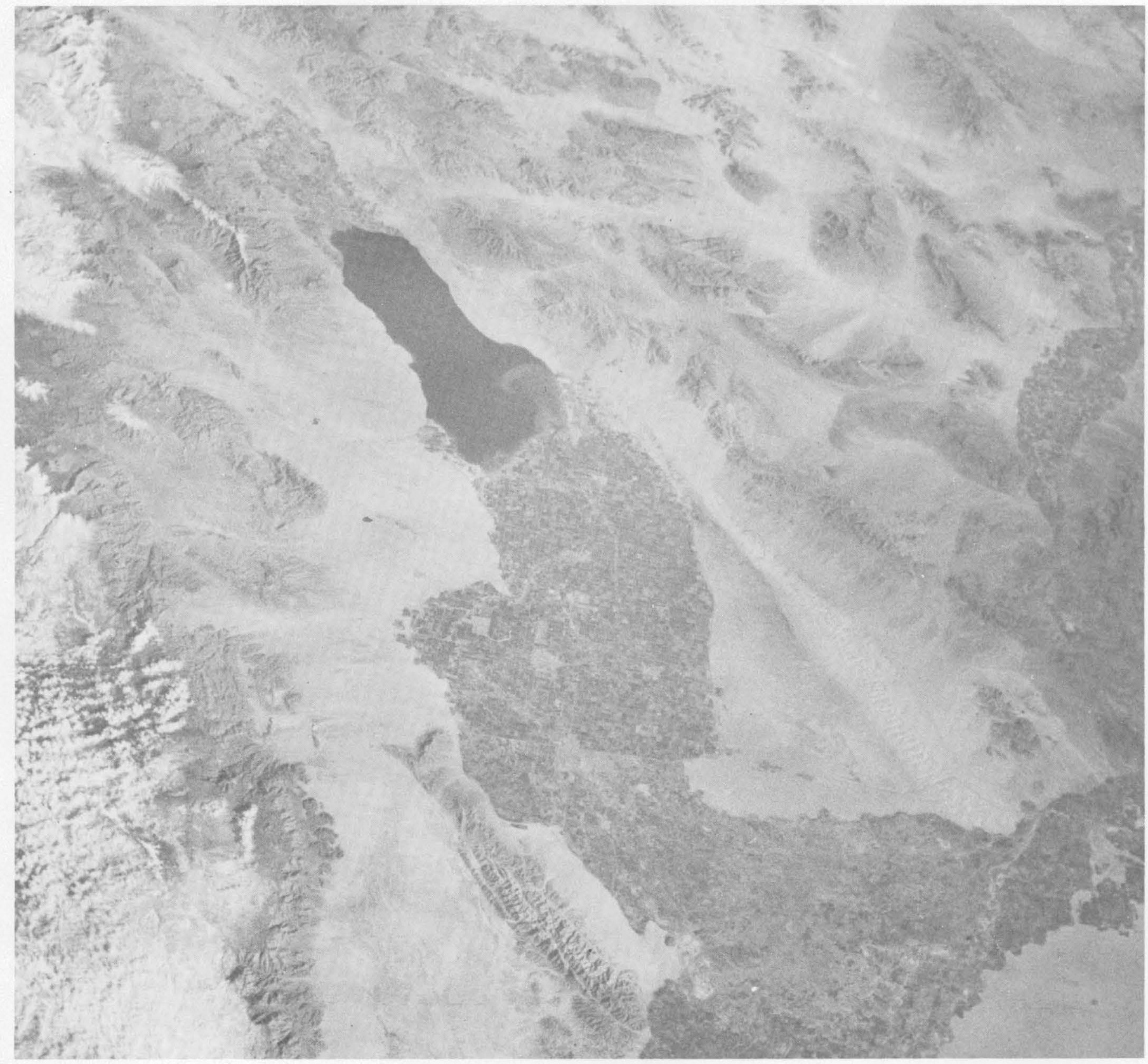

Oblique northward view of the Salton Trough in southern California and northwestern Mexico. Names of prominent indicate segments of faults that moved at the time of the 1968 Borrego Mountain earthquake. The distance 


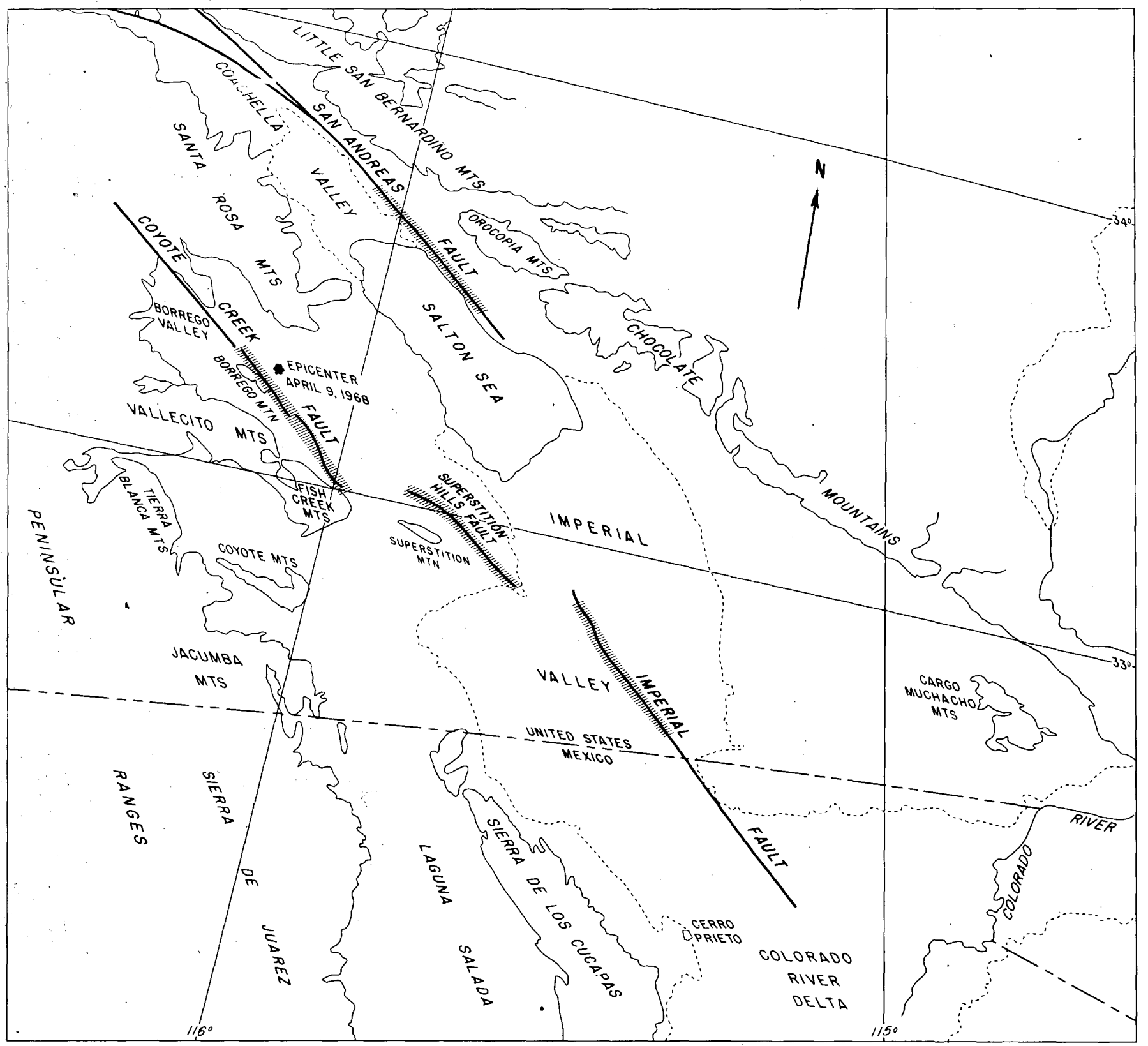

physiographic elements within and bordering the trough are shown in the adjoining line sketch. Lines within shaded areas between meridians shown is $93.5 \mathrm{~km}$ along the $33 \mathrm{~d}$ parallel. Apollo 9 photo AS9-23-3558, courtesy of NASA. 



\section{CONTENTS}

Introduction, by Robert V. Sharp, U.S. Geological Survey

Tectonic setting of the Salton Trough, by Robert V. Sharp, U.S. Geological Survey.

Foreshock, main shock, and larger aftershocks of the Borrego Mountain earthquake, by Clarence R. Allen and John M. Nordquist, Seismological Laboratory, California Institute of Technology....

Source parameters of the Borrego Mouuntain earthquake, by Max Wyss, Lamont-Doherty Geological Observatory of Columbia University, and Thomas C. Hanks, Seismological Laboratory, California Institute of Technology.

Aftershocks of the Borrego Mountain earthquake from April 12 to June 12, 1968, by Robert M. Hamilton, U.S. Geological Survey...

Surface rupture along the Coyote Creek fault, by Malcolm M. Clark, U.S. Geological Survey..

Displacements on the Imperial, Superstition Hills, and San Andreas faults triggered by the Borrego Mountain earthquake, by Clarence R. Allen, Seismological Laboratory, California Institute of Technology, Max Wyss, Lamont-Doherty Geological Observatory of Columbia University, James N. Brune, Institute of Geophysics and Planetary Physics, University of California, San Diego, and Arthur Grantz and Robert E. Wallace, U.S. Geological Survey...

Continued slip on the Coyote Creek fault after the Borrego Mountain earthquake, by R. O. Burford, U.S. Geological Survey.

Holocene activity of the Coyote Creek fault as recorded in sediments of Lake Cahuilla, by Malcolm M. Clark, Arthur Grantz, and Meyer Rubin, U.S. Geological Survey.

Geologic evidence of previous faulting near the 1968 rupture on the Coyote Creek fault, by Robert V. Sharp and Malcolm M. Clark, U.S. Geological Survey.

Intensity distribution and field effects, strong-motion seismograph records, and response spectra, by Seismological Field Survey, National Oceanic and Atmospheric Administration..

Engineering geology, by R. O. Castle and T. L. Youd, U.S. Geological Survey.

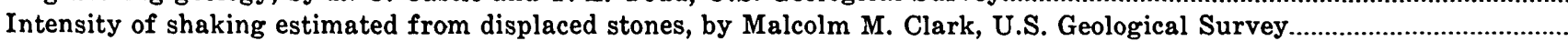

Water-resources effects, by A. O. Waananen and W. R. Moyle, Jr., U.S. Geological Survey....

Collapse fissures along the Coyote Creek fault, by Malcolm M. Clark, U.S. Geological Survey...

1

16

24

31

\section{ILLUSTRATIONS}

[Plates are in pocket]

Frontispiece. Oblique northward view of Salton Trough in southern California and northwestern Mexico, showing physiographic elements.

Plate 1. Map showing surface ruptures created at the time of and after the Borrego Mountain earthquake of April 9, 1968 (G.m.t.).

2. Map showing breaks along the Superstition Hills and San Andreas faults formed by displacement triggered by the Borrego Mountain earthquake of April 9, 1968.

3. Generalized geologic map of the area near the segment of the Coyote Creek fault that ruptured during the Borrego Mountain earthquake.

4. Map showing generalized geology and distribution of effects of the Borrego Mountain earthquake between Coronado and Glamis, Calif.

5. Map showing distribution of effects of the Borrego Mountain earthquake within the epicentral area.

Figure 1. Generalized geologic map of the Salton Trough, southern California.

2. Composite stratigraphic columns along the flanks of the northern part of the Salton Trough................. 6

3. Oblique aerial view of southern Borrego Badlands and West Butte, Borrego Mountain..............................

4. Map showing seismicity of the northern Salton Trough region ........................................................... 12

5. Map showing epicenter of the 1968 Borrego Mountain earthquake in relation to major faults and epicenters of other earthquakes of magnitude 6.0 and greater in southern California since 1912 ......

6. Index map showing location of Caltech seismograph stations that were operating at the time of the Borrego Mountain earthquake. 
FigURE 7. Map showing epicenters of shocks of the Borrego Mountain series of magnitude 3.0 and greater that occurred between April 9, 1968, and April 29, 1969.

8. Sketch showing focal-mechanism solution for the Borrego Mountain main shock.

9. Sketch showing epicenters of figure 7 separated into four consecutive time periods containing roughly equal number of shocks.

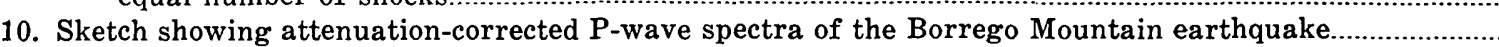

11. Sketch showing attenuation-corrected S-wave spectra of the Borrego Mountain earthquake from WWSSN recordings..

12. Map of the Borrego Mountain region showing location of seismograph stations, epicenter of main shock, aftershock epicenters, and active faults.

13. Detailed map of aftershock epicenters, ground breakage, epicenter of main shock, and lines of vertical sections.

14. Sketch showing variation of epicenter pattern with time.

15. Vertical sections through aftershock zone.

16. Vertical sections through aftershock zone...

17. Graph showing depth distribution of aftershocks with well-determined hypocenters.

18. Sketch showing equal-area projections of the firs

19. Map showing nodal-plane solutions, plotted at the epicenter, for 72 aftershocks that yielded well-de. termined solutions.

20. Graph showing average $\mathrm{P}$-magnitude residual plotted against average $\mathrm{X}$-magnitude residual for each seismograph station.

21. Graph showing average $P$-magnitude and average $X$-magnitude residuals plotted against station-time correction.

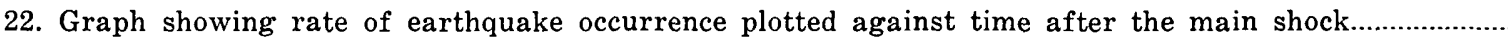

23. Vertical aerial photograph of the region of surface faulting associated with the Borrego Mountain earthquake..

24-28. Photographs showing:

24. Central break at location 19.7 showing en echelon fractures as much as $20 \mathrm{~m}$ long and $2 \mathrm{~m}$ apart within a zone about $2 \mathrm{~m}$ wide.

25. Band of complex fractures about $1 \mathrm{~m}$ wide across hardened silty sand crust in the wash at the eastern base of Borrego Mountain.

26. En echelon, parallel, and complex fractures in a zone $6-10 \mathrm{~m}$ wide on the north break at location 6.4

27. Time sequence on central break at location 22.5 .

28. En echelon fractures at the north end of Benson Lake

29. Photograph and sketch showing detail of symmetrical S-shaped overthrusts in the clay-silt crust of Benson Lake.

30. Photograph showing tire tracks at location 22.5 displaced about $180 \mathrm{~mm}$ across the single fracture at this location.

31. Photograph showing dune buggy crossing the north break in Benson Lake 6 days after the earthquake

32. Graph showing total right-lateral displacement along the north, central, and south breaks measured at different times.

33. Photograph showing segment of an extensional fracture showing dip slip

34. Photograph showing fractures with left-lateral displacement at location $\mathbf{1 7 . 5}$ northeast of the 'central break.

35. Map showing the location of recently active traces of the Coyote Creek fault in the vicinity of the surface rupture of 1968 .

36. Photograph showing view southeast along the central break, showing an old scarp $0.3-0.4 \mathrm{~m}$ high in alluvium at the northeast base of Ocotillo Badlands.

37. Photograph showing central break at Old Kane Spring Road.

38. Photograph showing view westward over south break in November 1956.

39. Map showing areas of postearthquake displacement along the surface rupture after April 14, 1968

40. Graph showing rainfall, estimates of dates of surface runoff on the central and south breaks, and dates of field investigations, January 1968 through December 1971

41. Map showing mean annual precipitation in the region of the 1968 break, 1931-60.

42. Photographs showing time sequence of collapse and eventual filling along a fracture of the central break at location 23.8 .

43. Map showing fractures that apparently formed near the central break between June 21, 1968, and March 20, 1969.

44. Photograph showing tire tracks at location 19.4 off set $240 \mathrm{~mm}$ horizontally

45. Photograph showing new breaks with $30 \mathrm{~mm}$ of right-lateral off set. 
47. Map showing horizontal displacement

Graph showing displacement from April 9, 1968, to March 1971 near location 19.4

49. Map showing special features of the south break.

50. Photograph showing sharp-edged fracture that formed after the channel that it crosses carried water in September 1969 .

51. Maps showing location of aftershocks and other earthquakes of $M \geqslant 3$ and areas of creep during three periods after April 8, 1968.

52. Index map showing relation of the Coyote Creek fault to the three distant faults, the San Andreas, Superstition Hills, and Imperial, upon which triggered movements occurred

53. Photograph showing fresh cracks in unconsolidated material at north edge of Highway 80

54. Map of Imperial fault trace.

55. Graph showing measurements of fault-crossing geodetic networks between May 1, 1967, and May 1, 1970, showing displacements at time of Borrego Mountain earthquake.

56-69. Photographs showing:

56. Fresh en echelon cracks crossing Ross Road

57. En echelon cracks on Meloland Road first observed following the Imperial earthquake of 1966 but slightly widened during the Borrego Mountain earthquake..

58. Fresh cracks across Heber Road

59. Right-lateral displacement of about $2 \mathrm{~cm}$ on the Superstition Hills fault in sec. 23, R. 12 E., T. 14 S.

60. En echelon cracks indicative of right-lateral displacement along Superstition Hills fault in sec. 9, R. 12 E., T. 14 S.

61. New en echelon cracks of right-lateral habit following the line of desert plants that marks the Superstition Hills fault in NW $1 / 4$ sec. 26, T. 13 S., R. 11 E.

62. Small collapse pits, alined vegetation, and variation in abundance of vegetation along the Superstition Hills fault in SW $1 / 4$ sec. 9, T. 14 S., R. 12 E...

63. En echelon cracks at base of scarplet along San Andreas fault in sec. 28, R. 9 E., T. 6 S.......

64. New fractures in old fracture zone along San Andreas fault in sec. 28, R. 9 E., T. 6 S..............

65. En echelon cracks along the San Andreas fault near Salt Creek, in center of sec. 28, T. 8 S., R. $11 \mathrm{E}$

66. En echelon fractures along the San Andreas fault approximately $2 \mathrm{~km}$ south of Salt Creek, in SW $1 / 4$ sec. $34, T .8$ S., R. 11 E.

67. En echelon fractures, sec. 28 , T. 6 S., R. 9 E.

68. Linear valley eroded along San Andreas fault, Mecca Hills.

69. View southeast along the San Andreas fault.

70. Map of the main surface breaks associated with the Borrego Mountain earthquake of April 1968, show-

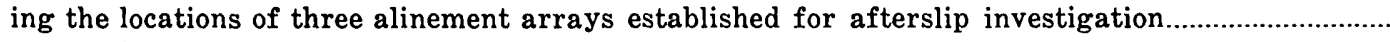

71. Plan view of typical alinement-array configuration.

72. Plan view of alinement changes at the Borrego Mountain array..

73. Plan view of incremental alinement changes at the Borrego Mountain array.....................

74. Terrain profile along the Borrego Mountain array, and profiles of relative elevation changes between sets of level readings.

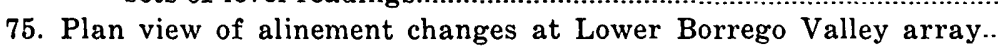

76. Graph showing cumulative right-lateral slip at the Lower Borrego Valley alinement array plotted with respect to time elapsed since main shock.

77. Graph showing cumulative right-lateral afterslip at the Lower Borrego Valley alinement array plotted with respect to logarithmic time scale.......

78. Terrain profile along the Lower Borrego Valley alinement array, and profiles of relative elevation changes between sets of level readings.

79. Plan view of alinement changes at Fish Creek Mountains array from December 10, 1969, to January 25,1971 .

80. Photographs showing central and branching breaks of the 1968 earthquake at Old Kane Spring Road, showing shallow graben between the breaks and the location of trenches 1, 2, and 4 and profiles 1 and 2 .......

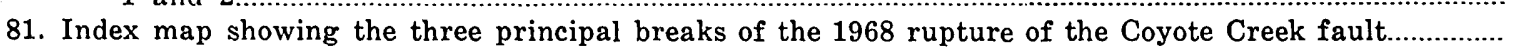

82. Structural profiles across the prominent scarp along the central break of 1968 .

83. Sketch showing south wall of trench 1 showing progressively greater offset of older strata and bending of strata (drag) across the branching break of the 1968 event.

84. Graphs showing relation of vertical offset to $\mathrm{C}^{14}$ age along a branching break of the 1968 earthquake, determined from late Holocene sediments exposed in trench 1. 
85. Graph showing deposition rates of sediments southwest of the branching break of 1968 in trenches 1 and 4 .

86. Map showing historic earthquakes of magnitude 6 and greater along the San Jacinto fault zone.........

87. Graph showing recurrence intervals at a point in the San Jacinto fault zone for different values of rupture length.

88. Sketch showing pre-1968 fractures and fissure collapse along the Coyote Creek fault as exposed in a natural cut at location 30.7 .

89. Sketch showing south wall of trench 4, showing a surface fracture associated with the 1968 earthquake that developed where there had never been a break before...

90. Regional map of the Coyote Creek fault, showing its relation to faults outside the 1968 rupture zone

91. Simplified geologic map of the area at the north end of 1968 Coyote Creek fault ruptures.

92. Geologic map of the area near Squaw Peak.

93. Geologic map of the Ocotillo Badlands.

94. Three-dimensional view of possible configuration for Coyote Creek fault beneath Ocotillo Badlands......

95. Isoseismal map of the Borrego Mountain earthquake.

96. Map showing location of strong-motion seismographs that were triggered by the Borrego Mountain earthquake.

97. Map showing location of strong-motion seismographs in the Los Angeles area that were triggered by the Borrego Mountain earthquake

98. El Centro accelerograph record from the Borrego Mountain earthquake

100. Graph showing maximum acceleration pountain earthquake.. earthquake.

101. Accelerograph record from the Central Engineering building at the Jet Propulsion Laboratory in Pasadena, ninth floor.

102. Map showing location of the ground-level displacement meters actuated by the Borrego Mountain earthquake

103. Graph showing maximum displacement plotted against epicentral distance for the Borrego Mountain earthquake

104. El Centro seismoscope records superimposed on a map of the city

105. Seismoscope records from San Fernando, San Gabriel, and Puddingstone dams.

106. Response spectra from El Centro

107. Response spectra from San Diego and San Onofre.

108-114. Photographs showing:

108. Rockfalls and soil falls

109. Soil falls along Fish Creek Wash...

110. Incipient slump north of Ocotillo Badlands.

111. Terrain in central Ocotillo Badlands.

112. Slump scarps generated in the epicentral area...

113. Headwall scarp of slump or block glide at Highway 80 crossing of New River

114. Slump in artificial fill

115. Sketch map of seismic-compaction ruptures in epicentral area

116-119. Photographs showing:

116. Seismic-compaction ruptures in epicentral area.

117. Scarp along edge of compaction-produced graben

118. Sand boils along the New River.

119. Shattered crusts overlying poorly indurated materials in epicentral area

120. Map of shattered brittle crust east of Ocotillo Wells

122. Damaged chimney in Ocotillo Wells................................................................................ 169

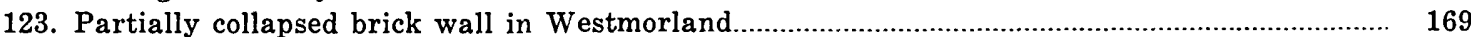

124. Collapsed fieldstone cellar east of Ocotillo Wells.................................................................... 169

125. Cracked concrete-block wall in Salton City...................................................................... 169

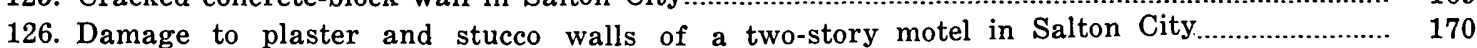

127. Cracked and spalled concrete bridge pier east of Ocotillo Wells............................................. 171

128. Cracked concrete railroad bridge abutment near Glamis.................................................. 171

129. Light truck that sank into sand southeast of Ocotillo Wells..................................................... 172

130. Projected and displaced light objects southeast of Ocotillo Wells.............................................. 172

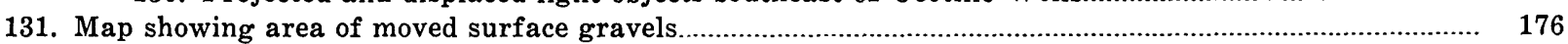

132. Photograph showing gravel on top of ridge A 
133. Stereo view of a 0.6 -m-high tetrahedron tipped over by shaking.

134. Photograph showing boulder propelled from its nearly horizontal seat and rotated $180^{\circ}$

135. Sketch showing forces acting between a block and the inclined surface on which it rests, as a result of friction, adhesion, and shaking of the surface.

136. Graph showing relation between coefficient of friction and minimum horizontal acceleration necessary to slide a block that rests on a horizontal plane.

137. Graph showing relation between coefficient of friction and the minimum horizontal acceleration necessary to slide a block that rests on an inclined plane..

138. Graph showing relation between height and excess acceleration between blocks and the surface on which they rest for different values of cohesive strength..

139. Map of selected observation wells, gaging stations, and compaction-recorder sites that showed effects of the Borrego Mountain earthquake......

140. Graph showing fluctuations recorded at observation wells in the Salton Sea basin during and after the Borrego Mountain earthquake.

141. Graph showing fluctuations recorded at observation wells in central and southern California during and after the Borrego Mountain earthquake.

142. Graph showing fluctuations recorded at gaging stations in response to the Borrego Mountain earthquake.

143-150. Photographs showing:

143. Collapse fissure on the south break that developed on a tectonic fracture after the rains of September 1969.

144. Aerial view southwestward of collapse fissures and channels leading into them on south break at location 31.4, January 1970 .

145. Detail of channel and collapse fissure shown at $a$ in figure 144

146. Detail of the collapse fissure at $b$, figure 144 .

147. Collapse across a silty mound.

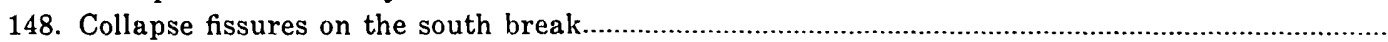

149. Collapse fissures on the south break, January 1970

150. Detail at $c$, figure 144 , October $1969 .$.

151. Sketch showing hypothetical development of a collapse fissure.

152. Map showing relict (preearthquake) collapse fissures near the south break of the 1968 rupture along the Coyote Creek fault.

153-156. Photographs showing:

153. Aerial view southward over part of the most prominent preearthquake fissure.

154. South end of the relict collapse fissure shown at $a$ in figure 153

155. A taut root across the relict collapse fissure at $b$ in figure 153 .

156. Concentric collapse fissures at location 18

\section{TABLES}

1. Caltech trailer-mounted stations in operation during the Borrego Mountain aftershock period.

2. Earthquakes of magnitude 3.0 and greater in the area from $32^{\circ} 45^{\prime}$ to $33^{\circ} 30^{\prime} \mathrm{N}$. and from $115^{\circ}$ to $116^{\circ} 30^{\prime}$ W. from April 9, 1968, through April 28, 1969.

3. Borrego Mountain earthquake data for stations of the Pasadena network

4. P- and $\mathrm{S}$-wave spectral data...

5. Source parameters.

6. Local observations of radiated seismic energies.

7. Seismograph station data...

8. Borrego Mountain aftershocks..

9. Width of the main rupture along the Coyote Creek fault.

10. Summary of creep at locations indicated in figure $39 .$.

11. Earthquakes of $\mathrm{M} \geqslant 3$ in the area from $32^{\circ} 45^{\prime}$ to $33^{\circ} 15^{\prime} \mathrm{N}$. and from $115^{\circ} 45^{\prime}$ to $116^{\circ} 15^{\prime} \mathrm{W}$. from April 29, 1969, through 1970

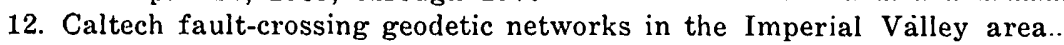

13. Vertical deformation along faults bounding the graben between the central break and branching break shown in figure 80 ...

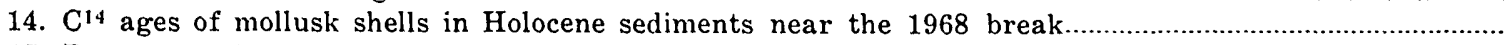

15. Recurrence intervals for 1968-size events along the Coyote Creek fault, based on the vertical component of late Holocene displacements along the central and branching breaks of 1968 . 
TABLE 16. Earthquakes of magnitude 6.0 and greater along the San Jacinto fault zone since 1899

17. Rate of late Holocene strike-slip deformation on the Coyote Creek fault based on 200-year recurrence interval for 1968-size events

18. Rate of late Holocene vertical deformation at selected points along the Coyote Creek fault based on 200-year recurrence interval for 1968-size events.

19. Rate of late Holocene strike-slip deformation on the Coyote Creek fault based on vertical deformation rates and ratio of horizontal to vertical displacement..

20. Strong-motion seismograph records from the Borrego Mountain earthquake.

21. Strong-motion seismographs not triggered by the Borrego Mountain earthquake but within potential operable range.

22. Locations of strong-motion seismographs triggered by the Borrego Mountain earthquake

23. General characteristics of strong-motion seismographs.

24. Maximum accelerations recorded at stations less than 120 miles from the epicenter.

25. General characteristics of displacement meters

26. Maximum amplitudes recorded by ground-level displacement meters

27. Relative maximum displacement spectra from the El Centro seismoscope records.

28. Maximum seismoscope amplitudes from selected dams in southern California..

29. Adhesion of cobbles to the surface of ridge $B$.

30. Fluctuations of water level in observation wells, April 8, 1968

31. Fluctuations and net changes in water level recorded at gaging stations in California, April 8, 1968 . 


\title{
THE BORREGO MOUNTAIN EARTHQUAKE OF APRIL 9, 1968
}

\author{
INTRODUCTION
}

By Robert V. Sharp

U. S. Geological Survey

Seismicity of the Imperial Valley region, perhaps the most exceptional of California's areas of active tectonism, has been dominated in this century by earthquakes of intermediate magnitude originating along two members of the San Andreas system: the San Jacinto fault zone and the Imperial fault. The most recent shock in the series of nine or more magnitude 6 and larger earthquakes along these faults since 1915 occurred at 2:29 G.m.t., ${ }^{1}$ April 9, 1968, near Borrego Mountain in northwestern Imperial Valley. The magnitude 6.4 shock, California's largest in the more than 18 years between the Arvin-Tehachapi earthquakes of 1952 and the San Fernando earthquake of 1971, was felt over most of southern California and adjacent parts of Nevada, Arizona, and northwestern Mexico. It caused little damage, mainly because it centered in a sparsely settled desert region. Horizontal (right-lateral) surface displacement accompanied the earthquake along several strands within the complex San Jacinto fault zone; the Coyote Creek fault branch nearest the epicenter moved a maximum of $38 \mathrm{~cm}$. Smaller surficial offsets occurred along the Superstition Hills fault and the Imperial fault, also within the San Jacinto zone but at considerable distance from the epicentral area, as well as along a segment of the San Andreas fault about $50 \mathrm{~km}$ northeast of the epicenter. (See frontispiece.) Although the Borrego Mountain earthquake cannot be regarded as a great seismic event either from the standpoint of magnitude or its effects on the works of man, the recognition of many significant geologic and seismologic consequences of this shock underscores the necessity of intensive documentation of all major earthquakes.

${ }^{1}$ Greenwich mean time. Corresponding Pacific standard time was $6: 29$ p.m., April 8, 1968.
Several organizations have cooperated on this detailed summary of investigations on the Borrego Mountain earthquake. Data on surface rupturing, including postearthquake creep, were obtained jointly by personnel from the U.S. Geological Survey's National Center for Earthquake Research and the California Institute of Technology. Seismographic studies of the main shock were conducted by the Seismological Laboratory at California Institute of Technology and by the National Oceanic and Atmospheric Administration (NOAA), and aftershock activity was recorded through joint efforts of seismologists of U.S. Geological Survey and California Institute of Technology. Engineering effects, disturbance of water resources, and the evidence of previous fault movement were documented by the U.S. Geological Survey. With the exception of investigations of continuing postearthquake surface creep and the history of Holocene displacement, most of the data reported here are essentially complete. Inasmuch as some of the conclusions on creep may be modified by future events, that part of this investigation should be regarded as a summary of progress as of January 1972. A preliminary report on the Borrego Mountain earthquake has been published in the Bulletin of the Seismological Society of America ${ }^{2}$ and reprinted in Mineral Information Service of the California Division of Mines and Geology. ${ }^{3}$

The papers assembled here report several important observations and conclusions, some of them unique, that help to clarify mechanisms of crustal strain release and its relationship to earthquakes.

'Allen, C. R., Grantz, A., Brune, J. N., Clark, M. M., Sharp, R. V., Theodore, T. G., Wolfe, E. W., and Wyss, M., 1968, The Borrego Mountain, California, earthquake of 9 April 1968; A preliminary report: Seismol. Soc. America Bull., v. 58, p. 1183-1186.

s1968, v. 21, no. 7, p. 103-106. 
Perhaps the most significant feature, heretofore unrecorded for any other earthquake in California, involves shaking-induced horizontal displacements along faults that lie relatively far from the earthquake hypocenter and the main body of aftershocks. Another unusual aspect of this seismic event was the uneven distribution of postearthquake displacement by creep along the principal Coyote Creek fault breaks; the fault segment that initially shifted the most at the time of the earthquake has subsequently crept very little, whereas on another sector where the initial displacement was relatively small, movement has continued and apparently has not yet stopped (1972). The observed aftershock distribution is likewise complex and widespread and is only partly associated with the positions of known steeply dipping surface faults, apparently reflecting complexity of the Sän Jacinto fault zone and perhaps other geologic structures at depth. Furthermore, the complexity of the aftershock distribution contrasts distinctly with that observed after the 1966 Parkfield-Cholame earthquakes, the only other shocks of intermediate magnitude associated with predominantly strike-slip surface movement that have occurred recently in the San Andreas system; the aftershock pattern for those events was confined within a narrow band along the zone of surface breakage. The epicenter of the main Borrego Mountain shock lies near the middle of the distribution of aftershocks, apparently reflecting bilateral faulting (fracturing propagating in two directions away from the point of initial rupture) that has not been observed in other recent California earthquakes. Another contrast to the 1966 Parkfield-Cholame earthquakes is the relatively great width of the zone of rupturing developed along the lines of surficial displacement along the Coyote Creek fault. The ground surface, moreover, broke almost exactly at those positions where prior movements are demonstrable by geologic and geomorphic evidence; a similar relationship of prehistoric repetitive offsets has been established in a number of studies elsewhere within the San Andreas system. Although displacement was principally horizontal along the Coyote Creek fault break, in several locations vertical offset also was evident but was variable in sense along the length of the fault; however, in every case the sense matched that of previous Quaternary movement shown by geomorphic features. That the history of vertical movement appeared to be this systematic has suggested the possibility of estimating the fre- quency of recurrent displacement through the study of local vertical offsets of datable flat-lying Quaternary beds transected by the Coyote Creek fault. Detailed followup studies on the record of Holocene vertical displacement were accomplished by sectioning the fault with several trenches; the data on recent fault movements serve as a basis for projections of future activity. Another study attempted was the field estimation of accelerations developed in the epicentral area during the earthquake through observations of dislodged stones mantling slopes and ridges. Aside from these considerations of general significance revealed by the study of the Borrego Mountain earthquake, the exact positions of active fault traces in the region of breakage also constitute new data of appreciable local importance.

Special mention should be made of the paper on source parameters of the Borrego Mountain earthquake, which presents a new technique for estimating the length of surface rupture and the amount of displacement based solely on teleseismic data. The capability of remote determination of these important measures now permits comparison of past, as well as future, tectonic ruptures that could not be investigated in the field, such as those that lie beneath ocean basins.

Although the San Andreas fault system is probably the most thoroughly studied zone of active faulting in the world, there have been relatively few opportunities in recent times to study major earthquakes originating along it. The reports on the 1971 San Fernando earthquake (U.S. Geological Survey Professional Paper 733, 1971), the 1966 ParkfieldCholame earthquakes (U.S. Geological Survey Professional Paper 579, 1967), and the 1952 ArvinTehachapi earthquakes (California Division of Mines and Geology, Bulletin 171, 1955) are the only others pertaining to the San Andreas system that are comparable in scope and detail. Because of the scientific interest now focused on the geologic and seismologic effects of large earthquakes, similar investigations are being made in tectonically active areas throughout the world. Probably the greatest value in the comparison of such studies lies in the recognition of the similarities and differences in regional tectonic mechanisms now prevalent in the mobile part of the earth's crust and their implications with regard to earthquake occurrence and crustal strain release. The papers presented here mark a significant step toward better understanding of these mechanisms. 


\title{
TECTONIC SETTING OF THE SALTON TROUGH
}

\author{
By Robert V. Sharp \\ U. S. Geological Survey
}

\begin{abstract}
The Salton Trough, an extension of the Gulf of California physiographic province, includes the Coachella and Imperial Valley areas of southern California and the Colorado River delta region of northwestern Mexico. Active right-lateral faults of the San Andreas system have dominated in the formation and subsequent development of the trough-gulf depression. Marine and nonmarine sediments have accumulated within the trough since it formed in Pliocene or possibly Miocene time. Tectonic activity is continuing at present at a high rate, as indicated by high levels of seismicity, geodetic measurement of crustal strain, geomorphic evidence of recent fault movements, and historic movements on faults in association with earthquakes, the latest having been the shocks in 1968 at Borrego Mountain and in 1971 at Superstition Hills.
\end{abstract}

\section{INTRODUCTION}

The Gulf of California physiographic province and its northern onland extension, the Salton Trough, together define a remarkably linear and narrow depression over $1,400 \mathrm{~km}$ long. The Salton Trough encompasses the low-lying areas of the Colorado River delta region in Mexico and the Imperial and Coachella Valley regions situated between the Peninsular Ranges of coastal southern California and the mountains northeast of the Salton Sea. (See frontispiece for a regional geomorphic view of the trough.) The land surface in the north-central section of the Salton Trough is below sea level, having been cut off from the Gulf of California by the aggrading deltaic cone of the Colorado River. In Holocene time this basinal segment of the Salton Trough has been periodically inundated by bodies of water, the latest having formed the Salton Sea in 1905. (See Mendenhall, 1909, for a brief summary of its formation.)

The present geographic limits of the Salton Trough correspond approximately to the boundaries of a late Cenozoic marine and nonmarine depositional basin that has been downwarped, downfaulted, and laterally translated between the bordering ranges along faults of the San Andreas system. (See fig. 1.) The basin extends southward the length of Baja California and is filled with as much as $6 \mathrm{~km}$ of sediments in central Imperial Valley (Tarbet, 1951 ; Biehler and others, 1964, fig. 6) ; most of the sediments may have accumulated in Quaternary time alone. The basin is underlain and bounded by Mesozoic and older crystalline rocks. On the west side of the trough, they consist predominantly of mid-Cretaceous granitic rocks of the southern California batholith, as well as prebatholithic metasedimentary and metavolcanic rocks. East of the trough, diverse types of Precambrian crystalline rocks, as well as Mesozoic granitic rocks and other rocks of unknown age, are exposed. In the axial part of the trough, sedimentation under intermittently marine and nonmarine conditions may have been essentially continuous since Pliocene time but perhaps began even earlier.

\section{TECTONIC CONTROL OF THE CONFIGURATION OF THE TROUGH}

Active fault zones of the San Andreas system parallel the northeast margin of the Salton Trough and obliquely transect its southwest flank. Intermittent right-lateral movement on some members of the system has continued to change the shape of the basin, and vertical components of offset on many of these faults have outlined the dominant physiographic elements within the trough or bordering on it.

Despite its overall northwest-trending linearity, the Salton Trough is not simply a narrow, grabenlike structure. South of the Santa Rosa Mountains, for example, where it is relatively broad, the trough is actually a complexly folded and faulted crustal downwarp. Structural complexity within the southwest flank of the trough is reflected physiographically by the projection of several structurally high basement blocks from which the Cenozoic cover has been stripped. In California these insular mountain masses include Borrego Mountain, Fish Creek Mountains, Superstition Mountains, and the Coyote Moun- 
tains (fig. 1), as well as Sierra de los Cucapas south of the International Boundary. Part of the irregularity of the west margin of the trough also is due to right-lateral offset on strike-slip fault zones that enter obliquely from the Peninsular Ranges to the west. For example, right-lateral translation amounting to $24 \mathrm{~km}$ on breaks within the San Jacinto fault zone (Sharp, 1967) has produced the jutting projections of the trough margin represented by Clark Valley and northern Borrego Valley.

\section{ORIGIN OF THE BASIN AND THE STRATIGRAPHIC SUCCESSION}

The origin of the Salton Trough, in particular the age of initial sedimentation, is difficult to pin down with the available evidence. The age of the trough is uncertain chiefly because the oldest strata known are exposed only in small areas along the perimeter. Moreover, in the axial part of the trough (Imperial Valley), basement lies at depths as great as $6 \mathrm{~km}$ (Biehler and others, 1964, p. 132), and the deepest

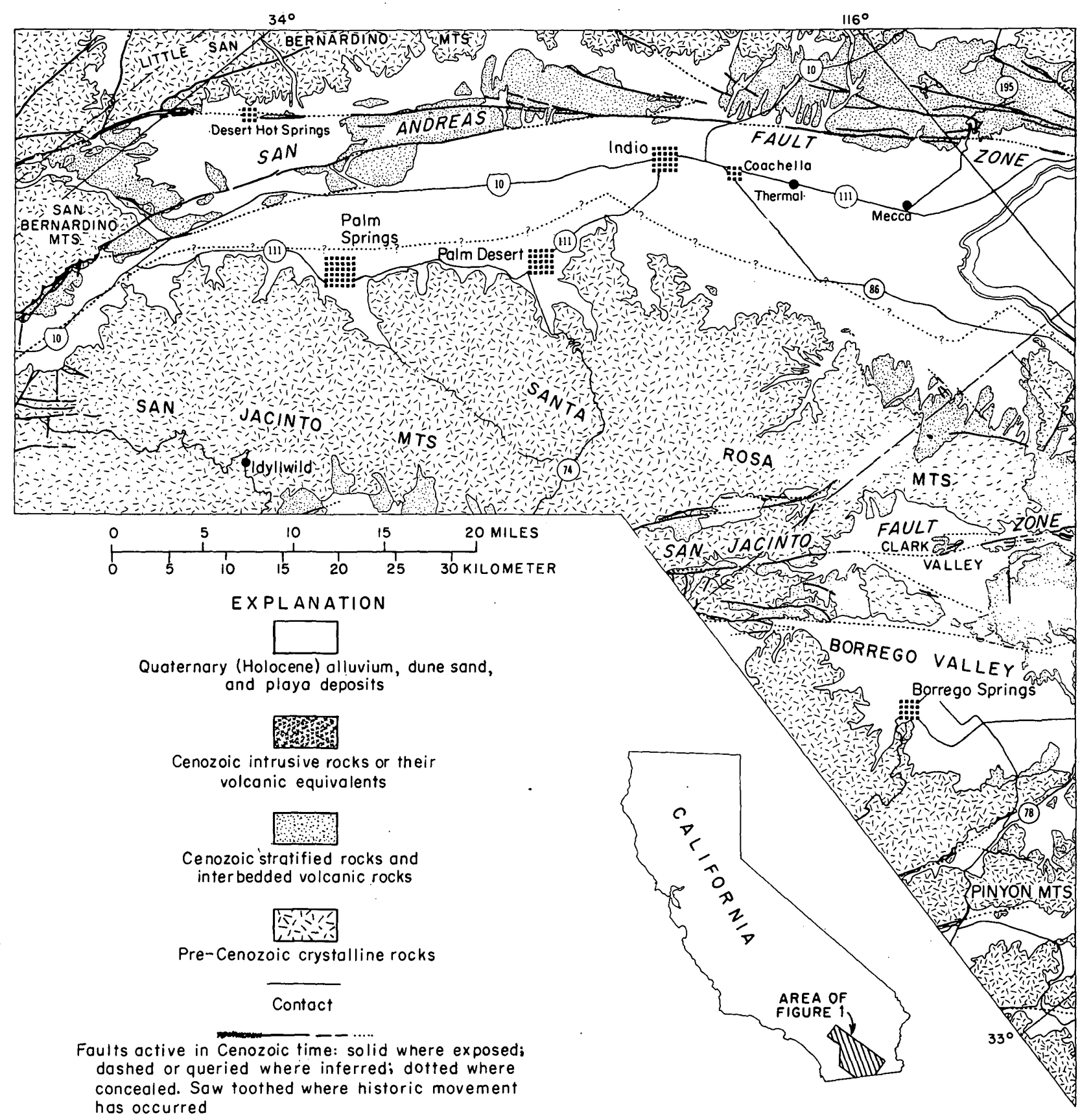

FIGURE 1. - Generalized geologic map of 
well $(4.1 \mathrm{~km})$ does not penetrate all the sedimentary fill (Muffler and White, 1969, p. 170). On the basis of stratigraphic and paleontologic evidence, all that can be said of the segment of the trough in California is that a structural depression was defined there by Pliocene time. However, pre-Pliocene basin fill may underlie the oldest exposed fossil-bearing beds or may be concealed in the axial part of the trough. By contrast, paleontologic evidence from the San Felipe area, on the Gulf of California, establishes the age of the trough at that latitude as Mio- cene or older (Hertlein, 1968, p. 405). Furthermore, the age of the gulf is postulated as about 4 million years (Pliocene) from rates of sea-floor spreading at the junction with the East Pacific Rise near the tip of Baja California, and a protogulf may have formed in the late Miocene (Larson and others, 1968; Moore and Buffington, 1968).

Early Cenozoic sedimentary deposits are unknown within the Salton Trough, although they occur very near both flanks. The early and middle Eocene marine Maniobra Formation of Crowell and Susuki

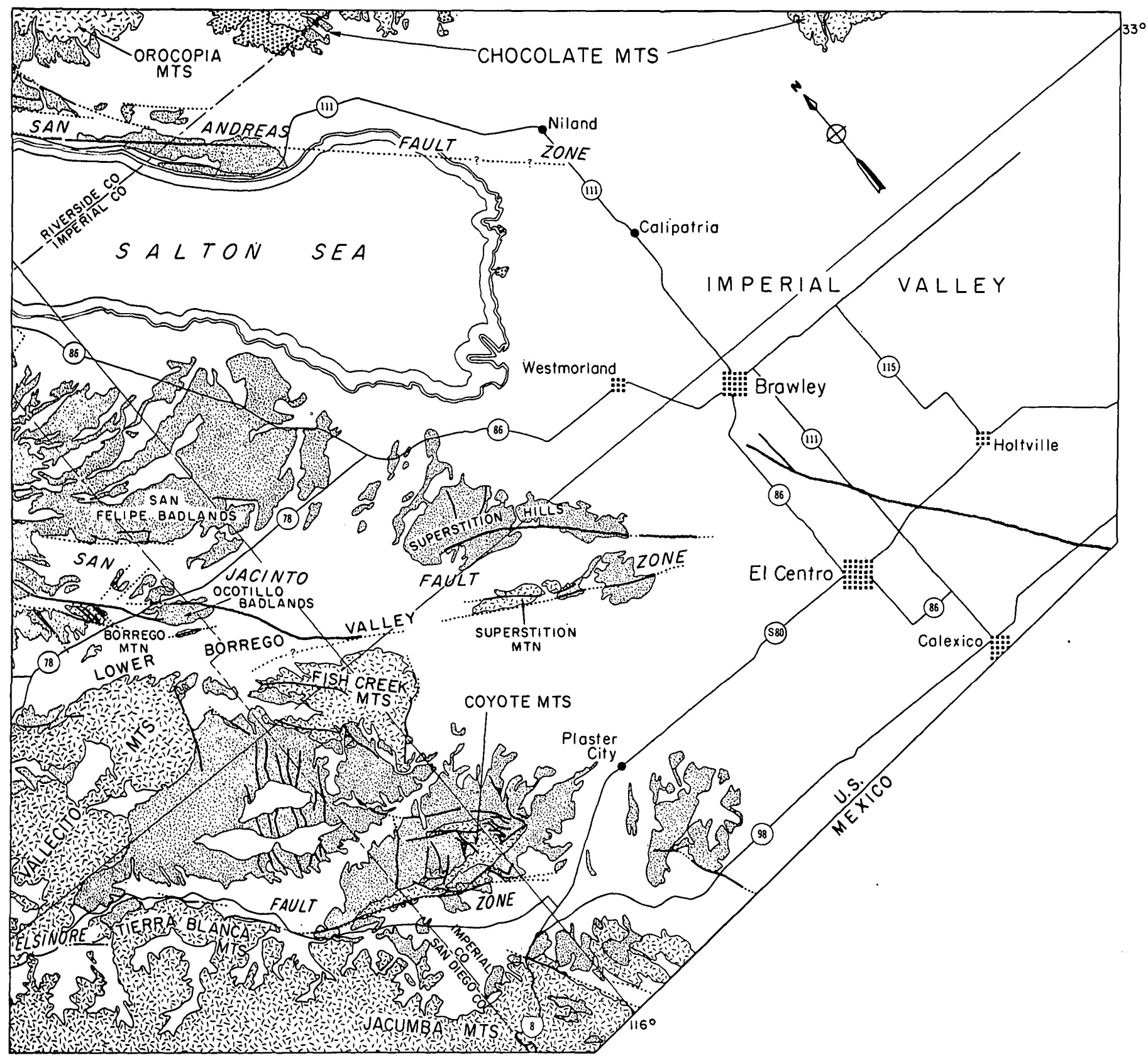

the Salton Trough, southern California.

$476 \cdot 2460-72-2$ 
(1959) and overlying nonmarine beds of probable Oligocene age (Crowell, 1962, p. 28) crop out in the Orocopia Mountains, northeast of the trough, but they lie east of the San Andreas fault and, according to the now widely accepted view, have been transported as much as a few hundred kilometers by right-lateral slip. Coarse nonmarine gravels probably correlative with conglomerate of the Eocene Poway Group (Kennedy and Moore, 1971) lie very near the southwest margin of the trough (Sharp, 1968) and may locally project under the upper Cenozoic deposits within the trough. The Poway Group, however, is widespread across the Peninsular Ranges west of the trough (see, for example, Woodford and others, 1968), and it is unlikely that these gravels represent Eocene basin fill.

Regional Cenozoic stratigraphy of the Salton Trough north of the Mexican border has been briefly summarized by Tarbet and Holman (1944), Tarbet (1951), and Dibblee (1954). Composites of their stratigraphic columns, together with modifications and additions to stratigraphic subdivisions and age designations that have resulted from subsequent work, are shown in figure 2. In Mexico, stratigraphic sequences have been outlined by Beal (1948) and Anderson (1950) in their pioneer studies on Baja California and the Gulf of California. Although stratigraphic descriptions for this southernmost

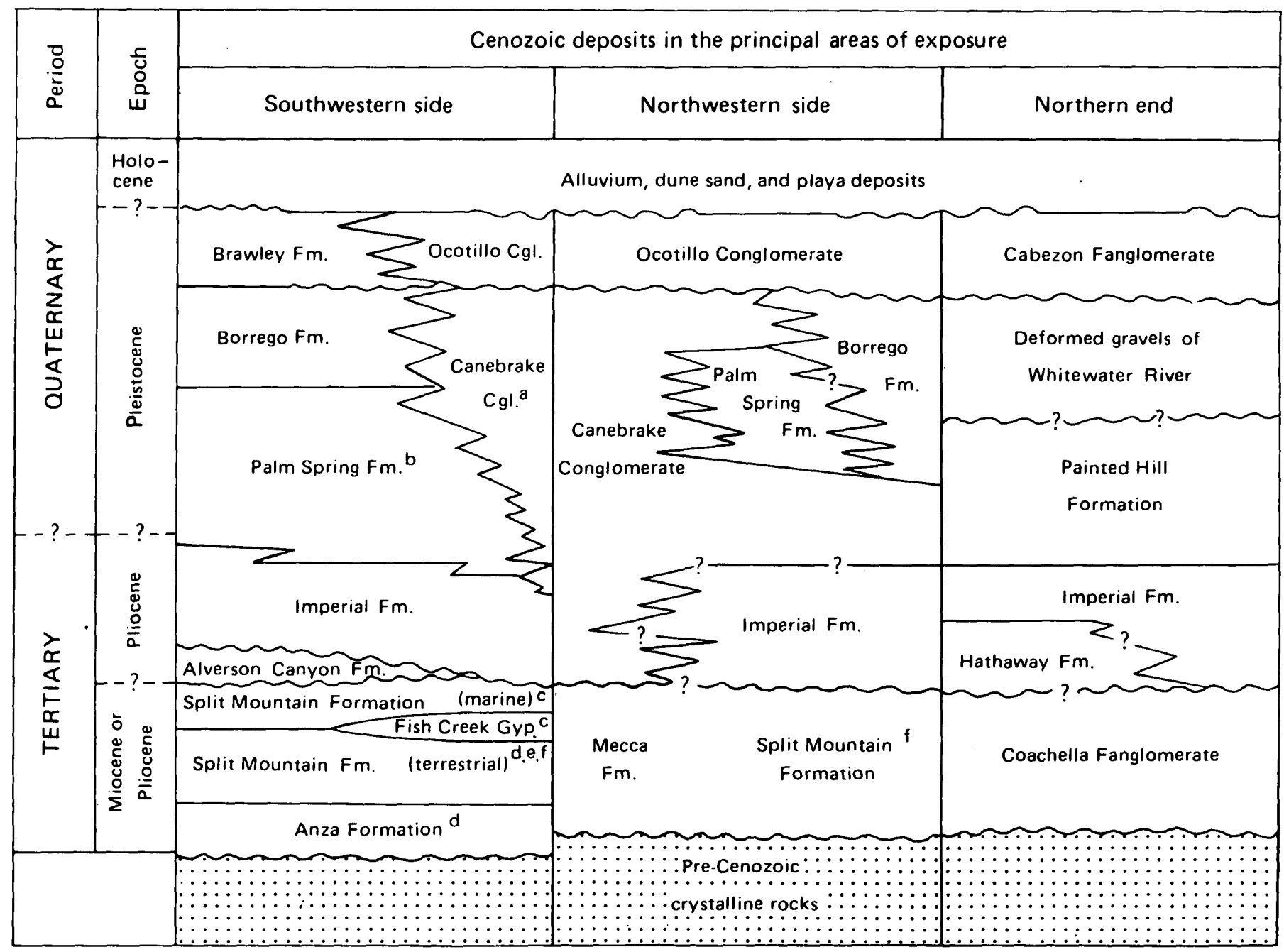

Figure 2. - Composite stratigraphic columns along the flanks of the northern part of the Salton Trough. Wavy lines indicate unconformable contacts, smooth lines indicate conformable contacts, queried lines indicate contacts not exposed. Section on southwestern side after Tarbet and Holman (1944) and Dibblee (1954), with modifications from (a) Downs (1957), (b) Downs and Woodard (1962) and Downs and White (1968), (c) Durham and Allison (1962), (d) Woodard $(1962 ; 1963)$, and (e) Weismeyer 1968). Section on northeastern side after Dibblee (1954), with modifications from (f) Proctor (1968). Section at northern end after Allen (1957). 
sector of the trough are not yet comprehensive, a number of recent studies in various parts of the region have compared or correlated sedimentary units with the Cenozoic sections exposed north of the border. (See Durham and Allison, 1960; Merriam, 1965; Hertlein, 1968; Slyker, 1969.)

Cenozoic sediments in the Salton Trough range from boulder gravel (or its consolidated conglomerate-fanglomerate equivalents) through predominantly sandy flood-plain and deltaic deposits to lacustrine or marine silts and clays. Continental conditions of sedimentation may have prevailed in some marginal parts of the trough throughout its history, although in much of the basin deposition has been alternately marine and nonmarine. Coarse clastic strata, mostly around the periphery of the basin, have been given the names Anza, Split Mountain, and Mecca Formations, Coachella Fanglomerate, Canebrake Conglomerate, Painted Hill Formation, deformed gravels of Whitewater River, Ocotillo Conglomerate, and Cabezon Fanglomerate (fig. 2). All these coarse deposits are continental in origin, and many have yielded vertebrate fossils. Strata consisting chiefly of sand but locally interbedded with gravel or silt constitute the upper, marine part of the Split Mountain Formation, the marine Imperial Formation, the terrestrial Hathaway Formation, and the mostly terrestrial but locally paralic Palm Spring Formation. The Borrego and Brawley Formations consist mostly of silt and clay beds of continental origin; however, the Borrego also contains some sediments deposited in brackish water. Other lithologies represented in the Cenozoic stratigraphy include bedded gypsum deposits (Ver Planck, 1952) termed the Fish Creek Gypsum (Dibblee, 1954) and mafic volcanic rocks that make up the Alverson Canyon Formation and isolated flows in the Coachella Fanglomerate and Painted Hill Formation (Allen, 1957).

The long-standing controversy regarding the age of the abundantly fossiliferous Imperial Formation bears on the problem of antiquity of the Salton Trough because the underlying strata of the trough generally lack fossil material. Although estimates of the age of the Imperial Formation have ranged from Cretaceous to Pliocene according to different investigators, the most recent interpretations of the faunal evidence by Durham and Allison (1960, p. 63) favor a Pliocene age. Moreover, despite the tropical affinities of the Imperial fauna that hinder comparison with the cooler water marine faunas found along the Pacific Coast of California, the local occurrence of one temporally restricted echinoid (Encope tenuis) in both the Imperial and the late Pliocene San Diego Formations suggests similarity in age of the two units (Allison, 1964, p. 19, 21). Only two definite occurrences of fossils from preImperial strata have been reported from the Salton Trough-one not diagnostic as to age (Tarbet and Holman, 1944; Durham and Allison, 1962; Allison, 1964, p. 21) and the other indicating pre-Imperial deposition possibly only back to late Pliocene time (Allen, 1957, p. 330 ; Allen, written commun., 1969). Pre-Imperial sediments of Miocene age, perhaps similar to the diatomite described near the head of the Gulf of California (Hertlein, 1968, p..405), may extend northward into the Salton Trough but have not yet been found on the surface or below.

All known fossiliferous deposits above the Imperial Formation in the Salton Trough are late Pliocene or younger. Although vertebrate fossils have been reported from a number of widely scattered locations on both sides of the northern part of the trough, extensively studied faunas indicating Blancan to Irvingtonian age have been recovered only from the Canebrake Conglomerate and the Palm Spring Formation west of the Fish Creek Mountains (Downs, 1957; Downs and Woodard, 1962; Woodard, 1962; Downs and White, 1965). An impoverished marine fauna also recovered in the Palm Spring Formation indicates a brackishwater environment (Downs and Woodard, 1962) that may have prevailed intermittently up through the deposition of fossil-bearing strata of the Borrego Formation (Durham, 1950, p. 23).

A large number of source areas around the Salton Trough probably were contributing clastic material at any given time. Coarse clastic units along the margins of the basin reflect local provenances both in their distribution and the lithology of the clasts, which can be correlated with different source terranes (Dibblee, 1954; Allen, 1957; Hays, 1957; Proctoor, 1968). Studies of provenance for fine detritus in the Salton Trough have suggested a more complex picture. Merriam and Bandy (1965) and Muffler and Doe (1968) showed that sandy Cenozoic strata in the southern and southeastern parts of the trough resemble material presently being transported down the Colorado River channel more than they do modern sediment derived from ranges peripheral to the trough. Nevertheless, sedimentary structures in some of the same formations elsewhere along the west margin of the Cenozoic basin indicate local provenance from the Peninsular Ranges.

\section{CENOZOIC IGNEOUS ROCKS}

Cenozoic intrusive rocks, or their volcanic equivalents, occur at several localities within the Salton Trough. In the largest area of exposure, in the 
northwestern Chocolate Mountains, hypabyssal intrusive rocks are largely silicic in composition (Morton and Jennings, in Jennings, 1967). Rhyolitic volcanic domes at the southeast corner of the Salton Sea protrude through Holocene sediments on the floor of Imperial Valley (Kelley and Soske, 1936; Robinson and Elders, 1970, 1971; Elders and Robinson, 1970). These domes and the dacitic volcano of Cerro Prieto in Baja California (see frontispiece for location) constitute the only known Holocene volcanism closely associated with the San Andreas fault system north of the Gulf of California. The domes consist of rhyolite, obsidian, and pumice that have been radiometrically dated at about 16,000 years (Muffler and White, 1969, p. 162). A body of Cenozoic extrusive rocks, named the Dos Palmas Rhyolite by Dibblee $(1954$, p. 24$)$, crops out in a very small area near the San Andreas fault east of Mecca. Other Cenozoic rocks, termed the Truckhaven Rhyolite by Dibblee $(1954$, p. 24), that are exposed near the west shore of the Salton Sea have been reinterpreted as silicified sediments by Weismeyer (1968, p. 30-35).

Although extensive basaltic and andesitic flows in the eastern Peninsular Ranges near Jacumba may continue eastward into the Salton Trough, such a relationship has not been established. These rocks are middle Miocene in age, according to a radiometric age by Hawkins (1970, p. 3326). On the basis of stratigraphic position, Dibblee (1954, fig. 1) assigned a Miocene age to mafic volcanic rocks of the Alverson Canyon Formation of Tarbet and Holman (1944) sporadically exposed within the adjoining southwest flank of the trough. Because of upward revision in the age of the Imperial Formation, however, the Alverson Canyon Formation may be younger than the volcanic rocks near Jacumba.

\section{CENOZOIC DEFORMATION IN THE SALTON TROUGH}

The Cenozoic deposits of the Salton Trough have been considerably folded and faulted, and both processes appear to be continuing at the present time. Faulting is dominated by three active right-lateral strike-slip zones, part of the very broad and complex San Andreas fault system in southern California. Major faults within the trough, as well as those farther south under the Gulf of California, define an en echelon pattern in which the separate strands trend more westerly than the axis of the depression. (See Rusnak and others, 1964; Biehler and others, 1964.) Much has been written regarding the amount of cumulative displacement on the various fault zones within the San Andreas system in California, but major disagreements remain. Current ideas relating to the history of movement within the fault system as a whole are discussed by Crowell (1962) and in Dickinson and Grantz (1968).

Folding of the Cenozoic deposits is evident throughout the marginal areas of the Salton Trough. The intensity of deformation by folding is especially pronounced along certain belts bordering on or bounded by the active faults traversing the trough. The pattern of modern sedimentary accumulation and the incipient development of anticlinal hills also reflect continuing diastrophism in the trough, particularly on the southwest side.

Many features of the stratigraphy of the Cenozoic deposits attest to regional crustal instability throughout the history of the basin. Although there was considerable initial relief on the basin floor, progressive diastrophism and erosion have allowed the youngest to the oldest sediments to rest directly on basement. Regional unconformities exist, but localized intraformational unconformities are far more common. Lack of lateral continuity, particularly in the coarser detritus and in lacustrine beds, is evident all along the margins of the trough. By contrast, sediments in the axial parts of the trough are almost uniformly fine grained; sediments penetrated by deep wells in the southern Imperial Valley cannot be directly correlated with the Cenozoic formations exposed along the edges of the trough (Dibblee, 1954 , p. 25 ; Muffler and Doe, 1968, p. 388). Widespread instability in the flanking regions of the trough is evident in the marked erosional truncation of thick sections of sediments that accumulated probably since Pleistocene time, such as those exposed in the southern Borrego Badlands (fig. 3). The detritus has been redeposited elsewhere in the axial part of the basin, perhaps beneath the Salton Sea. Features such as these suggest that the axial part of the trough may well have been continuously subsiding, whereas parts of the flanks have alternately received sediment and undergone erosion.

\section{CENOZOIC FOLDING}

Fault movement has outlined the Salton Trough, but major crustal downwarping not attributable to faulting has also taken place, particularly south of the middle of the Salton Sea where the trough widens markedly. Downwarping in the axial part of the trough is demonstrated by the distribution of various stratigraphic units and confirmed by data from wells and geophysical work. (See Biehler and others, 1964, fig. 7.)

Cenozoic sediments throughout the trough display a multitude of intermediate- and small-scale folds, 


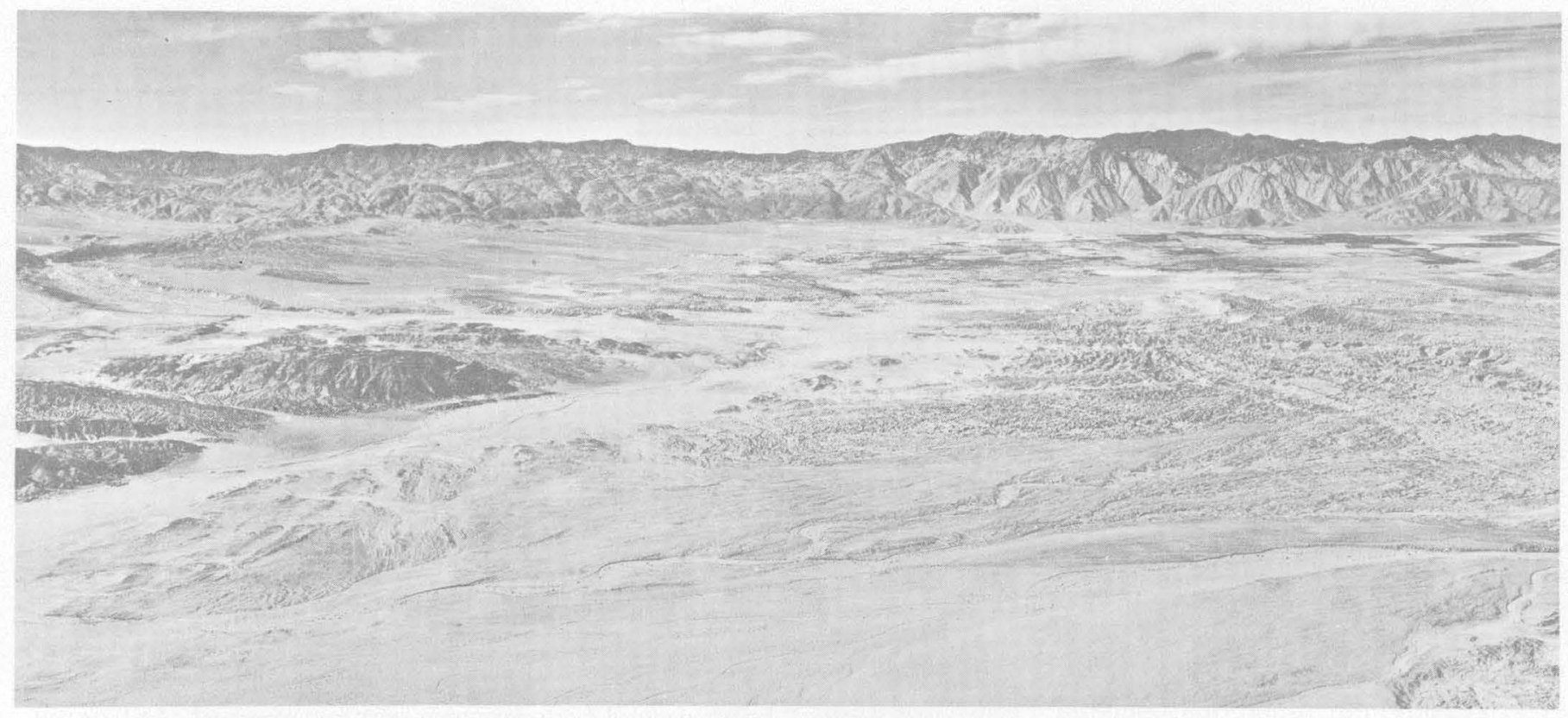

Figure 3. - Oblique aerial view of southern Borrego Badlands (central and right foreground) and West Butte, Borrego Mountain (left foreground). Poorly consolidated late Cenozoic strata in the badlands dip moderately to the north (right) and are beveled by a relatively flat erosional surface. A thickness of about $3.5 \mathrm{~km}$ of sediments probably has been eroded from the beds at the left side of the view since Pleistocene time.

as well as homoclinal dips over large areas. Generally the older strata are more deformed than the younger. Terrace gravels and alluvium, for example. show only local signs of folding. However, some of the most pronounced folding, including overturning and isoclinal folding, is not restricted to older strata but occurs in linear belts adjacent to fault zones, such as in the Mecca Hills east of the town of Mecca and in the Ocotillo Badlands. (See Sharp and Clark, this volume.) Other zones of strongly folded strata resembling diapirs with clay cores appear to degenerate along strike into faults. Examples of the latter occur in the Borrego Badlands.

That the land surface is being folded at present is demonstrated by small incipient hills that reflect anticlinal growth near at least one active fault zone and by closed playa basins scattered throughout the southwest side of the Salton Trough. (See Sharp and Clark, this volume.) Furthermore, warped shorelines of ancient Lake Cahuilla, which covered most of the trough earlier in Holocene time, indicate relatively recent large-scale diastrophic changes (Stanley, 1963, 1966).

\section{CENOZOIC FAULTING}

The principal fault zones in the Salton Trough consist of the San Andreas zone, near the northeast margin, a group of boundary faults that are concealed along the southwest edge of Coachella
Valley, the widely branching San Jacinto fault zone which transects the southwest flank of the trough, and the Elsinore fault zone along the southwest edge of the trough. (See fig. 1.) Except for the breaks on the southwest side of Coachella Valley, these faults all display the surficial features characteristic of the San Andreas system throughout California: linearity, northwest-southeast trend, physiographic evidence of recent activity, and clearcut surficial evidence of right-lateral strike-slip offset. Episodes of historic right-lateral movement on several of the faults, together with the rate of crustal strain accumulation and the seismicity, all indicate that the Salton Trough region is one of the most active areas along the San Andreas system.

Larson, Menard, and Smith (1968) as well as Moore and Buffington (1968) discussed the significance of the San Andreas fault system in terms of the transformation of ocean-floor spreading movements near the tip of Baja California to strike-slip movement within the Gulf of California and the Salton Trough. According to the transform-faulting model, coastal California and Baja California are drifting northwestward relative to the region east of the Salton Trough and the Gulf of California; movement is achieved by a combination of rightlateral slip along northwest-trending faults and horizontal spreading motions on offset segments of the East Pacific Rise situated between the fault 
strands within the trough and gulf. The distribution of recent small earthquakes in the Colorado River delta region (Lomnitz and others, 1970) is consistent with the transform model. Future geologic, seismologic and geodetic investigations may provide evidence that will indicate whether strike-slip faulting occurs in conjunction with spreading in the trough.

\section{SAN ANDREAS FAULT ZONE}

The San Andreas fault zone comprises two principal fractures in northern Coachella Valley. An eastern branch (Mission Creek fault of Allen, 1957, and Proctor, 1968) extends along a nearly straight line to the San Bernardino Mountains, and a western branch (Banning fault) curves to a nearly east-west trend at the north end of the trough. Near Indio, the zone includes a number of subsidiary breaks that lie on the northeast side of the main fault. Southeast of Indio, the San Andreas zone is relatively straight and continuous to the northeast shore of the Salton Sea. Throughout much of Coachella Valley, the fault zone is defined in alluvium by scarps and by groundwater and (or) vegetational effects (Hope, 1969) and in the areas of exposed Cenozoic strata by crushed sediments and gouge and by geologic discontinuities. Using gravity data, Biehler, Kovach, and Allen (1964) estimated that pre-Cenozoic basement rocks have been vertically downdropped at least $3.2 \mathrm{~km}$ on the southwest side of the San Andreas fault in Coachella Valley.

Surficial expression of the San Andreas fault zone dies out southeast. of the Salton Sea. Although sporadic surficial expression of breakage nearly on line with the fault-trace projection from the northwest is evident on the northern part of Imperial Valley (Babcock, 1971, fig. 2), the large displacement proposed for the San Andreas fault (for example, see Crowell, 1962) suggests that it may continue much farther southeastward in Imperial Valley (Biehler and others, 1964, p. 137). Gravity data (Kovach and others, 1962, p. 2867-2869) indicate a major concealed structural break along the northeast side of Imperial Valley that lies well east of a linear projection of the exposed fault trace northwest of the Salton Sea. However, on the basis of electrical resistivity profiles observed near Brawley, Tsvi Meidav (unpub. rept.) suggested that the San Andreas fault bends to a more southerly trend, bringing the break to a position closer to the axis of the trough. Moreover, the possibility of crustal spreading movement beneath the southeastern Salton Sea area and its transformation into horizontal slip on the San Andreas fault to the northwest has been recently suggested (Meidav, 1970; Lomnitz and others, 1970).
Historic movement on the San Andreas fault within the Salton Trough has not been recorded in conjunction with any earthquake prior to the Borrego Mountain event of 1968. Major seismic events at other times in the trough, however, may have produced ground breakage that escaped detection.

\section{CONCEALED FAULTS ON THE SOUTHWEST SIDE OF COACHELLA VALLEY}

A long generally southeast-trending fault zone is suspected to exist along the southwest side of Coachella Valley (queried dotted line in fig. 1). Several lines of evidence point to its existence: (1) The San Jacinto and Santa Rosa ranges appear to have been uplifted relative to the floor of the valley, and the slopes along the east flank of the highlands are steep (see Allen, 1957, p. 347 ; Proctor, 1968, p. 35) ; (2) there is a pronounced gravity gradient along this line (Biehler and others, 1964, p. 138); and (3) structural features in the crystalline rocks and overlying gravel beds in these ranges suggest that downfolding along the east margin of the ranges cannot account for the observed gravity gradient. The lack of scarps or other surficial features along the valley floor and the fact that many deeply incised canyons along the edge of the ranges have nearly flat alluvial floors indicate that the zone has been inactive for a relatively long period. Consequently, the only clues to the position of these faults are the gravity data; the fault zone shown in figure 1 is only a schematic representation of these data.

The breaks indicated by the gravity data appear to end near the southeast terminus of the Santa Rosa Mountains. Moreover, farther south in the basin the basement surface apparently slopes gently rather than abruptly eastward from points due south of the Santa Rosa Mountains to the deepest parts of the trough near its axis, as shown by well data (Oakeshott, 1952, p. 12) and basement depths determined by seismic and gravity methods (Biehler and others, 1964, p. 133).

\section{SAN JACINTO FAULT ZONE}

The San Jacinto fault zone enters the Salton Trough from the northwest at about the latitude of the Salton Sea and cuts diagonally into the basin. (See fig. 1.) Throughout its length in southern California the zone is characterized by southeastward-divergent branching of multiple strands (Dibblee, 1954; Sharp, 1967). Where it enters the trough, the zone is about $10 \mathrm{~km}$ wide and consists of three recognizable strands. The northernmost, the Clark fault, extends through Clark Valley and along the south tip of the Santa Rosa Mountains (Dibblee, 1954 ; Sharp, 1972). The middle break lies along the 
west edge of Clark Valley and extends southward from the Clark fault into the Borrego Badlands, where it apparently dies out. The third strand, the southwest one, is the Coyote Creek fault, along which the principal breakage associated with the 1968 Borrego Mountain earthquake occurred. The Coyote Creek fault follows the northeast edge of Borrego Valley and, with en echelon overlaps and branching, extends along the margins of Borrego Mountain and the Ocotillo Badlands southeastward to a point near the easternmost Fish Creek Mountains. Mapping in the area of the 1968 breakage (see Sharp and Clark, this volume) has demonstrated highly complex patterns of surficial fractures along the Coyote Creek fault.

Other faults lying farther southeast in western Imperial Valley and in the Colorado River delta region in Mexico have also been regarded as part of the San Jacinto fault zone (Beal, 1915; Dibblee, 1954; Biehler and others, 1964; Merriam, 1965; Sharp, 1967). The Superstition Mountain fault and the Superstition. Hills fault fall well within the southeastward projection of the zone encompassing all breaks near Borrego and Clark Valleys, as does the fault extending southeastward from Cerro Prieto in Baja California. (See frontispiece and fig. 4.) Moreover, the Superstition Mountain fault may join the Coyote Creek fault as a branch in the subsurface, although this cannot be proved yet. Alternatively, the principal fracture of the Coyote Creek fault could turn to a more southern trend and connect with recently observed cracks of possible tectonic origin near U.S. Highway 80 about $7 \mathrm{~km}$ east of Plaster City. (See Sharp and Clark, this volume.) The Imperial fault, as defined by the line of breakage during the major 1940 Imperial Valley earthquakes, may also belong to the San Jacinto zone. This fault lies nearly on the projection of the Clark fault, and the two could join at depth (Sharp, 1967, p. 726). However, it is also possible that movement on the Imperial fault is partly transferred northeastward to the San Andreas fault by crustal spreading near the southern Salton Sea (Lomnitz and others, 1970).

Displacement across the San Jacinto fault zone in the Peninsular Ranges northwest of the Salton Trough has been principally right lateral and has amounted to about $24 \mathrm{~km}$ since movement began (Sharp, 1967). About $19 \mathrm{~km}$ of this has been on breaks east of Coyote Mountain, and about $5 \mathrm{~km}$ has been along the Coyote Creek fault.

Vertical components of displacement. on various breaks along the Coyote Creek fault have produced a number of topographically high welts, some of which show separations probably in excess of $1 \mathrm{~km}$. The crystalline rocks and sediments exposed at Borrego Mountain were elevated in such a manner. In addition, sediments exposed in the Ocotillo Badlands have been squeezed up between en echelon breaks along the Coyote Creek fault. (See Sharp and Clark, this volume.)

Except for slippage on the San Andreas fault, all the lines of movement associated with the 1968 Borrego Mountain earthquake coincide with fault strands lying within the broad envelope of the San Jacinto zone. Ground displacement had been detected several times on some of the same fault strands prior to 1968. The first, and by far the largest, was right-lateral offset of as much as 4.3 meters that occurred on the Imperial fault during a series of major earthquakes on May 18, 1940, the largest of which was of magnitude 6.4 (Buwalda and Richter, 1941; Ulrich, 1941; Richter, 1958, p. 488; Trifunac and Brune, 1970). A small amount of right-lateral offset that was detected on the Superstition Hills fault in February 1951 probably originated in association with a magnitude 5.6 earthquake in the Superstition Hills on January 23, 1951 (Allen and others, 1965, p. 768 ). Very slight right-lateral movement recurred on part of the Imperial fault at the time of a magnitude 3.6 shock on March 4, 1966 (Brune and Allen, 1967b) and again after the magnitude 5.3 Superstition Hills earthquake of September 30, 1971 (C. R. Allen, oral commun., 1971); the 1966 event was the smallest earthquake ever known to have been associated with surface displacement.

\section{ELSINORE FAULT ZONE}

The Elsinore fault zone, a distinct yet surficially discontinuous zone of fractures, extends southeastward from the Peninsular Ranges into the Salton Trough near the Tierra Blanca Mountains (fig. 1). In the trough the zone is best defined and most continuous where it bounds the southwest sides of the Coyote Mountains and the Sierra de los Cucapas (frontispiece). As with breaks of the San Jacinto zone that are concealed by alluvium on the floor of Imperial Valley, continuity between many individual strands cannot be demonstrated.

The total strike-slip offset on the Elsinore fault probably is small compared with displacement estimated on the San Jacinto and San Andreas zones (Sharp, 1968; Baird and others, 1970). Historic movement has not been documented, and, indeed, within the Salton Trough only the sector south of the International Boundary has been characterized by appreciable seismicity in recent times. 


\section{SEISMICITY IN THE SALTON TROUGH}

A remarkably high rate of tectonic activity in the Salton Trough is signified by the record of seismic activity (fig. 4) and the accumulation of horizontal crustal shear strain, as well as by geomorphic evidence. In terms of earthquake frequency, both for minor to moderate-sized events and for smaller shocks down to the microearthquake range, the Salton Trough is evidently the most active area lying astride the San Andreas system (Biehler and others, 1964; Allen and others, 1965; Brune and Allen, 1967a). However, very great earthquakes have not occurred in the Salton Trough, at least within the period of the historic record (Allen and others, 1965, p. 783).

\section{MINOR TO MODERATE-SIZED EARTHQUAKES}

Although the regional level of seismic activity in the Salton Trough is at present the highest known in California, there is great diversity in apparent rates of seismicity associated with the different fault zones within its boundaries. The San Jacinto fault zone both within the trough and in its sector farther northwest is apparently the only break within the San Andreas system in southern California that is clearly delineated by post-1934 earthquake epicenters (Allen and others, 1965, p. 772) ; moreover, of the 10 historic earthquakes of magnitude greater than 6 located within the area of figure 4 , eight occurred on or near breaks within the San Jacinto zone. In contrast, the San Andreas fault zone has not in the period of the historical record been associated with a large number of earthquakes, at least along the zone north of the Salton Sea where physiographic expression of recent activity along the break is evident. Southward from the Salton Sea, a diffuse belt of seismicity converges with epicenters associated with the San Jacinto zone. In a similar way, the Elsinore fault zone north of the International Boundary is rather loosely associated with a belt of relatively small earthquakes, although in Mexico and northwest of the trough larger earthquakes have centered within this zone (Richter, 1958, p. 533; Biehler and others, 1964; Allen and others, 1965). A detailed study of seismicity in the Colorado River delta region (Lomnitz and others, 1970) showed that minor earthquakes are markedly concentrated along lines of recognized faulting, a characteristic not revealed by the earlier seismic records.

\section{MICROEARTHQUAKE ACTIVITY}

Brune and Allen (1967a) conducted a regional survey of the San Andreas fault system in southern California to measure microearthquake frequency and distribution for comparison with the levels of activity of larger earthquakes. In the Salton Trough, they found that there is virtually no microearthquake activity on the San Andreas fault zone between Desert Hot Springs and the Mexican border, an observation that agrees with the low level of seismic activity shown by the record of larger earthquakes since 1934 (Brune and Allen, 1967a, p. 291). In terms of microearthquake activity, the San Jacinto fault zone appears to be the most active zone

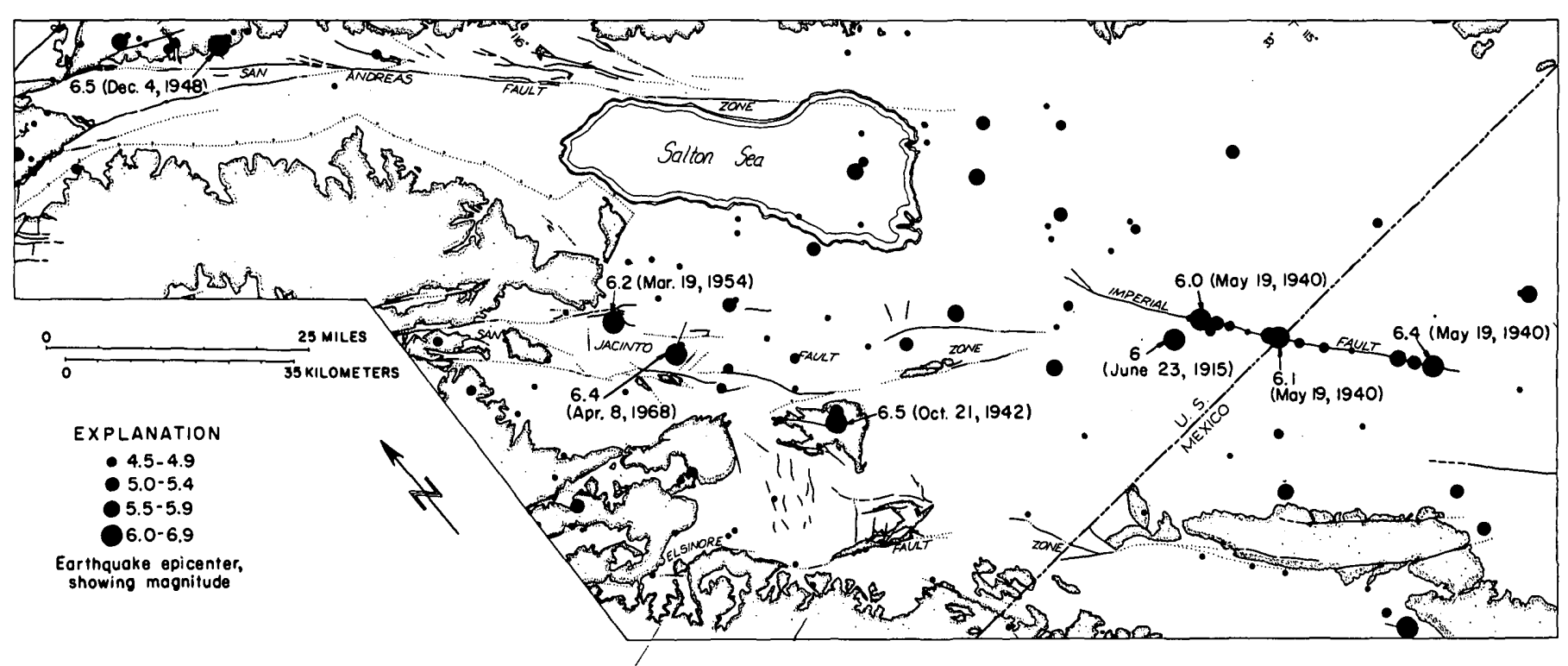

Figure 4. - Seismicity of the northern Salton Trough region. Seismic events less than magnitude 6 for period 1934-61 adapted from California Department of Water Resources (1964), with modifications from Trifunac and Bruce (1970). Seismic events of magnitude greater than 6 for period 1912-68 adapted from Allen, St. Amand, Richter, and Nordquist (1965) and Allen and Nordquist (this volume). 
in southern California, again in strong contrast with the San Andreas zone (Brune and Allen, 1967a, p. 291; Arabasz and others, 1970).

\section{RECENT CRUSTAL STRAIN}

Geodetic data obtained from repeated surveys by the U.S. Coast and Geodetic Survey in the northern part of the Salton Trough over the past third of a century enable an estimate of the rate at which right-lateral crustal strain has been accumulating across the San Andreas system (Whitten, 1956; 1960; Miller and others, 1970). According to recent syntheses of geodetic data (Miller and others, 1970, figs. 8, C8), buildup of right-lateral shear-strain component as measured across the entire Imperial Valley averaged about $2-3 \mathrm{~cm} / \mathrm{yr}$ between 1934 and 1967. Over shorter intervals of time (1934-41, 194154, and 1954-67) in this period, however, strain rates varied remarkably. Crustal strain near the Imperial fault in the central part of Imperial Valley has been consistently right lateral, averaging about $2 \mathrm{~cm} / \mathrm{yr}$ since the large 1940 earthquakes, whereas the data suggest that strain in the left-lateral sense has prevailed in other parts of the Imperial Valley region at least at certain times. For example, an area in eastern Imperial Valley along the southeastward projection of the San Andreas fault apparently shows strain accumulation in the left-lateral sense parallel to the trough axis after 1941 (Miller and others, 1970, fig. 6). Left-lateral strain buildup of about $1 \mathrm{~cm} / \mathrm{yr}$ across the San Andreas fault zone in the northernmost part of the Salton Trough has also been reported by Hofmann (1968, fig. 1) for the periods 1959-65 and 1965-67. The apparent temporal variability of strain accumulation suggests that time bases appreciably longer than a third of a century will be required to obtain a meaningful estimate of the average rate in the Salton Trough region. However, it is interesting to note that the average rate of strain accumulation measured over the longest term is comparable to recently measured rates of crustal strain across the San Andreas fault zone in central California, ranging from 0 to about $4 \mathrm{~cm} / \mathrm{yr}$ at different locations (Hofmann, 1968).

\section{REFERENCES CITED}

Allen, C. R.. 1957, San Andreas fault zone in San Gorgonio Pass, southern California: Geol. Soc. America Bull., v. 68, p. 315-350.

Allen, C. R., St. Amand, Pierre, Richter, C. F., and Nordquist, J. M., 1965, Relationship between seismicity and geologic structure in the southern California region: Seismol. Soc. America Bull., v. 55, p. 753-797.

Allison, E. C., 1964, Geology of areas bordering Gulf of California, in van Andel, T. H., and Shor, G. G., Jr., eds., Marine geology of the Gulf of California: Am. Assoc. Petroleum Geologists Mem. 3, p. 3-29.
Anderson, C. A., 1950, Geology of islands and neighboring land areas, pt. 1 of The 1940 E. W. Scripps cruise to the Gulf of California: Geol. Soc. America Mem. 43, $53 \mathrm{p}$.

Arabasz, W. J., Brune, J. N., and Engen, G. R., 1970, Locations of small earthquakes near the trifurcation of the San Jacinto fault southeast of Anza, California: Seismol. Soc. America Bull., v. 60, p: 617-627.

Babcock, E. A., 1971, Detection of active faulting using oblique infrared aerial photography in the Imperial Valley, California: Geol. Soc. America Bull., v. 82, p. 3189-3196.

Baird, A. K., Welday, E. E., and Baird, K. W., 1970, Chemical variations in batholithic rocks of southern California [abs.] : Geol. Soc. America Abs. with Programs, v. 2, no. 2, p. 69 .

Beal, C. H., 1915, The earthquake in Imperial Valley, California, June 22, 1915: Seismol. Soc. America Bull., v. 5, p. 130-149.

1948, Reconnaissance of the geology and oil possibilities of Baja California, Mexico: Geol. Soc. America Mem. $31,138 \mathrm{p}$.

Biehler, S., Kovach, R. L., and Allen, C. R., 1964, Geophysical framework of northern end of Gulf of California structural province, in van Andel, T. H., and Shor, G. G., Jr., eds., Marine geology of the Gulf of California: Am. Assoc. Petroleum Geologists Mem. 3, p. 126-143.

Brune, J. N., and Allen, C. R., 1967a, A micro-earthquake survey of the San Andreas fault system in southern California: Seismol. Soc. America Bull., v. 57, p. 277-296.

1967b, A low-stress-drop, low-magnitude earthquake with surface faulting: The Imperial, California, earthquake of March 4, 1966: Seismol. Soc. America Bull., v. 57, p. 501-514.

Buwalda, J. P., and Richter, C. F., 1941, Imperial Valley earthquake of May 18, 1940 [abs.]: Geol. Soc. America Bull., v. 52, p. 1944-1945.

California Department of Water Resources, 1964, Crustal strain and fault movement investigation: California Dept. Water Resources Bull. 116-2, 96 p.

Crowell, J. C., 1962, Displacement along the San Andreas fault, California: Geol. Soc. America Spec. Paper 71, 61 p.

Crowell, J. C., and Susuki, T., 1959, Eocene stratigraphy and paleontology, Orocopia Mountains, southeastern California: Geol. Soc. America Bull., v. 70, p. 581-592.

Dibblee, T. W., Jr., 1954, Geology of the Imperial Valley region, in Jahns, R. H., ed., Geology of southern California ; chap. 2, Geology of the natural provinces: California Div. Mines Bull. 170, p. 21-28.

Dickinson, W. R., and Grantz, Arthur, eds., 1968, Proceedings of conference on geologic problems of San Andreas fault system: Stanford Univ. Pub. Sci., v. 11, 377 p.

Downs, Theodore, 1957, Late Cenozoic vertebrates from the Imperial Valley region, California [abs.]: Geol. Soc. America Bull., v. 68, p. 1822.

Downs, Theodore, and White, J. A., 1965, Late Cenozoic vertebrates of the Anza-Borrego Desert area, southern California [abs.]: Geol. Soc. America, Program for AAAS Joint Mtg., Berkeley, p. 110-111.

1968, A vertebrate faunal succession in superposed sediments from late Pliocene to middle Pleistocene in California: Internat. Geol. Cong., 23d, Prague 1968, TertiaryQuaternary boundary, v. 10, p. 41-47. 
Downs, Theodore, and Woodard, G. D., 1962, Middle Pleistocene extension of the Gulf of California into the Imperial Valley [abs.] : Geol. Soc. America Spec. Paper 68, p. 21.

Durham, J. W., 1950, Megascopic paleontology and marine stratigraphy, pt. 2 of The 1940 E. W. Scripps cruise to the Gulf of California: Geol. Soc. America Mem. 43, $216 \mathrm{p}$.

Durham, J. W., and Allison, E. C.; 1960, The geologic history of Baja California and its marine faunas: Systematic Zoology, v. 9, p. 47-91.

1962, Stratigraphic position of the Fish Creek gypsum at Split Mountain Gorge, Imperial County, California [abs.] : Geol. Soc. America Spec. Paper 68, p. 22.

Elders, W. A., and Robinson, P. T., 1970, Possible sea-floor spreading in the Imperial Valley of California-a model for magma generation [abs.] : EOS (Am. Geophys. Union Trans.), v. 51, p. 422.

Hawkins, J. W., 1970, Petrology and possible tectonic significance of Late Cenozoic volcanic rocks, southern California and Baja California: Geol. Soc. America Bull., v. 81, p. 3323-3338.

Hays, W. H., 1957, Geology of the central Mecca Hills, Riverside County, California: Yale Univ. Ph. D. thesis.

Hertlein, L. G., 1968, Three late Cenozoic molluscan faunules from Baja California, with a note on diatomite from west of San Felipe: California Acad. Sci. Proc., v. 30, p. 401405.

Hofmann, R. B., 1968, Recent changes in California fault movement, in Dickinson, W. R., and Grantz, Arthur, eds., Proceedings of conference on geologic problems of San Andreas fault system: Stanford Univ. Pub. Geol. Sci., v. 11 , p. $89-93$.

Hope, R. A., 1969, Map showing recently active breaks along the San Andreas and related faults between Cajon Pass and Salton Sea, California: U.S. Geol. Survey open-file map, scale $1: 24,000$.

Jennings, C. W., 1967, Geologic map of California, Olaf P. Jenkins edition, Salton Sea sheet: California Div. Mines and Geology.

Kelley, V. C., and Soske, J. L., 1936, Origin of the Salton volcanic domes, Salton Sea, California: Jour. Geology, v. 44, p. $496-509$.

Kennedy, M. P., and Moore, G. W., 1971, Stratigraphic relations of upper Cretaceous and Eocene formations, San Diego coastal area, California: Am. Assoc: Petroleum Geologists Bull., v. 55, p. 709-722.

Kovach, R. L., Allen, C. R., and Press, Frank, 1962, Geophysical investigations in the Colorado Delta region: Jour. Geophys. Research, v. 67, p. 2845-2871.

Larson, R. L., Menard, H. W., and Smith, S. M., 1968, Gulf of California: a result of ocean-floor spreading and transform faulting: Science, v. 161, p. 781-784.

Lomnitz, Cinna, Mooser, Federico, Allen, C. R., Brune, J. N., and Thatcher, Wayne, 1970, Seismicity and tectonics of the northern Gulf of California region, Mexico, Preliminary results. Geofisica Internacional, v. 10, no. 2, p. 37-48.

Meidav, Tsvi, 1970, Possible sea-floor spreading in the Imperial Valley; I, Structural setting [abs.] : EOS (Am. Geophys. Union Trans.), v. 51, no. 4, p. 421.

Mendenhall, W. C., 1909, Groundwater of the Indio region, Calif.: U.S. Geol. Survey Water-Supply Paper 225, 56 p.

Merriam, Richard, 1965, San Jacinto fault in northwestern Sonora, Mexico: Geol. Soc. America Bull., v. 76, p. 10511054.
Merriam, Richard, and Bandy, O. L., 1965, Source of upper Cenozoic sediments in Colorado delta region: Jour. Sed. Petrology, v. 35, p. 911-916.

Miller, R. W., Pope, A. J., Stettner, H. S., and David, J. L., 1970, Crustal movement investigations - triangulation, Imperial Valley, vicinity of El Centro: U.S. Coast and Geodetic Survey Data Rept. 10, 117 p.

Moore, D. G., and Buffington, E. C., 1968, Transform faulting and growth of the Gulf of California since the late Pliocene: Science, v. 161, p. 1238-1241.

Muffler, L. J. P., and Doe, B. R., 1968, Composition and mean age of detritus of the Colorado River delta in the Salton trough, southeastern California: Jour. Sed. Petrology, v. 38, p. 384-399.

Muffler, L. J. P., and White, D. E., 1969, Active metamorphism of upper Cenozoic sediments in the Salton Sea geothermal field and the Salton trough, southeastern California: Geol. Soc. America Bull., v. 80, p. 157-182.

Oakeshott, G. B., 1952, Exploratory wells drilled outside oil and gas fields in California to December 31, 1950 : California Div. Mines Spec. Rep. 23, 77 p.

Proctor, R. J., 1968, Geology of the Desert Hot Springs-upper Coachella Valley area, California: California Div. Mines and Geology Spec. Rep. 24, 50 p.

Richter, C. F., 1958, Elementary seismology: San Francisco, W. H. Freeman, 768 p.

Robinson, P. T., and Elders, W. A., 1970, Possible sea-floor spreading in the Imperial Valley of California: Xenoliths in rhyolite volcanoes as samples of the basement [abs.]: EOS (Am. Geophys. Union Trans.), v. 51, p. 422.

1971, Late Cenozoic volcanism in the Imperial Valley, California [abs.] : Geol. Soc. America Abstracts with Programs, v. 3, no. 2, p. 185.

Rusnak, G. A., Fisher, R. L., and Shepard, F. P., 1964, Bathymetry and faults of Gulf of California, in van Andel, T. H., and Shor, G. G., Jr., eds., Marine geology of Gulf of California: Am. Assoc. Petroleum Geologists Mem. 3, p. 59-75.

Sharp, R. V., 1967, San Jacinto fault zone in the Peninsular Ranges of southern California: Geol. Soc. America Bull., v. 78, p. $705-730$.

1968, The San Andreas fault system and contrasting pre-San Andreas structures in the Peninsular Ranges of southern California [abs.], in Dickinson, W. R., and Grantz, Arthur, eds., Proceedings of conference on geologic problems of San Andreas fault system: Stanford Univ. Pub. Geol. Sci., v. 11, p. 292-293.

1972, Map showing recently active breaks along the San Jacinto fault zone between the San Bernardino area and Borrego Valley, California: U.S. Geol. Survey Misc. Geol. Inv. Map I-675, scale $1: 24,000$.

Slyker, R. G., 1969, Geologic and geophysical reconnaissance of the Valle de San Felipe region, Baja California, Mexico [abs.] : Geol. Soc. America Spec. Paper 121, p. 560.

Stanley, G. M., 1963, Prehistoric lakes in Salton Sea basin [abs.]: Geol. Soc. America Spec. Paper 73, p. 249-250.

1966, Deformation of Pleistocene Lake Cahuilla shoreline, Salton Sea basin, California [abs.]: Geol. Soc. America Spec. Paper 87, p. 165.

Tarbet, L. A., 1951, Imperial Valley, in Possible future petroleum provinces of North America: Am. Assoc. Petroleum Geologists Bull., v. 35, p. 260-263. 
Tarbet, L. A., and Holman, W. H., 1944, Stratigraphy and micropaleontology of the west side of Imperial Valley, California [abs.]: Am. Assoc. Petroleum Geologists Bull., v. 28, p. 1781-1782.

Trifunac, M. D., and Brune, J. N., 1970, Complexity of energy release during the Imperial Valley, California, earthquake of 1940: Seismol. Soc. America Bull., v. 60, p. 137-160.

Ulrich, F. P., 1941, The Imperial Valley earthquake of 1940 : Seismol. Soc. America Bull., v. 31, p. 13-31.

Ver Planck, W. E., 1952, Gypsum in California: California Div. Mines Bull. 163, 151 p.

Weismeyer, A. L., Jr., 1968, Geology of the northern portions of the Seventeen Palms and Fonts Point quadrangles, Imperial and San Diego Counties, California: Univ. Southern California M.A. thesis.
Whitten, C. A., 1956, Crustal movements in California and Nevada: Am. Geophys. Union Trans., v. 37, p. 393-398. 1960 , Horizontal movement in the earth's crust: Jour. Geophys: Research; v. 65, p. 2839-2844.

Woodard, G. D., 1962, Stratigraphic succession of the west Colorado Desert, San Diego and Imperial Counties, Southern California [abs.] : Geol. Soc. America Spec. Paper 68, p. $63-64$.

1963, The Cenozoic succession of the west Colorado Desert, San Diego and Imperial Counties, southern California: California Univ., Berkeley, Ph. D. thesis.

Woodford, A. O., Welday, E. E., and Merriam, Richard, 1968, Siliceous tuff clasts in the upper Paleocene of southern California: Geol. Soc. America Bull., v. 79, p. 1461-1486. 


\title{
FORESHOCK, MAIN SHOCK, AND LARGER AFTERSHOCKS OF THE BORREGO MOUNTAIN EARTHQUAKE ${ }^{1}$
}

\author{
By Clarence R. Allen and John M. Nordquist \\ Seismological laboratory, California Institute of Teghnology
}

\begin{abstract}
The Borrego Mountain earthquake, magnitude 6.4, occurred at $02: 28: 59.1$ G.m.t. on April 9, 1968 and has been assigned a hypocenter at $33^{\circ} 11.4^{\prime}$ N., $116^{\circ} 07.7^{\prime}$ W., $h=11.1 \mathrm{~km}$. The focal-mechanism solution indicates right-lateral slip on a fault striking N. $48^{\circ} \mathrm{W}$. and dipping $83^{\circ} \mathrm{NE}$., which is consistent with the field observations of faulting and the regional tectonic framework. A single foreshock of magnitude 3.7 preceded the main shock by one minute, but no other precursory activity has been identified. During the year following the event, 135 aftershocks of magnitude 3.0 and greater have been identified and located, outlining a broad zone of activity centered on but displaced 2-3 km northeast of the 33-km-long surface rupture on the Coyote Creek fault. Fracturing at depth during the aftershock period evidently occurred throughout the width of the San Jacinto fault zone, but initial surface faulting was localized along the Coyote Creek fault at the zone's southwestern margin. The area of aftershock activity enlarged progressively with time, and the region of the original epicenter became relatively inactive late in the aftershock period, leading to a doughnut-shaped epicentral distribution of late aftershocks. Inasmuch as the epicenter of the main shock was roughly midway along the zone of aftershock activity, the faulting presumably was bilateral. This kind of faulting is unusual in California.
\end{abstract}

\section{INTRODUCTION}

The earthquake of magnitude 6.4 that occurred near Borrego Mountain, Calif., was the largest earthquake to occur in the conterminous United States since 1959. It ranks among the larger shocks recorded in the southern California region since the establishment of modern seismographic stations (fig. 5). The earthquake was preceded by a minor foreshock, was followed by a series of aftershocks that lasted for several months, and was accompanied by right-lateral surface faulting along a $33-\mathrm{km}$ segment of the Coyote Creek fault (Clark, "Surface Rupture Along the Coyote Creek Fault," this volume). Probably the most surprising and significant phenomenon associated with the earthquake was the triggering of small

'Contribution No. 1953, California Institute of Technology, Division of Geological and Planetary Sciences, Pasadena, Calif. displacements on a number of distant faults far outside the aftershock area (Allen and others, this volume).

The Borrego Mountain earthquakes occurred in a fortunate location with respect to the distribution of seismographic stations that were in operation at the time of the main shock. Seven stations of the Caltech

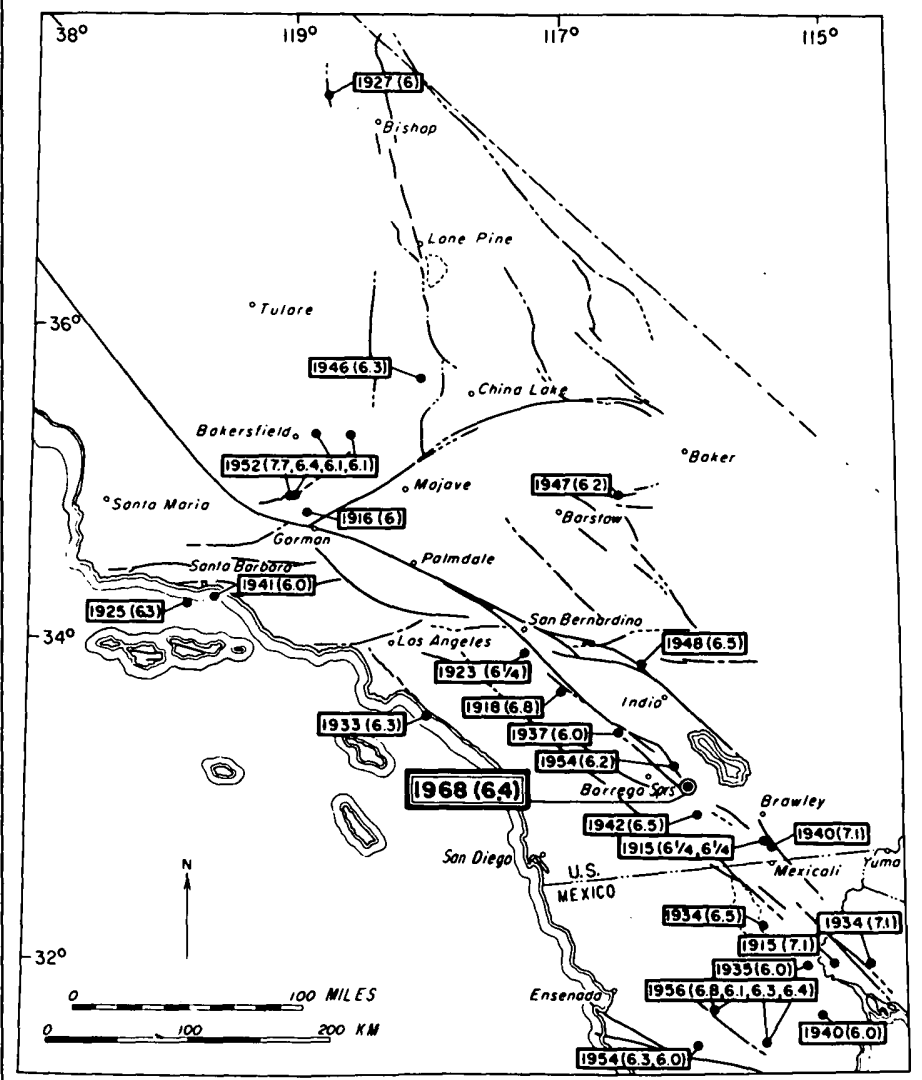

FiguRE 5. - Epicenter of the 1968 Borrego Mountain earthquake in relation to major faults, and epicenters of other earthquakes of magnitude 6.0 and greater in southern California since 1912. (Adapted from Allen and others, 1965.) 
network surrounded the epicenter within $150 \mathrm{~km}$, including a temporary trailer-mounted station $47 \mathrm{~km}$ distant at Obsidian Butte (OBB, fig. 6). Thus we

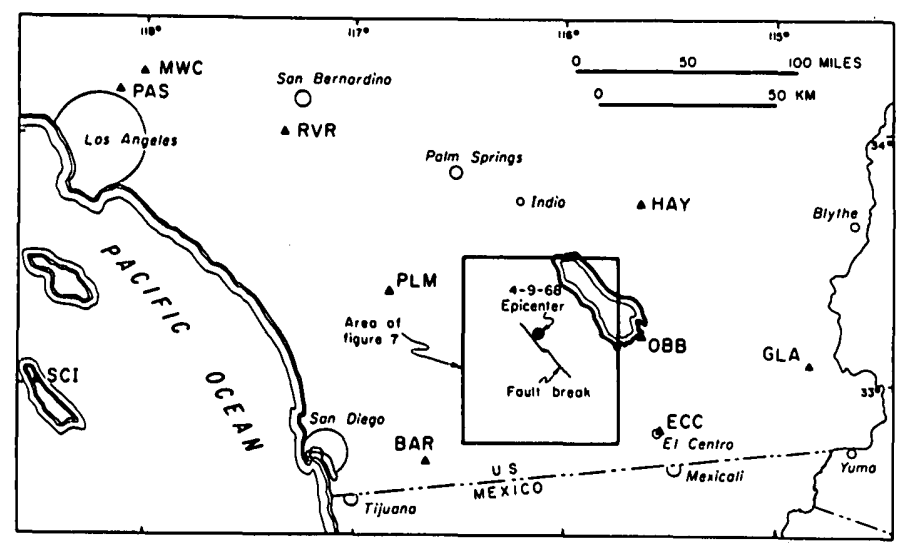

Figure 6. - Location of Caltech seismographic stations (solid triangles) that were operating at the time of the Borrego Mountain earthquake. The area of the detailed epicenter map (fig. 7) is also shown.

have some confidence in the epicentral locations of most of the larger shocks of the series, although depth control was minimal until the time that the first portable units were installed in the epicentral area.

Although the main shock occurred in the evening (6:29 p.m., April 8, 1968, local time), rapid computer location of the epicenter using the Caltech telemetered array allowed immediate planning of a field-recording program. Several field crews were dispatched to the epicentral area before 9:00 p.m., and the first backpack-mounted seismograph was installed within $5 \mathrm{~km}$ of the epicenter shortly after midnight. By 3:00 a.m., the first film-recording trailer-mounted unit was in operation at Ocotillo Wells (OCT, fig. 7), and two more trailer units were installed the next day (ELW and FCR, fig. 7) (table $1)$. These trailer units operated up to and following

TABLE 1. - Caltech trailer-mounted stations in operation during the Borrego Mountain aftershock period

\begin{tabular}{lcccc}
\hline & Station & Lat (N.) Long (W.) & Period of operation (G.m.t) \\
\hline Code & Name & Lat & & \\
\hline ELW & Ella Wash.............. $33^{\circ} 17.20^{\prime}$ & $116^{\circ} 08.95^{\prime}$ & $4-10-68(03: 05)$ & to $5-13-68$ \\
FCR & Fish Creek Range.. $33^{\circ} 01.82^{\prime}$ & $116^{\circ} 03.50^{\prime}$ & $4-9-68(19: 23)$ & to $6-12-68$ \\
OBB & Obsidian Butte........ $33^{\circ} 10.15^{\prime}$ & $115^{\circ} 38.17^{\prime}$ & $1-8-68$ & to $9-15-68$ \\
& & & $12-17-68$ & to $12-20-68$ \\
& & $3-21-69$ & to $6-14-69$ \\
OCT & Ocotillo Wells.......... $33^{\circ} 09.60^{\circ}$ & $116^{\circ} 09.04^{\prime}$ & $4-9-68(10: 12)$ & to $6-11-68$
\end{tabular}

the time that the much more extensive U.S. Geological Survey array began yielding high-precision hypocentral information on April 12 (Hamilton, this volume). This paper describes the events that took place prior to the installation of the U.S. Geological Survey array and summarizes the larger aftershocks

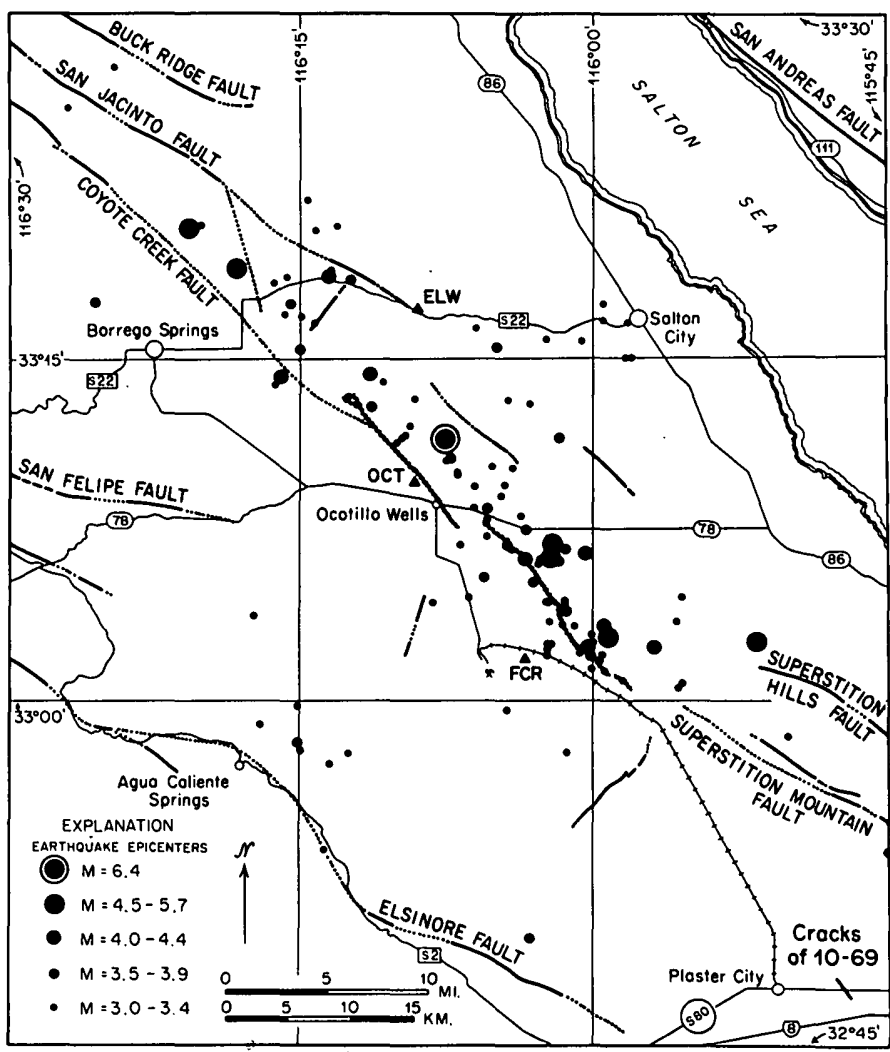

Figure 7. - Epicenters of shocks of magnitude 3.0 and greater that occurred between April 9, 1968 and April 28, 1969. All shocks listed in table 2 are included, except for those with " $D$ "-quality locations. Of the 126 epicenters shown, 69 were located by Caltech and 57 by the U.S. Geological Survey (Hamilton, this volume). Heavy solid lines are accurately located faults; dotted lines are concealed faults; the zigzag line is the approximate trace of the 1968 surface break along Coyote Creek fault (Clark, "Surface Rupture Along the Coyote Creek Fault," this volume).

that occurred during the entire aftershock period, including those aftershocks that followed the removal of the U.S. Geological Survey equipment in June.

\section{ACKNOWLEDGMENTS}

This study was supported by National Science Foundation Grants GA-1087 and GA-12868 to the California Institute of Technology. The authors appreciate the help of J. N. Brune, G. F. Davies, C. F. Richter, and W. T. Thatcher, and the critical comments of R. M. Hamilton and R. V. Sharp.

\section{SEISMIC HISTORY}

The San Jacinto fault zone, of which the Coyote Creek fault is a member (Sharp, this volume), has been the locus of repeated moderate seismic activity within the entire historical record (Allen and others, 1965). Assuming that the zone extends southeast to the Gulf of California, at least 10 shocks of magni- 
tude 6-7 have occurred along this line since 1912, and the fault zone is better delineated by seismic activity in this magnitude range than is any other individual fault in California. Many of the epicenters have been about equidistant along the fault (fig. 5), and the 1968 epicenter lies roughly midway between the epicenters of the 1942 Lower Borrego Valley earthquake $(M=6.5)$ and the 1954 Santa Rosa Mountains earthquake $(M=6.2)$.

\section{HYPOCENTRAL DETERMINATIONS}

Table 2 is a listing of all shocks of magnitude $\left(\mathrm{M}_{\mathrm{L}}\right) 3.0$ and greater that occurred from the time of the foreshock at 0227 G.m.t. on April 9, 1968 to the time of the Coyote Mountain earthquake on April 28, 1969, about 1 year later. The list was terminated with the Coyote Mountain earthquake

TABLE 2. - Earthquakes of magnitude 3.0 and greater in the area from $32^{\circ} 45^{\prime}$ to $33^{\circ} 30^{\prime} \mathrm{N}$. and from $115^{\circ}$ to $116^{\circ} 30^{\prime} \mathrm{W}$. from April 9, 1968 through April 28, 1969

[" $Q$ " indicates quality, as discussed in text. Depths indicated by " $R$ " were restricted to that depth in the computer solution. Source "T" is California
Institute of Technology ; source " $G$ " is U.S. Geological Survey. Quadrangle Institute of Technology; source "G" is U.S. Geological Survey. Quadrangle
names are those of USGS 15-minute quadrangles. All magnitudes are names are those of USGS 15-minute quadrangles. All magnitudes are
assigned by Pasadena. A-, B-, and C-quality epicenters are shown in the maps of figures 7 and 9$]$

\begin{tabular}{|c|c|c|c|c|c|c|}
\hline Time & $\widehat{z}$ & $\hat{\xi}$ & $\Rightarrow$ & $\widehat{\Phi}$ & & \\
\hline Yr Mo Da H M S & 嶌 & 荇. & 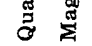 & $\stackrel{8}{\Omega}$ & $\stackrel{\Xi}{\mathscr{n}}$ & \\
\hline
\end{tabular}

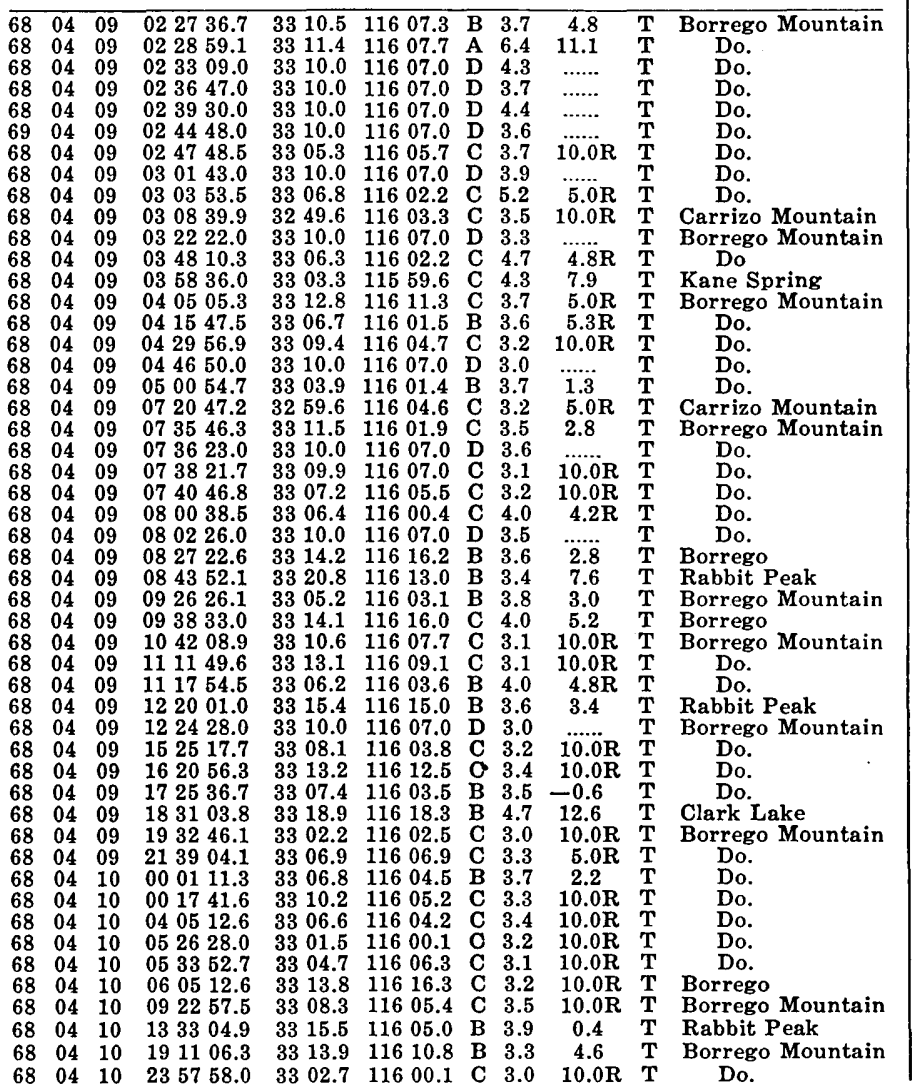

TABLE 2. - Earthquakes of magnitude 3.0 and greater in the area from $32^{\circ} 45^{\prime}$ to $33^{\circ} 30^{\prime} \mathrm{N}$. and from $115^{\circ}$ to $116^{\circ} 30^{\prime} \mathrm{W}$. from April 9, 1968 through April 28, 1969-Continued

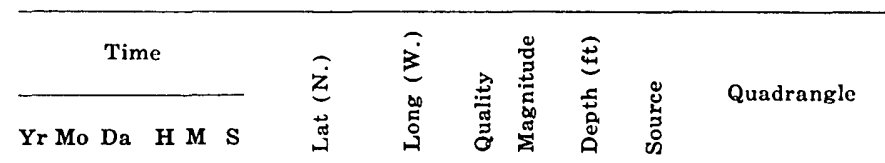

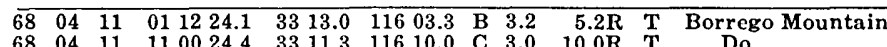

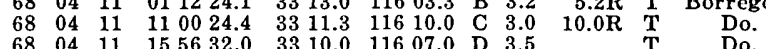

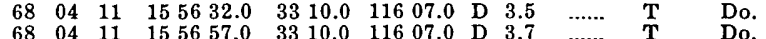

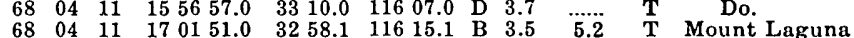

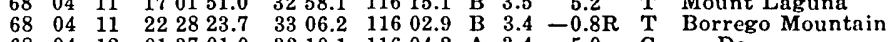

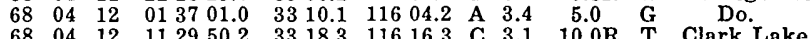

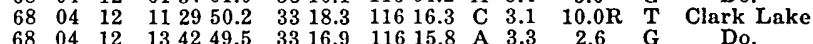

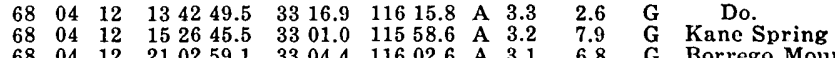

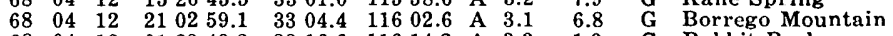

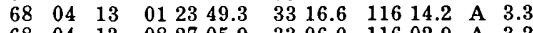

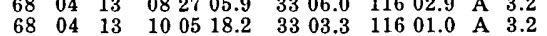

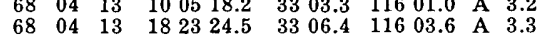

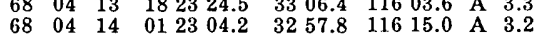

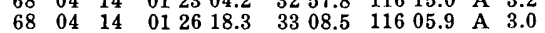

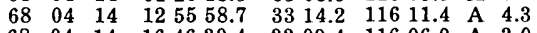

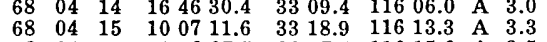

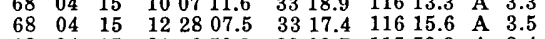

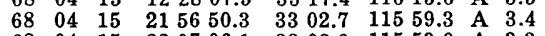

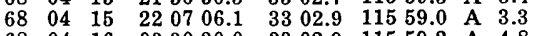

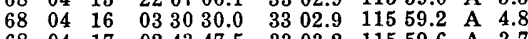

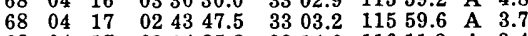

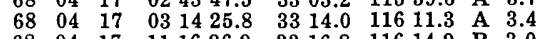
$\begin{array}{llllllllllll}68 & 04 & 17 & 11 & 16 & 26.9 & 33 & 16.8 & 116 & 14.9 & \text { B } & 3.0\end{array}$

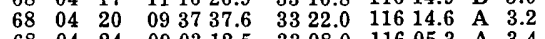
$\begin{array}{llllllllllll}68 & 04 & 24 & 09 & 03 & 12.5 & 33 & 08.0 & 116 & 05.3 & \text { A } & 3.4\end{array}$ $\begin{array}{llllllllll}68 & 04 & 24 & 22 & 09 & 54.2 & 33 & 03.4 & 116 & 01.7 \\ 68 & 04 & \text { A } & 3.0\end{array}$

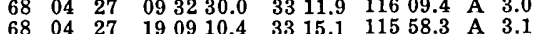

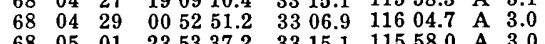

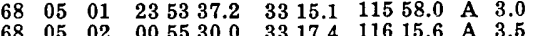

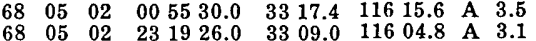

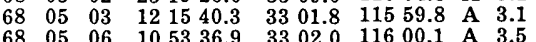

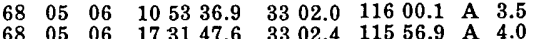

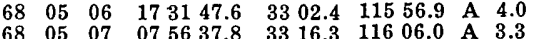

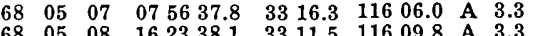

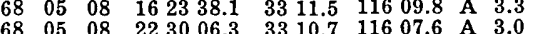
$\begin{array}{llllllllllll}68 & 05 & 08 & 22 & 30 & 06.3 & 33 & 10.7 & 116 & 07.6 & \text { A } & 3.0 \\ 68 & 05 & 09 & 10 & 21 & 46.2 & 33 & 06.1 & 116 & 01.9 & \text { A } & 3.6\end{array}$

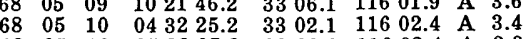
$\begin{array}{lllllllllllll}68 & 05 & 10 & 05 & 28 & 07.8 & 33 & 02.0 & 116 & 02.4 & \text { A } & 3.3 \\ 68 & 05 & 11 & 08 & 10 & 04.0 & 33 & 02.4 & 116 & 00.3 & \text { A } & 4.2\end{array}$

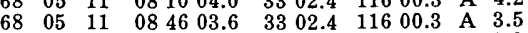

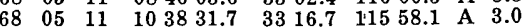
$\begin{array}{lllllllllll}68 & 05 & 12 & 1424 & 16.3 & 33 & 27.7 & 116 & 24.4 & \text { A } & 3.3\end{array}$

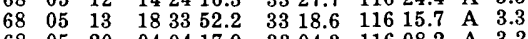

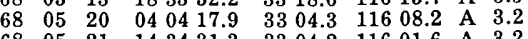

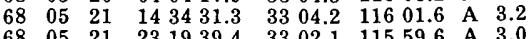

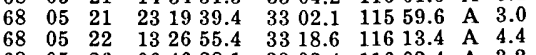

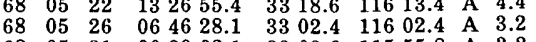

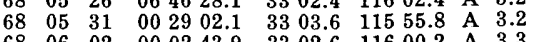

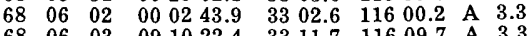

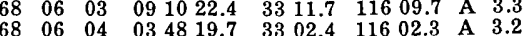

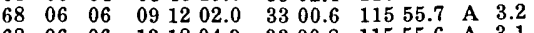

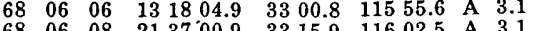

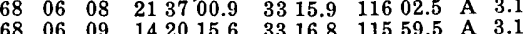

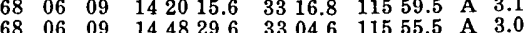

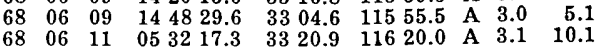

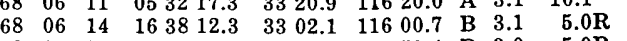

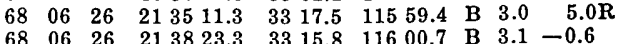

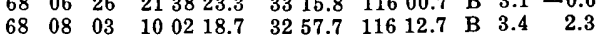
$\begin{array}{lllllllllllll}68 & 08 & 12 & 07 & 07 & 07.7 & 32 & 57.8 & 116 & 01.4 & \text { B } & 3.0 & 4.5\end{array}$

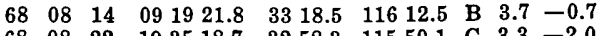

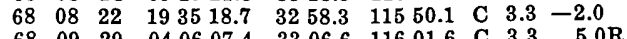

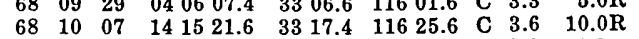

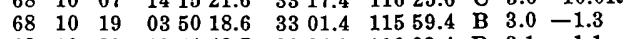

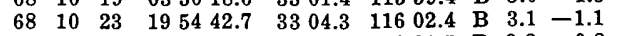

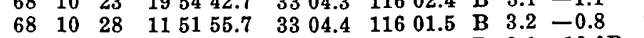

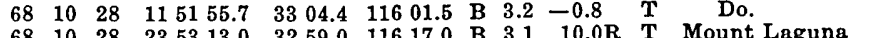

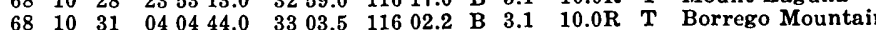

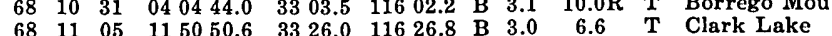

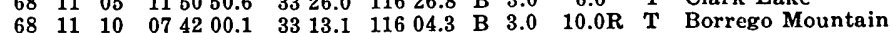

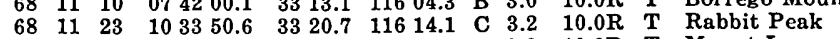

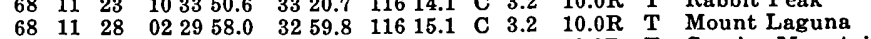

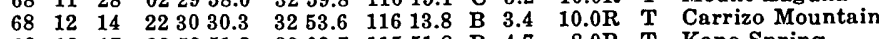

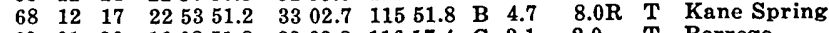

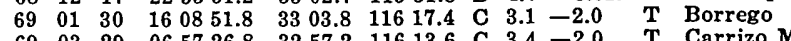

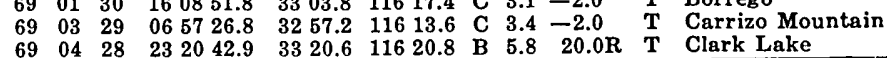


$(M=5.8)$ because it was larger than any earlier aftershock; it occurred somewhat farther northwest than most earlier aftershocks (fig. 7), and it had numerous local aftershocks of its own. Table 2 is thought to represent a relatively homogeneous coverage of earthquakes of magnitude 3.0 and greater during the 1-year period, except for a period of about 2 hours following the main shock, when some small aftershocks could have been missed because of the congested records. For the purpose of this study, aftershocks were arbitrarily assumed to include all shocks that occurred within the area of figure 7. This area includes the following U.S. Geological Survey 15-minute quadrangles (from left to right, top to bottom, in fig. 7) : Clark Lake, Rabbit Peak, Durmid, Borrego, Borrego Mountain, Kane Spring, Mt. Laguna, Carrizo Mountain, and Plaster City.

The establishment of precise time correction factors for the permanent stations of the Caltech network was made possible by the presence of the 20 -station USGS (U.S. Geological Survey) array in the epicentral area (Hamilton, this volume) and by the calibration of this array with three widely separated explosions (Hamilton, 1970). Fifty-five of the largest USGS-located shocks that occurred during the 2-month period between April 12, 1968 and June 12,1968 were used to establish station time corrections to be applied to the Caltech computer-location program (Nordquist, 1962). Average time corrections for P-arrivals of the aftershocks at the nearby stations are given in table 3 ; only those stations with time corrections shown were used in the routine

TABLE 3. - Borrego Mountain earthquake data for stations of the Pasadena network

\begin{tabular}{|c|c|c|c|c|c|c|c|}
\hline \multicolumn{2}{|r|}{ Station } & \multirow{2}{*}{\multicolumn{2}{|c|}{$\begin{array}{l}\text { P-wave } \\
\text { arrival time, } \\
\text { main shock } \\
\text { (G.m.t.) } \\
\end{array}$}} & \multirow{2}{*}{$\begin{array}{c}\text { Time } \\
\text { correc- } \\
\text { tion, } \\
\text { main } \\
\text { shock } \\
\text { (s) }\end{array}$} & \multirow{2}{*}{$\begin{array}{l}\text { Time } \\
\text { correc- } \\
\text { tion, } \\
\text { after- } \\
\text { shocks } \\
\text { (s) }\end{array}$} & \multirow{2}{*}{$\begin{array}{l}\text { Distance } \\
\text { to hypo- } \\
\text { center, } \\
\text { main } \\
\text { shock } \\
(\mathrm{km})\end{array}$} & \multirow{2}{*}{$\begin{array}{c}\text { First } \\
\text { motion, } \\
\text { main } \\
\text { shock } \\
\text { (quality) }\end{array}$} \\
\hline Code & Name & & & & & & \\
\hline & Barrett.. & $02: 29:$ & $\overline{11.3}$ & +0.3 & +0.1 & $\frac{1}{76.8}$ & \\
\hline $\begin{array}{l}\text { CLC } \\
\text { CWC }\end{array}$ & China Lake & & 44.1 & $\ldots$ & $\ldots$ & 320.5 & .... \\
\hline ECC & 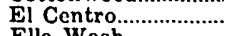 & & 10.7 & .0 & $\cdots$ & 70.2 & $\dddot{\mathrm{c}}$ \\
\hline ELW & $\begin{array}{l}\text { Ella Wash } \\
\text { (temporary) }\end{array}$ & & & & & 15.8 & \\
\hline FCR & Fish Creek Range & & & & & & \\
\hline FTC & Fort & & & $\cdots$ & -.2 & $\begin{array}{r}21.9 \\
315.9\end{array}$ & \\
\hline & $\ldots \ldots \ldots \ldots$ & & 17.6 & +1.2 & +.7 & 122.9 & $\mathbf{c}$ \\
\hline $\begin{array}{l}\text { GSC } \\
\text { HAY }\end{array}$ & $\begin{array}{l}\text { Goldstone.. } \\
\text { Hayfield.... }\end{array}$ & & $\begin{array}{l}04.4 \\
11.7\end{array}$ & \pm .9 & \pm .2 & $24:$ & $\begin{array}{l}\mathbf{D} \\
\mathbf{D}\end{array}$ \\
\hline ISA & 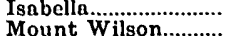 & & $\begin{array}{l}47.6 \\
30.8\end{array}$ & -1 & -1.1 & 212 & D \\
\hline & Obsic & & & & & & \\
\hline OCT & Ocotil & & 07.5 & -.6 & $\begin{array}{r}-.6 \\
-.3\end{array}$ & 47.3 & $\cdots$ \\
\hline $\begin{array}{l}\text { PLM } \\
\text { PAS } \\
\text { RVR }\end{array}$ & 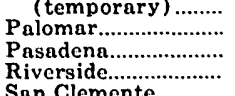 & & $\begin{array}{l}11.1 \\
30.0 \\
22.0\end{array}$ & $\begin{array}{r}-.2 \\
+. .9\end{array}$ & $\begin{array}{l}=.3 \\
=.1 \\
\mp 1.1 \\
+.5\end{array}$ & $\begin{array}{r}11.9 \\
41.8 \\
216.8 \\
146.4\end{array}$ & $\begin{array}{l}\text { C } \\
\text { C } \\
\text { C } \\
\text { D }\end{array}$ \\
\hline & nente & & & -.6 & +.2 & 226.7 & \\
\hline & bara.............. & & & .... & $\ldots$ & & \\
\hline SYI 1 & $\begin{array}{l}\text { Sawn } \\
\text { Sant }\end{array}$ & & & $\ldots .$. & $\cdots .$. & & $\mathbf{C}$ \\
\hline & & & & ..... & …. & & 5 \\
\hline & & & & & & & \\
\hline
\end{tabular}

locations of aftershocks. These corrections were used to determine the Caltech hypocenters listed in table 2 , except for the main shock, which is discussed separately below. Even with these corrections, not all shocks could be assigned good hypocenters, mainly because of poor arrivals masked by contemporaneous events or wind noise or because of sporadic station failures. " $B$ " quality epicenters are thought to be accurate within $5 \mathrm{~km}$, "C" quality within $15 \mathrm{~km}$, and " $D$ " quality greater than $15 \mathrm{~km}$, although the quality assignments in table 2 are generally conservative. "A" quality locations, such as those of the main shock and the U.S. Geological Survey epicenters, are the subject of special investigation and individual error assignments. A measure of the accuracy of hypocentral determinations from the Caltech stations alone (" $T$ " source in table 2), when the above corrections are applied, can be determined by comparing the depths assigned to all Caltech " $\mathrm{B}$ " hypocenters that were independently located by both networks. The average difference in depth assignments is $2.0 \mathrm{~km}$; this amount of difference suggests that most of the " $B$ " locations in table 2 are indeed accurate to within $5 \mathrm{~km}$ and many are much more accurate.

\section{FORESHOCK ACTIVITY}

A single foreshock of magnitude 3.7 preceded the main shock by about 1 minute, and its hypocenter can be considered identical with that of the main shock within the limits of location error. Perhaps the most significant feature of the foreshock activity, however, is the complete absence of any other foreshocks or preceding regional activity. Records of the 47-km-distant station at Obsidian Butte, for example, show no hint of any other earthquakes in the epicentral region within the previous few hours and days, and the general level of activity at this station had been unusually low during the preceding 4 months. Within the year preceding the main Borrego Mountain shock, only two earthquakes that might be considered within the zone of subsequent aftershock activity along the Coyote Creek fault are listed in the Caltech Local Bulletin.

\section{MAIN SHOCK LOCATION}

The main shock was located by using correction factors that were based on arrival-time residuals of only the 11 largest shocks that were recorded by both the USGS and Caltech networks - all of these shocks exceeded magnitude 3.4. The restriction to larger shocks permits a more direct comparison with the main shock, particularly for the more distant stations. These time corrections, as well as the 
$\mathrm{P}$-wave arrival times used in the solution, are given in table 3 ; only those stations with time corrections shown in table 3 were used in this solution. The computed hypocenter is $33^{\circ} 11.4^{\prime}$ N., $116^{\circ} 07.7^{\prime}$ W., $h=11.1 \mathrm{~km}$. Standard errors in the computer solution of the $\mathrm{x}, \mathrm{y}$, and $\mathrm{z}$ components are $0.65,0.96$, and 2.01 $\mathrm{km}$, respectively, but these are felt to be unrealistic in view of the somewhat arbitrary assignment of correction factors. On the basis of numerous attempts to locate the epicenter using a wide variety of assumptions and models, we believe that the computed epicenter is correct to within $3 \mathrm{~km}$, but because the nearest station (OBB) is $47 \mathrm{~km}$ away, depth control is poor. Nevertheless, the comparatively large hypocentral depth of the main shock relative to that of most aftershocks is consistent with the observations of Hamilton (this volume), who notes that all A- and B-quality USGS hypocenters of shocks exceeding magnitude 3.4 are at depths below $6 \mathrm{~km}$; their average depth is $8.06 \mathrm{~km}$, compared to an average depth of 4.97 for the hypocenters of all A- and B-quality aftershocks listed in table 8.

\section{MAGNITUDE}

A local magnitude $\left(M_{L}\right)$ of 6.4 has been assigned to the Borrego Mountain main shock by C. F. Richter, who used records of seven widely spaced stations of the Pasadena network. Most of the Wood-Anderson torsion seismometers of the network were driven off-scale by the main shock; to determine the magnitude it was necessary to compare the main shock with a well-recorded aftershock by using low-magnification seismometers (for example, $100 \times$ ). The magnitude 5.2 aftershock of 0304, April 9, was recorded on 12 torsion instruments and on 17 lowmagnification instruments that remained on-scale during the main shock; comparing the amplitudes on the various records is consistent with a magnitude assignment of 6.4 for the main shock. Berkeley reported a magnitude of 7.1-7.2 for the same shock (Niazi and others, 1969), and the U.S. Coast and Geodetic Survey $\mathrm{PDE}^{2}$ card reported a magnitude of 6.1 from the magnitude-determination system in use at that time.

\section{FOCAL MECHANISM}

The focal mechanism for the Borrego Mountain main shock is shown in figure 8 . The focal mechanism was solved by following the technique of Sykes (1967) ; a focal depth of $10 \mathrm{~km}$ and a crustal velocity of $6.0 \mathrm{~km}$ per sec were assumed. The two nodal planes are both relatively well defined and must be near vertical. It is reasonable to assume that the fault is represented by the nodal plane that strikes

${ }^{2}$ Preliminary determination of epicenter.

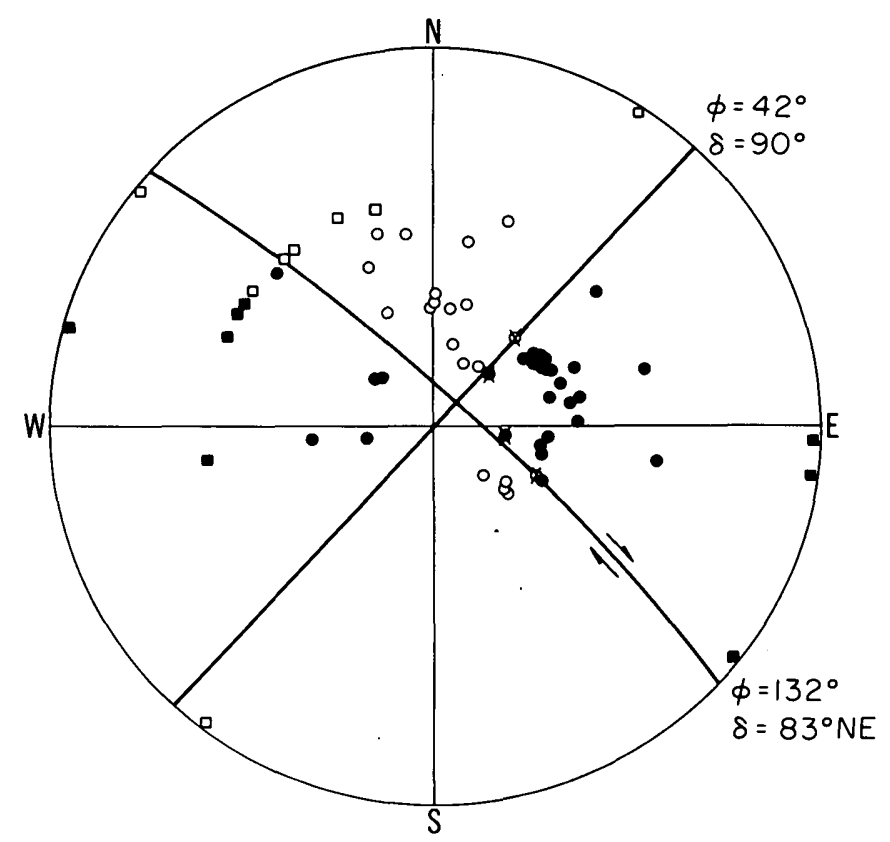

FiguRe 8. - Focal-mechanism solution for the Borrego Mountain main shock. Diagram is an equal-area projection of the lower hemisphere of the radiation field. Solid symbols represent compressions; open symbols, dilatations; square symbols, stations of the Pasadena network; circular symbols, distant stations of the Worldwide Standard Seismograph Network (WWSSN) and Canadian network; crosses, wave character indicating stations near nodal plane. $\theta$ and $\delta$ are strike and dip of nodal planes, and arrows indicate sense of shear on the plane chosen as the fault plane.

northwest and dips steeply to the northeast, at least $80^{\circ}$, and that has a right-lateral displacement of almost purely strike-slip character. The only inconsistencies in the solution are from nearby stations of the Pasadena network (table 3 ; square symbols in fig. 8), where there is a discrepancy between the first motions at Fort Tejon and Riverside compared with those at Berkeley. The more distant long-period arrival at Berkeley, which was clearly compressional, is given precedence in the solution. Although 15 of the 17 nearby stations are clearly consistent with the more distant stations, minor inconsistencies in the nearby stations are not surprising, particularly in view of the very complicated crustal structure in the epicentral region. Stations of the Pasadena network that are presumed to have received direct $P$ arrivals are arbitrarily plotted at the edge of the net in figure 8.

The fault-plane strike of $132^{\circ}$ (N. $48^{\circ}$ W.) corresponds within a few degrees to the trend of major aftershock activity (fig. 7) and to the trend of surface faulting (Clark, "Surface Rupture Along the Coyote Creek Fault," this volume). The steep dip, however, seems to preclude the possibility that the 
apparent offset of the line of major aftershocks 2-3 $\mathrm{km}$ northeast of the trace of surface fracturing can be caused by dip of the fault surface. Such a hypothesis would require a much shallower dip of the fault plane (for example, $70^{\circ}$ ) than is permitted by the focal-mechanism solution; furthermore, this hypothesis is not suggested by most of Hamilton's vertical cross sections (fig. 16).

Late in the aftershock period, a number of events occurred near the southeastern extremity of faulting that may be related to the creep history of surface displacement. During the aftershock period, little or no creep occurred on the northwestern segment of the surface trace (north of Highway 78), but considerable creep took place along the southeastern segment (Clark, "Surface Rupture Along the Coyote Creek Fault," this volume). When one compares the relative aftershock activity in the two areas, it seems reasonable that at least some of the creep in the southeastern segment may have been associated with the much more numerous aftershocks in this area, such as the increased activity that took place here shortly after April 14 (fig. 9C).

\section{EPICENTRAL DISTRIBUTION OF AFTERSHOCKS AS A FUNCTION OF TIME}

Inasmuch as it was not possible to assign accurate epicenters to a number of larger aftershocks that occurred within a few hours of the main event (table 2), arguments as to possible changes of aftershock activity with time are necessarily somewhat limited. Within 2 hours of the main shock, it is likely that aftershocks had occurred essentially over the entire length of the surface rupture as it was mapped in the days following the event. (Note, however, that most of the initial epicenters listed in table 3 are of C-quality.) Nevertheless, there is a definite suggestion of gradual enlargement of the zone of aftershock activity is the days and weeks following the earthquake. Figure 9 shows the distribution of epicenters during four successive time periods having roughly equal numbers of shocks of magnitude 3.0 and above, and the progressive expansion of areal activity is obvious, particularly late in the aftershock period (fig. 9D). For example, most of the numerous events near the southeast end of the fault trace, near $33^{\circ} 02^{\prime} \mathrm{N} .116^{\circ} 00^{\prime} \mathrm{W}$., occurred after April 14 (fig. 9C). Likewise, the two outlying areas of minor activity situated symmetrically with respect to the fault trace near Salton City and Agua Caliente Springs (fig. 7) became active primarily after April 27 (fig. 9D). Two of the largest aftershocks occurred near the extreme ends of the aftershock zone many months after the main event; these are the Coyote Mountain earthquake $(M=5.8)$ of April 28, 1969, which may not be an aftershock in the usual sense, and the shock of December 17, $1968(\mathrm{M}=4.7)$, which lies almost on the trace of the Superstition Hills fault (fig. 7, 9D). It is also significant that during the late period, when aftershocks were occurring over a wide area, almost no shocks exceeding magnitude 3.0 occurred in the area of original surface faulting except at its southeast end. Thus, the lateperiod aftershock distribution takes on almost a doughnut shape.

\section{TECTONIC IMPLICATIONS}

The hypocentral distribution of the Borrego Mountain earthquakes leads to several conclusions of tectonic significance: (1) Aftershocks are not limited to a single fault plane; instead they are distributed over a wide zone - in sharp contrast to the aftershock distribution of the Parkfield-Cholame earthquakes (Eaton, 1967); (2) the zone of most concentrated aftershock activity parallels but is somewhat northeast of the line of surface rupture along the Coyote Creek fault; (3) the epicenters of all shocks above magnitude 4.5 , including that of the main shock, seem to lie along a single line $2-3 \mathrm{~km}$ northeast of the Coyote Creek fault trace, with the exception of the shock of December 17, 1968 that is near the Superstition Hills fault; (4) the zone of rupturing at depth evidently extends farther northwest than does the zone of surface faulting; and (5) the epicenter of the main shock lies roughly midway along the zone of aftershock activity and thus seems to reflect bilateral faulting; the fracture propagates both northwest and southeast from the point of initial rupture.

As previously discussed, it seems unlikely that the dip of the fault plane is so shallow that the surface break represents the same fault plane as that indicated by the hypocenters of the larger aftershocks. Nor, in the light of the careful explosion calibration carried out by Hamilton (1970; this volume), is it likely that there is a major systematic error in the epicentral locations of the aftershocks.

The seeming lack of direct correlation between the trace of surface faulting and the aftershock distribution may be related to the complex local geology. It is obvious from the geologic map (fig. 7) that the San Jacinto fault zone in this region (as opposed to the San Jacinto fault itself) is not a simple fracture - not nearly so simple, for example, as the San Andreas fault in the Parkfield-Cholame area. The zone comprises many individual Quaternary breaks: northwest of the 1968 epicenters are the Buck Ridge, San Jacinto, and Coyote Creek faults, and to the southeast are the Superstition Hills and Superstition Mountain faults, as well as a possible extension of 


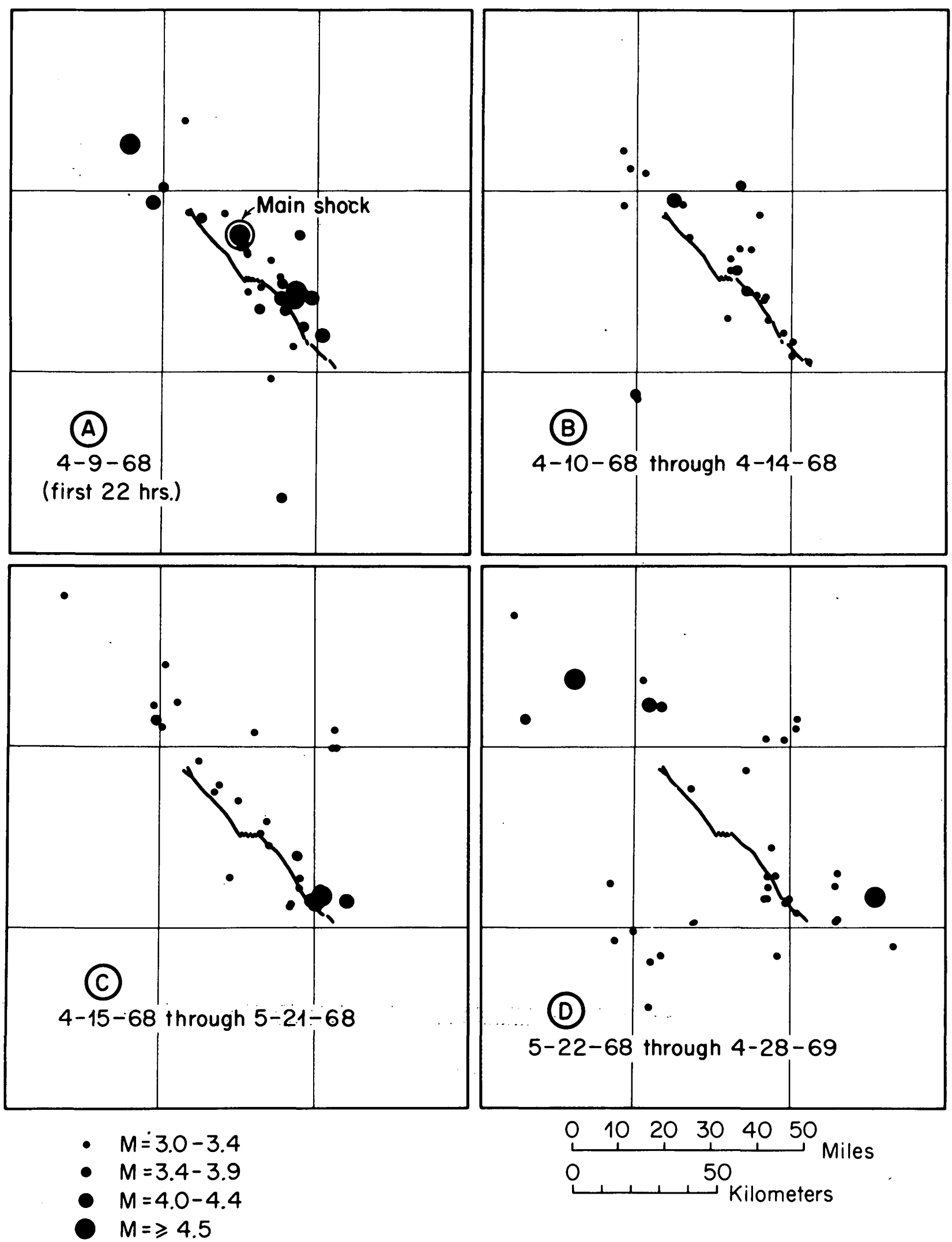

FIgURE 9. - Epicenters of figure 7, separated into four consecutive time periods (A to D) containing roughly equal numbers of shocks. Boundaries and coordinates of individual maps are the same as those of figure 7 . 
the Coyote Creek fault, which is represented by northwest-trending cracks across Highway $80,4 \mathrm{~km}$ east of Plaster City, that were first observed in October of 1969 (fig. 7). Therefore it is not surprising that a major earthquake within the zone is a complex event, with aftershocks distributed over a number of individual breaks. Indeed, the aftershock zone of the 1968 event alines rather well with the center of the entire band of Quaternary faulting along the San Jacinto fault zone, whereas the surface faulting lies at the southwest edge of it. However, the question of why the surface faulting is apparently localized only along the margin of the fractured zone remains unanswered.

It is interesting that although the Borrego Mountain and Parkfield-Cholame earthquakes are of somewhat comparable magnitude and displacement, there are significant mechanical differences between them. In addition to the markedly different patterns of aftershock distribution, Max Wyss (oral commun., 1970) has pointed out that the apparent stress at all depths is higher at Borrego Mountain than at Parkfield-Cholame; average values for 12 events in the two localities differ by two orders of magnitude. Despite the fact that both earthquakes are lowstress-drop events (Wyss and Brune, 1968; Wyss and Hanks, this volume), the absolute stress at Borrego Mountain was considerably higher, which may be related to the markedly different patterns of aftershock activity.

The geological conditions at Parkfield-Cholame, typified by a relatively simple fault break that was perhaps "lubricated" by the presence of abundant serpentine in the fault zone (Allen, 1968), might logically be associated with earthquakes at relatively low levels of absolute stress. In the Borrego Valley area, on the other hand, the complex and discontinuous fault pattern might be visualized as necessarily demanding a relatively high absolute stress for breaking to be initiated, so that the faulting then occurred over a wider zone of pervasive fracturing.

The epicenter of the Borrego Mountain main shock lies roughly midway along the zone of aftershock activity (fig. 7) and thus seems to reflect bilateral faulting - with the fracture propagating both northwest and southeast from the point of initial rupture. This relation is in marked contrast to most other recent earthquakes in California, in which the initial epicenter has been at one end of the aftershock zone, thus reflecting unilateral faulting. Among these earlier earthquakes that have been well documented are the 1933 Long Beach earthquake (Richter, 1958), the 1940 Imperial Valley earthquake (Trifunac and Brune, 1970), the 1948 Desert Hot Springs earthquake (Richter and others, 1958), the 1952 Kern County earthquake (Richter, 1955), and the 1966 Parkfield-Cholame earthquake (McEvilly, 1966; Eaton, 1967). Indeed, the 1906 San Francisco earthquake is perhaps the only other large earthquake in California for which there is good evidence of bilateral faulting (Bolt, 1968).

\section{REFERENCES CITED}

Allen, C. R., 1968, The tectonic environments of seismically active and inactive areas along the San Andreas fault system, in Proceedings of conference on geologic problems of San Andreas fault system, Stanford, Calif., 1967: Stanford Univ. Pub. Geol. Sci., v. 11, p. 70-80.

Allen, C. R., St. Amand, Pierre, Richter, C. F., and Nordquist, J. M., 1965, Relationship between seismicity and geologic structure in the southern California region: Seismol. Soc. America Bull., v. 55, p. 753-797.

Bolt, B. A., 1968, The focus of the 1906 California earthquake: Seismol. Soc. America Bull., v. 58, p. 457-471.

Eaton, J. P., 1967, Instrumental seismic studies, in Brown, R. D., and others, The Parkfield-Cholame, California, earthquakes of June-August 1966: U.S. Geol. Survey Prof. Paper 579, p. 57-66.

Hamilton, R. M., 1970, Time-term analysis of explosion data from the vicinity of the Borrego Mountain, California, earthquake of 9 April 1968: Seismol. Soc. America Bull., v. 60 , p. $367-381$.

McEvilly, T. V., 1966, Preliminary seismic data, June-July 1966, in Parkfield earthquakes of June 27-29, 1966, Monterey and San Luis Obispo Counties, California-preliminary report: Seismol. Soc. America Bull., v. 56, p. 967-971.

Niazi, M., Somcrville, M., and Dengler, L., 1969, Earthquakes and the registration of earthquakes from January 1, 1968, to June 30, 1968: California Univ., Berkeley, Seismog. Sta. Bull., v. 38 , no. 1, p. 1-161.

Nordquist, J. M., 1962, A special-purpose program for earthquake location with an electronic computer: Seismol. Soc. America Bull., v. 52, p. 431-437.

Richter, C. F., 1955, Foreshocks and aftershocks, in Earthquakes in Kern County, California, during 1952: California Div. Mines Bull. 171, p. 177-197.

1958, Elementary seismology: San Francisco, W. H. Freeman and Co., $768 \mathrm{p}$.

Richter, C. F., Allen, C. R., and Nordquist, J. M., 1958, The Desert Hot Springs earthquakes and their tectonic environment: Seismol. Soc. America Bull., v. 48, p. 315-337.

Sykes, L. R., 1967, Mechanism of earthquakes and nature of faulting on the mid-ocean ridges: Jour. Geophys. Research, v. 72, p. 2131-2153.

Trifunac, M. D., and Brune, J. N., 1970, Complexity of energy release during the Imperial Valley, California, earthquake of 1940 : Seismol. Soc. America Bull., v. 60, p. 137-160.

Wyss, M., and Brune, J. N., 1968, Seismic moment, stress, and source dimensions for earthquakes in the California-Nevada region: Jour. Geophys. Research, v. 73, p. 4681-4694. 


\title{
SOURCE PARAMETERS OF THE BORREGO MOUNTAIN EARTHQUAKE ${ }^{1}$
}

\author{
By MaX WYSS \\ Lamont-Doherty Geological Observatory of Columbia University \\ AND \\ Thomas C. Hanks \\ Seismological laboratory, California Institute of Teghnology
}

ABSTRACT

Spectral analysis of teleseismic body phases at several azimuths was used to determine the moment, fault length, dislocation, stress-drop, and radiated energy of the Borrego Mountain earthquake. The results agree well with the same parameters obtained from the surface fracture and aftershock distribution and local observations of radiated energy.

\section{INTRODUCTION}

The Borrego Mountain earthquake provided an ideal opportunity to check the reliability of teleseismic methods to estimate the moment, dimension, and dislocation of a seismic source. Although the event was large enough to be recorded by the WWSSN (Worldwide Standard Seismograph Network) at all epicentral distances, it was small enough to be recorded well on the Wood-Anderson instruments (magnification $=100$ ) operating at Pasadena and Riverside. More important, this earthquake produced a well-defined surface rupture with a measured right-lateral displacement (Allen and others, 1968; Clark, this volume). Detailed studies of the aftershock sequence provided further information on the extent of the source region (Hamilton, this volume).

In the equivalent double-couple representation of a seismic source (Burridge and Knopoff, 1964); the seismic moment $M_{0}$ (Maruyama, 1963; Haskell, 1964) was shown by Aki (1966) to be proportional to the product of the fault area $A$ and the average dislocation $\bar{u}$

$$
M_{0}=\mu A \bar{u}
$$

where $\mu$ is the shear modulus in the source region. With this relation, $M_{0}$ can be evaluated from field measurements of $\bar{u}$ and $A$, the latter calculated

'Contribution 1819, Lamont-Doherty Geological' Observatory and Contribution 1930. Division of Geological Sciences, California Institute of Technology, Pasadena, Calif. from rupture length and depth based on field mapping and aftershock studies. Provided that the dislocation theory models an earthquake source correctly, $M_{0}$ can be determined from the longperiod amplitude spectral density $\left(\Omega_{0}\right)$ of body or surface waves. The two independently obtained values of $M_{0}$ will be compared in order to check the validity of the dislocation theory. Previous comparisions of teleseismically estimated $M_{0}$ with that obtained from fault area and surface displacement suffered from considerable uncertainties in the field observations (Aki, 1966; Wyss and Brune, 1968).

Another important parameter that can be estimated from teleseismic spectral data is the source dimension $r$. Kasahara (1957) has related the corner/peak frequency $f_{0}$ of $\mathrm{P}$-wave spectra to the radius of a spherical source model. Berckhemer and Jacob (1968) have estimated the rupture area of deep earthquakes using $f_{0}$ of P-waves. Brune (1970) proposed a relation between $f_{0}$ of S-waves and the radius of a circular rupture area. The present results are a part of a larger study, one purpose of which is to check if any one of the proposed relations between $f_{01}$ and $r$ is correct for earthquakes with known rupture length.

\section{ACKNOWLEDGMENTS}

Helpful discussions with J. N. Brune, C. B. Archambeau, and W. R. Thatcher are gratefully acknowledged. We also thank L. R. Sykes, R. V. Sharp, B. Isacks, and R. M. Hamilton for critically reading the manuscript. Some of the work was completed while the senior author was at the Institute of Geophysics and Planetary Physics, University of California, La Jolla. This research was supported by National Science Foundation grants NSF GA-19473, NSF GA-12868, and NSF GA-22709. 


\section{DATA}

The vertical components of $P$-waves recorded at eight WWSSN locations (table 4) at distances between $37^{\circ}$ and $86^{\circ}$ were Fourier-analyzed; the resulting displacement spectra $\Omega(\omega)$ and their azimuthal distribution are shown in figure 10 . In order to cover as large a frequency range as possible, the spectra of the long-period records as well as of the short-period records have been determined and plotted on the same graph. $\Omega(\omega)$, as presented in figures 10 and 11 , has been corrected for the instrument response and for attenuation using the results of Julian and Anderson (1968) for an earth model MM8 (Anderson, Ben-Menahem, and Archambeau, 1965). The units of $\Omega(\omega)$ are given in centimeter-seconds for both $P$ - and S-waves.

The S-wave spectra of four stations are shown in figure 11. Only one station furnished a usable short-period record. The corner frequency $f_{0}$, however, is clearly defined by the long-period spectra alone. In order to avoid contamination of the $S$ phase by other phases, only stations between $63^{\circ}$ and $75^{\circ}$ were considered. The window length ranged from 30 to 60 seconds.

$\mathrm{P}$ - and $\mathrm{S}$-wave spectra were also recorded at local stations (Pasadena, $\Delta=220 \mathrm{~km}$; and Riverside, $\Delta=145 \mathrm{~km}$ ). The recordings were obtained from low-magnification Wood-Anderson instruments (gain=100). These data were considered useful only in the range $0.5 \leqslant f \leqslant 2.5 \mathrm{~Hz}$. Although these data will not be useful in the moment or source dimension determinations, they provide a lower bound check for the radiated seismic energy.

In order to estimate the moment and the total radiated energy, the following corrections of the spectral amplitudes measured at any station have. been made. For the combined displacement amplification by the free surface and the crust at the receiver, an average value of 2.5 for $P$ and $S$ has been assumed (Ben-Menahem and others, 1965). For the radiation pattern correction, a vertical right-lateral strike-slip source with strike azimuth of N. $48^{\circ}$ W. was used. The decrease in amplitude due to geometrical spreading was accounted for, and the spatial integration of the energy radiated in different directions was performed using Wu's (1966) results. Because of the presence of the free surface at the source, only half of the integral given by Wu (1966) for the whole space was taken for the energy integration.

\section{SEISMIC MOMENT}

At the long-period end, $50 \geqslant T \geqslant 10 \mathrm{sec}$, the spectral amplitudes are approximately constant and at shorter periods fall off approximately as $\omega^{-2}$, where $\omega=\frac{2 \pi}{T}$. The long-period levels have been approximated as indicated in figures 10 and 11 and given as $\Omega_{0}$ in table 4 . This approximation of the spectrum is in accordance with the results of dislocation theory (Aki, 1967; Brune, 1970), provided that the spectral information in this period range is a meaningful representation of the ultralongperiod level. On the other hand, the lack of data for $T>50 \mathrm{sec}$ admits the possibility that the chosen level may only be the maximum of a broad peak as required by Archambeau (1968). The seismic moment $M_{0}$ was estimated from the corrected $\Omega_{0}$ using equations for the far-field dynamic displacement by Keilis-Borok (1960) and BenMenahem, Smith, and Teng (1965).

The moments obtained from teleseismic spectra are in good agreement with the moments estimated from field observations. $M_{0}$ obtained from the different stations are given in table 4 .

TABLE 4. - P - and $S$ - wave spectral data

\begin{tabular}{|c|c|c|c|c|c|c|c|c|c|c|}
\hline $\begin{array}{l}\text { Station } \\
\text { code }\end{array}$ & $\begin{array}{c}\text { Dist. } \\
\Delta \\
\text { (deg) }\end{array}$ & $\begin{array}{c}\text { Az from } \\
\text { epicenter } \\
\text { (deg) }\end{array}$ & $\begin{array}{c}\text { Az from } \\
\text { station } \\
\text { (deg) }\end{array}$ & Phase & Component & $\begin{array}{c}\text { Long-period } \\
\text { amplitude } \\
\Omega_{0} \\
(\mathrm{~cm}-\mathrm{sec}) \\
\end{array}$ & $\begin{array}{c}\text { Corner } \\
\text { frequency } \\
f_{0} \\
(\mathrm{~Hz}) \\
\end{array}$ & $\begin{array}{c}\text { Dimension } \\
r=L / 2 \\
(\mathrm{~km})\end{array}$ & $\begin{array}{c}\text { Moment } \\
M_{0} \\
\left(10^{2 s} \text { dyne-cm) }\right.\end{array}$ & $\begin{array}{c}\text { Energy } \\
E \\
\left(10^{10} \mathrm{erg}\right)\end{array}$ \\
\hline 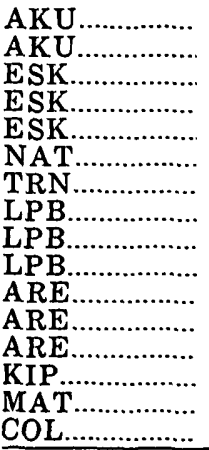 & $\begin{array}{l}63.4 \\
74.9 \\
\\
85.4 \\
54.8 \\
67.4 \\
\\
65.2\end{array}$ & $\begin{array}{r}99 \\
101 \\
129 \\
\\
132\end{array}$ & $\begin{array}{l}304 \\
303 \\
318 \\
\\
320\end{array}$ & $\begin{array}{l}\mathbf{S} \\
\mathbf{S} \\
\mathbf{P} \\
\mathbf{S} \\
\mathbf{S} \\
\mathbf{P} \\
\mathbf{P} \\
\mathbf{P} \\
\mathbf{S} \\
\mathbf{S} \\
\mathbf{P} \\
\mathbf{S} \\
\mathbf{S} \\
\mathbf{P} \\
\mathbf{P} \\
\mathbf{P}\end{array}$ & $\begin{array}{l}\text { EW } \\
\text { NS } \\
\text { Z } \\
\text { EW } \\
\text { NS } \\
\text { Z } \\
\mathbf{Z} \\
\text { Z } \\
\text { EW } \\
\text { NS } \\
\text { Z } \\
\text { EW } \\
\text { NS } \\
\mathbf{Z} \\
\text { Z } \\
\mathbf{Z}\end{array}$ & $\begin{array}{l}2.2 \cdot 10^{-2} \\
1.4 \cdot 10^{-2} \\
5.0 \cdot 10^{-4} \\
1.6 \cdot 10^{-2} \\
2.5 \cdot 10^{-2} \\
1.4 \cdot 10^{-3} \\
3.5 \cdot 10^{-3} \\
3.3 \cdot 10^{-3} \\
1.4 \cdot 10^{-2} \\
2.0 \cdot 10^{-2} \\
3.2 \cdot 10^{-3} \\
1.6 \cdot 10^{-2} \\
2.0 \cdot 10^{-2} \\
4.0 \cdot 10^{-3} \\
1.8 \cdot 10^{-3} \\
5.0 \cdot 10^{-3}\end{array}$ & $\begin{array}{l}0.069 \\
.05 \\
.20 \\
.063 \\
.063 \\
.22 \\
.126 \\
.12 \\
.052 \\
.05 \\
.16 \\
.063 \\
.047 \\
.16 \\
.21 \\
.126\end{array}$ & $\begin{array}{r}17.4 \\
24.0 \\
10.5 \\
19.0 \\
19.0 \\
9.5 \\
16.7 \\
17.5 \\
23.1 \\
24.0 \\
13.1 \\
19.0 \\
25.5 \\
13.0 \\
10.0 \\
16.7\end{array}$ & $\begin{array}{r}5.9 \\
5.9 \\
8.1 \\
8.1 \\
{[11 .]} \\
11 . \\
\overline{7.0} \\
7.0 \\
\overline{5.5} \\
5.5 \\
\overline{7.4} \\
\overline{12.0}\end{array}$ & $\begin{array}{l}3.8 \\
3.8 \\
\overline{8.0} \\
\overline{8.0} \\
\overline{1.6} \\
\overline{3.3} \\
3.3 \\
\overline{2.5} \\
2.5 \\
1.5 \\
\overline{1.9}\end{array}$ \\
\hline
\end{tabular}




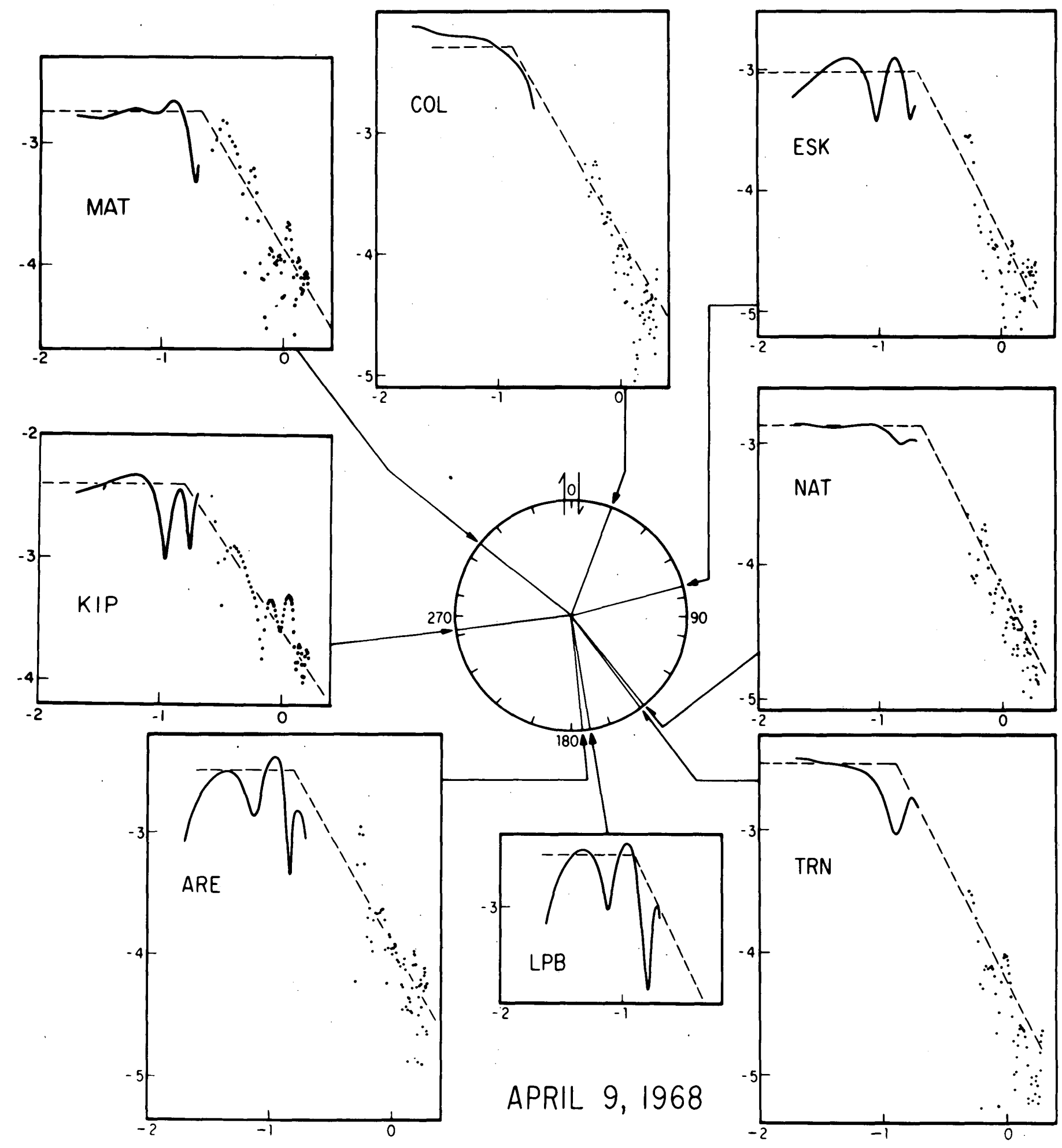

Figure 10. - Attenuation-corrected P-wave spectra of the Borrego Mountain earthquake. Solid line obtained from long-period vertical WWSSN instruments, and dots obtained from short-period vertical WWSSN instruments. Azimuths with respect to strike of fault. Vertical scale is $\log$ of displacement spectra, in centimeter-seconds; horizontal scale is log frequency, in Hertz. 


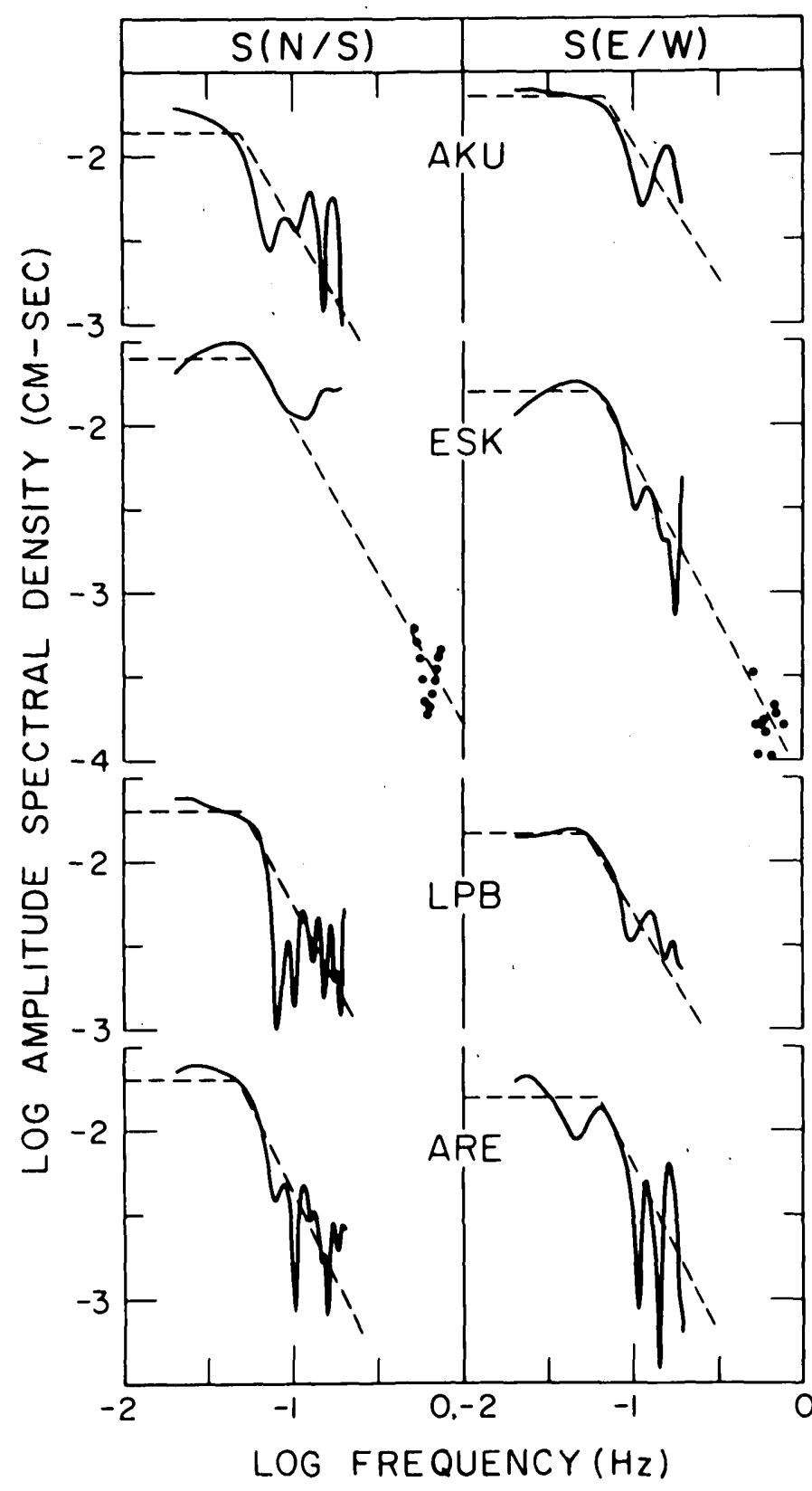

Figurf 11. - Attenuation-corrected S-wave spectra of the Borrego Mountain earthquake from WWSSN recordings.

Unfortunately, some stations were close to nodes in the $\mathrm{P}$-wave radiation pattern. If the correction due to the radiation pattern alone was larger than a factor of 10 , the moment was not computed, since small uncertainties in the focal mechanism could introduce large errors. The average of the P- and S-wave moments of table 4 is compared with $M_{0}$ derived from observations in the source area in table 5. The depth of faulting was assumed to be the depth to which the after- shock activity extended, $h=12 \mathrm{~km}$ (Hamilton, this volume). $M_{0}$, based on teleseismic (dynamic) measurements, agrees within approximately a factor of 2 with the field (static) observations.

The teleseismically determined moments are likely to be affected by reflections from the free surface. The inclusion of $p P$ in the spectral analysis is expected to cause an overestimate of $M_{0}$. This may explain why the teleseismic moments are larger than the static ones (table 5). The longperiod data, however, do not indicate the expected degradation of the radiation at periods long compared to the timelag $\Delta t$ of the reflected phase $\left(\Delta t \simeq \frac{2 h}{\alpha} \leqslant 3 \mathrm{sec} ; h=\right.$ source depth $\leqslant 10 \mathrm{~km}$, $\alpha=\mathrm{P}$-wave velocity $=6.0 \mathrm{~km} / \mathrm{sec}$ ). The facts that the Borrego Mountain earthquake ruptured the surface and had a depth of rupture comparable to the length of rupture undoubtedly complicates an image point source representation of reflected phases. Other error sources for individual stations are uncertainties in the fault plane solution and the effect of local upper mantle and crustal structure. These two effects could combine to form an error that in general should not exceed a factor of three in the moment determination for any one station. In the average moment determination from the several stations, some of these errors should be cancelled out; and it is encouraging to see that the RMS (root mean square) errors are small (table 5).

TABLE 5.-Source parameters

\begin{tabular}{|c|c|c|c|c|c|c|}
\hline $\begin{array}{l}\text { Field observation or } \\
\text { spectral estimate }\end{array}$ & $\begin{array}{c}\text { Moment } \\
M_{0} \\
\left(10^{2 s}\right. \\
\text { dyne-cm })\end{array}$ & $\begin{array}{c}\text { Length } \\
L=2 r \\
(\mathrm{~km})\end{array}$ & $\begin{array}{c}\text { A rea } \\
A_{0} \\
\left(\mathrm{~km}^{2}\right)\end{array}$ & $\begin{array}{c}\text { Depth } \\
h \\
(\mathbf{k m})\end{array}$ & $\begin{array}{c}\text { Dislo- } \\
\text { cation } \\
\bar{u} \\
(\mathrm{~cm})\end{array}$ & $\begin{array}{l}\text { Stress- } \\
\text { drop } \\
\Delta \sigma \\
\text { (bar) }\end{array}$ \\
\hline $\begin{array}{l}\text { Surface rupture........... } \\
\text { Aftershock zone .......... } \\
\text { P-wave (Brune)......... } \\
\text { S-wave (Brune).......... } \\
\text { P-wave (Kasahara)..... } \\
\text { P-wave (Berckhemer }\end{array}$ & $\begin{array}{c}3.6 \\
4.9(6.1) \\
10 . \pm 2 \\
6.6 \pm 1 \\
-\end{array}$ & $\begin{array}{c}33 \\
45(56) \\
26 \pm 6.5 \\
42 \pm 6 \\
8\end{array}$ & $\begin{array}{c}396 \\
540(672) \\
615 \\
1460 \\
53\end{array}$ & $\begin{array}{l}12 \\
12 \\
=\end{array}$ & $\begin{array}{l}30 \\
30 \\
30 \\
14 \\
-\end{array}$ & $\begin{array}{r}4 \\
20 \\
3 \\
-\end{array}$ \\
\hline and Jacob) ................. & - & 8.5 & 58 & - & - & - \\
\hline
\end{tabular}

'Assumed from aftershock distribution.

The moments obtained from field observations will be affected by errors in the estimates of the rupture area and the dislocation. The zone of intense aftershock activity in the figures shown by Hamilton (this volume) is $45 \mathrm{~km}$ long. If the sporadic aftershock activity toward the north is included, a length of $56 \mathrm{~km}$ is obtained. We feel, however, that the intense aftershock zone outlines the rupture area, whereas the more northern events were caused by strain accumulations beyond the fault end. This view is supported by the Coyote Mountain earthquake of April 28, 1969, and 
its aftershock sequence, which were located in this zone of sporadic aftershock activity, $33^{\circ} 20^{\prime}$ N., $116^{\circ} 21^{\prime}$ W. (R. M. Hamilton, oral commun., 1970; Thatcher and Hamilton, 1971). Even though we feel that $45 \mathrm{~km}$ is the value that should be used for the fault length as outlined by the aftershocks, the source parameters based on $56 \mathrm{~km}$ are also computed and given in table 5 in parentheses. The length of the surface rupture differs by a factor of 1.3. (1.7) from the extent of the intense (extended) aftershock activity. The depth of the main shock given by Allen and Nordquist (this volume) is $11 \mathrm{~km}$, which differs from the depth of the deepest aftershocks by a factor of 1.1. These factors may combine to an error of less than 2 in the area estimates. Insofar as no creep occurred on the northern branch of the fault, three-quarters (Brune and Allen, 1967) of the maximum surface displacement, $38 \mathrm{~cm}$ (Clark, "Surface Rupture Along the Coyote Creek Fault," this volume), was taken to represent the average displacement at depth. It is hard to imagine that the displacement could be in error by more than a factor of 3: Under this assumption, the statically estimated moment might be uncertain to a factor of. 5 . This is an extreme value, however, and we believe that the static moment is estimated to within a factor of 3 from the correct value.

The agreement in table 5 can be considered very good, and it is concluded that the moment of a double-couple source can be determined equally well from teleseismic body phases or from field observations in the source region.

\section{FAULT LENGTH}

The corner frequency $f_{0}$ was defined by the intersection of the horizontal line representing the long-period spectral level and the line drawn through the short-period data, if they were available. Otherwise, $f_{0}$ was estimated from the decay of the long-period data.

Brune (1970) related $f_{0}$ for S-waves to the source dimension $r$ by

$$
r=\frac{2.21 \beta}{2 \pi f_{0}(\mathrm{~S})}=\frac{1.2}{f_{0}(\mathrm{~S})}
$$

where $r$ is measured in kilometers.

Because the flat amplitude level at frequencies smaller than $f_{0}$ is due to interference of waves with wavelength $\lambda$ longer than the source size, it would seem reasonable to obtain a version of equation 2 valid for P-waves by keeping $\lambda_{0}$ constant and substituting $\alpha$, the $\mathrm{P}$-wave velocity, for $\beta$, the $\mathrm{S}$-wave velocity. To estimate the source dimension from the $P$-wave spectra, we use the relation

$$
r=\frac{2.21 \alpha}{2 \pi f_{0}(\mathrm{P})}=\frac{2.1}{f_{0}(\mathrm{P})}
$$

The source radii obtained from 2 and 3 and the appropriate $f_{0}$ are given in table 4 . The values from stations at different azimuths are generally consistent, indicating that such effects as focusing due to rupture propagation, substation crustal reverberations, and 6-second microseisms are not sufficient to disguise the gross behavior of bodywave spectra of the Borrego Mountain earthquake.

Averages for the $\mathrm{P}$ - and S-wave determinations of $L=2 r$ and of the ruptured area $A=\pi r^{2}$ are compared to surface rupture and aftershock length in table 5 . The agreement between the four length determinations is very close, if Brune's equations are used, and it is concluded that the source dimensions of an earthquake can be obtained with satisfactory accuracy from spectral P- and S-wave analysis using Brune's (1970) results. It is to be noted, however, that the source dimensions obtained from $\mathrm{P}$-wave spectra are consistently smaller than those from S-wave spectra. This fact may indicate that the step from equation 2 to equation 3 is not completely correct and needs clarification.

Kasahara's (1957) model relates the radius of a spherical source to $f_{0}$ by

$$
r=\frac{0.66}{f_{0}(\mathrm{P})} \text {. }
$$

If the average value for $f_{0}(\mathrm{P})$ from table 4 is used in equation 4 , we obtain $L=2 r=8.2 \mathrm{~km}$. In Berckhemer and Jacob's (1968) theory, the maximum rupture velocity $c$ has to be assumed; their relation is

$$
A_{0}=\frac{\mathrm{c}^{2}}{\mathrm{f}_{0}^{2}} \frac{1}{2 \pi} \text {. }
$$

Even if a rather high rupture velocity of $3 \mathrm{~km} / \mathrm{sec}$ is assumed, the average area $A_{0}$ from 5 is only $58 \mathrm{~km}^{2}$. The diameter of a circular fault with this area is approximately $8.5 \mathrm{~km}$.

The values for $A_{0}$ and $r$ that are estimated by equations 3,4 , and 5 are compared in table 5 to the values observed in the source region. It is evident that the theories of earlier authors (Kasahara, Berckhemer, and Jacob) underestimate the source dimensions, but Brune's relation gives results which are in excellent agreement with the field observations.

The use of body waves for source size determination has great advantages over the surface wave method (Ben-Menahem, 1961). The excitation of surface waves is strongly dependent on the crustal structure, and the source depth interference can be severe (Tsai, 1969). Also, for smaller events it is 
difficult to extend surface wave spectra to the short periods required without mode contamination, and deep earthquakes often do not excite measurable surface waves. A disadvantage of the body-wave method is that at a range of shallow depths the surface reflections $\mathrm{pP}$ and sS cannot be separated from the direct phase, and the interference of these phases with the direct waves can be expected to complicate the analysis.

\section{ENERGY ESTIMATES}

The energy in a body wave spectrum is proportional to $\varrho_{-\infty}^{\infty}|\Omega(\omega) \omega|^{2} d \omega$. For a theoretical spectrum as given by Haskell (1964), Aki (1967), or Brune (1970), the energy integral can be conveniently represented by $\Omega_{0}^{2} f_{0}^{3}$

$$
E_{\mathrm{s}}=k \Omega ; \mathrm{s} \text {. }
$$

The constant $k$ contains corrections for geometrical spreading, free surface and crustal effects at the station, the radiation pattern, integration of the radiation pattern over the focal sphere, rate of decay of the spectrum for $\omega>\omega_{0}$ (here assumed to be $\left.\omega^{-2}\right)$, as well as material constants. The energy radiated from a surface source into the halfspace below was taken to be half the integral given by $\mathrm{Wu}$ (1966) for a whole space. The total energy radiated by the Borrego Mountain earthquake as $\mathrm{P}$ - and $\mathrm{SH}$-waves is given in table 4 ; the average teleseismic estimates are $E(\mathrm{SH})=0.44 \cdot 10^{20} \mathrm{ergs}$; $E(\mathrm{P})=0.16 \cdot 10^{20} \mathrm{ergs}$. The teleseismic .observations indicate that the total radiated energy, including $\mathrm{SV}$, was approximately $0.69 \cdot 10^{20} \mathrm{ergs}$.

The radiated seismic energy has also been estimated from the seismograms written by the WoodAnderson torsion seismograph operating at a gain of 100 at Pasadena and Riverside. Table 6 gives estimates of the seismic energy radiated for all components of $\mathrm{P}$ - and $\mathrm{SH}$-waves in the frequency range $0.5 \leqslant f \leqslant 2.5 \mathrm{~Hz}$. The bulk of the seismic energy, however, is not included in the above estimate, because the corner frequency occurs outside of the frequency range available for analysis. We can check whether the locally recorded spectra agree approximately with the teleseismic ones by

TABLE 6. - Local observations of radiated seismic energies

\begin{tabular}{|c|c|}
\hline Station and Phase & Energy, $10^{19} \mathrm{ergs}^{1}$ \\
\hline \multicolumn{2}{|l|}{ Pasadena: } \\
\hline $\mathrm{P}(\mathrm{Z}) \ldots \ldots$ & .1 .3 \\
\hline $\mathrm{S}(\mathrm{NS}) \ldots$ & $\ldots 2.3$ \\
\hline $\mathrm{S}(\mathrm{EW}) \ldots$ & $\ldots 2.5$ \\
\hline \multicolumn{2}{|l|}{ Riverside: } \\
\hline $\mathrm{P}(\mathrm{Z}) \ldots \ldots$ & .2 .6 \\
\hline $\mathrm{S}(\mathrm{NS}) \ldots$ & .. .9 \\
\hline $\mathrm{S}(\mathrm{EW}) \ldots$ & 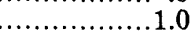 \\
\hline
\end{tabular}

extrapolating the slope of the local spectra as a straight line to lower frequencies. At the average corner frequency, this straight line should intersect with the long-period flat level of the spectrum, which at that particular station corresponds to the average moment. The agreement is fairly good. If we assume that the long-period level corresponding to the average moment, the corner frequency, and the slope of spectral decay to high frequencies are given, we estimate the total radiated energy based on the Pasadena record to be approximately $2 \cdot 10^{20} \mathrm{ergs}$.

The energy estimated from the energy-magnitude relationship (Gutenberg and Richter, 1956) amounts to several times $10^{21}$ ergs, depending on the relation used. The spectral estimates discussed above are an order of magnitude smaller. The energies estimated by integration agree with the energy estimated by DeNoyer (1959) for earthquakes with comparable magnitude. The problem of energy should be studied in detail, but for this task complete spectra, including the corner frequency from nearby stations, are needed.

An interesting result is that the ratio of the $\mathrm{S}$-wave to the $\mathrm{P}$-wave energy is 3.3 despite the considerably larger long-period S-wave amplitudes. Since the energy is a function of the ratio of amplitude over period, the larger long-period Swave amplitudes are partly offset by a smaller $f_{0}(\mathrm{~S})$. This relation is best understood in terms of equation 6 , where $\Omega_{0}$ is proportional to $\frac{1}{v^{3}}$, while $f_{0}$ and $k$ are proportional to $v$ ( $v=$ respective propagation velocity). Therefore, the ratio of $S$ to $P$ wave energy should be $(\alpha / \beta)^{2}=3$. Gutenberg and Richter (1956) assumed a ratio of 2 ; this ratio agrees with our observation.

\section{STRESS-DROP}

The stress-drop $\Delta \sigma=\sigma_{1}-\sigma_{2}\left(\sigma_{1}=\right.$ stress prior to rupture, $\sigma_{2}=$ stress after completion of the event) can be estimated from the moment and the source radius through the relation (Brune, 1970)

$$
\Delta \sigma=\frac{7}{16} \frac{M_{0}}{r^{3}}
$$

From the field observations, the stress-drop was also estimated using Knopoff's (1958) equation $\Delta \sigma=\frac{u \mu}{2 h}$, where $u=$ dislocation and $h=$ depth. The three different estimates are given in table 5 . They agree well, and the average stress-drop is $\Delta \sigma=9$ bar. This value is much smaller than the stresses that the earth's crust is able to maintain. 
From the stress-drops of large shallow earthquakes, Chinnery (1964) estimated that the crust can accumulate 100 bar shear stresses. Brune, Henyey, and Roy (1969) obtained an upper bound of 200 bars for the stresses accumulated on the San Andreas fault, based on heat-flow measurements. Evidently, the Borrego Mountain earthquake is another low stress-drop event like the Parkfield (Aki, 1967; Wyss and Brune, 1968) and the Imperial (Brune and Allen, 1967) earthquakes. This result lends further support to the observation that the stress-drop of small earthquakes is in general smaller than that of large earthquakes (King and Knopoff, 1968; Wyss, 1970).

\section{CONCLUSIONS}

With the assumption of a double-couple source model, the use of teleseismically observed bodywave spectra has been successful in determining the fault dimension and seismic moment (and, equivalently, total offset and stress-drop) of the Borrego Mountain earthquake. The results are in close agreement with independently obtained values resulting from local field observations. The average fault length is $37 \mathrm{~km}$, the average moment is $6.3 \cdot 10^{25} \mathrm{dyne} \mathrm{cm}$, and the average stress-drop is 9 bars.

The radiated seismic energy estimated from teleseismic observations is $0.69 \cdot 10^{20} \mathrm{erg}$ and from local observations, extrapolated to include the whole frequency range, is $2 \cdot 10^{20} \mathrm{erg}$.

\section{REFERENCES CITED}

Aki, K., 1966, Generation and propagation of $G$ waves from the Niigata earthquake of June 16, 1964. Part 2. Estimation of earthquake moment, released energy, and stress drop from the $G$ wave spectrum: Tokyo Univ. Earthquake Research Inst. Bull., v. 44, p. 73-88.

1967 , Scaling law of seismic spectrum: Jour. Geophys. Research, v. 72, p. 1217-1231.

Allen, C. R., Grantz, A., Brune, J. N., Clark, M. M., Sharp, R. V., Theodore, T. G., Wolfe, E. W., and Wyss, M., 1968, The Borrego Mountain, California, earthquake of 9 April 1968: A preliminary report: Seismol. Soc. America Bull., v. 58 , p. $1183-1186$.

Anderson, D. L., Ben-Menahem, A., and Archambeau, C. B., 1965, Attenuation of seismic energy in the upper mantle: Jour. Geophys. Research, v. 70, p. 1441-1448.

Archambeau, C. B., 1968, General theory of elastodynamic source fields: Rev. Geophyics, v. 6, p. 241-287.

Ben-Menahem, A., 1961, Radiation of seismic surface waves from finite moving sources: Seismol. Soc. America Bull., v. 51, p. 401-435.

Ben-Menahem, A., Smith, S. W., and Teng, T. L., 1965, A procedure for source studies from spectrums of long-period seismic waves: Seismol. Soc. America Bull., v. 55. p. 203-235.
Berckhemer, H., and Jacob, K. H., 1968, Investigation of the dynamical process in earthquake foci by analyzing the pulse shape of body waves: Final Sci. Rept. Contract AF61 (052) -801, Institute of Meteorology and Geophysics, University of Frankfurt, Germany, 85 p.

Brune, J. N., 1970, Tectonic stress and the spectra of seismic shear waves: Jour. Geophys. Research, v. 75, p. 4997-5009.

Brune, J. N., and Allen, C. R., 1967, A low stress-drop, low magnitude earthquake with surface faulting-The Imperial, California, earthquake of March 4, 1966: Seismol. Soc. America Bull., v. 57, p. 501-514.

Brune, J. N., Henyey, T. L., and Roy, R. F., 1969, Heat flow, stress, and rate of slip along the San Andreas fault, California: Jour. Geophys. Research, v. 74, p. 3821-3827.

Burridge, R., and Knopoff, L., 1964, Body force equivalents for seismic dislocations: Seismol. Soc. America Bull., v. 54, p. 1875-1888.

Chinnery, M. A., 1964, The strength of the earth's crust under horizontal shear stress: Jour. Geophys. Research, v. 69, p. 2085-2089.

DeNoyer, J., 1959, Determination of the energy in body and surface waves (Part II): Seismol. Soc. America Bull., v. 49 , p. $1-10$.

Gutenberg, Beno, and Richter, C. F., 1956, Earthquake magnitude, intensity, energy; and acceleration: Seismol. Soc. America Bull., v. 46, p. 105-145.

Haskell, N. A., 1964, Total energy and energy spectral density of elastic wave radiation from propagating faults: Seismol. Soc. America Bull., v. 54, p. 1811-1841.

Julian, B. R., and Anderson, D. L., 1968, Travel times, apparent velocities, and amplitudes of body waves: Seismol. Soc. America Bull., v. 58, p. 339-366.

Kasahara, K., 1957, The nature of seismic origins as inferred from seismological and geodetic observations: Tokyo Univ. Earthquake Research Inst. Bull., v. 35, p. 473-530.

Keilis-Borok, V. I., 1960, Investigation of the mechanism of earthquakes: Akad. Nauk. SSSR Geofiziki Inst. Trudy, no. 4 (166), Soviet Research in Geophysics, v. 4, (trans. by American Geophysical Union, Consultants Bureau Enterprises, New York).

King, C. Y., and Knopoff, L., 1968, Stress drop in earthquakes: Seismol. Soc. America Bull., v. 58, p. 249-257.

Knopoff, Leon, 1958, Energy release in earthquakes: Geophys. Jour., v. 1, p. 44-52.

Maruyama, T., 1963, On the force equivalent of dynamic elastic dislocations with reference to the earthquake mechanism: Tokyo Univ. Earthquake Research Inst. Bull., v. 41, p. 467-486.

Thatcher, W. R., and Hamilton, R. M., 1971, Spatial distribution and source parameters of the Coyote Mountain aftershock sequence, San Jacinto fault zone [abs.], in Geol. Soc. America Abs. with Programs, v. 3, no. 2, 229 p.

Tsai, Y. B., 1969, Determination of focal depths of earthquakes in the mid-oceanic ridges from amplitude spectra of surface waves: Massachusetts Inst. Technology $\mathrm{Ph}$. D. thesis.

Wu, F. T., 1966, Lower limit of the total energy of earthquakes and partitioning of energy among seismic waves: California Inst. Technology, Pasadena, Ph. D. thesis.

Wyss, M., 1970, Observation and interpretation of tectonic strain release mechanisms: California Inst. Technology, Pasadena, Ph. D. thesis.

Wyss, M., and Brune, J. N., 1968, Seismic moment, stress, and source dimensions for earthquakes in the California-Nevada region: Jour. Geophys. Research, v. 73, p. 4681-4694 


\title{
AFTERSHOCKS OF THE BORREGO MOUNTAIN EARTHQUAKE FROM APRIL 12 TO JUNE 12, 1968
}

\author{
By Robert M. Hamilton \\ U.S. Geological SURVEY
}

\begin{abstract}
Epicenters determined for 533 of the Borrego Mountain aftershocks form a complex pattern consisting of a main band $60 \mathrm{~km}$ long, subparallel and near the Coyote Creek fault, and two clusters centered $16 \mathrm{~km}$ northeast and 22 southwest of the main band. Foci range in depth from near surface to $12 \mathrm{~km}$; the zone of shallow foci is partly associated with the zone of surface fractures. All well-located aftershocks with a magnitude over 3.5 occurred below $6 \mathrm{~km}$ depth. The southeast fault strand was the site of much of the aftershock activity; the epicenter pattern along this strand exhibited a southeastward extension of about $3 \mathrm{~km}$ after the first week. Focal mechanisms are mostly of the strike-slip type. In the region of faulting, the right-lateral nodal plane is subparallel to the fault trend. At individual recording sites, average magnitude residuals are directly related to $\mathbf{P}$-wave traveltime delays and thus to basement depth.
\end{abstract}

\section{INTRODUCTION}

Aftershocks of the Borrego Mountain, California, earthquake were monitored by 20 portable seismographs of the U.S. Geological Survey (USGS) from April 12, 3 days after the magnitude 6.4 event, to June 12, 1968. Also in operation in the aftershock region during this 2-month period were four seismographs of the California Institute of Technology (Caltech). The main goal of the monitoring was to determine the relationship between the spatial distribution and focal mechanisms of the aftershocks, on the one hand, and the surface breakage and mapped faults, on the other.

In conjunction with the aftershock monitoring, a seismic refraction experiment was conducted involving three explosions that were recorded at the portable seismograph stations and at 10 truck-mounted seismic refraction units. The purpose of this experiment was to provide information about $\mathrm{P}$-wave velocities in the upper crust in the Borrego Mountain region in order to locate the aftershock hypocenters more accurately. Details of this refraction study were reported previously (Hamilton, 1970).

The combination of a large number of seismographs deployed, their careful distribution with respect to the aftershock zone, and the special seismic velocity study made possible unusually precise hypo- center and focal mechanism determinations. The only previous study of similar scope was done after the Parkfield-Cholame earthquake of 1966 (Eaton, O'Neill, and Murdock, 1970).

\section{ACKNOWLEDGMENTS}

The portable seismographs were installed and serviced by J. M. Coakley, E. E. Criley, and J. O. Ellis. Valuable help in selecting sites and in establishing the first seismograph stations was received from C. R. Allen, California Institute of Technology, who, with J. N. Brune of University of California at San Diego and Max Wyss of Lamont-Doherty Geological Observatory, provided arrival times and magnitude estimates for many aftershocks. J. C. Savage and Wayne R. Thatcher, U.S. Geological Survey, made helpful suggestions concerning the interpretation of the aftershock pattern.

\section{SEISMOGRAPH STATIONS}

The Borrego Mountain earthquake occurred at $6: 29$ p.m. local time (P.s.t.). The deployment of the Caltech seismograph stations during the first day of the aftershock sequence has been described by Allen and Nordquist (this volume). The U.S. Geological Survey dispatched 11 portable seismographs on the day after the main shock, and they arrived in the area of the earthquake a day later. At that time, the approximate extent of ground breakage along the Coyote Creek fault was known. It was assumed that the aftershocks would occur in the vicinity of this fracture zone, so some of the seismograph stations were placed along the zone and the others were distributed to surround it (sites 1-11, fig. 12).

About 10 days after the main shock, the seismograph at site 5 was moved to site 12 , and four other seismographs that had become available were installed at sites 13-16 to expand coverage. Near the end of April, 3 weeks after the main shock, the density of the network was increased by station additions at sites 17-19. About 3 weeks later, after two earthquakes were detected southeast of the network, the seismograph at site 13 was moved to site 


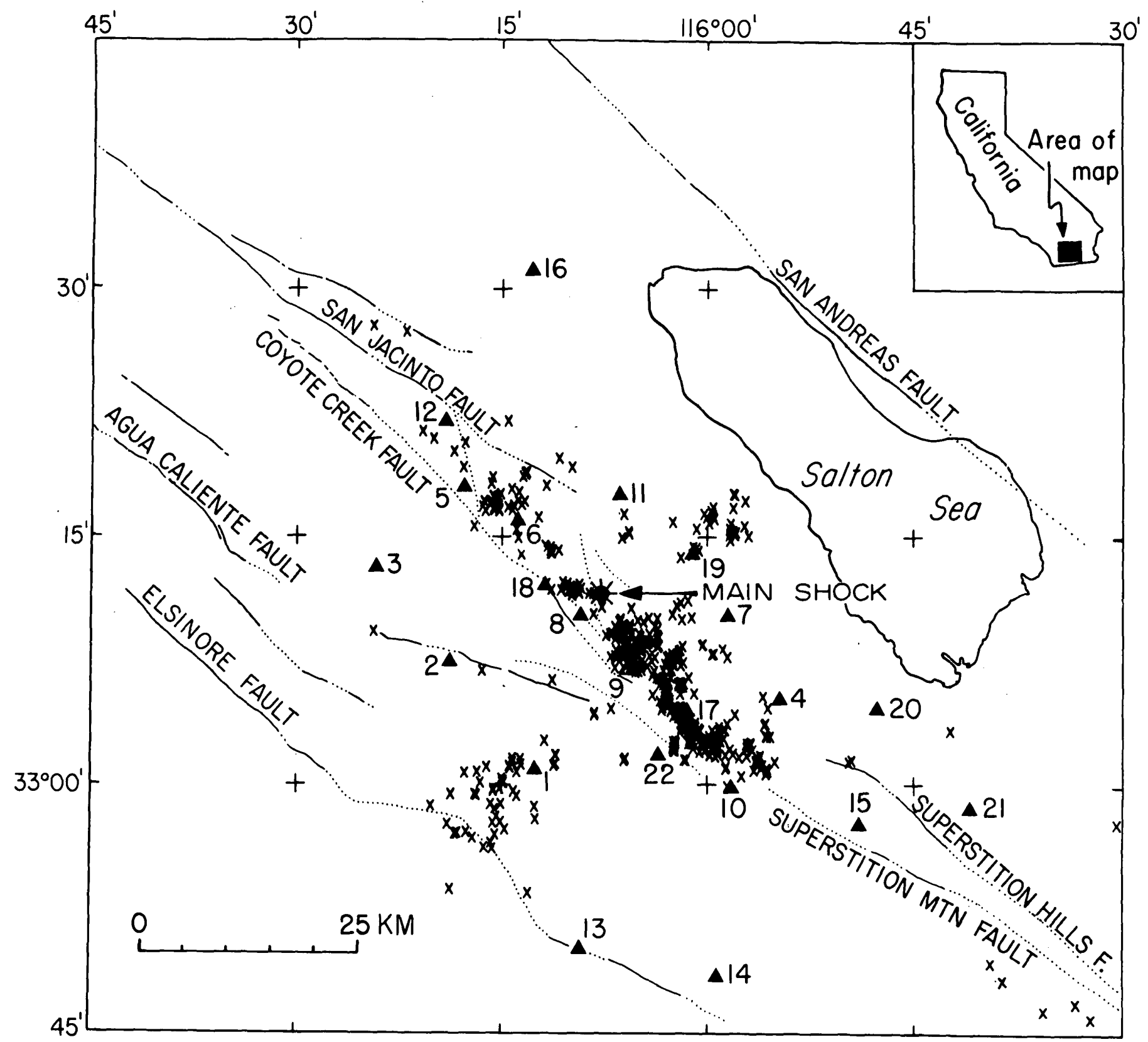

Figure 12. - Map of the Borrego Mountain region showing location of seismograph stations (triangles), epicenter of main shock (Allen and Nordquist, this volume), aftershock epicenters (crosses), and active faults. Faults are solid where there is evidence of recent or historic surface breakage, dotted where evidence is less conclusive.

20 , and station 21 was established. The final change in the network was the reoccupation of site 5 , bringing the number of stations to a total of 20 . The coordinates of the USGS seismograph stations have been published elsewhere (Hamilton, 1970), except for those of station 13 , which are $32^{\circ} 50.06^{\prime}$ N., $116^{\circ}$ $09.33^{\prime} \mathrm{W}$. Table 7 gives the period of operation for each station.

The portable seismographs installed in the Borrego Mountain region are the same units that were used by the U.S. Geological Survey to record the Parkfield-
Cholame aftershocks of 1966 . The recording system, which uses magnetic tape, the playback system, and the seismograms, have been described in detail by Eaton, O'Neill, and Murdock (1970). Briefly, the portable units all employ a short-period, verticalcomponent seismometer; six of them are also equipped with two horizontal-component seismometers. The system is particularly sensitive to frequencies ranging from 1 to $25 \mathrm{~Hz}$ (Hertz), with peak response at $17 \mathrm{~Hz}$. Maximum magnification, for a typical attenuation setting, is about 5 million. 
TABLE 7. - Seismograph station data

The station correction is subtracted from the observed arrival time; thus positive correction means waves are typically late. A positive magnitude pesidual means that for a particular earthquake the specified station yields magnitude that is larger than the average magnitude using all stations. $P$ wave and the other waves, respectively.]

\begin{tabular}{|c|c|c|c|c|c|}
\hline \multirow{2}{*}{ Station } & \multicolumn{2}{|c|}{ Recording Period } & \multirow{2}{*}{$\begin{array}{c}\text { Station } \\
\text { correction } \\
\text { (sec) }\end{array}$} & \multirow{2}{*}{$\begin{array}{l}\text { P-magnitude } \\
\text { residual }\end{array}$} & \multirow{2}{*}{ 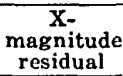 } \\
\hline & from & to & & & \\
\hline 11. & $4 / 11$ & $6 / 12$ & -0.20 & -0.25 & -0.18 \\
\hline 12. & $4 / 11$ & $6 / 12$ & -.15 & -.30 & -.27 \\
\hline 13. & $4 / 11$ & $6 / 13$ & -.36 & -.28 & -.27 \\
\hline 14. & $4 / 11$ & $6 / 12$ & .39 & .41 & .42 \\
\hline $5 .$. & $\begin{array}{l}4 / 12 \\
5 / 24\end{array}$ & $\begin{array}{l}4 / 18 \\
6 / 13\end{array}$ & -.23 & -.01 & .06 \\
\hline 6. & $4 / 12$ & $6 / 12$ & .37 & .16 & .13 \\
\hline & $4 / 12$ & $6 / 12$ & .10 & .22 & .08 \\
\hline 8. & $4 / 12$ & $6 / 12$ & -.14 & -.24 & -.16 \\
\hline 9. & $4 / 12$ & $6 / 12$ & .27 & .21 & .27 \\
\hline 110. & $4 / 12$ & $6 / 12$ & -.24 & -.13 & -.15 \\
\hline 111. & $4 / 12$ & $6 / 12$ & .09 & .05 & .09 \\
\hline $12 \ldots$ & $4 / 18$ & $6 / 13$ & -.34 & .25 & .33 \\
\hline $13 \ldots$ & $4 / 18$ & $5 / 18$ & 2.00 & & \\
\hline $14 \ldots \ldots \ldots$ & $4 / 18$ & $6 / 12$ & -.26 & .00 & .08 \\
\hline $16 \ldots \ldots \ldots$ & $4 / 19$ & $6 / 12$ & -.06 & -.09 & -.19 \\
\hline $16 \ldots \ldots \ldots . . .$. & $4 / 20$ & $6 / 12$ & -.42 & -.05 & .00 \\
\hline $17 \ldots$ & $4 / 28$ & $6 / 12$ & .52 & .07 & -.14 \\
\hline $18 \ldots \ldots \ldots . . . .$. & $4 / 29$ & $6 / 12$ & -.07 & -.13 & -.21 \\
\hline $19 .$. & $4 / 30$ & $6 / 12$ & .20 & .21 & .13 \\
\hline $20 \ldots$ & $5 / 19$ & $6 / 12$ & .71 & .45 & .38 \\
\hline 21. & $5 / 19$ & $6 / 12$ & .89 & .42 & .18 \\
\hline $322 \ldots \ldots \ldots . .$. & $4 / 09$ & $6 / 11$ & -.27 & $\ldots \ldots$ & $\ldots \ldots$ \\
\hline
\end{tabular}

13-component stration.

IStation correction derived from average of $P$-wave arrival time residuals Magnitudes not determined because seismograph was not satisfactorily calibrated.

${ }^{3}$ Caltech station.

\section{DATA PROCESSING}

In the 2 months that the seismograph network was in operation, well over 10,000 earthquakes were recorded. Locating all of these events was not feasible, so it was decided to locate 500 , a manageable number to process with current techniques. The principal goal in the analysis was to define the nature and extent of the aftershock activity. The more formidable task of producing a uniform data set including, for example, all earthquakes above a certain magnitude, was not undertaken.

Attention in locating the aftershocks was focused on the larger events. Besides involving a disproportionately large part of the energy released by the sequence, the larger aftershocks were recorded more clearly at more stations and therefore could be located more accurately than the smaller ones. Also, the direction of first motion on each record was generally clearer.

The earthquakes were selected from slow-speed $(37.5 \mathrm{~mm} / \mathrm{min})$ playbacks of the tapes from station 1. Station 1 was chosen as the primary source because it was located to the side of the main aftershock band; distance effects were thereby minimized, and it was easier to distinguish the larger from the smaller earthquakes. Earthquakes were selected for analysis if their maximum amplitude exceeded a certain value; this value was varied with the S-P interval or with the shape of the signal envelope to allow for amplitude attenuation with epicentral distance. The amplitude threshold was set to limit the number of events selected to about 500. Slow-speed (60 $\mathrm{mm} / \mathrm{min}$ ) playbacks from stations $6,9,12$, and 15 were used to fill periods when no record was obtained at station 1 .

Although most of the larger aftershocks were selected for analysis by this procedure, not all of those selected were successfully located. A poor or nonexistent radio-time-code signal from National Bureau of Standards station WWVB and interference from an earlier earthquake were two reasons for not determining a hypocenter.

The times of the events selected were noted to the nearest minute. High-speed playbacks $(75 \mathrm{~cm} / \mathrm{min})$ were then made covering a span of several minutes including each time. From these playbacks, readings were made of the $\mathrm{P}$-wave arrival time, direction of first motion, maximum amplitude in the $P$-wave group, and maximum amplitude in the signal after the $\mathrm{P}$-wave group. Sharp onsets of $\mathrm{P}$ could, in general, be read with a precision of 0.02 and $0.03 \mathrm{sec}$. The readings were made for all events that appeared on the playbacks and seemed large enough to be locatable, even for smaller events that had not been previously selected but that occurred within several minutes of a selected event. Extensive use was made of readings from the Caltech station at site 22 (fig. 12). Coordinates for this station have been given previously (Hamilton, 1970), and they are also listed in Allen and Nordquist (this volume).

The aftershock parameters (origin time, focal coordinates, and magnitude) were computed using a program written by Eaton (1969). Features of this program are also discussed by Eaton, O'Neill, and Murdock (1970). Locations, derived from P-wave arrival times, were determined only for those events read at six or more stations. Aftershock parameters and data indicating the reliability of the parameters are given in table 8 .

The precision of location of each earthquake depends mainly upon where the event occurred with respect to the seismograph stations. Epicenters for aftershocks within the net are thought to be accurate within $1 \mathrm{~km}$; this opinion is based on comparison of the computed and actual epicenters of the explosions (Hamilton, 1970) and on success in defining narrow lineations in epicenter patterns and relating them to recognized faults in a variety of similar studies elsewhere. As the margin of the net is approached, the epicenter precision declines rapidly. Experience indicates that just outside a net like the one in the Borrego Mountain area, epicenter errors of several kilometers are not unusual. Within the net, uncertainty in focal depth, in general, is probably about 
TABLE 8.-Borrego Mountain aftershocks

[For explanation of column heads see notes at end of table]

1968 HR MN SEC LAT N LONG W DEPTH MAG NO GAP DMIN ERT

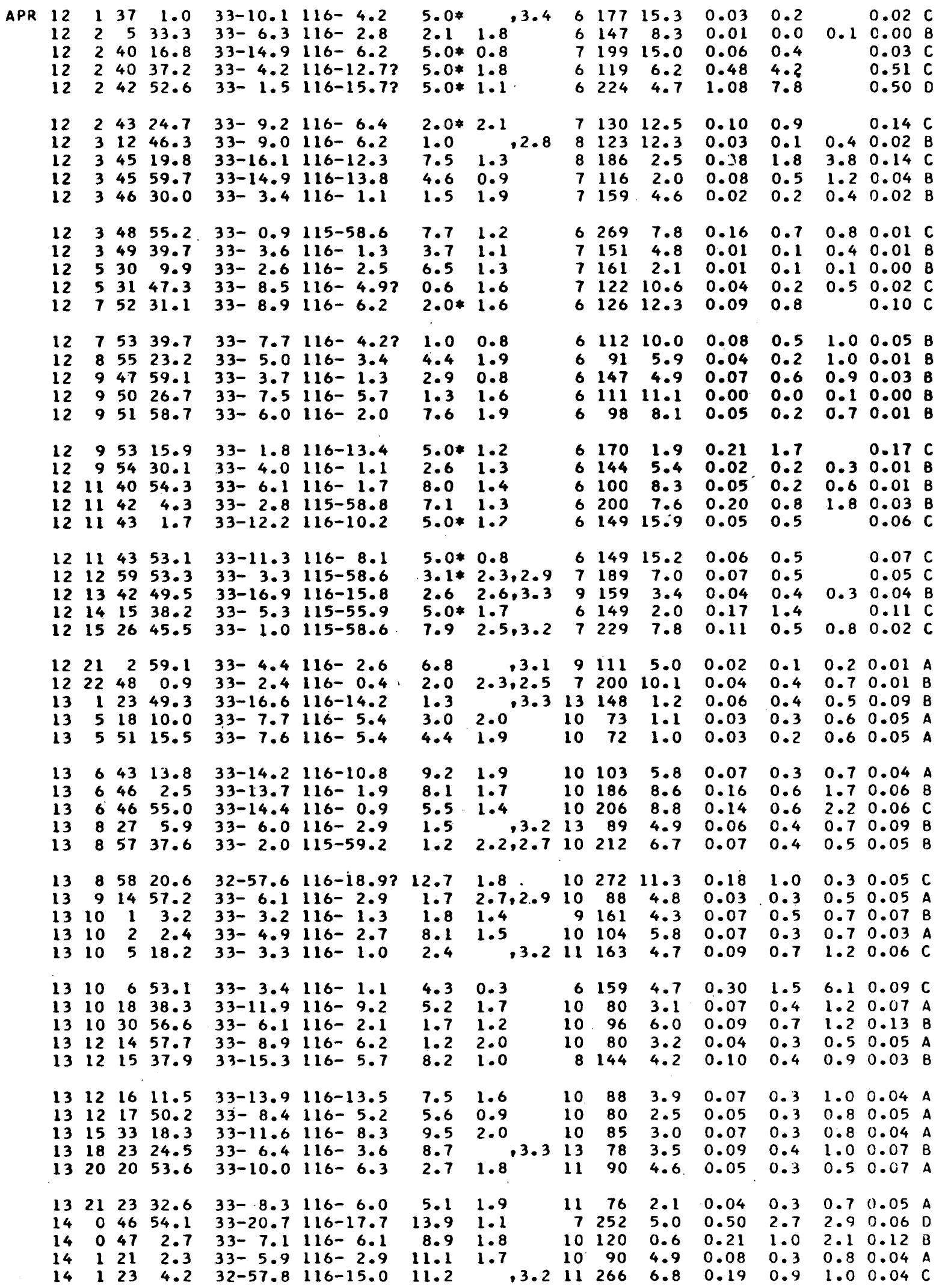


TABLE 8.-Borrego Mountain aftershocks-Continued

1968 HR MN SEC LAT N LONG W DEPTH MAG NO GAP DMIN ERT ERH ERZ MD 0

\begin{tabular}{|c|c|c|c|c|c|c|c|c|c|c|c|c|c|c|}
\hline $\begin{array}{l}14 \\
14 \\
14 \\
14 \\
14\end{array}$ & $\begin{array}{l}1 \\
1 \\
1 \\
1 \\
1\end{array}$ & $\begin{array}{l}24 \\
25 \\
26 \\
26 \\
27\end{array}$ & $\begin{array}{l}36.4 \\
53.1 \\
4.8 \\
18.3 \\
34.6\end{array}$ & $\begin{array}{l}33-15.2 \\
33-9.2 \\
33-17.2 \\
33-8.5 \\
33-8.4\end{array}$ & $\begin{array}{l}116-5.7 \\
116-6.9 \\
116-15.3 \\
116-5.9 \\
116-5.9\end{array}$ & $\begin{array}{r}10.2 \\
3.4 \\
2.1 \\
4.9 \\
2.9\end{array}$ & $\begin{array}{l}0.5 \\
0.4^{.3 .0} \\
0.6^{.3 .0}\end{array}$ & $\begin{array}{r}8 \\
7 \\
8 \\
11 \\
9\end{array}$ & $\begin{array}{r}143 \\
79 \\
156 \\
77 \\
77\end{array}$ & $\begin{array}{l}4.4 \\
4.1 \\
3.1 \\
2.5 \\
6.2\end{array}$ & $\begin{array}{l}0.18 \\
0.03 \\
0.10 \\
0.03 \\
0.06\end{array}$ & $\begin{array}{l}0.7 \\
0.2 . \\
0.8 \\
0.2 \\
0.5\end{array}$ & $\begin{array}{l}1.7 \\
0.8 \\
0.9 \\
0.5 \\
5.1\end{array}$ & $\begin{array}{l}0.07 \\
0.03 \\
0.10 \\
0.03 \\
0.07\end{array}$ \\
\hline $\begin{array}{l}14 \\
14 \\
14 \\
14 \\
14\end{array}$ & $\begin{array}{l}1 \\
3 \\
3 \\
7 \\
7\end{array}$ & $\begin{array}{l}29 \\
30 \\
51 \\
14 \\
15\end{array}$ & $\begin{array}{l}22.7 \\
52.0 \\
4.6 \\
45.3 \\
34.8\end{array}$ & $\begin{array}{l}33-7.8 \\
33-8.4 \\
33-5.4 \\
33-15.4 \\
33-11.6\end{array}$ & $\begin{array}{l}116-6.7 \\
116-5.8 \\
116-2.8 \\
116-13.9 \\
116-8.6\end{array}$ & $\begin{array}{l}2.6 \\
4.6 \\
1.4 \\
3.5 \\
6.6\end{array}$ & $\begin{array}{l}.0 \\
.6 \\
.6 \\
.3 \\
.8\end{array}$ & $\begin{array}{r}10 \\
11 \\
10 \\
6 \\
11\end{array}$ & $\begin{array}{l}69 \\
88 \\
89 \\
71 \\
82\end{array}$ & $\begin{array}{l}1.9 \\
2.2 \\
5.6 \\
1.0 \\
2.7\end{array}$ & $\begin{array}{l}.05 \\
.04 \\
.02 \\
.04 \\
.05\end{array}$ & $\begin{array}{l}0.4 \\
0.3 \\
0.2 \\
0.3 \\
0.3\end{array}$ & $\begin{array}{l}0.4 \\
0.7 \\
0.3 \\
0.6 \\
0.8\end{array}$ & $\begin{array}{l}0.06 \\
0.06 \\
0.04 \\
0.02 \\
0.05\end{array}$ \\
\hline $\begin{array}{l}14 \\
14 \\
14 \\
14 \\
14\end{array}$ & $\begin{array}{l}7 \\
12 \\
13 \\
13 \\
16\end{array}$ & $\begin{array}{l}45 \\
55 \\
13 \\
14 \\
46\end{array}$ & $\begin{array}{l}21.1 \\
58.7 \\
7.4 \\
47.6 \\
30.4\end{array}$ & $\begin{array}{l}33-7.7 \\
33-14.2 \\
33-3.4 \\
32-53.7 \\
33-9.4\end{array}$ & $\begin{array}{l}116-6.6 \\
116-11.4 \\
115-59.17 \\
116-18.7 \\
116-6.0\end{array}$ & $\begin{array}{c}0.5 \\
10.8 \\
2.9 \\
5.0 * \\
4.9\end{array}$ & $\begin{array}{l}1.2 \\
2.2 .2 .3 \\
1.5 \\
.3 .0\end{array}$ & $\begin{array}{r}10 \\
12 \\
10 \\
7 \\
8\end{array}$ & $\begin{array}{l}112 \\
108 \\
102 \\
301 \\
111\end{array}$ & $\begin{array}{r}1.6 \\
5.1 \\
6.7 \\
28.0 \\
4.2\end{array}$ & $\begin{array}{l}0.07 \\
0.09 \\
0.04 \\
1.53 \\
0.07\end{array}$ & $\begin{array}{l}0.4 \\
0.6 \\
0.6 \\
7.1 \\
0.5\end{array}$ & $\begin{array}{l}0.9 \\
0.8 \\
1.1 \\
1.3\end{array}$ & $\begin{array}{l}0.08 \\
0.08 \\
0.05 \\
0.10 \\
0.06\end{array}$ \\
\hline $\begin{array}{l}14 \\
14 \\
15 \\
15 \\
15\end{array}$ & $\begin{array}{r}21 \\
21 \\
9 \\
10 \\
12\end{array}$ & $\begin{array}{r}6 \\
6 \\
50 \\
7 \\
28\end{array}$ & $\begin{array}{r}22.8 \\
32.6 \\
11.9 \\
11.6 \\
7.5\end{array}$ & $\begin{array}{l}33-7.2 \\
33-7.1 \\
33-4.6 \\
33-18.9 \\
33-17.4\end{array}$ & $\begin{array}{l}116-5.6 \\
116-4.8 \\
116-2.2 \\
116-13.3 \\
116-15.6\end{array}$ & $\begin{array}{l}2.9 \\
2.0 \\
1.3 \\
3.0 * \\
3.0\end{array}$ & $\begin{array}{l}0.5 \\
1.6 \\
1.7 \\
.3 .3 \\
.3 .5\end{array}$ & $\begin{array}{r}6 \\
7 \\
9 \\
12 \\
11\end{array}$ & $\begin{array}{r}80 \\
107 \\
115 \\
207 \\
210\end{array}$ & $\begin{array}{l}0.2 \\
1.4 \\
5.5 \\
5.4 \\
3.7\end{array}$ & $\begin{array}{l}15 \\
10 \\
04 \\
08 \\
12\end{array}$ & $\begin{array}{l}1.5 \\
0.9 \\
0.3 \\
0.5 \\
0.7\end{array}$ & $\begin{array}{l}1.2 \\
1.1 \\
0.6 \\
2.4\end{array}$ & $\begin{array}{l}0.09 \\
0.12 \\
0.05 \\
0.07 \\
0.08\end{array}$ \\
\hline $\begin{array}{l}15 \\
15 \\
15 \\
15 \\
16\end{array}$ & $\begin{array}{r}15 \\
21 \\
21 \\
22 \\
3\end{array}$ & $\begin{array}{l}50 \\
56 \\
58 \\
7 \\
30\end{array}$ & $\begin{array}{r}55.5 \\
50.3 \\
0.2 \\
6.1 \\
29.9\end{array}$ & $\begin{array}{l}33-9.1 \\
33-2.7 \\
33-8.6 \\
33-2.9 \\
33-2.9\end{array}$ & $\begin{array}{l}116-5.9 \\
115-59.3 \\
116-5.7 \\
115-59.0 \\
115-59.2\end{array}$ & $\begin{array}{l}3.9 \\
7.3 \\
1.2 \\
8.2 \\
8.3\end{array}$ & $\begin{array}{r}.9 \\
.3 .4 \\
.3 .3 \\
.4 .8\end{array}$ & $\begin{array}{r}9 \\
10 \\
8 \\
11 \\
13\end{array}$ & $\begin{array}{r}110 \\
219 \\
84 \\
186 \\
186\end{array}$ & $\begin{array}{l}3.5 \\
8.4 \\
2.7 \\
7.3 \\
7.0\end{array}$ & & $\begin{array}{l}0.3 \\
0.5 \\
1.1 \\
0.9 \\
0.6\end{array}$ & $\begin{array}{l}0.9 \\
1.1 \\
2.4 \\
1.7 \\
1.4\end{array}$ & $\begin{array}{l}0.05 \\
0.04 \\
0.12 \\
0.08 \\
0.08\end{array}$ \\
\hline $\begin{array}{l}16 \\
16 \\
16 \\
16 \\
16\end{array}$ & $\begin{array}{r}4 \\
6 \\
6 \\
9 \\
10\end{array}$ & $\begin{array}{l}56 \\
35 \\
37 \\
49 \\
51\end{array}$ & $\begin{array}{l}45.5 \\
31.3 \\
33.4 \\
37.6 \\
34.0\end{array}$ & $\begin{array}{l}33-2.4 \\
33-11.7 \\
33-8.9 \\
33-12.1 \\
33-6.0\end{array}$ & $\begin{array}{l}116-0.3 \\
116-10.1 \\
116-3.5 \\
116-9.3 \\
116-2.1\end{array}$ & $\begin{array}{l}2.9 \\
4.5 \\
4.7 \\
8.3 \\
6.7\end{array}$ & $\begin{array}{l}1.8 \\
1.1 \\
2.0 \\
2.2 \\
1.8\end{array}$ & $\begin{array}{r}11 \\
9 \\
10 \\
10 \\
9\end{array}$ & $\begin{array}{r}197 \\
71 \\
106 \\
77 \\
142\end{array}$ & $\begin{array}{l}5.2 \\
3.1 \\
4.7 \\
3.6 \\
6.0\end{array}$ & $\begin{array}{l}.04 \\
.05 \\
.07 \\
.04 \\
.06\end{array}$ & $\begin{array}{l}0.3 \\
0.3 \\
0.4 \\
0.2 \\
0.3\end{array}$ & $\begin{array}{l}0.3 \\
1.1 \\
1.4 \\
0.5 \\
0.8\end{array}$ & $\begin{array}{l}0.03 \\
0.06 \\
0.06 \\
0.03 \\
0.03\end{array}$ \\
\hline $\begin{array}{l}17 \\
17 \\
17 \\
18 \\
18\end{array}$ & $\begin{array}{r}2 \\
3 \\
22 \\
3 \\
6\end{array}$ & $\begin{array}{r}43 \\
14 \\
1 \\
21 \\
8\end{array}$ & $\begin{array}{r}47.5 \\
25.8 \\
17.2 \\
9.1 \\
25.6\end{array}$ & $\begin{array}{l}33-3.2 \\
33-14.0 \\
33-8.3 \\
33-2.7 \\
33-6.8\end{array}$ & $\begin{array}{l}115-59.6 \\
116-11.3 \\
116-4.9 \\
115-59.4 \\
116-5.0\end{array}$ & $\begin{array}{r}7.9 \\
11.4 \\
7.0 \\
9.2 \\
3.4\end{array}$ & $\begin{array}{l}{ }^{.3 .7} \\
2.2^{.3 .4} \\
2.1 \\
0.2\end{array}$ & $\begin{array}{r}12 \\
12 \\
9 \\
12 \\
8\end{array}$ & $\begin{array}{r}178 \\
80 \\
96 \\
102 \\
72\end{array}$ & $\begin{array}{l}6.6 \\
5.4 \\
7.5 \\
5.5 \\
1.3\end{array}$ & & $\begin{array}{l}0.3 \\
0.3 \\
0.2 \\
0.4 \\
0.2\end{array}$ & $\begin{array}{l}0.7 \\
0.4 \\
0.6 \\
1.1 \\
0.4\end{array}$ & $\begin{array}{l}0.04 \\
0.04 \\
0.03 \\
0.05 \\
0.02\end{array}$ \\
\hline $\begin{array}{l}18 \\
18 \\
18 \\
18 \\
18\end{array}$ & $\begin{array}{l}6 \\
6 \\
6 \\
8 \\
9\end{array}$ & $\begin{array}{r}8 \\
17 \\
18 \\
2 \\
9\end{array}$ & $\begin{array}{l}43.6 \\
25.9 \\
10.5 \\
21.7 \\
13.8\end{array}$ & $\begin{array}{l}33-5.4 \\
33-2.1 \\
33-7.6 \\
33-6.8 \\
33-2.4\end{array}$ & $\begin{array}{l}116-4.0 \\
115-58.3 \\
116-5.3 \\
116-3.8 \\
116-1.3\end{array}$ & $\begin{array}{r}10.8 \\
8.0\end{array}$ & $\begin{array}{l}1.5 \\
1.3 \\
1.6 \\
1.7 \\
1.5\end{array}$ & $\begin{array}{l}12 \\
12 \\
12 \\
12 \\
12\end{array}$ & $\begin{array}{r}65 \\
133 \\
71 \\
65 \\
116\end{array}$ & $\begin{array}{l}4.1 \\
1.0 \\
3.0 \\
3.6\end{array}$ & & $\begin{array}{l}0.2 \\
1.9 \\
0.5 \\
0.1 \\
0.3\end{array}$ & $\begin{array}{l}0.6 \\
4.4 \\
1.3 \\
0.4 \\
0.6\end{array}$ & $\begin{array}{l}0.04 \\
0.10 \\
0.08 \\
0.02 \\
0.03\end{array}$ \\
\hline $\begin{array}{l}18 \\
18 \\
18 \\
18 \\
18\end{array}$ & $\begin{array}{r}9 \\
9 \\
17 \\
19 \\
21\end{array}$ & $\begin{array}{l}28 \\
31 \\
46 \\
28 \\
23\end{array}$ & $\begin{array}{r}26.2 \\
47.9 \\
46.4 \\
8.8 \\
35.7\end{array}$ & $\begin{array}{l}33-2.6 \\
33-15.6 \\
33-11.8 \\
33-9.4 \\
33-8.1\end{array}$ & $\begin{array}{l}115-59.6 \\
116-17.0 \\
116-10.0 \\
116-3.6 \\
116-1.9\end{array}$ & $\begin{array}{l}7.9 \\
0.1 * \\
5.6 \\
5.6 \\
1.2\end{array}$ & $\begin{array}{l}1.8 \\
0.6 \\
1.5 \\
1.6 \\
1.6\end{array}$ & $\begin{array}{r}12 \\
6 \\
10 \\
10 \\
13\end{array}$ & $\begin{array}{r}98 \\
155 \\
84 \\
96 \\
78\end{array}$ & $\begin{array}{l}5.5 \\
4.4 \\
3.3 \\
5.2 \\
6.2\end{array}$ & & $\begin{array}{l}0.4 \\
1.0 \\
0.3 \\
0.1 \\
0.3\end{array}$ & $\begin{array}{l}1.0 \\
0.7 \\
0.6 \\
0.5\end{array}$ & $\begin{array}{l}0.04 \\
0.12 \\
0.04 \\
0.03 \\
0.07\end{array}$ \\
\hline $\begin{array}{l}18 \\
19 \\
19 \\
19 \\
19\end{array}$ & $\begin{array}{l}23 \\
1 \\
6 \\
6 \\
6\end{array}$ & $\begin{array}{l}24 \\
46 \\
11 \\
12 \\
54\end{array}$ & $\begin{array}{r}15.9 \\
15.5 \\
51.1 \\
50.6 \\
7.0\end{array}$ & $\begin{array}{l}32-56.2 \\
33-9.1 \\
33-1.5 \\
33-0.5 \\
33-2.8\end{array}$ & $\begin{array}{r}-16.3 \\
-4.9 \\
-13.6 \\
-57.5 \\
-59.2\end{array}$ & $\begin{array}{c}10.4 \\
7.8 \\
0.2 * \\
4.3 \\
4.8\end{array}$ & $\begin{array}{l}1.1 \\
1.8 \\
1.4 \\
1.2 \\
0.6\end{array}$ & $\begin{array}{l}13 \\
14 \\
14 \\
14 \\
13\end{array}$ & $\begin{array}{r}201 \\
87 \\
159 \\
84 \\
62\end{array}$ & $\begin{array}{r}10.2 \\
3.8 \\
1.7 \\
1.7 \\
5.6\end{array}$ & & $\begin{array}{l}0.5 \\
0.2 \\
0.5 \\
0.2 \\
0.2\end{array}$ & $\begin{array}{l}1.2 \\
0.6 \\
0.5 \\
0.8\end{array}$ & $\begin{array}{l}0.06 \\
0.05 \\
0.10 \\
0.04 \\
0.04\end{array}$ \\
\hline $\begin{array}{l}19 \\
19 \\
19 \\
19 \\
19\end{array}$ & $\begin{array}{r}6 \\
7 \\
8 \\
11 \\
11\end{array}$ & $\begin{array}{r}55 \\
7 \\
7 \\
34 \\
35\end{array}$ & $\begin{array}{r}20.3 \\
27.0 \\
13.5 \\
4.1 \\
57.7\end{array}$ & $\begin{array}{l}32-58.7 \\
33-0.9 \\
33-7.8 \\
32-56.8 \\
33-7.7\end{array}$ & $\begin{array}{l}116-20.1 \\
115-56.6 \\
116-2.1 \\
116-17.1 \\
116-3.8\end{array}$ & $\begin{array}{c}11.0 \\
7.5 \\
1.7 \\
5.3 \\
0.1 *\end{array}$ & $\begin{array}{l}1.2 \\
1.3 \\
1.3 \\
0.8 \\
1.2\end{array}$ & $\begin{array}{r}15 \\
15 \\
15 \\
8 \\
15\end{array}$ & $\begin{array}{r}212 \\
96 \\
73 \\
204 \\
73\end{array}$ & $\begin{array}{r}12.2 \\
3.2 \\
5.6 \\
10.1 \\
3.1\end{array}$ & $\begin{array}{l}0.10 \\
0.06 \\
0.03 \\
0.10 \\
0.04\end{array}$ & $\begin{array}{l}0.5 \\
0.3 \\
0.2 \\
0.6 \\
0.3\end{array}$ & $\begin{array}{l}1.4 \\
0.7 \\
0.4 \\
2.5\end{array}$ & $\begin{array}{l}0.06 \\
0.06 \\
0.06 \\
0.05 \\
0.09\end{array}$ \\
\hline $\begin{array}{l}19 \\
19 \\
19 \\
19 \\
19\end{array}$ & $\begin{array}{l}12 \\
12 \\
12 \\
14 \\
14\end{array}$ & $\begin{array}{l}14 \\
32 \\
34 \\
33 \\
34\end{array}$ & $\begin{array}{l}48.2 \\
28.0 \\
32.0 \\
19.9 \\
15.4\end{array}$ & $\begin{array}{l}33-1.2 \\
33-2.2 \\
33-8.2 \\
33-15.5 \\
33-4.6\end{array}$ & $\begin{array}{l}116-11.2 \\
115-59.2 \\
115-58.8 \\
116-0.8 \\
116-6.9\end{array}$ & $\begin{array}{c}3.4 \\
8.4 \\
4.5 \\
0.3 * \\
10.1\end{array}$ & $\begin{array}{l}0.9 \\
1.9 \\
1.1 \\
1.7 \\
0.6\end{array}$ & $\begin{array}{l}15 \\
15 \\
15 \\
15 \\
15\end{array}$ & $\begin{array}{r}87 \\
65 \\
121 \\
218 \\
52\end{array}$ & $\begin{array}{l}2.5 \\
4.6 \\
3.7 \\
9.5 \\
5.2\end{array}$ & $\begin{array}{l}0.04 \\
0.04 \\
0.07 \\
0.09 \\
0.05\end{array}$ & $\begin{array}{l}0.3 \\
0.2 \\
0.4 \\
0.6 \\
0.2\end{array}$ & $\begin{array}{l}1.1 \\
0.4 \\
1.3\end{array}$ & $\begin{array}{l}0.07 \\
0.03 \\
0.06 \\
0.09 \\
0.05\end{array}$ \\
\hline
\end{tabular}


TABLE 8. - Borrego Mountain aftershocks-Continued

\begin{tabular}{|c|c|c|c|c|c|c|c|c|c|c|c|c|c|c|c|}
\hline & $R$ & $M N$ & SEC & LAT N & LONG $W$ & EPTH & MAG & NO & GAP & DMIN & ERT & RH & ERZ & MD & \\
\hline $\begin{array}{l}R \quad 19 \\
19 \\
19 \\
19 \\
20\end{array}$ & $\begin{array}{l}16 \\
20 \\
20 \\
20 \\
0\end{array}$ & $\begin{array}{l}19 \\
21 \\
22 \\
23 \\
41\end{array}$ & $\begin{array}{r}43.3 \\
36.8 \\
3.6 \\
5.4 \\
21.6\end{array}$ & $\begin{array}{l}33-17.1 \\
33-2.2 \\
33-14.4 \\
33-7.4 \\
33-6.6\end{array}$ & $\begin{array}{l}116-13.8 \\
116-0.9 \\
116-11.4 \\
116-1.7 \\
116-4.0\end{array}$ & $\begin{array}{l}7.4 \\
2.7 \\
8.1 \\
6.2 \\
1.8\end{array}$ & $\begin{array}{l}2.0 \\
1.2 \\
1.4 \\
2.1 \\
1.4\end{array}$ & $\begin{array}{l}13 \\
11 \\
13 \\
13 \\
14\end{array}$ & $\begin{array}{r}202 \\
146 \\
94 \\
66 \\
64\end{array}$ & $\begin{array}{l}2.2 \\
4.0 \\
4.9 \\
7.3 \\
2.9\end{array}$ & $\begin{array}{l}0.09 \\
0.07 \\
0.06 \\
0.04 \\
0.03\end{array}$ & $\begin{array}{l}0.4 \\
0.5 \\
0.3 \\
0.2 \\
0.2\end{array}$ & $\begin{array}{l}0.7 \\
0.7 \\
0.8 \\
0.9 \\
0.4\end{array}$ & $\begin{array}{l}0.05 \\
0.07 \\
0.06 \\
0.04 \\
0.06\end{array}$ & \\
\hline $\begin{array}{l}20 \\
20 \\
20 \\
20 \\
20\end{array}$ & $\begin{array}{l}1 \\
1 \\
1 \\
2 \\
4\end{array}$ & $\begin{array}{r}10 \\
41 \\
56 \\
3 \\
23\end{array}$ & $\begin{array}{l}2.1 \\
44.3 \\
49.1 \\
12.6 \\
30.9\end{array}$ & $\begin{array}{l}33-6.8 \\
33-4.9 \\
33-1.8 \\
33-1.6 \\
33-1.3\end{array}$ & $\begin{array}{l}116-2.7 \\
116-2.8 \\
116-11.0 \\
115-57.6 \\
116-11.1\end{array}$ & $\begin{array}{l}2.0 \\
7.2 \\
2.5 \\
9.2 \\
3.7\end{array}$ & $\begin{array}{l}1.2 \\
1.5 \\
1.2 \\
2.2 \\
0.5\end{array}$ & $\begin{array}{r}14 \\
14 \\
14 \\
15 \\
8\end{array}$ & $\begin{array}{r}64 \\
54 \\
77 \\
83 \\
113\end{array}$ & $\begin{array}{l}4.7 \\
5.8 \\
3.1 \\
3.4 \\
2.8\end{array}$ & $\begin{array}{l}0.03 \\
0.08 \\
0.05 \\
0.03 \\
0.11\end{array}$ & $\begin{array}{l}.2 \\
.5 \\
.3 \\
0.2 \\
.6\end{array}$ & $\begin{array}{l}0.3 \\
1.3 \\
0.6 \\
0.3 \\
1.7\end{array}$ & $\begin{array}{l}0.04 \\
0.08 \\
0.06 \\
0.04 \\
0.04\end{array}$ & \\
\hline $\begin{array}{l}20 \\
20 \\
20 \\
20 \\
20\end{array}$ & $\begin{array}{r}4 \\
4 \\
9 \\
10 \\
14\end{array}$ & $\begin{array}{r}23 \\
23 \\
37 \\
56 \\
7\end{array}$ & $\begin{array}{l}53.6 \\
55.7 \\
37.6 \\
29.4 \\
21.8\end{array}$ & $\begin{array}{l}33-5.1 \\
33-7.1 \\
33-22.0 \\
33-7.2 \\
33-9.3\end{array}$ & $\begin{array}{l}116-2.7 \\
116-5.5 \\
116-14.6 \\
116-1.9 \\
116-5.7\end{array}$ & $\begin{array}{r}8.0 \\
2.9 \\
10.1 \\
2.7 \\
2.3\end{array}$ & $\begin{array}{l}0.9 \\
1.7 \\
2.6 .3 .2 \\
1.5 \\
1.4\end{array}$ & $\begin{array}{l}15 \\
13 \\
15 \\
15 \\
15\end{array}$ & $\begin{array}{r}55 \\
67 \\
114 \\
65 \\
86\end{array}$ & $\begin{array}{l}0 \\
7 \\
6\end{array}$ & $\begin{array}{l}0.03 \\
0.03 \\
0.05 \\
0.04 \\
0.04\end{array}$ & $\begin{array}{l}.2 \\
.2 \\
.4 \\
.2 \\
.2\end{array}$ & $\begin{array}{l}0.5 \\
0.3 \\
0.4 \\
0.4 \\
0.4\end{array}$ & $\begin{array}{l}0.04 \\
0.04 \\
0.07 \\
0.06 \\
0.05\end{array}$ & \\
\hline $\begin{array}{l}20 \\
20 \\
20 \\
20 \\
20\end{array}$ & $\begin{array}{l}14 \\
14 \\
15 \\
16 \\
16\end{array}$ & $\begin{array}{r}8 \\
57 \\
19 \\
38 \\
40\end{array}$ & $\begin{array}{l}53.7 \\
15.9 \\
47.9 \\
35.2 \\
17.6\end{array}$ & $\begin{array}{l}33-1.6 \\
33-8.4 \\
33-5.4 \\
33-16.7 \\
33-16.6\end{array}$ & $\begin{array}{l}115-56.3 \\
116-3.6 \\
116-1.9 \\
116-15.4 \\
116-15.6\end{array}$ & $\begin{array}{l}7.5 \\
1.7 \\
1.1\end{array}$ & $\begin{array}{l}0.6 \\
1.7 \\
1.3 \\
1.3 \\
1.7\end{array}$ & $\begin{array}{l}11 \\
15 \\
15 \\
15 \\
15\end{array}$ & $\begin{array}{r}101 \\
83 \\
62 \\
85 \\
87\end{array}$ & $\begin{array}{l}2.7 \\
2.8\end{array}$ & $\begin{array}{l}0.06 \\
0.03 \\
0.04 \\
0.04 \\
0.03\end{array}$ & & $\begin{array}{l}.2 \\
.6 \\
.6 \\
.5 \\
.3\end{array}$ & $\begin{array}{l}0.06 \\
0.05 \\
0.05 \\
0.06 \\
0.05\end{array}$ & \\
\hline $\begin{array}{l}20 \\
20 \\
20 \\
21 \\
21\end{array}$ & $\begin{array}{r}16 \\
19 \\
23 \\
4 \\
6\end{array}$ & $\begin{array}{r}40 \\
38 \\
21 \\
25 \\
0\end{array}$ & $\begin{array}{r}52.9 \\
3.6 \\
47.4 \\
34.1 \\
49.6\end{array}$ & $\begin{array}{l}33-16.7 \\
33-5.5 \\
33-12.3 \\
33-9.9 \\
33-1.3\end{array}$ & $\begin{array}{l}116-15.6 \\
116-0.9 \\
116-10.5 \\
116-1.6 \\
116-14.2\end{array}$ & $\begin{array}{l}3.7 \\
3 .-1 * \\
7.2\end{array}$ & $\begin{array}{l}1.3 \\
1.8 \\
1.5 \\
2.0 \\
1.3\end{array}$ & $\begin{array}{l}14 \\
15 \\
13 \\
13 \\
15\end{array}$ & $\begin{array}{r}87 \\
71 \\
85 \\
110 \\
164\end{array}$ & $\begin{array}{l}5.0 \\
2.3\end{array}$ & $\begin{array}{l}0.05 \\
0.04 \\
0.03 \\
0.04 \\
0.04\end{array}$ & $\begin{array}{l}.3 \\
.2 \\
.3 \\
.3 \\
.3\end{array}$ & $\begin{array}{l}0.6 \\
0.6 \\
1.5 \\
0.6\end{array}$ & $\begin{array}{l}0.07 \\
0.05 \\
0.07 \\
0.08 \\
0.04\end{array}$ & B \\
\hline $\begin{array}{l}21 \\
21 \\
21 \\
21 \\
21\end{array}$ & $\begin{array}{r}6 \\
7 \\
7 \\
13 \\
14\end{array}$ & $\begin{array}{l}50 \\
48 \\
48 \\
12 \\
12\end{array}$ & $\begin{array}{r}21.2 \\
7.1 \\
38.5 \\
10.1 \\
0.1\end{array}$ & $\begin{array}{l}33-4.1 \\
33-11.6 \\
32-59.6 \\
33-14.0 \\
33-4.1\end{array}$ & $\begin{array}{l}116-2.4 \\
116-9.3 \\
116-14.1 \\
116-11.4 \\
116-2.0\end{array}$ & $\begin{array}{r}8.5 \\
7.8 \\
6.1 \\
11.0 \\
1.1\end{array}$ & $\begin{array}{l}2.3 \\
1.6 \\
0.7 \\
1.9 \\
0.3\end{array}$ & $\begin{array}{r}15 \\
15 \\
11 \\
15 \\
6\end{array}$ & $\begin{array}{r}53 \\
81 \\
171 \\
80 \\
118\end{array}$ & $\begin{array}{l}3.2 \\
5.3 \\
4.8\end{array}$ & $\begin{array}{l}0.03 \\
0.04 \\
0.0 .7 \\
0.03 \\
0.06\end{array}$ & $\begin{array}{l}0.2 \\
0.2 \\
0.4 \\
0.2 \\
0.5\end{array}$ & $\begin{array}{l}0.4 \\
0.5 \\
0.9 \\
0.4 \\
0.9\end{array}$ & $\begin{array}{l}0.04 \\
0.05 \\
0.05 \\
0.05 \\
0.04\end{array}$ & 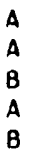 \\
\hline $\begin{array}{l}21 \\
21 \\
21 \\
21 \\
21\end{array}$ & $\begin{array}{l}14 \\
16 \\
16 \\
21 \\
23\end{array}$ & $\begin{array}{r}12 \\
15 \\
16 \\
0 \\
13\end{array}$ & $\begin{array}{r}13.4 \\
1.8 \\
35.5 \\
48.9 \\
28.0\end{array}$ & $\begin{array}{l}33-7.1 \\
33-7.1 \\
33-8.4 \\
33-16.1 \\
33-6.8\end{array}$ & $\begin{array}{l}116-5.1 \\
116-2.5 \\
116-4.6 \\
116-16.1 \\
116-5.2\end{array}$ & & $\begin{array}{l}1.4 \\
0.9 \\
1.6 \\
1.8 \\
1.4\end{array}$ & $\begin{array}{l}15 \\
14 \\
14 \\
13 \\
14\end{array}$ & $\begin{array}{l}67 . \\
66 \\
80 \\
91 \\
64\end{array}$ & $\begin{array}{l}3 \\
8\end{array}$ & & & $\begin{array}{l}0.3 \\
0.5 \\
0.5 \\
2.0 \\
2.9\end{array}$ & $\begin{array}{l}0.05 \\
0.06 \\
0.06 \\
0.17 \\
0.09\end{array}$ & A \\
\hline $\begin{array}{l}21 \\
21 \\
21 \\
22 \\
22\end{array}$ & $\begin{array}{r}23 \\
23 \\
23 \\
3 \\
3\end{array}$ & $\begin{array}{l}14 \\
15 \\
39 \\
42 \\
42\end{array}$ & $\begin{array}{l}33.4 \\
33.9 \\
30.8 \\
11.1 \\
32.6\end{array}$ & $\begin{array}{l}33-7.6 \\
33-1.7 \\
3.3-2.6 \\
33-7.7 \\
33-2.7\end{array}$ & $\begin{array}{l}116-2.5 \\
115-59.3 \\
115-59.0 \\
115-58.5 \\
116-1.3\end{array}$ & & $\begin{array}{l}0.4 \\
0.2 \\
1.7 \\
0.6 \\
1.4\end{array}$ & $\begin{array}{r}8 \\
7 \\
13 \\
12 \\
14\end{array}$ & $\begin{array}{r}102 \\
73 \\
65 \\
127 \\
49\end{array}$ & $\begin{array}{l}7.9 \\
3.8\end{array}$ & & & $\begin{array}{l}1.1 \\
1.2 \\
0.4 \\
1.1 \\
0.3\end{array}$ & $\begin{array}{l}0.06 \\
0.05 \\
0.03 \\
0.05 \\
0.05\end{array}$ & $\begin{array}{l}\text { A } \\
B \\
A\end{array}$ \\
\hline $\begin{array}{l}22 \\
22 \\
22 \\
22 \\
22\end{array}$ & $\begin{array}{l}4 \\
4 \\
6 \\
6 \\
6\end{array}$ & $\begin{array}{r}1 \\
5 \\
36 \\
37 \\
37\end{array}$ & $\begin{array}{l}55.1 \\
12.8 \\
28.5 \\
45.0 \\
47.2\end{array}$ & $\begin{array}{l}33-1.8 \\
33-8.5 \\
33-2.9 \\
33-1.5 \\
33-1.3\end{array}$ & $\begin{array}{r}59.2 \\
6.1 \\
59.3 \\
49.4 \\
49.6\end{array}$ & & $\begin{array}{l}1.3 \\
1.4 \\
1.1 \\
0.5 \\
2.5 .2 .7\end{array}$ & $\begin{array}{l}15 \\
14 \\
11 \\
10 \\
12\end{array}$ & $\begin{array}{r}66 \\
78 \\
60 \\
208 \\
205\end{array}$ & & & & $\begin{array}{l}0.5 \\
0.7 \\
0.5 \\
0.6 \\
0.5\end{array}$ & $\begin{array}{l}0.04 \\
0.04 \\
0.03 \\
0.06 \\
0.07\end{array}$ & \\
\hline $\begin{array}{l}22 \\
22 \\
22 \\
22 \\
22\end{array}$ & $\begin{array}{l}7 \\
7 \\
13 \\
17 \\
18\end{array}$ & $\begin{array}{l}9 \\
35 \\
17 \\
33 \\
59\end{array}$ & $\begin{array}{r}6.0 \\
0.1 \\
14.3 \\
35.7 \\
55.5\end{array}$ & $\begin{array}{l}33-2.5 \\
33-7.8 \\
33-3.7 \\
33-3.5 \\
33-7.5\end{array}$ & $\begin{array}{l}116-0.1 \\
116-6.2 \\
116-1.3 \\
116-1.8 \\
116-5.4\end{array}$ & $\begin{array}{l}1.2 \\
3.8\end{array}$ & $\begin{array}{l}2.2,2 . \\
1.2 \\
1.6 \\
1.0 \\
1.1\end{array}$ & $\begin{array}{l}15 \\
15 \\
14 \\
13 \\
14\end{array}$ & $\begin{array}{l}56 . \\
72 \\
56 \\
96 \\
71\end{array}$ & 0.8 & $\begin{array}{l}0.03 \\
0.02 \\
0.02 \\
0.04 \\
0.02\end{array}$ & & $\begin{array}{l}0.6 \\
0.5 \\
0.8 \\
0.5 \\
0.5\end{array}$ & $\begin{array}{l}0.05 \\
0.04 \\
0.04 \\
0.05 \\
0.04\end{array}$ & 4 \\
\hline $\begin{array}{l}22 \\
23 \\
23 \\
23 \\
23\end{array}$ & $\begin{array}{r}22 \\
3 \\
8 \\
8 \\
8\end{array}$ & $\begin{array}{r}27 \\
1 \\
23 \\
27 \\
29\end{array}$ & $\begin{array}{r}35.4 \\
26.9 \\
30.3 \\
41.1 \\
4.4\end{array}$ & $\begin{array}{l}33-8.6 \\
33-1.8 \\
33-11.6 \\
33-1.7 \\
33-9.0\end{array}$ & $\begin{array}{l}116-4.5 \\
116-11.0 \\
116-9.3 \\
115-56.1 \\
116-7.4\end{array}$ & & $\begin{array}{l}1.4 \\
0.9 \\
1.2 \\
0.4 \\
0.7\end{array}$ & $\begin{array}{l}15 \\
14 \\
16 \\
16 \\
16\end{array}$ & $\begin{array}{r}83 \\
70 \\
77 \\
105 \\
75\end{array}$ & 3.6 & & & $\begin{array}{l}0.6 \\
0.6 \\
0.6 \\
1.2 \\
0.4\end{array}$ & $\begin{array}{l}0.04 \\
0.07 \\
0.06 \\
0.07 \\
0.07\end{array}$ & \\
\hline $\begin{array}{l}23 \\
23 \\
23 \\
23 \\
23\end{array}$ & $\begin{array}{r}8 \\
9 \\
9 \\
10 \\
11\end{array}$ & $\begin{array}{l}32 \\
32 \\
32 \\
19 \\
19\end{array}$ & $\begin{array}{r}52.3 \\
6.8 \\
21.7 \\
7.0 \\
5.9\end{array}$ & $\begin{array}{l}33-13.9 \\
33-1.1 \\
33-1.6 \\
33-1.0 \\
33-1.9\end{array}$ & $\begin{array}{l}116-14.4 \\
116-14.2 \\
116-14.4 \\
116-0.5\end{array}$ & $\begin{array}{l}1.4 \\
0.2 \\
2.1 \\
5.1\end{array}$ & $\begin{array}{l}1.7 \\
1.1 \\
0.9 \\
1.3 \\
.2\end{array}$ & $\begin{array}{l}16 \\
16 \\
15 \\
16 \\
16\end{array}$ & $\begin{array}{l}178 \\
166 \\
164 \\
166 \\
56\end{array}$ & $\begin{array}{l}2.6 \\
2.5 \\
2.5 \\
4.7\end{array}$ & $\begin{array}{l}0.05 \\
0.05 \\
0.08 \\
0.06 \\
0.02\end{array}$ & $\begin{array}{l}0.4 \\
0.3 \\
0.6 \\
0.3 \\
0.1\end{array}$ & $\begin{array}{l}0.5 \\
0.5\end{array}$ & $\begin{array}{l}0.07 \\
0.05 \\
0.11 \\
0.07 \\
0.04\end{array}$ & A \\
\hline
\end{tabular}


TABLE 8. - Borrego Mountain aftershocks-Continued

1968 HR MN SEC LAT N LONG W DEPTH MAG NO GAP DMIN ERT ERH ERZ MD 0

APR $23 \quad 1120 \quad 42.6 \quad 33-2.6 \quad 115-59.7$ $231317 \quad 54.4 \quad 33-16.2116-0.0$ $\begin{array}{llllllll}23 & 13 & 20 & 23.1 & 33-2.9 & 115-55.4\end{array}$ $2313 \quad 3748.2 \quad 33-0.2 \quad 116-14.9$ $23162122.8 \quad 33-8.1116-2.0$

$5.6 \quad 0.7$

$\begin{array}{ll}0.4 & 0.9 \\ 5.0 & 1.7\end{array}$

$5.0 \quad 1.7$

$0.0 * 0.8$

$2.0 \quad 0.3$

$\begin{array}{llllllll}23 & 16 & 46 & 9.7 & 33-9.6 & 116-6.7\end{array}$ $23 \quad 1648 \quad 35.1 \quad 33-16.5 \quad 116-16.2$ $23 \quad 164954.2 \quad 33-1.8 \quad 115-58.0$ $\begin{array}{llllll}23 & 19 & 12 & 3.4 & 32-56.5 & 116-15.7\end{array}$ $\begin{array}{lllllll}23 & 19 & 39 & 13.9 & 33-2.4 & 115-59.7\end{array}$

$232041 \quad 51.3 \quad 33-10.3 \quad 116-8.3$ $\begin{array}{llllll}23 & 20 & 45 & 9.6 & 33-7.6 & 116-2.2\end{array}$ $\begin{array}{llllll}23 & 22 & 8 & 37.2 & 33-7.6 & 116-2.2\end{array}$ $24 \quad 0 \quad 28 \quad 42.0 \quad 33-9.1 \quad 116-4.2$ $2435946.9 \quad 33-7.6 \quad 116-2.6$

$\begin{array}{llllll}24 & 4 & 1 & 1.7 & 33-18.2 & 116-15.8\end{array}$

$\begin{array}{lllllll}24 & 4 & 12 & 22.7 & 33-0.6 & 115-55.7 \\ 24 & 4 & 12 & 41.6 & 33-6.0 & 116-3.4\end{array}$

$\begin{array}{rrrrrrr}24 & 4 & 12 & 41.6 & 33-6.0 & 116-3.4 \\ 24 & 4 & 13 & 2.4 & 33-0.4 & 116-15.0\end{array}$

$24 \quad 4 \quad 16 \quad 12.4 \quad 33-0.3 \quad 116-14.8$

$24 \quad 4 \quad 17 \quad 31.9 \quad 33-0.1 \quad 116-14.9$

$\begin{array}{llllllll}24 & 7 & 29 & 34.7 & 33-8.1 & 116-4.9\end{array}$

$2473027.6 \quad 33-6.9116-5.1$

$2474923.2 \quad 33-16.6 \quad 116-15.2$

$2474934.5 \quad 33-11.9 \quad 116-9.6$

$2474956.3 \quad 33-7.6 \quad 116-2.2$

$\begin{array}{llllll}24 & 8 & 23 & 4.2 & 33-16.9 & 116-13.5\end{array}$

$\begin{array}{lllllll}24 & 8 & 23 & 58.5 & 33-17.1 & 116-13.3\end{array}$

$2490015.1 \quad 33-6.4 \quad 116-3.2$

$24912.23 .3-8.0116-5.3$

$2493 \quad 12.5 \quad 33-8.0116-5.3$

$24994 \quad 3.7 \quad 33-8.1116-5.5$

$2495 \quad 6.5 \quad 33-8.1116-5.1$

$24 \quad 10 \quad 13 \quad 13.4 \quad 33-1.5 \quad 116-1.6$

$\begin{array}{llllll}24 & 12 & 33 & 46.7 & 33-17.5 & 115-57.9\end{array}$

$\begin{array}{llllll}24 & 13 & 6 & 26.7 & 33-17.6 & 115-58.0\end{array}$

$24 \quad 13 \quad 6 \quad 55.6 \quad 33-3.4 \quad 116-1.1$

$2422954.2 \quad 33-3.4 \quad 116-1.7$

$\begin{array}{llllll}24 & 22 & 10 & 31.8 & 33-3.1 & 116-1.9\end{array}$

$\begin{array}{lllllll}24 & 23 & 39 & 18.0 & .33 & 8.6 \quad 116-3.9\end{array}$

$24233941.6 \quad 33-0.8 \quad 116-13.8$

2540.55 .5

2541048.8

$\begin{array}{llll}25 & 4 & 34 & 44.5\end{array}$

$\begin{array}{llll}25 & 4 & 35 & 48.7\end{array}$

$\begin{array}{lllll}25 & 6 & 57 & 46.1\end{array}$

$\begin{array}{llll}25 & 7 & 33 & 16.3\end{array}$

$\begin{array}{lllll}25 & 10 & 11 & 30.7\end{array}$

$\begin{array}{lllll}25 & 10 & 12 & 36.6\end{array}$

$\begin{array}{llll}25 & 10 & 13 & 38.4\end{array}$

$\begin{array}{llll}25 & 10 & 14 & 24.4\end{array}$

$\begin{array}{llll}25 & 20 & 59 & 26.3\end{array}$

25210045.1

$\begin{array}{llll}25 & 23 & 5 & 12.3\end{array}$

$\begin{array}{llll}25 & 23 & 7 & 27.8\end{array}$

$\begin{array}{llll}27 & 9 & 32 & 30.0\end{array}$

$\begin{array}{llll}27 & 19 & 9 & 10.4\end{array}$

$\begin{array}{llll}27 & 20 & 41 & 24.2\end{array}$

$\begin{array}{llll}27 & 22 & 50 & 32.7\end{array}$

$28 \quad 4 \quad 1113.3$

6.01 .4

$3.6 \quad 0.8$

$8.7 \quad 0.4$

10.51 .5

5.31 .7

5.10 .9

$6.7 \quad 1.5$

$1.8 \quad 1.5$

8.11 .9

1.20 .5

$10.0 \quad 1.8$

$\begin{array}{ll}7.4 & 0.7\end{array}$

$2.8 \quad 1.0$

6.10 .7

$\begin{array}{ll}2.4 & 0.2\end{array}$

$2.5 \quad 1.2$

$5.2 \quad 1.5$

$2.6 \quad 0.9$

2.80 .5

7.10 .9

$1.2 \quad 1.9$

$0.2 * 0.7$

3.70 .3

$2.0 * 1.6$

$\begin{array}{llll}0.8 & 116-13.8 & 2.8 & 1.0 \\ 8.3 & 116-4.7 & 3.2 & 0.0\end{array}$

$33-19.2116-9.8 \quad 10.0 \quad 1.6$

33-16.8 116-14.5 $3.2 \quad 2.2$

$3.3-5.2115-49.9 ? 2.0 * 0.9$

$33-9.9116-6.4$

$33-10: 8 \quad 116-2.2 ?$

$33-7.8116-2.8$

$2.7 \quad 1.4$

$1.8 \quad 1.6$

$8.6 \quad 0.3$

$\begin{array}{ll}2.1 & 1.7\end{array}$

$33-1.9116-1.1 ? \quad 7.8 \quad 1.6$

$33-8.1 \quad 116-4.7$

$33-1.7115-57.0$

$33-8.4116-4.3$

$33-5.3116-3.1$

5.9. 0.5

$\begin{array}{ll}5.9 & 0.5 \\ 6.4 & 1.5\end{array}$

$2.9-0.0$

$33-11.9116-9.4$

$33-15.1115-58.3$

$32-56.1116-15.6$

$33-2.5116-1.1$
$5.4 \cdot 1.6$

$\begin{array}{lll}16 & 59 & 5.5\end{array}$

$\begin{array}{lll}16 & 189 & 10.2\end{array}$

$\begin{array}{lll}7116 & 6.0\end{array}$

0.13
0.07

$\begin{array}{llll}16 & 83 & 4.1 & 0.03\end{array}$

$\begin{array}{llll}12 & 92 & 3.7 & 0.03\end{array}$

$\begin{array}{llll}12 & 81 & 3.6 & 0.09\end{array}$

$\begin{array}{llll}16 & 197 & 9.4 & 0.07\end{array}$

$\begin{array}{llll}16 & 59 & 5.3 & 0.03\end{array}$

$$
16
$$

$\begin{array}{llll}16 & 79 & 1.5 & 0.03 \\ 15 & 71 & 5.5 & 0.04\end{array}$

$\begin{array}{llll}16 & 72 & 5.6 & 0.03\end{array}$

$\begin{array}{llll}16 & 89 & 4.2 & 0.04\end{array}$

$\begin{array}{llll}16 & 72 & 4.9 & 0.04\end{array}$

$\begin{array}{llll}16 & 88 & 5.0 & 0.03\end{array}$

$\begin{array}{llll}15 & 108 & 4.3 & 0.06\end{array}$

$\begin{array}{llll}16 & 58 & 4.1 & 0.02\end{array}$

$\begin{array}{llll}10 & 173 & 3.6 & 0.07\end{array}$

11172

$5.7 \quad 1.2$

$\begin{array}{llll}16 & 174 & 3.6 & 0.07\end{array}$

$\begin{array}{llll}16 & 78 & 2.2 & 0.02\end{array}$

$\begin{array}{llll}16 & 66 & 1.0 & 0.02\end{array}$

$\begin{array}{llll}14 & 83 & 2.3 & 0.05\end{array}$

$\begin{array}{llll}15 & 75 & 3.2 & 0.03\end{array}$

$\begin{array}{llll}13 & 72 & 5.5 & 0.07\end{array}$

$\begin{array}{llll}16 & 82 & 1.8 & 0.03\end{array}$

$\begin{array}{llll}6 & 118 & 2.3 & 0.05\end{array}$

$1460 \quad 4.1 \quad 0.03$

$\begin{array}{llll}16 & 74 & 1.6 & 0.03\end{array}$

$\begin{array}{lllllll}5.7 & 03.4 & 16 & 75 & 1.6 & 0.02\end{array}$

$\begin{array}{llllll}5.3 & 2.0 & 14 & 76 & 1.8 & 0.03\end{array}$

$\begin{array}{llllll}5.5 & 1.3 & 16 & 76 & 1.9 & 0.02\end{array}$

$\begin{array}{llllll}7.8 & 1.6 & 16 & 57 & 3.0 & 0.03\end{array}$

$$
16
$$

$2.0 * 0.5 \quad 15201 \quad 12.9 \quad 0.13$

$\begin{array}{llllll}5.3 & 1.7 & 16 & 55 & 4.7 & 0.02\end{array}$

$\begin{array}{lllllll}1.9 & .3 .0 & 16 & 52 & 4.1 & 0.03\end{array}$

$\begin{array}{llllll}1.4 & 1.9 & 15 & 50 & 8.3 & 0.06\end{array}$

$\begin{array}{llllll}1.9 & 0.1 & 12 & 84 & 3.8 & 0.06\end{array}$

$\begin{array}{llll}15 & 165 & 1.7 & 0.06\end{array}$

$\begin{array}{llll}8 & 135 & 2.6 & 0.05\end{array}$

$\begin{array}{llll}16 & 132 & 6.2 & 0.04\end{array}$

$\begin{array}{llll}16 & 78 & 1.7 & 0.04\end{array}$

$\begin{array}{llll}8 & 281 & 16.3 & 1.88\end{array}$

$\begin{array}{llll}16 & 88 & 4.5 & 0.04\end{array}$

$\begin{array}{llll}16 & 126 & 6.0 & 0.06\end{array}$

$\begin{array}{llll}15 & 74 & 4.7 & 0.06\end{array}$

$\begin{array}{llll}16 & 71 & 1.2 & 0.03\end{array}$

$\begin{array}{llll}16 & 54 & 3.7 & 0.23\end{array}$

$\begin{array}{lll}2.6 & 1.5 & \\ 1.6 & .2 .9 & 16\end{array}$

$\begin{array}{llll}10 & 77 & 2.3 & 0.04\end{array}$

$1590 \quad 3.9 \quad 0.04$

$1181 \quad 3.0 \quad 0.04$

$\begin{array}{llll}16 & 53 & 5.4 & 0.08\end{array}$

$\begin{array}{lllllll}5.1 & 2.3 .3 .0 & 15 & 76 & 3.3 & 0.03\end{array}$

$0.3 * \quad .3 .116 \quad 193 \quad 9.2 \quad 0.13$

$2.8 * 1.0 \quad 15198 \quad 9.90 .06$

$2.1 \quad 1.9,2.9 \quad 15 \quad 51 \quad 3.9 \quad 0.03$

$\begin{array}{llllll}7.6 & 1.8 & 14 & 64 & 6.3 & 0.04\end{array}$ $\begin{array}{lllll}0.3 & 0.9 & 0.06 \quad A\end{array}$ $0.6 \quad 1.00 .08 \mathrm{C}$ $\begin{array}{llll}0.5 & 1.6 & 0.09 & 8\end{array}$ $\begin{array}{lllll}0.6 & 0.8 & 0.06 & B\end{array}$

$\begin{array}{lllll}0.2 & 0.6 & 0.05 \quad A\end{array}$ $\begin{array}{lllllllllll}0.2 & 0.9 & 0.05 & A\end{array}$ $\begin{array}{llllll}0.5 & 1.0 & 0.06 & B\end{array}$ $\begin{array}{lllll}0.4 & 0.6 & 0.06 \quad 8\end{array}$

$\begin{array}{llll}0.2 & 0.5 & 0.05 \quad A\end{array}$ $\begin{array}{llllllllllll}0.2 & 0.7 & 0.05 & A\end{array}$ $\begin{array}{llll}0.2 & 0.3 & 0.04 & B\end{array}$ $\begin{array}{llllllll}0.2 & 0.5 & 0.05 & A\end{array}$ $\begin{array}{llllll}0.2 & 0.4 & 0.07 & A\end{array}$

$\begin{array}{llll}0.2 & 0.3 & 0.04 \quad A\end{array}$ $\begin{array}{llllll}0.4 & 0.8 & 0.06 & A\end{array}$ $\begin{array}{lllll}0.2 & 1.4 & 0.05 & A\end{array}$ $\begin{array}{llll}0.5 & 1.0 & 0.05 & B\end{array}$ $\begin{array}{llll}0.4 & 0.4 & 0.04 \mathrm{~B}\end{array}$

$\begin{array}{llll}C .4 & 0.5 & 0.06 \quad B\end{array}$ $\begin{array}{lllll}0.2 & 0.5 & 0.05 & A\end{array}$ $0.10 .20 .04 \mathrm{~A}$ $\begin{array}{lllll}0.3 & 0.4 & 0.06 & A\end{array}$ $\begin{array}{lllll}0.2 & 0.7 & 0.06 & \text { A }\end{array}$

$\begin{array}{lllll}0.4 & 1.0 & 0.07 \quad A\end{array}$ $\begin{array}{llll}0.2 & 0.3 & 0.06 & \text { A }\end{array}$ $0.4 \quad 0.05 \mathrm{C}$ $\begin{array}{llll}0.2 & 1.0 & 0.05 & \mathrm{~A}\end{array}$ $\begin{array}{lll}2 & 0.5 & 0.05 A\end{array}$

$\begin{array}{llll}0.2 & 0.5 & 0.05 \text { A }\end{array}$ $\begin{array}{lllll}0.2 & 0.6 & 0.05 & A\end{array}$ $\begin{array}{lllll}0.2 & 0.5 & 0.04 & \text { A }\end{array}$ $\begin{array}{llllll}0.2 & 0.4 & 0.04 & A\end{array}$ $0.6 \quad 0.09 \mathrm{C}$

$0.11 \mathrm{C}$ $0.50 .03 \mathrm{~A}$ 20.30 .05 A $\begin{array}{llll}.4 & 0.7 & 0.07 & B\end{array}$ $\begin{array}{llll}.4 & 0.6 & 0.08 \text { A }\end{array}$

$\begin{array}{llll}0.3 & 0.4 & 0.06 & B\end{array}$ $\begin{array}{lll}0.4 & 1.1 & 0.0 .5\end{array}$ $\begin{array}{llll}0.3 & 0.4 & 0.06 & B\end{array}$ $\begin{array}{lll}0.3 & 0.8 & 0.06 \mathrm{~A}\end{array}$ 0.330

$\begin{array}{llll}.3 & 0.4 & 0.06 & A\end{array}$ $\begin{array}{llll}0.3 & 0.6 & 0.08 & B\end{array}$ $\begin{array}{llll}0.3 & 0.9 & 0.08 & A\end{array}$ $\begin{array}{llll}.2 & 0.3 & 0.06 \quad A\end{array}$ .23 .10 .26 B 
TABLE 8. - Borrego Mountain aftershocks-Continued

1968 HR MN SEC LAT $N$ LONG $W$ DEPTH MAG NO GAP DMIN ERT ERH ERZ MD 0

APR $28 \quad 1930 \quad 51.6$ 28194852.3 28194951.9 28195012.0 $281951 \quad 15.6$

$2823 \quad 3335.5$ $\begin{array}{llll}29 & 0 & 52 & 51.2\end{array}$ $\begin{array}{llll}29 & 1 & 3 & 0.5\end{array}$ $\begin{array}{llll}29 & 2 & 5 & 31.9\end{array}$ $\begin{array}{llll}29 & 3 & 25 & 31.7\end{array}$

$\begin{array}{llll}29 & 3 & 25 & 59.7\end{array}$ $\begin{array}{llll}29 & 3 & 26 & 49.0\end{array}$ $\begin{array}{lllr}29 & 3 & 27 & 9.9\end{array}$ $\begin{array}{llll}29 & 11 & 49 & 58.4\end{array}$

$\begin{array}{llll}29 & 12 \quad 37 \quad 16.5\end{array}$ $\begin{array}{lllll}29 & 12 & 58 & 13.6\end{array}$ $\begin{array}{llll}29 & 2033 & 24.5\end{array}$ 29203349.6 $292036 \quad 36.2$

$2923 \quad 0 \quad 20.0$ $\begin{array}{llrrr}29 & 23 & 0 & 52.9 \\ 30 & 4 & 14 & 46.6\end{array}$ $\begin{array}{llll}30 & 9 & 56 & 27.4\end{array}$ $\begin{array}{llll}30 & 12 & 39 & 49.0\end{array}$

$\begin{array}{llll}30 & 12 & 40 & 42.2\end{array}$ $\begin{array}{lllll}30 & 16 & 46 & 5.7\end{array}$ $\begin{array}{lllll}30 & 16 & 48 & 16.5\end{array}$ MAY $11136 \quad 24.7$

1113637.1

$\begin{array}{llll}120 & 13 & 12.7\end{array}$ $\begin{array}{llll}12014 & 0.2\end{array}$

$\begin{array}{llll}1 & 20 & 16 & 19.1\end{array}$

$\begin{array}{llll}1 & 20 & 17 & 39.7\end{array}$

1222756.7

1233024.1

123536.1

123.5337 .2

$\begin{array}{llll}1 & 2354 & 20.9\end{array}$

$\begin{array}{llll}2 & 0 & 35 & 6.4\end{array}$

$\begin{array}{llll}2 & 0 & 39 & 18.0\end{array}$ $\begin{array}{llll}2 & 0 & 55 & 30.0\end{array}$ $\begin{array}{llll}2 & 3 & 11 & 17.7\end{array}$ $\begin{array}{llll}2 & 23 & 19 & 26.0\end{array}$

$\begin{array}{llll}3 & 5 & 9 & 44.6\end{array}$

$\begin{array}{llll}3 & 5 & 10 & 25.1\end{array}$ $\begin{array}{llll}3 & 7 & 21 & 29.0\end{array}$ $\begin{array}{llll}3 & 10 & 42 & 7.6\end{array}$ $\begin{array}{llll}3 & 12 & 15 & 40.3\end{array}$

$\begin{array}{llll}3 & 23 & 27 & 3.5\end{array}$

$\begin{array}{llll}3 & 23 & 29 & 27.8\end{array}$ $\begin{array}{llll}3 & 23 & 31 & 7.5\end{array}$

415851.5

444044.6

$4 \quad 4 \quad 42 \quad 9.9$

$4 \quad 83545.0$ $\begin{array}{llll}4 & 15 & 18 & 11.2\end{array}$

$\begin{array}{llll}4 & 1519 & 58.5\end{array}$

$\begin{array}{llll}4 & 152138.0\end{array}$

$42156 \quad 48.1$
$33-15.8 \quad 115-59.8$

$33-1.0 \quad 115-56.0$

33- $9.3116-5.7$

$33-9.4116-5.7$

$33-9.1116-5.5$

$33-3.6 \quad 116-1.1$ $33-6.9116-4.7$ $33-6.4116-3.4$ $33-9.1116-5.4$ $33-4.5116-3.2$

$33-2.4 \quad 115-57.0$ $33-6.0116-3.2$ $33-7.1116-5.3$ ? $33-5.9116-1.7$ $3.3-16.9116-14.1$

33- $1.3 \quad 115-56.4$ $33-9.8116-4.6$ $33-6.3116-2.8$ $33-9.4116-6.3$ $33-8.0116-6.5$

33-14.8 115-58.3 $33-15.1115-57.9$ $33-4.6116-3.2$ $33-16.8 \quad 116-16.4$ 33- $2.9115-55.6$

33- $2.9115-55.7$ $33-5.3116-3.0$ 33-12.0 116-9.9 $33-8.6116-4.2$ $33-8.6116-4.2$

33-19.7 116-10.8 $33-2.3115-56.9$ $33-7.5116-4.7$ $33-2.4115-56.8$ $33-4.2116-1.4$

$33-4.6116-2.1$ $33-1.4116-1.5$ 33-15.1 115-58.0 $33-15.2115-58.0$ $33-17.3116-15.5$

33-15.4 115-58.2 $33-17.4 \quad 116-15.6$ $33-15.5115-58.3$ $33-9.0116-4.8$ $33-7.7116-7.0$

$33-3.0116-0.9$ $33-2.8116-0.7$ 33- $1.8115-59.7$ $33-1.8 \quad 115-59.8$ $33-6.1116-1.9$

33- $1.3 \quad 115-56.2$ $33-20.1116-18.5$ $33-7.9115-59.6$ $33-7.8116-2.3$ $33-17.1 \quad 116-15.7$

33-11.7 116-9.7 $33-18.8 \quad 116-13.3$ $33-2.3116-2.3$ $33-17.6 \quad 116-15.1$ $33-2.0116-0.1$

\begin{tabular}{|c|c|c|c|c|c|c|c|c|c|}
\hline $\begin{array}{l}1.2 \\
7.9 \\
2.1 \\
2.1 \\
2.0\end{array}$ & $\begin{array}{r}1.6 \\
0.7 \\
0.3 \\
1.1 \\
-0.0\end{array}$ & $\begin{array}{r}15 \\
14 \\
9 \\
15 \\
7\end{array}$ & $\begin{array}{r}188 \\
104 \\
98 \\
87 \\
150\end{array}$ & $\begin{array}{r}10.6 \\
4.1 \\
4.0 \\
4.2 \\
3.5\end{array}$ & $\begin{array}{l}0.09 \\
0.06 \\
0.04 \\
0.03 \\
0.05\end{array}$ & $\begin{array}{l}0.5 \\
0.3 \\
0.3 \\
0.2 \\
0.4\end{array}$ & $\begin{array}{l}0.7 \\
0.7 \\
0.4 \\
0.3 \\
0.5\end{array}$ & $\begin{array}{l}0.07 \\
0.05 \\
0.04 \\
0.05 \\
0.04\end{array}$ & $\begin{array}{l}B \\
A \\
A \\
A \\
B\end{array}$ \\
\hline $\begin{array}{l}3.0 \\
3.3 \\
2.7 \\
7.4 \\
5.3\end{array}$ & $\begin{array}{l}1.4 \\
1.2^{3.0} \\
1.3 \\
-0.1\end{array}$ & $\begin{array}{l}14 \\
16 \\
15 \\
16 \\
9\end{array}$ & $\begin{array}{r}56 \\
66 \\
113 \\
85 \\
124\end{array}$ & $\begin{array}{l}1.5 \\
1.6 \\
3.8 \\
3.6 \\
2.5\end{array}$ & $\begin{array}{l}0.02 \\
0.02 \\
0.03 \\
0.03 \\
0.05\end{array}$ & $\begin{array}{l}0.2 \\
0.2 \\
0.2 \\
0.2 \\
0.3\end{array}$ & $\begin{array}{l}0.2 \\
0.5 \\
0.2 \\
0.5 \\
0.6\end{array}$ & $\begin{array}{l}0.04 \\
0.04 \\
0.04 \\
0.05 \\
0.04\end{array}$ & B \\
\hline $\begin{array}{l}.99 \\
.6 \\
.6 \\
.09 \\
.5\end{array}$ & $\begin{array}{l}1.9 \\
0.1 \\
0.0 \\
1.7^{.2 .7}\end{array}$ & $\begin{array}{r}17 \\
6 \\
12 \\
18 \\
18\end{array}$ & $\begin{array}{r}88 \\
100 \\
110 \\
65 \\
80\end{array}$ & $\begin{array}{l}5.0 \\
4.1 \\
0.6 \\
3.1 \\
1.6\end{array}$ & $\begin{array}{l}0.05 \\
0.04 \\
0.16 \\
0.03 \\
0.04\end{array}$ & $\begin{array}{l}0.3 \\
0.3 \\
0.4 \\
0.2 \\
0.3\end{array}$ & $\begin{array}{l}0.7 \\
0.4 \\
0.5 \\
0.5 \\
0.3\end{array}$ & $\begin{array}{l}0.05 \\
0.03 \\
0.10 \\
0.05 \\
0.05\end{array}$ & $\begin{array}{l}A \\
A\end{array}$ \\
\hline $\begin{array}{l}6.0 \\
0.3 \\
2.3 \\
1.9 \\
1.4\end{array}$ & $\begin{array}{l}1.6 \\
1.6 \\
0.7 \\
0.8 \\
1.2\end{array}$ & $\begin{array}{l}18 \\
18 \\
17 \\
17 \\
18\end{array}$ & $\begin{array}{r}100 \\
97 \\
59 \\
84 \\
71\end{array}$ & $\begin{array}{l}4.0 \\
5.2 \\
4.1 \\
4.1 \\
1.9\end{array}$ & $\begin{array}{l}0.03 \\
0.04 \\
0.02 \\
0.03 \\
0.03\end{array}$ & $\begin{array}{l}0.2 \\
0.2 \\
0.2 \\
0.2 \\
0.2\end{array}$ & $\begin{array}{l}0.5 \\
0.5 \\
0.2 \\
0.3 \\
0.3\end{array}$ & $\begin{array}{l}0.04 \\
0.05 \\
0.05 \\
0.06 \\
0.05\end{array}$ & $\begin{array}{l}A \\
A \\
A\end{array}$ \\
\hline $\begin{array}{l}0.3 * \\
0.2 \\
6.1 \\
4.9 \\
4.8\end{array}$ & $\begin{array}{l}1.0 \\
1.9 \\
1.5 \\
1.9 \\
0.6\end{array}$ & $\begin{array}{l}17 \\
18 \\
18 \\
18 \\
18\end{array}$ & $\begin{array}{r}193 \\
195 \\
51 \\
131 \\
114\end{array}$ & $\begin{array}{l}8.6 \\
9.1 \\
2.5 \\
4.1 \\
4.2\end{array}$ & $\begin{array}{l}0.08 \\
0.12 \\
0.03 \\
0.03 \\
0.06\end{array}$ & $\begin{array}{l}0.5 \\
0.6 \\
0.2 \\
0.2 \\
0.3\end{array}$ & $\begin{array}{l}0.9 \\
0.4 \\
0.6 \\
1.0\end{array}$ & $\begin{array}{l}0.08 \\
0.09 \\
0.04 \\
0.04 \\
0.07\end{array}$ & . \\
\hline $\begin{array}{l}5.2 \\
4.8 \\
3.0 \\
2.2 \\
1.6\end{array}$ & $\begin{array}{l}1.7 \\
1.4 \\
0.7 \\
1.1 \\
1.5\end{array}$ & $\begin{array}{l}18 \\
13 \\
11 \\
17 \\
18\end{array}$ & $\begin{array}{r}111 \\
85 \\
108 \\
46 \\
47\end{array}$ & $\begin{array}{l}4.3 \\
2.8 \\
2.9 \\
3.4 \\
3.6\end{array}$ & $\begin{array}{l}0.04 \\
0.06 \\
0.04 \\
0.03 \\
0.03\end{array}$ & $\begin{array}{l}0.2 \\
0.4 \\
0.4 \\
0.2 \\
0.2\end{array}$ & $\begin{array}{l}0.6 \\
1.2 \\
1.1 \\
0.3 \\
0.4\end{array}$ & $\begin{array}{l}0.04 \\
0.08 \\
0.07 \\
0.06 \\
0.07\end{array}$ & $\begin{array}{l}\text { A } \\
\text { B } \\
\text { A } \\
\text { A } \\
\text { A }\end{array}$ \\
\hline $\begin{array}{l}.4 \\
.0 \\
.0 \\
0 \\
.1 \\
.1\end{array}$ & $\begin{array}{l}0.2 \\
2.3,2.7 \\
-0.0 \\
0.2 \\
1.3\end{array}$ & $\begin{array}{l}13 \\
18 \\
15 \\
16 \\
18\end{array}$ & $\begin{array}{r}130 \\
90 \\
64 \\
91 \\
59\end{array}$ & $\begin{array}{l}8.0 \\
4.9 \\
1.7 \\
5.2 \\
0.3\end{array}$ & $\begin{array}{l}0.10 \\
0.05 \\
0.05 \\
0.10 \\
0.02\end{array}$ & $\begin{array}{l}0.4 \\
0.3 \\
0.3 \\
0.6 \\
0.1\end{array}$ & $\begin{array}{l}1.2 \\
0.6 \\
0.6 \\
1.9 \\
0.4\end{array}$ & $\begin{array}{l}0.06 \\
0.05 \\
0.11 \\
0.13 \\
0.04\end{array}$ & $\begin{array}{l}B \\
A \\
B \\
B \\
A\end{array}$ \\
\hline $\begin{array}{l}1.6 \\
8.3 \\
0.3 * \\
0.3 * \\
4.2\end{array}$ & $\begin{array}{l}1.3 \\
1.2 \\
2 \cdot 3,3 \cdot 0 \\
2 \cdot 1 \cdot 2 \cdot 8 \\
1.9\end{array}$ & $\begin{array}{l}17 \\
18 \\
19 \\
19 \\
19\end{array}$ & $\begin{array}{r}57 \\
57 \\
195 \\
195 \\
87\end{array}$ & $\begin{array}{l}0.9 \\
3.3 \\
5.2 \\
5.2 \\
3.5\end{array}$ & $\begin{array}{l}0.04 \\
0.04 \\
0.07 \\
0.08 \\
0.03\end{array}$ & $\begin{array}{l}0.4 \\
0.2 \\
0.4 \\
0.5 \\
0.2\end{array}$ & $\begin{array}{l}0.5 \\
0.5\end{array}$ & $\begin{array}{l}0.09 \\
0.05 \\
0.06 \\
0.08 \\
0.05\end{array}$ & $\begin{array}{l}B \\
A \\
C \\
C \\
A\end{array}$ \\
\hline $\begin{array}{l}0.3 * \\
6.6 \\
0.3 * \\
7.6 \\
8.4\end{array}$ & $\begin{array}{l}1.6 \\
2.5,3.5 \\
1.8 \\
2.1,3.1 \\
1.6\end{array}$ & $\begin{array}{l}19 \\
19 \\
19 \\
19 \\
19\end{array}$ & $\begin{array}{r}194 \\
88 \\
194 \\
46 \\
53\end{array}$ & $\begin{array}{l}5.1 \\
3.7 \\
5.0 \\
3.6 \\
2.3\end{array}$ & $\begin{array}{l}0.08 \\
0.03 \\
0.07 \\
0.03 \\
0.03\end{array}$ & $\begin{array}{l}0.5 \\
0.2 \\
0.5 \\
0.2 \\
0.2\end{array}$ & $\begin{array}{l}0.5 \\
0.5 \\
0.4\end{array}$ & $\begin{array}{l}0.08 \\
0.04 \\
0.07 \\
0.05 \\
0.04\end{array}$ & $\begin{array}{l}\text { C } \\
A \\
A\end{array}$ \\
\hline $\begin{array}{l}4.7 \\
3.1 \\
4.5 \\
3.8 \\
7.6\end{array}$ & $\begin{array}{l}0.5 \\
1.1 \\
2.1,2.8 \\
2.1,3.1 \\
1.6\end{array}$ & $\begin{array}{l}11 \\
19 \\
18 \\
18 \\
17\end{array}$ & $\begin{array}{l}72 \\
52 \\
95 \\
93 \\
63\end{array}$ & $\begin{array}{l}2.6 \\
3.1 \\
4.1 \\
4.2 \\
3.5\end{array}$ & $\begin{array}{l}0.05 \\
0.02 \\
0.04 \\
0.03 \\
0.05\end{array}$ & $\begin{array}{l}0.3 \\
0.2 \\
0.2 \\
0.2 \\
0.3\end{array}$ & $\begin{array}{l}0.8 \\
0.6 \\
0.7 \\
0.7 \\
0.7\end{array}$ & $\begin{array}{l}0.06 \\
0.05 \\
0.04 \\
0.05 \\
0.06\end{array}$ & $\begin{array}{l}A \\
A \\
A \\
A \\
A\end{array}$ \\
\hline $\begin{array}{l}8.0 \\
9.7 \\
3.5 \\
5.6 \\
2.8\end{array}$ & $\begin{array}{l}1.1 \\
1.5 \\
1.6 \\
1.2^{.2 .8}\end{array}$ & $\begin{array}{l}17 \\
17 \\
17 \\
18 \\
14\end{array}$ & $\begin{array}{r}171 \\
128 \\
100 \\
59 \\
89\end{array}$ & $\begin{array}{l}4.2 \\
3.7 \\
4.6 \\
5.4 \\
3.4\end{array}$ & $\begin{array}{l}0.08 \\
0.07 \\
0.03 \\
0.03 \\
0.05\end{array}$ & $\begin{array}{l}0.4 \\
0.4 \\
0.2 \\
0.2 \\
0.3\end{array}$ & $\begin{array}{l}0.7 \\
0.6 \\
1.1 \\
0.6 \\
0.5\end{array}$ & $\begin{array}{l}0.06 \\
0.07 \\
0.05 \\
0.05 \\
0.06\end{array}$ & $\begin{array}{l}\text { B } \\
\text { B } \\
\text { A } \\
A \\
\text { A }\end{array}$ \\
\hline $\begin{array}{l}7.4 \\
3.8 \\
6.0 \\
6.9 \\
8.8\end{array}$ & $\begin{array}{r}1.4 \\
0.7 \\
1.7 \\
-0.0 \\
1.6\end{array}$ & $\begin{array}{r}18 \\
16 \\
18 \\
7 \\
18\end{array}$ & $\begin{array}{r}48 \\
101 \\
58 \\
82 \\
59\end{array}$ & $\begin{array}{r}2.8 \\
5.3 \\
2.1 \\
10.5 \\
4.8\end{array}$ & $\begin{array}{l}0.03 \\
0.08 \\
0.04 \\
0.11 \\
0.04\end{array}$ & $\begin{array}{l}0.2 \\
0.5 \\
0.2 \\
0.4 \\
0.2\end{array}$ & $\begin{array}{l}0.4 \\
3.1 \\
0.5 \\
2.0 \\
0.4\end{array}$ & $\begin{array}{l}0.04 \\
0.10 \\
0.05 \\
0.04 \\
0.05\end{array}$ & $\begin{array}{l}A \\
B \\
A \\
B \\
A\end{array}$ \\
\hline
\end{tabular}


TABLE 8. - Borrego Mountain aftershocks-Continued

1968 HR MN SEC LAT $N$ LUNG $W$ DEPTH MAG NO GAP DMIN ERT ERH ERL MD $Q$

\begin{tabular}{|c|c|c|c|c|c|c|c|c|c|c|c|c|c|c|}
\hline $\begin{array}{l}4 \\
5 \\
5 \\
5 \\
5\end{array}$ & $\begin{array}{r}23 \\
5 \\
5 \\
10 \\
13\end{array}$ & $\begin{array}{r}50 \\
8 \\
43 \\
14 \\
34\end{array}$ & $\begin{array}{l}45.7 \\
50.8 \\
50.9 \\
56.0 \\
10.8\end{array}$ & $\begin{array}{l}33-2.2 \\
33-16.5 \\
33-17.7 \\
33-3.1 \\
33-8.8\end{array}$ & $\begin{array}{l}116-2.5 \\
116-15.2 \\
116-13.7 \\
116-1.4 \\
116-6.7\end{array}$ & $\begin{array}{l}4.5 \\
3.1 \\
5.0 \\
1.1 \\
4.0\end{array}$ & $\begin{array}{l}1.8,2.6 \\
1.8 \\
2.2,2.9 \\
1.5 \\
1.2\end{array}$ & $\begin{array}{l}18 \\
17 \\
18 \\
17 \\
17\end{array}$ & $\begin{array}{l}56 \\
83 \\
89 \\
69 \\
49\end{array}$ & $\begin{array}{l}1.8 \\
2.2 \\
3.1 \\
2.2 \\
4.7\end{array}$ & $\begin{array}{l}0.02 \\
0.03 \\
0.04 \\
0.04 \\
0.02\end{array}$ & $\begin{array}{l}0.2 \\
0.2 \\
0.3 \\
0.3 \\
0.2\end{array}$ & $\begin{array}{l}0.5 \\
1.2\end{array}$ & $\begin{array}{l}0.04 \\
0.05 \\
0.05 \\
0.09 \\
0.05\end{array}$ \\
\hline $\begin{array}{l}5 \\
6 \\
6 \\
6 \\
6\end{array}$ & $\begin{array}{r}16 \\
2 \\
2 \\
2 \\
2\end{array}$ & $\begin{array}{r}47 \\
0 \\
3 \\
4 \\
42\end{array}$ & $\begin{array}{r}37.3 \\
5.0 \\
42.4 \\
48.5 \\
31.2\end{array}$ & $\begin{array}{l}33-7.3 \\
33-2.5 \\
33-2.1 \\
33-16.4 \\
32-59.9\end{array}$ & $\begin{array}{l}116-3.0 \\
115-56.8 \\
115-57.0 \\
116-0.2 \\
115-58.5\end{array}$ & $\begin{array}{l}1.7 \\
6.4 \\
7.1 \\
0.3 * \\
8.7\end{array}$ & $\begin{array}{l}1.8,2.5 \\
0.9 \\
1.8,2.2 \\
0.8 \\
1.8\end{array}$ & $\begin{array}{l}18 \\
18 \\
18 \\
15 \\
17\end{array}$ & $\begin{array}{r}54 \\
152 \\
153 \\
194 \\
86\end{array}$ & $\begin{array}{l}4.2 \\
5.4 \\
4.6 \\
4.5 \\
0.4\end{array}$ & $\begin{array}{l}0.02 \\
0.08 \\
0.11 \\
0.10 \\
0.04\end{array}$ & $\begin{array}{l}.1 \\
.4 \\
.6\end{array}$ & $\begin{array}{r}.2 \\
.9 \\
.1\end{array}$ & $\begin{array}{l}0.04 \\
0.07 \\
0.08 \\
0.09 \\
0.04\end{array}$ \\
\hline $\begin{array}{l}6 \\
6 \\
6 \\
6 \\
6\end{array}$ & $\begin{array}{r}6 \\
7 \\
8 \\
10 \\
12\end{array}$ & $\begin{array}{l}24 \\
34 \\
32 \\
53 \\
42\end{array}$ & $\begin{array}{r}9.7 \\
26.0 \\
28.8 \\
36.9 \\
21.0\end{array}$ & $\begin{array}{l}33-2.4 \\
33-2.4 \\
33-2.2 \\
33-2.0 \\
33-2.4\end{array}$ & $\begin{array}{l}115-56.9 \\
115-56.8 \\
116-2.3 \\
116-0.1 \\
115-57.1\end{array}$ & $\begin{array}{l}6.3 \\
6.4 \\
5.3 \\
9.2 \\
6.3\end{array}$ & $\begin{array}{l}1.7 \\
1.8 \\
1.4 \\
1.6^{.3 .5}\end{array}$ & $\begin{array}{l}17 \\
17 \\
16 \\
18 \\
17\end{array}$ & $\begin{array}{r}152 \\
153 \\
58 \\
87 \\
149\end{array}$ & $\begin{array}{l}2.0 \\
4.7 \\
5.1\end{array}$ & $\begin{array}{l}.06 \\
.08 \\
.03 \\
.05 \\
.05\end{array}$ & $\begin{array}{l}0.3 \\
0.4 \\
0.2 \\
0.3 \\
0.3\end{array}$ & $\begin{array}{l}.7 \\
.8 \\
.4 \\
.5 \\
.6\end{array}$ & $\begin{array}{l}0.05 \\
0.06 \\
0.04 \\
0.05 \\
0.04\end{array}$ \\
\hline $\begin{array}{l}6 \\
7 \\
7 \\
7 \\
7\end{array}$ & $\begin{array}{r}17 \\
1 \\
1 \\
3 \\
7\end{array}$ & $\begin{array}{r}31 \\
7 \\
26 \\
17 \\
49\end{array}$ & $\begin{array}{l}47.6 \\
16.9 \\
38.6 \\
16.7 \\
41.8\end{array}$ & $\begin{array}{l}33-2.4 \\
33-9.9 \\
33-2.8 \\
33-8.4 \\
33-3.2\end{array}$ & $\begin{array}{l}115-56.9 \\
116-5.0 \\
116-1.1 \\
116-0.3 \\
116-1.2\end{array}$ & $\begin{array}{r}6.7 \\
10.3 \\
7.3 \\
4.8 \\
2.3\end{array}$ & $\begin{array}{l}1.5^{, 4.0} \\
1.9,2.6 \\
1.6 \\
1.6\end{array}$ & $\begin{array}{l}18 \\
19 \\
19 \\
18 \\
17\end{array}$ & $\begin{array}{r}152 \\
48 \\
51 \\
83 \\
72\end{array}$ & $\begin{array}{l}5.0 \\
5.1 \\
2.7 \\
4.3 \\
2.0\end{array}$ & $\begin{array}{l}.07 \\
.05 \\
.04 \\
.04 \\
.02\end{array}$ & $\begin{array}{l}0.3 \\
0.2 \\
0.2 \\
0.2 \\
0.2\end{array}$ & $\begin{array}{l}.7 \\
.6 \\
.5 \\
.8 \\
.8\end{array}$ & $\begin{array}{l}0.05 \\
0.07 \\
0.05 \\
0.05 \\
0.05\end{array}$ \\
\hline $\begin{array}{l}7 \\
7 \\
7 \\
8 \\
8\end{array}$ & $\begin{array}{r}7 \\
7 \\
16 \\
10 \\
10\end{array}$ & $\begin{array}{r}50 \\
56 \\
17 \\
0 \\
54\end{array}$ & $\begin{array}{r}39.8 \\
37.8 \\
3.6 \\
46.2 \\
46.2\end{array}$ & $\begin{array}{l}33-9.0 \\
33-16.3 \\
33-2.4 \\
33-14.4 \\
33-10.3\end{array}$ & $\begin{array}{l}116-3.6 \\
116-6.0 \\
115-56.9 \\
116-11.7 \\
116-2.5\end{array}$ & $\begin{array}{r}10.0 \\
2.9 \\
7.0 \\
10.8 \\
6.3\end{array}$ & $\begin{array}{l}0.5 \\
1.8^{.3 .3} \\
1.9 \\
1.7\end{array}$ & $\begin{array}{l}14 \\
17 \\
16 \\
17 \\
16\end{array}$ & $\begin{array}{r}52 \\
133 \\
152 \\
58 \\
74\end{array}$ & $\begin{array}{l}4.8 \\
2.2 \\
5.2 \\
4.6 \\
6.4\end{array}$ & & $\begin{array}{l}3 \\
3 \\
4\end{array}$ & $\begin{array}{l}0.8 \\
0.5 \\
0.8 \\
0.3 \\
2.4\end{array}$ & $\begin{array}{l}0.06 \\
0.07 \\
0.06 \\
0.04 \\
0.15\end{array}$ \\
\hline $\begin{array}{l}8 \\
8 \\
8 \\
8 \\
8\end{array}$ & $\begin{array}{l}13 \\
14 \\
14 \\
16 \\
17\end{array}$ & $\begin{array}{l}21 \\
54 \\
54 \\
23 \\
28\end{array}$ & $\begin{array}{l}39.4 \\
22.4 \\
50.9 \\
38.1 \\
15.6\end{array}$ & $\begin{array}{l}33-2.4 \\
33-12.2 \\
33-11.4 \\
33-11.5 \\
33-8.3\end{array}$ & $\begin{array}{l}116-2.4 \\
116-11.7 \\
116-9.8 \\
116-9.8 \\
116-5.5\end{array}$ & $\begin{array}{l}5.1 \\
1.0- \\
3.8 \\
3.1 \\
6.0\end{array}$ & $\begin{array}{l}1.5 \\
-0.1 \\
1.8 \\
1.8^{.3 .3}\end{array}$ & $\begin{array}{r}17 \\
9 \\
17 \\
17 \\
18\end{array}$ & $\begin{array}{l}51 \\
98 \\
63 \\
62 \\
75\end{array}$ & 2.0 & $\begin{array}{l}.02 \\
.05 \\
.03 \\
.02 \\
.03\end{array}$ & & $\begin{array}{l}0.4 \\
0.6 \\
0.8 \\
0.8 \\
0.5\end{array}$ & $\begin{array}{l}0.04 \\
0.06 \\
0.06 \\
0.05 \\
0.05\end{array}$ \\
\hline $\begin{array}{l}8 \\
9 \\
9 \\
9 \\
9\end{array}$ & $\begin{array}{r}22 \\
0 \\
6 \\
10 \\
20\end{array}$ & $\begin{array}{l}30 \\
16 \\
47 \\
21 \\
16\end{array}$ & $\begin{array}{r}6.3 \\
54.1 \\
57.8 \\
46.2 \\
1.5\end{array}$ & $\begin{array}{l}33-10.7 \\
33-17.9 \\
33-2.2 \\
33-6.1 \\
33-6.9\end{array}$ & $\begin{array}{l}116-7.6 \\
116-14.3 \\
115-57.1 \\
116-1.9 \\
116-3.1\end{array}$ & $\begin{array}{l}6.9 \\
4.4 \\
6.2 \\
7.3 \\
1.3\end{array}$ & $\begin{array}{l}2.1,3.0 \\
2.2,2.5 \\
1.8 \\
1.0,3.6\end{array}$ & $\begin{array}{l}17 \\
17 \\
18 \\
18 \\
18\end{array}$ & $\begin{array}{l}63 \\
88 \\
88 \\
74 \\
75\end{array}$ & $\begin{array}{l}3.5 \\
4.7 \\
.3 .4 \\
4.0\end{array}$ & & & $\begin{array}{l}.5 \\
.6 \\
.5 \\
.5 \\
.5\end{array}$ & $\begin{array}{l}0.05 \\
0.04 \\
0.04 \\
0.05 \\
0.08\end{array}$ \\
\hline $\begin{array}{l}9 \\
10 \\
10 \\
10 \\
10\end{array}$ & $\begin{array}{r}23 \\
4 \\
5 \\
6 \\
6\end{array}$ & $\begin{array}{l}35 \\
32 \\
28 \\
28 \\
28\end{array}$ & $\begin{array}{r}17.8 \\
25.2 \\
7.8 \\
24.8 \\
46.6\end{array}$ & $\begin{array}{l}33-7.0 \\
33-2.1 \\
33-2.0 \\
33-8.4 \\
33-7.8\end{array}$ & $\begin{array}{l}116-6.7 \\
116-2.4 \\
116-2.4 \\
116-3.5 \\
116-5.0\end{array}$ & $\begin{array}{r}10.6 \\
5.3 \\
4.8 \\
4.4 \\
0.7\end{array}$ & $\begin{array}{l}1.6 \\
2.2: 3.4 \\
0.5 \\
1.4\end{array}$ & $\begin{array}{l}17 \\
18 \\
17 \\
17 \\
18\end{array}$ & $\begin{array}{l}84 \\
47 \\
56 \\
48 \\
44\end{array}$ & $\begin{array}{l}1.5 \\
1.8 \\
1.7 \\
4.2 \\
1.6\end{array}$ & & & $\begin{array}{l}.6 \\
.4 \\
.4 \\
.8\end{array}$ & $\begin{array}{l}0.06 \\
0.05 \\
0.04 \\
0.05 \\
0.06\end{array}$ \\
\hline $\begin{array}{l}10 \\
10 \\
10 \\
10 \\
11\end{array}$ & $\begin{array}{r}7 \\
7 \\
20 \\
20 \\
8\end{array}$ & $\begin{array}{l}24 \\
57 \\
16 \\
17 \\
10\end{array}$ & $\begin{array}{r}9.3 \\
49.1 \\
23.5 \\
49.6 \\
4.0\end{array}$ & $\begin{array}{l}33-7.8 \\
33-9.4 \\
33-5.6 \\
33-7.7 \\
33-2.4\end{array}$ & $\begin{array}{l}116-5.1 \\
116-5.6 \\
116-3.1 \\
116-2.9 \\
116-0.3\end{array}$ & $\begin{array}{l}0.3 * \\
0.5 \\
7.7 \\
4.6 \\
8.8\end{array}$ & $\begin{array}{l}0.9 \\
1.1 \\
1.4 \\
1.5 \\
.4 .2\end{array}$ & $\begin{array}{l}17 \\
17 \\
15 \\
15 \\
18\end{array}$ & $\begin{array}{l}48 \\
70 \\
83 \\
74 \\
56\end{array}$ & $\begin{array}{l}1.5 \\
4.1 \\
3.4 \\
4.5 \\
4.0\end{array}$ & & $\begin{array}{l}0.3 \\
0.2 \\
0.3\end{array}$ & $\begin{array}{l}0.4 \\
0.5 \\
0.6 \\
0.5\end{array}$ & $\begin{array}{l}0.07 \\
0.05 \\
0.05 \\
0.04 \\
0.05\end{array}$ \\
\hline $\begin{array}{l}11 \\
11 \\
11 \\
11 \\
11\end{array}$ & $\begin{array}{l}8 \\
8 \\
8 \\
8 \\
8\end{array}$ & $\begin{array}{l}13 \\
13 \\
15 \\
15 \\
45\end{array}$ & $\begin{array}{l}14.0 \\
33.7 \\
10.2 \\
19.2 \\
55.2\end{array}$ & $\begin{array}{l}33-1.1 \\
33-2.4 \\
33-0.1 \\
33-2.5 \\
33-14.3\end{array}$ & $\begin{array}{l}115-55.9 \\
116-0.2 \\
116-1.0 ? \\
116-0.1 \\
116-0.7 ?\end{array}$ & $\begin{array}{r}6.4 \\
7.3 \\
11.6 \\
7.9 \\
0.2\end{array}$ & $\begin{array}{l}0.7 \\
1.4 \\
0.5 \\
0.5 \\
0.6\end{array}$ & $\begin{array}{r}15 \\
17 \\
8 \\
8 \\
12\end{array}$ & $\begin{array}{l}106 \\
56 \\
122 \\
138 \\
180\end{array}$ & $\begin{array}{l}4.0 \\
5.0 \\
4.0 \\
0.8\end{array}$ & & $\begin{array}{l}0.7 \\
0.6\end{array}$ & $\begin{array}{l}1.3 \\
0.6 \\
6.1 \\
1.3 \\
0.8\end{array}$ & $\begin{array}{l}0.11 \\
0.06 \\
0.33 \\
0.08 \\
0.07\end{array}$ \\
\hline $\begin{array}{ll}1 & 1 \\
1 & 1 \\
1 & 1 \\
11 \\
11\end{array}$ & $\begin{array}{r}8 \\
10 \\
15 \\
15 \\
8\end{array}$ & $\begin{array}{l}46 \\
38 \\
22 \\
22 \\
43\end{array}$ & $\begin{array}{l}3.6 \\
31.7 \\
26.0 \\
38.2 \\
31.1\end{array}$ & $\begin{array}{l}33-2.4 \\
33-16.7 \\
33-8.3 \\
33-7.4 \\
33-2.5\end{array}$ & $\begin{array}{l}116-0.3 \\
115-58.1 \\
116-1.8 \\
116-4.0 \\
116-0.2\end{array}$ & $\begin{array}{l}8.5 \\
1.3 \\
6.7 \\
1.4 \\
7.0\end{array}$ & $\begin{array}{l}2.3 .5 \\
2.1,3.0 \\
0.2 \\
0.9 \\
0.1\end{array}$ & $\begin{array}{r}18 \\
18 \\
17 \\
9 \\
7\end{array}$ & $\begin{array}{r}56 \\
199 \\
62 \\
83 \\
109\end{array}$ & $\begin{array}{l}4.0 \\
6.8 \\
6.2 \\
2.7 \\
3.9\end{array}$ & & $\begin{array}{l}0.3 \\
0.5 \\
0.2 \\
0.3 \\
0.6\end{array}$ & $\begin{array}{l}0.5 \\
0.6 \\
0.6 \\
0.8 \\
1.3\end{array}$ & $\begin{array}{l}0.05 \\
0.07 \\
0.06 \\
0.06 \\
0.06\end{array}$ \\
\hline $\begin{array}{l}12 \\
12 \\
12 \\
12 \\
12\end{array}$ & $\begin{array}{r}8 \\
8 \\
8 \\
9 \\
14\end{array}$ & $\begin{array}{l}44 \\
47 \\
48 \\
55 \\
24\end{array}$ & $\begin{array}{l}54.3 \\
51.4 \\
35.6 \\
21.2 \\
16.3\end{array}$ & $\begin{array}{l}33-7.9 \\
33-7.9 \\
33-4.6 \\
33-5.0 \\
33-27.7\end{array}$ & $\begin{array}{l}116-2.2 \\
116-2.6 \\
116-1.9 \\
116-2.9 \\
116-24.4\end{array}$ & $\begin{array}{l}0.1 * \\
1.5 \\
7.7 \\
6.3\end{array}$ & $\begin{array}{l}0.4 \\
0.7 \\
1.7 \\
2.4 .3\end{array}$ & $\begin{array}{l}18 \\
15 \\
18 \\
18 \\
19\end{array}$ & $\begin{array}{r}60 \\
56 \\
58 \\
53 \\
252\end{array}$ & $\begin{array}{l}5.6 \\
5.1 \\
0.9 \\
2.4 \\
3.3\end{array}$ & $\begin{array}{l}0.03 \\
0.06 \\
0.03 \\
0.03 \\
0.20\end{array}$ & $\begin{array}{l}0.2 \\
0.4 \\
0.2 \\
0.2 \\
1.0\end{array}$ & $\begin{array}{l}0.3 \\
0.5 \\
0.7\end{array}$ & $\begin{array}{l}0.06 \\
0.13 \\
0.07 \\
0.05 \\
0.08\end{array}$ \\
\hline
\end{tabular}


TABLE 8. - Borrego Mountain aftershocks-Continued

1968 HR MN SEC LAT N LONG W DEPTH MAG NO GAP DMIN ERT ERH ERZ MD 6

MAY $12 \quad 17 \quad 10 \quad 49.6 \quad 33-6.1 \quad 116-2.8$

$\begin{array}{lllllll}13 & 2 & 25 & 8.3 & 33-6.1 & 116-2.8\end{array}$

$\begin{array}{lllllll}13 & 2 & 27 & 7.6 & 33-11.8 & 116-10.6\end{array}$

$13846 \quad 28.0 \quad 33-9.2 \quad 116-6.5$

$\begin{array}{llllll}13 & 10 & 40 & 59.7 & 33-7.4 & 116-5.4\end{array}$

$\begin{array}{lllllll}13 & 11 & 22 & 19.5 & 33-9.6 & 116-3.8\end{array}$

$\begin{array}{llllll}13 & 13 & 43 & 39.5 & 33-4.0 & 116-2.0\end{array}$

$\begin{array}{lllllll}13 & 13 & 45 & 4.6 & 33-4.1 & 116-2.1\end{array}$

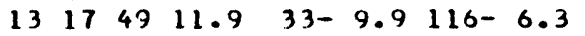

$\begin{array}{llllll}13 & 17 & 50 & 26.9 & 33-10.0 & 116-6.3\end{array}$

$131832 \quad 45.6 \quad 33-1.2115-56.3$

$1.31833 \quad 38.9 \quad 33-11.0 \quad 116-1.9$

$1318 \quad 3352.2 \quad 33-18.6 \quad 116-15.7$

$14022 \quad 47.5 \quad 33-2.0116-2.3$

$14153 \quad 22.9 \quad 33-19.2 \quad 116-17.8$

$14 \quad 15424.3 \quad 33-15.6 \quad 115-57.3$

$\begin{array}{lllllll}14 & 9 & 45 & 27.0 & 32-59.4 & 116-18.7\end{array}$

$14 \quad 16 \quad 1345.7 \quad 33-2.5 \quad 116-1.0$

$141920 \quad 23.8 \quad 33-11.8 \quad 116-11.4$

$\begin{array}{lllllll}15 & 0 & 9 & 7.0 & 33-7.9 & 115-59.5\end{array}$

$1524653.4 \quad 33-8.1116-6.6$

$154242.0 \quad 33-8.5116-4.6$

$15 \quad 4 \quad 3 \quad 18.8 \quad 33-11.1 \quad 116-1.4$

$\begin{array}{lllllll}15 & 20 & 1 & 13.9 & 33-0.7 & 116-17.7\end{array}$

4.21 .3

$\begin{array}{lllllllll}2.2 & 0.9 & 18 & 57 & 3.9 & 0.02 & 0.2 & 0.6 & 0.05\end{array}$

$\begin{array}{lllllllll}16 & 57 & 3.9 & 0.02 & 0.1 & 0.2 & 0.04 & 0\end{array}$

$\begin{array}{llllllllll}3.0 & 1.2 & 19 & 47 & 4.0 & 0.10 & 0.6 & 0.9 & 0.12 & \mathrm{P}\end{array}$

$\begin{array}{llllllllll}3.2 & 1.2 & 18 & 44 & 0.5 & 0.02 & 0.2 & 0.5 & 0.05 & \Delta\end{array}$

$\begin{array}{lllllllll}6.9 & 1.4 & 19 & 56 & 5.3 & 0.03 & 0.2 & 0.5 & 0.05\end{array}$

$\begin{array}{lllllllll}1.6 & 1.5 & 19 & 40 & 0.5 & 0.03 & 0.2 & 0.3 & 0.06\end{array}$

$\begin{array}{llllllllll}1.6 & 1.0 & 19 & 41 & 0.6 & 0.02 & 0.2 & 0.3 & 0.06 & A\end{array}$

$\begin{array}{lllllllll}0.9 & -0.0 & 8 & 109 & 0.7 & 0.08 & 0.6 & 0.5 & 0.07\end{array}$

$\begin{array}{llllllllll}2.8 & 1.0 & 18 & 47 & 4.6 & 0.03 & 0.2 & 0.3 & 0.05 & 4\end{array}$

$\begin{array}{llllllllll}2.6 & 1.4 & 16 & 48 & 4.6 & 0.04 & 0.2 & 0.4 & 0.06 & \Delta\end{array}$

$\begin{array}{llllllllll}5.6 & 0.5 & 12 & 101 & 3.9 & 0.06 & 0.4 & 1.0 & 0.06 & \Delta\end{array}$

$\begin{array}{llllllllll}4.4 & 0.1 & 8 & 93 & 5.7 & 0.08 & 0.5 & 1.8 & 0.06 & B\end{array}$

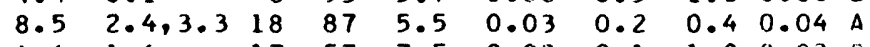

$\begin{array}{llllllllll}4.6 & 1.6 & 17 & 57 & 7.5 & 0.03 & 0.1 & 1.0 & 0.03 & \mathrm{E}\end{array}$

$\begin{array}{llllllllll}10.4 & 0.8 & 14 & 117 & 5.7 & 0.07 & 0.5 & 0.6 & 0.08 & A\end{array}$

$\begin{array}{llllllllll}2.9 & 1.4 & 17 & 199 & 6.6 & 0.11 & 0.5 & 0.7 & 0.07 & B\end{array}$

$\begin{array}{llllllllll}7.0 & 1.2 & 17 & 201 & 9.6 & 0.06 & 0.4 & 1.0 & 0.05 & B\end{array}$

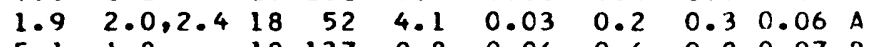

$\begin{array}{llllllllll}5.1 & 1.8 & 10 & 137 & 0.8 & 0.06 & 0.6 & 0.8 & 0.07 & \text { 8 }\end{array}$

$\begin{array}{llllllllll}4.5 & 1.7 & 19 & 103 & 4.5 & 0.03 & 0.2 & 0.8 & 0.05 & A\end{array}$

$\begin{array}{llllllllll}4.0 & 1.3 & 19 & 49 & 2.2 & 0.02 & 0.1 & 0.5 & 0.04 & A\end{array}$

$\begin{array}{llllllllll}1.1 & 0.8 & 18 & 83 & 3.1 & 0.03 & 0.2 & 0.3 & 0.05 & A\end{array}$

$\begin{array}{llllllllll}6.6 & 0.3 & 9 & 137 & 4.9 & 0.08 & 0.5 & 1.3 & 0.07 & 8\end{array}$

$\begin{array}{lllllllll}3.6 & 1.0 & 19 & 189 & 7.7 & 0.05 & 0.3 & 2.3 & 0.06 \mathrm{C}\end{array}$

$\begin{array}{llllllll}16 & 5 & 48 & 35.7 & 33-2.1 & 116-2.3\end{array}$

$\begin{array}{llllll}16 & 8 & 40 & 59.8 & 32-59.7 & 116-15.6\end{array}$

$16 \quad 8 \quad 41 \quad 17.0 \quad 32-59.6 \quad 116-15.6$

$\begin{array}{llllll}16 & 22 & 9 & 24.8 & 33-1.4 & 116-6.0\end{array}$

$\begin{array}{ll}1622945.8 & 33-1.6116-5.9\end{array}$

5.01 .5

$9.4 \quad 0.4$

9.51 .3

$4.2 \quad 0.8$

3.31 .0

$\begin{array}{llll}17 & 50 & 1.9 & 0.03\end{array}$

$17180 \quad 4.9 \quad 0.07$

$19180 \quad 5.0 \quad 0.06$

$\begin{array}{lrrr}19 & 70 & 4.0 & 0.03 \\ 19 & 69 & 3.8 & 0.04\end{array}$

$\begin{array}{llll}0.2 & 0.4 & 0.05 \quad A\end{array}$

$2.8 \quad 1.4$

$\begin{array}{llll}17 & 50 & 3.9 & 0.03\end{array}$

$\begin{array}{llll}19 & 101 & 3.9 & 0.04\end{array}$

$7.2 \quad 1.5$

$0.1 * 0.6$

4.11 .6

$\begin{array}{llll}18 & 48 & 3.6 & 0.03\end{array}$

$17215239.1 \quad 33-1.1115-56.3$

$1834024.0 \quad 33-9.0116-6.5$

$1855830.4 \quad 33-1.5116-6.0$

$1854149.1 \quad 33-15.3 \quad 115-57.9$

$18 \quad 19 \quad 16 \quad 34.5 \quad 33-3.5 \quad 116-1.6$

$\begin{array}{llllllll}18 & 21 & 45 & 44.0 & 33-3.2 & 115-42.3\end{array}$

$19 \quad 04126.6 \quad 33-9.6 \quad 116-6.1$

$194522.4 \quad 33-12.0 \quad 116-10.2$

$194636.4 \quad 33-15.4 \quad 115-58.1$

$1917 \quad 0 \quad 0.7 \quad 33-5.9116-1.9$

$191930 \quad 19.6 \quad 33-3.4 \quad 116-0.9$

$20 \quad 0 \quad 5547.2 \quad 33-2.6 \quad 116-0.3$

$20 \quad 0 \quad 57 \quad 43.4 \quad 33-18.1 \quad 116-11.7$

$20 \quad 0 \quad 59 \quad 14.8 \quad 32-59.3 \quad 116-13.9$

$0.3 * 1.7$

$\begin{array}{llll}19 & 195 & 5.4 & 0.08\end{array}$

$6.4 \quad 1.2$

$18.64 \quad 1.3$

0.03

$\begin{array}{lllll}0 * 0 * 2.1 .3 .0 & 18 & 267 & 14.8\end{array}$

$\begin{array}{lllll}1.9 & 1.4 & 18 & 45 & 4.5\end{array}$

10

2.90 .4

$\begin{array}{lll}14 & 78 & 2.5\end{array}$

0.18

0.03

0.03

$\begin{array}{llll}19 & 179 & 5.3 & 0.06\end{array}$

$\begin{array}{llll}0.4 & 0.8 & 0.06 & B\end{array}$

$\begin{array}{llll}0.4 & 0.6 & 0.07 & B\end{array}$

$\begin{array}{llll}0.2 & 1.3 & 0.07 & A\end{array}$

$\begin{array}{llll}0.3 & 1.8 & 0.09 A\end{array}$

6.11 .4

$2.8 \quad 1.4$

$8.1 \quad 1.7$

6.50 .5

6.10 .4

$\begin{array}{llll}15 & 75 & 3.0 & 0.03\end{array}$

$\begin{array}{rrrr}17 & 90 & 1.9 & 0.03 \\ 18 & 111 & 3.8 & 0.03\end{array}$

$\begin{array}{llll}18 & 111 & 3.8 & 0.03 \\ 12 & 101 & 5.1 & 0.07\end{array}$

$\begin{array}{llll}13201 & 16.7 & 0.06\end{array}$

$\begin{array}{llll}0.2 & 0.3 & 0.05 \quad A\end{array}$

$\begin{array}{llll}0.2 & 0.5 & 0.04 \mathrm{~A}\end{array}$

$0.3 \quad 0.10 \mathrm{~B}$

$\begin{array}{llll}0.2 & 1.5 & 0.07 \quad A\end{array}$

0.07 A
0.08 C

$201050.6 \quad 33-16.5 \quad 115-59.7$

$3.1 * 1.2$

$7.1-0.1$

$\begin{array}{llll}19 & 177 & 5.2 & 0.06\end{array}$

$\begin{array}{llll}10 & 84 & 2.8 & 0.05\end{array}$

2.21 .2

$2.0 \quad 1.0$

$4.8-0.1$

$\begin{array}{lll}18 & 46 & 3.5\end{array}$

$\begin{array}{llll}17 & 46 & 3.5 & 0.03\end{array}$

$\begin{array}{llll}9 & 84 & 3.3 & 0.03\end{array}$

$\begin{array}{lllll}0.2 & 0.4 & 0.05 & A\end{array}$

$0.9 \quad 0.06 \mathrm{D}$

$\begin{array}{llll}0.2 & 0.3 & 0.05 & \text { A }\end{array}$

$\begin{array}{llll}0.2 & 0.3 & 0.05 & \text { A }\end{array}$

$0.4 \quad 0.07 \mathrm{C}$

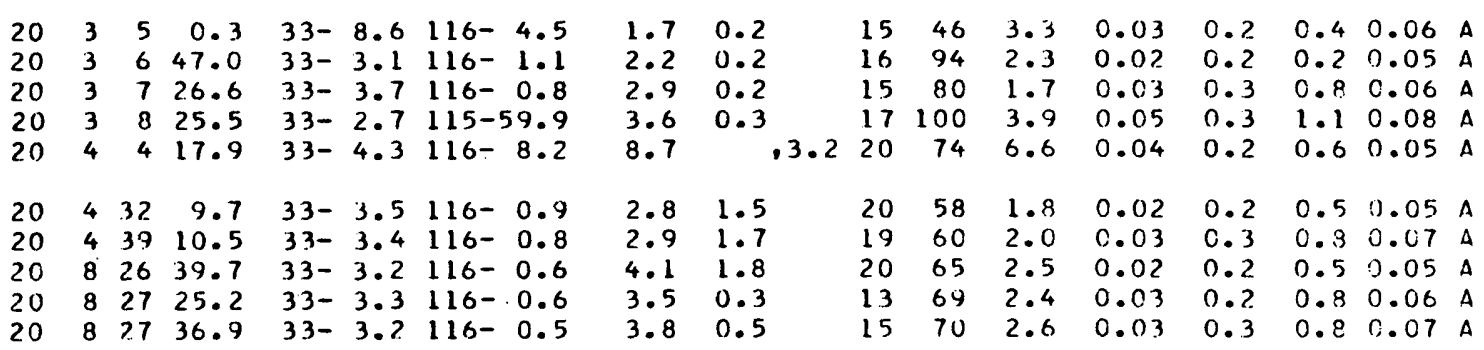


TABLE 8. - Borrego Mountain aftershocks-Continued

\begin{tabular}{|c|c|c|c|c|c|c|c|c|c|c|c|c|c|c|}
\hline & $1 / R$ & MN & $\mathrm{EC}$ & $A T N$ & LONG $W$ & EP & MAG & NO & $A P$ & DMIN & RT & RH & $\mathrm{R} 2$ & D \\
\hline $\begin{array}{l}20 \\
20 \\
21 \\
21 \\
21\end{array}$ & $\begin{array}{r}8 \\
20 \\
5 \\
9 \\
13\end{array}$ & $\begin{array}{r}33 \\
22 \\
44 \\
46 \\
7\end{array}$ & $\begin{array}{l}38.4 \\
32.3 \\
48.4 \\
16.8 \\
39.0\end{array}$ & $\begin{array}{l}32-57.3 \\
33-17.4 \\
33-4.2 \\
33-15.0 \\
33-3.4\end{array}$ & $\begin{array}{l}116-14.7 \\
116-15.3 \\
116-8.2 \\
115-56.9 \\
116-1.8\end{array}$ & $\begin{array}{l}10.3 \\
3.9 \\
8.6 \\
0.3 * \\
6.5\end{array}$ & $\begin{array}{l}1.3 \\
1.6 \\
1.2 \\
1.6 \\
1.7\end{array}$ & $\begin{array}{l}19 \\
19 \\
18 \\
19 \\
19\end{array}$ & $\begin{array}{r}209 \\
85 \\
75 \\
181 \\
51\end{array}$ & $\begin{array}{l}7.3 \\
3.4 \\
8.5 \\
6.7 \\
1.7\end{array}$ & $\begin{array}{l}.08 \\
.03 \\
.04 \\
.07 \\
.03\end{array}$ & $\begin{array}{l}.5 \\
.2 \\
.2\end{array}$ & $\begin{array}{l}.5 \\
.7 \\
.6\end{array}$ & $\begin{array}{l}0.07 \\
0.04 \\
0.05 \\
0.09 \\
0.06\end{array}$ \\
\hline $\begin{array}{l}21 \\
21 \\
21 \\
21 \\
21\end{array}$ & $\begin{array}{l}14 \\
14 \\
14 \\
14 \\
22\end{array}$ & $\begin{array}{l}34 \\
55 \\
56 \\
59 \\
17\end{array}$ & $\begin{array}{l}31.3 \\
25.2 \\
21.3 \\
15.3 \\
56.1\end{array}$ & $\begin{array}{l}33-4.2 \\
33-3.9 \\
33-4.1 \\
33-4.0 \\
32-59.8\end{array}$ & $\begin{array}{l}116-1.6 \\
116-1.6 \\
116-1.6 \\
116-2.18 \\
116-15.1\end{array}$ & & $\begin{array}{l}0.7^{.3 .2} \\
1.4^{3} \\
0.2 \\
1.3\end{array}$ & $\begin{array}{l}19 \\
15 \\
18 \\
10 \\
18\end{array}$ & $\begin{array}{r}58 \\
79 \\
78 \\
109 \\
200\end{array}$ & & & & $\begin{array}{l}.4 \\
.6 \\
.4 \\
.7 \\
.3\end{array}$ & $\begin{array}{l}0.10 \\
0.10 \\
0.09 \\
0.11 \\
0.09\end{array}$ \\
\hline $\begin{array}{l}21 \\
22 \\
22 \\
22 \\
22\end{array}$ & $\begin{array}{l}23 \\
4 \\
13 \\
16 \\
16\end{array}$ & $\begin{array}{l}19 \\
58 \\
26 \\
51 \\
53\end{array}$ & $\begin{array}{r}39.4 \\
4.3 \\
55.4 \\
23.2 \\
38.9\end{array}$ & $\begin{array}{l}33-2.1 \\
33-3.5 \\
33-18.6 \\
33-18.8 \\
33-1.1\end{array}$ & $\begin{array}{l}115-59.6 \\
116-1.0 \\
116-13.4 \\
116-13.2 \\
116-16.1\end{array}$ & $\begin{array}{l}4.8 \\
2.9 \\
7.5 \\
5.6 \\
0.3 *\end{array}$ & $\begin{array}{l}1.6^{.3 .0} \\
1.4^{.4 .4} \\
0.9\end{array}$ & $\begin{array}{l}19 \\
15 \\
13 \\
15 \\
12\end{array}$ & $\begin{array}{r}85 \\
81 \\
112 \\
113 \\
198\end{array}$ & .7 & & & $\begin{array}{l}0.6 \\
0.2 \\
1.2 \\
1.1\end{array}$ & $\begin{array}{l}0.05 \\
0.04 \\
0.07 \\
0.06 \\
0.11\end{array}$ \\
\hline $\begin{array}{l}22 \\
23 \\
23 \\
23 \\
23\end{array}$ & $\begin{array}{l}19 \\
12 \\
12 \\
13 \\
15\end{array}$ & $\begin{array}{r}12 \\
4 \\
41 \\
13 \\
16\end{array}$ & $\begin{array}{r}2.9 \\
0.1 \\
22.9 \\
47.5 \\
58.7\end{array}$ & $\begin{array}{l}33-9.2 \\
32-58.8 \\
32-53.5 \\
32-57.1 \\
33-0.8\end{array}$ & $\begin{array}{l}116-24.4 \\
116-15.8 \\
116-13.1 ? \\
116-18.2 \\
116-16.8\end{array}$ & $\begin{array}{l}9 \\
7 \\
7\end{array}$ & $\begin{array}{l}1.7 \\
1.3 \\
1.7 \\
1.4 \\
0.9\end{array}$ & $\begin{array}{l}13 \\
18 \\
19 \\
19 \\
18\end{array}$ & $\begin{array}{l}227 \\
206 \\
221 \\
224 \\
203\end{array}$ & $\begin{array}{r}7.1 \\
6.0 \\
13.7\end{array}$ & & & $\begin{array}{l}.4 \\
.0 \\
.7 \\
.6 \\
.7\end{array}$ & $\begin{array}{l}0.04 \\
0.07 \\
0.10 \\
0.07 \\
0.08\end{array}$ \\
\hline $\begin{array}{l}23 \\
24 \\
24 \\
24 \\
24\end{array}$ & $\begin{array}{r}23 \\
7 \\
7 \\
11 \\
12\end{array}$ & $\begin{array}{r}6 \\
7 \\
7 \\
41 \\
39\end{array}$ & $\begin{array}{l}4.6 \\
10.7 \\
43.8 \\
25.6 \\
57.1\end{array}$ & $\begin{array}{l}32-59.4 \\
33-4.7 \\
33-6.4 \\
33-6.9 \\
33-3.1\end{array}$ & $\begin{array}{r}16.8 \\
1.9 \\
2.6 \\
2.6 \\
.59 .1\end{array}$ & & $\begin{array}{l}1.4 \\
0.3 \\
1.0 \\
1.5 \\
2.1 .2 .6\end{array}$ & $\begin{array}{l}20 \\
19 \\
19\end{array}$ & $\begin{array}{r}209 \\
58 \\
55 \\
54 \\
69\end{array}$ & & & & $\begin{array}{l}.6 \\
.9 \\
.3 \\
.8\end{array}$ & $\begin{array}{l}0.07 \\
0.11 \\
0.06 \\
0.07 \\
0.04\end{array}$ \\
\hline $\begin{array}{l}24 \\
25 \\
25 \\
25 \\
25\end{array}$ & $\begin{array}{r}12 \\
0 \\
8 \\
8 \\
13\end{array}$ & $\begin{array}{l}49 \\
21 \\
22 \\
23 \\
42\end{array}$ & $\begin{array}{l}22.2 \\
55.8 \\
48.5 \\
16.5 \\
41.5\end{array}$ & $\begin{array}{l}33-3.1 \\
33-18.8 \\
32-59.4 \\
33-0.1 \\
33-2.6\end{array}$ & $\begin{array}{l}116 \\
116 \\
116\end{array}$ & & $\begin{array}{l}2.0,2.5 \\
1.8 \\
1.4 \\
1.0 \\
1.2\end{array}$ & $\begin{array}{l}19 \\
20 \\
19 \\
19 \\
20\end{array}$ & $\begin{array}{l}70 \\
101 \\
210 \\
204 \\
104\end{array}$ & & & & $\begin{array}{l}0 \\
1 \\
.6 \\
3 \\
8\end{array}$ & $\begin{array}{l}0.07 \\
0.05 \\
0.06 \\
0.09 \\
0.06\end{array}$ \\
\hline $\begin{array}{l}25 \\
25 \\
25 \\
25 \\
25\end{array}$ & $\begin{array}{l}14 \\
16 \\
16 \\
16 \\
17\end{array}$ & $\begin{array}{l}55 \\
32 \\
33 \\
37 \\
43\end{array}$ & $\begin{array}{l}36.8 \\
37.2 \\
32.7 \\
36.3 \\
56.8\end{array}$ & $\begin{array}{l}33-14.5 \\
32-57.1 \\
32-57.1 \\
33-7.4 \\
33-6.9\end{array}$ & $\begin{array}{l}116 \\
116 \\
116 \\
116 \\
116\end{array}$ & $\begin{array}{r}8 \\
10 \\
9 \\
6 \\
2\end{array}$ & $\begin{array}{l}1.7 \\
1.8 \\
1.1 \\
1.9 \\
1.6\end{array}$ & $\begin{array}{l}20 \\
20 \\
20 \\
20\end{array}$ & $\begin{array}{r}59 \\
225 \\
225 \\
52 \\
129\end{array}$ & 11 & & & $\begin{array}{l}7 \\
2 \\
5 \\
7\end{array}$ & $\begin{array}{l}0.08 \\
0.10 \\
0.05 \\
0.07\end{array}$ \\
\hline $\begin{array}{l}26 \\
26 \\
26 \\
26 \\
28\end{array}$ & $\begin{array}{r}6 \\
16 \\
23 \\
23 \\
0\end{array}$ & $\begin{array}{l}46 \\
57 \\
28 \\
28 \\
34\end{array}$ & $\begin{array}{r}28.1 \\
8.6 \\
17.9 \\
25.8 \\
27.3\end{array}$ & $\begin{array}{l}33-2.4 \\
33-17.3 \\
33-8.4 \\
33-4.6 \\
33-2.9\end{array}$ & $\begin{array}{l}16-2.4 \\
16-15.3 \\
16-3.6 \\
16-1.7 \\
15-59.8\end{array}$ & & $\begin{array}{l}1.6^{.3 .} \\
0.1 \\
1.1 \\
1.3\end{array}$ & $\begin{array}{l}17 \\
19 \\
17 \\
18 \\
19\end{array}$ & $\begin{array}{l}91 \\
85 \\
57 \\
72 \\
73\end{array}$ & & & & $\begin{array}{l}6 \\
.5 \\
0 \\
2 \\
6\end{array}$ & $\begin{array}{l}0.04 \\
0.04 \\
0.06 \\
0.05 \\
0.06\end{array}$ \\
\hline $\begin{array}{l}29 \\
29 \\
29 \\
29 \\
30\end{array}$ & $\begin{array}{r}0 \\
8 \\
10 \\
19 \\
6\end{array}$ & $\begin{array}{l}46 \\
48 \\
19 \\
38 \\
12\end{array}$ & $\begin{array}{l}57.3 \\
26.6 \\
13.1 \\
30.3 \\
55.1\end{array}$ & $\begin{array}{l}33-2.6 \\
33-9.4 \\
33-7.9 \\
33-17.2 \\
33-7.1\end{array}$ & $\begin{array}{l}116-0.2 \\
116-5.8 \\
116-5.1 \\
116-15.4 \\
116-4.3\end{array}$ & & $\begin{array}{l}1.3 \\
2.1 \\
1.0\end{array}$ & $\begin{array}{l}21 \\
20 \\
19 \\
18 \\
18\end{array}$ & $\begin{array}{l}48 \\
86 \\
56\end{array}$ & & & & $\begin{array}{l}0.4 \\
0.5 \\
0.5 \\
0.5 \\
0.4\end{array}$ & $\begin{array}{l}0.04 \\
0.05 \\
0.05 \\
0.04 \\
0.07\end{array}$ \\
\hline $\begin{array}{l}30 \\
30 \\
31 \\
31 \\
31\end{array}$ & $\begin{array}{r}6 \\
22 \\
0 \\
1 \\
3\end{array}$ & $\begin{array}{l}13 \\
12 \\
29 \\
34 \\
51\end{array}$ & $\begin{array}{r}15.4 \\
58.2 \\
2.1 \\
6.0 \\
3.3\end{array}$ & $\begin{array}{l}33-1.2 \\
33-2.6 \\
33-3.6 \\
33-10.0 \\
33-3.7\end{array}$ & $\begin{array}{l}116-13.6 \\
116-0.3 \\
115-55.8 \\
116-3.6 \\
115-55.7\end{array}$ & & $\begin{array}{r}-0.2 \\
1.9 \\
1.7 \\
1.8\end{array}$ & $\begin{array}{l}6 \\
17 \\
18 \\
17 \\
17\end{array}$ & $\begin{array}{r}209 \\
78 \\
55 \\
60 \\
56\end{array}$ & & & & $\begin{array}{l}2.8 \\
0.6 \\
0.8 \\
0.7 \\
0.8\end{array}$ & $\begin{array}{l}0.03 \\
0.05 \\
0.04 \\
0.05 \\
0.04\end{array}$ \\
\hline $\begin{array}{l}31 \\
31 \\
31 \\
31 \\
31\end{array}$ & $\begin{array}{l}8 \\
15 \\
15 \\
15 \\
15\end{array}$ & $\begin{array}{l}13 \\
22 \\
23 \\
38 \\
38\end{array}$ & $\begin{array}{r}32.4 \\
50.1 \\
7.2 \\
43.4 \\
60.0\end{array}$ & $\begin{array}{l}33-10.0 \\
32-57.7 \\
32-58.1 \\
33-9.7 \\
33-9.6\end{array}$ & $\begin{array}{l}116-6 . \\
116-15 . \\
116-15 . \\
116-5 . \\
116-5 .\end{array}$ & & $\begin{array}{l}1.2 \\
1.2 \\
1.1 \\
0.4 \\
1.4\end{array}$ & $\begin{array}{l}18 \\
18 \\
15 \\
16 \\
16\end{array}$ & $\begin{array}{r}48 \\
211 \\
208 \\
57 \\
57\end{array}$ & תם & & 0.2 & $\begin{array}{l}0.4 \\
1.4 \\
1.9 \\
0.7 \\
0.5\end{array}$ & $\begin{array}{l}0.06 \\
0.07 \\
0.09 \\
0.06 \\
0.04\end{array}$ \\
\hline 1 & $\begin{array}{r}7 \\
0 \\
3 \\
5 \\
15\end{array}$ & $\begin{array}{r}22 \\
2 \\
55 \\
21 \\
34\end{array}$ & $\begin{array}{l}43.9 \\
13.5 \\
38.8 \\
57.8\end{array}$ & $\begin{array}{l}33-2.6 \\
33-2.6 \\
33-5.1 \\
33-5.2\end{array}$ & $\begin{array}{l}116-1.3 \\
116-0.2 \\
116-0.2 \\
116-2.9 \\
116-2.8\end{array}$ & $\begin{array}{l}7.2 \\
7.8 \\
5.1 \\
6.2\end{array}$ & $\begin{array}{l}1.7 \\
1.3 \\
1.4\end{array}$ & $\begin{array}{l}19 \\
20 \\
20 \\
19\end{array}$ & $\begin{array}{l}84 \\
77 \\
54 \\
55\end{array}$ & $\begin{array}{l}3.8 \\
3.7 \\
2.5 \\
2.4\end{array}$ & $\begin{array}{l}0.03 \\
0.04 \\
0.04 \\
0.03\end{array}$ & $\begin{array}{l}0.2 \\
0.3 \\
0.2 \\
0.2\end{array}$ & $\begin{array}{l}0.5 \\
0.6 \\
0.7 \\
0.6\end{array}$ & $\begin{array}{l}0.04 \\
0.05 \\
0.05 \\
0.06 \\
0.06\end{array}$ \\
\hline
\end{tabular}


TABLE 8. - Borrego Mountain aftershocks-Continued

\begin{tabular}{|c|c|c|c|c|c|c|c|c|c|c|c|c|c|c|c|}
\hline & HR & MN & SEC & LAT $N$ & LONG $W$ & EPTH & MAG & NO & GAP & DMIN & ERT & ERH & $R 2$ & MD & \\
\hline $\begin{array}{l}3 \\
3 \\
3 \\
3 \\
3\end{array}$ & $\begin{array}{l}6 \\
6 \\
6 \\
9 \\
9\end{array}$ & $\begin{array}{r}2 \\
4 \\
6 \\
10 \\
11\end{array}$ & $\begin{array}{l}19.6 \\
56.6 \\
26.1 \\
22.4 \\
42.4\end{array}$ & $\begin{array}{l}33-3.6 \\
33-18.9 \\
33-11.4 \\
33-11.7 \\
33-11.9\end{array}$ & $\begin{array}{l}115-58.5 \\
116-13.2 \\
115-55.0 \\
116-9.7 \\
116-9.9\end{array}$ & $\begin{array}{l}4.6 \\
3.0 * \\
7.3 \\
7.7 \\
6.4\end{array}$ & $\begin{array}{l}1.2 \\
2.1 .2 .5 \\
0.6 \\
0.2^{.3 .3}\end{array}$ & $\begin{array}{l}21 \\
21 \\
20 \\
20 \\
13\end{array}$ & $\begin{array}{r}66 \\
102 \\
68 \\
47 \\
79\end{array}$ & $\begin{array}{l}5.1 \\
5.5 \\
5.7 \\
3.0 \\
2.9\end{array}$ & $\begin{array}{l}0.08 \\
0.03 \\
0.05 \\
0.04 \\
0.08\end{array}$ & $\begin{array}{l}0.5 \\
0.2 \\
0.3 \\
0.2 \\
0.5\end{array}$ & $\begin{array}{l}2.0 \\
0.8 \\
0.5 \\
1.1\end{array}$ & $\begin{array}{l}0.09 \\
0.05 \\
0.07 \\
0.06 \\
0.08\end{array}$ & \\
\hline $\begin{array}{l}3 \\
3 \\
3 \\
3 \\
3\end{array}$ & $\begin{array}{l}10 \\
12 \\
14 \\
21 \\
21\end{array}$ & $\begin{array}{l}47 \\
31 \\
16 \\
28 \\
31\end{array}$ & $\begin{array}{l}17.4 \\
37.3 \\
56.9 \\
34.9 \\
36.8\end{array}$ & $\begin{array}{l}33-4.2 \\
33-11.7 \\
32-52.0 \\
33-7.8 \\
33-7.9\end{array}$ & $\begin{array}{l}115-57.9 \\
116-9.5 \\
116-1.03 \\
116-2.1 \\
116-2.1\end{array}$ & $\begin{array}{l}5.5 \\
7.7 \\
5.0 * \\
1.8 \\
2.0\end{array}$ & $\begin{array}{l}1.7 \\
2.1 .2 .6 \\
2.0 \\
1.1 \\
1.1\end{array}$ & $\begin{array}{l}21 \\
21 \\
15 \\
20 \\
21\end{array}$ & $\begin{array}{r}77 \\
47 \\
155 \\
52 \\
53\end{array}$ & $\begin{array}{l}5.3 \\
2.9 \\
7.1 \\
6.7 \\
5.8\end{array}$ & $\begin{array}{l}0.03 \\
0.04 \\
0.33 \\
0.03 \\
0.03\end{array}$ & $\begin{array}{l}0.2 \\
0.2 \\
2.6 \\
0.2 \\
0.2\end{array}$ & $\begin{array}{l}0.7 \\
0.6\end{array}$ & $\begin{array}{l}0.05 \\
0.06 \\
0.47 \\
0.06 \\
0.06\end{array}$ & \\
\hline $\begin{array}{l}3 \\
3 \\
3 \\
3 \\
4\end{array}$ & $\begin{array}{r}21 \\
21 \\
21 \\
22 \\
2\end{array}$ & $\begin{array}{l}32 \\
34 \\
36 \\
25 \\
14\end{array}$ & $\begin{array}{r}31.4 \\
56.4 \\
0.9 \\
51.0 \\
16.7\end{array}$ & $\begin{array}{l}33-7.8 \\
33-11.7 \\
33-7.8 \\
33-16.1 \\
33-2.5\end{array}$ & $\begin{array}{l}116-2.1 \\
116-9.9 \\
116-2.0 \\
115-59.5 \\
116-2.3\end{array}$ & $\begin{array}{l}1.7 \\
7.0 \\
1.7 \\
3.4\end{array}$ & $\begin{array}{l}0.2 \\
0.8 \\
0.6 \\
1.6 \\
1.6\end{array}$ & $\begin{array}{l}16 \\
19 \\
20 \\
20 \\
20\end{array}$ & $\begin{array}{r}61 \\
47 \\
53 \\
176 \\
67\end{array}$ & $\begin{array}{l}5.7 \\
3.0 \\
5.8 \\
4.6 \\
2.3\end{array}$ & $\begin{array}{l}0.02 \\
0.03 \\
0.03 \\
0.06 \\
0.03\end{array}$ & $\begin{array}{l}.2 \\
.2 \\
.2 \\
.4 \\
.2\end{array}$ & $\begin{array}{l}0.3 \\
0.4 \\
0.3 \\
1.9 \\
0.5\end{array}$ & $\begin{array}{l}0.05 \\
0.05 \\
0.06 \\
0.07 \\
0.05\end{array}$ & $B$ \\
\hline $\begin{array}{l}4 \\
4 \\
4 \\
5 \\
5\end{array}$ & $\begin{array}{r}3 \\
13 \\
13 \\
1 \\
23\end{array}$ & $\begin{array}{l}48 \\
37 \\
38 \\
18 \\
23\end{array}$ & $\begin{array}{l}19.7 \\
42.2 \\
44.4 \\
49.1 \\
39.2\end{array}$ & $\begin{array}{l}33-2.4 \\
33-16.4 \\
33-15.8 \\
32-58.8 \\
33-0.9\end{array}$ & $\begin{array}{l}116-2.3 \\
115-59.6 \\
115-58.3 \\
116-15.0 \\
115-55.6\end{array}$ & $\begin{array}{l}5.1 \\
0.9 \\
1.9 \\
7.6 \\
9.6\end{array}$ & $\begin{array}{l}2.0^{.3 .2} \\
0.5 \\
0.8 \\
1.7\end{array}$ & $\begin{array}{l}21 \\
19 \\
16 \\
16 \\
20\end{array}$ & $\begin{array}{r}69 \\
176 \\
180 \\
203 \\
76\end{array}$ & $\begin{array}{l}2.2 \\
5.0 \\
5.4 \\
5.2 \\
7.7\end{array}$ & $\begin{array}{l}0.03 \\
0.09 \\
0.10 \\
0.07 \\
0.05\end{array}$ & $\begin{array}{l}0.2 \\
0.4 \\
0.4 \\
0.4 \\
0.3\end{array}$ & $\begin{array}{l}0.4 \\
0.7 \\
0.6 \\
0.8 \\
0.6\end{array}$ & $\begin{array}{l}0.04 \\
0.07 \\
0.07 \\
0.05 \\
0.07\end{array}$ & $\begin{array}{l}B \\
B \\
B \\
A\end{array}$ \\
\hline $\begin{array}{l}6 \\
6 \\
6 \\
6 \\
6\end{array}$ & $\begin{array}{r}5 \\
6 \\
9 \\
13 \\
16\end{array}$ & $\begin{array}{l}27 \\
13 \\
12 \\
18 \\
28\end{array}$ & $\begin{array}{r}46.4 \\
48.5 \\
2.0 \\
4.9 \\
11.2\end{array}$ & $\begin{array}{l}33-8.4 \\
33-2.4 \\
33-0.6 \\
33-0.8 \\
32-57.9\end{array}$ & $\begin{array}{l}116-0.4 \\
116-2.4 \\
115-55.7 \\
115-55.6 \\
116-12.5\end{array}$ & $\begin{array}{l}4.7 \\
4.6 \\
6.4 \\
7.6 \\
2.4\end{array}$ & $\begin{array}{l}1.6 \\
1.8 \\
.3 .2 \\
0.4^{.3 .1}\end{array}$ & $\begin{array}{r}20 \\
20 \\
18 \\
20 \\
8\end{array}$ & $\begin{array}{r}67 \\
66 \\
77 \\
76 \\
200\end{array}$ & $\begin{array}{l}4.4 \\
2.1 \\
8.3 \\
8.0 \\
5\end{array}$ & $\begin{array}{l}0.03 \\
0.03 \\
0.04 \\
0.04 \\
0.29\end{array}$ & $\begin{array}{l}0.2 \\
0.2 \\
0.2 \\
0.2 \\
1.6\end{array}$ & $\begin{array}{l}.7 \\
.6 \\
.8 \\
.7 \\
.8\end{array}$ & $\begin{array}{l}0.06 \\
0.05 \\
0.04 \\
0.04 \\
0.13\end{array}$ & $B$ \\
\hline $\begin{array}{l}6 \\
6 \\
7 \\
7 \\
7\end{array}$ & $\begin{array}{r}16 \\
22 \\
2 \\
13 \\
13\end{array}$ & $\begin{array}{r}29 \\
41 \\
18 \\
1 \\
36\end{array}$ & $\begin{array}{r}6.9 \\
24.9 \\
13.1 \\
56.8 \\
50.1\end{array}$ & $\begin{array}{l}32-58.7 \\
33-7.8 \\
33-8.7 \\
33-1.4 \\
33-6.3\end{array}$ & $\begin{array}{l}116-12.6 \\
116-6.0 \\
116-3.6 \\
115-56.4 \\
116-11.3\end{array}$ & $\begin{array}{l}2.7 \\
1.5 \\
6.1 \\
7.7 \\
8.2\end{array}$ & $\begin{array}{l}0.5 \\
1.0 \\
1.3 \\
1.7 \\
1.6\end{array}$ & $\begin{array}{l}11 \\
19 \\
20 \\
21 \\
20\end{array}$ & $\begin{array}{r}194 \\
56 \\
50 \\
68 \\
86\end{array}$ & $\begin{array}{l}4.1 \\
1.1 \\
4.3 \\
4.1 \\
7.8\end{array}$ & $\begin{array}{l}0.12 \\
0.03 \\
0.03 \\
0.03 \\
0.04\end{array}$ & $\begin{array}{l}0.6 \\
0.2 \\
0.2 \\
0.2 \\
0.2\end{array}$ & $\begin{array}{l}0.9 \\
0.3 \\
0.6 \\
0.5 \\
0.6\end{array}$ & $\begin{array}{l}0.07 \\
0.07 \\
0.06 \\
0.04 \\
0.05\end{array}$ & A \\
\hline $\begin{array}{l}7 \\
7 \\
8 \\
8 \\
8\end{array}$ & $\begin{array}{l}13 \\
16 \\
16 \\
16 \\
20\end{array}$ & $\begin{array}{l}46 \\
13 \\
14 \\
19 \\
51\end{array}$ & $\begin{array}{r}39.2 \\
55.3 \\
10.0 \\
6.3 \\
18.8\end{array}$ & $\begin{array}{l}33-27.4 \\
33-8.3 \\
33-1.4 \\
33-1.5 \\
32-58.5\end{array}$ & $\begin{array}{l}116- \\
116- \\
115- \\
115 \\
116\end{array}$ & $\begin{array}{l}9.8 \\
4.6 \\
6.4 \\
6.2 \\
7.9\end{array}$ & $\begin{array}{l}1.7 \\
1.7 \\
2.0,2.6 \\
1.6 \\
1.5\end{array}$ & $\begin{array}{l}19 \\
19 \\
20 \\
20 \\
21\end{array}$ & $\begin{array}{r}237 \\
47 \\
69 \\
68 \\
207\end{array}$ & $\begin{array}{r}10.8 \\
3.9 \\
4.2 \\
4.4 \\
6.1\end{array}$ & $\begin{array}{l}0.23 \\
0.02 \\
0.03 \\
0.04 \\
0.09\end{array}$ & $\begin{array}{l}1.3 \\
0.2 \\
0.2 \\
0.2 \\
0.5\end{array}$ & $\begin{array}{l}0.7 \\
0.6 \\
0.5 \\
0.6 \\
1.0\end{array}$ & $\begin{array}{l}0.12 \\
0.05 \\
0.04 \\
0.05 \\
0.07\end{array}$ & $\begin{array}{l}C \\
A\end{array}$ \\
\hline $\begin{array}{l}8 \\
9 \\
9 \\
9 \\
9\end{array}$ & $\begin{array}{r}21 \\
0 \\
6 \\
14 \\
14\end{array}$ & $\begin{array}{r}37 \\
15 \\
0 \\
20 \\
48\end{array}$ & $\begin{array}{r}0.9 \\
11.8 \\
49.1 \\
15.6 \\
29.6\end{array}$ & $\begin{array}{l}33-15.9 \\
33-14.1 \\
33-25.2 \\
33-16.8 \\
33-4.6\end{array}$ & $\begin{array}{l}116-2.5 \\
116-0.6 \\
116-21.3 \\
115-59.5 \\
115-55.5\end{array}$ & $\begin{array}{c}1.3 \\
0.4 * \\
10.6 \\
2.9 \\
5.1\end{array}$ & $\begin{array}{l}2.3 .3 .1 \\
1.5 \\
2.0 \\
.3 .1 \\
.3 .0\end{array}$ & $\begin{array}{l}21 \\
21 \\
17 \\
18 \\
19\end{array}$ & $\begin{array}{r}162 \\
162 \\
290 \\
211 \\
84\end{array}$ & $\begin{array}{l}0.8 \\
6.6 \\
5.6 \\
1.6\end{array}$ & $\begin{array}{l}0.05 \\
0.04 \\
0.31 \\
0.14 \\
0.03\end{array}$ & $\begin{array}{l}0.3 \\
0.3 \\
1.6 \\
0.6 \\
0.2\end{array}$ & $\begin{array}{l}.8 \\
.8 \\
.5\end{array}$ & $\begin{array}{l}0.07 \\
0.07 \\
0.09 \\
0.09 \\
0.04\end{array}$ & $\begin{array}{l}B \\
C \\
C \\
B \\
A\end{array}$ \\
\hline $\begin{array}{ll}1 & 1 \\
1 & 1 \\
1 & 1 \\
1 & 1 \\
11\end{array}$ & $\begin{array}{r}2 \\
2 \\
5 \\
5 \\
19\end{array}$ & $\begin{array}{l}22 \\
22 \\
32 \\
33 \\
28\end{array}$ & $\begin{array}{r}7.0 \\
34.7 \\
17.3 \\
37.8 \\
11.1\end{array}$ & $\begin{array}{l}33-1.8 \\
33-2.6 \\
33-20.9 \\
33-21.3 \\
32-57.2\end{array}$ & $\begin{array}{l}115-58.6 \\
116-2.3 \\
116-20.0 \\
116-20.8 \\
116-17.5\end{array}$ & $\begin{array}{r}6.6 \\
5.3 \\
10.1 \\
13.0\end{array}$ & $\begin{array}{l}-0.2 \\
1.8 \\
2.5 .3 .1 \\
0.6 \\
1.3\end{array}$ & $\begin{array}{r}11 \\
20 \\
21 \\
8 \\
11\end{array}$ & $\begin{array}{r}92 \\
63 \\
185 \\
194 \\
220\end{array}$ & $\begin{array}{l}3.5 \\
2.4 \\
2.5 \\
2.8 \\
10.1\end{array}$ & $\begin{array}{l}0.08 \\
0.03 \\
0.10 \\
0.20 \\
0.11\end{array}$ & $\begin{array}{l}0.4 \\
0.2 \\
0.6 \\
0.8 \\
0.7\end{array}$ & $\begin{array}{l}1.0 \\
0.5 \\
0.5 \\
1.5 \\
0.8\end{array}$ & $\begin{array}{l}0.06 \\
0.05 \\
0.10 \\
0.06 \\
0.07\end{array}$ & $\begin{array}{l}C \\
B \\
B\end{array}$ \\
\hline $\begin{array}{l}11 \\
12 \\
12 \\
12 \\
12\end{array}$ & $\begin{array}{r}21 \\
1 \\
1 \\
12 \\
12\end{array}$ & $\begin{array}{r}41 \\
9 \\
9 \\
31 \\
31\end{array}$ & $\begin{array}{r}7.6 \\
47.9 \\
52.5 \\
8.0 \\
33.3\end{array}$ & $\begin{array}{l}33-2.1 \\
33-17.1 \\
33-16.3 \\
33-0.1 \\
32-57.0\end{array}$ & $\begin{array}{l}115-59.2 \\
116-16.3 \\
115-59.6 \\
116-14.8 \\
116-15.4\end{array}$ & $\begin{array}{l}8 . \\
6 . \\
0 . \\
6 . \\
5 .\end{array}$ & $\begin{array}{l}1.5 \\
0.1 \\
1.6 \\
0.4 \\
0.6\end{array}$ & $\begin{array}{l}20 \\
12 \\
21 \\
15 \\
18\end{array}$ & $\begin{array}{r}84 \\
75 \\
176 \\
196 \\
213\end{array}$ & $\begin{array}{l}4 . \\
3 . \\
8\end{array}$ & $\begin{array}{l}0.04 \\
0.08 \\
0.09 \\
0.09\end{array}$ & $\begin{array}{l}0.3 \\
0.2 \\
0.3 \\
0.5 \\
0.5\end{array}$ & $\begin{array}{l}0.6 \\
0.5 \\
0.6 \\
1.1 \\
1.8\end{array}$ & $\begin{array}{l}0.06 \\
0.04 \\
0.07 \\
0.09 \\
0.06\end{array}$ & C \\
\hline $\begin{array}{l}12 \\
12 \\
12\end{array}$ & $\begin{array}{l}12 \\
13 \\
13\end{array}$ & $\begin{array}{l}34 \\
30 \\
38\end{array}$ & $\begin{array}{l}26.4 \\
29.3 \\
11.6\end{array}$ & $\begin{array}{l}33-17.1 \\
33-2.1 \\
33-10.6\end{array}$ & $\begin{array}{l}115-57.2 \\
115-56.6 \\
116-5.5\end{array}$ & $\begin{array}{l}1.1 \\
4.9 \\
9.8\end{array}$ & $\begin{array}{l}1.0 \\
0.9 \\
1.2\end{array}$ & $\begin{array}{l}19 \\
33 \\
21\end{array}$ & $\begin{array}{r}219 \\
53 \\
54\end{array}$ & $\begin{array}{l}8.4 \\
2.0 \\
5.9\end{array}$ & $\begin{array}{l}0.02 \\
0.04\end{array}$ & $\begin{array}{l}0.7 \\
0.2 \\
0.2\end{array}$ & $\begin{array}{l}1.0 \\
0.4 \\
0.5\end{array}$ & $\begin{array}{l}0.10 \\
0.05 \\
0.06\end{array}$ & \\
\hline
\end{tabular}


THE BORREGO MOUNTAIN EARTHQUAKE OF APRIL 9, 1968

TABLE 8. - Borrego Mountain aftershocks-Continued

EARTHQUAKES SOUTHEAST OF PORTABLE SEISMOGRAPH NETWORK

\begin{tabular}{crrrrrrrrrrrr}
\multicolumn{1}{c}{1968} & HR & MN & SEC & LAT N & LONG W & DEPTH & MAG & NO & ERT & ERH & MD \\
APR & 18 & 0 & 0 & 25.2 & $32-55.6$ & $115-40.5$ & $5.0 *$ &, 3.2 & 14 & 0.11 & 1.4 & 0.11 \\
& 23 & 15 & 42 & 41.1 & $32-45.9$ & $115-32.2$ & $5.0 *$ & & 16 & 0.43 & 3.0 & 0.09 \\
23 & 16 & 23 & 52.4 & $32-48.2$ & $115-38.7$ & $5.0 *$ &, 3.1 & 18 & 0.14 & 1.4 & 0.14 \\
& 23 & 16 & 24 & 09.1 & $32-46.3$ & $115-35.8$ & $5.0 *$ &, 4.1 & 17 & 0.15 & 1.4 & 0.15 \\
23 & 16 & 50 & 41.1 & $32-46.8$ & $115-33.3$ & $5.0 *$ & & 16 & 0.60 & 4.0 & 0.11 \\
& 24 & 12 & 32 & 31.1 & $32-49.2$ & $115-39.5$ & $5.0 *$ &, 3.4 & 18 & 0.22 & 2.1 & 0.26 \\
MAY & 14 & 9 & 11 & 18.7 & $32-57.6$ & $115-30.3$ & $5.0 *$ &, 2.6 & 21 & 0.15 & 1.7 & 0.23
\end{tabular}

NoTE. - List of Borrego Mountain aftershocks for which locations were determined. For each event the following data are given:

Origin time in Greenwich mean time: date, hour $(\mathrm{Hr})$, minute $(\mathrm{Mn})$, and second (Sec).

Epicenter in degrees and minutes of north latitude (Lat (N.)) and west longitude (Long (W.)). Poor convergence of the episolution is indicated by "?".

DEPTH $=$ depth of focus, in kilometers. Assumed depth is indicated by "*".

$\mathrm{X}-\mathrm{MAG}=$ earthquake magnitude. Value on left determined from USGS data (see discussion in text); value on right quoted from Caltech bulletin (Nordquist and others, 1972).

$\mathrm{NO}=$ number of stations used in locating earthquake.

GAP $=$ largest azimuthal separation, in degrees, between stations.

DMIN=epicentral distance, in kilometers, to the nearest station.

$E R T=$ standard error of the origin time, in seconds.

$\mathrm{ERH}=$ standard error of the epicenter, in kilometers.

$$
\left[=\sqrt{\left(\mathrm{SDX}^{2}+\mathrm{SDY}^{2}\right) / 2}\right]
$$

where SDX and SDY are the standard errors in latitude and longtitude, respectively, of the epicenter.

$E R Z=$ standard error of the depth, in kilometers.

$\mathrm{MD}=$ mean deviation of the time residuals. $\left[=\frac{\sum \cdot R_{i}}{\mathrm{NO}}\right]$, where $R_{i}$ is the observed seismic wave arrival time less the computed time at the ith station.

$2 \mathrm{~km}$, but this uncertainty depends strongly on the distance from an epicenter to the nearest station.

The crustal model used in locating the aftershocks, which was derived from the special refraction survey (Hamilton, 1970), is summarized below:

\begin{tabular}{|c|c|c|c|}
\hline Layor & $\begin{array}{c}\text { Volocity } \\
(\mathrm{km} / \mathrm{scc})\end{array}$ & $\begin{array}{c}\text { Thickness } \\
(\mathrm{km})\end{array}$ & $\begin{array}{c}\text { Depth to top } \\
(\mathrm{km})\end{array}$ \\
\hline 5 & 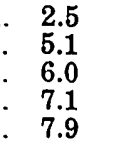 & $\begin{array}{r}0.4 \\
2.5 \\
11.1 \\
11.0 \\
\ldots \ldots .\end{array}$ & $\begin{array}{r}0.0 \\
.4 \\
2.0 \\
14.0 \\
25.0\end{array}$ \\
\hline
\end{tabular}

$Q=$ solution quality of the hypocenter. This measure is intended to indicate the general reliability of each solution:

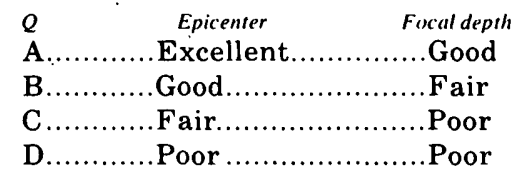

$Q$ is based on both the nature of the station distribution with respect to the earthquake and the statistical measures of the solution. These two factors are each rated independently according to the following scheme:

Station distribution

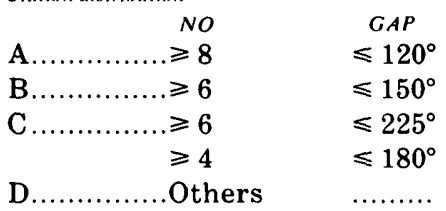

Statistical measures

$\begin{array}{lccr}E R H(\mathrm{~km}) & E R Z(\mathrm{~km}) & M D(\mathrm{sec}) & R M A X(\mathrm{sec})^{\prime} \\ \mathrm{A} \ldots \ldots \ldots \ldots \ldots \ldots \ldots \ldots 1.0 & \leqslant 2.0 & \leqslant 0.10 & \leqslant 0.25 \\ \mathrm{~B} \ldots \ldots \ldots \ldots \ldots \ldots \ldots \ldots \leqslant 2.5 & \leqslant 5.0 & \leqslant 0.20 & \leqslant 0.50 \\ \mathrm{C} \ldots \ldots \ldots \ldots \ldots \ldots \ldots \ldots \leqslant 5.0 & \ldots \ldots \ldots & \leqslant 0.30 & \leqslant 0.75 \\ \mathrm{D} \ldots \ldots \ldots \ldots \ldots \ldots \ldots \ldots & \ldots \ldots \ldots \ldots\end{array}$

' $R M A X$ is the maximum residual.

$Q$ is taken as the average of the ratings from the two schemes; that is, an A and a C yield a B, and two B's yield a B. When the two ratings are only one level apart, the lower one is used; that is, an A and a B yield a B.

Station time corrections against this model are given in table 7 ; a correction is subtracted from the observed arrival time. The corrections were applied to all arrivals, a procedure that is not strictly accurate because the corrections were obtained from refracted $P_{g}$ rays. Thus, direct $P$-wave arrivals would not be appropriately corrected; therefore, this method leads to errors mainly in focal-depth estimates for earthquakes above the $P_{g}$ refractor and in the vicinity of a station having a large correction. For example, shallow shocks near station 17 probably are actually 
somewhat deeper than was computed. It does not appear, though, that the method of applying station corrections seriously distorted the hypocenter pattern.

\section{AFTERSHOCK DISTRIBUTION}

The general distribution of the Borrego Mountain aftershocks in relation to mapped faults is shown in figure 12. The aftershock distribution with respect to surficial fracturing is shown in figures 13 and 14 . In these three figures, epicenters are plotted for all earthquakes with a mean deviation of the time residuals (MD) $\leq 0.05$ sec that are given in table 8 .

Figure 12 shows that a large part of the aftershock activity occurred in a band subparallel to the northwest-trending fault system. The epicenter of the main shock is near the center of this band, although many more aftershocks occurred to the southeast of it than to the northwest. The concentrated activity in the band southeast of the main shock ends at a point about $5 \mathrm{~km}$ east of station 10 and about $28 \mathrm{~km}$ southeast of the main shock. Northwest of the main shock, the band extends to near station 12. The epicenter $3 \mathrm{~km}$ west-southwest of station 12 lies $28 \mathrm{~km}$ from the main shock.

At its southeast end, the main seismic zone swings toward the Superstition Hills fault, where movement exceeding $1 \mathrm{~cm}$ occurred near the time of the main earthquake (Allen and others, this volume). Three earthquakes occurred on the Superstition Hills fault. Five approximately colinear epicenters were located about $5 \mathrm{~km}$ southwest of the Superstition Mountain fault. The seven epicenters in the azimuthal quadrant southeast of station 15, outside the portable seismograph net, were located using readings from both the portable stations and Caltech regional stations. The time corrections for the Caltech stations that were derived by Allen and Nordquist (this volume) were used. The earthquakes are given at the end of table 8. No earthquakes were detected near the San Andreas fault; this fault also moved on the order of $1 \mathrm{~cm}$ (Allen and others, this volume).

Most of the aftershocks that are not in the main band lie in two clusters centered about $16 \mathrm{~km}$ northeast and $22 \mathrm{~km}$ southwest of the middle of the main band.

A more detailed view of the epicenter pattern and its relation to the surface faulting is presented in figure 13. The surface faulting associated with the Borrego Mountain earthquake can be divided into two more or less continuous strands that come together in a complex pattern of faulting at the Oco- tillo Badlands, a group of low hills in the vicinity of station 9. Initial maximum horizontal displacement, which was in the right-lateral sense, was $38 \mathrm{~cm}$ on the northwest strand, near the main-shock epicenter, and $20 \mathrm{~cm}$ on the southeast strand (Allen and others, 1968; Clark, this volume). Movement on the southeast strand continued long after the main shock: postearthquake displacement of $8 \mathrm{~cm}$ occurred between April 25, 1968 and May 4, 1969 (Burford, this volume), and surface cracks continued to develop at least until January 1970 (Clark, "Surface Rupture Along the Coyote Creek Fault," this volume). No evidence of continued movement was found on the northwest strand.

The aftershocks in the main band are mostly along the surface fracture or to the northeast of it. The concentrated activity along the southeast fault strand extends about $4 \mathrm{~km}$ beyond the surface break. To the northwest, the earthquake $3 \mathrm{~km}$ west-southwest of station 12 lies about $20 \mathrm{~km}$ from the nearest surface fracture.

The southeast fault strand was far more active seismically than the northwest strand during the period of this study. Along the southeast strand, the epicentral zone varies in width from over $5 \mathrm{~km}$, near the region of complex faulting, to about $1 \mathrm{~km}$ at the center of the strand (near station 17). Farther to the southeast, the zone is several kilometers wide. On its southwest margin, the seismic zone along the southeast strand is rather sharply defined.

Seismic activity on the northwest fault strand is concentrated primarily in one cluster near the northwest end (near station 18). Another cluster of epicenters lies about $10 \mathrm{~km}$ northwest of the limit of recognized surface faulting, and there was scattered activity even farther to the northwest. Relatively few aftershocks were located near the epicenter of the main shock.

Variations in the epicenter pattern with time are shown in figure 14. The pattern was fairly stable; however, two variations are evident, both of which occurred after the first week of monitoring. One is the increased level of activity in the areas northeast and southwest of the main aftershock band; the other is the eastward extensions of the southeast end of the main band by about $3 \mathrm{~km}$.

The depth distribution of the aftershocks is shown in figures 15 and 16, where earthquakes having ERH $<1 \mathrm{~km}$ and $\mathrm{ERZ}<2 \mathrm{~km}$ (table 8 ) are plotted in vertical sections along the lines indicated in figure 13. The aftershocks are distributed from near surface to about $12 \mathrm{~km}$. Depth determinations for after- 


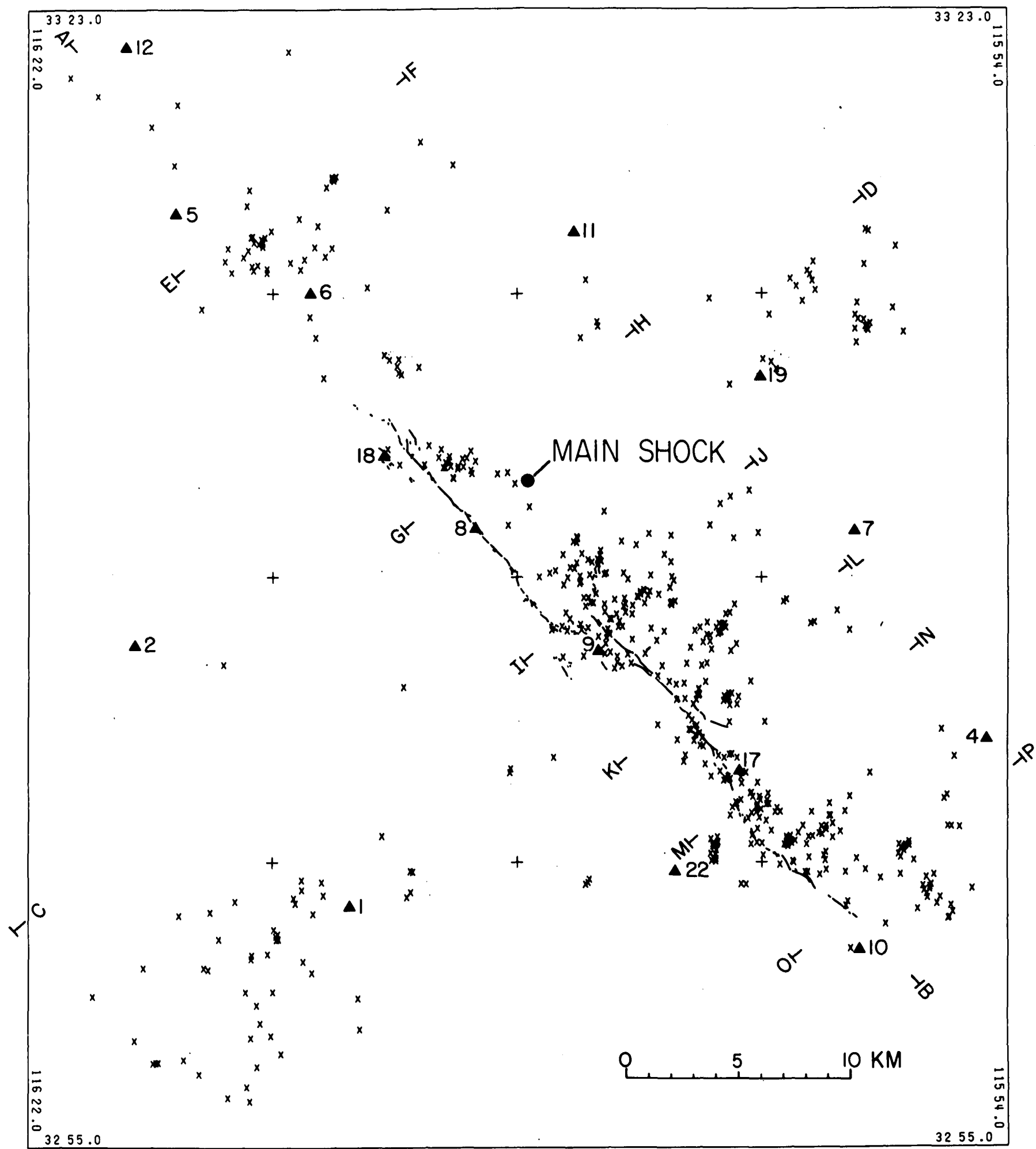

Figure 13. - Detailed map of aftershock epicenters (crosses), ground breakage (after Clark, this volume), epicenter of main shock (Allen and Nordquist, this volume), and lines of vertical sections for figure 15 (A-B, C-D) and figure 16 $(\mathrm{E}-\mathrm{F}$, etc.) 


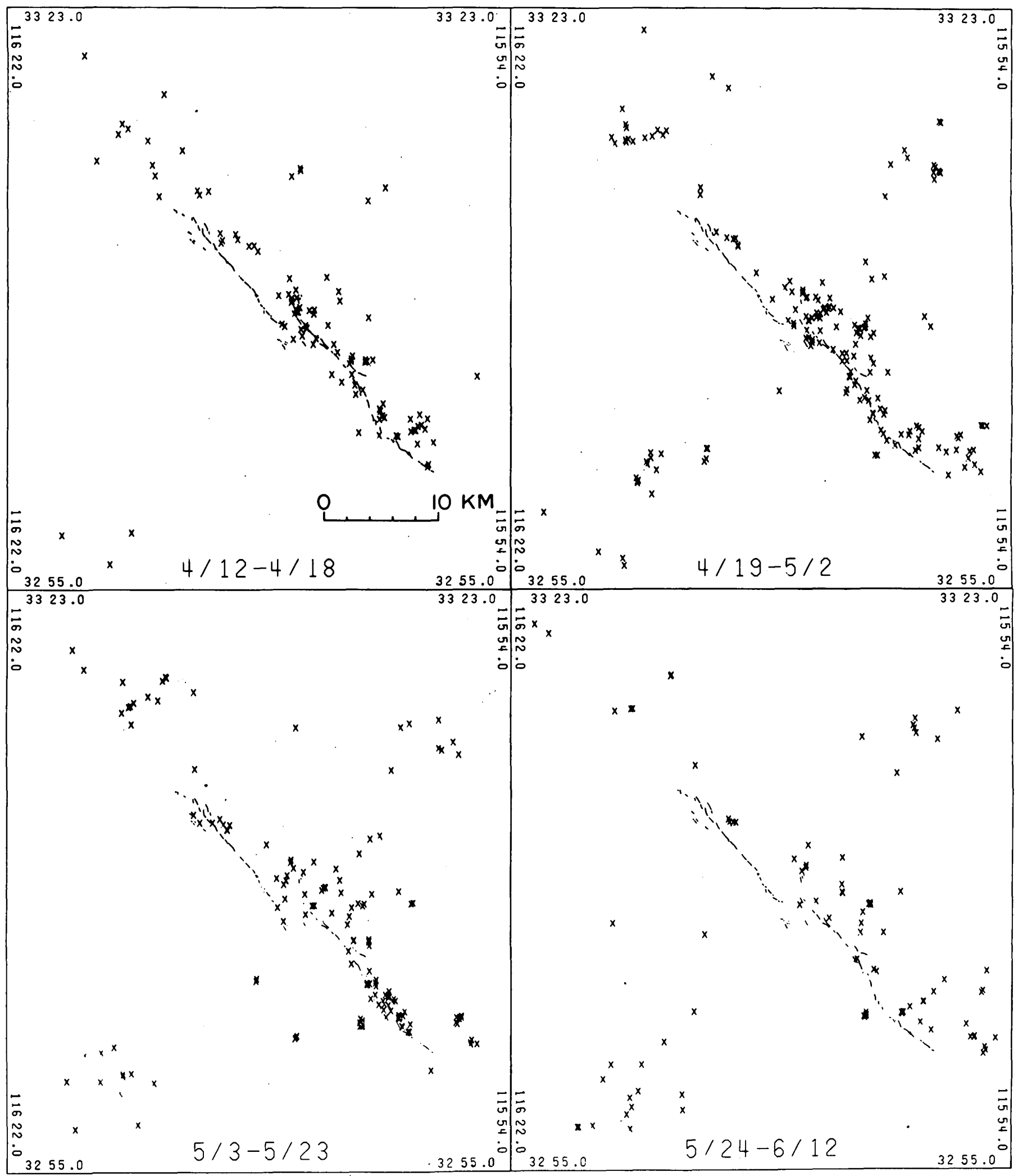

Figure 14. - Variation of epicenter pattern with time. 


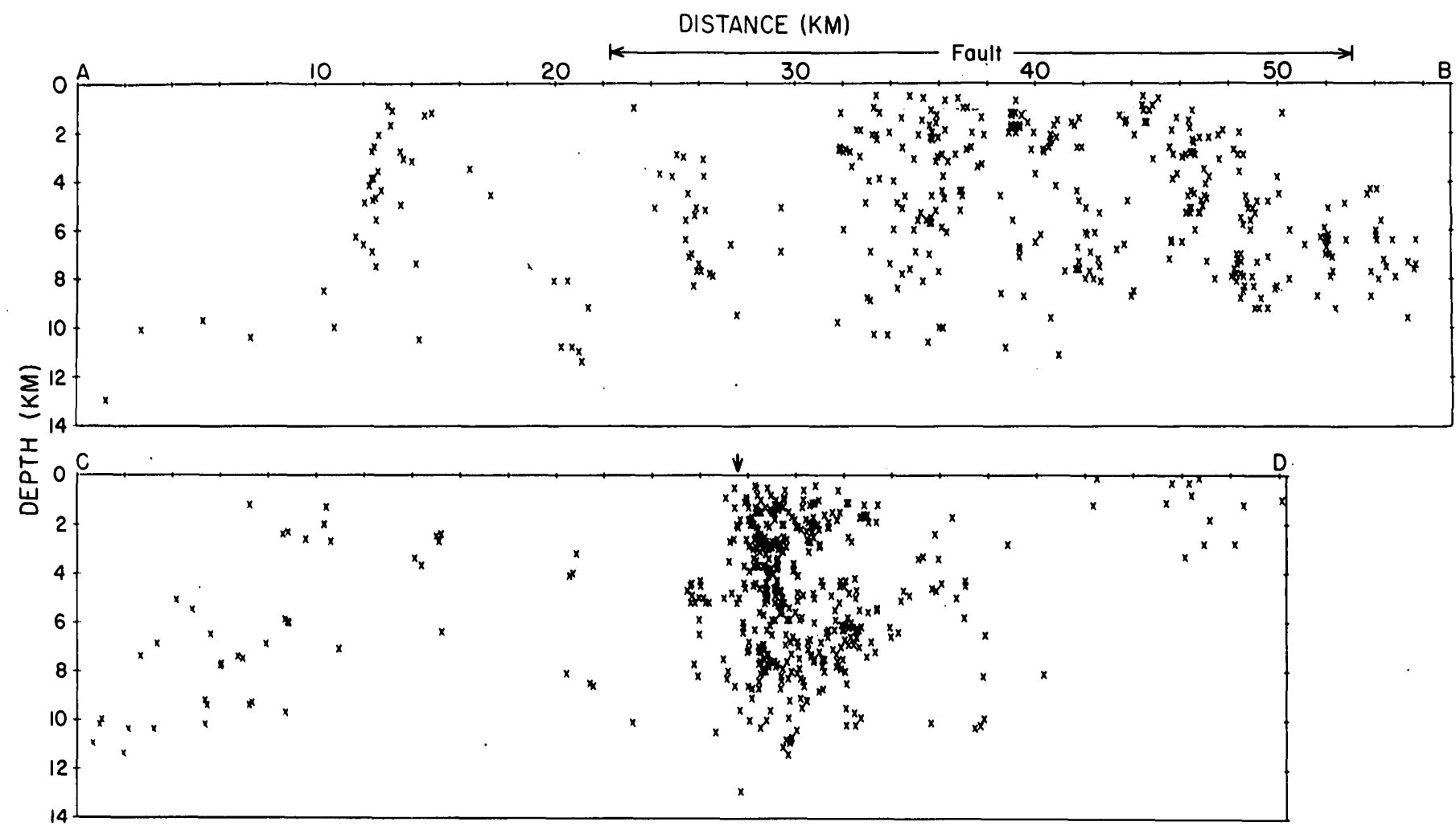

FIGURE 15. - Vertical sections through aftershock zone. Arrows indicate the approximate position of ground breakage on the line of section. Maximum projection distances of foci are: A-B, $5.00 \mathrm{~km} ; \mathrm{C}-\mathrm{D}, 30.00 \mathrm{~km}$. Lines of section are shown in figure 13.

shocks in the main band of activity along the fault are generally good; they are thought to have a precision of better than $2 \mathrm{~km}$. The depths for the shocks in the side clusters, on the margin of the seismograph net, are not so reliable.

The minimum depth of aftershock occurrence varies and is related to the surface faulting. On the southeast fault strand (fig. 15, section A-B, distance range $30-50 \mathrm{~km}$ ), where continued movement was observed, aftershocks occurred near the surface. The aftershocks beyond the southeastern limit of observed faulting occurred only as shallow as about $4 \mathrm{~km}$. The aftershocks just beyond the northwestern limit of observed faulting are below $7 \mathrm{~km}$. Farther northwest (between stations 5 and 6 ), foci are again near the surface, but no surface breakage was observed. This zone of activity lies in a thick section of Tertiary sedimentary rocks. At the northwest boundary of this zone the minimum depth of foci sharply increases to about $10 \mathrm{~km}$ under Clark Valley.

The vertical sections perpendicular to the main epicenter trend (fig. 16) reveal several interesting characteristics of the aftershock distribution. The aftershock zone of each section generally lies toward the right, that is, toward the northeast of the surface break, and usually ascends to its minimum depth near the surficial fractures. In section I-J, which crosses the zone of complex surface faulting, the aftershock zone is about $4 \mathrm{~km}$ thick and dips about $60^{\circ} \mathrm{NE}$. In section $\mathrm{M}-\mathrm{N}$, except for the small cluster of earthquakes at $5 \mathrm{~km}$ depth near the left-hand margin, the aftershock zone is within about $1 \mathrm{~km}$ of a vertical line extending to a depth of $9 \mathrm{~km}$.

A histogram of the depth distribution of all the aftershocks in table 8 that are assigned a quality of $\mathrm{A}$ or $\mathrm{B}$ is shown in figure $17 \mathrm{~A}$. An $\mathrm{A}$ or $\mathrm{B}$ rating means that the focal depth is relatively well determined, probably with a precision better than $2 \mathrm{~km}$. The histogram shows that the foci are fairly evenly distributed to a depth of about $8 \mathrm{~km}$, below which there is a steady decline to none below $13 \mathrm{~km}$. The relatively low number of foci above $1 \mathrm{~km}$ could be the result of a systematic error in the hypocenter determinations.

The possibility that larger and smaller earthquakes have different depth distributions is examined 


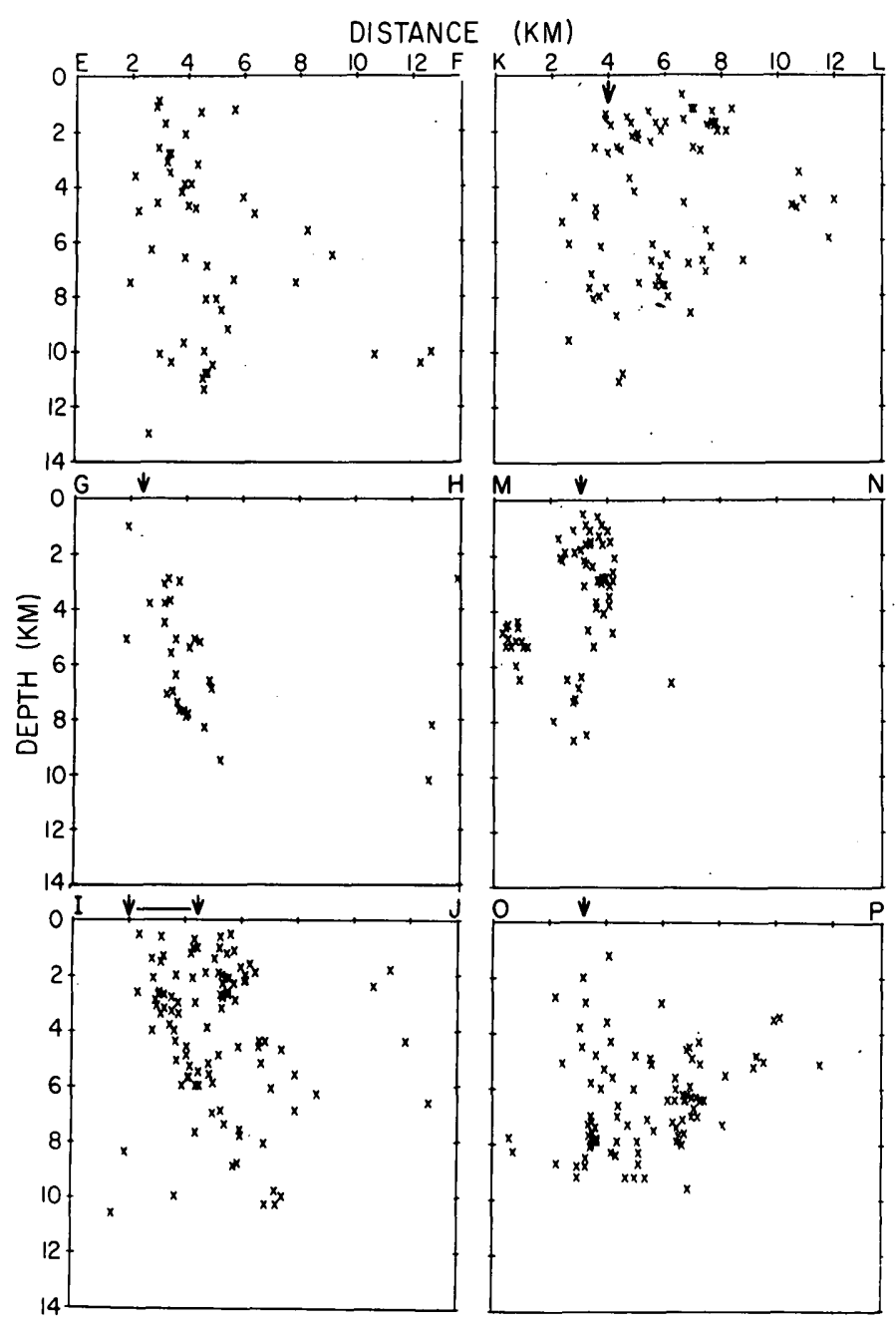

Figure 16. - Vertical sections through aftershock zone. Arrows indicate the approximate position of ground breakage on the line of section. Maximum projection distances of foci are: E-F, $11.42 \mathrm{~km}$; G-H, $4.24 \mathrm{~km}$; I-J, $3.92 \mathrm{~km}$; K-L, $2.47 \mathrm{~km} ; \mathrm{M}-\mathrm{N}, 2.40 \mathrm{~km}$; and $\mathrm{O}-\mathrm{P}, 4.73 \mathrm{~km}$. Lines of section are shown in figure 13 .

in figures $17 B$ and $C$. In figure $17 B$, the A- or B-rated earthquakes that are assigned a magnitude by the U.S. Geological Survey are plotted in four magnitude categories. No strong trend is apparent. The A- or B-rated earthquakes that are assigned a magnitude by the California Institute of Technology are plotted in figure $17 C$. Although there are few earthquakes in each category, the data show an increase in average depth with magnitude. Most impressive is the fact that none of the nine earthquakes of magnitude 3.5 or larger occurred shallower than $6 \mathrm{~km}$.

One must question whether any variation observed could result from some systematic error in the hypocenter determination procedure that depends on magnitude. The main difference in the data between

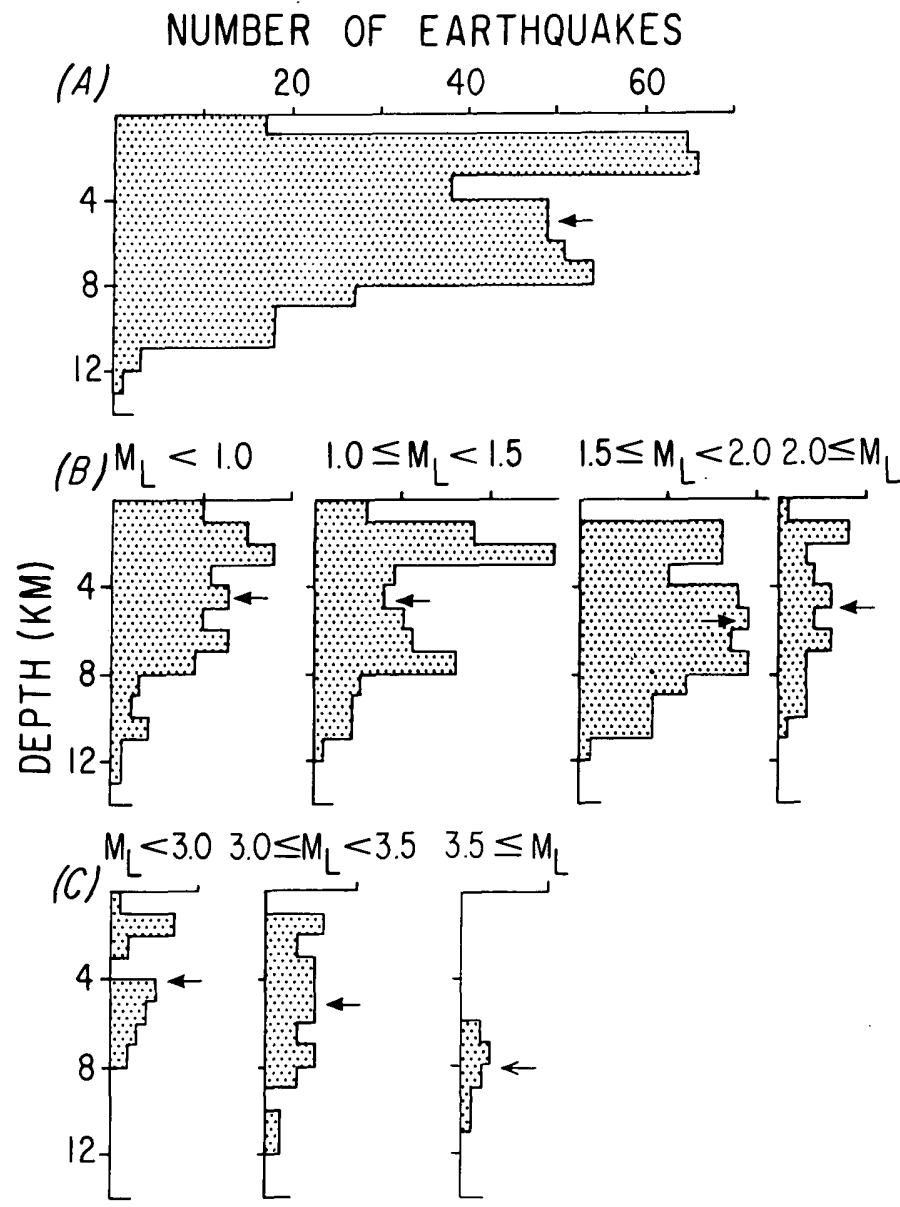

Figure 17. - Depth distribution of aftershocks with well-determined hypocenters $(\mathrm{Q}=\mathrm{A}$ or $\mathrm{B}$ in table 8$)$. $A$, All earthquakes. $B$, Earthquakes assigned a magnitude by the U.S. Geological Survey. $C$, Earthquakes assigned a magnitude by California Institute of Technology. The arrows indicate the average depth.

large and small earthquakes is that the larger earthquakes are generally observed at more stations. In figure 17 , the earthquakes with magnitude $\geqslant 1.8$ were located by using an average of 16.2 readings, whereas the smaller ones were located by using an average of 13.6 readings. The variations in number of stations used seems to be insignificant.

\section{AFTERSHOCK FOCAL MECHANISMS}

The nature of the focal mechanisms of the Borrego Mountain aftershocks was determined from the first-motion patterns of a large number of events. The main problem in obtaining a nodal-plane solution for the first-motion pattern from a network of closein stations (epicentral distance less than $50 \mathrm{~km}$ ) lies in determining the angle of incidence of a ray at the focus. Given the focal depth, epicentral distance, and crustal model, the computation is straightforward; however, if the focus is near a layer bound- 
ary or if a station is near a crossover distance, particularly the crossover from the direct to a refracted ray, large discontinuous changes in the firstmotion pattern can result from small changes in the parameters of the focus or model. The general effect of errors in the parameters is to confuse the pattern, except for the strike-slip mechanism. Very few patterns for the Borrego Mountain aftershocks appeared to be so affected, and most of those that were affected could be cleared up by a minor parameter shift. This success in obtaining good nodal-plane solutions probably is a result of the relatively good crustal velocity data, the precise hypocenter determinations, and the predominance of strike-slip mechanisms. Each of the first-motion patterns studied was plotted on an equal-area projection of the lower half of the focal sphere. Rays leaving the focus upward were plotted as if they had left in the opposite direction.

In determining many of the nodal planes, the nature of the first motion as well as its direction was considered. In checking the seismograms for earthquakes yielding well-controlled solutions, it was found that stations near nodal planes often recorded either an emergent onset or a sharp short excursion in one direction followed by a much larger excursion in the opposite direction. Such onsets were noted on the first-motion plots ; for poorly controlled solutions, a nodal plane passing near them was favored.

Some examples of the better determined nodalplane solutions, chosen to show the variety of solutions, are presented in figure $18 A-G$. Figure $18 H$ shows the type of pattern obtained for events in the side clusters of activity; such patterns are ambiguous but are consistent with right-lateral strike-slip motion in a direction subparallel to the regional fault trend. Figure 18I shows a pattern that is consistent with a variety of fault mechanisms.

Equal-area projections of the first-motion pattern were made for each shock that was located; nodalplane solutions were then determined for the 50 largest aftershocks. The solutions that were unambiguous and well determined were plotted on a map at their epicenters (fig. 19); areas where no solutions were plotted were filled using good solutions from among the smaller aftershocks. The map of the nodal-plane solutions (fig. 19) was then checked against the remaining solutions to assure that significant variations had not been omitted.

Figure 19 indicates that strike-slip displacement was the dominant mechanism for the aftershocks. There were only a few patterns indicating thrust, or reverse, faulting. Most of the patterns indicating strike-slip displacement that are near the zone of observed faulting have one nodal plane that sub-
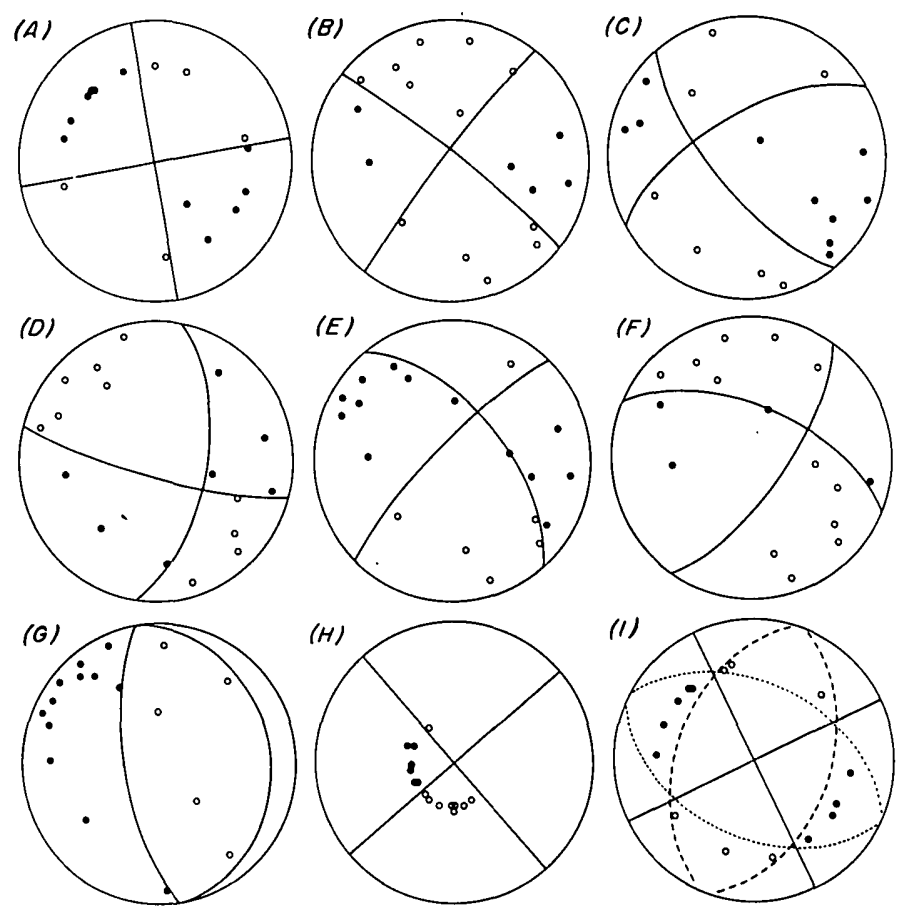

FIGURE 18. - Equal-area projections (lower hemisphere) of the first-motion pattern for nine aftershocks. Compressional first motion, solid dot; dilatational first motion, open circle; nodal planes, solid lines. Examples $A-G$ show the variety of patterns in the main aftershock zone, $H$ shows the type of pattern obtained for aftershocks in the side clusters, and $I$ is an ambiguous pattern.

parallels the fault trend and indicates right-lateral displacement, which is consistent with the observed surface displacement. Many of the patterns for earthquakes east of the surface faulting are rotated with respect to the patterns along the fault; for some aftershocks the nodal plane corresponding to the right-lateral displacement strikes nearly east. Such a variation in strike is found in many of the patterns at the southeast end of the seismic zone.

The first-motion patterns reveal some interesting details about the southeast end of the main seismic band. In the vicinity of station 17 (fig. 13), where the seismic band is narrowest and most closely associated with the surficial fractures, the patterns are remarkably similar and have a nodal plane indicating right-lateral displacement closely alined with the strike of the fractures. On the other hand, 3-10 km east of station 17, eight patterns were found with a distinctly more easterly strike for the right-lateral nodal plane. Thus, the transition in the epicenter pattern at the southeast end of the main band from close alinement with the faulting to dispersal correlates with a transition in strike of the focal mechanism. 


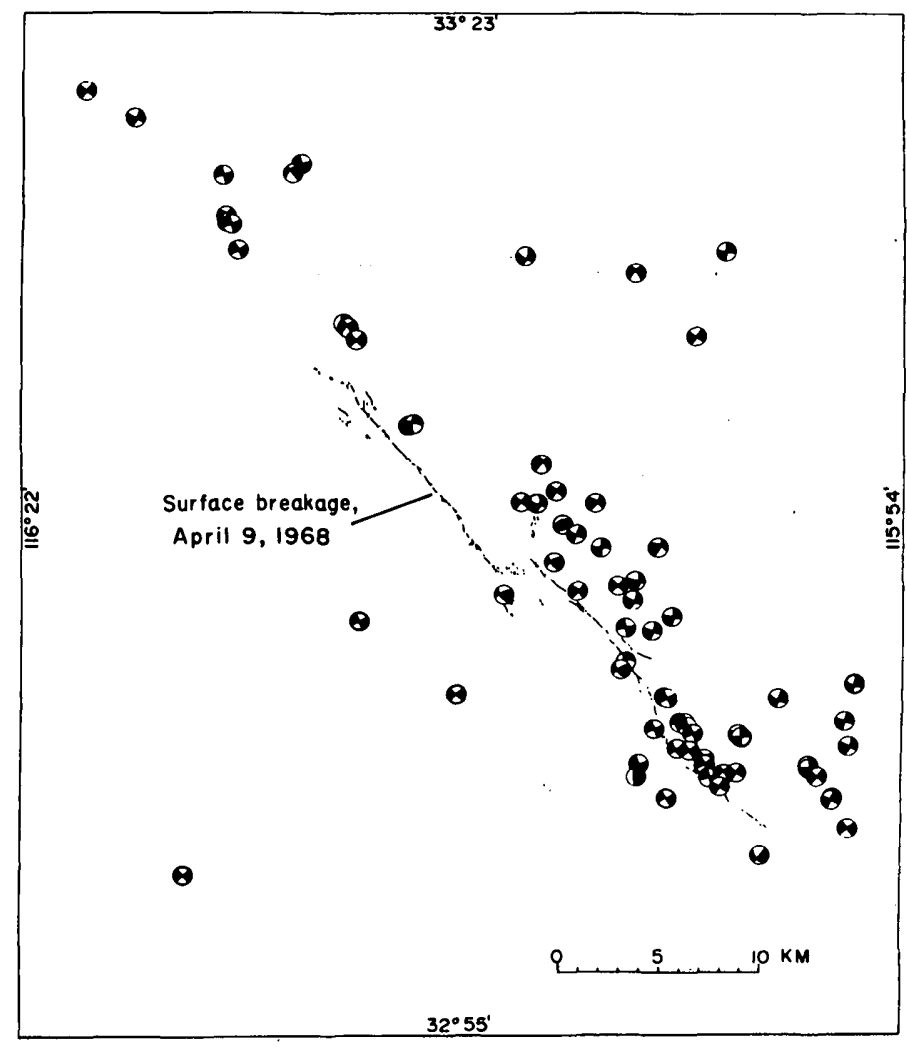

Figure 19. - Nodal-plane solutions, plotted at the epicenter, for 72 aftershocks that yielded well-determined solutions. Dark areas represent compressional quadrants and light areas dilatational quadrants.

The more easterly striking patterns at the south end of the seismic zone suggest that the zone of tectonic activity associated with the Borrego Mountain event swings toward the Superstition Hills fault (fig. 12). The mapped trace of this fault veers westerly: at its northwest end toward the aftershock area. It was mentioned earlier that three aftershocks were detected on the Superstition Hills fault and that the fault displaced about $1 \mathrm{~cm}$ near the time of the main shock.

The first-motion patterns were examined for all 14 events in the aftershock group centered at $33^{\circ} .02 .3^{\prime}$ $\mathrm{N}$. and $116^{\circ} 02.4^{\prime} \mathrm{W}$. (See fig. 13 , about $1 \mathrm{~km}$ southeast of vertical section reference "M".) Events in the northern half of the group all had strike-slip movement, and those in the southern half all had either reverse- or thrust-fault movement. These two pattern types are represented by two nodal-plane diagrams at the corresponding positions in figure 19 .

\section{MAGNITUDE RESIDUALS AND TRAVELTIME DELAYS}

Magnitudes were calculated for the aftershocks, following the procedure devised by Eaton, O'Neill, and Murdock (1970), using both the maximum am- plitude in the first few oscillations of the signal, referred to as "P magnitude," and the maximum amplitude in the rest of the signal, referred to as "X magnitude." In the computations, a period of 0.1 sec was uniformly used. The magnitude assigned to each event is the average of the various station magnitudes. In general, the estimates of the P- and $\mathrm{X}$-magnitudes agreed within a few tenths of a magnitude unit. Only the $\mathrm{X}$ magnitude is listed in table 8 .

Magnitudes could not be determined for the larger earthquakes that caused all seismographs to be overdriven. The California Institute of Technology bulletin (Nordquist and others, 1972) gives magnitudes for 84 of the earthquakes given in table 8 , including all those that were too large for magnitude determination by the U.S. Geological Survey. For the 39 earthquakes given in table 8, magnitudes were determined by both California Institute of Technology and U.S. Geological Survey. The Caltech magnitudes are higher by an average of 0.7 . The magnitude computation scheme of Eaton, O'Neill, and Murdock (1970) was designed to be consistent with the local magnitude $\left(\mathrm{M}_{\mathrm{L}}\right)$ scale devised at Caltech. Thus, the systematic difference is unexpected.

The USGS seismograms were reexamined to seek an explanation of the magnitude difference. It was found that for some aftershocks the amplitude was read for a signal that possibly was clipped, or so near to being clipped that the instrument response might have been nonlinear. Reading this kind of signal could have resulted at most in a factor-of-two error in the measured amplitude, with a corresponding error of 0.3 in the magnitude.

Another possible source of error lies in uniformly using a period of $0.1 \mathrm{sec}$. If the appropriate period is actually $0.2 \mathrm{sec}$, the magnitude estimate would be low by 0.3 . The signal associated with the maximum signal amplitude, however, was found to differ from $0.1 \mathrm{sec}$ by less than a factor of two. Moreover, variations from that period occur in both ways and do not seem to depend on magnitude.

It seems then that the systematic difference between the USGS and Caltech magnitude estimates is not just an error in applying the procedure of Eaton, O'Neill, and Murdock (1970). A possible explanation has been developed through discussions with Wayne $R$. Thatcher. The Caltech magnitudes are derived from Wood-Anderson seismograms, and the maximum signal amplitude would have an associated frequency of 1 to $3 \mathrm{~Hz}$. This associated frequency would lie near the corner frequency in the source spectrum of a magnitude 3 earthquake (Wayne R. Thatcher, oral commun., 1972). The USGS magnitudes are based on waves with frequencies near $10 \mathrm{~Hz}$, which are over three times higher 
than those of Caltech. These frequencies occur in a part of the spectrum where the amplitude is inversely proportional to frequency or frequency squared. Assuming a fall-off in amplitude with frequency raised to the power 1.5, the magnitude discrepancy of 0.7 can be accounted for by a frequency ratio of about 3 .

The magnitude residual, that is, the difference between the individual station magnitude and the average magnitude, was found for each station for each earthquake. Both the average P-magnitude and average $\mathrm{X}$-magnitude residuals for each station are given in table 7 .

The average magnitude residuals exhibit a range of over 0.8 magnitude units. The P-magnitude and $\mathrm{X}$-magnitude average residuals were consistent at each station (fig. 20). Examination of table 7 sug-

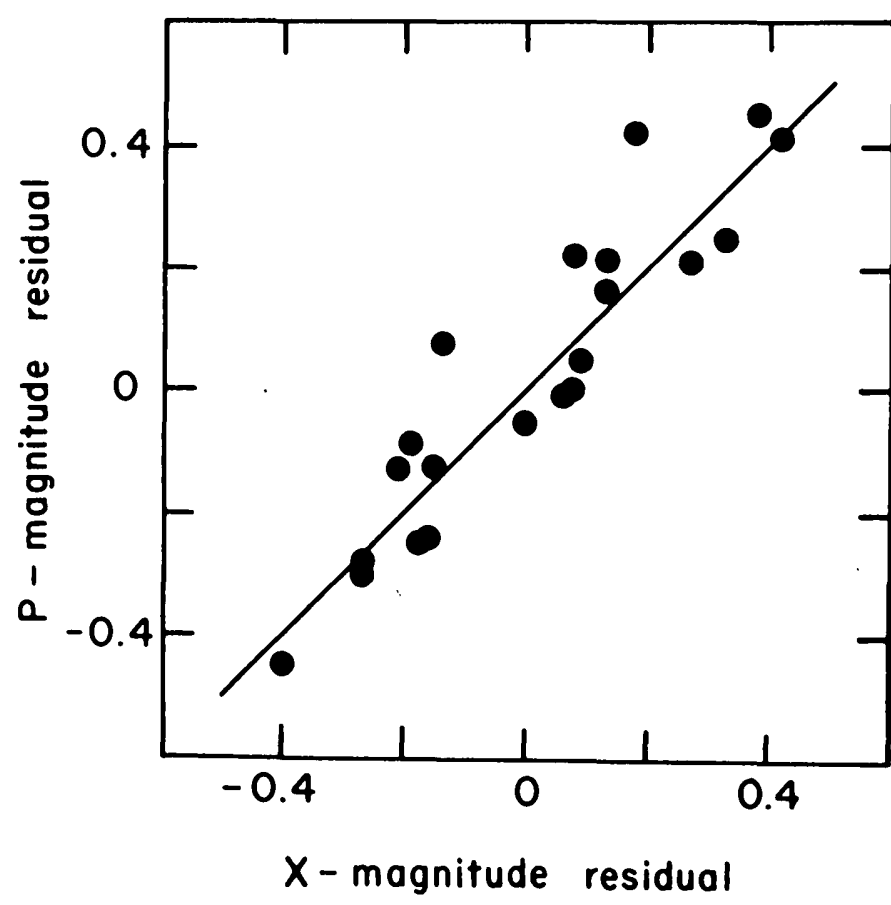

Figure 20. - Average P-magnitude residual plotted against average $\mathrm{X}$-magnitude residual for each seismograph station. The line indicates $\mathrm{P}$-magnitude residual equals $\mathrm{X}$-magnitude residual.

gests that there is a direct relation between average magnitude residual and station time correction. These data are plotted in figure 21. Eaton, O'Neill, and Murdock (1970) observed a similar relation in the Parkfield-Cholame region, but with a stronger dependence of the magnitude residual on the time correction. Their plot of magnitude residual against station-time correction was fitted with a straight line with slope 2.0 ; the lines for Borrego Mountain have

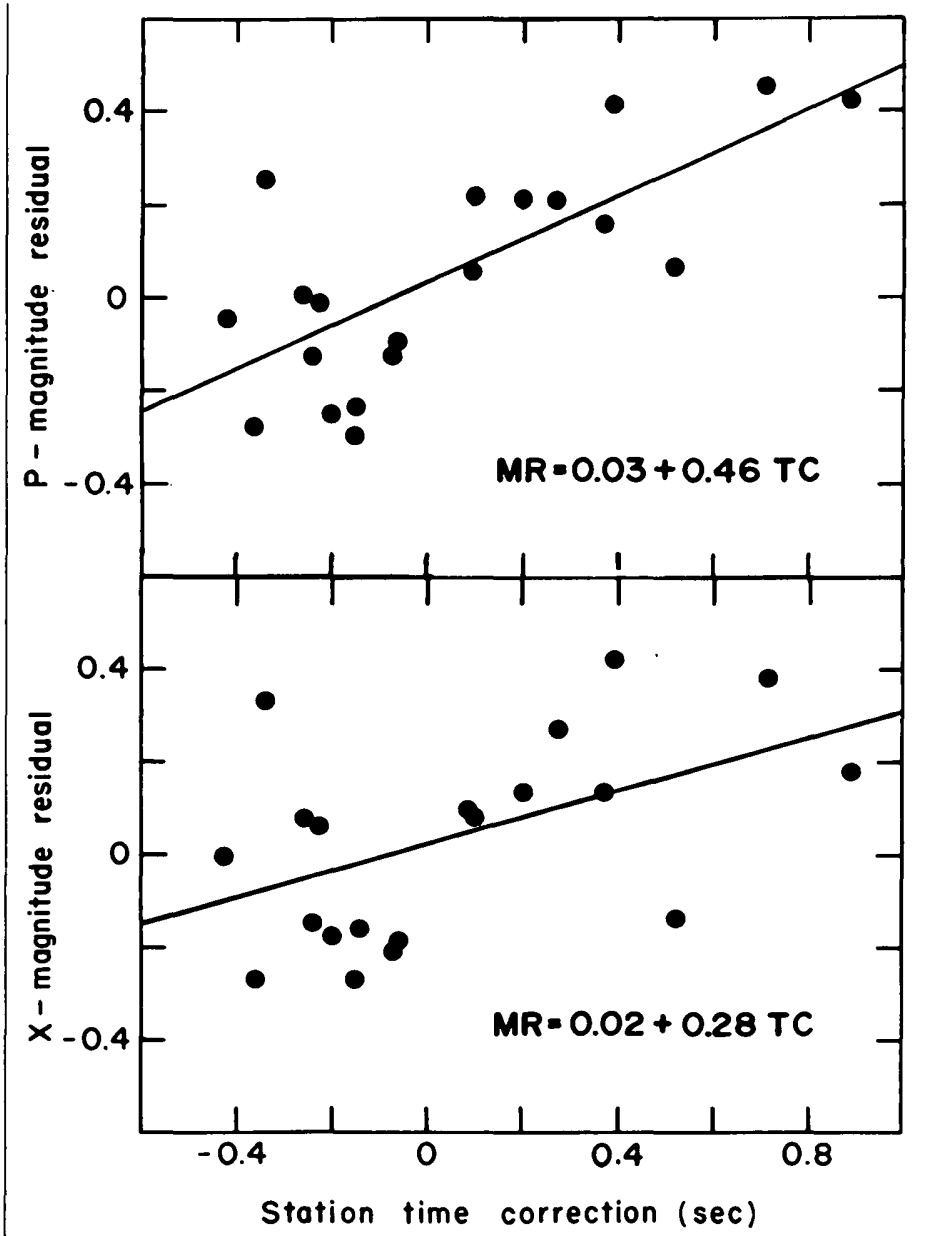

Figure 21. - Average P-magnitude and average X-magnitude residuals (MR) plotted against station time correction (TC). A large time correction means that waves arrive late. The lines were fitted by the least-squares method.

slopes of 0.46 and 0.28 for the $\mathrm{P}$ and $\mathrm{X}$ magnitudes, respectively.

Variations in the station-time correction are related directly to variations in basement depth in the Borrego Mountain region (Hamilton, 1970). The relation in figure 21 suggests that the magnitude variations may also be related to basement depth. Although depth to basement is not the sole control on magnitude anomalies, it is nevertheless a very important one. The importance of the effects of near-surface lithologic variations on controlling the excitation in the coda of the seismic signal was demonstrated by Aki (1969). Variations in thickness of sediments in the San Francisco Bay region have been related directly to variations in amplification of horizontal and, to a lesser degree, vertical motion from underground nuclear explosions at the Nevada Test Site (Borcherdt, 1970). 


\section{RATE OF AFTERSHOCK OCGURRENCE VERSUS TIME}

The rate of earthquake activity in the Borrego Mountain sequence was analyzed by using a continuous slow-speed $(37.5 \mathrm{~mm} / \mathrm{min})$ playback of the tapes from station 1. This station was chosen because it had three components of motion recorded, thus making S-wave identification easier, and because it was situated to the side of the main aftershock area (fig. 12), thus minimizing effects arising from variations in epicentral distance. Almost all seismic activity in the sequence was within $40 \mathrm{~km}$ of station 1 and, except for the cluster of activity southwest of the main zone, was at distances beyond $15 \mathrm{~km}$.

The earthquakes counted had an S-P time between 1 and 10 seconds. This restriction eliminated events that occurred outside the aftershock region and those that occurred very near station 1 . Events that produced a signal so large that the $S$ waves could not be recognized were counted; though some of them may have had an S-P time outside the range specified, such shocks made up less than 1 percent of the total. The events counted were also required to have an amplitude above a specified level; the amplitude threshold corresponded to a magnitude 1 earthquake at an epicentral distance of $20 \mathrm{~km}$, the distance to the main aftershock area.

Owing to recorder stoppages, a continuous record was not obtained. The longest stoppage was for 5 days; other stoppages were less than 1 day's duration. The total time lost was 10.6 percent of the recording period. If less than 24 hours of record was obtained in a day, the count was adjusted by dividing by the fraction of the record obtained.

A total of 13,412 earthquake signals was counted. There were 529 other signals that may have been caused by earthquakes, but they also could have been noise; therefore, they were not included. Plots were made of $\log n$ against $t$, where $n$ is the number of earthquakes per day and $t$ is the day after the main shock, and of $\log n$ against $\log t$. Straight lines fitted by least squares to these plots had the equations

$$
\begin{aligned}
& n=836 \exp (-0.185 t), \text { and } \\
& n=5,130 t^{-0.98} .
\end{aligned}
$$

The observed variations in the rate of aftershock occurrence lies between these two relations, but the second one is the better approximation of the two. The data for equation 2 are shown in figure 22. Such a time dependence has been observed for the rate of aftershock occurrence in numerous other sequences.

\section{RELATED STUDIES IN OTHER AREAS}

Detailed earthquake studies using dense seismograph networks have been carried out in recent

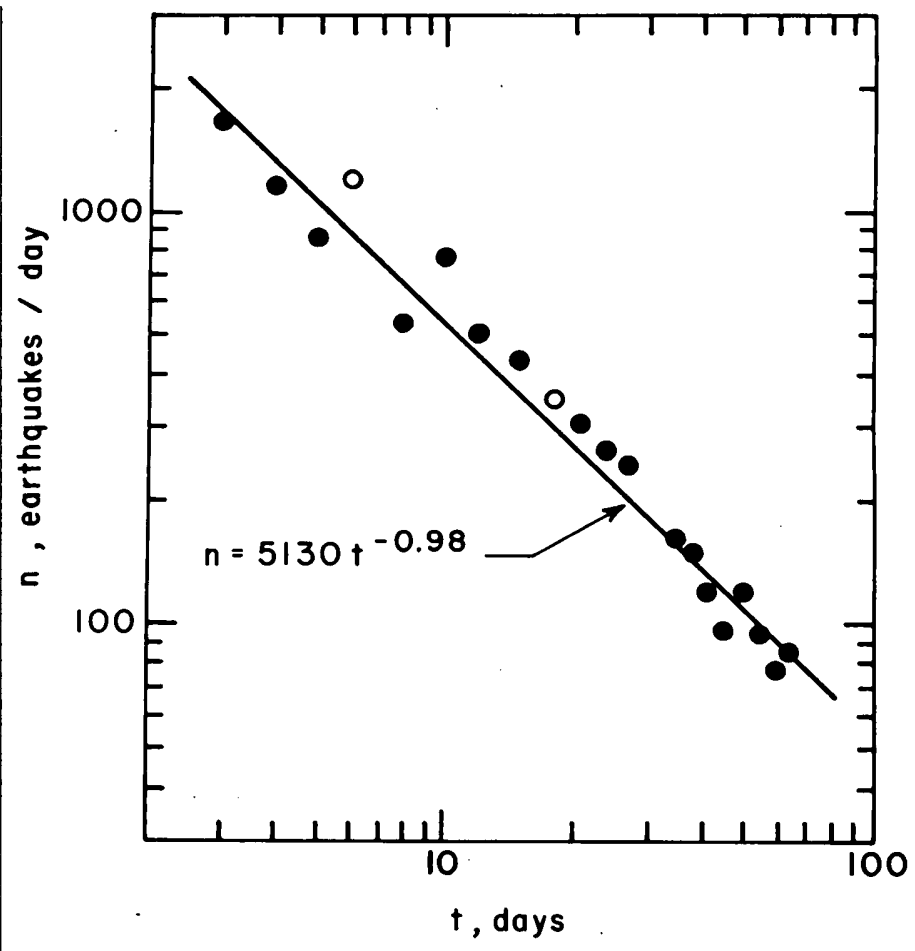

FIGURE 22. - Rate of earthquake occurrence, $n$, plotted against time after the main shock, $t$. Open circle indicates that $n$ is based on less than half a day's record and is therefore of lower reliability.

years along a number of other sections of the San Andreas fault system in California from the Imperial Valley to north of San Francisco Bay. The maximum. depth determined for hypocenters in all but one of these studies is within a few kilometers of the 12-km maximum depth found for the Borrego Mountain aftershocks. The areas studied include (1) southeast of Anza (Arabasz and others, 1970), (2) Lake Hughes and Cajon Pass (Brune and Allen, 1967), (3) Parkfield-Cholame (Eaton, O'Neill, and Murdock, 1970), (4) Bear Valley (Eaton, Lee and Pakiser, 1970), (5) Watsonville (Udias, 1965), (6) Corralitos (McEvilly, 1966), (7) the San Andreas, Sargent, Hayward, and Calaveras faults in the San Francisco Bay area (Eaton, Lee, and Pakiser, 1970), (8) Antioch (McEvilly and Casaday, 1967), and (9) Santa Rosa (Unger and Eaton, 1970). Exceptional focal depths were found in the San Gorgonio Pass area, where earthquakes occur as deep as $21 \mathrm{~km}$ (Hanks and Brune, 1970).

The previously studied area nearest the Borrego Mountain area lies at the trifurcation of the San Jacinto fault about $10 \mathrm{~km}$ southeast of Anza and $50 \mathrm{~km}$ northwest of the main Borrego Mountain earthquake (Arabasz and others, 1970). One hundred earthquakes, located by using as many as six 
seismograph stations, occurred in a dispersed pattern extending several kilometers outside the area outlined by mapped faults. The complexity of the epicenter pattern indicates activity on more than one fault in the area. The aftershock and creep patterns in the Borrego Mountain area reveal a similar behavior. The dispersed tectonic activity observed near Anza and Borrego Mountain reflects the locally complex and splaying nature of the San Andreas fault system in southern California. In contrast, the aftershocks near Parkfield in central California (Eaton, O'Neill, and Murdock, 1970) mostly occurred within $1 \mathrm{~km}$ of a near-vertical plane; the intersection of this plane with the surface almost coincides with the surface faulting along the relatively simple and single-stranded trace of the San Andreas fault in that area.

\section{SUMMARY AND TECTONIC IMPLICATIONS}

The epicenters of the Borrego Mountain aftershocks define a rather complex pattern consisting of a main band subparallel to the regional fault trend and two side clusters. Most of the earthquakes in the main band occurred in a distance interval of $56 \mathrm{~km}$ and are approximately centered on the epicenter of the main shock. The symmetrical extent of the epicenter band with respect to the main-shock epicenter suggests that the Borrego Mountain earthquake resulted from bilateral faulting (Allen and Nordquist, this volume). It also seems that the rupture started near the bottom of the fault: the aftershocks suggest that the fault extends to a depth of about $12 \mathrm{~km}$, and the computed depth of the main shock was $11.1 \mathrm{~km}$ (Allen and Nordquist, this volume). This depth determination cannot be considered very reliable because the nearest station was $47 \mathrm{~km}$ from the main shock; however, it is supported by the observation that all nine aftershocks with magnitudes over 3.5 and with well-determined focal depths occurred below $6 \mathrm{~km}$ deep.

Although the extent of the aftershock zone was apparently symmetrical with respect to the main shock, the level of aftershock activity was not. Most of the aftershocks occurred southeast of the main shock, where part of the zone of activity was closely associated with surficial faulting. The southeast section of the surficial faulting was the site of continued displacement long after the main shock (Burford, this volume; Clark, "Surface Rupture Along the Coyote Creek Fault," this volume).

The continued fault displacement following the 1966 Parkfield earthquake was thought by Smith and Wyss (1968) to result from the viscous properties of the near-surface materials. This explanation could probably also be applied to the Borrego Mountain earthquake; however, the southeasterly growth of the aftershock zone, with earthquakes in the depth range of 4 to $10 \mathrm{~km}$, indicates further fault movement in the basement. The additional displacement on the surface fractures could then have resulted from growth of the original faulting.

Although part of the aftershock activity was associated with surficial fractures, most of the earthquakes occurred northeast of the fractures. To a certain extent, the surficial fractures bound the main aftershock band on the southwest. Wyss and Brune (1971) found that the Borrego Mountain aftershocks had higher stresses associated with them than did the Parkfield aftershocks. Noting the dispersed Borrego Mountain aftershock pattern, they inferred that at Parkfield the fault surface is a well-developed zone of weakness, whereas at Borrego it is less well defined.

Relatively few aftershocks were detected in the vicinity of the main shock. The surficial fracturing near the main-shock epicenter exhibited the greatest initial displacement but showed no later displacement.

Beyond the northwestern extent of surface faulting, only a few aftershocks were detected. However, approximately a year after the Borrego Mountain earthquake, on April 28, 1969, a magnitude 5.8 earthquake occurred near the site of station 12 (fig. 12). Its aftershocks, monitored in a joint project by California Institute of Technology and the U.S. Geological Survey (Thatcher and Hamilton, 1971), define a zone that is essentially a northwestward extension of the Borrego Mountain aftershock zone.

Thus, aftershock studies reveal that the initial bilateral faulting from the Borrego Mountain earthquake extended in markedly different ways at its two ends. On the southeast end, fault creep and gradual growth are indicated, whereas on the northwest end; sudden extension more than a year later appears to have occurred.

The curious distribution of the main aftershock band and the epicentral clusters northeast and southwest of it suggests that the zones may be related in a special way. One possibility is that the clusters are associated with a fault system that is conjugate with the northwest-trending system. However, the lack of continuity between the two clusters detracts from this suggestion, and such a system of active faults is unknown in the cluster areas or in any other nearby part of southern California. The shear-stress changes resulting from the main shock may have controlled the aftershock pattern. The stress changes 
from a strike-slip dislocation in a halfspace were calculated by Chinnery (1963). His results show that the greatest increase in shear stress in the horizontal direction parallel to the dislocation plane occurs in a complicated pattern near the ends of the dislocation. However, the shear stress also increases in areas centered on the order of one dislocation length perpendicular to the center of the dislocation. Such a stress redistribution resulting from the Borrego Mountain faulting may account for the unusual epicenter pattern.

An important result of this study is the correlation between the aftershock distribution and the surface faulting. In the area where the aftershock zone is narrowest, along the southeast fault strand near station 17 (fig. 12), the surface faulting runs through the epicenter region (fig. 16, M-N). Near station 9, where the fracture zone is widest, the aftershock zone is wide (fig. 16, I-J). Beyond the southeastern limit of faulting, the minimum depth of foci increases to below $2 \mathrm{~km}$ (fig. 16, O-P). The minimum depth of foci also increases at the northwestern limit of faulting (fig. 15, A-B). The faultplane solutions also correlate well with the aftershock distribution. Where the aftershock zone is narrowest, near station 17, the fault-plane solutions are all essentially identical, and one of the nodal planes passes through the aftershock zone.

There is good evidence that aftershocks occurred in the sedimentary rocks above the granitic basement. Because of the uncertainties inherent in focaldepth determination, such a conclusion would generally be difficult to draw with confidence. For the Borrego Mountain aftershock sequence, though, the close-in distribution of seismographs and the explosion-refraction study (Hamilton, 1970) provided critical information. Along the southeast fault strand, foci were computed to lie within $1 / 2 \mathrm{~km}$ of the surface. The explosion data indicate basement depths of about $13 / 4 \mathrm{~km}$ below station $9,23 / 4 \mathrm{~km}$ below station $17,21 / 4 \mathrm{~km}$ below station 4 , and $31 / 4$. $\mathrm{km}$ in the area several kilometers northeast of the surface faulting.

\section{REFERENCES CITED}

Aki, Keiiti, 1969, Analysis of the seismic coda of local earthquakes as scattered waves: Jour. Geophys. Research, v. 74, p. 615-631.

Allen, C. R., Grantz, A., Brune, J. N., Clark, M. M., Sharp, R. V., Theodore, T. G., Wolfe, E. W., and Wyss, M., 1968, The Borrego Mountain, California, earthquake of 9 April 1968: a preliminary report: Seismol. Soc. America Bull., v. 58 , p. $1183-1186$.
Arabasz, W. J., Brune, J. N., and Engen, G. R., 1970, Locacations of small earthquakes near the trifurcation of the San Jacinto fault southeast of Anza, California: Seismol. Soc. America Bull., v. 60, p. 617-627.

Borcherdt, R. D., 1970, Effects of local geology on ground motion near San Franscisco Bay: Seismol. Soc. America Bull., v. 60, p. 29-61.

Brune, J. N., and Allen, C. R., 1967, A microearthquake survey of the San Andreas fault system in southern California: Seismol. Soc. America Bull., v. 57, p. 277-296.

Chinnery, M. A., 1963, The stress changes that accompany strike-slip faulting: Seismol. Soc. America Bull., v. 53, p. 921-932.

Eaton, J. P., 1969, HYPOLAYR, a computer program for determining hypocenters of local earthquakes in an earth consisting of uniform flat layers over a half space: U.S. Geol. Survey open-file report, $106 \mathrm{p}$.

Eaton, J. P., Lee, W. H. K., and Pakiser, L. C., 1970, Use of microearthquakes in the study of the mechanics of earthquake generation along the San Andreas fault in central California: Tectonophysics, v. 9, p. 259-282.

Eaton, J. P., O’Neill, M. E., and Murdock, J. N., 1970, Detailed study of the aftershocks of the 1966 Parkfield-Cholame, California, earthquake: Seismol. Soc. America Bull., v. 60 , p. 1151-1197.

Hamilton, R. M., 1970, Time-term analysis of explosion data from the vicinity of the Borrego Mountain, California, earthquake of 9 April 1968: Seismol. Soc. America Bull., v. 60 , p. $367-381$.

Hanks, T. C., and Brune, J. N., 1970, Seismicity of the San Gorgonio Pass [abs.]: EOS (Trans. Am. Geophys. Union), v. 51, p. 352 .

McEvilly, T. V., 1966, The earthquake sequence of November 1964 near Corralitos, California: Seismol. Soc. America Bull., v. 56, p. 755-773.

McEvilly, T. V., and Casaday, K. B., 1967, The earthquake sequence of September, 1965 near Antioch, California: Seismol. Soc. America Bull., v. 57, p. 113-124.

Nordquist, J. M., Allen, C. R., Brune, J. N., Richter, C. F., and Taylor, V., 1972, Local bulletin of earthquakes in the southern California region, 1 January 1968 to 31 December 1969: California Inst. Technology, Pasadena, 38 p.

Smith, S. W., and Wyss, Max, 1968, Displacement on the San Andreas fault subsequent to the 1966 Parkfield earthquake: Seismol. Soc. America Bull., v. 58, p. 1955-1973.

Thatcher, W. R., and Hamilton, R. M., 1971, Spatial distribution and source parameters of the Coyote Mountain aftershock sequence, San Jacinto fault zone [abs.]: Geol. Soc. America Abs. with Programs, v. 3, no. 2, p. 208.

Udias, Augustin, 1965, A study of the aftershocks and focal mechanism of the Salinas-Watsonville earthquakes of August 31 and September 14, 1963 : Seismol. Soc. America Bull., v. 55, p. 85-106.

Unger, J. D., and Eaton, J. P., 1970, Aftershocks of the October 1969 Santa Rosa, California, earthquakes [abs.]: Geol. Soc. America Abs. with Programs, v. 2, no. 2, p. 155.

Wyss, Max, and Brune, J. N., 1971, Regional variations of source properties in southern California estimated from the ratio of short- to long-period amplitudes: Seismol. Soc. America Bull., v. 61, p. 1153-1168. 


\title{
SURFACE RUPTURE ALONG THE COYOTE CREEK FAULT
}

\author{
By Malcolm M. Clark \\ U.S. Geological Survey
}

\begin{abstract}
The nearly linear $31-\mathrm{km}$-long surface rupture that accompanied the Borrego Mountain earthquake of April 9, 1968, along the Coyote Creek fault comprised three well-defined bands of fractures - the north, central, and south breaks - each of which consisted of en echelon, complex, and simple right-lateral fractures that cut Pliocene-Pleistocene and Holocene sediments at the surface. Associated minor diverging and isolated fractures and zones of fractures developed as far as $3 \mathrm{~km}$ from the well-defined breaks. A few of these displayed dominant vertical or left-lateral displacement.

Maximum right-lateral displacement was $380 \mathrm{~mm}$ on the north break and, including postearthquake creep into 1971, $250-300 \mathrm{~mm}$ on the central break, and $80-140 \mathrm{~mm}$ on the south break. Subordinate vertical slip (230 $\mathrm{mm}$ maximum) accompanied right-lateral slip along nearly half of the north and central breaks and without exception was in the same sense as earlier uplift of scarps and hills.

Some of the outlying fractures that displayed significant left-lateral displacement have orientations that are compatible with some theories and observations of secondary fracturing associated with strike-slip faulting. However, others of these that also display large vertical displacement appeared to be normal faults trending obliquely to the local direction of tectonic extension.

Surviving older scarps and vegetation boundaries extended along roughly one-third of the entire length of the 1968 rupture and were in all places coincident with new fractures. However, where vertical displacements and vegetation boundaries were absent, other surface evidence that would have betrayed the position of the fault was scanty. Few offset drainage channels survived from previous displacements, because erosion in the poorly consolidated sediments proceeds more rapidly than tectonic displacement. Along about 50 percent of the length of the rupture, the position of the main fractures could have been predicted before the earthquake to within $\pm 100 \mathrm{~m}$ or closer.

The width of the band of fracturing along the three main breaks exceeded $50 \mathrm{~m}$ along about 35 percent of the length of the 1968 rupture and exceeded $100 \mathrm{~m}$ along 25 percent of the length of the rupture. However, most of the ground displacement along the main breaks occurred within a band less than $20 \mathrm{~m}$ wide. Associated fractures with potentially damaging displacements extended more than $100 \mathrm{~m}$ from the main breaks in some locations and as much as $500 \mathrm{~m}$ from the main breaks in a few locations. Because not all these fractures followed obvious lines of earlier faulting, sub-
\end{abstract}

surface investigations should be mandatory around the site of any proposed large or multistory structure within about $1 \mathrm{~km}$ of the 1968 rupture.

Fault creep and rain-induced collapse maintained or enlarged some fractures as late as 1971 , more than $3 \frac{1}{2}$ years after the earthquake. No significant postearthquake creep took place on the north break, but simple measurements made at irregular intervals since April 1968 reveal that postearthquake movement along the central and south breaks has increased original displacements by as much as 100 percent. Some of this creep was apparently continuous, beginning immediately after the earthquake, but several parts of the central break did not start moving until at least several months after the earthquake, and the south break may not have started moving until about 1 year after the earthquake.

Reconstruction of the creep history since the earthquake at one place on the central break employed repeated measurements of displaced tire tracks and new offsets in crusts formed in material filling 1968 fractures. The reconstruction is compatible with the exponential decay in creep rate reported for other earthquakes, except for an apparent delay of several months after the earthquake before the onset of creep. The net result of creep on the central break has been to increase initial displacements to amounts comparable to those on the north break at the time of the earthquake.

The south break not only experienced smaller displacements and a greater delay (perhaps more than 1 year) before the onset of creep than did the central break, but it also includes four sets of tectonic fractures that predate the 1968 earthquake by a few months to more than 15 years. This activity, combined with postearthquake creep and a recent history of earthquakes that includes one of magnitude 6.5 , suggests that the south break may behave more like the Superstition Hills and Imperial faults than adjacent parts of the Coyote Creek fault.

\section{INTRODUCTION}

This paper describes the surface rupture and displacements that formed along the Coyote Creek fault during and after the Borrego Mountain earthquake of April 9, 1968. The fault is part of the San Jacinto fault zone, a major branch of the San Andreas system of right-lateral strike-slip faults. (See Sharp, "Tectonic Setting of the Salton Trough," this volume.) Conditions along the 1968 rupture have revealed some characteristics of surface faulting that are not obvious at many other places along the San 


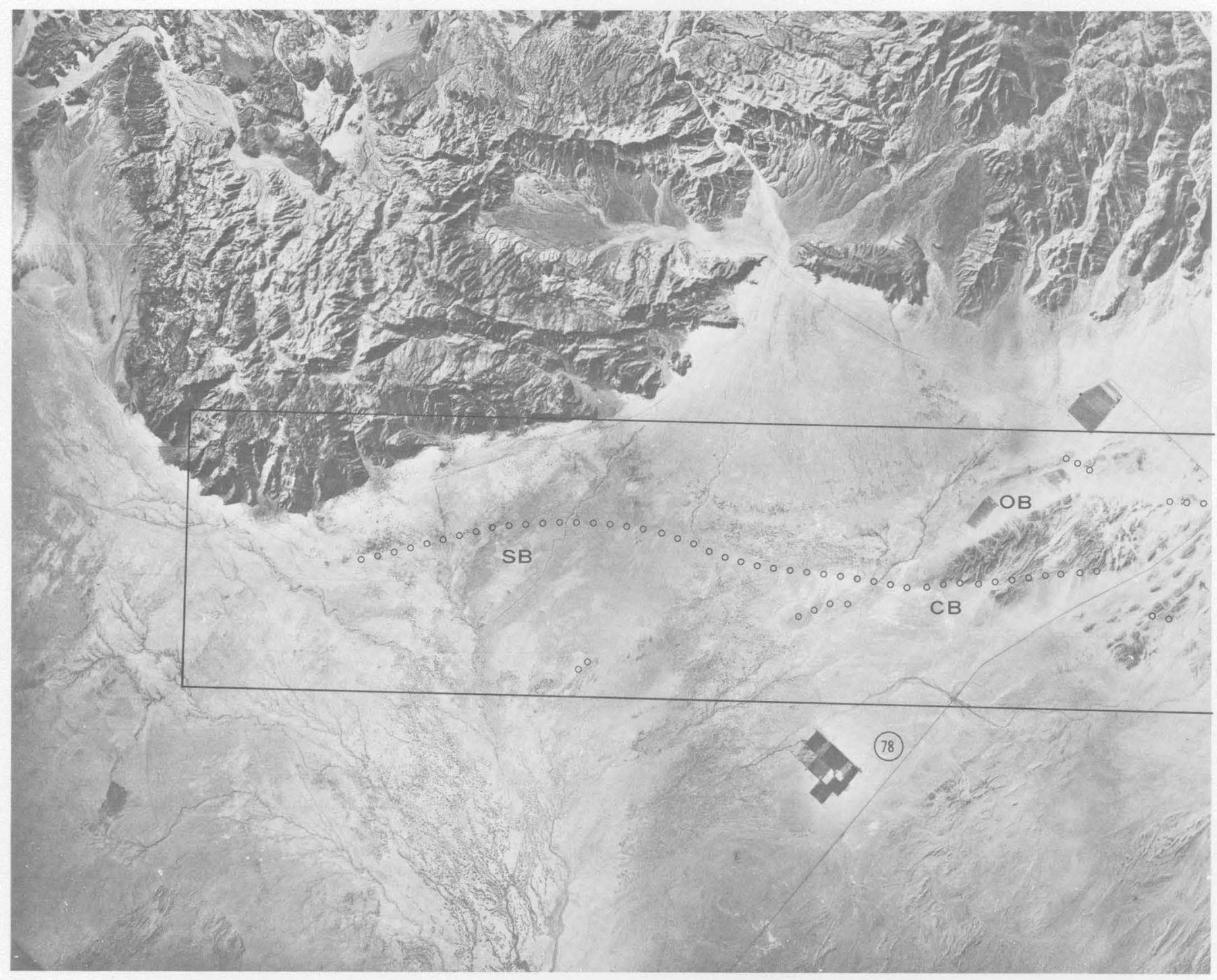

FIGURE 23.- Vertical aerial photograph of the region of surface faulting associated with the Borrego Mountain earthquake. The Lake; CM, Coyote Mountain; NB, north break; OB, Ocotillo Badlands; OW, Ocotillo Wells; SB, south break. Other features

Andreas fault system. The combination of scant vegetation, low rainfall, low relief, and predominantly fine-grained sediment along the 1968 rupture has resulted in preservation of many details of the surface fractures and allowed continuing study of changes in the rupture more than 3 years after the earthquake. Many aspects of the surface faulting are similar to patterns produced by earlier earthquakes along the San Andreas system. Most of the rupture consisted of right-lateral en echelon fractures that coincided with locally surviving traces of earlier faulting. The investigation also documented the association of significant and consistent vertical and minor left-lateral components of displacement on fractures associated with the main breaks, characteristics not generally recorded or observed after earlier earthquakes in the San Andreas system. Furthermore, the investigation has pieced together a complex history of postearthquake creep that contrasts with the simpler relations found between initial displacements, creep, and aftershocks of the San Andreas fault at Parkfield-Cholame after the earthquake of 1966.

Field investigation of the surface rupture started about 8 hours after the earthquake and is still being conducted at this writing (November 1971). The initial effort to record fractures began at 2 a.m. (P.s.t.) April 9, 1968, when C. R. Allen of California Institute of Technology measured the rupture where it crossed Highway 78. By April 14, most of the fractures had been mapped. Allen was joined in mapping the rupture by several U.S. Geological Sur- 


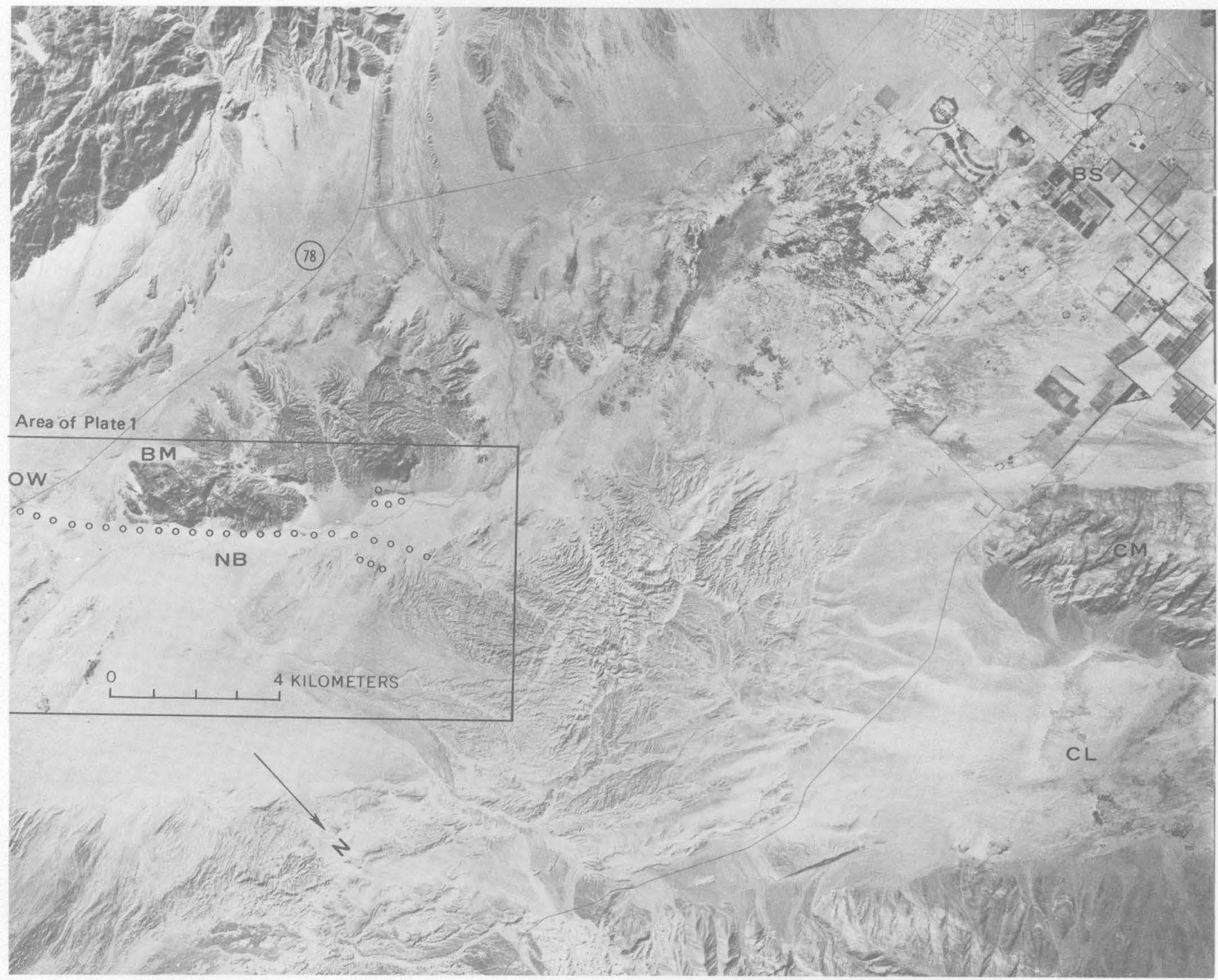

surface ruptures are shown as rows of open circles. BM, Borrego Mountain; BS, Borrego Spring's; CB, central break; CL, Clark can be identified from plate 1. USGS aerial photographs USAF 665V 037 and 039, taken by U.S. Air Force, November 1967.

vey geologists (Arthur Grantz, T. G. Theodore, R. V. Sharp, E. W. Wolfe, and myself). Later in April and in May, Survey geologists E. W. Wolfe, R. O. Castle, and T. L. Youd recorded additional fractures at some distance away from the main breaks. In June 1968, I noticed the first obvious evidence of continued movement along the rupture and mapped additional fractures that were either overlooked or not present in April. Thereafter, I was accompanied at times by A. Grantz, R. V. Sharp, M. Rubin, M. G. Bonilla, and J. Kahle in recording increases in displacement, new fractures, and other changes in the surface rupture during visits in October 1968, March, April, and October-November 1969, January and December 1970, and March and November 1971. Allen also monitored creep during other visits.
Many people in addition to the aforementioned have contributed to this investigation. J. P. Lockwood helped identify and record ground ruptures during the first few days of field study. Light Photographic Squadron 63 of the U.S. Navy at Miramar, Calif., made excellent large-scale aerial photographs of the surface rupture in 1968 and 1969. I especially thank Commanders Hegrat and Dunkin for their cooperation and expertise in getting these photographs. Mr. and Mrs. Earl Cartier and Mr. and Mrs. John Balch of Ocotillo Wells provided important information and assistance during our fieldwork. Bob Densmore of Borrego Springs supplied logistic support and took photographs of creep localities in March 1970. H. B. Goldis helped compile many of the illustrations of this report. Arthur Grantz, R. V. 
Sharp, M. G. Bonilla, and R. F. Yerkes offered helpful suggestions regarding the text.

Field parties used a variety of base materials for mapping fractures. They recorded most of those found in April and May 1968 on aerial photographs taken by the U.S. Department of Agriculture in 1953 at $1: 20,000$ scale; they plotted the remaining fractures found during this period on $1: 25,000$-scale U.S. Geological Survey photographs or $1: 24,000$ scale topographic maps. Subsequent investigations used aerial photographs at $1: 10,000$ and larger scales taken in May 1968 and April 1969 by U.S. Navy aircraft from Miramar, Calif., and 1:8,000scale U.S. Geological Survey color aerial photographs taken in April 1969.

\section{DESCRIPTION OF THE SURFACE BREAKS}

The principal surface rupture defines a narrow roughly linear band about $31 \mathrm{~km}$ long (pl. 1; fig. 23). The band consists mainly of right-lateral en echelon fractures and trends roughly $\mathrm{N} .40^{\circ} \mathrm{W}$. across lower Borrego Valley and along the northeastern base of Borrego Mountain. Minor isolated and diverging fractures formed as much as $3 \mathrm{~km}$ away from the main zone of breakage. The surface fractures formed entirely in Holocene alluvium and lake beds, except in the vicinity of Ocotillo Badlands and at the extreme north end of the main rupture. In both these places, fractures traversed uplifted and folded Pliocene-Pleistocene sediments.

Two interruptions in the continuity of the rupture divide it into three main breaks (fig. 23), the north (locs. 1-14, pl. $1^{1}$ ), central (locs. 14-26), and south (locs. 26-33). The north and central breaks are en echelon and are separated by the uplifted and tightly folded sediments of Ocotillo Badlands. The central and south breaks are connected by a narrow zone of widely separated small fractures. Displacements on each of these three breaks reached a maximum near the center and diminished toward the ends. Maximum right-lateral displacement on the entire rupture was $380 \mathrm{~mm}$ (15 in.) on the north break near the base of Borrego Mountain.

Fractures of the 1968 rupture can be divided into three categories: (1) nearly all fractures of the three main breaks, (2) all diverging and isolated tectonic fractures that lay more than about $100 \mathrm{~m}$ from the centers of the three main breaks, and (3) nontectonic fractures produced by differential compaction of sediment and by local slumping and sliding on steep slopes bordering washes or in hills. Although we intended to map only tectonic fractures,

\footnotetext{
1Throughout this and several subsequent chapters, locations along the fault zone will be given by the horizontal coordinate system (kilometers) of pl. 1 .
}

a few of the fractures on plate 1 (for example, those $1 \mathrm{~km}$ east of the north break at loc. 3 and within Ocotillo Badlands at loc. 15) may actually be in the nontectonic category.

Fractures of the three principal breaks (the first category) shared characteristics that differed from most of the fractures of the second category. All fractures of the principal breaks showed right-lateral displacement, except for minor extension and compression fractures oriented nearly perpendicular to the trend of the zones. Many fractures of the principal breaks also exhibited vertical displacement that was nearly always smaller than the associated horizontal movement. The principal breaks trend more or less uniformly $\mathrm{N}$. $30^{\circ}-50^{\circ} \mathrm{W}$. and displayed an essential continuity of fracturing within each break; they also contain prominent scarps from previous faulting, indicating continuity of position with respect to past tectonic events.

In contrast, outlying and diverging fractures (the second category) as a group showed different characteristics. Although some showed right-lateral offset, others showed vertical offset only (loc. 3.4) or vertical offset greater than horizontal (loc. 3.6), and on a few, displacements were left lateral (locs. 3.6, $12.4,14.0,15.2,15.8,17.4)$. The outlying and diverging fractures also displayed a greater range of orientations and, obviously, a lesser degree of continuity. Maximum displacements in this group of fractures were far less than the maximum displacements along the main breaks. Many of the outlying and diverging fractures had large vertical components of displacement but did not follow older scarps, suggesting randomness of position with respect to earlier fracturing.

Despite these differences, most of the diverging and outlying fractures appeared to be tectonic, rather than the secondary effects of shaking. Most either connected with fracures of the main breaks or bordered low uplifted hills and ridges underlain by tectonically deformed Pleistocene and Holocene strata, such as those southwest of the principal rupture zone at locations 3 and 15 to 16 , or northeast of the zone at locations 13 to 14,22 , and 27 .

Some of the isolated and diverging fractures of the 1968 rupture probably escaped detection. Many shown on plate 1 had small displacements (less than $20 \mathrm{~mm}$ ) and were quite inconspicuous. These are not visible on our largest scale vertical aerial photographs and were found only during careful traverses on foot. In addition, the wide bands flanking the 1968 rupture were not inspected as thoroughly as the immediate areas of the main breaks and the larger diverging fractures. 
Nontectonic fractures (the third category) were common. The 140 -m-high hills of Ocotillo Badlands, made up of unconsolidated and poorly consolidated muds, sands, and gravels, were shattered by hundreds of slide and slump fractures from a few meters to hundreds of meters long. Small subsidence fractures also surrounded the many irrigated alfalfa fields south and southeast of Ocotillo Wells.

\section{PATTERNS OF GROUND BREAKAGE}

Displacement along the three principal breaks was commonly distributed along a zone of fractures, en echelon, parallel, or complex, rather than along a single fracture. Most of the north break and much of the central and south breaks consisted of en echelon fractures from a few meters to tens or hundreds of meters long that occupied a band ranging in width from less than $1 \mathrm{~m}$ to more than $100 \mathrm{~m}$. A small part of the three main breaks consisted of bands of complex fractures as much as $10 \mathrm{~m}$ wide or two or more subparallel fractures in a zone as much as $100 \mathrm{~m}$ wide. Other parts of the central and south breaks consisted of single fractures as much as hundreds of meters long.

As noted by Allen, Grantz, Brune, Clark, Sharp, Theodore, Wolfe, and Wyss (1968), even in the wide zones of complex fractures most of the displacement took place within a narrower zone that was generally less than $20 \mathrm{~m}$ across (figs. 24-28). This band of maximum displacement did not occupy a consistent position within the rupture zones and would have been the location of greatest damage had manmade structures been present along the rupture.

The en echelon pattern of fracturing persisted on various scales in remarkable similarity to patterns of surface faulting reported from such places as Parkfield-Cholame on the San Andreas fault (Brown and others, 1967) and Iran (Tchalenko and Abraseys, 1970). En echelon separation ranged in size from $2 \mathrm{~km}$ between the north and central breaks to a few millimeters between en echelon hairline cracks developed locally on smooth parts of the surface.

The geometry of en echelon pattern requires compression and distortion in the area between fractures, which is shown clearly by the folded sediments of Ocotillo Badlands, lying between the north and central breaks. (See Sharp and Clark, this volume.) However, compression was also displayed over much smaller distances by regular patterns of thrusting between en echelon fractures developed in surface flakes and slabs of the muddy silt playa of Benson Lake. Here fractures about $3 \mathrm{~m}$ apart displayed a notable regularity of spacing and behavior; each was connected to the next by paired S-shaped

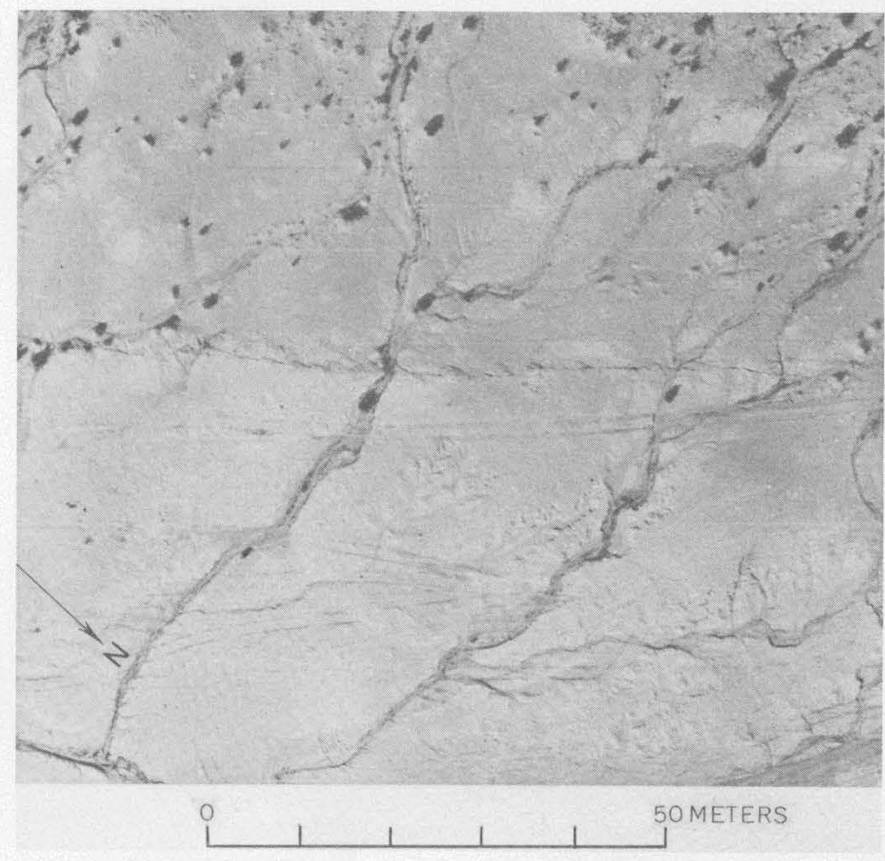

Figure 24. - Central break at location 19.7 showing en echelon fractures as much as $20 \mathrm{~m}$ long and $2 \mathrm{~m}$ apart within a zone about $2 \mathrm{~m}$ wide (sets of vehicle tracks are about $1.6 \mathrm{~m}$ wide). The fractures follow an old scarp that is $0.5-1 \mathrm{~m}$ high (southwest side up). Displacements on this segment of the break at the time of the earthquake reached $160 \mathrm{~mm}$ right lateral and $100 \mathrm{~mm}$ vertical. Photograph by B. W. Troxel, California Div. Mines and Geology, April 19, 1968.

overthrusts (figs. 28, 29). Thrusting here appeared to be limited to a relatively brittle layer about $0.1-$ $0.2 \mathrm{~m}$ thick at the surface. Presumably, compression below this layer caused distortion or flowage of the material between the en echelon fractures.

In general, individual en echelon fractures trended $5^{\circ}-40^{\circ}$ clockwise from the strike of the breaks ( $\mathrm{pl}$. 1 ; fig. 24), with $30^{\circ}$ the most common difference. Thus, along the predominant N. $40^{\circ} \mathrm{W}$. strike of the north and central breaks, individual fractures trended nearly north-south. However, in the vicinity of Fish Creek Wash (loc. 25), where the central break runs nearly north-south, individual fractures strike N. $5^{\circ}-30^{\circ} \mathrm{E}$.

Although the position and relation of the three main breaks probably reflect the configuration of the fault in bedrock, the factors that control the detailed appearance of the surface rupture itself remain unknown. Magnitude of displacement does not obviously control the character of surface fracturing, inasmuch as both small and large displacements occurred across single, multiple, complex, and en echelon fractures that occupied either narrow or wide bands. Depth to bedrock may slightly influence 


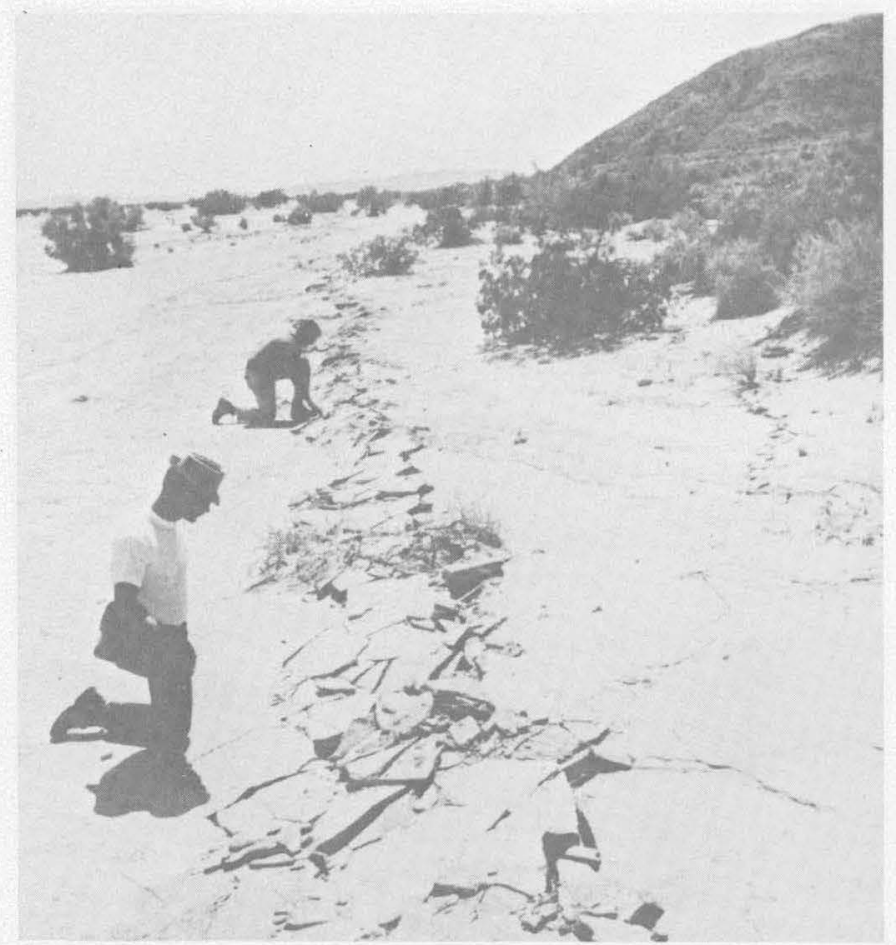

FIgure 25. - Band of complex fractures about $1 \mathrm{~m}$ wide across hardened silty sand crust in a wash at the eastern base of Borrego Mountain (near loc. 6). Right-lateral displacement across the rupture reached $300 \mathrm{~mm}$ in the foreground; right (southwest) side was raised about 100 $\mathrm{mm}$. A subsidiary fracture is in the right center. Photograph by C. R. Allen, April 13, 1968.

the surface fractures, because most long single fractures were limited to the central break, which lies entirely in a 2-4-km thickness of late Cenozoic sediment (Hamilton, 1970; Sharp, "Tectonic Setting of the Salton Trough," this volume), whereas the north break, which essentially lacked long single fractures, presumably crosses very shallow sediment alongside Borrego Mountain. In contrast, however, en echelon and complex breaks similar to those on the north break next to Borrego Mountain were abundant in the thick sediment of the central break. It seems more reasonable to suppose that patterns of surface fracturing tens to hundreds of meters wide are controlled by physical properties within a surface layer of comparable depth, rather than to suppose that such patterns are transmitted unchanged upward from bedrock through sediments tens to hundreds of times thicker than the dimensions of these patterns. An explanation for the varied appearance of the surface trace probably requires further study of subsurface material of the fault zone.

\section{DISPLACEMENTS}

Nearly all the displacements recorded on plate 1 were measured directly from irregularities on or in

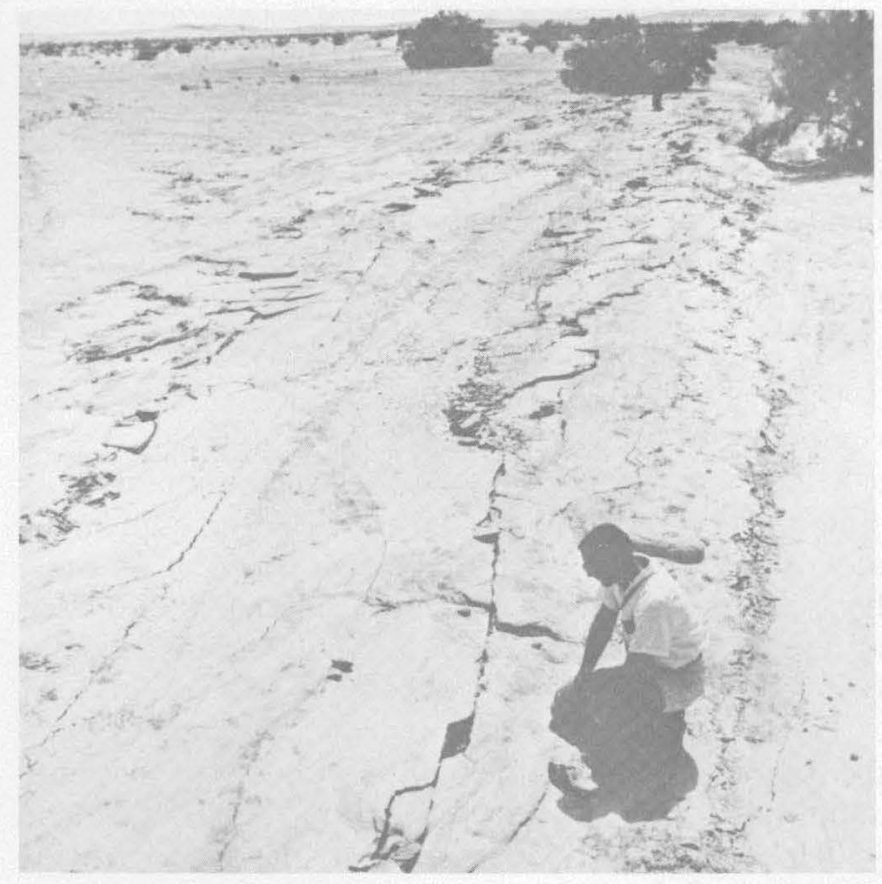

Figure 26. - En echelon, parallel, and complex fractures in a zone $6-10 \mathrm{~m}$ wide on the north break at location 6.4 . Cumulative horizontal displacement here was $380 \mathrm{~mm}$, the maximum measured along the 1968 rupture. Photograph by C. R. Allen, April 13, 1968.

the ground itself, rather than from manmade objects such as fences, pipelines, or buildings, few of which are present in the fault zone. Vertical measurements were simple to make because most of the rupture crossed nearly flat terrain, but horizontal displacements were more difficult to determine. We were able to measure a few such displacements in places where matching irregularities across fractures survived; small channels across fractures in some other places recorded horizontal offsets. However, the best and most abundant indicators of horizontal displacement were tire tracks. Hundreds of motorcycles, dune buggies, and four-wheel-drive vehicles frequent this region every weekend and holiday period during the cooler seasons. Tracks laced the fault at the time of the earthquake and became one of our most common indicators of lateral displacement (figs. 30, 31). Unfortunately, drivers continued to drive on and across the break after the earthquake, often obliterating evidence of displacement.

\section{FRACTURES WITH RIGHT-LATERAL COMPONENTS OF DISPLACEMENT}

Horizontal displacement along any individual fracture that does not connect with other fractures increases from zero at each end to a maximum value somewhere in between, usually near the center. This distribution of displacement holds for each of the three major breaks as well as for diverging or out- 


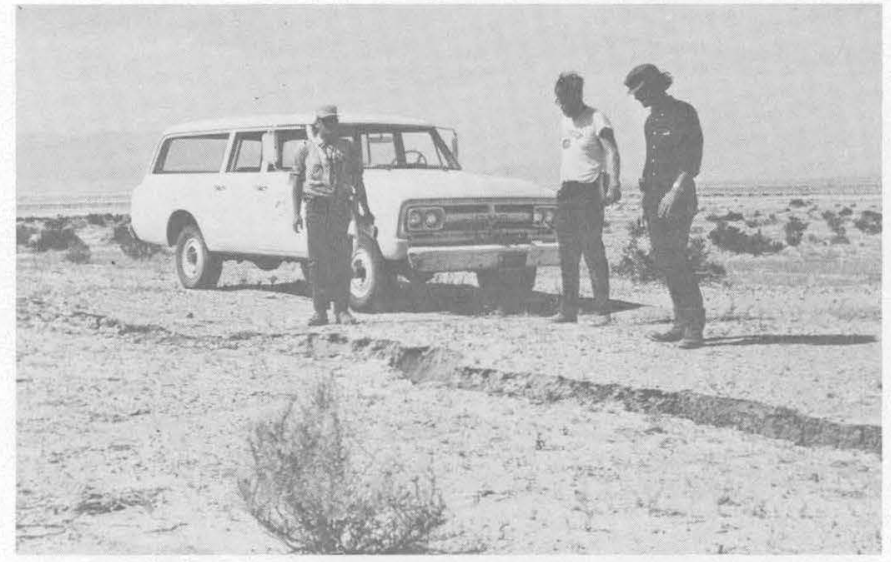

$A$

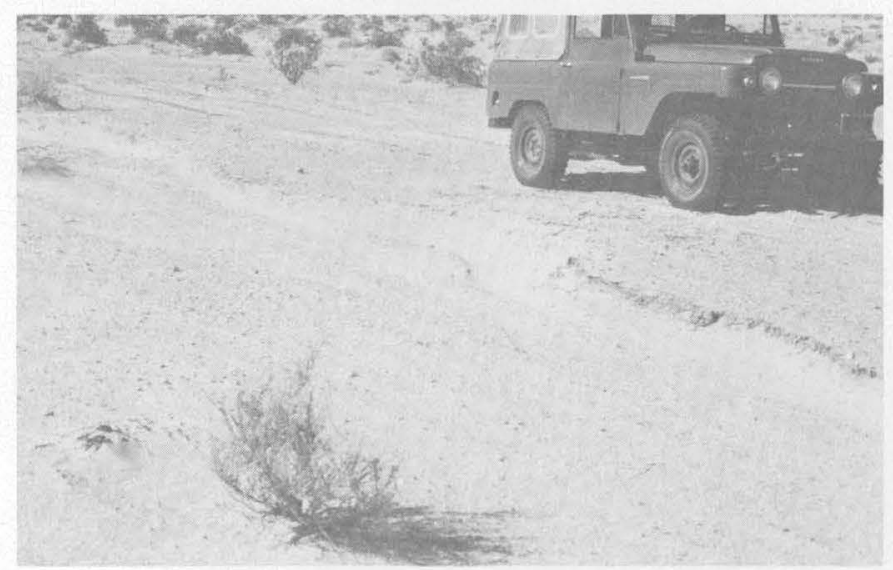

C

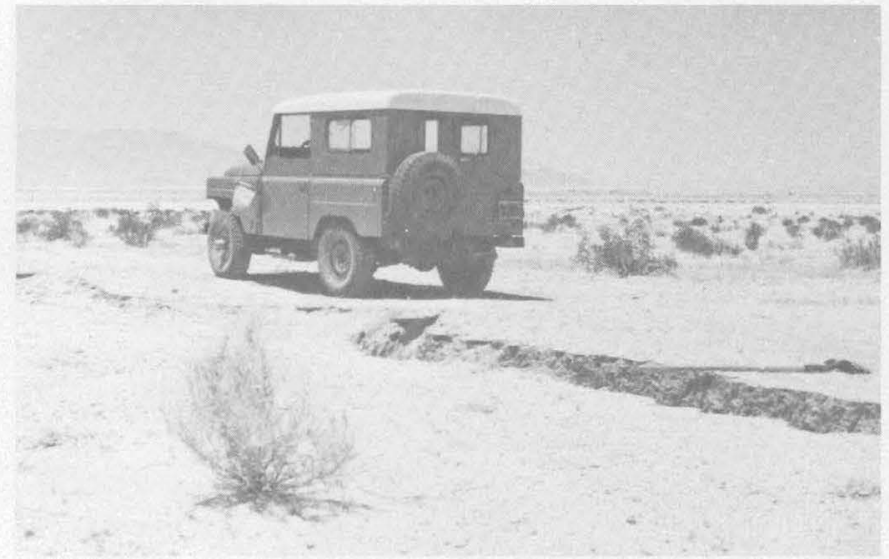

$B$

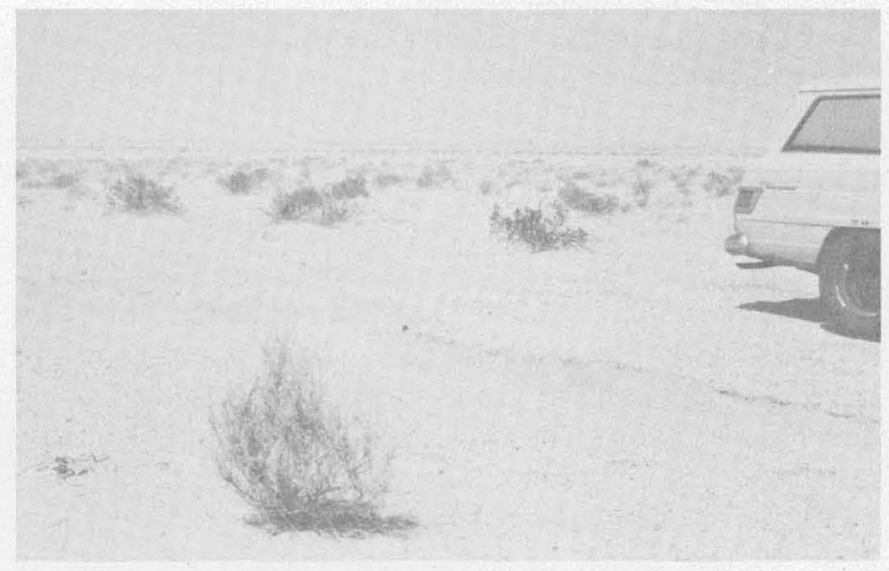

$D$

FIgURE 27. - Time sequence on central break at location 22.5. Here the central break was a single fracture with very few en echelon fractures. For $100 \mathrm{~m}$ along the single fracture at this location, the vertical displacement in 1968 and in the past raised the northeast side of the fault as shown here, in contrast to adjacent sections of the break, along which the southwest side has moved up. (See fig. 24.) A large amount of postearthquake creep occurred on this section of the break. A, April 12, 1968. Horizontal displacement about $180 \mathrm{~mm}$, vertical, 100-150 mm. B, June 20, 1968. Horizontal displacement has increased to $250-300 \mathrm{~mm}$ and vertical displacement to $150 \mathrm{~mm}$ at the shovel handle and 230 $\mathrm{mm}$ between the shovel and jeep. No further displacement occurred after this date. C, March 1969. Drifting sand fills the fracture and is banked against the scarp. D, October 1969. Scarp is gradually being destroyed by erosion and deposition.

lying zones and individual en echelon fractures. (See fig. 32.) Maximum displacement on the north break, $380 \mathrm{~mm}$, occurred in the central third of that zone at the base of Borrego Mountain. Similarly, the maximum displacements measured on the central and south breaks, $230-300 \mathrm{~mm}$ and $50-80 \mathrm{~mm}$, respectively, occurred in the central part of each.

Some of the pronounced variability in displacement shown in figure 32 along each break probably results from measurement errors. Generally, the larger and smaller measurements of displacement on each break are fairly accurate. The larger displacements received special attention because we were interested in the maximum displacements caused by the earthquake. Smaller displacements (less than $30-50 \mathrm{~mm}$ ) nearly always occurred on clean single fractures and were easy to measure accurately. However, some intermediate values of displacement may be too low, because total cumulative slip was not recognized and measured, particularly across wide zones of multiple, complex, or en echelon fractures.

The central and south breaks appeared to have a continuous yet poorly developed connection, shown on plate 1 between locations 25 and 28 as a series of widely spaced fractures with small displacements. There were probably more fractures in this zone immediately after the earthquake than the map shows. Unfortunately, strong winds drifted sand along this and other parts of the central and south breaks before mapping was completed. The sand almost certainly filled some unmapped fractures at the junction of the central and south breaks because 


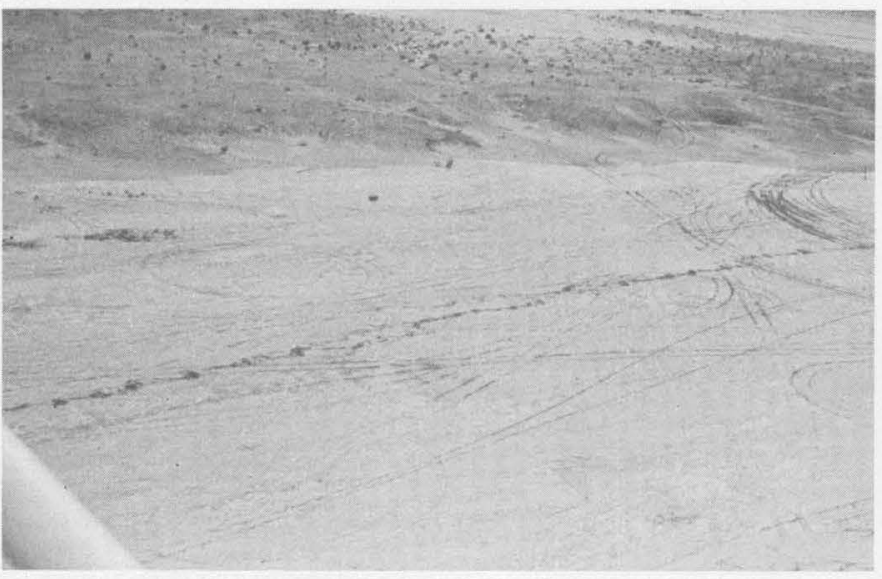

Figure 28. - En echelon fractures at the north end of Benson Lake (loc. 10.2). Fractures are connected by S-shaped overthrusts shown in detail in figure 29. Photograph by Arthur Grantz, April 10, 1968.

it filled some fractures in nearby areas that had already been mapped. Thus, we probably missed many of the small fractures in this region. However the small displacements that characterize this section also indicate that the connection at the surface between the central and south break was poorly developed, despite the fact that sand probably buried much of the evidence. Mapping to the north and south before the wind filled some of the fractures and later to the south in areas not greatly affected by blown sand indicated that displacements diminished to very small values (about $10 \mathrm{~mm}$ ) toward the junction of the central and south breaks.

Horizontal slip along nearly all right-lateral fractures of the three main breaks appeared to be parallel to the N. $30^{\circ}-40^{\circ} \mathrm{W}$. trend of the entire rupture, regardless of the orientation of individual fractures. Thus, fractures trending N. $30^{\circ}-40^{\circ} \mathrm{W}$. showed only strike slip, but those with increasingly divergent trends showed an increasing component of opening normal to the trend of the fracture. Fractures oriented more than $45^{\circ}$ from the general trend of the rupture showed larger components of opening than components of strike slip. Inasmuch as most parts of the three main breaks nearly parallel the N. $40^{\circ}$ $\mathrm{W}$. trend of the 1968 rupture, most individual rightlateral fractures trend about $30^{\circ}$ clockwise from this direction and show roughly twice as much strike slip as opening slip. However, near location 24, where the central break runs roughly north-south, individual fractures diverge about $60^{\circ}$ from the general N. $40^{\circ}$ W. slip direction and show twice as much opening slip as strike slip.
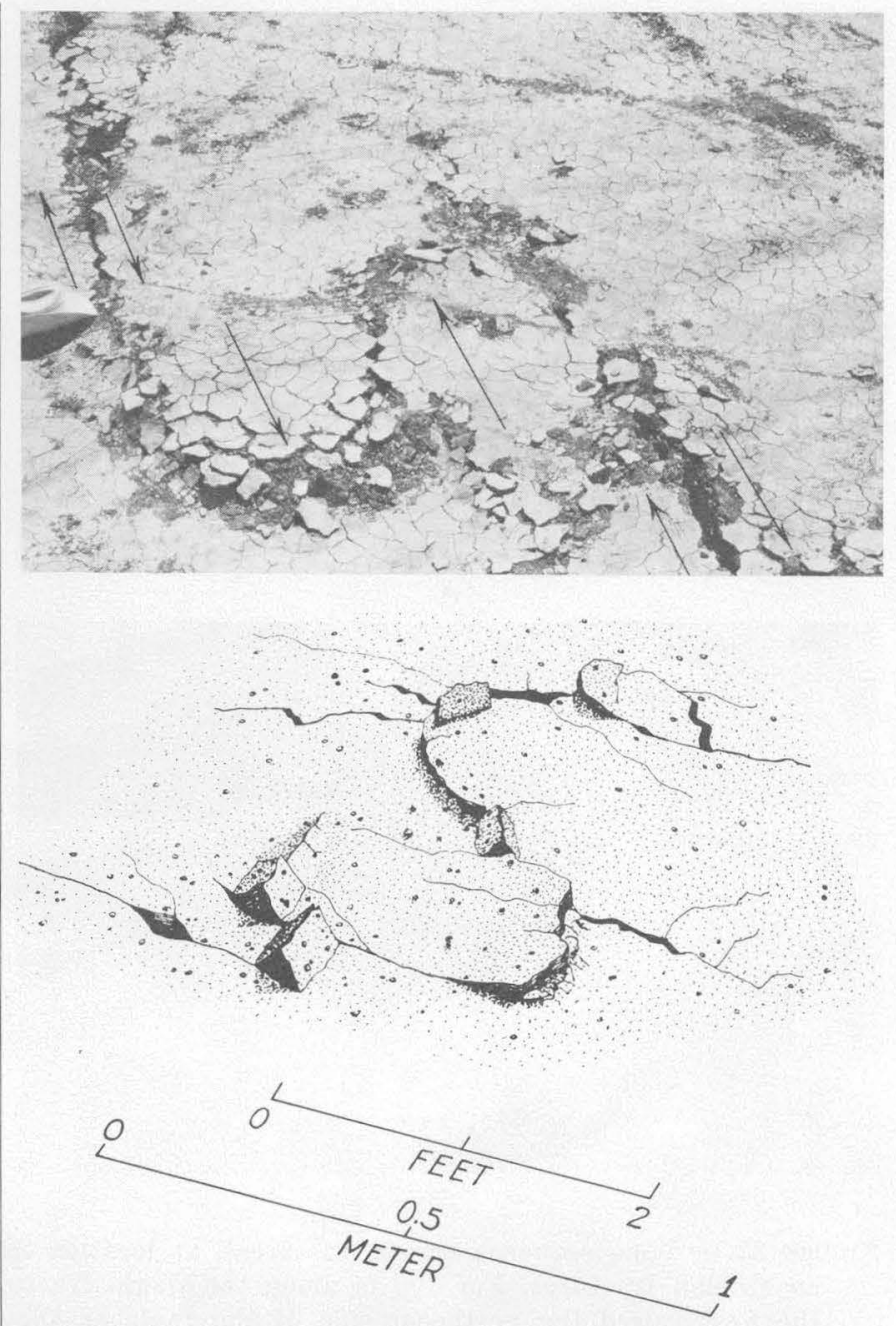

Figure 29. - Detail of symmetrical S-shaped overthrusts in the clay-silt crust of Benson Lake. These thrusts connect en echelon fractures of the main rupture shown in figure 28. Sketch by R. V. Sharp.

\section{FRACTURES WITH VERTICAL COMPONENTS OF DISPLACEMENT}

Nearly all significant vertical displacement across tectonic fractures occurred at locations showing clear evidence of vertical displacement in the past. Evidence of such displacement was found along scarps, at the base of hills or mountains, or alongside uplifted beveled strata. Moreover, vertical displacement in 1968 everywhere continued the sense of past vertical movements. For example, vertical displacement on the north break occurred only along the base of Borrego Mountain (mountain side up) and along Ocotillo Badlands (badlands side up). On the flat terrain north of Borrego Mountain and at Benson Lake, the north break showed no vertical 


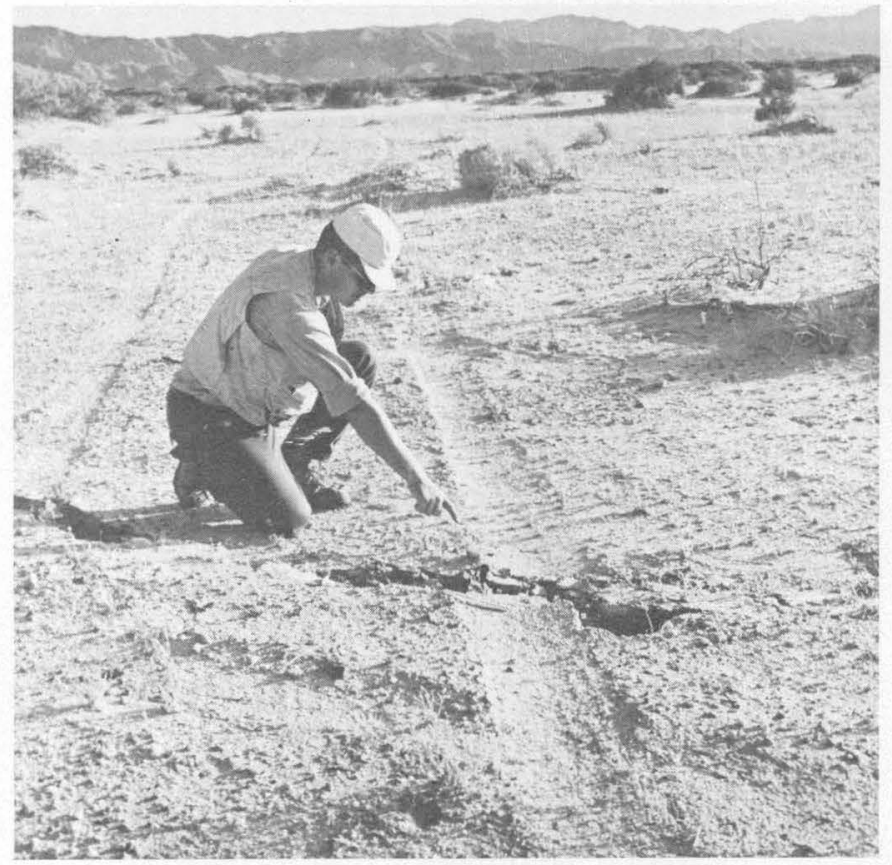

Figure 30. - Tire tracks at location 22.5 displaced about $180 \mathrm{~mm}$ across the single fracture at this location. Photograph by C. R. Allen, April 10, 1968.

displacement. The central break displayed vertical offset of 20-100 mm (southwest side up) nearly continuously for $8 \mathrm{~km}$ from its north end southeastward along the base of Ocotillo Badlands and beyond (description of an exception follows) along a $1 / 2-1$-m-high east-facing scarp as far as location 23 , where the break diverged from the scarp. Farther southeast, the break crossed flat terrain and showed no vertical offset. The south break followed no significant scarps and showed very little vertical displacement.

A localized zone of anomalous vertical displacement occurred along the scarp of the central break at location 22.5. Along this prominent east-facing scarp, vertical displacement in 1968 reversed along a segment about $100 \mathrm{~m}$ long and created a westfacing scarp that was $100-150 \mathrm{~mm}$ high on April 8 but rose to a height of $230 \mathrm{~mm}$ by June 1968 (fig. 27 ). No old west-facing scarp existed here, but color aerial photographs taken in April 1969 and ground examination revealed uplifted and beveled strata on the east side of the break exactly at this location. Evidently, there has been reversed uplift on this short segment many times in the past.

New fractures were associated with upwarped sediments in two other places, both east of the main rupture. Near location $22,1 \mathrm{~km}$ east of the central break, a prominent secondary break traversed the steeper, north slope of low hills formed by uplifted unconsolidated sediments in an area of otherwise

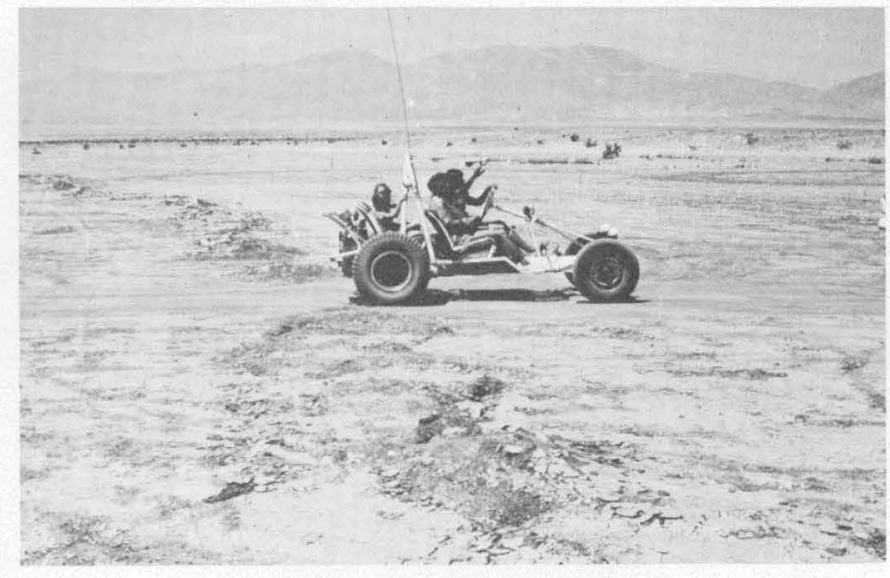

Figure 31. - Dune buggy crossing the north break in Benson Lake 6 days after the earthquake. Desert vehicles supplied many tire tracks for measurements of horizontal displacement; however, they also caused a small but significant part of the destruction of the surface rupture.

undisturbed surficial strata. Gentle dips evident on some of the strata reveal a pattern of upwarping and beveling very similar to that of the reversely uplifted section of the central break just described. Near location $27.4,5 \mathrm{~km}$ farther to the southeast and some $3 \mathrm{~km}$ distant from the south break, the sand-filled remnants of fresh cracks traverse the scarp on the southwest side of a low hill. Again, gently dipping beds are present at this scarp. The fractures most likely formed during or after the April earthquake, and as at the two locations just mentioned and at Ocotillo Badlands, the vertical displacements of the earthquake were continuing to uplift a hill here that evidently owed its existence to episodic tectonism. (See Sharp and Clark, this volume.)

\section{FRACTURES WITH LEFT-LATERAL COMPONENTS OF DISPLACEMENT}

A fascinating aspect of the outlying fractures was that some of them displayed left-lateral offset. We found this apparently anomalous displacement at four separate locations: (1) a series of fractures lying as much as $1 \mathrm{~km}$ west of Ocotillo Badlands (locs. 12-12.4; 15-16), (2) several fractures northeast of Highway 78 and $1-2 \mathrm{~km}$ from Ocotillo Badlands (locs. 13-14), (3) two small zones at the northeast base of Ocotillo Badlands (locs. 15.1, 17.5), and (4) a series of fractures near the north end of the north break at the base of Borrego Mountain (locs. 3-5). Unfortunately, the first two groups of left-lateral fractures, recorded by R. V. Sharp, R. O. Castle, and E. W. Wolfe, did not survive for further study. However, the left-lateral fractures at the other two locations did survive (those at the northeast base of Ocotillo Badlands were not discovered 
EXPLANATION

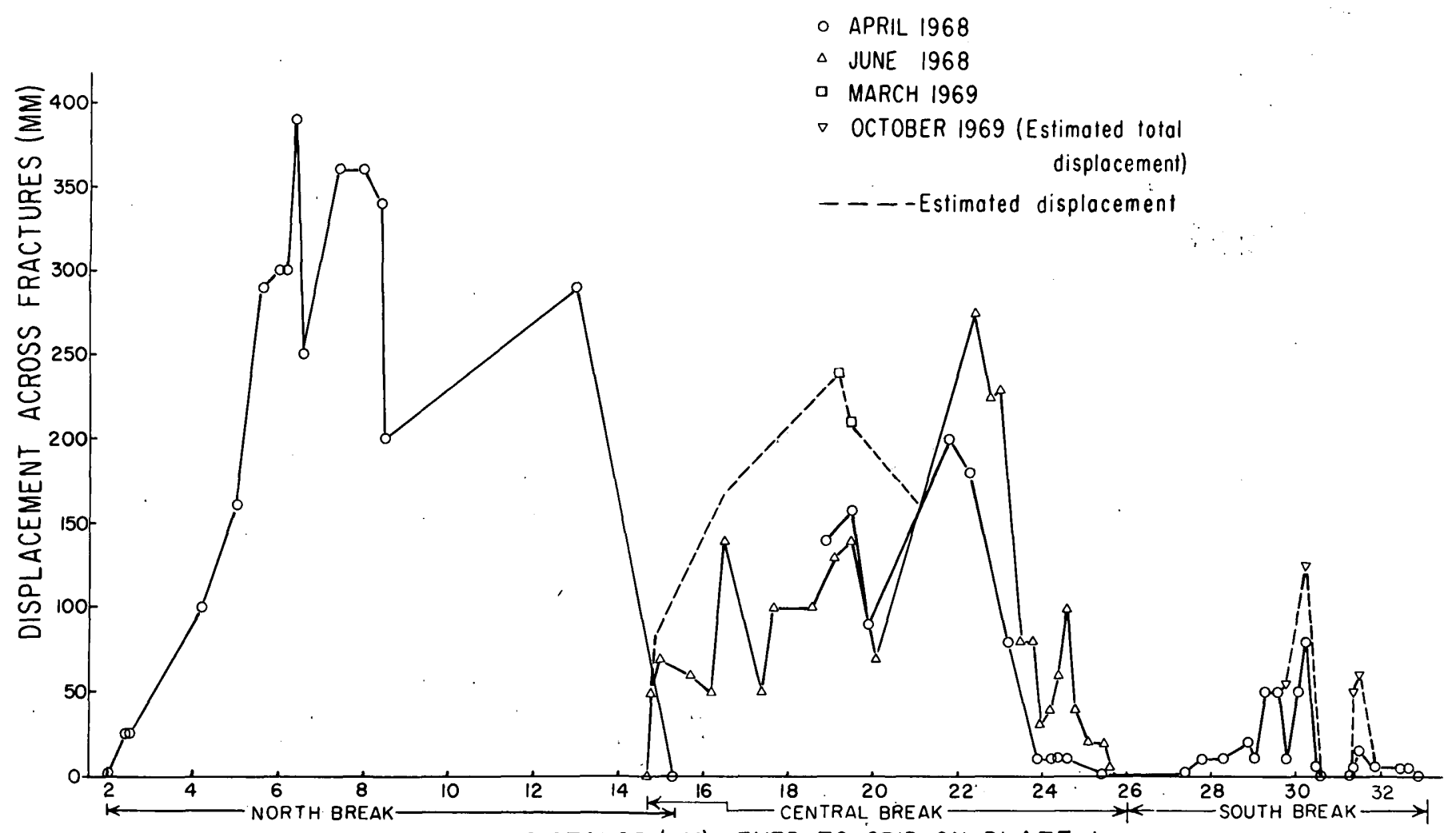

HORIZONTAL DISTANCE (KM) KEYED TO GRID ON PLATE I

Figure 32. - Total right-lateral displacement along the north, central, and south breaks measured at different times (data from pl. 1). The fact that June measurements near locations 18-20 are smaller than measurements made the preceding April has no significance. The same features were not measured each time, and the differences are within the

until June 1968), and much of the discussion of left-lateral displacement is based on study of these fractures.

Three generalizations can be made about the fractures showing left-lateral offset. First, the three groups in the vicinity of Ocotillo Badlands trended northeast, rather than northwest or north as did the right-lateral fractures in the same area. Second, at least some of the fractures in each group displayed a left-lateral en echelon arrangement. Third, all fractures except those northeast of Highway 78 and those southwest of the highway at locations 12-12.4 displayed about as much vertical as left-lateral displacement. Indeed, most of the left-lateral fractures at the base of Borrego Mountain had more vertical than horizontal displacement. In contrast, almost nowhere along the right-lateral fractures did vertical displacement exceed horizontal.

Those left-lateral fractures that trend northeast are compatible with patterns of faulting reported by Dibblee (1954) and Tchalenko (1970) and with accuracy of measurements of this amount of displacement (roughly $\pm 10 \mathrm{~mm}$ ). However, comparable differences between measurements made in April and June between locations 23 and 26 are significant because the measurement error is smaller $(<5 \mathrm{~mm})$ for small displacements.

a theoretical analysis by Chinnery (1966). Dibblee ascribed the prevalence of minor left-lateral faults in this region to clockwise rotation of northeasttrending blocks, caused by drag along northwesttrending right-lateral faults. The northeast-trending left-lateral faults also fit the category of "conjugate Riedel shear" fractures as summarized from laboratory and field observations by Tchalenko (1970), although the northeast-trending faults along the 1968 rupture are not so widespread as those described by Tchalenko. Chinnery's (1966) model of the stress system at the end of a strike-slip fault also predicts left-lateral fractures.

Left-lateral fractures in the vicinity of Ocotillo Badlands (those of the first three locations described) fit Chinnery's model in that they lay near the ends of two strike-slip faults (the north and central breaks), and their directions (roughly northeast to southwest) generally fit the predicted trends of secondary fractures showing displacement opposite in sense to that of a primary break. 


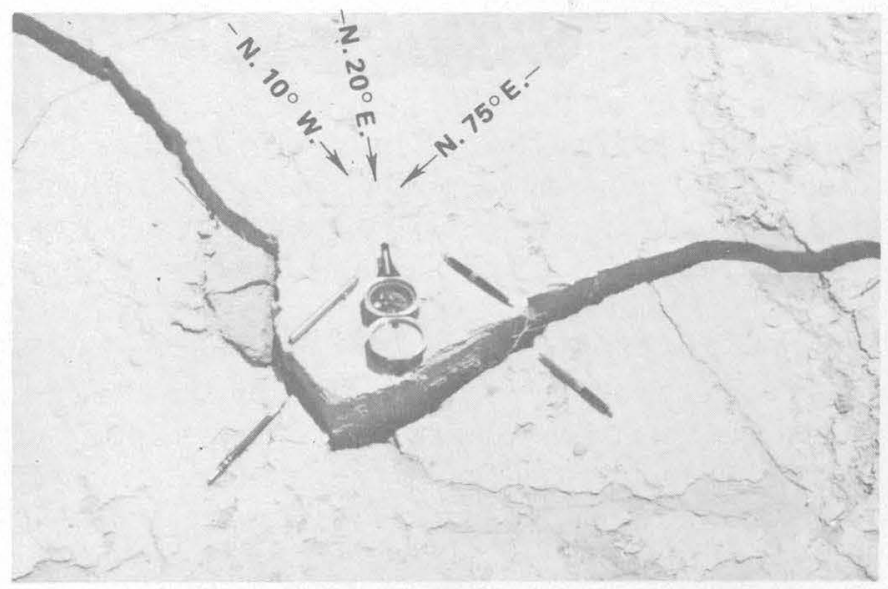

FIGURE 33. - Segment of an extensional fracture showing dip slip at location $3.3,1 \mathrm{~km}$ southwest of the north break. Compass is parallel to the direction of extension, N. $20^{\circ}$ E. Vertical displacement is roughly $50 \mathrm{~mm}$, and opening is about $20 \mathrm{~mm}$. Pencils and thermometer case point to matching irregularities. Those to the left of the compass, on a segment of the crack trending N. $10^{\circ}$ W., show leftlateral displacement, whereas those to the right, on a segment trending N. $75^{\circ}$ E., show right-lateral offset when viewed normal to each segment.

An alternative explanation for left-lateral displacement is suggested by the evidence at the north end of the north break, where vertical displacement exceeded left-lateral displacement on most breaks that survived for further study two months after the earthquake. Here fractures trended north or northwest, so that explanations based on the northeast strike of left-lateral fractures do not apply. These fractures separated a steep mountain front from an alluvial basin, and at least some of them resembled normal faults because they were not straight and they displayed a significant amount of displacement normal to the trend of the fractures, in addition to vertical and left-lateral displacement.

If normal faults are assumed to accommodate extensional strain, then a normal fault that is not perpendicular to the direction of extension will appear to have a strike-slip component in addition to the dominant vertical displacement. Figure 33 shows part of a normal fault trace in the zone of left-lateral fractures near the base of Borrego Mountain. This fracture trended generally perpendicular to the extension direction measured across it, about N. $20^{\circ} \mathrm{E}$. In detail, however, the fracture followed a zig-zag course and showed left-lateral displacement in some places and right-lateral in others, depending on the trend of the fracture at each place. Other nearby fractures showed the same phenomena. Unfortunately, all the fractures mapped in April 1968 showing left- and right-lateral displacements in this

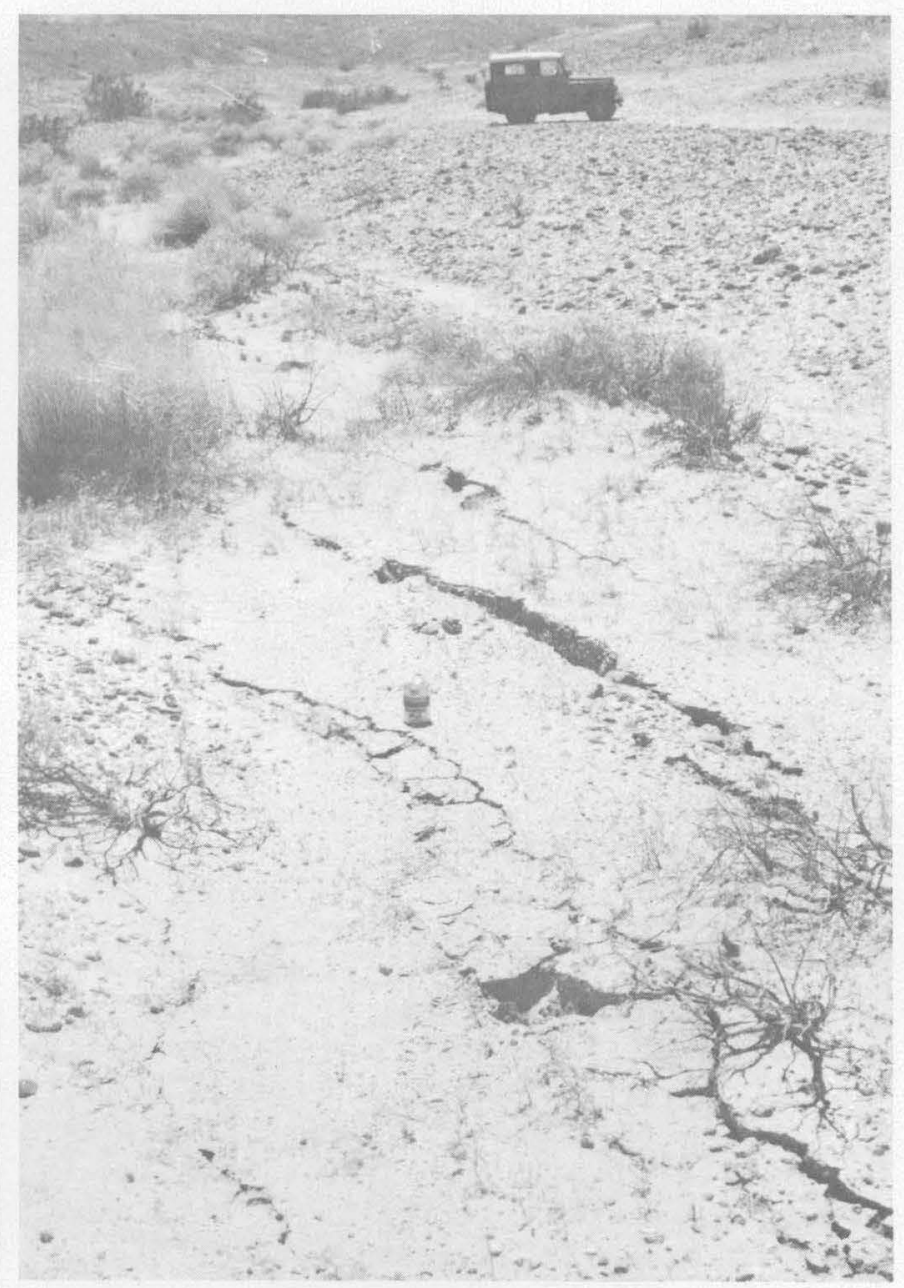

Figure 34. - Fractures showing left-lateral displacement at location 17.5, northeast of the central break. The en echelon arrangement of these fractures is opposite that of right-lateral fractures of the main breaks; that is, individual fractures here are oriented about $30^{\circ}$ counterclockwise from the trend of this fracture zone. Vertical and left-lateral displacements are about equal $(40-50 \mathrm{~mm})$.

location could not be found later to verify whether local changes in trend had caused the observed mix of readings ( $\mathrm{pl} .1$ ). However, since vertical displacements were consistently larger than horizontal ones, it seems at least possible, if not likely, that local changes did occur. Thus, displacement on outlying fractures at the north end of the 1968 break may result primarily from vertical adjustments near the boundary between a young range of crystalline rocks (Borrego Mountain) and an adjacent deep alluvial basin rather than from true left-lateral faulting. If this explanation is correct, the presence of minor left- and right-lateral displacements on these essentially dip-slip faults need not have fundamental tectonic significance. 
Some of the left-lateral fractures adjacent to the uplifted Ocotillo Badlands may also be due partly to the mechanism just described. These fractures lay at the northeast base of the badlands (loc. 17.5) and displayed roughly equal amounts of leftlateral and vertical displacement (about 20-50 $\mathrm{mm}$ ). In places, they followed older scarps that were as much as $150 \mathrm{~mm}$ high. However, some of the fractures displayed the en echelon pattern of left-lateral faults (fig. 34), suggesting that they did not result from normal faulting. Location of these fractures in an area of local tectonic uplift, plus the presence of significant vertical displacement on them, favors the normal-fault explanation, whereas the northeast strike and en echelon pattern of the fractures imply that horiontal displacements were most important.

The position of the remaining left-lateral fractures as much as $2 \mathrm{~km}$ away from Ocotillo Badlands, the northeast strike of these fractures, and the lack of older scarps adjacent to them suggest at least that they were created directly by horizontal displacements rather than as byproducts of normal faulting.

\section{ALTERATION OF THE 1968 RUPTURE WITH TIME}

Periodic reobservation of parts of the fault has revealed extensive modifications of the 1968 rupture. In general, blown silt and sand have filled fractures and smoothed out abrupt scarps (fig. 27). Fractures that were not filled or sealed by sand and silt were enlarged by the action of storm runoff, which caused walls to slump and collapse and carried the resulting debris deep into the fractures (fig. 42; Clark, "Collapse Fissures Along the Coyote Creek Fault," this volume). Most of the places where 1968 fractures still survived 1 year later were the same places that displayed obvious scarps or lines of vegetation before the earthquake. By April 1969, most of the north break north of Highway 78, a region lacking older scarps, was obliterated by blown silt and sand or by the action of San Felipe Creek. The same was true of the central and south breaks between the southeast end of the low scarp (loc. 22.6) and collapsed fractures at location 29.8. In contrast, as late as November 1971, many of the 1968 fractures and others subsequently created or enlarged by creep were still present and visible on the central break along the low scarp and Ocotillo Badlands. Moreover, many of the large collapse fissures of both the central and south break, some of which were still forming late in 1970, remained open in March 1971, and some were still open in November 1971. (See Clark, "Collapse Fissures Along the Coyote Creek Fault," this volume.)

\section{POSITION OF THE RUPTURE RELATIVE TO OLDER TRACES}

The position of the 1968 and later fractures with respect to older fractures is important because such information helps us to predict patterns of future ground breakage along active faults, which tend to break repeatedly along the same trace. (See Ross, 1969 ; Clark and others, this volume.) Accordingly, a map (fig. 35) was prepared showing preearthquake evidence at the surface indicating the presence of earlier fault traces along the principal rupture of 1968. This map, based partly on interpretations of aerial photographs, is similar to a series of strip maps produced by the Geological Survey showing recently active ruptures along the San Andreas and other active faults of California. (See Ross, 1969; Vedder and Wallace, 1970; Sharp, 1970; Clark, 1971; Brown and Wolfe, 1970.) Sharp and Clark (this volume) discussed the implications of the structural evidence for past faulting on and near the 1968 rupture.

Several different kinds of field evidence reveal the presence of traces of active faults. Physiographic evidence, such as scarps, offset channels, linear valleys, and other features caused by faulting, is the most common kind. These features are short lived and indicate fault movement no older than the lifetime of the landscape. Other evidence includes vegetation boundaries (caused by the influence of faults on ground water or soil) and the lithologic and structural criteria that ordinarily identify faults of any age. This nonphysiographic evidence is not restricted to active faults, because it merely indicates the presence of faults, whose most recent movements may be nearly as old as rocks they displace. However, most of the rupture of the Borrego Mountain earthquake is in Holocene deposits, and any fault that disrupts deposits this young must be considered active or potentially so.

All these types of evidence of active faulting are shown in figure 35. Roughly one-third of the 1968 rupture coincided with vegetation boundaries or the bases of scarps made by previous displacements

FIGURE 35. - Map showing the location of recently active traces of the Coyote Creek fault (heavy dashed line) in the vicinity of the surface rupture of 1968 inferred from visible evidence that existed just before the earthquake of April 9, 1968, and from knowledge gained during the postearthquake investigation concerning the association of this evidence with surface fractures. Prepared with the help of preearthquake aerial photographs. Collapse fissures lying 2-3 km southwest of main trace may not be tectonic. (See Clark, "Collapse Fissures Along the Coyote Creek Fault," this volume.) Light lines show 1968 surface ruptures. 

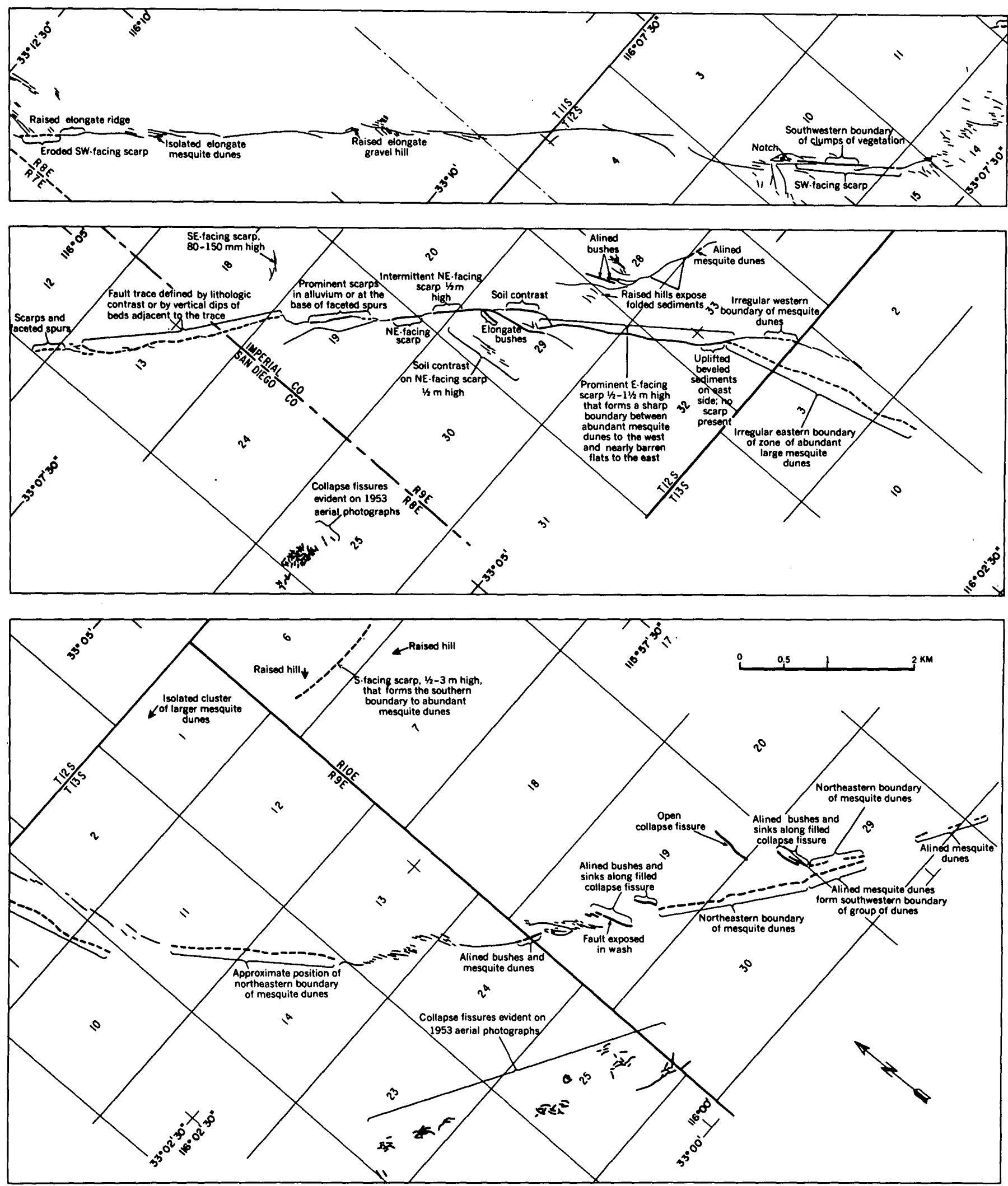


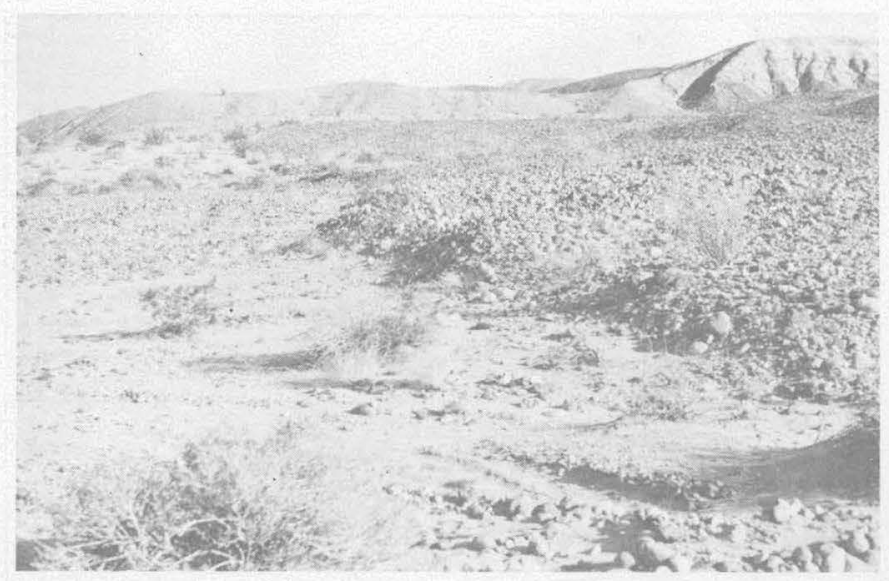

FIgURE 36. - View southeast along the central break showing an old scarp 0.3-0.4 $\mathrm{m}$ high in alluvium at the northeast base of Ocotillo Badlands. A single fracture of the 1968 rupture forms the base of this scarp but is not visible in this picture.

(figs. 36, 37). The scarps are best developed along the northwest and southeast flanks of Ocotillo Badlands and, as noted before, extend for more than $3 \mathrm{~km}$ beyond the southeast tip of Ocotillo Badlands. Less prominent scarps, also followed by isolated fractures, exist along and within some of the uplifted hills east of the central break.

There is no scarp along the base of Borrego Mountain, even though relatively large vertical displacements took place there in 1968 and Borrego Mountain itself may be a result of continuing episodic uplift along the Coyote Creek fault. The reason for this lack of a scarp almost certainly is that water in San Felipe Creek and other washes flows directly on the trace, leveling vertical displacements soon after they take place. By April 1969, flow in San Felipe Creek had effectively erased the scarp created in its channels in 1968. A prominent scarp has not formed immediately south of where San Felipe Creek leaves the trace and the mountain front (loc. 6.6) apparently because of a combination of circumstances: (1) Minor channels flow parallel to and on the break, (2) vertical displacement was distributed across a zone more than $15 \mathrm{~m}$ wide, and (3) between April and June 1968, drifting sand buried most of the trace and completely filled a nearby 1-m-deep stream channel.

Parts of the fault zone that showed no physiographic evidence of previous vertical displacement also lacked other significant visible geologic evidence of faulting. This observation emphasizes that in an area of active deposition on both sides of an active fault, sedimentation acts to conceal lithologic and structural changes caused by horizontal displace- ment, and horizontal faulting without vertical movement has virtually no topographic expressions. In addition, on a nearly featureless and horizontal surface there are no topographic features to be offset. These conditions obtain in parts of the north break and almost all the central and south breaks southeast of location 23.5.

Offset channels are virtually absent from the zone of the 1968 rupture, despite clear evidence of repeated late Holocene movement (Clark and others, this volume). Large channels incised $3-6 \mathrm{~m}$ into the surface cross the fault at locations 24.8 and 27.8 (visible in fig. 23) with no apparent deflection. (The prominent right-lateral offset in one of the washes about one-half $\mathrm{km}$ downstream from the south break at location 28 has no apparent tectonic cause.) Moreover, virtually none of the many small deep welldeveloped channels and gullies that cross the central break along the northeast base of Ocotillo Badlands are offset. Such offset is common elsewhere along the San Jacinto fault zone (Sharp, 1970) and along other active strike-slip faults in California, such as the San Andreas and Garlock faults. (See Vedder and Wallace, 1970; Clark, 1971.)

The primary reason that channels along the 1968 rupture are not offset is apparently that they are being enlarged by erosion faster than they are being offset by tectonism. The large channels southeast of Ocotillo Badlands at locations 24.8 and 27.8 lay below the maximum level of Holocene Lake Cahuilla (roughly the 40 -ft contour line on pl. 1) and must have developed after the lake dried up, about 800 years ago (see Clark and others, this volume), because the channels cut deposits of that lake. Since the lake disappeared the total lateral displacement across the fault in this location has probably been less than $1 \mathrm{~m}$, based on 1968 displacements (about $100 \mathrm{~mm}$ maximum) and probable recurrence intervals for 1968-type events (about 200 years). (See Clark and others, this volume.) Horizontal displacement of $1 \mathrm{~m}$ almost certainly would not be evident in walls of channels that have widened by 10-30 m in the same period. Channels in Ocotillo Badlands cross the fault above the highest levels of Holocene Lake Cahuilla and hence are much older. However, the significant vertical component of uplift of the soft sediments of the badlands that attended the 1968 earthquake (pl. 1) and earlier ones evidently has promoted deepening and widening of nearly all channels sufficient to erase horizontal offsets.

The central and south breaks evidently act as ground-water barriers, and zones of relatively abundant mesquite on the southwest (upslope) side of 


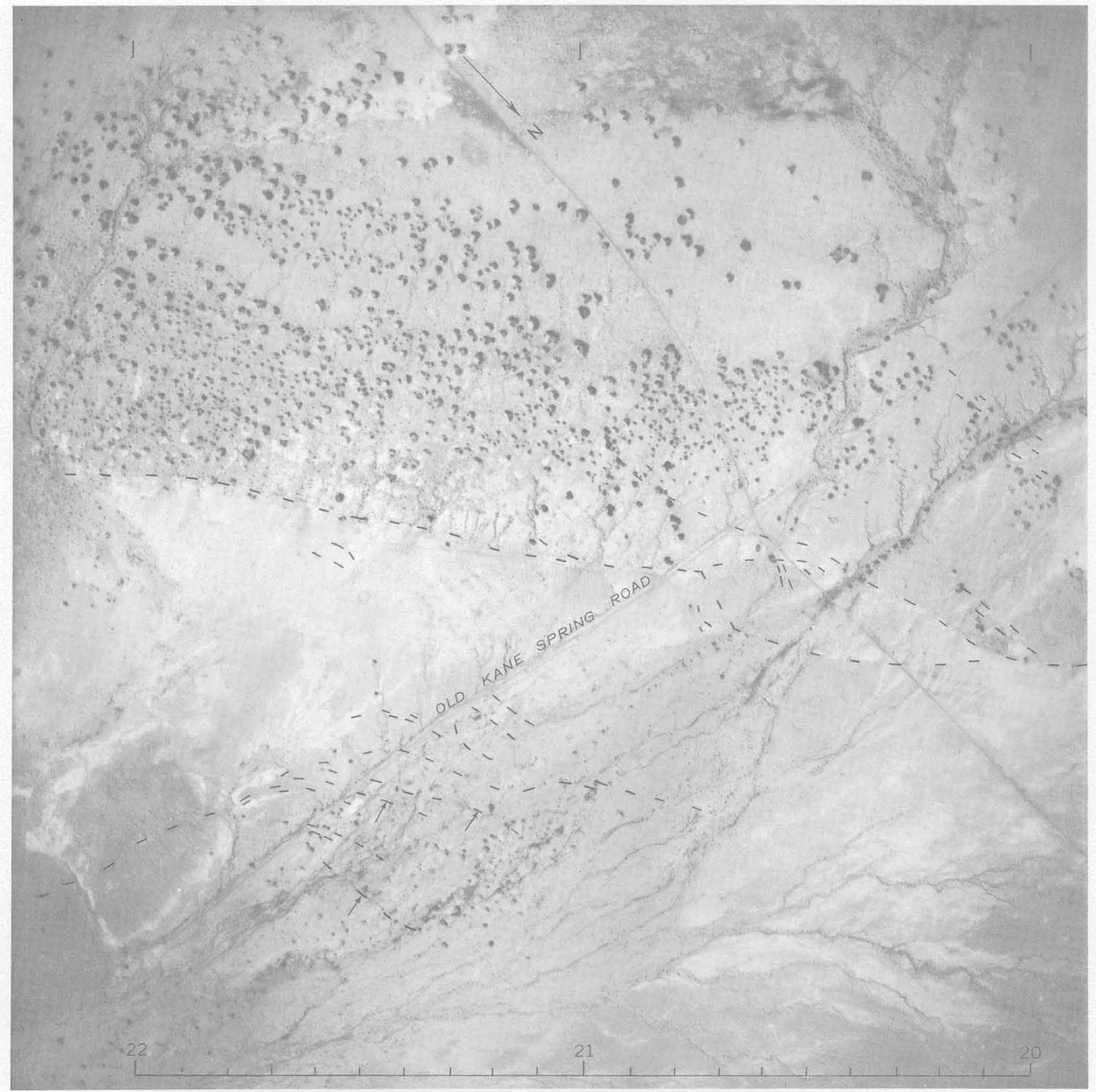

FIGURE 37. - Central break at Old Kane Spring Road. Dashed lines show position of breaks formed since the earthquake. The central break follows alined bushes (barely evident across the center of this photograph) along a northeast-facing scarp $1 / 2-1 \mathrm{~m}$ high. Small channels incise the relatively upthrown block (upper half of picture). The central break also forms the northeast boundary of prominent mesquite-stabilized dunes. Light surface tone indicates the white shelly youngest deposit of Holocene Lake Cahuilla. Removal of this layer from the relatively upthrown blocks defines a graben between the central break and a branching break from locations 20.120.5. Southeast of location 20.7 , the shelly layer is preserved on both sides of the central break. Arrows indicate fractures north of Old Kane Spring Road that follow alined creosote bushes (Larrea), apparently the location of previous fractures or collapse fissures. Faint northsouth lineaments across the central break from locations 20.8-21.4 are creosote bushes along old tracks of vehicles, probably from World War II maneuvers. Aerial photograph by Light Photographic Squadron 63, U.S. Navy, Miramar, Calif., May 1968. 


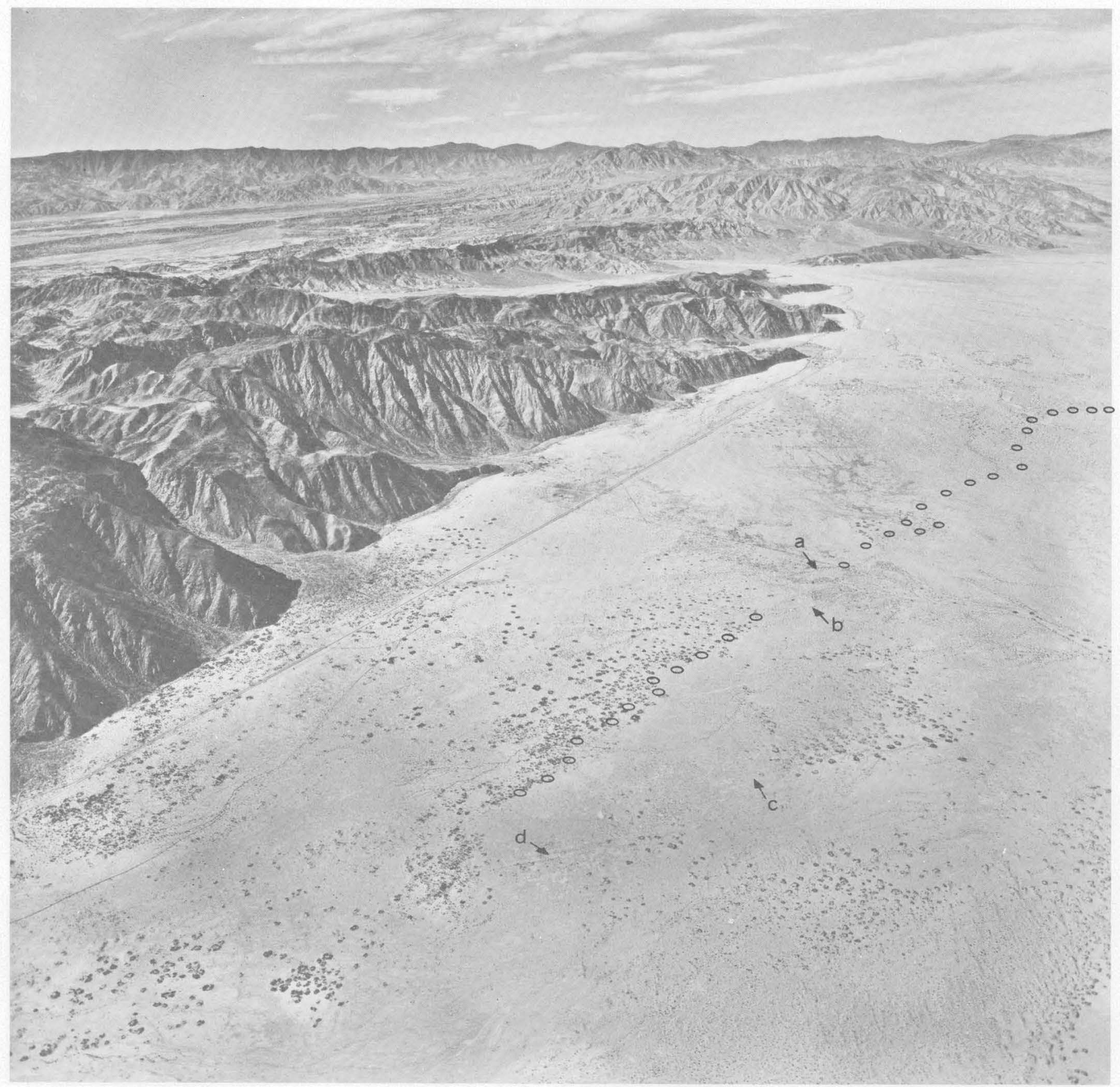

FIGURE 38. - View westward over south break (open circles) in November 1956. Mesquite-stabilized dunes are concentrated on the southwest side of the fault, which acts as a barrier to ground water. Relict collapse fissures visible as lines of bushes are at $a, b$, and $d$. A prominent collapse fissure developed later (but before the 1968 earthquake) at $c$. (See Clark, "Collapse Fissures Along the Coyote Creek Fault," this volume.) U.S. Geological Survey photograph OAJ 5-129, November 1956.

the fault reflect this (figs. 37, 38). Mesquite (Prosopis juliflora), a phreatophyte whose roots may penetrate to $20 \mathrm{~m}$ (Kearney and Peebles, 1960, p. $402)$, tends to accumulate blown silt and sand, creating prominent dunes as much as $5 \mathrm{~m}$ high from which unburied branches protrude. Because they are conspicuous, both on aerial photographs and on the ground, any linear arrangement of or boundary to the mesquite dunes is easy to recognize and to check for fractures. However, such linear aspects of the mesquite dunes that exist away from the rupture did not show fractures. Some may represent 
formerly active traces, but others may well result from other causes.

Creosote bush (Larrea), another plant very abundant in the area of the 1968 rupture, also commonly grows or flourishes along breaks. Indeed, since 1968, many creosote bushes along collapsed fractures have grown markedly and have become much fuller and greener than others nearby because the adjacent fractures have captured surface runoff. Several collapse fissures in the vicinity of Old Kane Spring Road and on the south break followed old lines of creosote bushes, presumably former breaks or collapse fissures (figs. 37, 38).

In many areas where older sediments are exposed, the usual geologic evidence of faulting also coincided with the 1968 rupture. Lithologic changes and drag effects on structural trends along the northeast base of Ocotillo Badlands (described in detail in Sharp and Clark, this volume) clearly coincide with the position of the 1968 rupture.

Along parts of the 1968 rupture, wide zones evidently must be considered vulnerable to future fracturing and large displacements, despite the lack of surficial evidence of similar broad bands in previous episodes of faulting. Although the 1968 rupture closely followed virtually all scarps and other evidence of former fracturing along its path, many individual fractures of the rupture formed where no obvious surficial evidence of previous breakage existed. Some of these fractures formed in sections of the rupture that entirely lacked such evidence, but others, including many with displacements greater than $50 \mathrm{~mm}$, extended away from or developed parallel to either side of obvious lines of earlier faulting for distances greater than $100 \mathrm{~m}$. Moreover, some fractures, including those with displacements greater than $50 \mathrm{~mm}$, formed along lines known to have remained unbroken during the last $300-1,600$ years, yet they were adjacent to traces on which movement had occurred many times in that same period (Clark and others, this volume).

Where a branching or subsidiary break intersects a primary break, the zone of fracturing may be much wider. In the region where the central break and its large subsidiary break cross Old Kane Spring Road, fractures occupy a zone as much as $1 \mathrm{~km}$ wide and more than $3 \mathrm{~km}$ long that trends roughly eastwest across the fault (locs. 19.8-22.8). Although some of the fractures in this area followed older scarps or alined bushes (fig. 37), many appeared to be randomly oriented.

With the information in figure 35 and knowing how the various types of surficial evidence generally are associated with faulting in this region, R. V.
Sharp and I estimated how well the position of the main breaks of 1968 could have been predicted before the earthquake (Youd and Castle, 1970, p. 1206-1207). The location of the principal fractures could have been predicted to within $\pm 3 \mathrm{~m}$ along about 10 percent of the length of the main 1968 rupture, within $\pm 10 \mathrm{~m}$ along an additional 20 percent of the length, within $\pm 100 \mathrm{~m}$ along another 20 percent of the length, but only within $\pm 500 \mathrm{~m}$ over most of the remaining 50 percent of the 1968 rupture. The predictions within \pm 3 and $10 \mathrm{~m}$ were along the prominent scarps of the central and north breaks. Those within $\pm 100 \mathrm{~m}$ were on parts of the central break and north break along or near scarps. Those within $\pm 500 \mathrm{~m}$ included places along the remainder of the rupture that lacked obvious or unambiguous surficial evidence of the presence of the fault.

\section{ENGINEERING IMPLICATIONS OF PATTERNS OF FRACTURING}

Patterns of fracturing and the distribution of displacement within those patterns must be considered in evaluating the potential hazard to manmade structures near the 1968 rupture. The preceding section describes the clear evidence of earlier faulting, such as scarps, that coincide with the main fractures along many parts of the 1968 rupture, but emphasizes that other fractures extend well beyond all surficial signs of earlier faulting. This section describes the variation in the width of the band of fracturing that constitutes the 1968 rupture and the possible widths of bands in which damaging displacements might occur. The engineering implications of other recently active faulting in terrain farther away from the 1968 rupture are discussed by Sharp and Clark (this volume).

The variation in width of the band of fracturing along the 1968 rupture is shown in table 9. The large subsidiary break near Old Kane Spring Road

TABLE 9. - Width of the main rupture along the Coyote
Creek fault

Estimated from fractures recorded on pl. 1 between locs. 1.8 and 32.8 including all diverging ruptures, all fractures between the central and north breaks in Ocotillo Badlands, and all fractures between locs. 19.8 and 22.7 . Isolated fractures or groups of fractures $1 / 2 \mathrm{~km}$ or more from the main breaks at locs. $2.9-3.8 ; 4.6-4.9 ; 13.1-14$ to the northeast and $15.0-16.3$ to the south west of Ocotillo Badlands; and 27.2 were not included in the rupture zone for these estimates.

${ }^{2}$ Measured normal to the coordinate axis of pl. 1 .

'Measured parallel to the coordinate axis of pl. 1 . 
is included as part of the band of fractures of the adjacent part of the central break for these estimates because it may connect with the central break at depth and the area between them contains scattered small fractures. Other isolated fractures more than one-half $\mathrm{km}$ from the main break have not been included in this band. (See table 9.) The estimates show that fractures occupied a band more than $50 \mathrm{~m}$ wide along about 35 percent of the length of the 1968 rupture, a band more than $100 \mathrm{~m}$ wide along about 25 percent of the length, and a band more than $500 \mathrm{~m}$ wide along 10 percent of the length. Bands of fracturing less than $500 \mathrm{~m}$ wide occur at many places along the 1968 rupture; wider bands occur in only a few places, and these generally displayed pre1968 geologic clues about the width of the zone of fracturing. For example, at locations 14-15.2 in Ocotillo Badlands and locations 20-23 along the central break, tectonically upwarped hills bounded on one or more sides by scarps mark the positions of these unusually wide bands of fracturing. Because of their more widespread occurrence (table 9 ), the bands less than $500 \mathrm{~m}$ wide seem to be the most important for estimating the probability that fractures would appear at a given distance from any part of the Coyote Creek fault. On the basis of the fractures that formed in 1968 and later, a 100500 -m-wide zone along the entire fault must be considered vulnerable to fracturing, inasmuch as 17 percent of the length of the 1968 rupture was between 100 and $500 \mathrm{~m}$ wide and 27 percent of the length was more than $100 \mathrm{~m}$ wide.

The fact that a band of fractures developed on one side or the other of the principal fractures at different places along the rupture in 1968 (pl. 1) indicates that estimates of the extent of future fracturing along an identified fault trace (generally a line only a few meters wide, such as a scarp) should include a band on each side that is as wide as the widest band of fracturing that could be anticipated from field evidence. Thus, the band vulnerable to future fracturing along a given fault will be twice as wide as the anticipated band of fracturing.

At most places along the 1968 rupture, the zone of potential damage to structures is narrower than the zone of fracturing because the outermost fractures in a zone tend to have small displacements. If we assume that displacements of $50 \mathrm{~mm}$ ( 2 in.) may damage structures, however, then the zone of potential damage along the main breaks, although narrower than the band of fracturing, is considerably wider than the $20-\mathrm{m}$-wide band that contained most of the displacement in 1968. Although most fractures with $50 \mathrm{~mm}$ or more of displacement lie within this $20-\mathrm{m}$ band, enough extend beyond in different places along the rupture to create a significant general risk to structures. In several places, fractures with $50 \mathrm{~mm}$ or more of displacement extended more than $100 \mathrm{~m}$ from the main break (for example, locs. $18,30,31.3$, pl. 1). In the vicinity of Old Kane Spring Road, such fractures were widely scattered over the 1 by $3 \mathrm{~km}$ area across the central break and the large subsidiary break to the east. The available observations, however, are far too meager to allow any quantitative estimates of variation in width of the band within which displacements of $50 \mathrm{~mm}$ occur along the 1968 rupture.

The degree of risk to structures near the Coyote Creek fault depends on how far fractures with some minimum amount of offset might extend from the principal line or lines of fracturing and displacement. To assess this risk we must know (1) the location of the most recently active principal traces, (2) the patterns of fracturing that are likely to occur during an earthquake, and (3) the distribution of displacement within these patterns.

Lacking data of the third sort, we cannot make specific assessments of risk along this fault. However, the prevalence of apparently active faults in the general region of the 1968 rupture (see Sharp and Clark, this volume), plus the information discussed in this and the previous sections, indicates that before any large structure or multistory building is built within that general region, the subsurface should be examined. Until we learn more about distribution of displacement next to the fault, such subsurface examination would seem to be mandatory before any such structures are built within about $1 \mathrm{~km}$ of the 1968 rupture or other identified recently active faults nearby.

\section{CREEP}

After the 1968 earthquake, displacement (fault creep) continued along parts of the central and south breaks but not along the north break. Through November 1971, this postearthquake movement had added as much as 50 percent to the displacements measured during the week after the earthquake (pl. 1 ; fig. 32). Fault creep has occurred along most of the central break (nearly continuously along some parts), beginning shortly after the earthquake. In contrast, creep apparently did not occur on the south break until about 1 year after the earthquake, and then it occurred only in the middle part of the break. Figure 39 shows areas affected by postearthquake creep along the 1968 break, and table 10 gives approximate dates of this activity. 


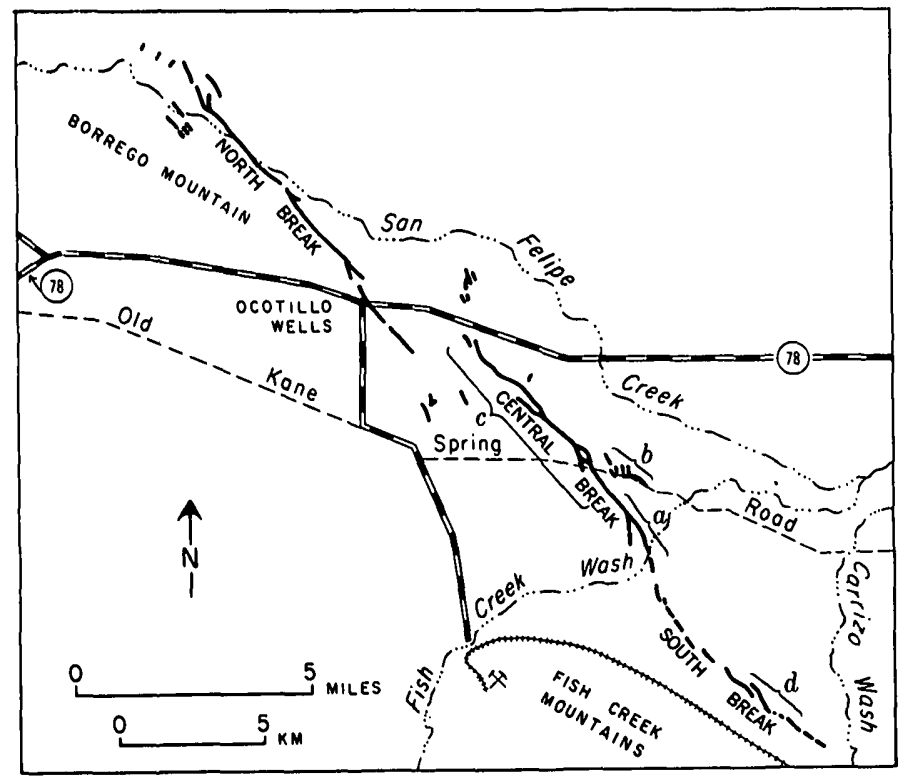

Figure 39. - Areas of postearthquake displacement $(a, b$, $c, d)$ after April 14, 1968, along the surface rupture. Summaries of dates and total creep are in table 10.

TABLE 10.- Summary of creep at locations indicated in figure 39

\begin{tabular}{|c|c|c|c|c|}
\hline Area & $\begin{array}{l}\text { Date creep } \\
\text { first noted }\end{array}$ & $\begin{array}{l}\text { Date creep } \\
\text { commenced }\end{array}$ & $\begin{array}{c}\text { Date creep } \\
\text { ceased } \\
\text { (as of } \\
\text { November 1971) }\end{array}$ & $\begin{array}{c}\text { Estimated total } \\
\text { horizontal creep } \\
\text { April 14, 1968, } \\
\text { to March 1971 } \\
\text { (mm) }\end{array}$ \\
\hline a....... & June $1968 \ldots . . .$. & $\begin{array}{l}\text { Probably after } \\
\text { April } 14, \text { and } \\
\text { definitely before }\end{array}$ & \multicolumn{2}{|c|}{ Before July 5, 1968...80-120 } \\
\hline & $. J u n e ~ 1968 \ldots . . .$. & $\begin{array}{l}\text { Probably between } \\
\text { May } 26 \text { and } \\
\text { June } 14,1968 .\end{array}$ & \multirow{3}{*}{$\begin{array}{l}\text { Probably after } \\
\text { Jan. 14, 1969; } \\
\text { definitely before } \\
\text { Mar. 22, 1969. } \\
\text { Still moving as of } \\
\text { November } 1971 . \\
\text { Latest movement } \\
\text { between Decem- } \\
\text { ber } 1970 \text { and } \\
\text { November } 1971 .\end{array}$} & $\begin{array}{l}\text { Approximately } \\
120\end{array}$ \\
\hline & March $1969 \ldots$ & $\begin{array}{l}\text { After June } 20 \text {, } \\
1968, \text { and before } \\
\text { Jan. 14, 1969. }\end{array}$ & & $80-110$ \\
\hline d...... & October 1969 & $\begin{array}{l}\text { Probably after } \\
\text { Jan. 14, } 1969 . \\
\text { Possibly as late } \\
\text { as August } 1969 .\end{array}$ & & $30-60$ \\
\hline
\end{tabular}

Fault creep first became apparent at several places during mapping along the central break in June 1968 and could still be detected by tape measurement in March 1971. Although a few precision strainmeasuring instruments and stations were established by other investigators on the north and central breaks shortly after the earthquake, only the array on the central break recorded large changes (Burford, this volume). Observations of this geodetic station have not been so frequent as the far less precise observations reported here, which although based in part on indirect evidence, offer a more complete record of both timing and location of creep along the 1968 break. Most changes in displacement were measured with pocket tapes between matching irregularities on opposite sides of fractures. Periods of creep could be determined only within broad limits defined by our infrequent visits to the area and by our ability to deduce when creep occurred with respect to sporadic heavy rains in the area.

Three general types of evidence indicate continued displacement along the rupture: (1) the appearance of new (postearthquake) fractures, (2) an increase in displacement measured at a specific point on the rupture (for example, at a certain tire track), and (3) a general increase in displacements measured along a segment of the rupture in which exact locations of previous measurements cannot be recovered.

The first two types of evidence are best for documenting creep. However, the second depends on rediscovery of individual displaced features, few of which were suitably recorded during the week following the earthquake. The creep discovered in June 1968 became evident only through observation of a general increase of $50-100 \mathrm{~mm}$ in displacements along specific areas of the fault (locs. 22.2, 24). As a result of the discovery of postearthquake creep, however, many individual tire tracks and fractures were photographed in June 1968, and some survived erosion to indicate the presence or absence of creep during ensuing months.

New postearthquake fractures, the most reliable sign of continued movement, can commonly be dated with respect to infrequent periods of intense rain. Several times during the 3-year period after the 1968 earthquake, hard rain created widespread surface runoff that eroded or caused collapse of the walls of many open fractures. (See Clark, "Collapse Fissures Along the Coyote Creek Fault," this volume.) Fractures that developed after rain were easy to distinguish from those that had been open during heavy rains or runoff. The former had sharp, angular edges; the latter rounded, eroded edges. Intense rains also formed a crust on windblown silt and sand that filled many fractures, and creep after the rain broke this crust.

\section{RUNOFF ALONG THE 1968 RUPTURE}

In order to establish dates of runoff that limit episodes of observed creep, we must combine rainfall records, which give dates when surface runoff was possible, with repeated observations along the fault to determine which storms produced runoff. (See fig. 40.) Most of the scant rainfall in this area (about $70 \mathrm{~mm}$ per yr) generally comes from widespread winter storms and is commonly of relatively low intensity; however, a few cloudbursts connected with thunderstorms usually occur during the summer months. Rain of sufficient intensity or amount to cause surface runoff and obvious modification of fractures falls only during the largest winter storms or during summer showers. Unfortunately, published 

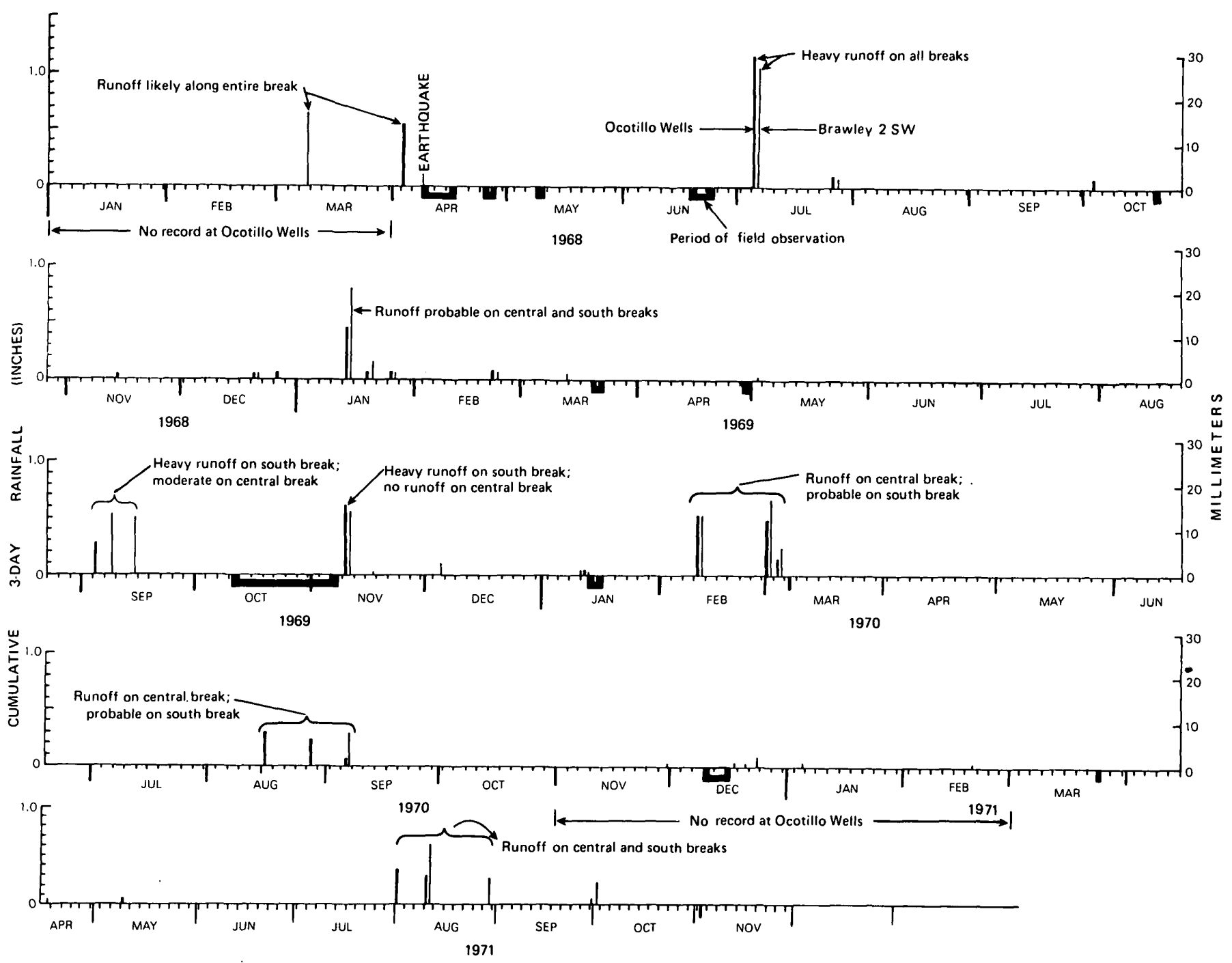

Figure 40. - Rainfall, estimates of dates of surface runoff on the central and south breaks, and dates of field investigations (heavy bars below time axis), January 1968 through November 1971. Rainfall is shown as cumulative totals for 3-day periods; from Environmental Data Service (1968-71), records for Ocotillo Wells (heavy line) and Brawley $2 \mathrm{SW}$ (light line).

data alone do not indicate when rainfall produced surface runoff. U.S. Environmental Data Service weather records for Ocotillo Wells list total daily precipitation but not intensity of precipitation for shorter periods. The nearest station that records hourly precipitation, El Centro, is too distant to yield reliable estimates of intensity in the area of the rupture. Thus, detailed before-and-after field observations of channels and other surficial forms are necessary to establish timing and location of episodes of runoff in the fault zone.

Rainfall reported at Ocotillo Wells is assumed to be representative of rainfall along the entire 1968 rupture. This assumption is supported by figure 41 and by the fact that vegetation is virtually uniform along the fault. The record from Brawley $2 \mathrm{SW}$, about $40 \mathrm{~km}$ east of the south break, is also included in figure 40 because parts of the Ocotillo Wells record in winter months are missing, and Brawley rainfall is generally quite close to that of Ocotillo Wells in winter.

Seven periods of runoff are crucial to our reconstruction of the timing of creep episodes after April 1968. Rain that definitely caused surface runoff and ensuing collapse of open fractures along the 1968 break fell on July 6, 1968, September 6-15 and November 9-10, 1969, February to March and August 1970, and August 1971. Rain on January 14, 1969, probably led to local runoff and collapse, but previous observations during the brief field investigation of October 1968 were insufficient to permit an unequivocal distinction between all effects of the 


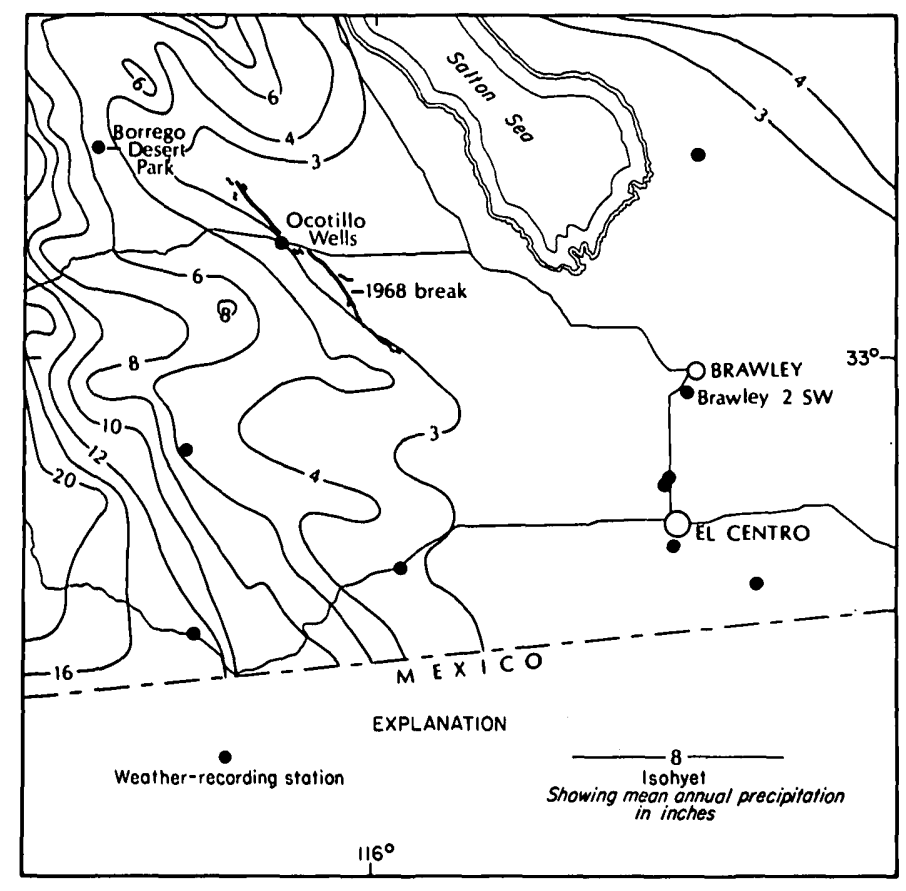

Figure 41. - Mean annual precipitation in the region of the 1968 break, 1931-60. From Hely and Peck (1964, pl. 3). 1 in. $=25.4 \mathrm{~mm}$.

rains of July 6, 1968 and January 14, 1969. Precipitation on July 6, 1968 (1.05 in.) was the highest amount recorded in a single day from 1967 through 1971 at Ocotillo Wells and resulted in heavy rain throughout the region and widespread surface runoff along the entire 1968 break.

\section{CREEP ON AND NEAR THE CENTRAL BREAK}

AREA $A$ (FIG. 39)

Obvious signs of continued displacement along the 1968 break were first noted in June 1968 near location 22.3, the part of the central break on which vertical displacement was reversed in sense for a short distance along the prominent scarp southeast of Ocotillo Badlands. Maximum vertical displacement increased from $100 \mathrm{~mm}$ in April 1968 to $150-230 \mathrm{~mm}$ 2 months later, and horizontal displacement increased from about $180 \mathrm{~mm}$ to $250-300 \mathrm{~mm}$ (fig. 27). This same site was measured during all subsequent visits to the fault zone, but no further displacement could be detected.

Creep also affected the central break from location 22.5 southeast as far as location 25.0. Although no repeat measurements were possible at individual sites (the only offset exactly located and measured in this section in April 1968 was obliterated by the following June), many measurements made in June were significantly larger than any made in April, implying additional movement (pl. 1; fig. 32).

Measurements in October 1968 and later at many sites marked carefully the previous June along this part of the fault showed no further displacement. The heavy rain of July 6, 1968, enlarged many fractures in this area by collapse (fig. 42). However, by March 1968, all the collapse fissures along this part of the fault had been filled with sand and silt, a further indication that movement had ceased.

\section{AREA $B$ (FIG. 39)}

Persistent and widespread postearthquake creep has taken place on many fractures along the subsidiary break east of the central break. This area provides clear documentation of formation of new fractures after the earthquake and their subsequent enlargement by collapse. The history of development of fractures in this zone is not complete because mapping in April 1968 did not cover all ground in which fractures were later found. Some fractures discovered as late as January 1970 might have developed as early as the time of the earthquake. Fortunately, detailed mapping in certain parts of this area in June 1968 documented later creation of new fractures.

The first indication of continuing displacement in this area after the earthquake was reported by $\mathrm{W}$. L. Cantrell, manager of a motel near Ocotillo Wells. On June 9, 1968, he discovered a prominent fracture on the low hill about $2 \mathrm{~km}$ east of the intersection of Old Kane Spring Road and the central break (loc. 21.9). He was certain that the fracture was not present 2 weeks earlier on May 26 when he visited the same hill (oral commun., 1968). On June 21, I mapped this creek and associated fractures north of Old Kane Spring Road and searched the area between it and the main break without finding other fractures. Subsequent field checks indicated that movement continued in this area east of the central break until sometime between January 14 and March 22, 1969. As determined from evidence of surface runoff, new fractures evidently formed in this area in each of three periods after June 21, 1968: June 21 to July 6, 1968, July 6, 1968 to January 14, 1969, and January 14 to March 22, 1969. Since March 1969, no new fractures have appeared here, nor have old fractures opened further.

Figure 43 shows new fractures that formed in this area after June 1968. Most individual fractures were oriented roughly north-south, as on the main break, but many others were oriented closer to N. $30^{\circ} \mathrm{E}$. In accord with observations made on the main break in April, these fractures showed significant components of opening movement as a result of their 


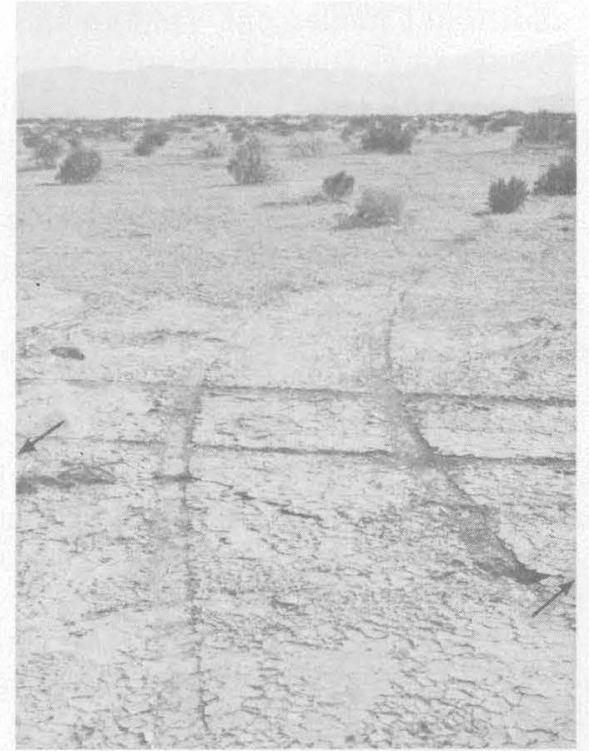

A

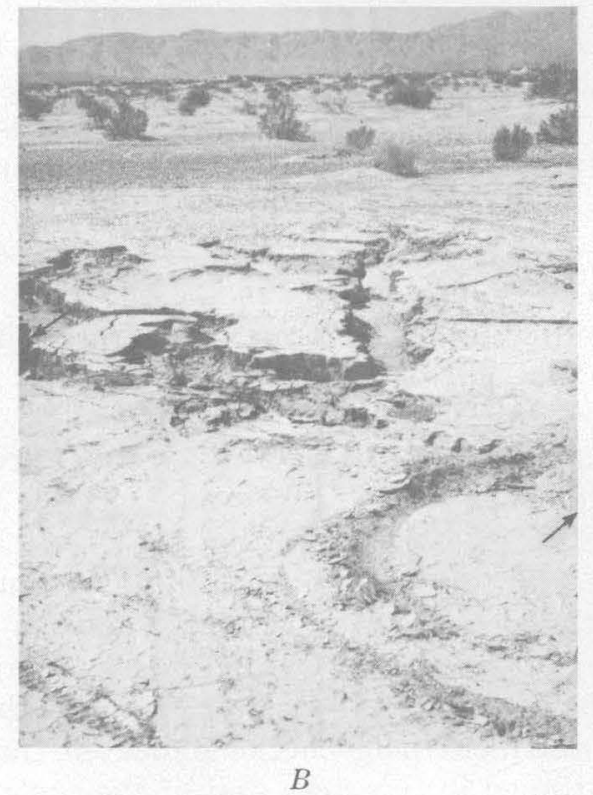

orientation $30^{\circ}-60^{\circ}$ from the regional trend of the central break. Maximum opening was about $50 \mathrm{~mm}$; however, most fractures opened 20-30 mm.

\section{AREA $C$ (FIG. 39)}

The most complete record of postearthquake creep on the central break comes from the section near Ocotillo Badlands. Creep commenced here between June 1968 and January 1969 and continued through November 1971. By March 22, 1969, displacement of tire tracks immediately north of the road that intersects the central break at location 19.6 had increased to $210-240 \mathrm{~mm}$ from the $130-160 \mathrm{~mm}$ measured in the same area in April and June of 1968 (fig. 44).

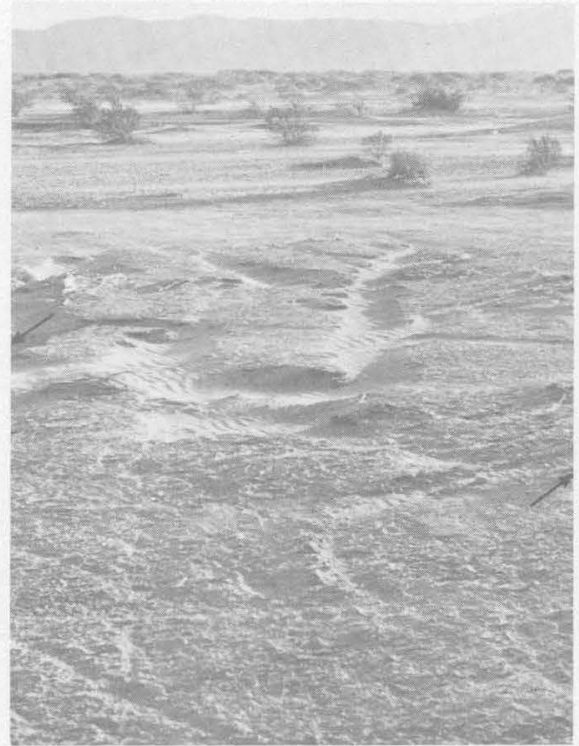

C

Figure 42. - Time sequence showing collapse and eventual filling along a fracture of the central break at location 23.8. $A$, June 23, 1968. Opening of the tectonic fracture (between arrows) has increased from about $10 \mathrm{~mm}$ in April to $20-30 \mathrm{~mm}$. $B$, October 20,1968 . Surface runoff from the heavy rain of July 6,1968 , caused slumping and collapse of the walls of the fracture, followed by partial filling when water could no longer flow readily into the depths of the fracture. A tire track made June 23 became a channel conducting water to the fracture. No measurable creep occurred at this location after June 23, 1968. C, April 29, 1969. Blown silt and sand fill most of the collapsed fracture and channel.

Creep after the rains of January 1969 left a clear record of offset crusts and channel deposits throughout the length of this segment of the central break (figs. 45-47). As measured in March 1969, offsets across these fresh, postrain fractures diminished steadily northwestward from about $40 \mathrm{~mm}$ near location 20.5 to $5 \mathrm{~mm}$ near location 15 (fig. 47). Postrain displacements to the southeast along the central break diminished to zero between locations 20.5 and 22.3 .

Subsequent observations in 1969, 1970, and 1971 revealed evidence of further small displacements. Silt and sand filled these new fractures after March 1969; then the rains of September 1969 formed new crusts on this material. New channel deposits of hard silt at location 17.6 and new crusts that formed in the main fracture between there and location 19.6 showed new fractures in October 1969 with about 1-2 $\mathrm{mm}$ of right-lateral displacement. (Cracks with 


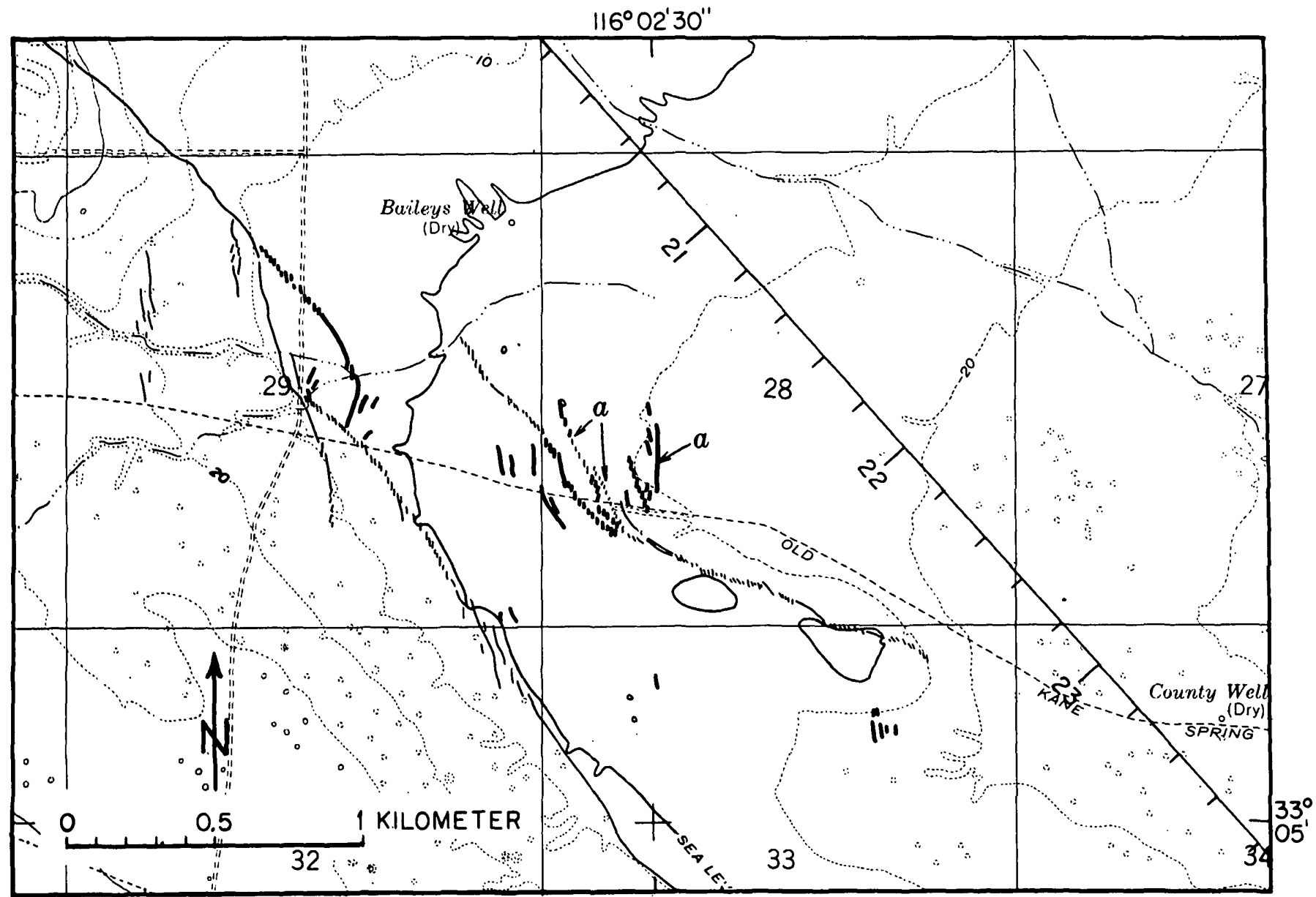

Bose from U.S. Geological Survey 1:24,000 Borrego Mountain SE, 1958

Figure 43. - Fractures (heavy lines) that apparently formed near the central break between June 21, 1968, and March 20, 1969 (light lines show fractures that formed at the time of the earthquake and afterward until June 21, 1968). Many of the fractures probably formed after October 1968. Postearthquake fractures labeled "a" follow alined vegetation (evident on 1953 aerial photographs) that apparently grew on earlier fractures. Base and kilometer grid from plate 1.

openings of less than $1 \mathrm{~mm}$ are easily visible in the hard silt of these channels. The tectonic fractures described here are precisely on the 1968 rupture, show en echelon patterns, and generally have larger openings than shrinkage cracks in the same deposits.) By January 1970, displacements on some of these same fractures had apparently increased by about $1 \mathrm{~mm}$ more. Heavy rains in February to March 1970 and high winds erased these small fractures by March 7, 1970. Whether or not new fractures formed between then and the heavy rains of August 1970 is unknown, but any displacement in that period probably did not exceed 10 or $20 \mathrm{~mm}$. New fractures developed again along the central break after August 1970 between locations 17.6 and 20.6, with as much as $10 \mathrm{~mm}$ of displacement, as observed in December 1970. At location 17.6, this displacement increased by several millimeters by March 1971 and by several more before November 1971.

Figure 48 summarizes the history of displacement measured near location 19.4 on the central break within area $c$ of figure 39 . These crude measurements of postearthquake creep are grossly compatible, except for an initial delay, with the exponential decay of creep found by Wallace and Roth (in Brown and others, 1967, fig. 25) and by Smith and Wyss (1968) after the Parkfield-Cholame earthquake on the San Andreas fault. Furthermore, Burford (this volume) inferred exponential decay of creep for the central break from a nearby alinement array. The data for the central break contrast with those for Parkfield-Cholame in that creep on this segment of the central break evidently did not begin immediately after the earthquake. 


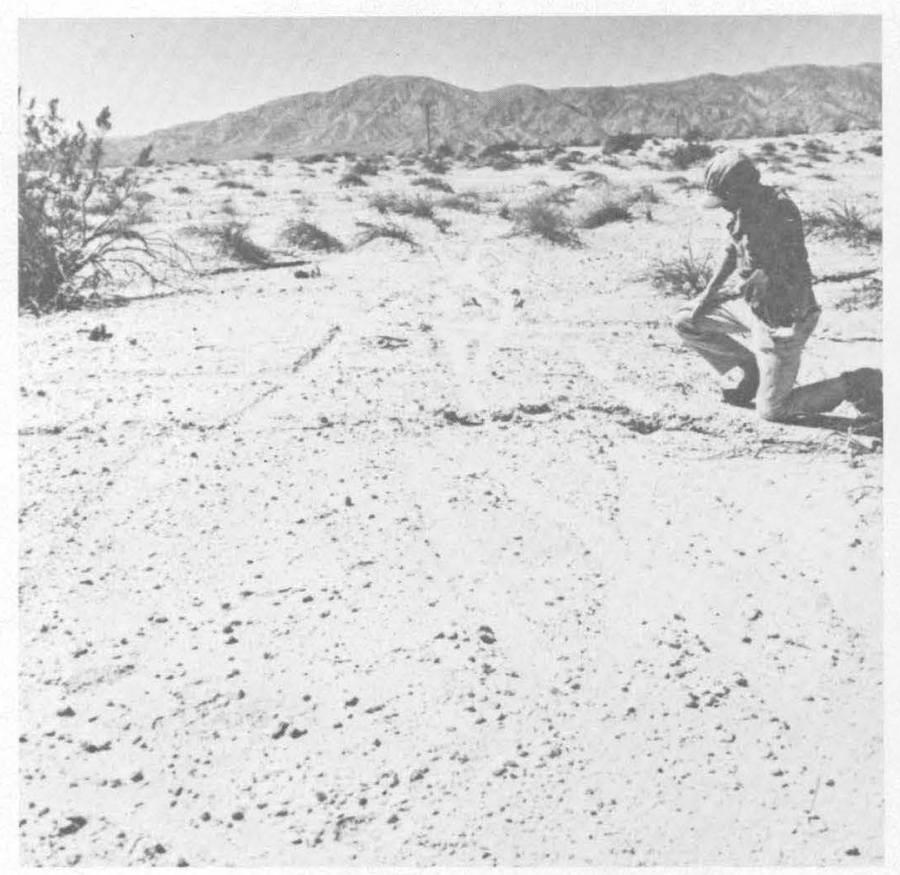

Figure 44. - Tire tracks at location 19.4 offset $240 \mathrm{~mm}$ horizontally. March 1969. Rain, blown sand, and newer tracks had virtually obliterated these tracks 1 year after this photograph was taken, preventing further measurement of total offset at this site. Photograph by C. R. Allen.

\section{CONTINUED DISPLACEMENT ON THE SOUTH BREAK} AREA $D$ (FIG. 39)

Probably the most unusual zone of continued displacement along the 1968 rupture is on the south break near location 31. (See fig. 49.) Large displacements did not occur on the south break during the earthquake, nor did it show any signs of continued movement in the months immediately following. Yet significant creep commenced between January and September of 1969 and was still apparent in a few places in December 1970. This creep reopened 1968 fractures, created some new fractures, and reopened a relict fracture that predated the earthquake. Because no displaced tracks or other evidence of the original offset of April 1968 survived after September 1969, we have no measurements of cumulative displacement after the earthquake. Creep on the south break has added an estimated $30-60 \mathrm{~mm}$ of horizontal displacement to the maximum of $80 \mathrm{~mm}$ measured in April 1968.

Careful examination during nearly every visit to the 1968 rupture has enabled us to reconstruct the history of postearthquake creep on the south break. A detailed check of the entire south break in June 1968 revealed no indication of continued displacement. Indeed, north of location 31.1, many of the

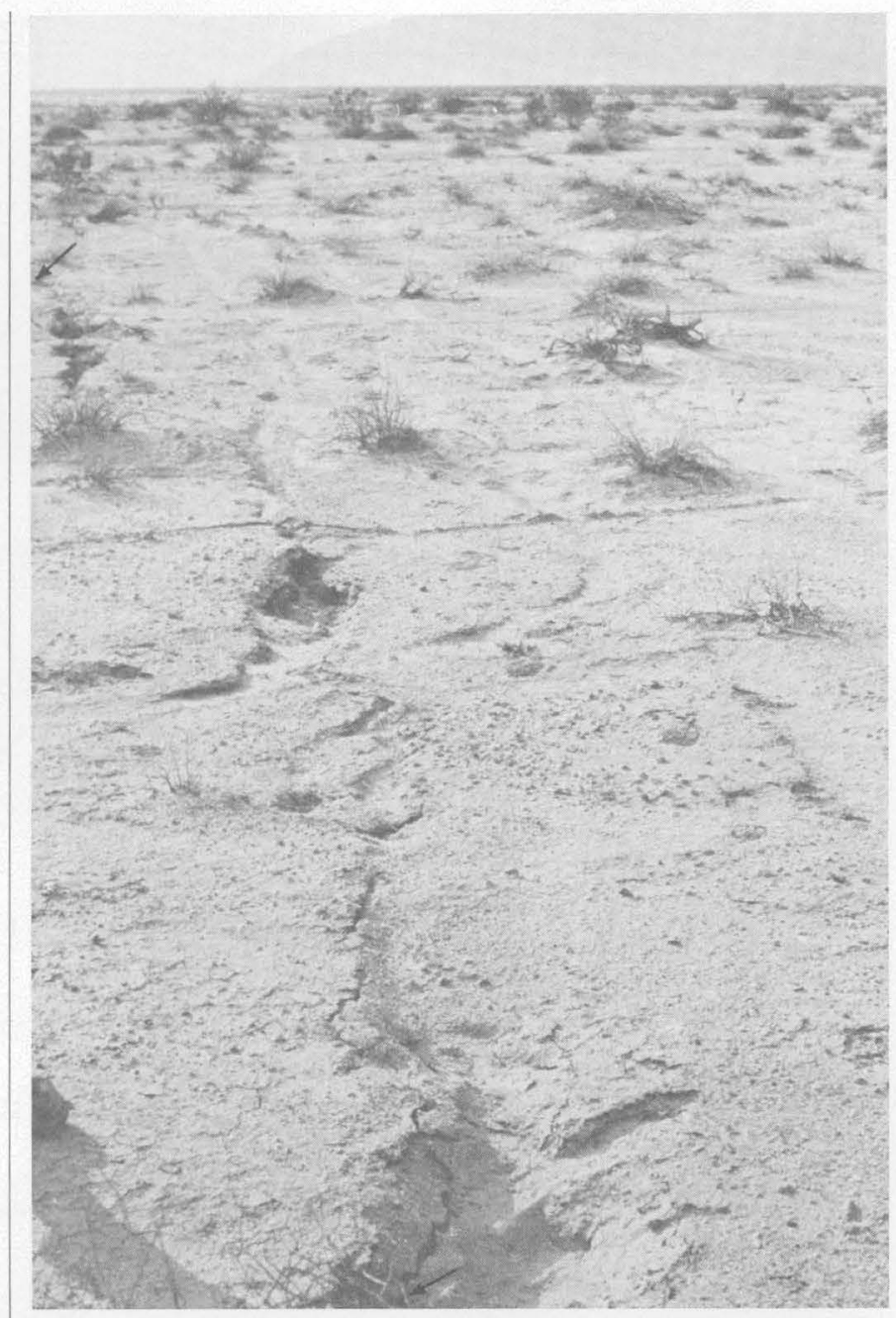

Figure 45. - New breaks with $30 \mathrm{~mm}$ of right-lateral offset. March 1969. These fractures broke the crust that was created by rains of January 1969 on blown silt and sand filling a large 1968 fracture (indicated by arrows). Location 19.4 .

fractures were no longer visible 2 months after the earthquake because they were filled by blown sand and silt, and between locations 29.4 and 25 all signs of the 1968 rupture were obliterated by drifted sand. However, a field check in October 1969 revealed renewed displacement along the 1968 fractures of the south break and creation of two new fractures diverging from it at location 31.4 (fig. 49 , e). The rains of September 1969 had caused large runoff and initiated major collapse of many of the newly opened and reopened fractures, including the two new fractures. Both these new fractures increased in length by more than $30 \mathrm{~m}$ after the rains of September, as shown by sharp-edged postrain extensions of each across channels that carried water in September (fig. 50). Triangular arrays of stakes placed across 


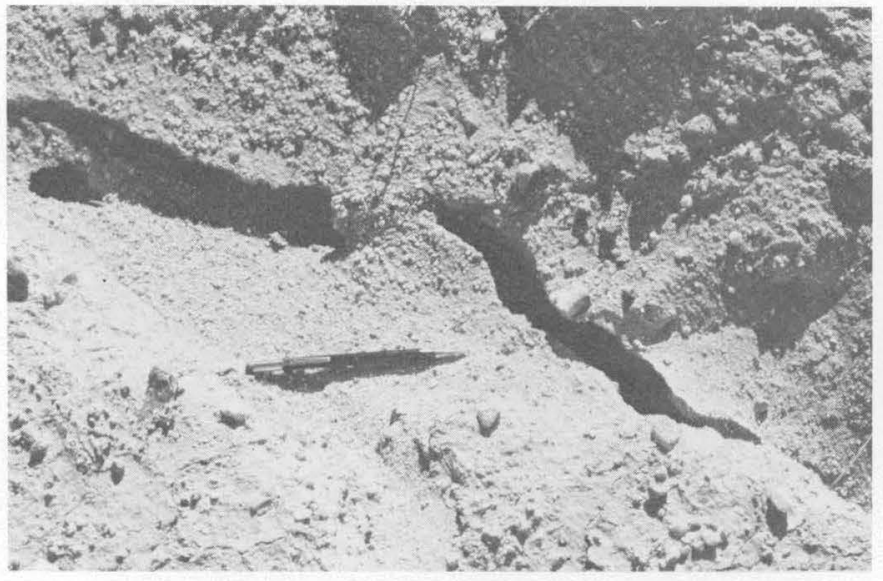

Figure 46. - Detail of the fracture shown in figure 45. March 1969. The pencil lies parallel to the direction of tectonic movement; displacement here is $30 \mathrm{~mm}$.

these two new fractures in October 1969 showed that the southernmost of the two fractures had opened another $10 \mathrm{~mm}$ by December 1970. In contrast, other nearby new and reopened fractures apparently ceased further significant movement late in 1969. Two additional new fractures (fig. 49, g) developed in this area after early November 1969 and continued to open and extend between January and December 1970. Movement also recurred on a prominent preearthquake fissure (fig. 49, c, described next) after January 1970 and continued in the period August-December 1970. Total opening on these latest fractures did not exceed $15 \mathrm{~mm}$, and movement apparently ceased by December 1970 .

The period during which the ruptures of April 1968 first reopened and the new fractures began to form on the south break is fairly well defined. These fractures were definitely not open at the time of the field check in June 1968. The field check of March 1969 did not include parts of the south break that displayed renewed displacement the following October. However, large-scale aerial photos taken in April 1969 along the entire rupture show only one section of minor collapse on the south break, about $3 \mathrm{~m}$ long at location 30.4 , in the center of the area of later extensive collapse. (This same series of photographs clearly shows all the collapse fissures elsewhere along the 1968 break.) Lack of collapse in April 1969 means that the fractures had not reopened at the time of the previous runoff, very probably January 1969 but certainly July 1968. Thus, renewed displacement on this part of the south break definitely began between July 1968 and September 1969 and probably began between January and September 1969. The fact that the two new fractures (fig. 49, e) continued to extend after the rains of September 1969 suggests that the tectonic activity had begun shortly before those rains.

In addition to renewed displacement after perhaps 1 year or more of stability subsequent to the earthquake, the south end of the 1968 rupture is notable for its association with preearthquake fractures. A fresh collapse fissure and at least three prominent groups of relict collapse fissures nearby all predate the 1968 earthquake. Of these fissures, shown in figure $49, a-d, a$ and $d$ developed before 1953, b developed after 1953-56 but ceased movement several years before 1968, and c evidently opened or reopened less than 1 year and possibly just a few months before the earthquake (Clark, "Collapse Fissures Along the Coyote Creek Fault," this volume). All these fissures almost surely developed from tectonic fractures. In addition, several small (less than several meters long) alined sinks that predated the earthquake were present on the south break at locations 28.4 and 32.0 .

The relict fissures, creep behavior, and small total displacement since 1968 suggest that the region at the south end of the 1968 rupture is one of more frequent tectonic activity than the areas to the north along the 1968 rupture. The older fissures at a and $d$ appear to represent tectonic activity perhaps 15-20 years before the earthquake, whereas $b$ seems to have developed, or at least collapsed, after 1953 but well before 1968. Fissure c strongly indicates tectonic movement in this area shortly before the earthquake. The reactivation of the 1968 break and creation of new fissures resulted from creep that has continued for more than 2 years after the earthquake.

\section{PATTERNS OF CREEP ALONG THE 1968 RUPTURE}

Ready explanations are not apparent for the pattern of postearthquake creep indicated for the 1968 rupture. Creep did not simply migrate from one end of the rupture to the other, as at Parkfield in 1966-67 (Smith and Wyss, 1968). It started first on the southeast end of the central break and on the outlying fractures along Old Kane Spring Road but then shifted by unknown steps to the northwestern part of the central break. The net result of creep on the central break has been to increase the amount of displacement closer to that of the north break than it was immediately after the earthquake. No clear relation exists between creep and the pattern of larger local aftershocks. Figure 51 shows the locations of aftershocks of $M \geqslant 3.0$ through 1970. (See also table 11.) Although the delayed onset of creep along the south break might be connected with the 


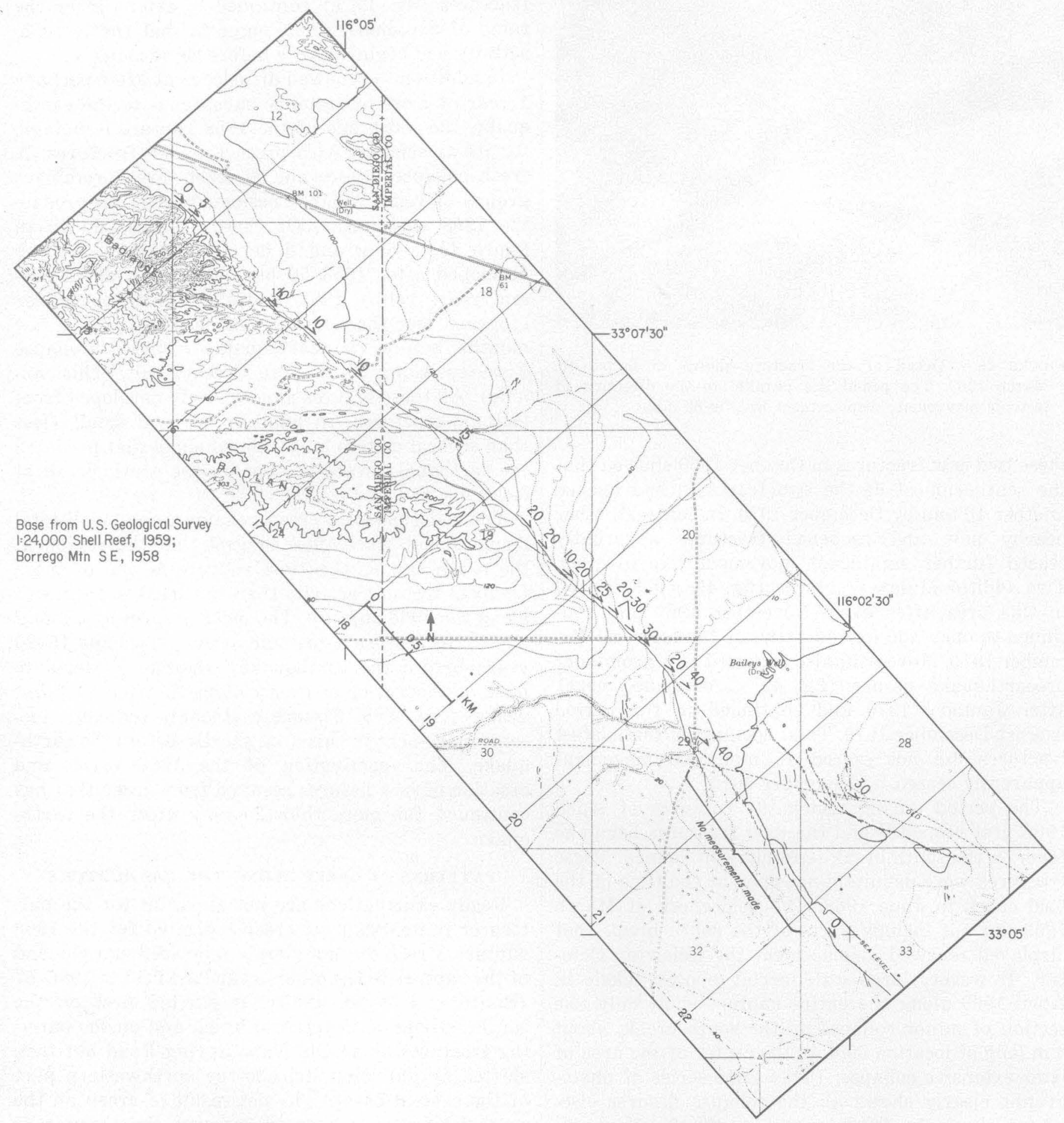

Figure 47. - Horizontal displacement, in millimeters, measured March 21-22, 1969, on either (1) post-January 1969 fractures in crusted deposits filling the 1968 fracture, (2) new channel deposits laid across the 1968 fractures in January 1969, or (3) new post-January 1969 fractures in sediments that were not broken in 1968. Direction of offset shown by arrows. Base and kilometer grid from plate 1 .

enlarging "doughnut" of aftershocks noted by Allen | creep along the central break is not obviously related and Nordquist (this volume), the persistence of to any pattern of recorded local aftershocks. 


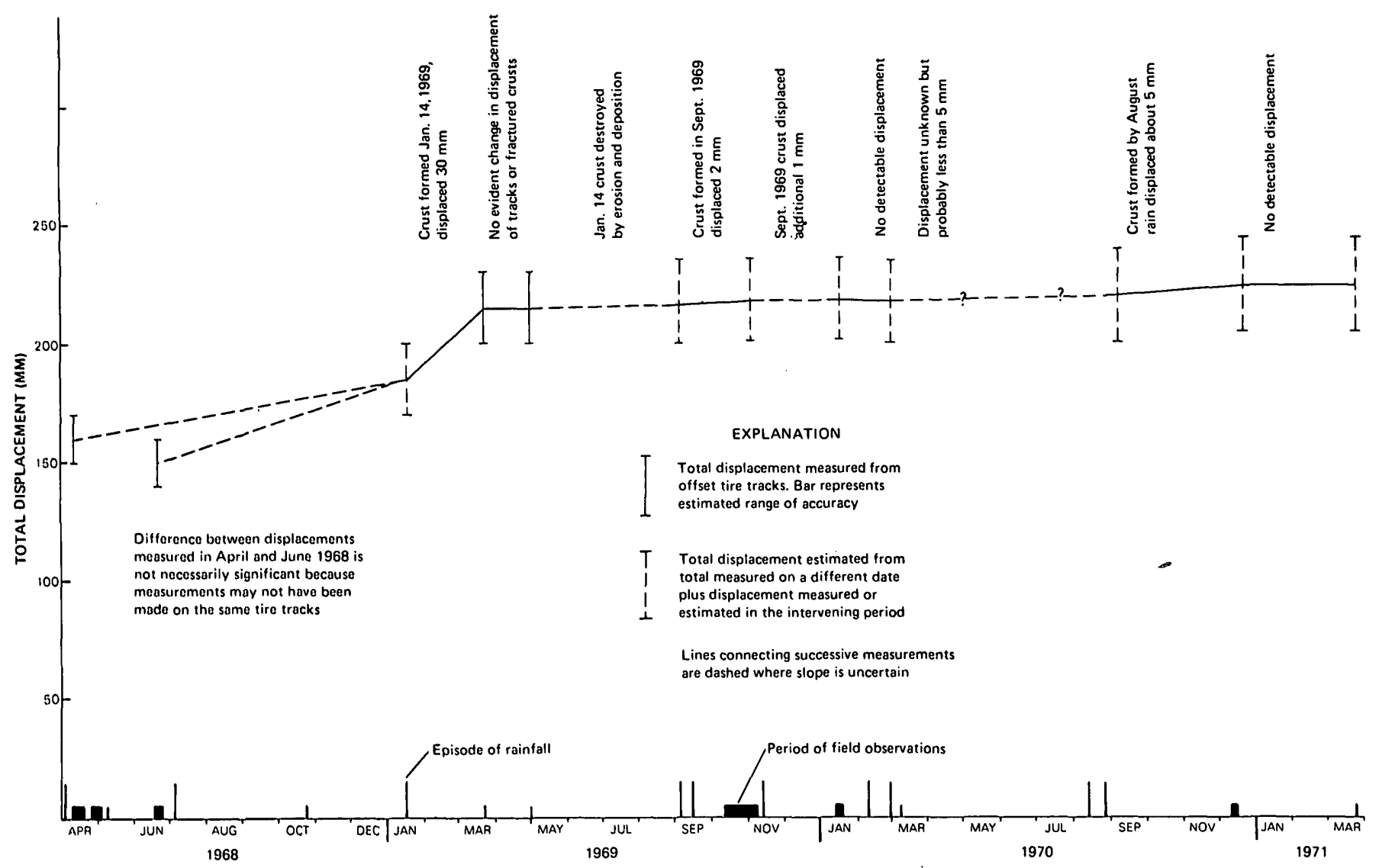

Figure 48. - Displacement from April 9, 1968, to March 1971 near location 19.4. Offsets were measured on tire tracks and rain-consolidated crusts along a segment of the central break about $100 \mathrm{~m}$ long.

TABLE 11. - Earthquakes of $M \geq 3$ in the area from $32^{\circ} 45^{\prime}$ to $38^{\circ} 15^{\prime} N$. and from $115^{\circ} 45^{\prime}$ to $116^{\circ} 15^{\prime} W$. from April 29, 1969 , through 1970

[Epicenters are plotted in fig. 51 . See Allen and Nordquist, table 2, this volume, for an explanation of symbols. From preliminary lists of shocks in Bouthern California during 1969 and 1970 supplied by C. R. Allen, California Inst. Technology]

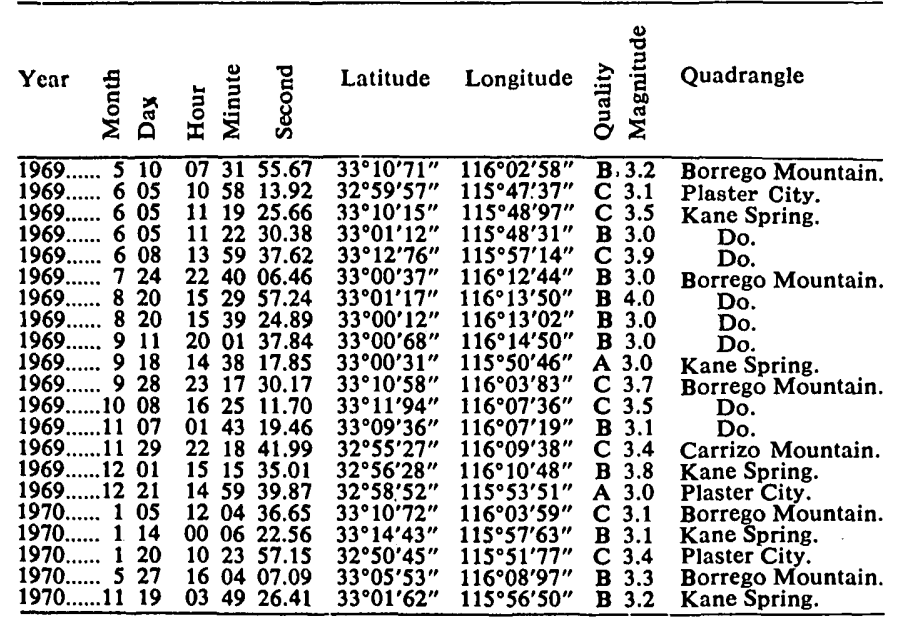

The observed creep conceivably is related to aftershocks of $\mathrm{M}<3$, for which there is no reliable cover- age during the period considered here, or it may reflect a delayed response to the initial or subsequent displacement in underlying rocks, as discussed in this volume by Burford and by Allen, Wyss, Brune, Grantz, and Wallace.

\section{THE UNUSUAL STATUS OF THE SOUTH BREAK}

The history of seismicity and displacement along the south break has only a few characteristics in common with that of the central and north breaks, in addition to the rupturing of all three in 1968. A major similarity of the south and central breaks is that both experienced postearthquake creep, although at different times and in different amounts. The major common characteristic of the south and north breaks probably is their proximity to epicenters of large earthquakes. A magnitude 6.5 earthquake occurred on or near the southeast end of.. the south break in October 1942. (See Allen and Nordquist; Clark and others, this volume.) No surface investigation has been reported for the epicentral area of the 1942 earthquake, but if the location is correct, 


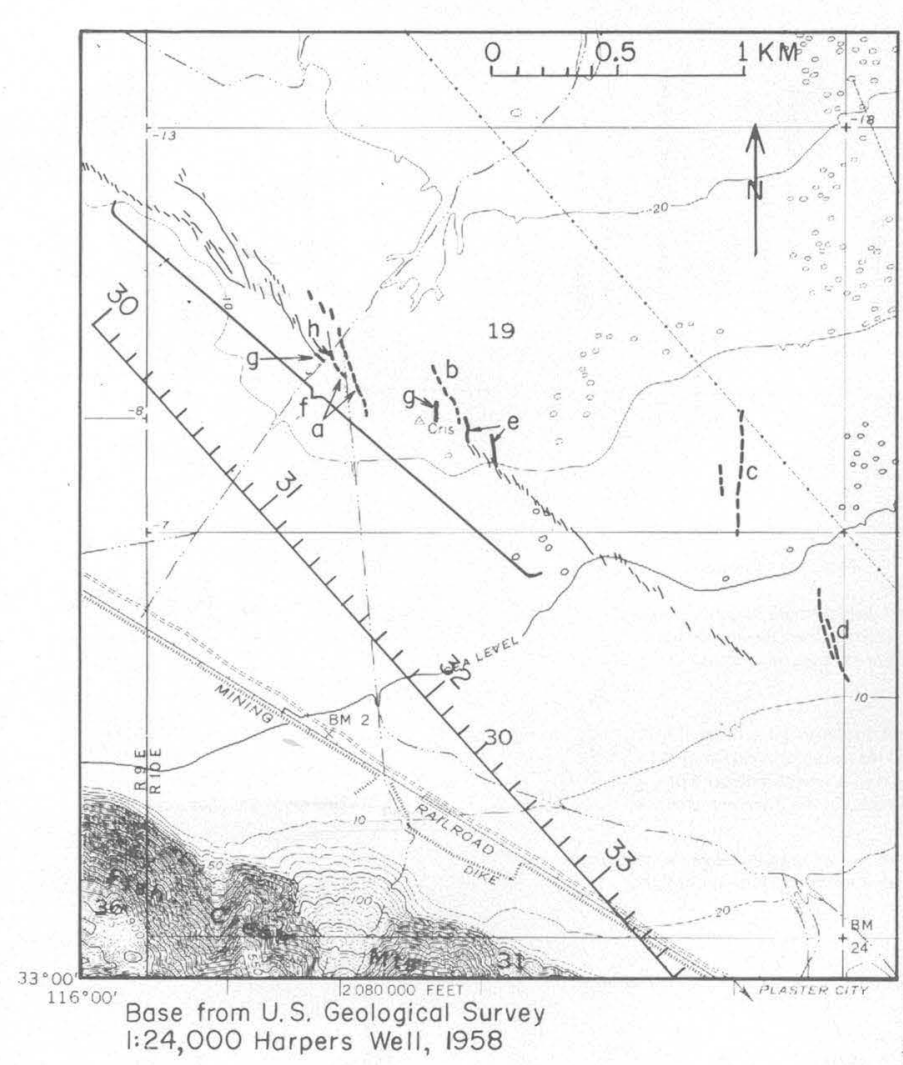

Figure 49. - Special features of the south break (light lines). a-d (dashed lines), relict collapse fissures; e, fractures that probably formed after January 1969 and extended after September 1969; f, area of renewed movement after January 1969 and collapse of new and reopened fractures after the rains of September 1969 and later; g, fractures that developed after November 1969 (parts of relict fissure at $c$ also reopened after January 1970); h, old collapse features exposed in gully. Base and kilometer grid from plate 1.

surface ruptures may have formed at that time near or on the south break and, perhaps, on the Superstition Hills or other nearby faults. Indeed, the older collapse fissures evident near the south break (fig. 49 , d) may be remnants of breaks formed during the large earthquake of 1942 . In strong contrast to the south break, however, the north break displays no comparable history of displacement immediately before the 1968 earthquake and, so far, no significant postearthquake creep. Lack of evidence of preearthquake movement on the north break could be simply the result of poor preservation of the evidence. Nevertheless, lack of postearthquake creep is an established and important difference, particularly if it is a typical difference after other earthquakes.

Contrasts in displacement history and geometry between the south break and the central and north

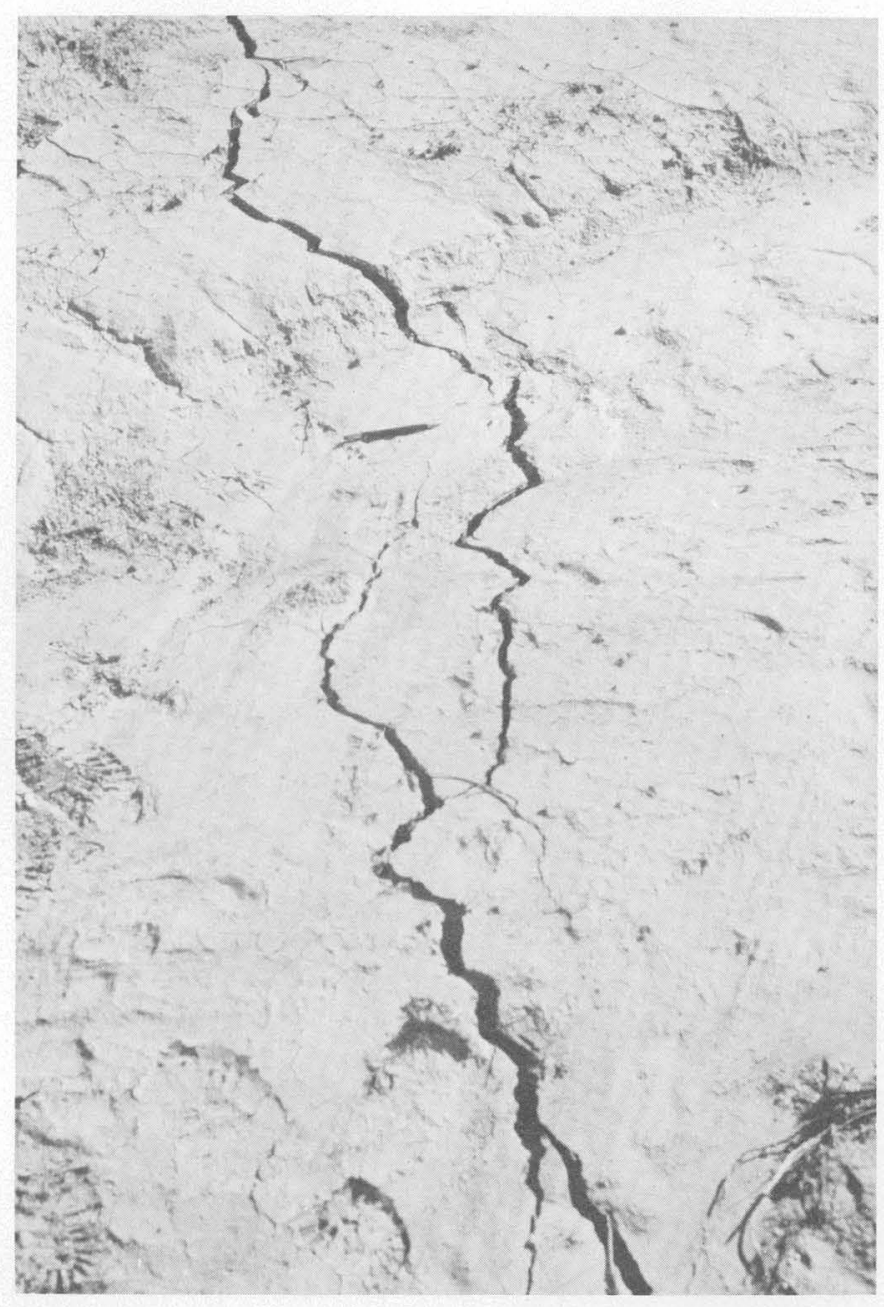

FIGURE 50. - October 1969. Sharp-edged fracture (fig. 49, e) that formed after the channel that it crosses carried water in September 1969. Pencil is parallel to the direction of net movement.

breaks seem to be more important than similarities. In addition to the completely different history of creep, the south break showed much smaller displacement in 1968 and was shorter. It trends roughly N. $50^{\circ} \mathrm{W}$., whereas the central and north breaks each trend about N. $40^{\circ} \mathrm{W}$. The south break connects to the central break at an anomalous north-south segment of the 1968 rupture, whereas the central and north breaks join by an en echelon overlap that repeats the pattern shown throughout the 1968 rupture for smaller fractures. These differences suggest that the south break is fundamentally distinct from the central and north breaks and that it will continue to behave differently from the remainder of the 1968 rupture.

The south break is possibly more a transitional element between the Coyote Creek fault and the 


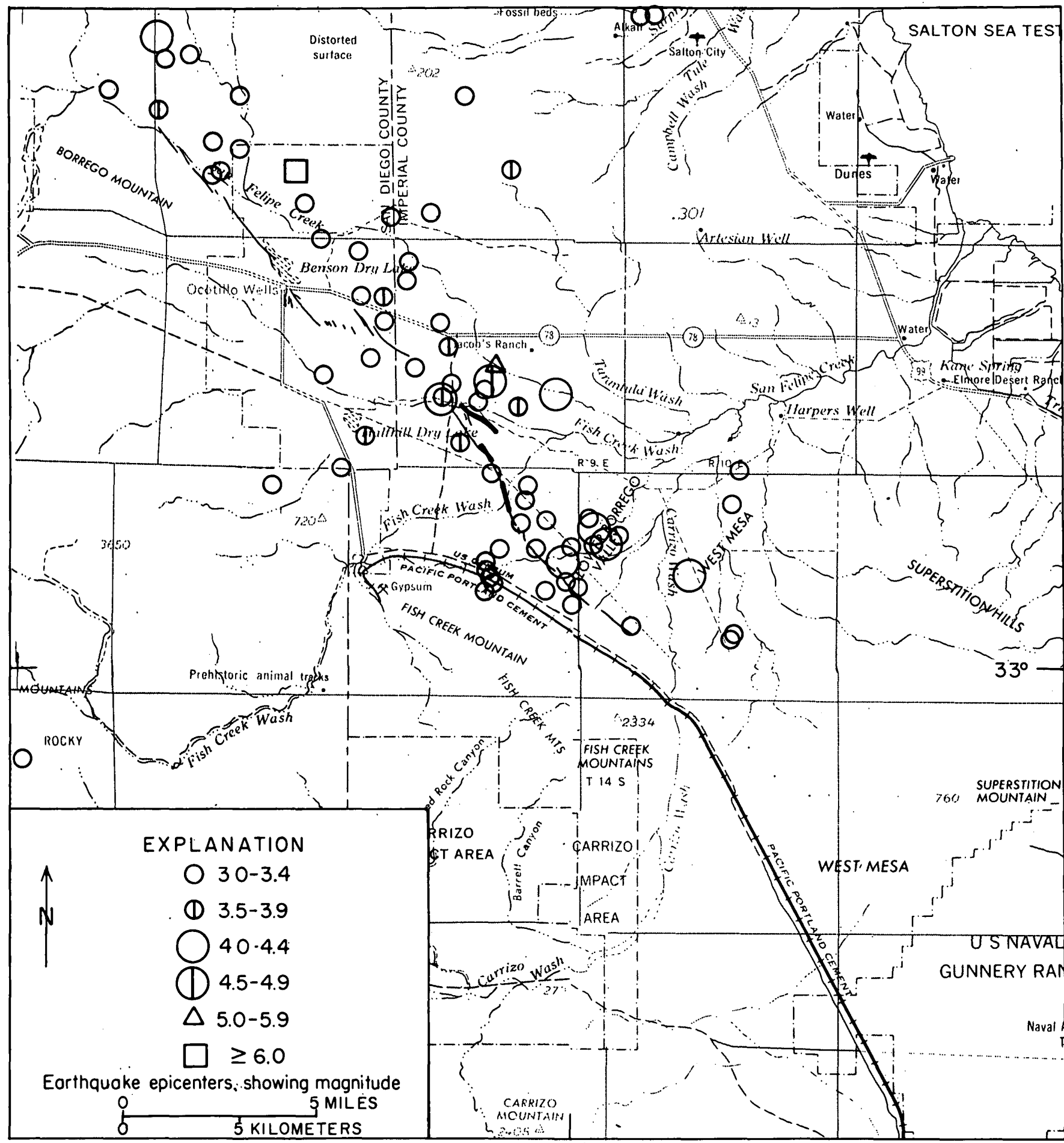

Bose from U.S. Geological Survey Solton Seo 1959-67, Sonto Ano, 1959, Son Diego, 1958-64, and El Centro, 1958

\section{April 9 - June 30, 1968}

FIGURE 51. - Location of aftershocks and other earthquakes of $M \geq 3$ and areas of creep (heavy line) during three periods after April 8, 1968. Locations and dates of seismic events from table 11 and from figure 9. Locations and dates of creep from figure 39 and table 10 . Light line shows 1968 surface rupture. 


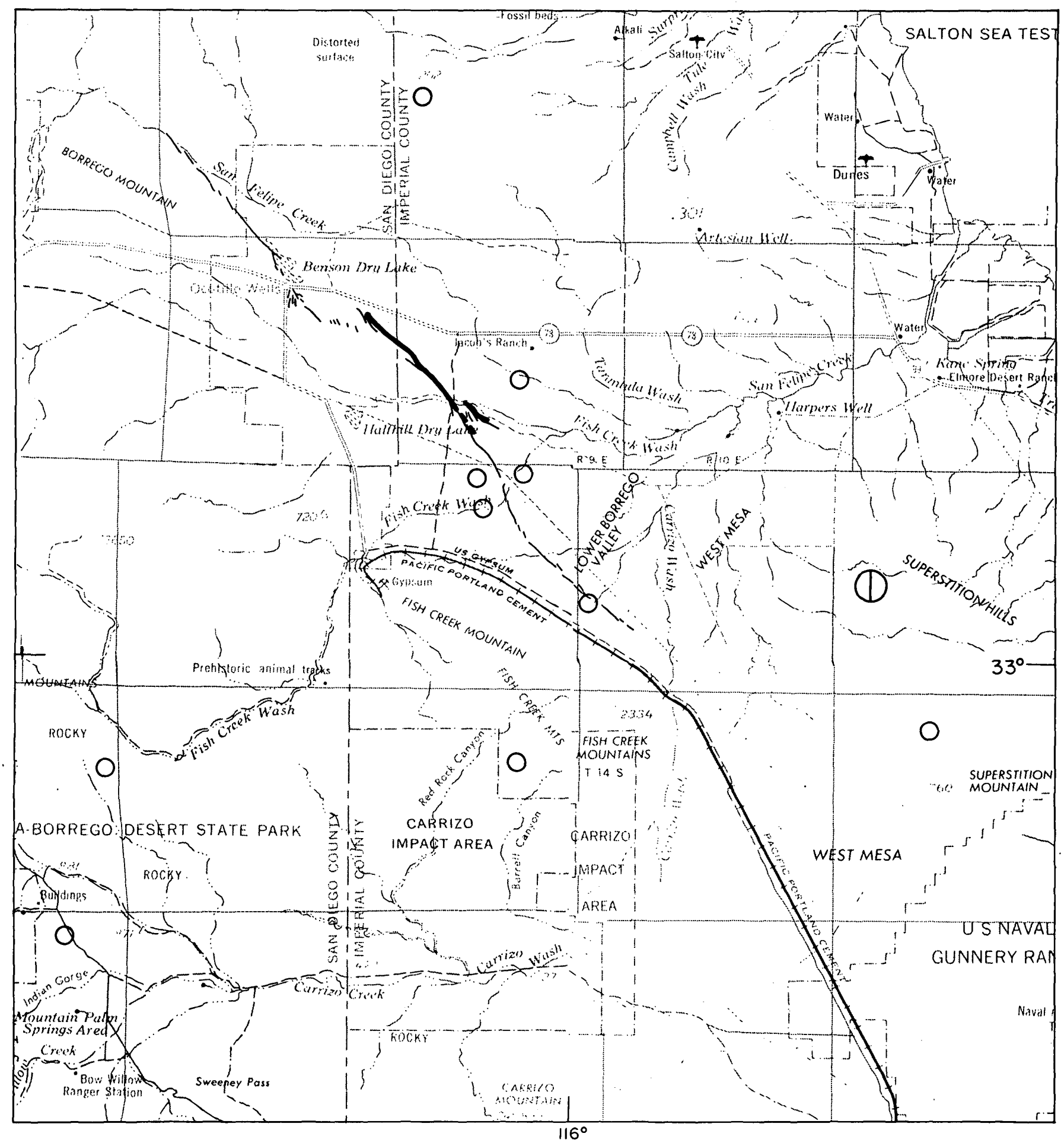

July I-December 3I, 1968

Figuké 51. - Continued. 


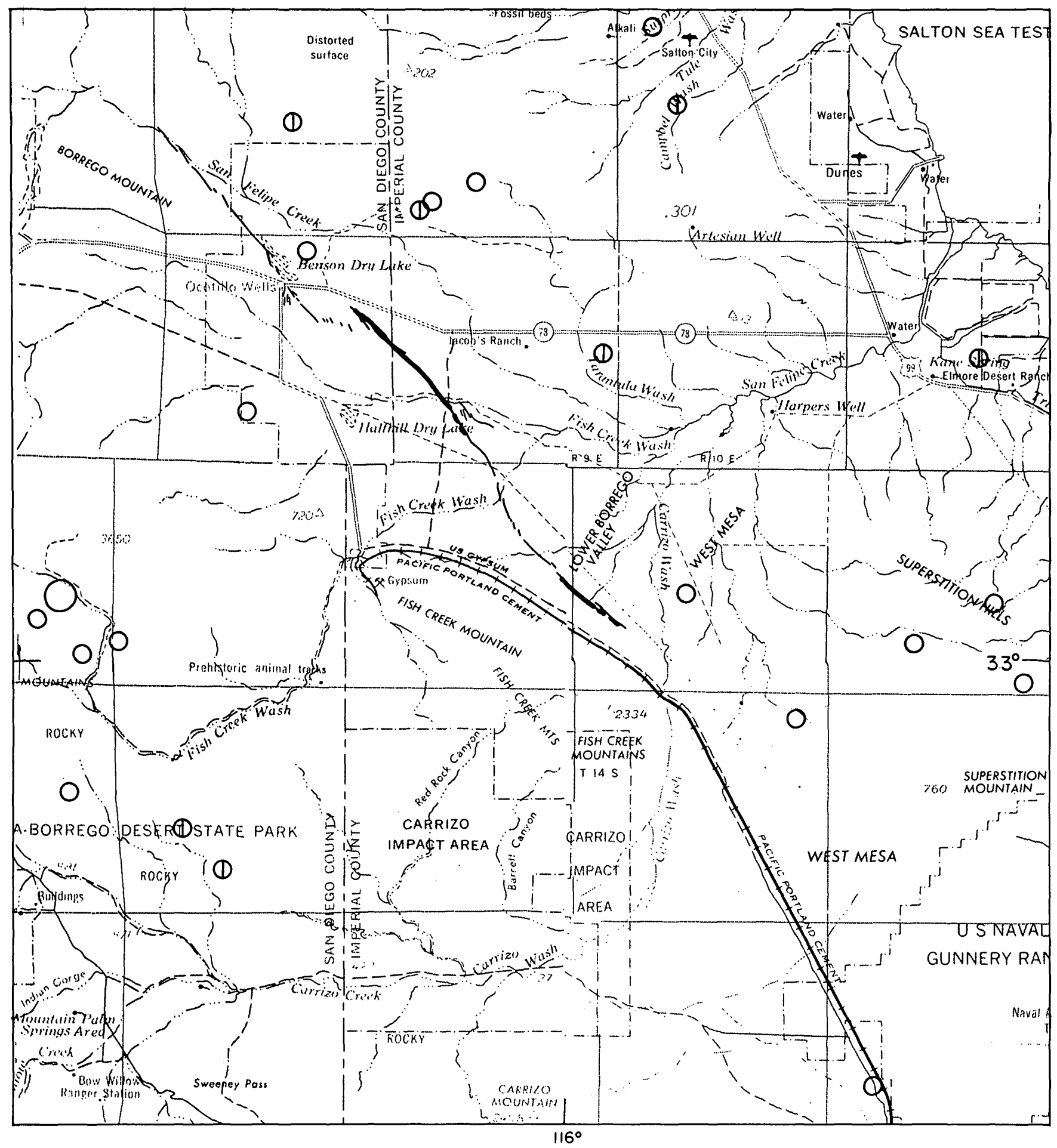

January I, 1969 through 1970

Figure 51. - Continued. 
Superstition Hills and Imperial faults to the southeast than it is an integral part of the Coyote Creek fault. Its history of displacement and seismicity has intriguing similarities to that of the Superstition Hills and the Imperial faults.

Except for the lack of comparably large historic earthquakes near it, the Superstition Hills fault has several characteristics in common with the south break. Creep since the 1968 earthquake and the inferred creep during the preceding 20 years or so along the south break resemble the intermittent small displacements recorded along the Superstition Hills fault that are evidently related to or triggered by local or distant earthquakes. (See Allen and others, 1965 ; Allen and others, this volume.) Since 1934 , the region common to these two faults has also experienced several earthquakes of magnitude 5-5.6 (see Sharp, "Tectonic Setting of the Salton Trough," this volume, fig. 4), at least one of which was apparently associated with small displacements on the Superstition Hills fault. Although neither the south break nor the Superstition Hills fault appeared to move during the latest nearby moderate earthquake $(M=5.3$ in Superstition Hills in September 1971; see Allen and others, this volume), it seems reasonable to suppose that the south break may have moved during one or more of the earlier moderate earthquakes, some of which were closer than that of 1971 .

Historic behavior of the Imperial fault perhaps comes closer to that observed and deduced for the south break. In 1940, there was a swarm of major earthquakes along the Imperial fault (Ulrich, 1941; Trifunac and Brune, 1970) and associated large displacements, followed by later displacements, including delayed creep, that were apparently associated with small or distant earthquakes (Brune and Allen, 1967; Allen and others, this volume). Although both faults combine major earthquakes with displacement at other times, their behavior certainly is not identical. The earthquake of September 1971, for example, was roughly equidistant between the faults, but displacement then occurred only on the Imperial fault.

\section{REFERENCES CITED}

Allen, C. R., St. Amand, Pierre, Richter, C. F., and Nordquist, J. M., 1965, Relationship between seismicity and geologic structure in the southern California region: Seismol. Soc. America Bull., v. 55, no. 4, p. 753-797.

Allen, C. R., Grantz, Arthur, Brune, J. N., Clark, M. M., Sharp, R. V., Theodore, T. G., Wolfe, E. W., and Wyss, Max, 1968, The Borrego Mountain California earthquake of 9 April 1968 - a preliminary report: Seismol. Soc. America Bull., v. 58, no. 3, p. 1183-1186.

Brown, R. D., Jr., Vedder, J. G., Wallace, R. E., Roth, E. R., Yerkes, R. F., Castle, R. O., Waananen, A. O., Page, R. W., and Eaton, J. P., 1967, The Parkfield-Cholame
California, earthquakes of June-August 1966 - surface geologic effects, water resources aspects, and preliminary seismic data: U.S. Geol. Survey Prof. Paper 579, 66 p.

Brown, R. D., Jr., and Wolfe, E. W., 1970, Map showing recently active breaks along the San Andreas fault between Point Delgada and Bolinas Bay, California: U.S. Geol. Survey open-file map.

Brune, J. R., and Allen, C. R., 1967, A low stress-drop, lowmagnitude earthquake with surface faulting - The Imperial California earthquake of March 4, 1966: Seismol. Soc. America Bull., v. 57, no. 3, p. 501-514.

Chinnery, M. A., 1966, Secondary faulting; I and II: Canadian Jour. Earth Sciences, v. 3, p. 163-190.

Clark, M. M., 1971, Map showing recently active breaks along the Garlock and associated faults, California: U.S. Geol. Survey open-file map.

Dibblee, T. W., Jr., 1954, Geology of the Imperial Valley region, in Jahns, R. H., ed., Geology of southern California; chap. 2, Geology of the natural provinces: California Div. Mines and Geol. Bull. 170, p. 21-28.

Environmental Data Service, 1968-71, Climatological data, California: Natl. Oceanog. and Atmospheric Adm., v. $72-75$.

Hamilton, R. M., 1970, Time-term analysis of explosion data from the vicinity of the Borrego Mountain, California, earthquake of 9 April 1968: Seismol. Soc. America Bull., v. 60 , no. 2 , p. $367-381$.

Hely, A. G., and Peck, E. L., 1964, Precipitation, runoff, and water loss in the lower Colorado River-Salton Sea area: U.S. Geol. Survey Prof. Paper 486-B, 16 p.

Kearney, T. H., Peebles, R. H., and others, 1960, Arizona flora: Berkeley, Univ. California Press, 1085 p.

Ross, D. C., 1969, Map showing recently active breaks along the San Andreas fault between Tejon Pass and Cajon Pass, southern California: U.S. Geol. Survey Misc. Geol. Inv. Map I-553.

Sharp, R. V., 1970, Map showing recently active breaks along the San Jacinto fault zone between San Bernardino and Borrego Valley, California: U.S. Geol. Survey openfile map.

Smith, S. W., and Wyss, Max, 1968, Displacement on the San Andreas fault subsequent to the 1966 Parkfield earthquake: Seismol. Soc. America Bull., v. 58, p. 19551973.

Tchalenko, J. S., 1970, Similarities between shear zones of different magnitudes: Geol. Soc. America Bull., v. 81, p. $1625-1640$.

Tchalenko, J. S., and Ambraseys, N. N., 1970, Structural analysis of the Dasht-e Bayaz (Iran) earthquake fractures: Geol. Soc. America Bull., v. 81, p. 41-60.

Trifunac, M. O., and Brune, J. N., 1970, Complexity of energy release during the Imperial Valley, California earthquake of 1940: Seismol. Soc. America Bull., v. 60, p. 137-160.

Ulrich, F. P., 1941, The Imperial Valley earthquakes of 1940 : Seismol. Soc. America Bull., v. 31, p. 13-31.

Vedder, J. G., and Wallace, R. E., 1970, Map showing recently active breaks along the San Andreas and related faults between Cholame Valley and Tejon Pass, California: U.S. Geol. Survey Misc. Geol. Inv. Map I-574.

Youd, T. L., and Castle, R. O., 1970, Borrego Mountain earthquake of April 8, 1968: Am. Soc. Civil Engineers Proc., Jour. Soil Mechanics and Found. Div., v. 96, no. SM4, p. 1201-1219. 


\title{
DISPLACEMENTS ON THE IMPERIAL, SUPERSTITION HILLS, AND SAN ANDREAS FAULTS TRIGGERED BY THE BORREGO MOUNTAIN EARTHQUAKE ${ }^{1}$
}

\author{
By Clarence R. Alien, \\ Seismological Laboratory, California Institute of Technology, \\ Max WySS, \\ Lamont-Doherty Geological Observatory of Columbia University, \\ JAMES N. BRUNE, \\ Institute of Geophysics and Planetary Physics, University of California, San Diego, \\ AND \\ Arthur Grantz and Robert E. Wallace, \\ U.S. Geological Survey
}

\begin{abstract}
The Borrego Mountain earthquake of April 9, 1968, triggered small but consistent surface displacements on three faults far outside the source area and zone of aftershock activity. Right-lateral displacement of $1-2 \frac{1}{2} \mathrm{~cm}$ occurred along 22,23 , and $30 \mathrm{~km}$ of the Imperial, Superstition Hills, and San Andreas (Banning-Mission Creek) faults, respectively, at distances of 70,45 , and $50 \mathrm{~km}$ from the epicenter. Although these displacements were not noticed until 4 days after the earthquake, their association with the earthquake is suggested by the freshness of the resultant en echelon cracks at that time and by the absence of creep along most of these faults during the year before or the year after the event. Dynamic strain associated with the shaking is a more likely cause of the distant displacements than is the static strain associated with the faulting at Borrego Mountain because (1) the dynamic strain was much larger and (2) the static strain at the San Andreas fault was in the wrong sense for the observed displacement. The principal surface displacements on the Imperial fault took place within 4 days of the earthquake and may have occurred simultaneously with the passage of the seismic waves, but the possibility of delayed propagation to the surface is indicated by a 1971 event on the Imperial fault in which the surface displacement followed the triggering earthquake by 3-6 days. All three of the distant faults are "active" in that they show evidence of repeated Quaternary movement, and surface displacements occurred only along those segments where the fault trace is well delineated in surface exposures, at least in uncultivated areas. This is the first documented example of fault displacement triggered by seismic shaking far from the source area, although such displacement has probably gone undetected many previous times here and in similar tectonic environmments. This phenomenon forces us to be much more conservative in estimating the probabilities of damage from surface displacements along active faults in seismic regions.
\end{abstract}

${ }^{1}$ Contribution 1833, California Institute of Technology, Division of Geological and Planetary Sciences, Pasadena, Calif.

\section{INTRODUCTION}

The Borrego Mountain earthquake of April 9, 1968 (magnitude 6.4) was associated not only with a conspicuous surface break in its source region along the Coyote Creek fault (Clark, "Surface Rupture Along the Coyote Creek Fault," this volume), but also with displacements far outside the epicentral region along three major faults in the Imperial Valley region to the east and southeast of the epicenter (fig. 52). The Imperial, Superstition Hills, and San Andreas ${ }^{2}$ faults broke along segments at least 22,23 , and $30 \mathrm{~km}$ long, respectively, at distances of 70,45 , and $50 \mathrm{~km}$ from the epicenter. Remeasurements of several small-scale geodetic networks that had been established before the earthquake, as well as observations of en echelon cracking, showed that right-lateral displacements of $1-21 / 2 \mathrm{~cm}$ had occurred on these three distant faults. This is the first documented case of an earthquake apparently causing fault displacements well outside the epicentral region. A similar phenomenon may have occurred along a segment of the Garlock fault as a result of the 1952 Kern County earthquake on the White Wolf fault (Buwalda and St. Amand, 1955, p. 53), but the Garlock fault is relatively close to the White Wolf fault and was almost within the zone of aftershock activity.

This paper presents evidence that the displacements on the Imperial, Superstition Hills, and San

2The branch of the San Andreas fault system northeast of the Salton Sea has sometimes been called the Banning-Mission Creek fault because it represents the combined Banning and Mission Creek faults southeast of their point of coalescence near Indio and because of this fault's debatable continuity with the San Andreas fault farther north. The name San Andreas is used in this report for the sake of brevity and in keeping with usage by Dibblee (1954) and Hope (1969). 


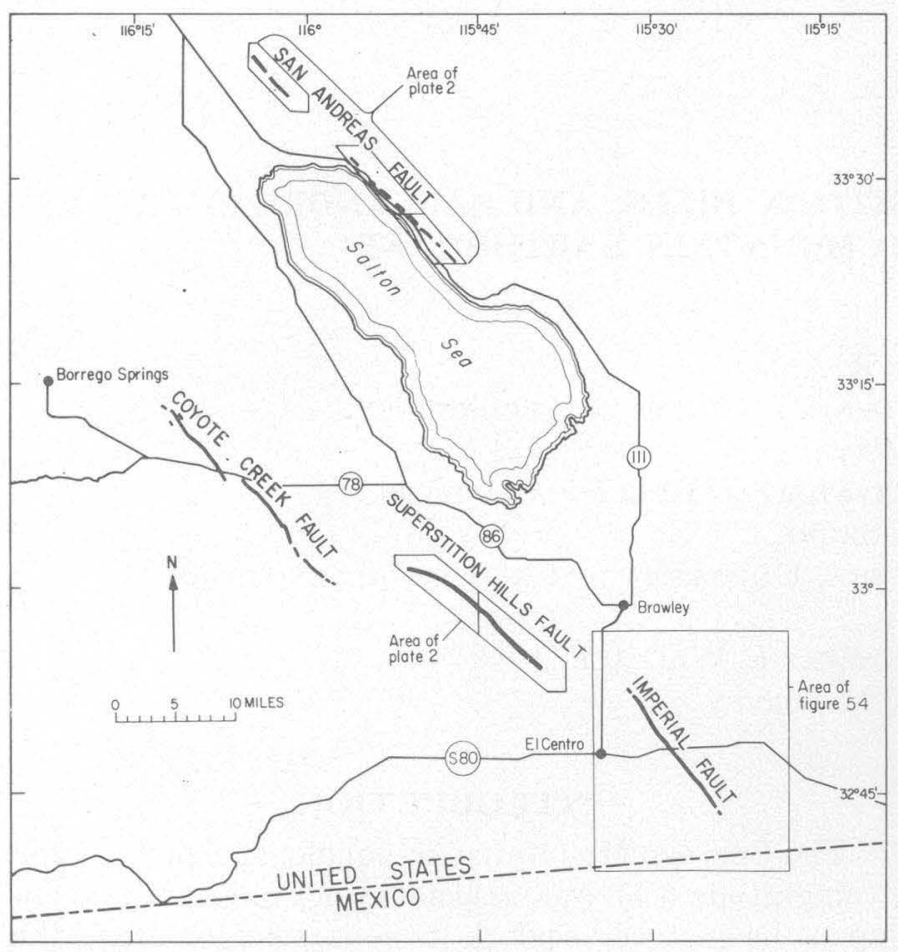

FIGURE 52. - Index map showing relation of the Coyote Creek fault (locus of the 1968 Borrego Mountain earthquake) to the three distant faults, the San Andreas, Superstition Hills, and Imperial, upon which triggered movements occurred. Heavy lines represent approximate segments which broke at that time.

Andreas faults were in fact triggered by the seismic shaking of the distant Borrego Mountain earthquake, that these displacements were not associated with normal aftershocks, and that they were not caused by the change in the regional static strain field that resulted from the fault displacements of the Borrego Mountain earthquake.

\section{ACKNOWLEDGMENTS}

Participation in this study by the California Institute of Technology was supported by National Science Foundation Grants GA-1087 and GA-12863. $\mathrm{Mr}$. James Hileman assisted in the interpretation of the data from the small geodetic networks, Dr. Walter Arabasz studied the microearthquakes that followed the main shock, and Dr. R. D. Nason first observed the cracks at Highway 80 that stimulated the subsequent investigations. The authors appreciate the critical comments of R. V. Sharp.

\section{OBSERVATIONS \\ IMPERIAL FAULT}

Although a number of auxiliary faults near Borrego Mountain were examined for possible surface displacements on the day after the April 9 earthquake, the Imperial fault, $70 \mathrm{~km}$ distant, was not visited until April 13. At that time, Wyss and R. D. Nason noticed fresh en echelon cracks along the trace of the fault at Highway $80^{3}$ (fig. 53) suggesting at least one-third centimeter of right-lateral displacement. It was this discovery that then stimulated the careful examination of other distant faults and led to the subsequent documentation of surface displacements on the Superstition Hills and San Andreas faults, as well as at other localities along the Imperial fault.

Because of the unusual fault displacement along the Imperial fault in March 1966 (Brune and Allen, 1967a) and the suspicion that creep might be occurring along this and related faults, Brune and Allen in 1966 and 1967 established a series of small geo-

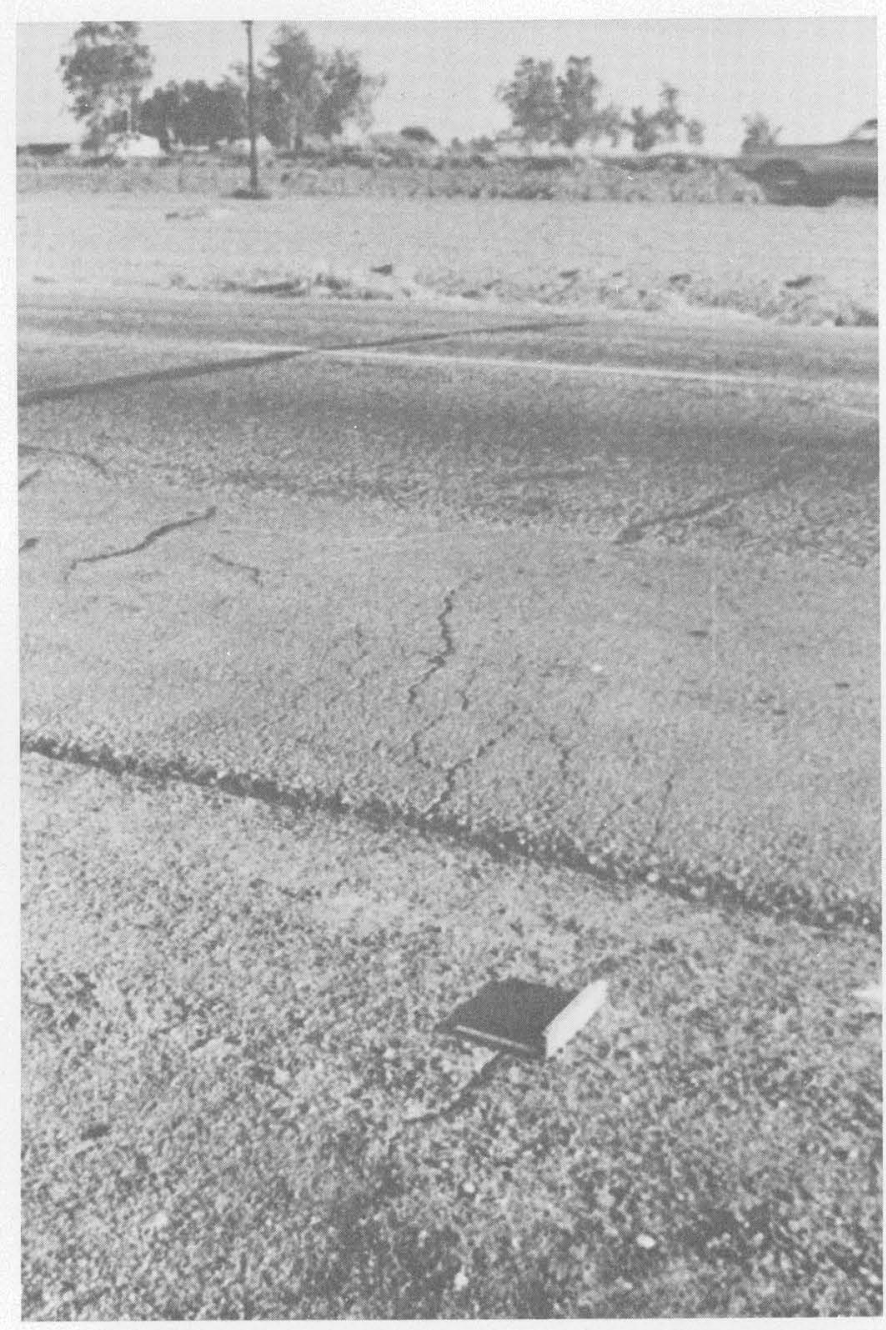

FIGURE 53. - Fresh cracks in unconsolidated material (beneath ruler) at north edge of Highway 80. Ołder tar-filled cracks in pavement resulted from earlier movements at this locality. (See Brune and Allen, 1967a.) Photograph by R. D. Nason, April 13, 1968.

3Since completion of the adjacent freeway in 1971, the former Highway 80 is now known as Evan Hewes Highway, Imperial County route 80S. 
detic networks that straddled the Imperial, Superstition Hills, and San Andreas faults. These networks, each comprising a single theodolite station and 5-10 markers within a few hundred meters on both sides of the fault trace, were patterned on the similar networks that had been established earlier across the San Andreas fault near Parkfield (Smith and Wyss, 1968). The locations of these stations are shown in figure 54 and on plate 2 , and their coordinates are given in table 12 .

TABLE 12. - Caltech fault-crossing geodetic networks in the Imperial Valley area

\begin{tabular}{|c|c|c|c|c|}
\hline Station & Lat (N.) & Long (W.) & $\begin{array}{c}\text { First } \\
\text { observation }\end{array}$ & $\begin{array}{c}\text { Number of } \\
\text { observa- } \\
\text { tions to } \\
1-1-71\end{array}$ \\
\hline All-American Imperial............... & $32^{\circ} 40.55^{\prime}$ & $115^{\circ} 21.45^{\prime}$ & $5-10-67$ & 9 \\
\hline 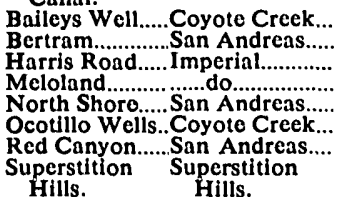 & $\begin{array}{l}33^{\circ} 06.20^{\prime} \\
33^{\circ} 24.68^{\prime} \\
32^{\circ} 53.02^{\prime} \\
32^{\circ} 48.21^{\prime} \\
33^{\circ} 31.71^{\prime} \\
33^{\circ} 09.65^{\prime} \\
33^{\circ} 37.62^{\prime} \\
32^{\circ} 55.60^{\prime}\end{array}$ & $\begin{array}{l}116^{\circ} 03.63^{\prime} \\
115^{\circ} 47.54^{\prime} \\
115^{\circ} 32.34^{\prime} \\
115^{\circ} 28.01^{\prime} \\
115^{\circ} 56.21^{\prime} \\
116^{\circ} 07.85^{\prime} \\
116^{\circ} 02.91^{\prime} \\
115^{\circ} 41.84^{\prime}\end{array}$ & $\begin{array}{l}3-31-69 \\
5-5-68 \\
1-5-68 \\
6-19-66 \\
6-20-69 \\
4-9-68 \\
5-11-67 \\
5-7-67\end{array}$ & $\begin{array}{r}3 \\
6 \\
10 \\
12 \\
3 \\
12 \\
8 \\
9\end{array}$ \\
\hline $\begin{array}{l}\text { Worthington Imperial.................... } \\
\text { Road. }\end{array}$ & $32^{\circ} 50.85^{\prime}$ & $115^{\circ} 30.69^{\prime}$ & $5-10-67$ & 11 \\
\hline
\end{tabular}

Figure $55 C$ shows that about $2 \mathrm{~cm}$ of right-lateral displacement took place along the Imperial fault at Highway 80 between January 5, 1968, and April 19, 1968 , and evidence is presented in a later section to indicate that this displacement took place at about the time of the Borrego Mountain earthquake and not as creep distributed throughout the 3-month interval. Three additional geodetic networks had been established across the Imperial fault prior to the earthquake (figs. 54, 55). The southernmost network, at the All-American Canal, showed no obvious displacement during the 3 -year period shown in figure 55. The northernmost station, at Harris Road (fig. 55A), showed a clear displacement of about $0.8 \mathrm{~cm}$ at about the time of the earthquake. However, the intervening network at Worthington Road (fig. $55 \mathrm{~B}$ ) showed a displacement of about $1.1 \mathrm{~cm}$ between May 1 and December 29, 1967 (the year before the earthquake) and no displacement at the time of the earthquake. We feel that these findings are consistent with our argument that the Borrego Mountain earthquake triggered the release of elastic strain along most of the fault; if the strain had already been relieved shortly prior to that time, as it was near Worthington Road, then no further displacement took place.

The geodetic observations were supported by field evidence of intermittent surface faulting along more than $22 \mathrm{~km}$ of the Imperial fault, extending distinctly farther both to the north and to the south than the $10-\mathrm{km}$ segment broken during the 1966 Imperial earthquake (Brune and Allen, 1967a). Clear en eche- lon cracks were observed at several localities from Harris Road on the north to Heber Road on the south (figs. 54, 56-58), although they could not be followed continuously throughout the intervening area because of extensive and continuing cultivation; cracks were not present near Worthington Road, where geodetic data indicated no movement. Fresh cracks in soil were generally observed only on the shoulders of roads between cultivated fields. En echelon cracks showed up particularly well in many asphalt roads that were crossed by the fault (fig. 56 ), although in some places it was difficult to distinguish cracks still existing from the 1966 earthquake from further cracking caused by the 1968 event (fig. 57). The absence of obvious cracking at some localities suggests that not everywhere did the fracture reach the surface as a discrete plane, which is not surprising in view of the small displacement and the known discontinuous nature of the much larger 1940 displacement in some localities (fig. 54). It is significant however, that wherever fresh cracks were observed, they followed precisely the trace of the 1940 break, which in some areas is well documented to within $1 \mathrm{~m}$ (J. P. Buwalda, unpub. data) . Although the full extent of the 1968 trace is not known because of agricultural developments at the north end and sand dunes at the south end, careful examination revealed no fresh cracks where the Imperial fault crosses Keystone Road on the north and Highway 98 on the south (fig. 54), thus giving a total length of between 22 and $30 \mathrm{~km}$.

For more than 3 years after the Borrego Mountain earthquake, no further slip on the Imperial fault at Highway 80 was observed visually or indicated by surveying records. On September 30, 1971, however, a further displacement was observed after an earthquake of magnitude 5.3 in the Superstition Hills, $37 \mathrm{~km}$ northwest of the Highway 80 locality; this earthquake represents the heaviest shaking the region had experienced since the Borrego Mountain event of 1968. Six days after the shock, right-lateral displacements of about $11 / 2 \mathrm{~cm}$ were clearly visible in the pavement of Highway 80, and a resurvey of the geodetic network on October 13 showed a displacement at this time of $1.4 \mathrm{~cm}$ (calculated on the same basis as that used for the earlier measurements shown in fig. 55C). As discussed in a later section, however, there is some evidence that the observed displacement did not take place exactly at the time of the earthquake, but between 3 and 6 days later.

The Imperial fault was first noticed and named at the time of the $1940 \mathrm{El}$ Centro earthquake, but a history of repeated Quaternary displacements along the fault is indicated by (1) a conspicuous scarp as 


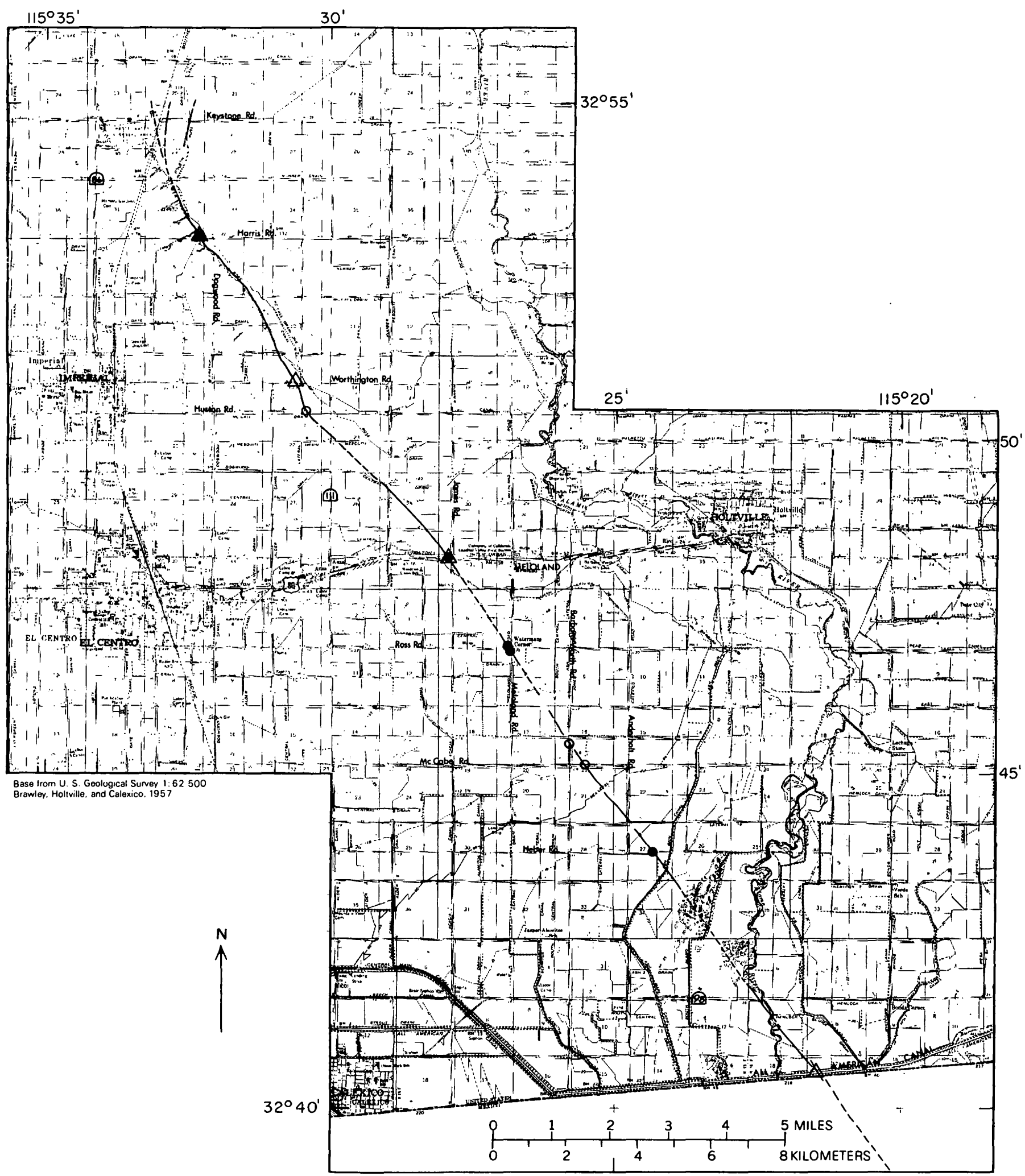

FigURE 54. - Map of Imperial fault trace based primarily on aerial photographs taken shortly after the 1940 El Centro earthquake and on the unpublished 1940 field notes of J. P. Buwalda. Triangles show locations of Caltech fault-crossing geodetic networks that were surveyed before and after the Borrego Mountain earthquake of April 9, 1968. Solid circles show localities where fresh cracks were observed in loose soil on April 19-28, 1968, presumably resulting from triggered fault displacement on April 9. Open circles are localities where freshness or significance of cracks was questionable. Trace is dashed where projected across areas where surficial evidence of faulting (scarps, ground-water barriers, or lineaments on aerial photographs) is indistinct or absent. See figure 52 for location. 


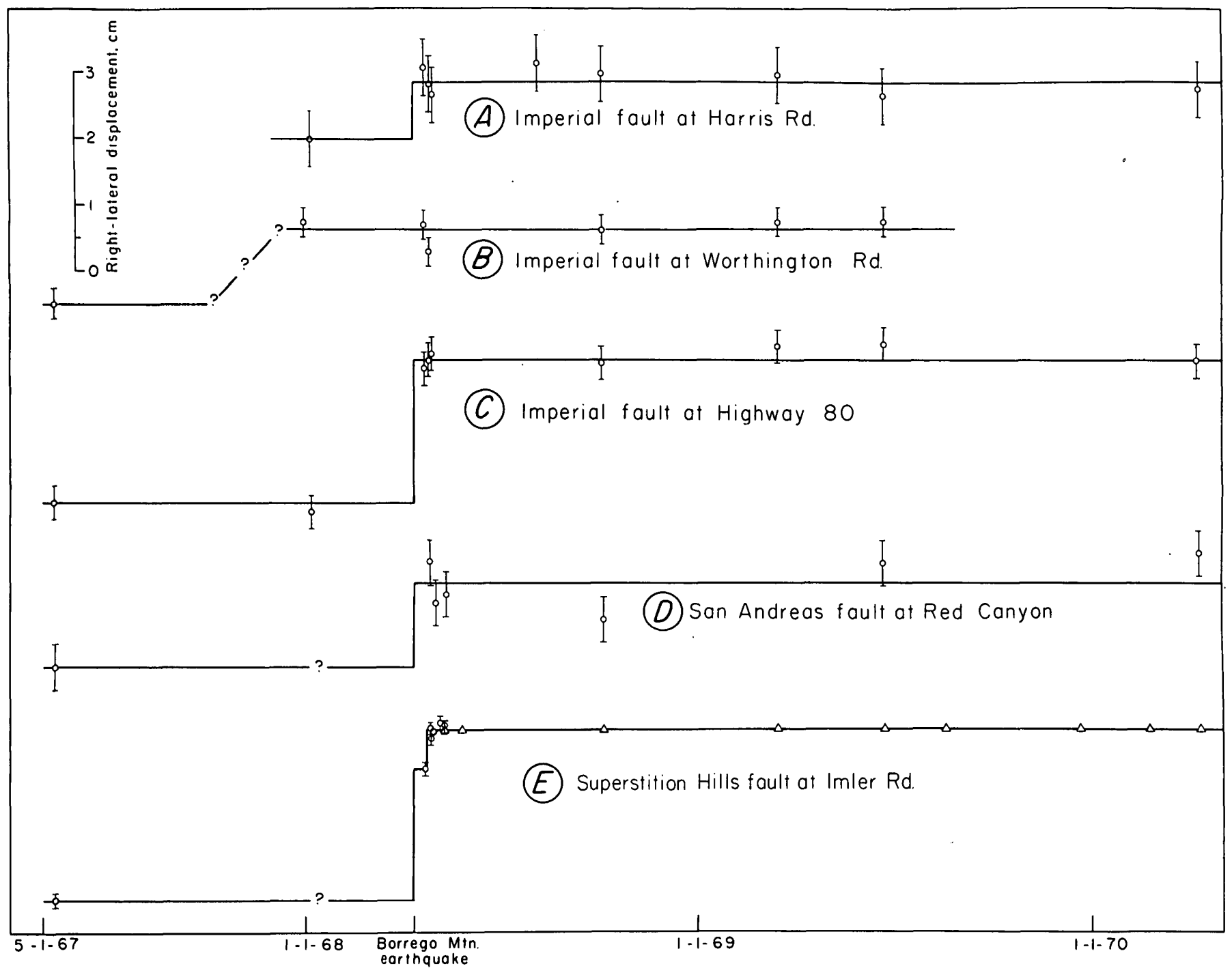

Figure 55. - Measurements of fault-crossing geodetic networks between May 1, 1967, and May 1, 1970, showing displacements at time of Borrego Mountain earthquake. Localities are shown in figure 54 and on plate 2. Circles represent averages of measurements of $2-4$ independent lines crossing the fault at each locality, and error bars indicate twice the standard deviation of repeated measure-

much as $7 \mathrm{~m}$ high that delineates the northern $11 \mathrm{~km}$ of the fault trace, (2) a marked ground-water barrier that is sometimes visible from the air even in cultivated fields, and (3). a pronounced gravity anomaly along the fault trace (Kovach and others, 1962). However, the total extent of the Imperial fault beyond the $60-\mathrm{km}$ segment broken in 1940 is not known; there is as yet no good geological or geophysical evidence of the fault north of Keystone Road, where the 1940 trace died out, or south of a point near Tortuosa Check (Mexico), at the south end of the 1940 break. Possibly, the Imperial fault is a transform fault whose active segments indeed do not extend much beyond its presently known length (Lomnitz and others, 1970). ments of individual lines in the surveys following the earthquake. Survey lines average $90 \mathrm{~m}$ long. Triangles in diagram $E$ show readings of taut-wire creepmeter located $31 / 2 \mathrm{~km}$ north of Imler Road, showing lack of further displacement there after installation of creepmeter on May 7, 1968.

\section{SUPERSTITION HILLS FAULT}

On May 11, 1967, Allen and Brune had established a small geodetic network across the Superstition Hills fault where it crosses Imler Road (pl. 2) ; reoccupation of this station on April 19, 1968, 10 days after the Borrego Mountain earthquake, revealed about $21 / 2 \mathrm{~cm}$ of right-lateral displacement (fig. 55E). At the same time, fresh en echelon cracks showing as much as $1.5 \mathrm{~cm}$ of right-lateral displacement were discovered along the Quaternary fault trace in the same vicinity (figs. 59,60). On April 25, these cracks were followed northwest for about $8 \mathrm{~km}$ along the fault trace, and on May 13-15, Grantz and Wyss mapped the entire broken zone, which extended for $23 \mathrm{~km}$ (pl. 2). 


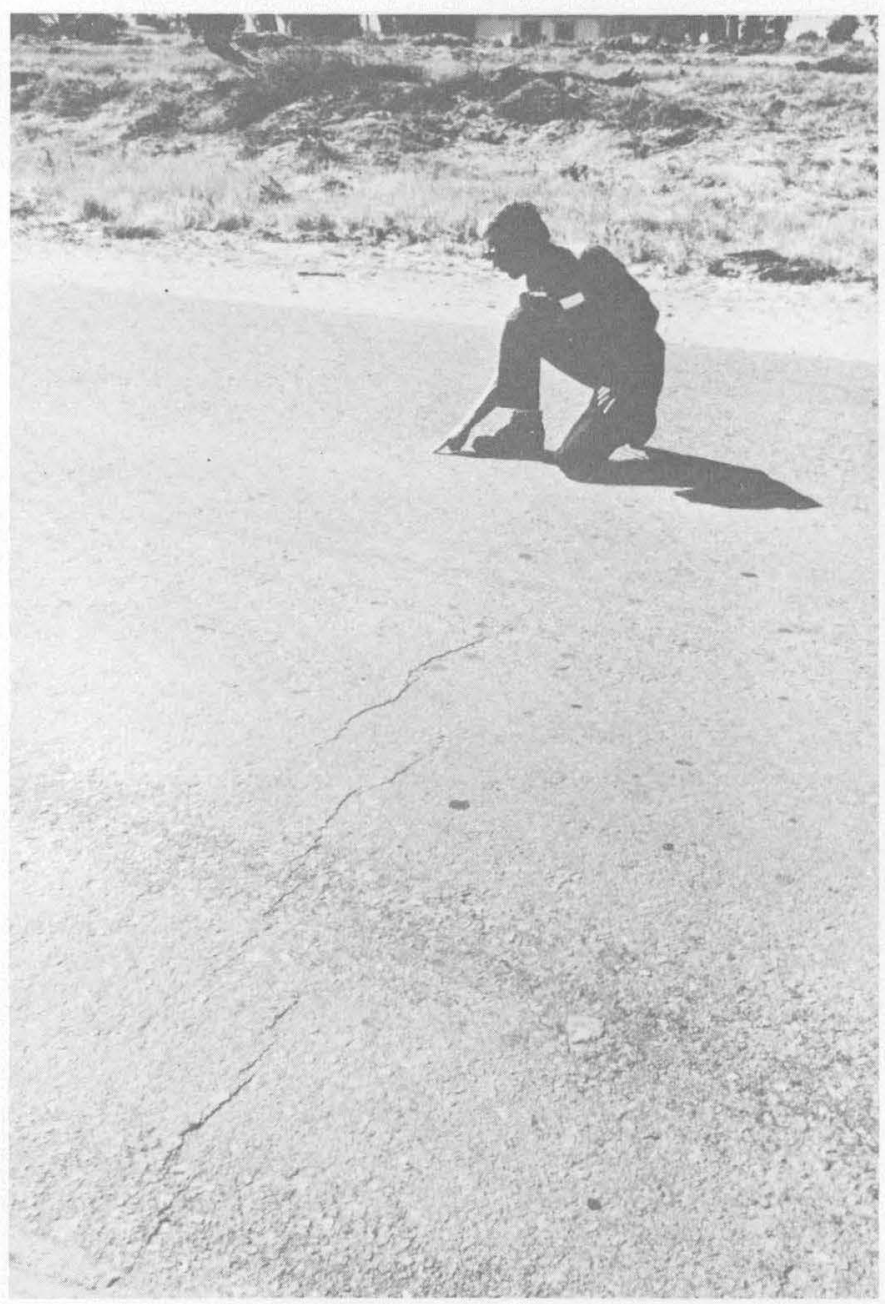

Figure 56. - Fresh en echelon cracks crossing Ross Road. View southeast. Photograph taken April 28, 1968.

The cracks, as mapped on May 13-15, varied from a single, narrow well-defined break several centimeters to a meter wide, the most common type, to zones of en echelon cracks as much as $7-10 \mathrm{~m}$ wide in which the individual cracks stepped to the left in the manner of ground cracking along right-lateral strike-slip faults. Their dip, as recorded at four near-vertical exposures, were $80^{\circ}-90^{\circ}$. The cracks followed very closely the trace of the Superstition Hills fault across the strongly folded Pleistocene sedimentary rocks of the Superstition Hills and vicinity. Commonly, they lay within the $1 / 3-12 / 3-m$-wide gouge zone of the Quaternary Superstition Hills fault, but in a number of places, the cracks occurred in the Pleistocene sediments as much as $7 \mathrm{~m}$ from the Quaternary fault. Where they departed from the Quaternary fault gouge zone, the cracks usually, but not always, lay northeast of it.

Where the cracks formed a narrow well-defined zone, they commonly coincided exactly with a single

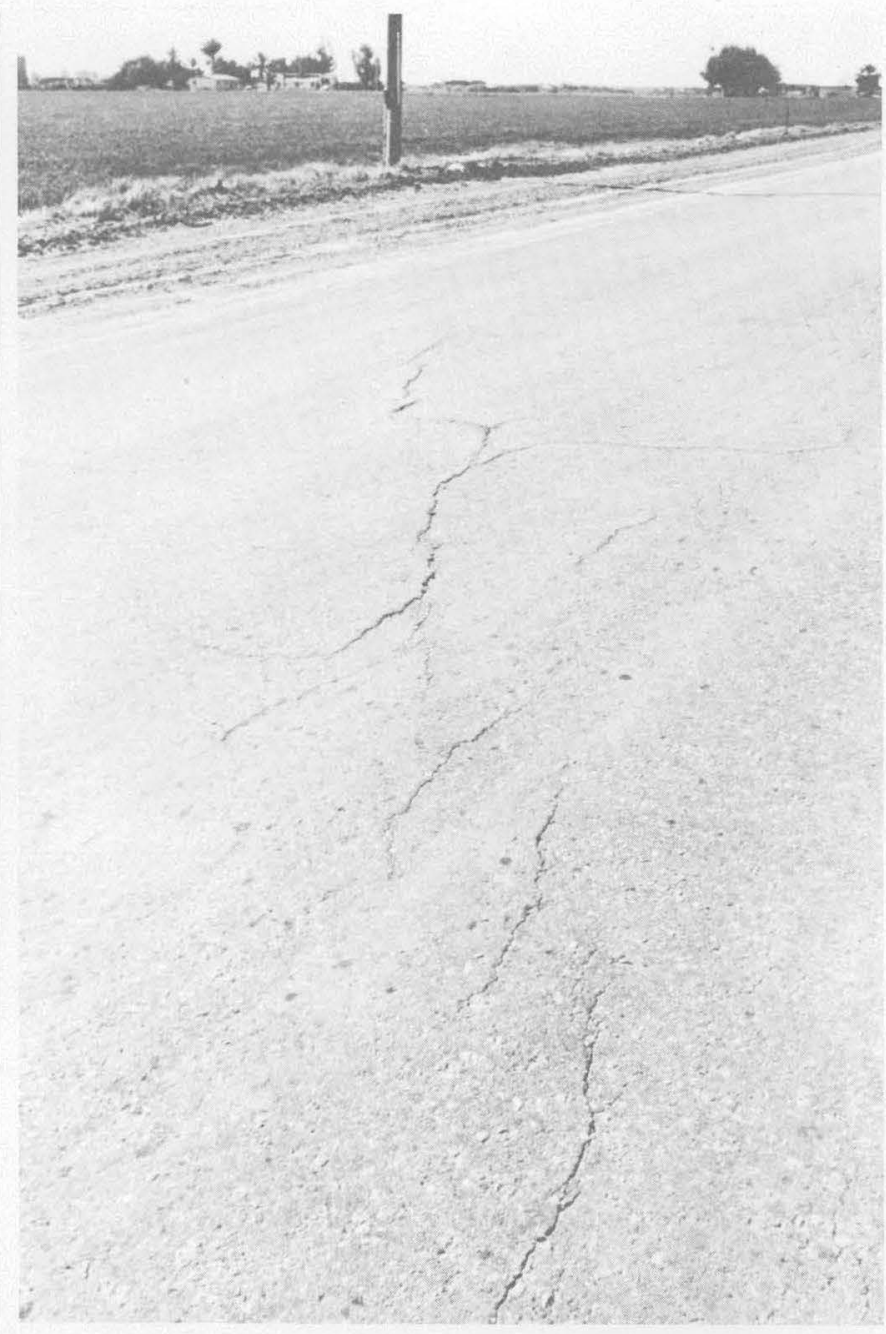

FIGURE 57. - En echelon cracks on Meloland Road first observed following the Imperial earthquake of 1966 (Brune and Allen, 1967a) but slightly widened during the Borrego Mountain earthquake. Fault displacement of about onehalf $\mathrm{m}$ occurred at this same locality in 1940. Photograph taken April 28, 1968.

fault-controlled line of desert shrubs (fig. 61), small drainage sumps and collapse pits or trenches (fig. $62)$, local ground-water barriers, small fault scarps in soft sediments, or, at a few places, right-laterally offset small ridges and gullies. This alinement and the character of the alined features indicate that the fault cracks of April 1968 follow a well-established line of latest Holocene faulting as well as a Quaternary bedrock fault.

The displacements measured on May 13-15 along the partly wind-eroded and sand-filled fault cracks indicated dominantly right-lateral strike slip, with only local vertical displacement and no instances of left-lateral slip. The right slip was as much as $1.8 \mathrm{~cm}$ but averaged about 0.8 or $0.9 \mathrm{~cm}$. Of only possible significance, because of the erosion and filling of the 


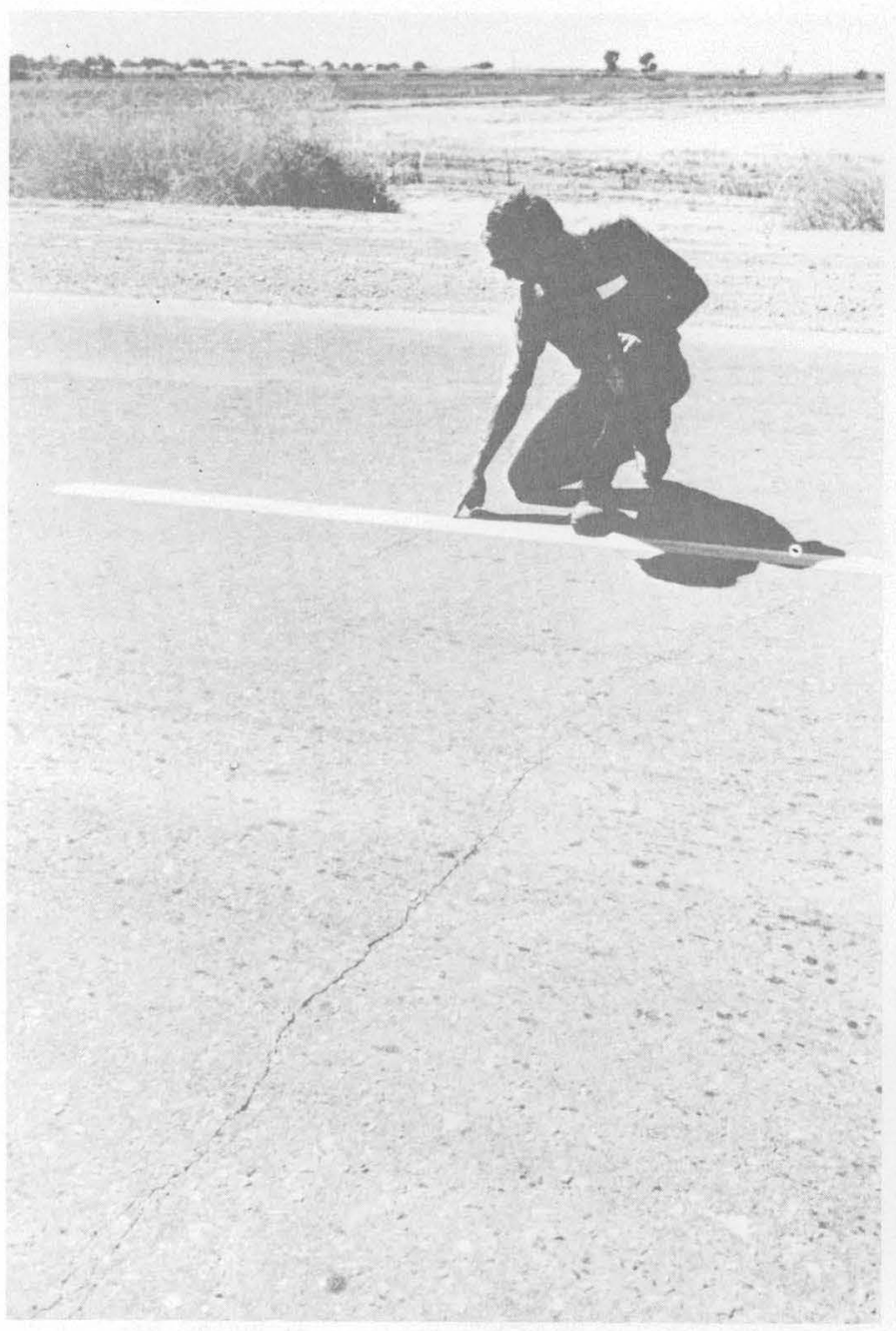

Figure 58. - Fresh cracks across Heber Road. Displacement of about $1 \frac{1}{2} \mathrm{~m}$ took place here in 1940 , but no further displacements are known to have occurred here until 1968. Photograph taken April 28, 1968.

cracks, is the fact that most of the largest right-lateral displacements were recorded near, although not at, the ends of the fault break of April 1968. Most displacements of $11 / 4 \mathrm{~cm}$ and greater occurred between 1.7 and $5.8 \mathrm{~km}$ of the southeast end and between 2.8 and $4.7 \mathrm{~km}$ of the northwest end of the 23-km-long fault break. Displacements of between 0.4 and $1.0 \mathrm{~cm}$ were common along the central part of the break. Vertical displacements, along three short $(\approx 75 \mathrm{~m})$ segments of the fault, were as much as $2 \frac{1}{2} \mathrm{~cm}$. Two, at the northwest end of the break, were up on the northeast; one, near the middle of the break, was up on the southwest. In all three places, the uplifted side is also the topographically higher side, and in two of them the uplifted side holds up a low scarp cut in soft Pleistocene sediments. These relations indicate that the local vertical components

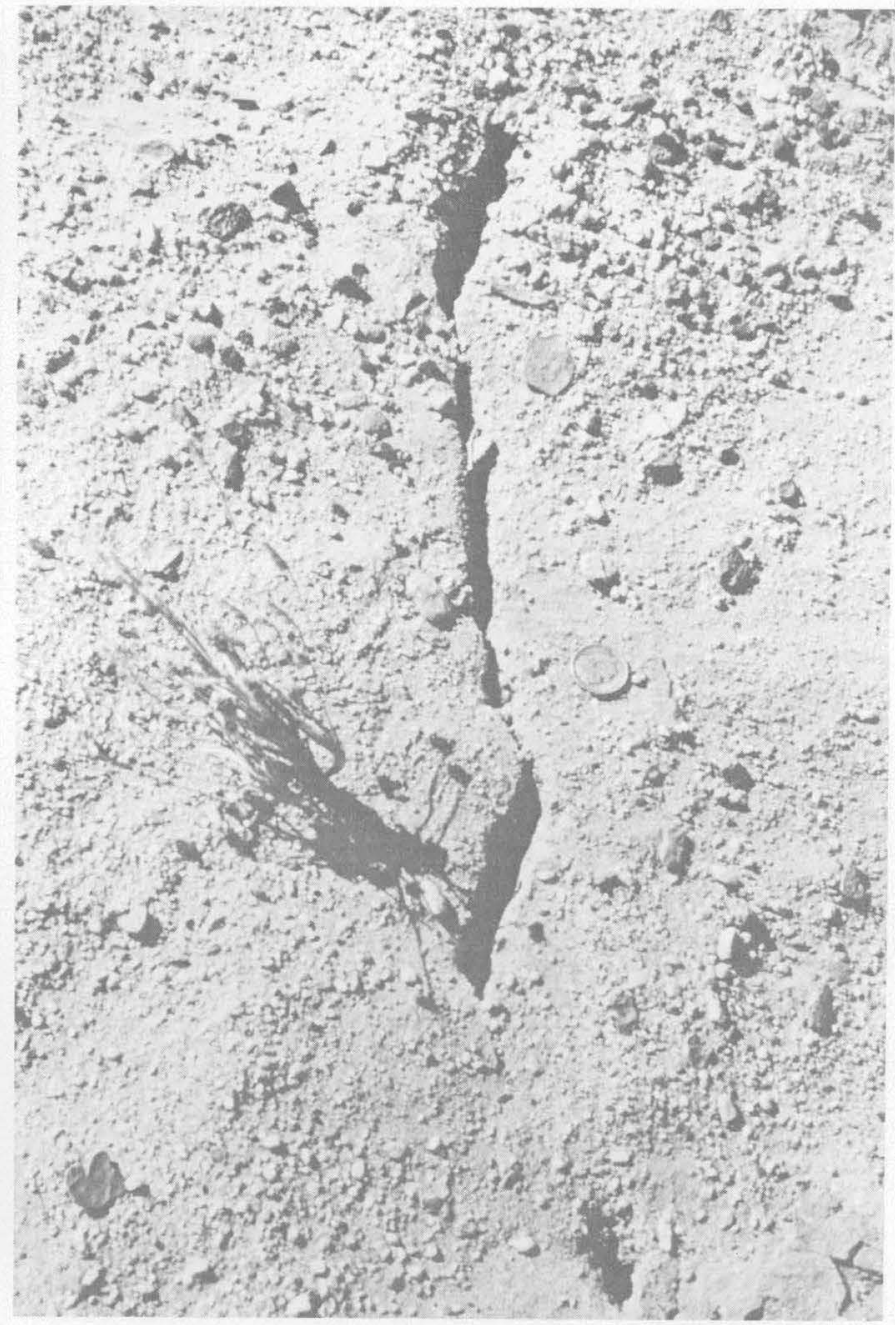

FIGURE 59. - Right-lateral displacement of about $2 \mathrm{~cm}$ on the Superstition Hills fault in sec. 23, R. 12 E., T. 14 S. Diameter of coin is $1.7 \mathrm{~cm}$. Photograph taken April 22, 1968; note that crack has filled in since time of probable formation on April 9.

of slip in April 1968 acted in the same sense as the latest Holocene displacements at those places.

When the cracks were first observed on April 19, they were relatively fresh appearing, but by the time mapping was completed on May 15 , windblown sand had already obscured much of the fault trace. We conclude that the cracks could not have come into existence long before April 19, and their origin in association with the Borrego Mountain earthquake on April 9 seems highly probable. Because of suspicion that creep might be taking place on this fault following the earthquake, a creepmeter was installed across the fault on May 7 at a locality midway along its length (pl. 2). A recorder registered the displacement as measured by a taut $10-\mathrm{m}$ Invar wire, similar to an instrument previously used at Parkfield (Smith and Wyss, 1968). Seventeen subsequent readings of 


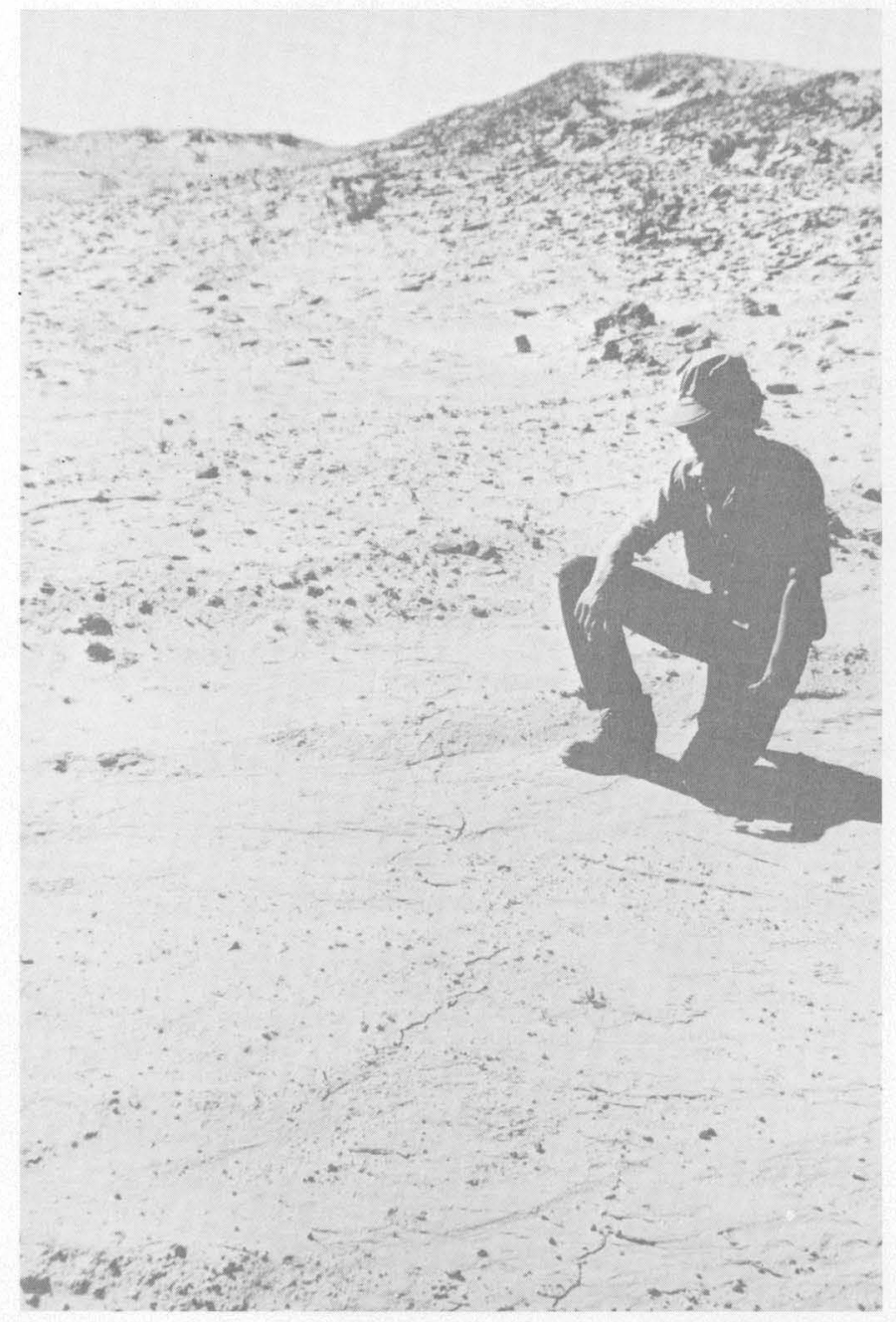

FIGURE 60. - En echelon cracks indicative of right-lateral displacement along Superstition Hills fault in sec. 9, R. 12 E., T. 14 S. Photograph taken April 27, 1968.

the creepmeter, through November 1971, revealed no evidence of further significant movement on this segment of the Superstition Hills fault, nor have any further cracks appeared in the nearby paved road where it is crossed by the fault. These observations further support the inference that the displacement observed on April 19, 1968, occurred relatively suddenly in association with the Borrego Mountain earthquake.

Minor displacements on the Superstition Hills fault similar to those observed in 1968 have occurred at least three other times in recent years. In 1951, Joseph Ernst (written commun.) noted fresh en echelon cracks along about $3 \mathrm{~km}$ of the Superstition Hills fault, approximately centered within the segment that broke in 1968 and along exactly the same trace. A magnitude 5.6 earthquake had been centered in this area about 2 weeks earlier, and Ernst concluded that the rate at which windblown sand was

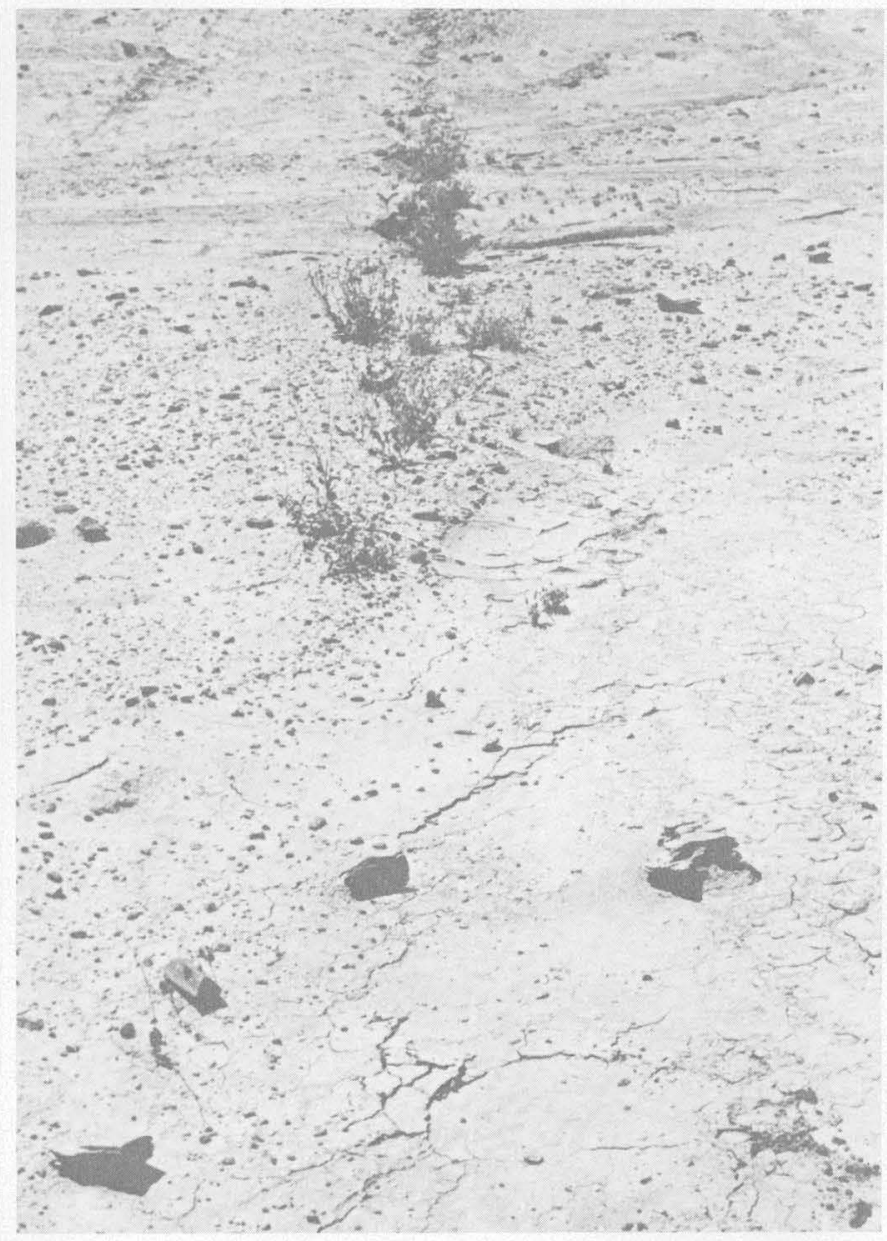

Figure 61. - New en echelon cracks of right-lateral habit following the line of desert plants that marks the Superstition Hills fault in NW $1 / 4$ sec. 26 , T. 13 S., R. 11 E. The larger plants are about 1 foot high. Photograph taken May 13, 1968.

filling the cracks demanded their origin within about this period. Similarly, in late December of 1965 , Brune noticed fresh en echelon cracks along the Superstition Hills fault at Imler Road, and he and Allen subsequently followed these for about $1 \mathrm{~km}$ north and south of the road; these cracks may have been triggered by a nearby magnitude 4.0 shock on November 30,1965 . The cracks were quickly obscured by blowing sand, and it is clear from repeated subsequent visits to the area that significant creep is not occurring continuously. Similarly, fresh cracks were noted along the fault south of Imler Road during a visit to the area on December 20, 1969, although they could not be correlated with a specific local earthquake. The movement did not extend far enough north to affect the creepmeter. The movement in April 1968 is evidently only one in a series of small episodic surface displacements that have characterized this fault in recent years; the same behavior may well be true of the segments of the 


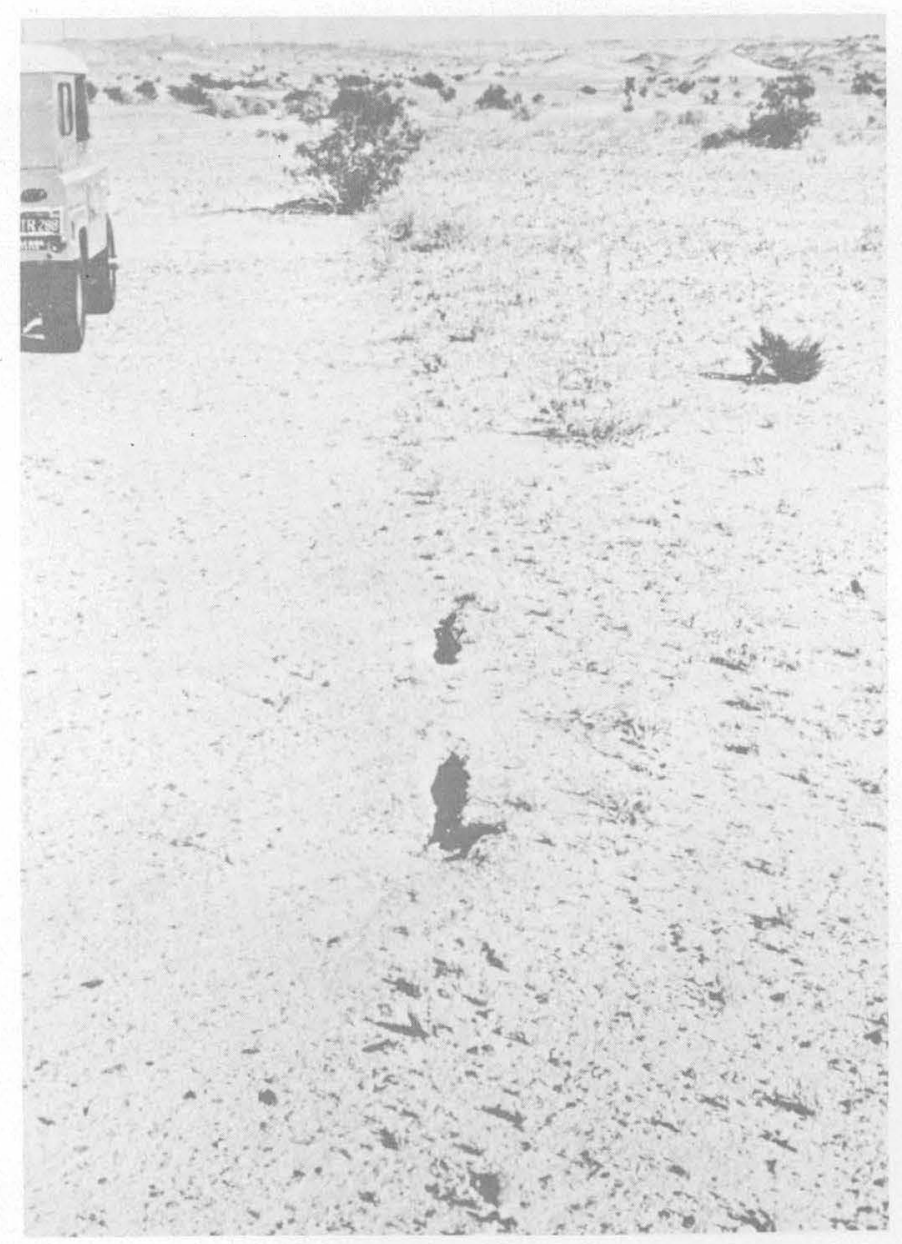

Figure 62. - Small collapse pits, alined vegetation, and variation in abundance of vegetation along the Superstition Hills fault in SW $1 / 4$ sec. 9, T. 14 S., R. 12 E. New hairline cracks follow the alined features but are largely obscured by windblown sand and silt. Photograph taken May 14, 1968.

Imperial fault and San Andreas fault that broke at about the same time.

Like the Imperial fault, little is known about the possible extent, if any, of the Superstition Hills fault beyond the segment broken in 1968. On the southeast, the 1968 fractures ended about $1 \mathrm{~km}$ north of Edgar Road, at very nearly the same point that the Quaternary trace disappears as observed on aerial photographs and in the field. Likewise, the fractures continued northwest only about as far as the mapped trace of the Quaternary fault (Dibblee, 1954; unpub. data). The Superstition Hills fault, together with the nearby Superstition Mountain fault, appear to be branches of the San Jacinto fault zone, and if projected still farther northwest, they would join the Coyote Creek fault - also a branch of the San Jacinto zone - on which the Borrego Mountain earthquake occurred.

\section{SAN ANDREAS FAULT}

In February 1967, A. G. Sylvester (oral commun.) found fresh cracks along the trace of the San Andreas fault in the Mecca Hills north of the Salton Sea; they were particularly evident in the $4-\mathrm{km}$ segment between Painted Canyon and the unnamed canyon (locally called Red Canyon) in sec. 28, T. 6 S., R. 9 E. (Thermal Canyon quadrangle). We were not convinced at that time that the cracks necessarily reflected tectonic movements, because among other reasons there has been very little historic seismicity (Allen and others, 1965) or microearthquake activity (Brune and Allen, 1967b) along this segment of the San Andreas fault zone. However, to check this possibility, we established a small geodetic network across the fault in the unnamed canyon noted (station "Red Canyon" in table 12 ; pl. 2) on May 11 , 1967. Resurvey of this network on April 24, 1968, indicated $1.3 \mathrm{~cm}$ of right-lateral displacement, and since that time there has been no significant additional change (fig. $55 \mathrm{D}$ ). A second fault-crossing geodetic network was established across the fault near Bertram (station "Bertram" in table 12; pl. 2) on May 5, 1968, and it likewise has shown no significant change in six subsequent surveys through August 7, 1970.

Despite the low seismicity along this segment of the San Andreas fault, the fault trace is so clearly marked in the field that displacements along it must have occurred in very recent years. Small scarplets as much as $50 \mathrm{~cm}$ high and only slightly eroded are abundant along the fault (fig. 63). Zones of en echelon fractures, eroded and distinctly older than the recent fractures (fig. 64), appear to be too transient to have persisted for much more than a decade; conceivably, all the erosion along the older fractures could have been accomplished by one heavy rainstorm. Similarly, piping of rainwater into the fracture zone in places appears very recent. From this evidence, it would appear that more than one episode of recent fault displacement has occurred in this area despite the scarcity of historic seismic activity. Whether such previous displacements likewise accompanied distant earthquakes is unknown.

When this area was first visited after the Borrego Mountain earthquake on April 24, fresh en echelon cracks were observed at the base of the scarplets (fig. 65) and at several other localities along the fault trace. Wallace and Wyss subsequently mapped the fresh break for more than $30 \mathrm{~km}$ from near Bertram on the south to Thermal Canyon on the north (pl. 2), although it is significant that surface fracturing was by no means continuous throughout the $30-\mathrm{km}$ segment. The average right-lateral dis- 


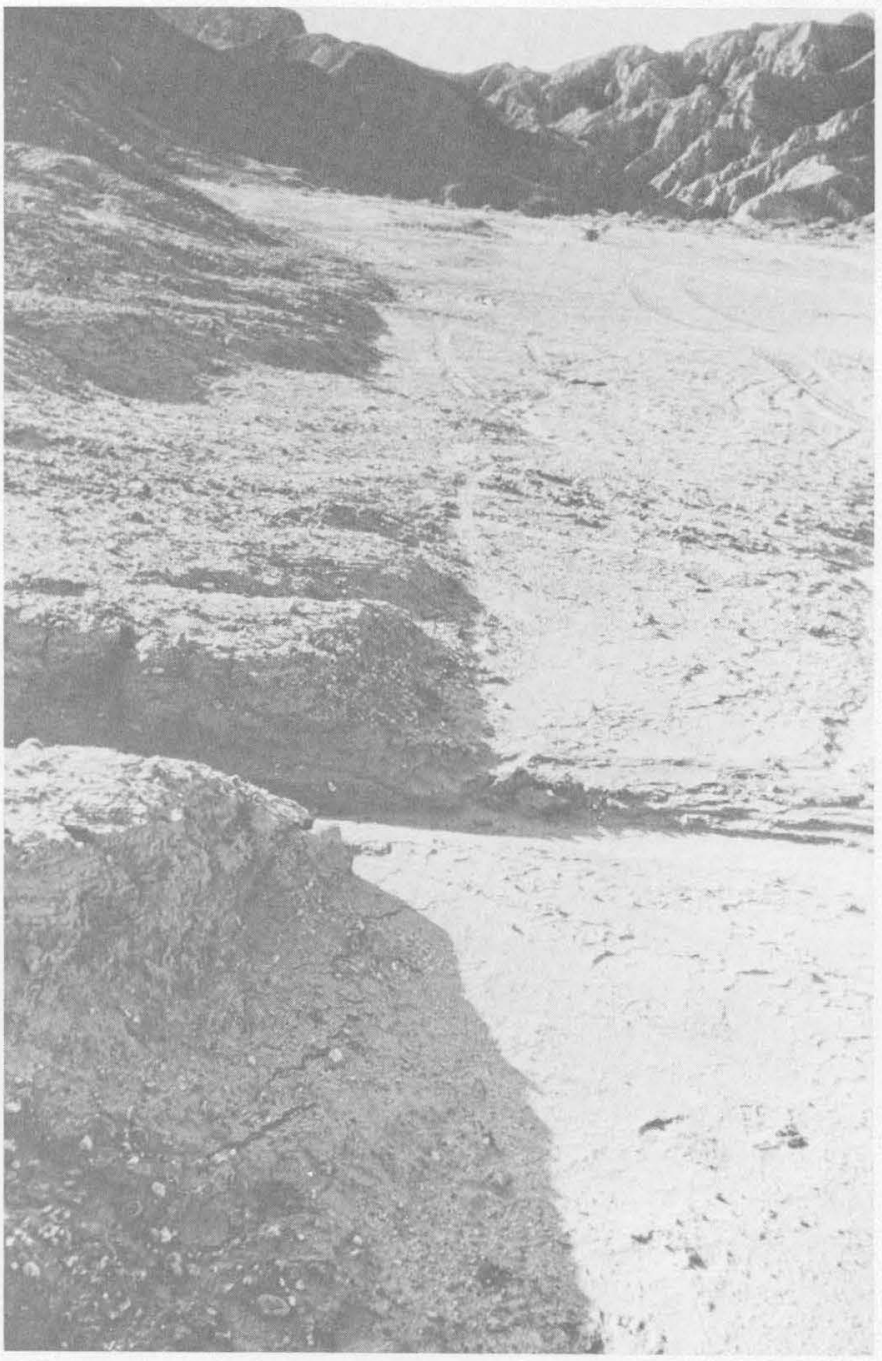

Figure 63. - En echelon cracks (in shadow, foreground) at base of scarplet along San Andreas fault in sec. 28, R. 9 E., T. 6 S. View northwest. Geodetic data at nearby station "Red Canyon" indicated about $1.3 \mathrm{~cm}$ right-lateral displacement (fig. 55D). Photograph taken April 28, 1968.

placement was estimated to be between 0.5 and $1.0 \mathrm{~cm}$.

One of the puzzling features of the San Andreas fault in this region is its apparent termination opposite the south end of the Salton Sea, particularly because this segment of the fault may have as much as $260 \mathrm{~km}$ of post-early-Miocene strike-slip displacement (Crowell, 1962). The apparent termination might be explained simply by concealment of the fault trace southeast of this point by Quaternary sediments, including those of Lake Cahuilla that inundated all this region for 1,300 years, ending perhaps as recently as 300 years ago (Lake LeConte in Hubbs and others, 1960). Likewise, there is some geophysical evidence suggesting continuity of the fault farther southeast (Kovach and others, 1962;

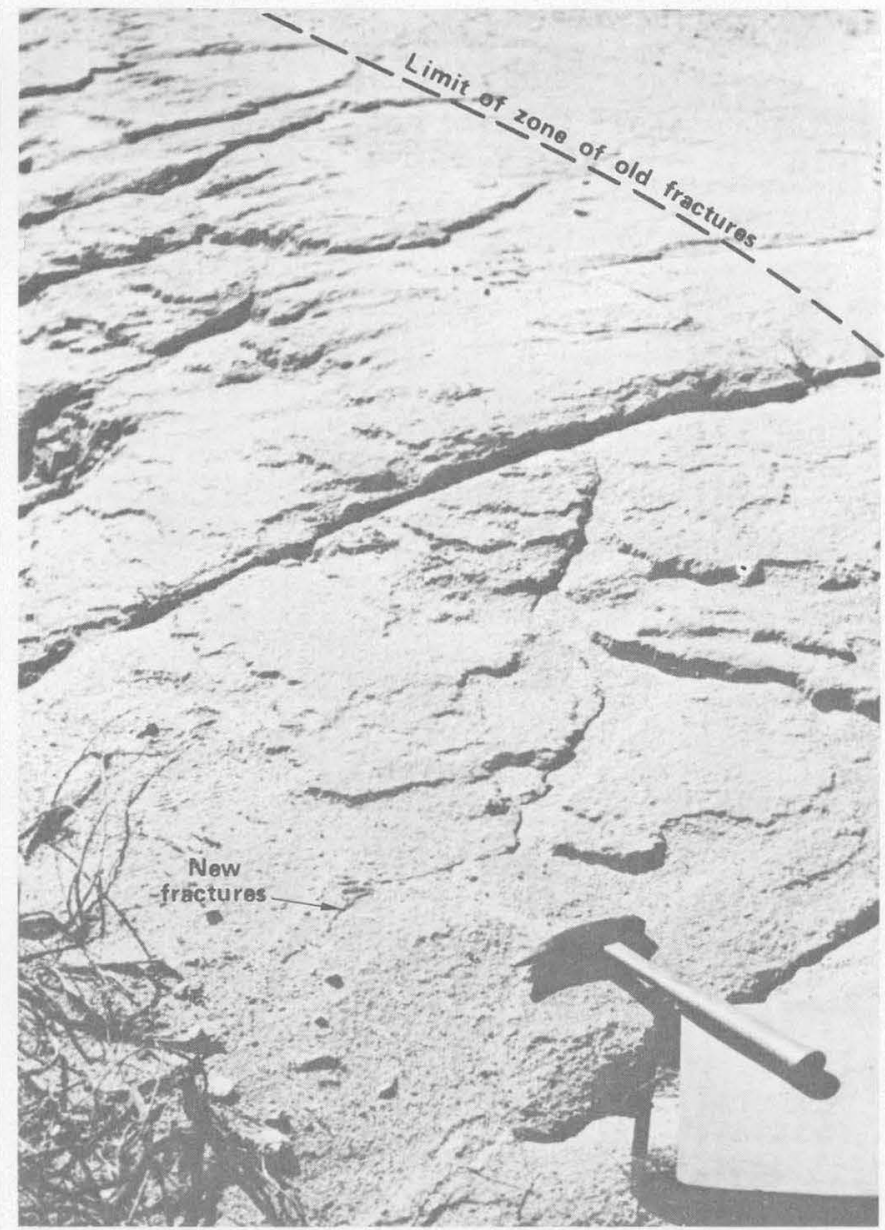

Figure 64.- New fracture in old fracture zone along San Andreas fault in sec. 28 , R. 9 E., T. 6 S. New en echelon fractures are subparallel to old en echelon fractures. Note that erosion has progressed along old fractures. Photograph taken May 9, 1965.

S. Biehler, unpub. data). On the other hand, the San Andreas fault may be a transform fault whose active segment does indeed terminate near the Salton Sea. Adding weight to such a hypothesis is the presence of the northeast-trending Salton volcanic domes that might be a manifestation of a ridge segment in a transform fault system (Lomnitz and others, 1970). Nonetheless, it is significant that the 1968 fractures on the San Andreas fault extended southeast to almost exactly the same point where the continuous Quaternary trace disappears (Hope, 1969).

\section{DESCRIPTION OF THE SURFACE DISPLACEMENTS}

En echelon cracking oriented so as to clearly indicate right-lateral displacement was the typical style of surface rupture along the Imperial, Superstition Hills, and Sari Andreas faults (figs. 65-67). Individual cracks rarely gaped more than 1 or $2 \mathrm{~mm}$ when fresh. Individual en echelon breaks were typi- 


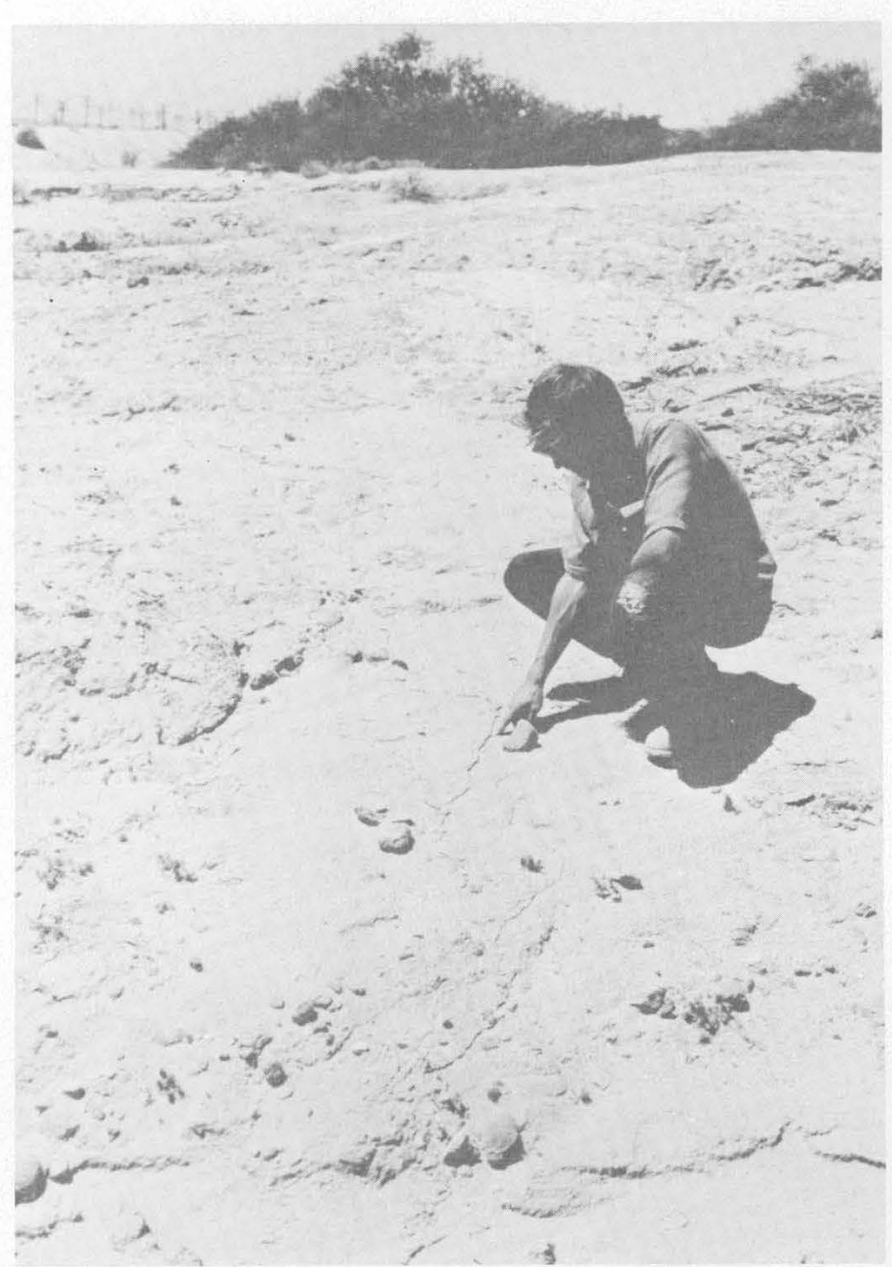

Figure 65. - En echelon cracks along the San Andreas fault near Salt Creek, in the center of sec. 28, T. 8 S., R. 11 E. View northwest. The fault here brings rocks of the Palm Spring Formation (right) into contact with those of the Borrego Formation (left), and the contact is visible in line with the cracks in the middle distance. Photograph taken April 28, 1968.

cally less than $1 \mathrm{~m}$ long where the zone of cracks was several centimeters to less than $1 \mathrm{~m}$ wide, but they ranged in length from 2 to $30 \mathrm{~m}$ in those less common places where the fractured zone was $1-7 \mathrm{~m}$ or more wide. The fractures showed up not only in undisturbed soil but also in asphalt roads that were crossed by each of the three faults. All the broken sections of the Superstition Hills and San Andreas faults were traversed in their entirety by one or more of the authors. This was not possible along the Imperial fault because of intensive cultivation of most of the area; instead, each road and canal crossing was checked.

The newly formed fractures along the three distant faults were such minor features that they would easily have escaped detection if we had not specifically looked for them and if we had not known from

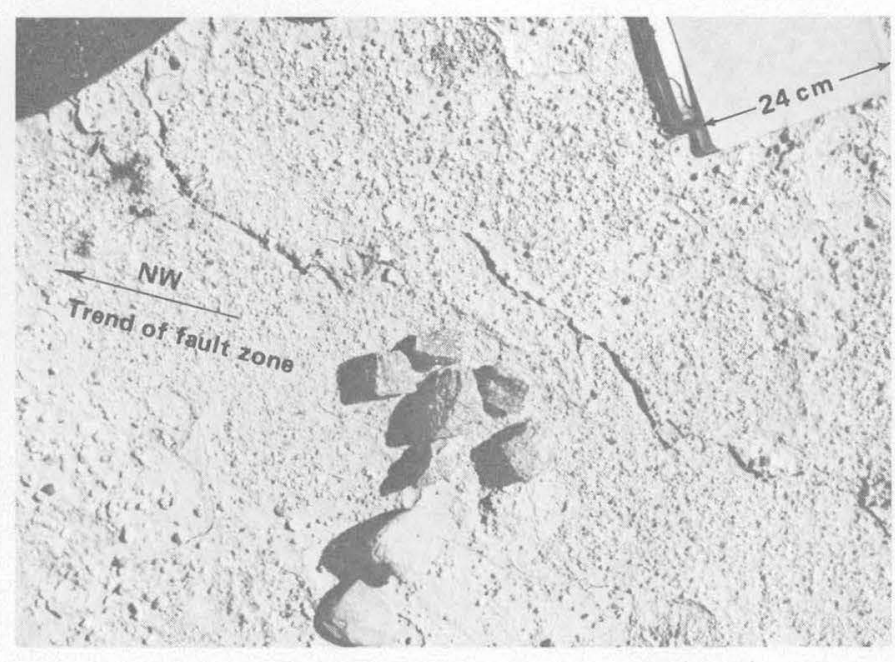

Figure 66. - En echelon fractures along the San Andreas fault approximately $2 \mathrm{~km}$ south of Salt Creek, in SW $1 / 4$ sec. 34, T. 8 S., R. 11 E. Photograph taken May 10, 1968.

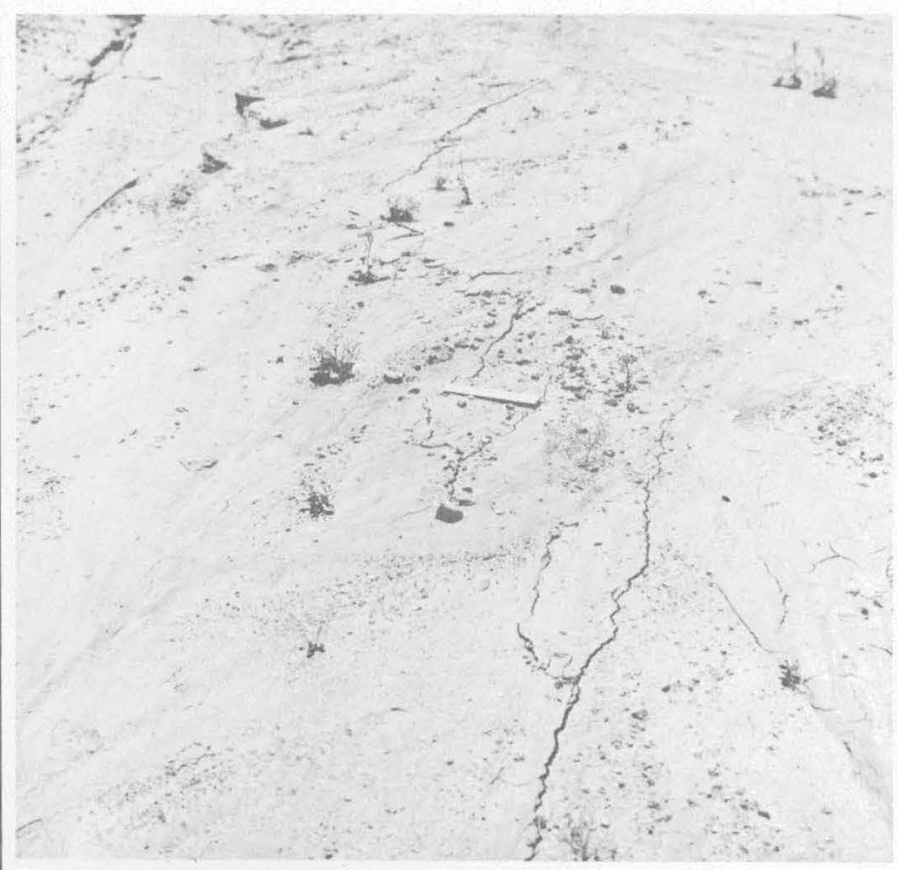

FIGURE 67. - En echelon fractures, sec. 28, T. 6 S., R. 9 E. Scale is $19 \mathrm{~cm}$ long. View southeast. Photograph taken May 9, 1968.

other geologic evidence the exact locations of the active fault traces to within a very few meters. The precise trace of the Imperial fault, despite its location within the heavily cultivated floor of the Imperial Valley, was known from J. P. Buwalda's very detailed notes on the much larger 1940 earthquake displacements, as well as from the subsequent displacements along part of the trace in 1966 (Brune and Allen, 1967a). The detailed trace of the Superstition Hills fault is clear on large-scale aerial photo- 
graphs and on the ground because of the abrupt truncation of well-exposed and highly deformed Pleistocene sedimentary rocks at the fault. Similarly, segments of the San Andreas fault are well delineated on detailed aerial photographs because of ground-water damming and the truncation of young sedimentary rocks (figs. 68, 69) (Hope, 1969). Both the Superstition Hills and San Andreas faults traverse desert areas that, for the most part, have never been cultivated or otherwise culturally modified. Considerable stretches of both faults are mantled with a veneer of sediments dating from the last major filling of the Salton depression by Lake Cahuilla between about 300 and 1,600 years ago.

Seldom have such well-defined faults been examined in such great detail after a major nearby earthquake, and although this may be the first documentation of fault displacements caused by seismic shaking, the same phenomenon may have happened many times before, not only here, but also on other active faults in similar tectonic environments.

It is particularly significant that, with minor exceptions, surface fracturing occurred in 1968 only along those segments of the Superstition Hills and San Andreas faults where examination of aerial photographs and field exposures could clearly delineate preexisting active breaks. Several examples have already been cited in the preceding section, but perhaps the most intriguing localities are along the San Andreas fault northeast of the Salton Sea. In this area, some segments of the fault are much more clearly delineated by features of recent displacement than others, and it is only on the clearly delineated parts that fresh fractures were found after the Borrego Mountain earthquake, despite careful searches in some of the intervening "inactive" segments (for example, Box Canyon Wash, Salton Sea State Park headquarters area, Highway 111 northeast of Bombay Beach). This distribution is well illustrated by Hope's (1969) map, which shows both the 1968 fractures and the Quaternary breaks that were visible on $1: 14,000$-scale aerial photographs flown in 1966. Either (1) contemporary movements are taking place along a discrete fault plane at the surface in some segments and throughout a distributed zone in other segments or (2) contemporary displacements are limited to certain weak segments of the fault in contrast to other stronger segments that are temporarily locked, to be broken through during a larger earthquake at some time in the future. The second argument is analogous to that sometimes used for the San Andreas fault as a whole (for example, Allen, 1968), but whether this kind of reasoning is applicable on such a small scale is unknown.
It should also be noted that the geodetically measured displacements (fig. 55) were consistently larger than those estimated from field observations of the faulting. Inasmuch as the geodetic lines used for the calculations of figure 55 were typically about $100 \mathrm{~m}$ long, this variation suggests that some shear deformation was taking place that was not expressed as discrete visible fractures at the surface. Indeed, in attempting to interpret the geodetic data at localities where many stations were surveyed, one could not escape the conclusion that, at least at some localities, deformation took place in a distributed fashion rather than entirely along a single fault plane. The accumulation of such distributed deformation during repeated fault displacements is the mechanism that produces the drag so commonly observed along faults in layered rocks. At the Coyote Creek fault rupture of April 9, 1968, this mechanism produced pronounced drag in late Holocene sedimentary rocks and in places produced more than half the total late Holocene deformation. (See Clark and others, this volume.)

\section{OTHER FAULTS}

At the same time that fresh displacements were being discovered on the Imperial, Superstition Hills, and San Andreas faults, a number of other faults in the region were carefully checked in the field and found to show no evidence of surface displacements. These include the Superstition Mountain fault, Elsinore fault, Laguna Salada fault, Earthquake Valley fault, San Felipe fault, and branches of the San Jacinto fault system north of Borrego Valley. One feature that distinguishes these faults is that they are predominantly in crystalline rocks, whereas the parts of the three faults that moved are all in deep alluvium or late Cenozoic sedimentary deposits. The estimated minimum distance to crystalline basement based on seismic work (Kovach and others, 1962; Biehler and others, 1964) is $3,500 \mathrm{~m}$ along the Superstition Hills fault, $6,000 \mathrm{~m}$ along the Imperial fault, and perhaps $2,000 \mathrm{~m}$, but locally only $400 \mathrm{~m}$ (Babcock, 1970), along the San Andreas fault. It is also probably significant that the only three faults in southeastern California for which we had some evidence of slippage before the earthquake (and which had therefore been straddled with small geodetic networks) were the same three faults that moved during the Borrego Mountain earthquake.

\section{MECHANISM OF DISPLACEMENT}

Three lines of evidence lead us to believe that the observed fault displacements took place on or about April 9, 1968: (1) The geodetic measurements that were made during the year before and the year after 


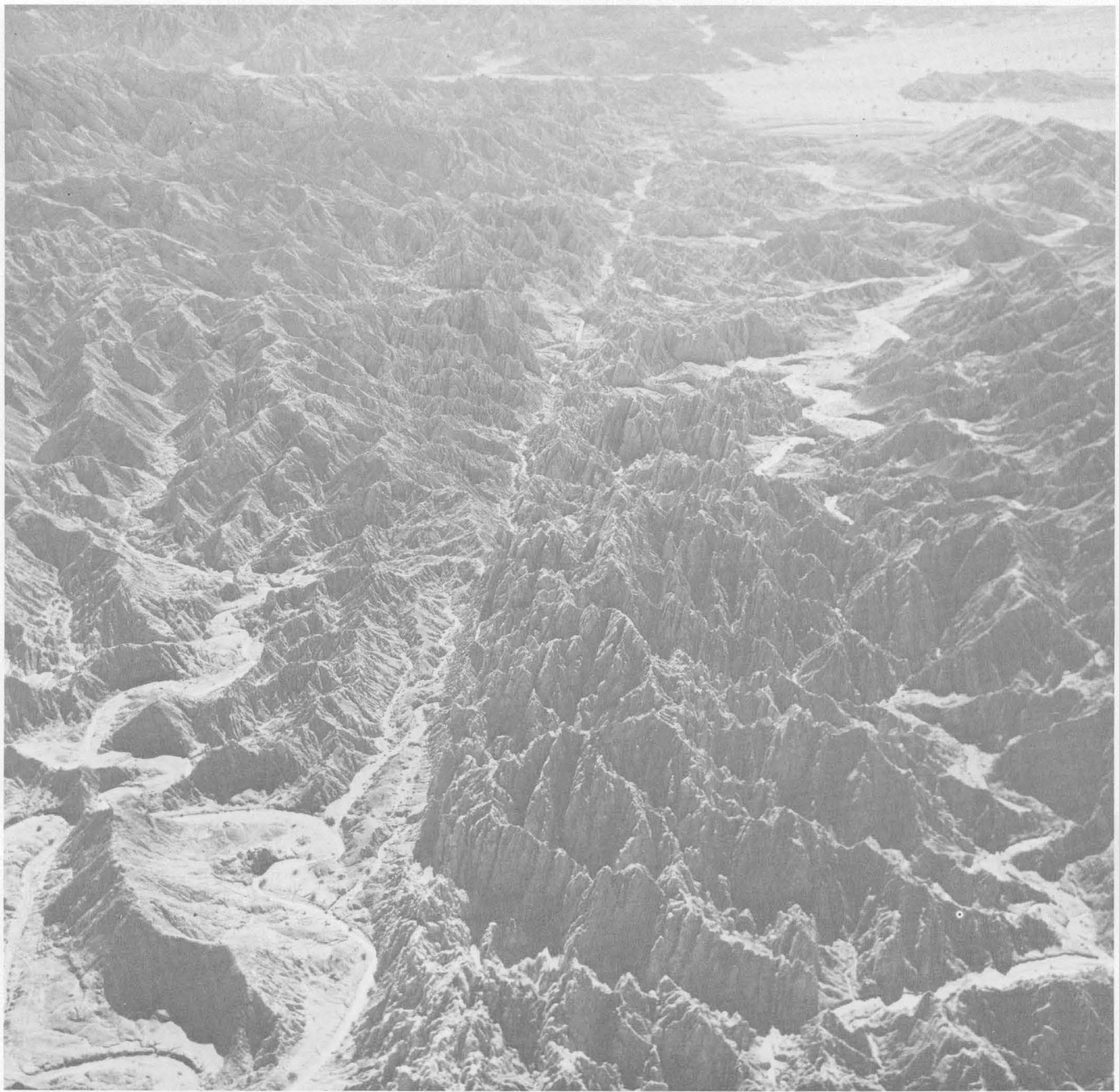

FIgURE 68. - Linear valley eroded along San Andreas fault, Mecca Hills. New fractures followed this valley but could not be found in the dry alluvium of Painted Canyon (far background).

the earthquake indicate little or no creep, except at Worthington Road. It thus seems unlikely that creep should have characterized only the short interval that included the earthquake, unless triggered by it. (2) On March 8, 1968, 1 month before the earthquake, a heavy and unusual rainstorm brought approximately $5 \mathrm{~cm}$ of precipitation to the entire Imperial Valley-Coachella Valley area, causing considerable runoff and local flooding. All the fresh cracks observed after the earthquake must have originated after this rainstorm. (3) Blowing dust and sand are characteristic of the entire region, and everyone who studied the displacements on these three faults, as well as the main break near Ocotillo Wells, was impressed with the rate at which fresh features disappeared. Within 2 weeks of the earthquake, many of the cracks along the main break had become barely recognizable because of windblown 


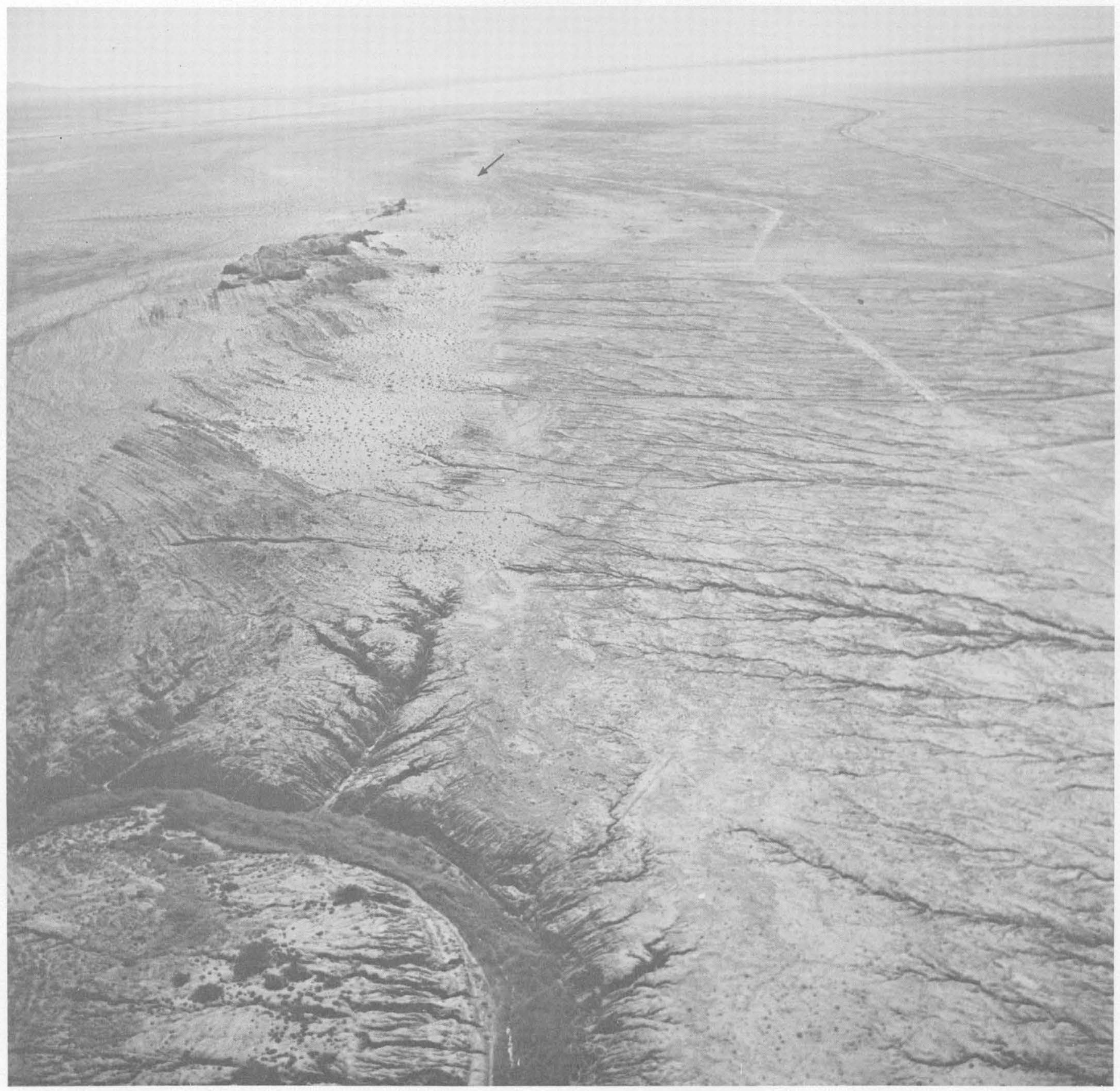

FIgURe 69. - View southeast along San Andreas fault. New fractures closely followed this lineament to point shown by arrow in distance. Bat Caves Buttes on left; Salton Sea in distance; Salt Creek in foreground.

sand. It is our judgment, based on field experience in this area, that the fractures first observed between April 13 and April 24 must have come into existence during the first 2 weeks in April. Particularly along the Imperial fault, the fresh cracks in powdery alluvium that were first observed on April 13 must have originated within the preceding few days. It seems to us to be a reasonable, indeed a highly likely, conclusion that all the fractures came into existence at the approximate time of the Borrego Mountain earthquake on April 9.

\section{STATIC VERSUS DYNAMIC STRAIN}

If the hypothesis is accepted that the breaks on the Imperial, Superstition Hills, and San Andreas faults were caused by the Borrego Mountain earthquake, the important question still remains as to whether the displacements were caused by the dy- 
namic strain associated with the shaking or by the static strain associated with the main fault break. The static strain is the permanent strain field caused by the 33-km-long break on the Coyote Creek fault; the dynamic strain is the transitory strain associated with the seismic waves generated by the earthquake.

For an estimate of the static strain, we use the diagrams of Press (1965). The length of the surface break is taken as $33 \mathrm{~km}$, and the hypocentral depth now assigned by the Seismological Laboratory, California Institute of Technology, is $11 \mathrm{~km}$. For an upper limit of the static strain at distance, we therefore use Press's case in which $L=D$; the far-field strains were calculated by scaling down Press's figures to correspond to an average fault displacement of $30 \mathrm{~cm}$. The resulting static strains at distances of 45,70 , and $50 \mathrm{~km}$ in the directions of the Superstition Hills, Imperial, and San Andreas faults are $4 \times 10^{-7}, 1 \times 10^{-7}$, and $-3 \times 10^{-7}$ respectively, assuming these faults to be parallel to the Coyote Creek fault. The minus sign for the San Andreas fault indicates that the residual static strain induced by the Coyote Creek fault displacement was left lateral (Dr. John McGinley, California Inst. Technology, oral commun., 1968).

The dynamic strain caused by $\mathrm{S}$ waves with a period of approximately $4.3 \mathrm{sec}$, recorded at El Centro near the Imperial fault, was about $1.1 \times 10^{-5}$ (corresponding to a trace amplitude of $4.9 \mathrm{~cm}$ at a period of $4.3 \mathrm{sec}$ on the strong-motion Wood-Anderson instruments). This dynamic strain is two orders of magnitude larger than the static strain at this distance. This fact, in addition to the persuasive argument that the static strain would have led to the opposite sense of displacement on the San Andreas fault, strongly indicates that the dynamic (vibratory) strains rather than the static strain induced the observed ruptures on the distant faults.

\section{SUDDEN DISPLACEMENT VERSUS CREEP}

Even granting that the dynamic strains caused the displacements, the question remains as to whether these displacements took place suddenly or during a period of creep lasting several minutes, hours, or days. After the distant displacements were first noticed, geodetic measurements were repeated at closely spaced intervals to determine if creep was perhaps still taking place (fig. 55); it appears that within the accuracy of the measurements, no creep was occurring at this time on any of the three distant faults except for one possible increment at Superstition Hills (fig. $55 E$ ). The sensitive creepmeter installed subsequently at Superstition Hills further substantiates this conclusion. The observations at Highway 80 indicate that if displacements occurred during a period of creep after the earthquake, this period must have been shorter than 4 days.

If any of the three distant breaks had occurred as sudden ruptures, however, seismic waves would have been radiated. Because the seismographic records of most southern California stations were off scale for several minutes following the Borrego Mountain earthquake, we cannot state with assurance that earthquakes did not occur at the three distant localities immediately following the main event, although it seems unlikely that the magnitudes of such events could have exceeded 4.5 without being detected. However, three lines of evidence suggest that such sudden displacements, if they did occur, were not in any sense "normal" earthquakes :

1. The fault lengths of 22,23 , and $30 \mathrm{~km}$ are much longer than could typically be associated with earthquakes of magnitude less than 4.5 (Wyss and Brune, 1968).

2. Only a very few possible aftershocks could be associated with the three distant faults, in sharp contrast to the usual high aftershock activity accompanying fault breaks of this length. Despite a careful search of seismic records from several stations close to the distant faults, including temporary stations within the Imperial Valley at Obsidian Butte and near Westmoreland, only six small shocks could be found that might possibly have been associated with the Superstitution Hills fault within a month after the earthquake, one small shock that might have been associated with the Imperial fault, and none in the area of the San Andreas fault. A particular search was made of the Hayfield ${ }^{4}$ records for events with short S-P intervals, because the San Andreas fault is much closer to this station than to the Coyote Creek fault, near which the principal aftershock activity occurred. The absence of aftershocks in the vicinities of the distant faults is substantiated by microearthquake surveys in two of these areas by Walter Arabasz on April 20-21. Using a backpack instrument that recorded on smoked paper and operated at a magnification of 100,000 at $20 \mathrm{cps}$, he found no nearby microearthquakes during 13 hours of continuous recording at the Imperial fault near the south end of the fractured segment. Similar but shorter periods of recording farther north along the Imperial fault and in a granite quarry at Superstition Mountain ( $5 \mathrm{~km}$ from the Superstition Hills fault) like-

'For the location of the Hayfield station, see fig. 6 . 
wise revealed little or no microearthquake activity.

3. Another obvious peculiarity of these displacements is the unusually low ratio of average offset to length of rupture. For most earthquakes, when the faulting length is about 20 $30 \mathrm{~km}$, the average offset is about $10-100 \mathrm{~cm}$, whereas the average offset observed here is only $1-21 / 2 \mathrm{~cm}$. In addition, it appears that the breaks may not have been continuous on the San Andreas and Imperial faults.

After the Borrego Mountain earthquake, we concluded (1) that the displacements on the three distant faults occurred rapidly, but with a mechanism of strain release different from that of typical earthquakes associated with fault breaks of these lengths, (2) that this relatively rapid motion commenced with the arrival of the first intense seismic energy from the Borrego Mountain earthquake, and (3) that it probably lasted at most only as long as the strong shaking persisted. The displacements were visualized to have been triggered by strong seismic shaking, which is a previously undocumented mechanism of strain release on active faults.

These three conclusions must be tempered, however, by subsequent events along the Imperial fault. As was mentioned in an earlier section, there was no further movement along the Imperial fault after the Borrego Mountain earthquake until September 30,1971 , when a magnitude 5.3 shock in the Superstition Hills, $37 \mathrm{~km}$ away, was followed by about $1.4 \mathrm{~cm}$ of right-lateral displacement on the Imperial fault at the Highway 80 locality. Fresh cracks were observed along a segment of the fault at least $10 \mathrm{~km}$ long extending from south of Ross Road to north of Robinson Road. But evidence suggests that the faulting did not occur at the same time as the earthquake: 3 days after the earthquake, Brian Tucker (oral commun., 1971) examined the Highway 80 locality and noticed no fresh cracks despite his familiarity with the locality and its history. However, 3 days later, on October 6, 1971, fresh cracks and lateral offsets were obvious to several visiting parties. Repeated subsequent visits to the site revealed no increase in displacement, so we must conclude that virtually all the surface faulting took place between 3 and 6 days after the earthquake. One might argue, of course, that the near coincidence of the two events was fortuitous, but this seems unlikely in view of the two earlier movement episodes, both of which $(1966,1968)$ also occurred in close association with large local shocks. As for the Borrego Mountain earthquake, we are forced to conclude that this later faulting was triggered by the dynamic waves of the nearby shock, but the mechanism of local strain release and its apparent delay remain problematical. Perhaps the Imperial fault was triggered only at depth at the time of the September 30 earthquake, and this dislocation then extended surfaceward only gradually, something like the situation visualized by Smith and Wyss (1968) for the Parkfield displacements and by Burford (this volume) for the Coyote Creek fault displacements. We must now recognize that the same sort of delay may have characterized the 1968 displacement as well; the actual triggering may have occurred at a depth of perhaps $4 \mathrm{~km}$ in the elastically strained sedimentary rocks, with the displacement then propagating to the surface within the following 4 days. Only continuously recording creepmeters will resolve this problem in future events.

Many additional aspects of the mechanics of displacements on the three distant faults remain unexplained. We assume that elastic strain was released by the displacements, but the depth at which this elastic strain had accumulated is problematical. An attractive but unproved hypothesis is that creep is taking place continually in the crystalline basement rocks along these three faults, partly reflecting the unusual semioceanic crust and complex fault pattern of the region (Allen, 1968). Elastic strain is visualized to accumulate only in the overlying thick section of indurated sedimentary rocks, to be relieved intermittently either by episodic creep, by very shallow small earthquakes such as the Imperial earthquake of 1966 (Brune and Allen, 1967a), or by occasional intermediate-sized shocks such as the El Centro earthquake of 1940 on the Imperial fault. Such events may in turn be triggered by externally caused shaking.

What determined the amount of displacement on the three distant faults is another unanswered question. Scholz, Wyss, and Smith (1969) argued that the amount of episodic creep displacement is not a function of the nature of the instigating event, but instead is related to the stress-drop between a constant rupture stress and a constant frictional stress on the fault. Had the tectonic stress accumulated to a critical value, the creep presumably would have started even without instigation by the earthquake, as presumably happened at Worthington Road before the earthquake (fig. $55 B$ ). On the other hand, field evidence may suggest that the total displacements on the three distant faults might have been at least partly a function of the strength of shaking; the larger displacement on the Superstition Hills fault as compared to the Imperial fault may be an indication not of higher stress accumulation but, at least in part, of stronger shaking closer to the source. 
STRESS-DROPS

In order to estimate the stress-drops associated with the displacements on the distant faults, a fault depth must be assumed. We arbitrarily assume a depth of $4 \mathrm{~km}$, equal to about half the thickness of the sedimentary section in the center of the Imperial Valley (Biehler and others, 1964) and corresponding to the depth of transition between stable sliding and stick-slip in the Parkfield model of Scholz, Wyss, and Smith (1969). If the average displacement is assumed to be $1.5 \mathrm{~cm}$, the stress-drop is 0.5 bar, close to (that is one-half of) the value obtained by Brune and Allen (1967a) for the Imperial earthquake of 1966.

\section{TRIGGERING}

We have chosen to use the word "trigger" in connection with the displacements on the Imperial, Superstition Hills, and San Andreas faults in recognition of the fact that one event initiated other events. However, it must be recognized in these examples that the displacements were initiated by a much larger event than the displacements themselves. As was indicated in a previous section, the maximum dynamic strain at El Centro was about $1.5 \times 10^{-5}$, and assuming a fault depth of $4 \mathrm{~km}$ and an average displacement of $1.5 \mathrm{~cm}$ on the nearby Imperial fault, the calculated strain associated with the displacement was only $2.5 \times 10^{-6}$. In contrast, a different type of seismic triggering might be the multiple ruptures of the Alaska earthquake, where small events apparently triggered larger events (Wyss and Brune, 1967).

\section{ENGINEERING IMPLICATIONS}

In the planning of engineering structures adjacent to or across active faults, it has usually been assumed in the past that significant fault displacements would occur only infrequently - perhaps about once every few hundred years on even the most active faults. The documentation of semicontinuous creep along parts of the San Andreas fault has tended to modify this kind of thinking (for example, Wallace, 1970), and the present study emphasizes still further the difficult problems of estimating probabilities of fault displacements. A few years ago, for example, one might have estimated that the San Andreas fault opposite the Salton Sea would break perhaps once every hundred years, the inferred recurrence time for large earthquakes on this segment of the fault. However, if one now assumes, on the basis of the Borrego Mountain experience, that an earthquake of magnitude 6 or greater anywhere in the Imperial Valley area might cause a small displacement on parts of the San Andreas fault, then the estimated chances of fault displacement are considerably higher. The recurrence curve for the Imperial Valley region based on 1934-63 records (Allen and others, 1965) suggests that an earthquake of magnitude 6 or greater should occur about once every $61 / 2$ years. Perhaps the reason that some of the scarplets along the faults look so fresh (fig. 63) is that displacements indeed take place with about this frequency.

Although it does not necessarily follow that active faults in all parts of California would behave in the same manner as the Imperial, Superstition Hills, and San Andreas faults when heavily shaken, it seems clear, nevertheless, displacements occur more frequently than previously recognized along the myriad of active faults that underlie California and other tectonically similar regions. Although many types of engineering structures have sufficient flexibility to withstand fault displacements of a centimeter or two without significant damage, it should be remembered that the disastrous failure of the Baldwin Hills Reservoir in 1963 was caused by a fault displacement of a comparably small amount (Hudson and Scott, 1965).

Lest the impression be left that all faults in earthquake-prone regions should now be suspect of possible small but frequent displacements, we emphasize once again that the three distant faults that moved because of shaking generated by the Borrego Mountain earthquake were all "active" faults in that they showed abundant evidence of repeated Quarternary displacements. Furthermore, the only segments on which displacements took place were clearly delineated by surface exposures (at least in uncultivated areas), and each of the three faults had histories that suggested similar movements within the previous few years. These findings point out the need for thorough geologic studies prior to engineering developments in faulted areas but also give some confidence that even in highly faulted regions dangerous areas can reasonably be differentiated from safe areas.

\section{REFERENCES CITED}

Allen, C. R., 1968, The tectonic environments of seismically active and inactive areas along the San Andreas fault system: Stanford Univ. Pub. Geol. Sci., v. 11, p. 70-82.

Allen, C. R., St. Amand, Pierre, Richter, C. F., and Nordquist, J. M., 1965, Relationship between seismicity and geologic structure in the southern California region: Seismol. Soc. America Bull., v. 55, p. 753-797.

Babcock, E. A., 1970, Basement structure and faulting along the northeast margin of the Salton Sea [abs.]: Geol. Soc. America Abs. with Programs, v. 2, p. 68.

Biehler, Shawn, Kovach, R. L., and Allen, C. R., 1964, Geophysical framework of northern end of Gulf of California structural province: Am. Assoc. Petroleum Geologists Mem. 3, p. 126-143.

Brune, J. N., and Allen, C. R., 1967a, A low stress-drop, lowmagnitude earthquake with surface faulting: The Imperial, California, earthquake of March 4, 1966: Seismol. Soc. America Bull., v. 57, p. 501-514. 
1967b, A micro-earthquake survey of the San Andreas fault system in southern California: Seismol. Soc. America Bull., v. 57, p. 277-296.

Buwalda, J. P., and St. Amand, Pierre, 1955, Geologic effects of the Arvin-Tehachapi earthquake: California Div. Mines Bull. 171, p. 41-56.

Crowell, J. C., 1962, Displacement along San Andreas fault, California: Geol. Soc. America Spec. Paper 71, 61 p.

Dibblee, T. W., Jr., 1954, Geology of the Imperial Valley region, in Jahns, R. H., ed., Geology of southern California; chap. 2, Geology of the natural provinces: California Div. Mines Bull. 170, p. 21-28.

Hope, R. A., 1969, Map showing recently active breaks along the San Andreas and related faults between Cajon Pass and Salton Sea, California: U.S. Geol. Survey open-file rept.

Hubbs, C. L., Bien, G. S., and Suess, H. E., 1960, La Jolla natural radiocarbon measurements I: Am. Jour. Science, Radiocarbon Supplement, v. 2, p. 197-223.

Hudson, D. E., and Scott, R. F., 1965, Fault motions at the Baldwin Hills Reservoir site: Seismol. Soc. America Bull., v. 55, p. $165-180$.
Kovach, R. L., Allen, C. R., and Press, F., 1962, Geophysical investigations in the Colorado delta region: Jour. Geophys. Research, v. 67, p. 2845-2871.

Lomnitz, C., Mooser, F., Allen, C. R., Brune, J. N., and Thatcher, W., 1970, Seismicity and tectonics of the northern Gulf of California region, Mexico - Preliminary results: Geofisica Internacional, v. 10, p. 37-48.

Press, F., 1965, Displacements, strains, and tilts at teleseismic distances: Jour. Geophys. Research, v. 70, p. 2395-2412.

Scholz, C. H., Wyss, Max, and Smith, S. W., 1969, Seismic and aseismic slip on the San Andreas fault: Jour. Geophys. Research, v. 74, p. 2049-2069.

Smith, S. W., and Wyss, Max, 1968, Displacement on the San Andreas fault initiated by the 1966 Parkfield earthquake: Seismol. Soc. America Bull., v. 58, p. 1955-1974.

Wallace, R. E., 1970, Earthquake recurrence intervals on the San Andreas fault: Geol. Soc. America Bull., v. 81, p. 2875-2889.

Wyss, Max, and Brune, J. N., 1967, The Alaska earthquake of 28 March 1964: A complex multiple rupture: Seismol. Soc. America Bull., v. 57, p. 1017-1023.

1968, Seismic moment, stress and source dimensions for earthquakes in the California-Nevada region: Jour. Geophys. Research, v. 73, p. 4681-4694. 


\title{
CONTINUED SLIP ON THE COYOTE CREEK FAULT AFTER THE BORREGO MOUNTAIN EARTHQUAKE
}

\author{
BY R. O. BurFord
}

U.S. Geological Survey

\begin{abstract}
Two alinement arrays designed for the precise determination of continued slip or deformation were established across surface breaks associated with the Borrego Mountain earthquake within a few days of the main shock. One array was established on the north break near the point of maximum initial offset (about $38 \mathrm{~cm}$ ), another on the central break about 6 miles southeast of Ocotillo Wells where the initial offset was about $13 \mathrm{~cm}$. Measurements made over a 33month period show that a complex pattern of relatively small displacement $(0.5 \mathrm{~cm})$ developed at the north site, compared with a simple pattern of larger continued slip (18 cm) at the central site. Apparent logarithmic decrease in slip rate at the central site, a similar decrease in frequency of aftershock occurrence, and the presence of a thick sedimentary cover $(3 \mathrm{~km})$ over basement rocks along the central trace in Lower Borrego Valley indicate that continued postearthquake slip on the central break is largely due to delay in upward propagation of sudden basement-rock displacement through the sediments. Studies of continued slip on the San Andreas fault within Cholame Valley after the 1966 Parkfield earthquakes led other investigators to similar conclusions.
\end{abstract}

\section{INTRODUCTION}

A few days after the Borrego Mountain earthquake, two alinement arrays were installed across the surface fractures. One was placed across the north break at Borrego Mountain, and the other across the central break in Lower Borrego Valley about 6 miles southeast of Ocotillo Wells (fig. 70). The arrays were designed for long-term periodic monitcring of continuing slip on fracture surfaces (termed "afterslip" by Nason, 1969, 1971) and of shallow elastic strain changes in the vicinity of the surface breaks. Initial observations were completed at the Borrego Mountain site on April 19 and at the Lower Borrego Valley site on April 25, 1968. A third array, installed slightly beyond the southeast end of the south break near the Fish Creek Mountains on December 10, 1969, was resurveyed on January 25, 1971. All three alinement arrays will be remeasured at approximately yearly intervals.
A typical alinement-array configuration is shown in figure 71. Experience with similar installations in central California has demonstrated that monuments installed this way are stable enough under usual surface conditions, but the stability of individual monuments has not been tested. A general indication of monument stability is evident in the final results. The contribution of possible monument instability to the total noise in the alinement-survey data is thought to be negligible compared with the noise level introduced by random errors in instrument positioning, pointing, and scale reading.

Angles and alinements were measured with a Wild T-3 theodolite. Typical standard deviations of the changes in mean angle values obtained by comparison of theodolite pointings on different dates cluster about \pm 2 seconds, as determined according to the following formula:

$$
\sigma_{\Delta}=\left(\sigma_{1}^{2}+\sigma_{2}^{2}\right)^{1 / 2}
$$

where $\sigma_{1}$ and $\sigma_{2}$ are the standard deviations of the mean angle values at time 1 and time 2 , and $\sigma_{\Delta}$ is the standard deviation of the change in the mean value of the angle. This error corresponds to an uncertainty of about $\pm 1 \mathrm{~mm}$ in the value of lateral shift at an end station $100 \mathrm{~m}$ from the instrument station. Estimated average positioning errors for theodolite and targets are $\pm 0.1 \mathrm{~mm}$; this estimated error adds to the uncertainty in the value of lateral shift by only an insignificant amount. The average error in the determination of alinement changes for stations between the instrument station and the end stations is estimated to be about $\pm 0.2 \mathrm{~mm}$.

\section{ACKNOWLEDGMENTS}

Installation and measurements of the alinement arrays were completed with the help of field crews and other U.S. Geological Survey personnel. The sites were selected on the basis of preliminary fracture maps that were compiled by geologists of the 


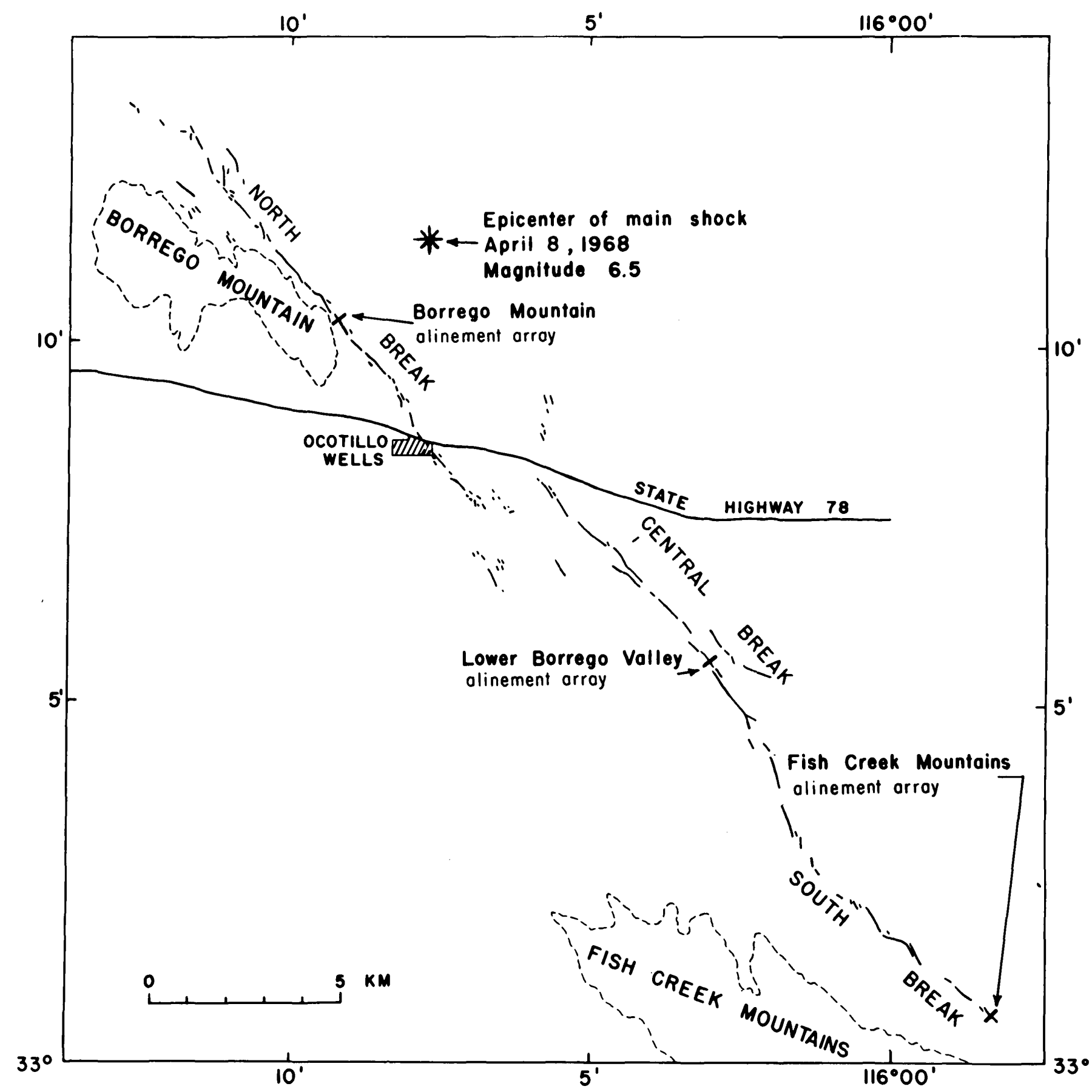

FIGURE 70. - Main surface breaks associated with the Borrego Mountain earthquake of April 1968, and the locations of three alinement arrays established for afterslip investigation.

California Institute of Technology and the U.S. Arthur Grantz, and James C. Savage and from the Geological Survey. The author profited from discus- critical reviews of the manuscript by Robert 0 . sions with Robert M. Hamilton, Malcolm M. Clark, Castle and Robert V. Sharp. This work was sup- 


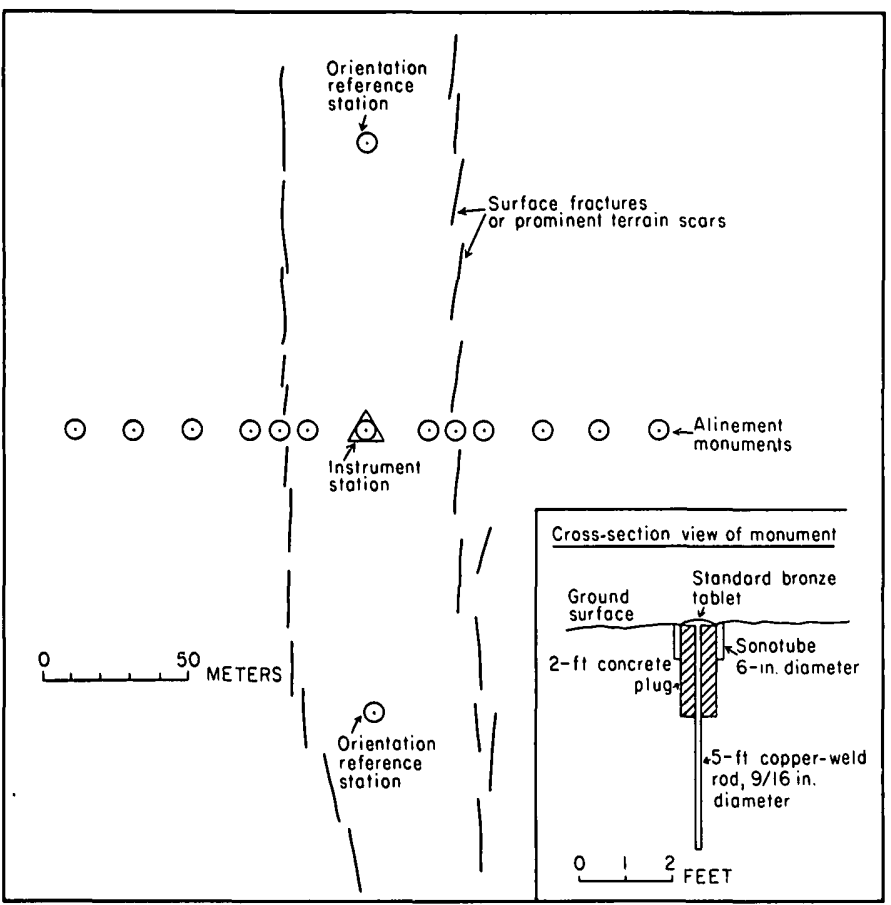

FIGURE 71. - Typical alinement-array configuration. In the inset is a cross section of the monument construction.

ported in part by research funds from the Atomic Energy Commission, Division of Reactor Development and Technology.

\section{RESULTS OF MEASUREMENTS BORREGO MOUNTAIN SITE}

The initial alinement of the Borrego Mountain array was determined on April 19, the 11th day after the main shock. Observations were repeated on April 24, 1968, and again on February 15, May 5, and December 11, 1969. Comparison of each set of repeat measurements with the initial set yielded the results shown in figure 72 .

Abrupt steplike offsets between stations are interpreted as being due to fault slip, whereas lesser offsets comprising a pattern of gradual bending across the terrain are thought to be due either to changes in elastic shear or perhaps to permanent deformation. No attempt has been made to establish definite displacement limits to distinguish between the possible cases.

The general displacement pattern developed during the course of the repeated measurements shows that slight right-lateral slip continued to accumulate across the zone of surface fractures

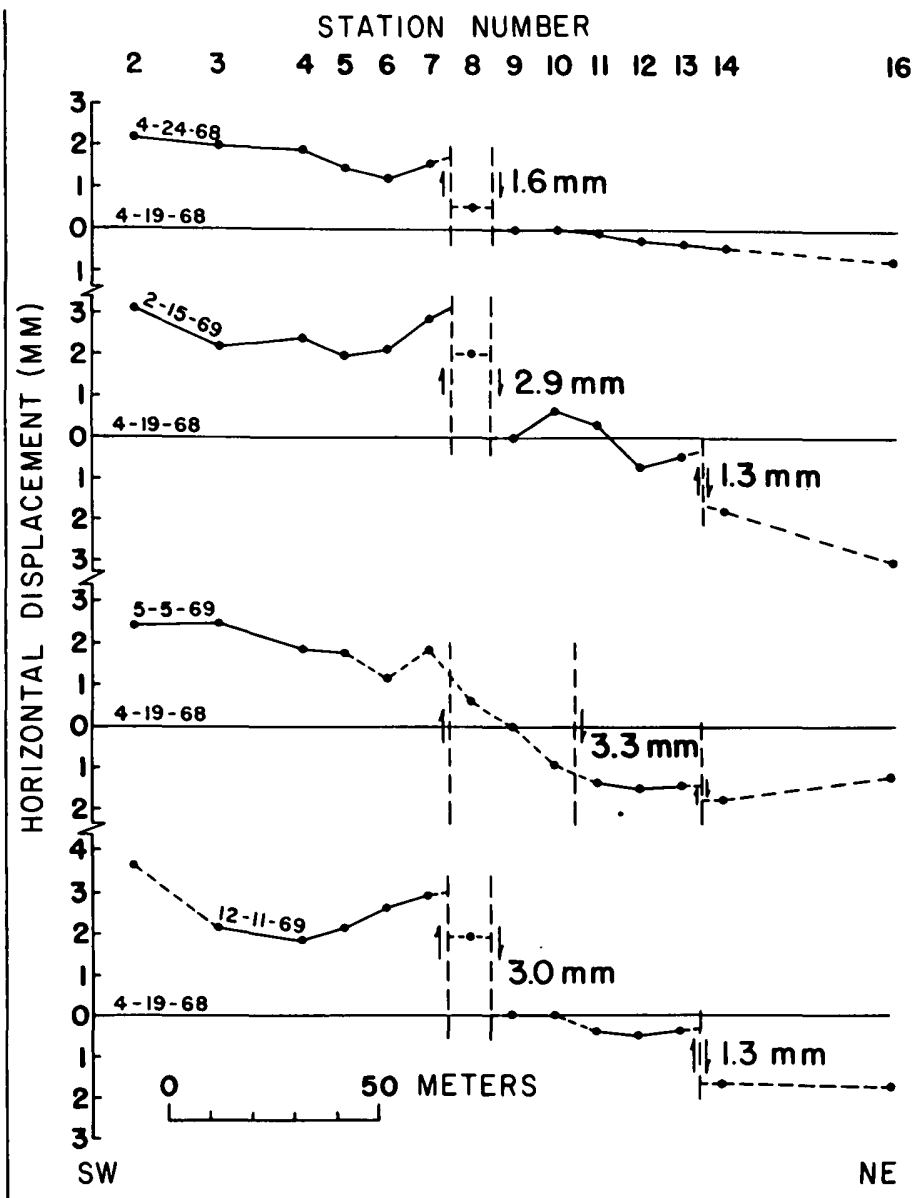

Figure 72. - Plan view of alinement changes at the Borrego Mountain array. Each profile shows the change between April 19, 1968, and the date of the repeat survey shown. Approximate slip values are listed to the right of prominent offsets of the monument line; these offsets are indicated by slip arrows. Dashed lines indicate uncertainty about actual distribution of displacement between points.

generated during the main shock. The slip rate was higher, and slip was concentrated across a relatively narrow zone occupied by faint surface fractures between stations 7 and 9 during the period of the first three sets of observations.

Deformation after the third set of observations on February 15, 1969, was much more complex. The slip rate decreased, and the width of the active slip zone increased to at least $70 \mathrm{~m}$ by the time of the fourth set of observations on May 5, 1969. Right and left slips with frequent reversals through time are evident on a number of possible fractures distributed across the array (fig. 73). The cumulative effect of 


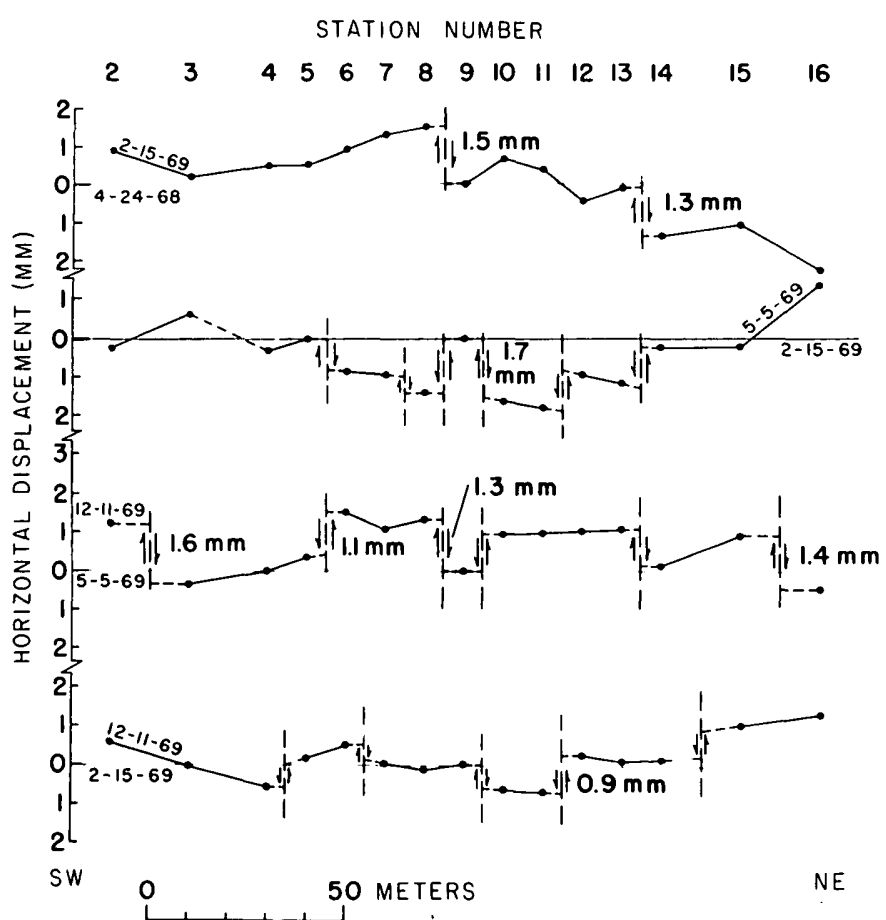

Figure 73. - Incremental alinement changes at the Borrego Mountain array. Approximate slip values are listed to the right of prominent off sets of the monument line; these offsets are indicated by slip arrows. Dashed lines indicate uncertainty about actual distribution of displacement between points.

this period of readjustment across the laterally expanding slip zone was a smoothing-out of the displacement profile and an apparent slight decrease in net right-lateral shift across the entire array (figs. $72,73)$. The results suggest that overfling occurred during the initial offset and early afterslip; it was followed by a readjustment that was presumably produced by partial relaxation of elastic compression in the southwest block and elastic expansion in the northeast block imposed by gradual decay of right-lateral slip along the fault between the array and the northwest end of the surface break.

We began monitoring level changes along the Borrego Mountain alinement array on February 14, 1969. The terrain profile across the fracture zone that is shown in figure 74 is based on the measured heights of the monuments $(1-3 \mathrm{~cm}$ above the ground surface) relative to station 9 . Approximate positions of the surface fractures that were associated with the main shock and the additional lines of afterslip that were detected by alinement changes are indicated. The most obvious surface break associated with the main shock was only slightly active during the period of measurements, while a faint surface fracture noted between stations 7 and 8 on April

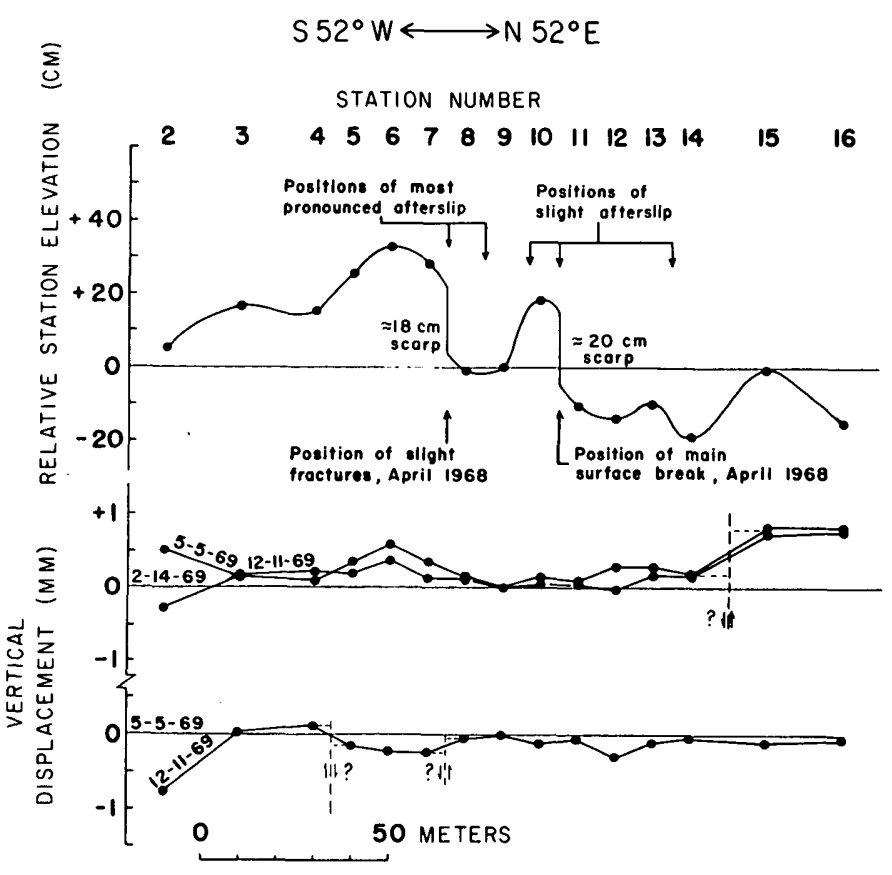

FIGURE 74. - Terrain profile along the Borrego Mountain array, and profiles of relative elevation changes between sets of level readings. Dashed lines indicate uncertainty about actual distribution of displacement between points.

19,1968 , developed into a prominent line of activity (figs. 72-74).

Results of comparisons of successive sets of level readings are shown in the lower two profiles of figure 74. The maximum elevation changes shown are barely significant; most of the changes are within the estimated average error of $\pm 0.25 \mathrm{~mm}$. Pressure ridges or scarps with crests near stations 6 and 15 may have been accentuated by slight upward movement during the period between February 14 and May 5, 1969.

\section{LOWER BORREGO VALLEY SITE}

Initial alinement observations were completed at the Lower Borrego Valley site on April 25, the 17th day after the main shock. Measurements were repeated on February 14, May 4, and December 11, 1969. Results of comparisons of repeated alinement measurements with the initial measurements are shown in figure 75 . In contrast to the results obtained for the Borrego Mountain array, afterslip on the central break accumulated at a high rate. The slip activity was confined to the prominent surface fractures formed during the main shock.

Surficial material in the northeast block shows very little distortion, except for a possible slight left-lateral slip between stations 9 and 10 which developed late in the course of the measurements. An increase in displacement near the fractures on 


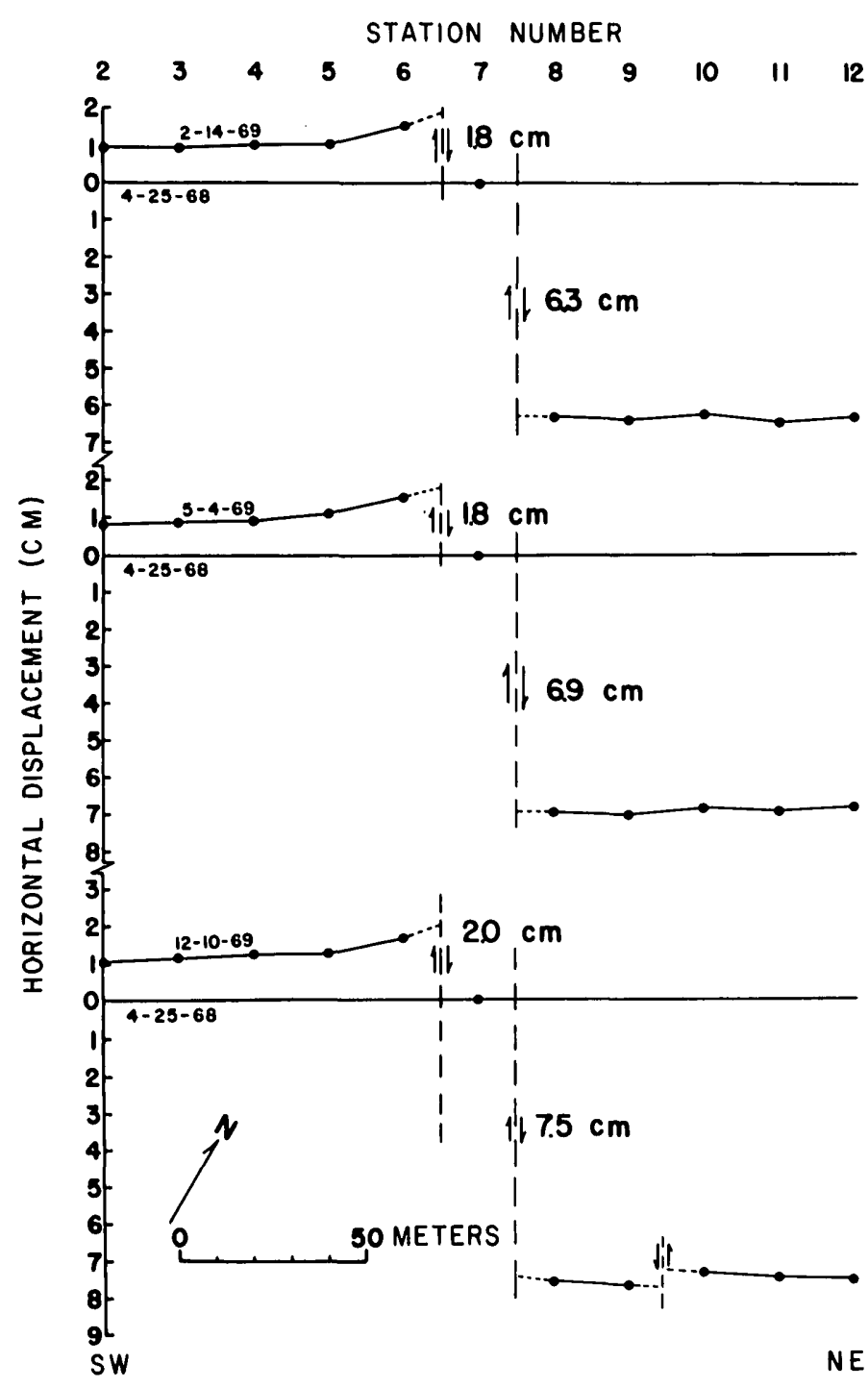

Figure 75. - Plan view of alinement changes at Lower Borrego Valley array. Each profile shows the change between April 25, 1968, and the date of the repeat survey shown. Approximate slip values are listed to the right of prominent offsets of the monument line; these offsets are indicated by slip arrows. Dashed lines indicate uncertainty about actual distribution of displacement between points.

the southwest side is consistent with elastic rebound within surface material, a conclusion that is based on dislocation models developed by Chinnery (1961). Lack of evidence for elastic rebound in the northeast block and the resulting asymmetry in the displacement pattern across the fracture zone remain unexplained; the pattern may be associated with a shallower depth to crystalline basement rocks on the southwest side of the fault.

Cumulative right-lateral slip across the Lower Borrego Valley array is plotted with respect to time elapsed since the main shock in figure 76 . The

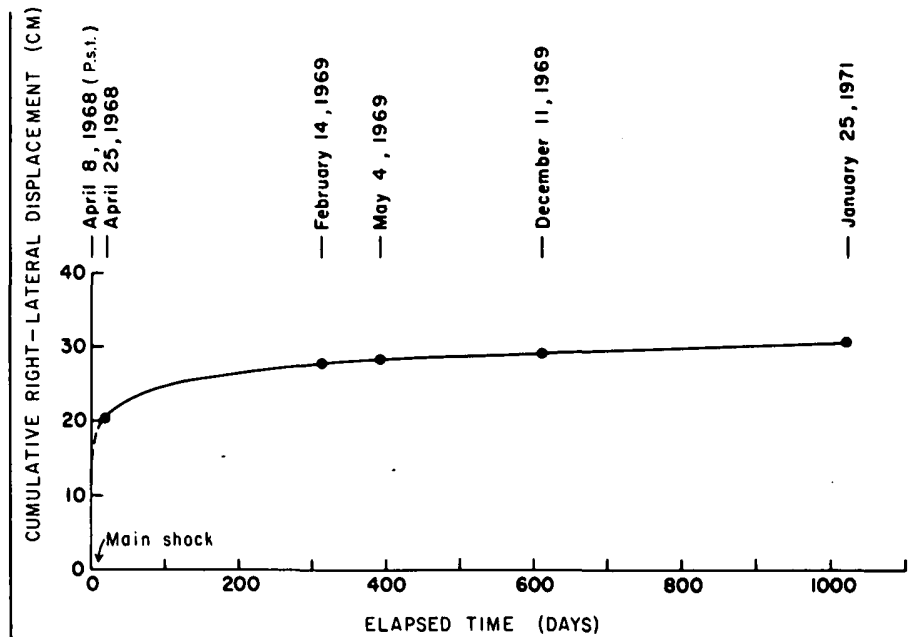

FIgURE 76. - Cumulative right-lateral slip at the Lower Borrego Valley alinement array plotted with respect to time elapsed since main shock.

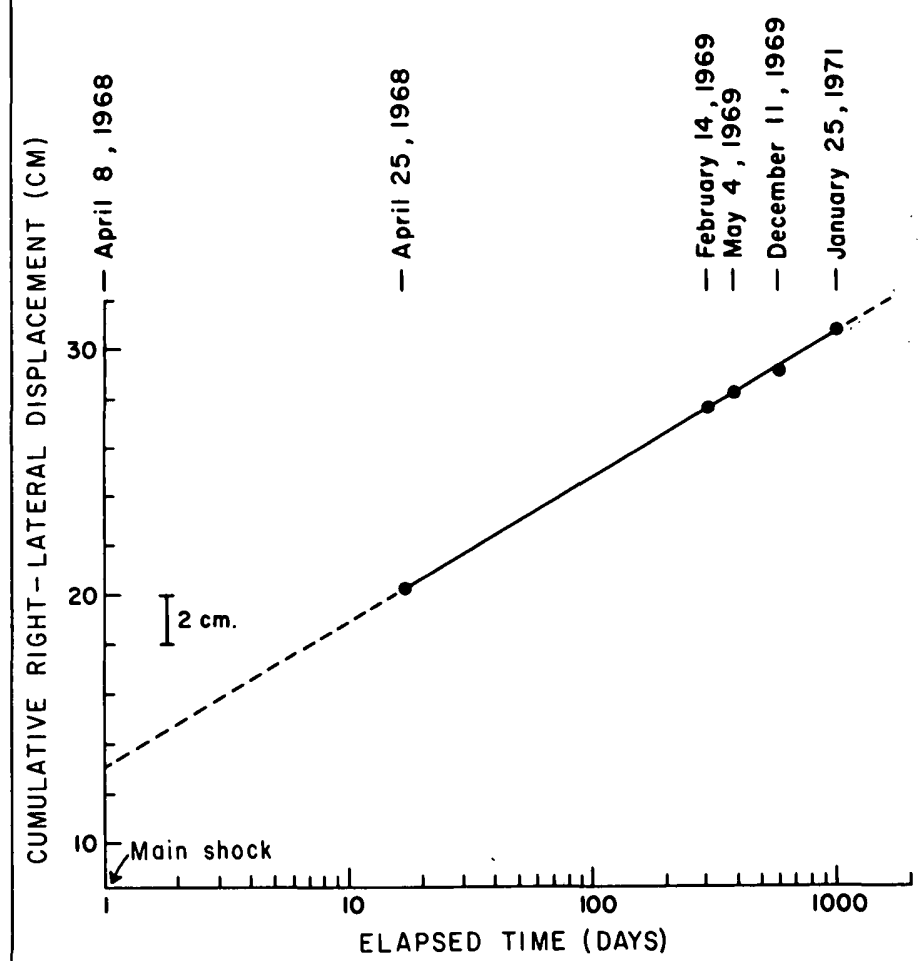

FIGURE 77. - Cumulative right-lateral afterslip at the Lower Borrego Valley alinement array plotted with respect to logarithmic time scale.

initial offset is based on measured slip a day or so after the main shock (Clark, "Surface Rupture along the Coyote Creek Fault," this volume). It is assumed that offsets measured on the fractures at that time took place during a brief period of mainshock slippage, although early afterslip at a rate of about $2 \mathrm{~cm} /$ day probably had already begun (fig. 77). The shapes of the time-slip curves shown 


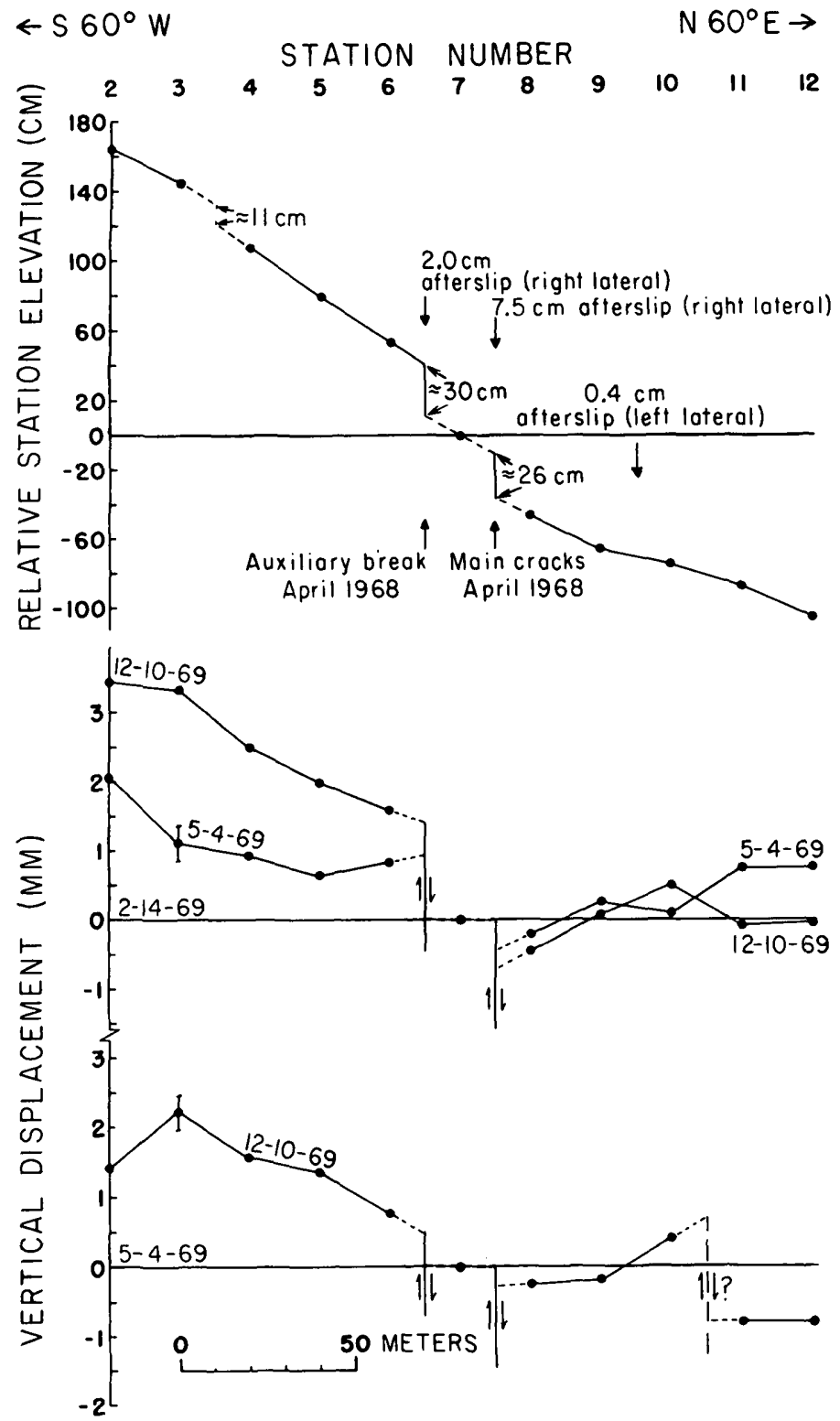

Figure 78. - Terrain profile along the Lower Borrego Valley alinement array, and profiles of relative elevation changes between sets of level readings. Error bars at station 3 indicate the estimated average error of $\pm 0.25 \mathrm{~mm}$ applicable to the measured vertical displacement at each station. Dashed lines indicate uncertainty about actual distribution of displacement between points.

in figures 76 and 77 indicate that the rate decreased logarithmically after the main shock in a manner similar to that demonstrated for slip on the San Andreas fault in Cholame Valley after the 1966 Parkfield earthquakes (Wallace and Roth, 1967; Smith and Wyss, 1968). A data point based on a fifth set of measurements, completed on January 25,1971 , was added to both figures.

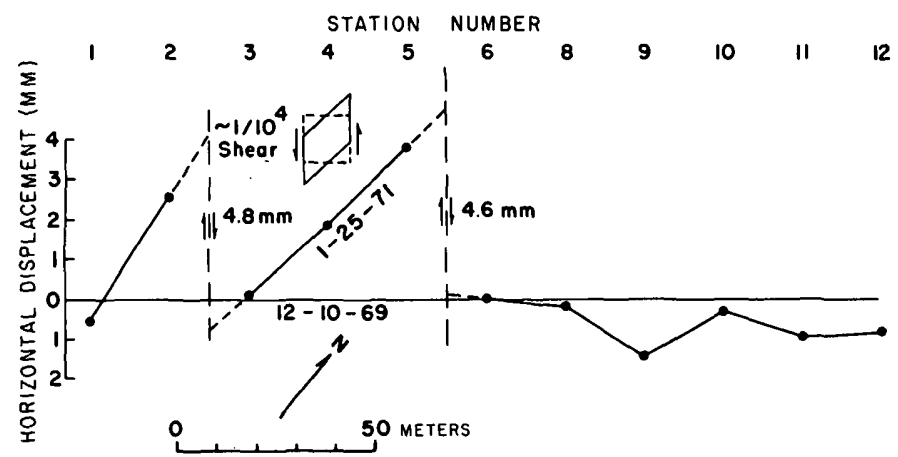

Figure 79. - Plan view of alinement changes at Fish Creek Mountains array from December 10, 1969, to January 25, 1971. Approximate slip values are listed to the right of possible offsets of the monument line; these possible offsets are indicated by slip arrows.

A terrain profile across the fractures at the array site and elevation changes during the period of February 14 to December 10, 1969, are shown in figure 78. A nearly uniform surface slope to the northeast along the array is interrupted by northeast-facing scarps at the lines of prominent surface fractures. Scarp heights compare with those at the Borrego Mountain site. A third minor scarplet may lie between stations 3 and 4 , but its existence was unconfirmed by noticeable surface fractures or by afterslip. A slight broad swell with a crest near station 10 suggests the development of a pressure fold.

Significant vertical slip components accompanied the right-lateral slip recorded during 1969 (fig. 78). Relative vertical movement was generally down on the northeast sides of fractures, a continuation of the prominent Holocene trend developed along most of the Coyote Creek fault. (See pl. 1.)

\section{FISH CREEK MOUNTAINS SITE}

The Fish Creek Mountains array was installed, and initial measurements were completed on December 10, 1969, after Malcolm Clark (oral commun., 1969) observed that fresh surface cracks along the south break. were enlarged by continued slip after the rainy season of the previous spring. The array was positioned slightly beyond the southeast end of the obvious surface fractures, in anticipation of possible southeastward propagation of afterslip along the same trend. Results of the first remeasurement, completed on January 25, 1971, suggest rightlateral slip of about $4 \mathrm{~mm}$ during 1970 , accompanied by possible elastic rebound of about $1 \times 10^{-4}$ within a sliver of material bounded by apparent slip surfaces. The results, shown in figure 79 , remain ques- 
tionable; additional measurements are needed to confirm or clarify the pattern.

\section{TECTONIC IMPLICATIONS}

The marked difference in the level of afterslip activity at the north and central alinement array sites is representative of the general difference in behavior of the two separate breaks. The north break is apparently characterized by a general lack of significant afterslip; this fact confirms the results of early postearthquake measurements by Allen, Grantz, Brune, Clark, Sharp, Theodore, Wolfe, and Wyss, (1968) and by Nason (1969) at a number of sites. In contrast, there is abundant evidence of significant afterslip at a number of localities along the central break (Clark, "Surface Rupture along the Coyote Creek Fault," this volume). The difference in afterslip behavior correlates with differences in thickness of poorly consolidated sediment within the two areas. The available evidence indicates that a blanket of sediment about $3 \mathrm{~km}$ thick covers basement rocks along the central break within Lower Borrego Valley. Basement rocks are exposed immediately southwest of the north break, and the maximum thickness of sediment in the basin on the northeast side of the north break is probably about $1 \mathrm{~km}$ (Hamilton, 1970).

The slip-date decay on the central break corresponds in a general way to the decay in aftershock activity (Hamilton, this volume). A similar history was demonstrated for afterslip and aftershocks of the 1966 Parkfield earthquakes (Eaton, 1967; Wallace and Roth, 1967; Smith and Wyss, 1968). Thus, afterslip on the central break probably was due chiefly to delay in propagation of slip from the granitic basement upward through the layer of sediments.

\section{CONCLUSIONS}

During a 33-month period following the Borrego Mountain earthquake, the initial slip of about $13 \mathrm{~cm}$ on the central break was increased to perhaps as much as $31 \mathrm{~cm}$ through afterslip activity. During this same period, only about $0.5 \mathrm{~cm}$ of additional slip accumulated across the north break adjacent to Borrego Mountain, where the initial slip amounted to about $38 \mathrm{~cm}$.

The pattern of afterslip distribution at the Borrego Mountain site was particularly complex compared to the simple pattern of continued slip across well-defined lines of surface fractures at the Lower Borrego Valley site on the central break.

The afterslip rate at the array site on the central break apparently decreased logarithmically during the period of repeated measurements; this decrease corresponded to a similar decrease in frequency of aftershock occurrence.

The presence of a thick blanket of sedimentary cover over basement rocks along the central break in Lower Borrego Valley and an afterslip and aftershock history similar to that established for the Parkfield earthquakes of 1966 indicate that afterslip on the central break probably was due largely to a delay in propagation of sudden basement-rock displacement through the sediments.

\section{REFERENCES CITED}

Allen, C. R., Grantz, Arthur, Brune, J. N., Clark, M. M., Sharp, R. V., Theodore, T. G., Wolfe, E. W., and Wyss, Max, 1968, The Borrego Mountain, California, earthquake of 9 April 1968 - a preliminary report: Seismol. Soc. America Bull., v. 58, no. 3, p. 1183-1186.

Chinnery, M. A., 1961, The deformation of the ground around surface faults: Seismol. Soc. America Bull., v. 51, no. 3 , p. $355-372$.

Eaton, J. P., 1967, Instrumental seismic studies, in The Parkfield-Cholame, California, earthquakes of June-August 1966: U.S. Geol. Survey Prof. Paper 579, p. 57-65.

Hamilton, R. M., 1970, Time-term analysis of explosion data from the vicinity of the Borrego Mountain, California, earthquake of 9 April 1968: Seismol. Soc. America Bull., v. 60 , no. 2 , p. $367-381$.

Nason, R. D., 1969, Continuing fault movement after earthquakes: EOS (Am. Geophys. Union Trans.), v. 50, no. 4 , p. 252 .

1971, Instrumental monitoring of postearthquake fault movements (afterslip), in The San Fernando, California, earthquake of February 9, 1971: U.S. Geol. Survey Prof. Paper 733, p. 89-90.

Smith, S. W., and Wyss, Max, 1968, Displacement on the San Andreas fault subsequent to the 1966 Parkfield earthquake: Seismol. Soc. America Bull., v. 58, no. 6, p. 1955-1973.

Wallace, R. E., and Roth, E. F., 1967, Rates and patterns of progressive deformation, in The Parkfield-Cholame, California, earthquakes of June-August 1966: U.S. Geol. Survey Prof. Paper 579, p. 23-40. 


\title{
HOLOCENE ACTIVITY OF THE COYOTE CREEK FAULT AS RECORDED IN SEDIMENTS OF LAKE CAHUILLA
}

\author{
By Malcolm M. Clark, Arthur Grantz, and Meyer Rubin \\ U.S. Geological SURVEY
}

\begin{abstract}
Much of the late Holocene history of the southern part of the Coyote Creek fault, including the part that ruptured during the magnitude 6.4 Borrego Mountain earthquake of 1968 , can be inferred from progressive vertical deformation of the flat-lying sediments deposited in Holocene Lake Cahuilla, which covered parts of the fault until at least 800 years ago.

The recurrence interval for tectonic events like that of 1968 along the Coyote Creek fault was determined by comparing vertical components of displacement of 1968 with earlier movement recorded in offset sediments of Lake Cahuilla. Pre-1968 displacements were measured from two structural profiles across the central break of 1968 and from a trench across a branch of this break. The offset strata were dated by $\mathrm{C}^{14}$. Fault off sets measured in the two profiles suggest that during the last 860 years, 1968 -size events occurred every 160 or 190 years. Total vertical deformation (fault off set plus adjacent bending) measured across the branching break suggests that during the last 3,080 years, 1968-size events occurred every 205 years. Vertical fault offsets alone across this same branching break suggest that during the last 1,230 years, 1968-size events occurred every 195 years. Thus, three sets of $\mathrm{C}^{14}$-controlled structural measurements suggest a recurrence interval of roughly 200 years for tectonic events like that of 1968 . This recurrence interval is compatible with those intervals estimated from historic seismicity of the San Jacinto fault zone and from long-term slip rates and historic faulting of the San Andreas fault north of the Transverse Ranges.

Rates of late Holocene strike-slip displacement along the Coyote Creek fault were derived in two different ways from measured rates of vertical displacement. Both methods suggest that the strike-slip rate is about $3 \mathrm{~mm} / \mathrm{yr}$, a value compatible with Sharp's (1967) estimated minimum rate of $2.5 \mathrm{~mm} / \mathrm{yr}$ for the San Jacinto fault zone during the Pleistocene.

Structural profiles and trenches also show that total vertical deformation is a combination of slip at the fault and inelastic bending of equal or greater amount within a zone $2-50 \mathrm{~m}$ wide or more on either side of the fault. Thus, at least in poorly consolidated deposits, estimates of seismic moment or stress-drop at faults during earthquakes may be in error by a factor of 2 or more if they are based solely on fault off sets.

The trenches and natural exposures reveal that most late Holocene faulting occurred repeatedly within zones $1 \mathrm{~m}$ or

less in width along the same traces. Two exposures show recurrent fault displacement along a single trace during the last 2,400-3,000 years. In one location at least three and possibly 17 episodes of faulting have taken place during the last 3,000 years along a planar zone only a few tens of millimeters wide. A few new fractures, however, do appear in the fault zone. A conspicuous new fracture that branches from a trace showing repeated late Holocene movement broke 1,650-year-old sediments for the first time in 1968 .

\section{INTRODUCTION}

Tectonic deformation of Holocene strata amenable to $\mathrm{C}^{14}$ dating has yielded valuable information about timing, position, and rates of Holocene activity along those parts of the Coyote Creek fault that ruptured during the Borrego Mountain earthquake of April 9, 1968 (fig. 80, 81; pl. 1). Most of the information was obtained from three trenches excavated across the central break and its branches (see Clark, "Surface Rupture Along the Coyote Creek Fault," this volume) in November 1969. These trenches showed progressively greater vertical offset of older strata. The oldest dated layer (about $3,000 \mathrm{yr}$ B.P.) is offset nearly three times as much as the base of the youngest ( $860 \mathrm{yr}$ B.P.). Natural exposures of Holocene strata elsewhere along the 1968 rupture show evidence of pre-1968 faulting in some places and evidence of lack of pre-1968 movement in others.

Displacement rates and recurrence intervals described here are based on local vertical components of movement along a predominantly strike-slip fault. This approach was taken for several reasons: (1) We could not find offset geologic features from which pre-1968 horizontal displacements could be dated; (2) vertical displacements are locally large along the Coyote Creek fault (Clark, "Surface Rupture Along the Coyote Creek Fault," this volume), and the vertical component of displacement in 1968, although variable, was everywhere in the same sense as in the recent past; (3) shelly sediments deposited in
\end{abstract}


Holocene Lake Cahuilla provide a means of dating small, very recent progressive vertical offsets along the central and south breaks of the Coyote Creek fault.

The use of vertical offset as a proportional measure of horizontal or net displacement rates and as a basis for determining recurrence intervals for slip on the Coyote Creek fault rests on the assumption that the pattern of slip in 1968 was typical of prior late Holocene events. This assumption can, at present, only be evaluated by the internal consistency of our data and by their agreement with other kinds of information on displacement rates on the Coyote Creek and related faults. Although we feel that this assumption is probably valid for the Coyote Creek fault, we recognize that it cannot be rigorously tested until other sorts of data become available. (For example, the results of long-term geodetic monitoring of vertical and horizontal movement across the fault.) We also tacitly assume that all displacements at the fault result from sudden slip accompanied by earthquakes and by associated preearthquake and postearthquake tectonic creep similar to that associated with the earthquake of April 1968. Regardless of the validity of these assumptions, our data provide useful information on the history of vertical deformation along the Coyote Creek fault.

The shelly strata in which recent vertical displacements along the 1968 breaks could be measured were deposited in Holocene Lake Cahuilla. This fresh-water lake occupied the Salton Basin and covered part of Lower Borrego Valley from at least 1,900 years ago until perhaps as recently as $220 \pm 100$ years ago, according to $\mathrm{C}^{14}$ dates of charcoal, pelecypods, and calcareous tufa associated with shorelines at high stands of the lake (reported in Hubbs and others, 1960, 1963, and 1965 and Hubbs and Bien, 1967). Prominent well-preserved beaches and waterlines of high stands lie 12.8-13.7 $\mathrm{m}$ above sea level (fig. $81 ;$ pl. 3 ; Stanley, 1963). This highest shoreline roughly follows the 40 -foot contour on plate 1 . Shorelines of an older lake, considered to be late Pleistocene by Stanley $(1963,1966)$, have been detected south of the area of the 1968 rupture at about $49 \mathrm{~m}$ above sea level. We have not identified the deposits and shorelines of this older lake in the area of the 1968 rupture, nor recognized its deposits in the trenches.

The highest shoreline of Holocene Lake Cahuilla is not obviously displaced where it crosses the 1968 rupture near location 19 on plate 1 . However, the uncertainty of the horizontal position of the shoreline at this place is on the order of 10-15 m, so any horizontal displacement of the shoreline less than about $15 \mathrm{~m}$ would be difficult to recognize.
The youngest deposit of Lake Cahuilla (860 yr B.P.) in Lower Borrego Valley, called layer $A$ in this report, is an important datum for measuring and estimating the latest Holocene activity of the Coyote Creek fault. This deposit is a prominent whitish shelly layer as much as $0.5 \mathrm{~m}$ thick that is conspicuous both in the field and on aerial photographs (fig. 80). In spite of its youthfulness, much of it has been reworked or entirely removed by erosion or buried by waterborne and windborne mud, silt, and sand. Where layer A is exposed, pelecypod and gastropod shells are abundant on the surface. Erosion of layer A from the top of the locally upwarped sediments on the northeast side of the central break of 1968 at location 22.5 on plate 1 indicates uplift of this small area between the time that Lake Cahuilla withdrew, less than 860 years ago, and 1968. Similarly, prominent scarps cutting layer $A$ at the central break of 1968 clearly reveal vertical displacement along the fault between 860 years ago and 1968.

The displacement rates and recurrence intervals derived in this report are based on $\mathrm{C}^{14}$ ages with analytical uncertainties that range from \pm 200 to \pm 600 years, or from \pm 10 percent to \pm 23 percent of the nominal ages. These uncertainties are a measure of analytical precision. They cannot tell us how close the stated ages are to actual ages, nor do they necessarily encompass the actual ages. For these reasons, only the nominal $\mathrm{C}^{14}$ ages, without uncertainty ranges, were used in the calculations described here.

\section{ACKNOWLEDGMENTS}

Many people contributed to this investigation and report. R. V. Sharp helped sketch the walls of all trenches, and J. E. Kahle, of the California Division of Mines and Geology, helped collect shells for $\mathrm{C}^{14}$ dating and assisted in surveying the profiles. Tom J. Allen of Borrego Springs kindly loaned us a surveyor's level for the profiles. The U.S. Bureau of Land Management, the Southern Pacific Land Co., and its lessee, the Landmark Corp. of San Diego, were very cooperative and readily gave permission to dig trenches on their lands. We greatly appreciate the ideas and comments of our Geological Survey colleagues, K. R. Lajoie, R. V. Sharp, J. H. Healy, R. D. Brown, Jr., and R. E. Wallace.

\section{HISTORY OF FAULT DISPLACEMENTS AND RECURRENCE INTERVALS CENTRAL BREAK}

Displacements of the Coyote Creek fault during the last 860 years are recorded by offset and warping of layer $A$ at the central break of the 1968 earthquake. (See fig. 82.) For a distance of about $4 \mathrm{~km}$ 


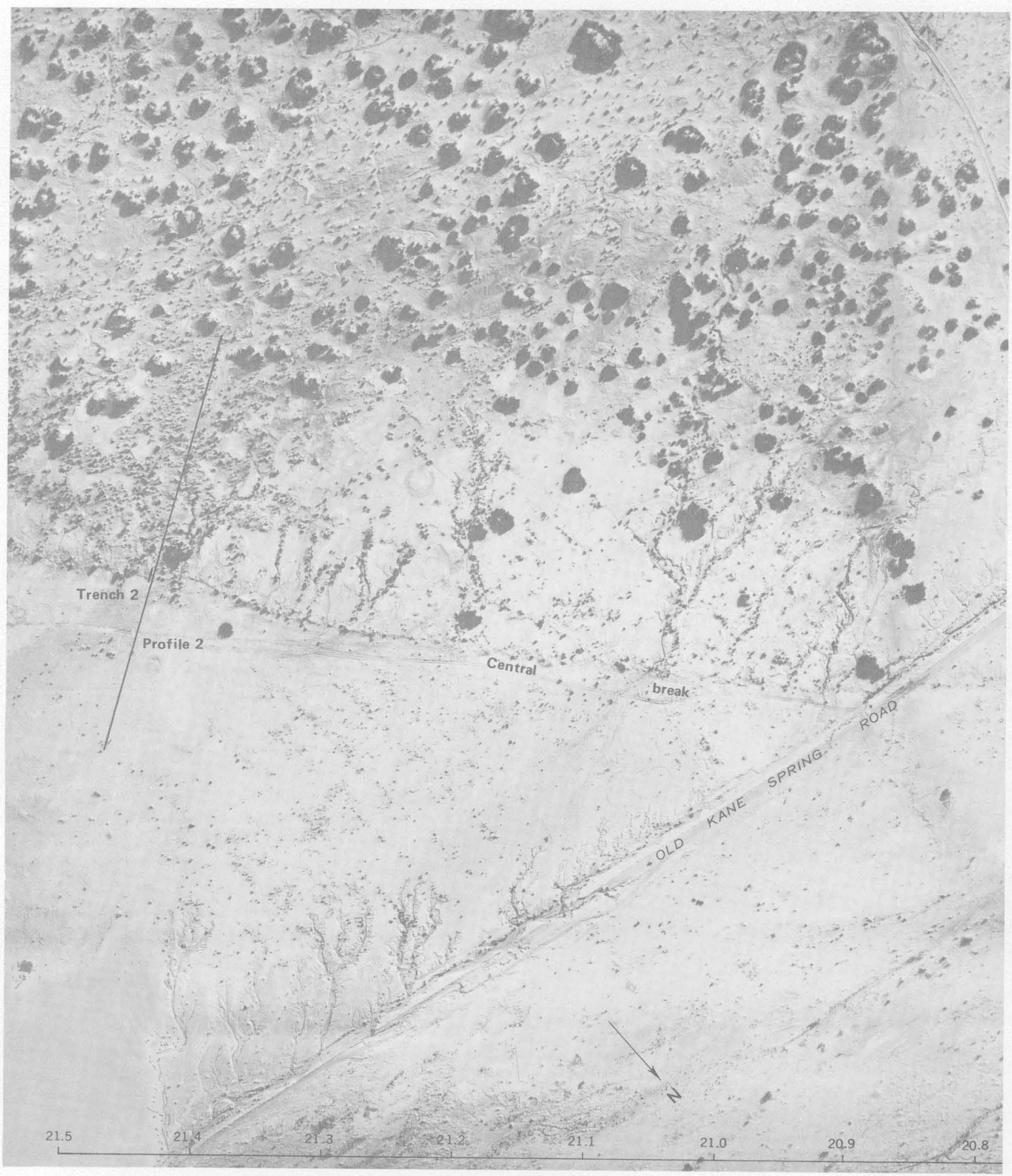

FIGURE 80. - Vertical aerial photographs of central and branching breaks of the 1968 earthquake at Old Kane Spring Road showing a shallow graben between the breaks and the location of trenches 1, 2, and 4 and profiles 1 and 2 . (These photographs were taken in April 1969, about 1 year after the earthquake. Fig. 37 shows the same area a few weeks after the earthquake and gives a general description of this scene. Numbers along the bottom of the photographs refer to the coordinate system of pl. 1.) Heavy rains of July 1968 and January 1969 enlarged tectonic fractures of the branching break to gaping fissures (dark lines). The sediments in trench 1 record repeated late Holocene offsets along the branching break, whereas the 


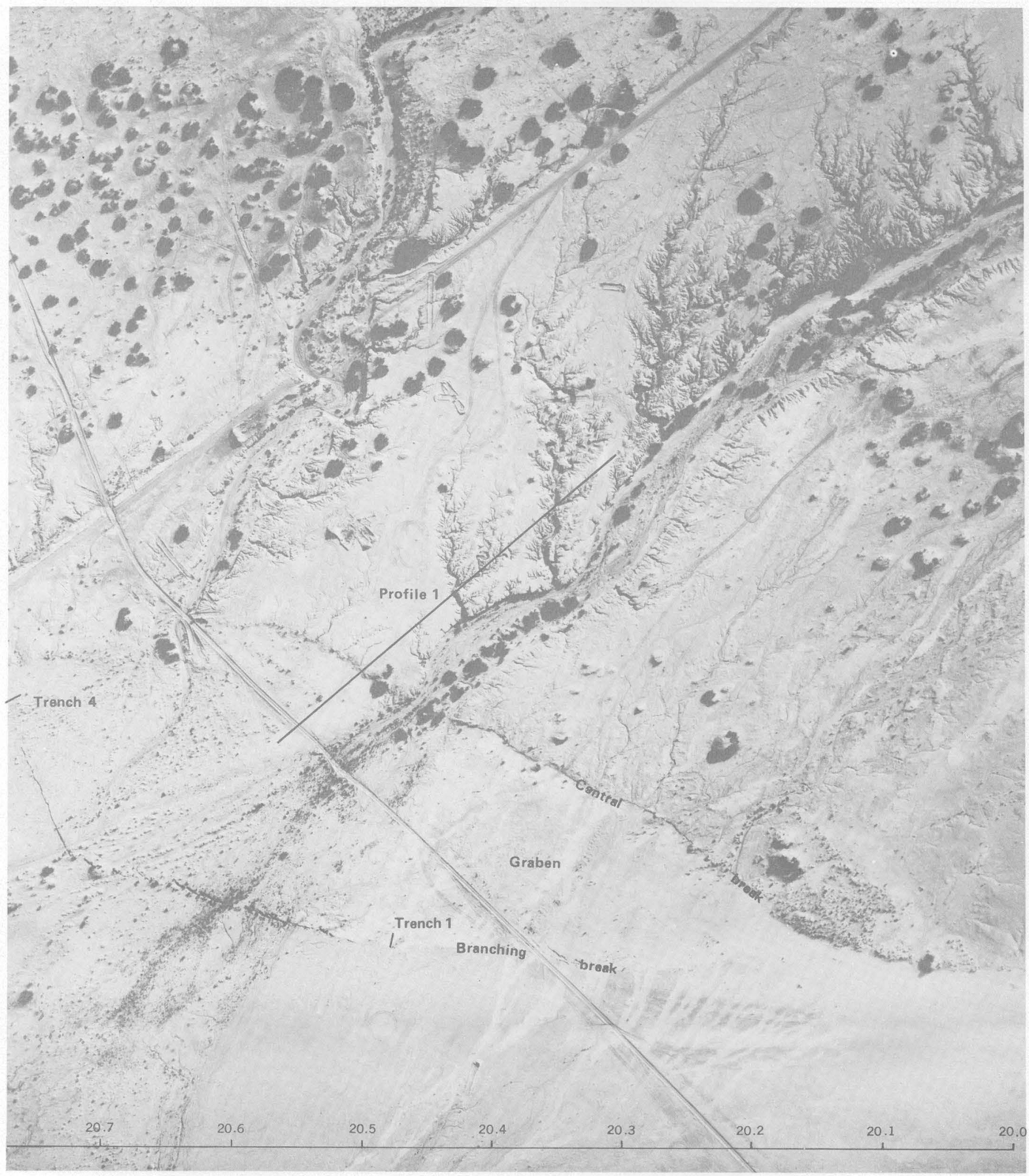

100 METERS

1968 fracture crossed by trench 4 broke the upper $3 \mathrm{~m}$ of sediments for the first time. Dendritic light-toned strips northeast of the branching break in the bottom part of the right-hand photograph are topographically high remnants of the youngest deposit (layer A) of Lake Cahuilla. This whitish shelly layer stands as much as $0.5 \mathrm{~m}$ above the adjacent surface, which has been lowered by wind erosion. These strips may represent topography inverted from channels beneath which the whitish lake beds became slightly indurated. Aerial photographs by Light Photographic Squadron 63, U.S. Naval Air Station, Miramar, Calif. 


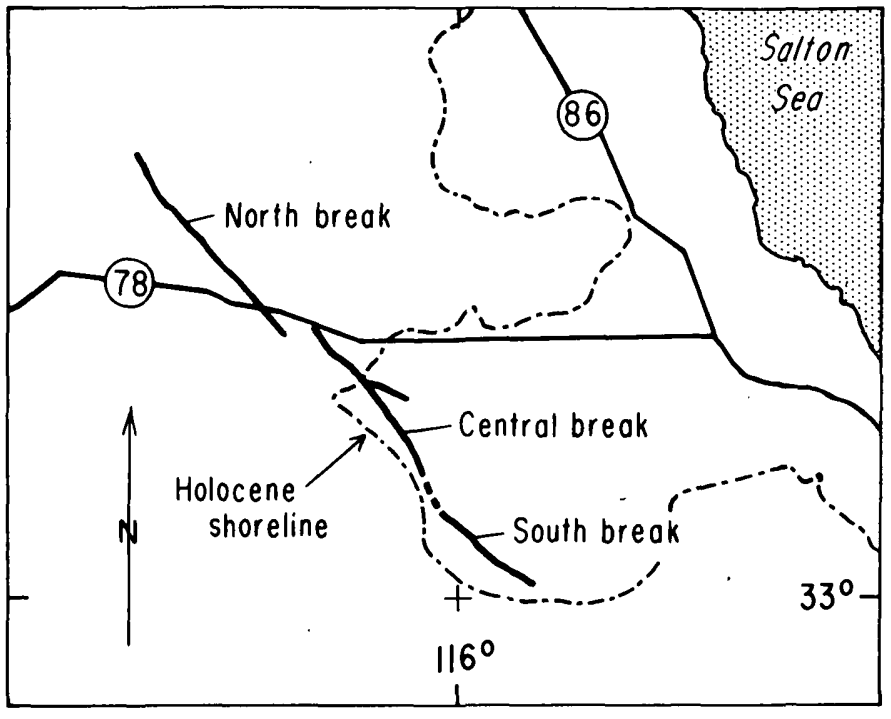

$0 \quad 10 \quad 20 \quad 30 \mathrm{KM}$

FIGURE 81. - Index map showing the three principal breaks of the 1968 rupture of the Coyote Creek fault. Dashed line shows the highest shoreline of Holocene Lake Cahuilla (modified from Strand, 1962; Rogers, 1965; and Jennings, 1967).

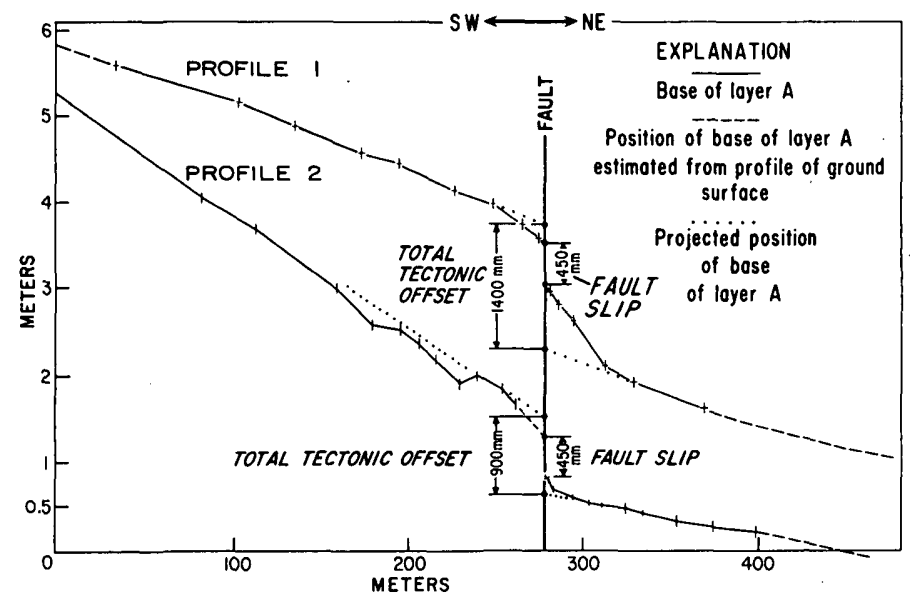

Figure 82. - Structural profiles across the prominent scarp along the central break of 1968 . The profiles are $930 \mathrm{~m}$ apart and show the base of the youngest bed of Holocene Lake Cahuilla (layer A); locations are shown in figure 80. Of the total height of the scarp, $450 \mathrm{~mm}$ at both profiles is estimated to be due to fault slip at the face of the scarp during the last 860 years. (Measured with the help of J. E. Kahle, California Div. Mines and Geology.)

southeast from its intersection with the Lake Cahuilla shoreline (locs. 19-23, pl. 1), the central break of 1968 follows a low pre-1968 scarp at which layer A was broken and deformed during both the 1968 and earlier tectonic events. The scarp itself was probably very low or nonexistent when layer
A was deposited. If it had been as high then as it is now, wave and current action and perhaps slumping would probably have caused local changes in thickness or texture of clay and silt strata deposited on it, but no obvious evidence of such changes has been found in layer $\mathrm{A}$.

Two structural profiles across this scarp (fig. 82), drawn on the base of layer A, show that both fault slip and tectonic bending (drag) within about $50 \mathrm{~m}$ of the fault contributed to the vertical displacement of the layer at the break. If layer $A$ was indeed planar when deposited, one of these profiles indicates $900 \mathrm{~mm}$, and the other $1400 \mathrm{~mm}$, of total vertical deformation across the scarp since the layer was deposited some 860 years ago. For both profiles, about $450 \mathrm{~mm}$ of this vertical movement is due to slip at the fault itself. (See fig. 82.) The ratios of vertical drag to vertical fault slip in layer $A$ at the central break in profiles 1 and 2 are $2: 1$ and $1: 1$, respectively. The difference between them evidently represents variation in the vertical component of past slip along the Coyote Creek fault analogous to that found along the surface breaks of 1968. (See pl. 1.) The average rate of vertical fault slip at these profiles during the last 860 years is $0.5 \mathrm{~mm} / \mathrm{yr}$. For this period the rate of total vertical deformation, slip plus drag, at profile 1 is $1 \mathrm{~mm} / \mathrm{yr}$ and at profile $2,1.6 \mathrm{~mm} / \mathrm{yr}$.

The number of 1968-size events required to form the present scarp since layer A was deposited some 860 years ago, and the recurrence interval for such events during the last several hundred years, can be approximated from the estimated vertical fault slip measured at the base of layer $A$ at profiles 1 and 2 $(450 \mathrm{~mm})$ and the vertical component of slip produced at the fault scarp during the 1968 earthquake. The vertical component of slip on the central break resulting from the 1968 event, as measured in 12 places near profiles 1 and 2 (that is, between locations 19.5 and 22.1 in pl. 1), ranges from 0 to $100 \mathrm{~mm}$. (See pl. 1.) Most readings, however, are between 30 and $100 \mathrm{~mm}$, and the readings of $0 \mathrm{~mm}$ are on fractures with displacements that elsewhere are as much as 80 and $100 \mathrm{~mm}$. The variations in slip are due mainly to the en echelon arrangement of the individual fractures that constitute much of the fault break in this area. The mean of the average values of slip at each of the 12 field stations near profiles 1 and 2 is about $60 \mathrm{~mm}$, the mean of the maximum values of slip is about $70 \mathrm{~mm}$, and the largest individual offsets measured were $100 \mathrm{~mm}$. Because of the en echelon character of the fractures and because small vertical offsets are difficult to recognize and measure, the mean of the maximum 
values $(70 \mathrm{~mm})$ and in particular the maximum observed values $(100 \mathrm{~mm})$ are thought to be more representative of the total vertical component of slip than the mean of average values $(60 \mathrm{~mm})$. By using $450 \mathrm{~mm}$ as the vertical fault slip of layer $A$ in the last 860 years and $100 \mathrm{~mm}$ as the vertical fault slip produced by the 1968 event, the number of 1968-size events required to produce the scarp at the central break of 1968 is calculated to be $4.5(450 \mathrm{~mm} / 100 \mathrm{~mm})$. If $70 \mathrm{~mm}$ is used as the 1968 fault slip, the number of required events would be $6.4(450 \mathrm{~mm} / 70 \mathrm{~mm})$. From these figures the recurrence interval for 1968-size events during the last 860 years near profiles 1 and 2 would be either 190 years $(860 \mathrm{yr} / 4.5)$ or 130 years $(860 \mathrm{yr} / 6.4)$. (See table 15.)

We have no objective way to evaluate. which of the preceding is the better value, but because of the difficulty of measuring total slip on en echelon fractures, we tentatively favor the 190-year interval or the average of the 190- and 130-year intervals, which is 160 years. A similar calculation for the total vertical tectonic deformation (slip plus drag) across the central break cannot be made because the total vertical deformation that occurred at profiles 1 and 2 in 1968 cannot be distinguished from the total deformation of layer $A$ in the absence of precise pre-1968 level lines across the central break.

\section{BRANCHING BREAK}

Data on fault displacements older than layer A ( 860 yr B.P.) were obtained at the branching fault break near profile 1 (locs. 20.02-20.67, fig. 80). The terrane between this and the central break has been relatively downdropped, and layer $\mathrm{A}$ has been preserved in the shallow graben between the breaks. It has also been preserved west of the graben, and its change in level at the central break is reflected topographically in a $0.5-1.5-\mathrm{m}$-high east-facing scarp. It has been stripped, however, from most of the relatively upthrown terrane east of the graben. The change in level at the branching break is now represented topographically by isolated mounds east of the graben with caps of layer $A$ that survive as erosional remnants as much as $0.5 \mathrm{~m}$ higher than the general surface. Some of these mounds lie within $1 \mathrm{~m}$ of the branching break.

Late Holocene recurrence intervals for seismotectonic events on the branching break are probably applicable to the central break because these breaks share the downward displacement of the graben between them and because the branching break of 1968 is relatively short $(700 \mathrm{~m})$ and rejoined the central break at both ends. The graben appears to move regularly downward along both bounding faults during each seismotectonic event. The evidence for this coordinated movement is that the ratio of total vertical deformation of the base of layer $\mathbf{A}$ to measured or reconstructed total vertical deformation in 1968 across each bounding fault is essentially the same. (See table 13.) This means that the total vertical deformation along both faults in 1968 was typical of vertical deformation since deposition of layer $\mathrm{A}$.

TABLE 13. - Vertical deformation along faults bounding the graben between the central break and branching break shown in figure 80

Ratio of vertical drag to vertical fault slip ("offset

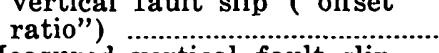

Measured vertical fault slip in 1968 (see text)

Measured total vertical deformation in 1968 (see text).

mation in 1968 (see text)
Total vertical deformation in

1968 estimated from off set

ratio and fault slip in $1968 \ldots .210-300 \mathrm{~mm} \quad 100 \mathrm{~mm}$

Total vertical deformation since

deposition of layer A..............

Ratio of total vertical de-

formation, postlayer $\mathrm{A}: 1968$.

$\begin{array}{cc}\begin{array}{c}\text { Central break } \\ \text { at profile 1 } \\ \text { (fig. 82) }\end{array} & \begin{array}{c}\text { Branching break } \\ \text { at trench } 1 \\ \text { (fig. 8s) }\end{array} \\ 2: 1 & 1: 1 \\ 70-100 \mathrm{~mm} & 50 \mathrm{~mm} \\ \cdots \cdots & 100 \mathrm{~mm} \\ & \\ 210-300 \mathrm{~mm} & 100 \mathrm{~mm} \\ 1,400 \mathrm{~mm} & 560 \mathrm{~mm} \\ 4.7-6.7: 1 & 5.6: 1\end{array}$

At the branching break, vertical slip associated with the 1968 earthquake was about $50 \mathrm{~mm}$, and the land surface east of the break appeared to be roughly 50-150 $\mathrm{mm}$ higher than the surface of the depressed block west of it. Precise determination of the difference in elevation is difficult because the smoothest parts of the surface in the vicinity of the break contain at least $100 \mathrm{~mm}$ of relief. The pattern of surface fractures also suggested that the horizontal was comparable to the vertical displacement in 1968, but this movement could not be accurately measured. Because pre-1968 microdrainage patterns showed no obvious signs of the difference in elevation of the land surface across the branching break, it appears that the 1968 event caused the 50$150 \mathrm{~mm}$ of vertical displacement found across the break after the earthquake. Here, as near profiles 1 and 2, the uncertainty in the total 1968 displacement results from our inability to determine the configuration of the pre-1968 surface.

Trench 1 (fig. 80), excavated across the branching break in November 1969, revealed a clear record of pre-1968 vertical deformation. It exposed the base of layer $\mathrm{A}$, which was found to have been offset vertically a total of $560 \mathrm{~mm}$. Part of the offset was due to fault slip at the break (including about $50 \mathrm{~mm}$ of offset from 1968), and part was due to bending of the strata within a zone $2 \mathrm{~m}$ wide on either side of the break. (See fig. 83.) Deeper strata exposed in the trench are offset and flexed progressively greater amounts. 


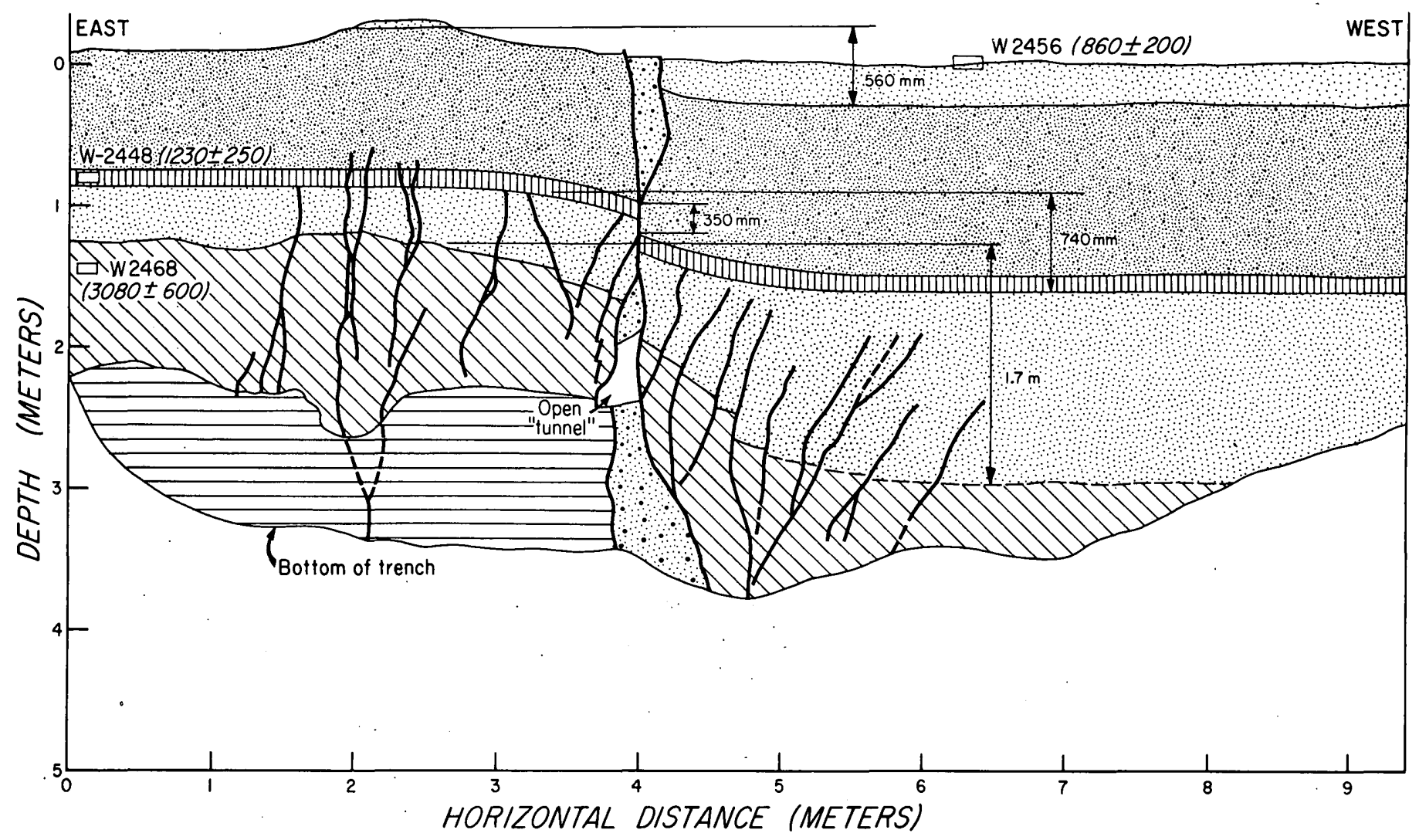

\section{EXPLANATION}

sand filin

Clay, silt, and sand filling fracture

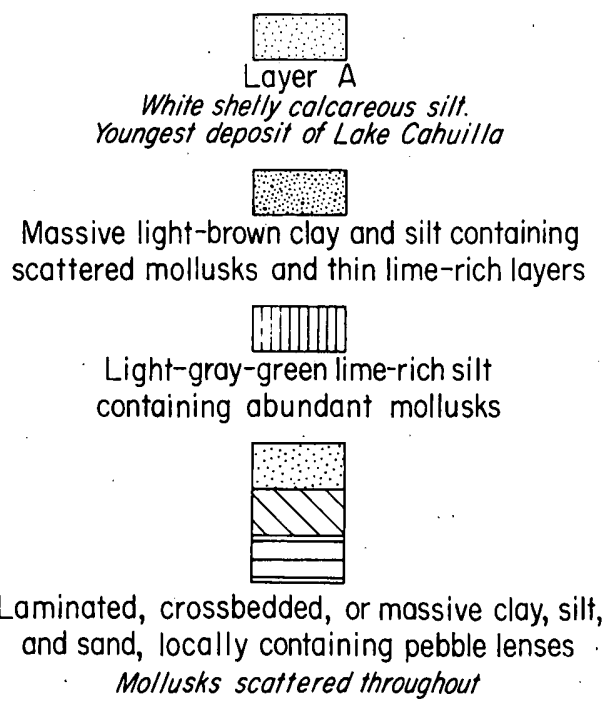

FIGURE 83. - South wall of trench 1 showing progressively greater offset of older strata and bending of strata (drag) across the branching break of the 1968 event. All deposits appear to be lacustrine, although some are possibly fluvial or eolian. Some deposits east of the break have been removed by erosion. Surface water that entered this fracture in July 1968 eroded the tunnel and caused surface collapse along the break within $30 \mathrm{~m}$ of the trench. Sample W-2456 was collected from the surface of the youngest lake deposit $1 \mathrm{~km}$ southeast of this. trench. (From sketches by M. M. Clark and R. V. Sharp.) 
The vertical offset of these beds at the break is not the result of large horizontal offset of slightly inclined beds but is due to a vertical component of displacement. The same strata are exposed in the upper parts of trenches 1 and 4 and are within $2 \mathrm{~m}$ of the same elevation. Because these trenches are more than $300 \mathrm{~m}$ apart (fig. 80), the tangent of the dip of these strata is less than 0.01 . With this dip, if slip were entirely horizontal, the ratio of true horizontal slip to apparent vertical displacement of the youngest bed in trenches 1 and 4 would exceed $100: 1$. Judging from the length and opening of individual fractures, however, horizontal displacement at trench 1 in 1968 could not have been more than about two or three times as large as the vertical offset and probably was approximately equal to it.

Table 14 gives $\mathrm{C}^{14}$ ages of shells from trench 1 and other places along the 1968 break. (See figs. $83,88,89$.) Pelecypod shells collected from the surface of layer $\mathrm{A}$, which is vertically offset 560 $\mathrm{mm}$ at trench 1, yield an age of $860 \pm 200$ years B.P. (W-2456). Gastropods in a deeper layer (W-2448) exposed in trench 1 and offset $350 \mathrm{~mm}$ at the break $(740 \mathrm{~mm}$ total) yield an age of $1,230 \pm$ 250 years B.P. This layer in turn is underlain by a lithologic contact that has been offset about $1.7 \mathrm{~m}$ total and lies some $200 \mathrm{~mm}$ above gastropod shells yielding an age of $3,080 \pm 600$ years B.P. (W-2468).

TABLE 14. - $\mathrm{C}^{1+}$ ages of mollusk shells in Holocene sediments near the 1968 break

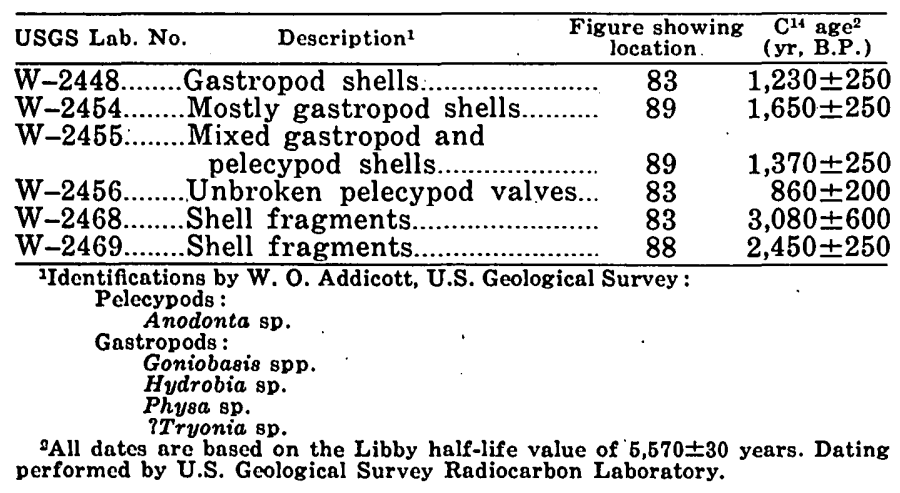

These shells supply limiting ages for the strata they occupy. The gastropods tend to occur in local concentrations within the sediments, indicating reworking after death; hence they possibly were eroded from older strata and therefore supply maximum ages for the enclosing strata. Some of the pelecypod shells collected from the surface of the thin shell-rich layer A may also have been eroded from older strata; however, very few could have been added to layer $A$ because the fragile valves, many of which were still articulated, could not have survived much transport. Nevertheless, the age given for layer A must be considered an average for the entire stratum, inasmuch as we do not know from which part of the layer the shells came.

The relation of the 860 -year-B.P. date for the pelecypod shells in layer $A$ to the history of the latest stages of Lake Cahuilla is somewhat uncertain. In the area of the 1968 rupture the youngest bed extends to the highest shoreline of Holocene Lake Cahuilla. Therefore its age should be within the range of ages for this shoreline reported by Hubbs and Bien (1967) and Hubbs, Bien, and Suess (1960, $1963,1965)$. The $\mathrm{C}^{14}$ ages range from $1,800 \pm 200$ and $1,890 \pm 500$ down to $220 \pm 100$ years B.P. Some of these ages cited for the highest shoreline, which lies at $13.7 \mathrm{~m}$ above sea level, overlap the date of the first reported visit by Europeans to the Salton Trough in 1774 (Bolton, 1930). These visitors crossed within the area of the lake defined by the highest shorelines to a point $45 \mathrm{~m}$ below sea level but did not report the presence of a lake at all. Therefore, the lake had either dried up or retreated to near the low point of the trough at $84.7 \mathrm{~m}$ below sea level by that date. In addition, an Indian estimate, cited somewhat dubiously by Mendenhall (1909, p. 19-20), placed the retreat of the lake some 400-500 years prior to 1900 . In view of (1) the conflict between the youngest possible $\mathrm{C}^{14}$ ages for the highest shoreline and the admittedly meager historical data before 1774, (2) the continuity of 860 -year-old layer $\mathrm{A}$ with the highest shoreline, and (3) the probability that the 860 -year date for layer A represents an average age for a layer that took at least several tens of years to accumulate, we believe that the youngest dates for the highest Lake Cahuilla shorelines do not invalidate the $860 \pm 200$-B.P. date obtained for layer $A$ by direct measurement of its pelecypods. This age is near but younger than the median of 18 ages for Holocene Lake Cahuilla shorelines and related deposits in and near the Salton Trough cited by Hubbs, Bien, and Suess $(1965$, p. 89).

A potentially more serious problem is the possibility that the dated fresh-water mollusk shells were not in isotopic equilibrium with atmospheric carbon. Hubbs, Bien, and Suess $(1965$, p. 69) cross-checked dates based on the fresh-water mussel Anodonta from Holocene Lake Cahuilla and elsewhere with dates on charcoal and calcareous tufa and stated that "Empirical data lead us to place considerable reliance, at least as approximations, on dates based on Anodonta. when solid, lustrous shell is. used and when any powdery, chalky coating is 
removed by initial treatment with weak acid." The shells dated in the present study (including Anodonta) were treated with acid to remove outer layers and any chalky material present, and the $\mathrm{C}^{14}$ dates from three stratigraphic positions indicate the same rate of structural deformation along the Coyote Creek fault for the last 3,000 years or so (see succeeding text); however, the possibility that some disequilibrium existed between the shells when living and the atmosphere cannot be entirely discounted.

Figure 84 shows the $\mathrm{C}^{14}$ dates plotted against the total vertical deformation of the dated beds in trench 1 and against the vertical offset produced in the 1,230-year-B.P. layer at the fault alone. The slopes of the resulting curves represent the rate of total vertical deformation (curve $A$ ) and the rate of vertical offset due to fault slip alone (curve $B$ ) across the branching break. The rate of total vertical deformation is $0.52 \mathrm{~mm} / \mathrm{yr}$, whereas the rate of vertical fault slip alone is $0.24 \mathrm{~mm} / \mathrm{yr}$. The first rate is based on four points $(3,080,1,230$, and $860 \mathrm{yr}$ B.P. and 1968 A.D.), and the second on only two points $(1,230$ B.P. and 1968 A.D.). The alinement of the four data points on curve $A$ and the continuity of the curve on passing from the segment controlled by $\mathrm{C}^{14}$ dates to the point controlled by the event of known date (1968) and approximately known vertical deformation suggest that the data are indeed valid. The fact that curve $A$ is straight indicates that the gross deformation rate here has been essentially constant for the last 3,000 years and that the timing and displacement of the 1968 event are compatible with the past deformation rate.

In view of the analytical uncertainty in the $\mathrm{C}^{14}$ dates and the range in the structural data against which they are plotted in figure 84 , curve $A$ might not be straight; however, the alinement of the median values of the data points is striking, particularly when compared with the plots of median points of $\mathrm{C}^{14}$ dates versus thicknesses of sedimentary deposits in the graben bounded by the branching break in trenches 1 and 4. (See fig. 85.) Not only is the deposition rate for each trench in figure 85 apparently variable, but the two curves are also dissimilar, even though the trenches are only about $330 \mathrm{~m}$ apart.

It should be noted that sample W-2468 was taken almost $200 \mathrm{~mm}$ below the deepest contact in trench 1 that could be traced across the fault with certainty. In view of the unconformities east of the fault revealed by trench 1 , sample $\mathrm{W}-2468$ may be significantly older than the contact above it, which has been tectonically offset $1.7 \mathrm{~m}$. If it is, the slope

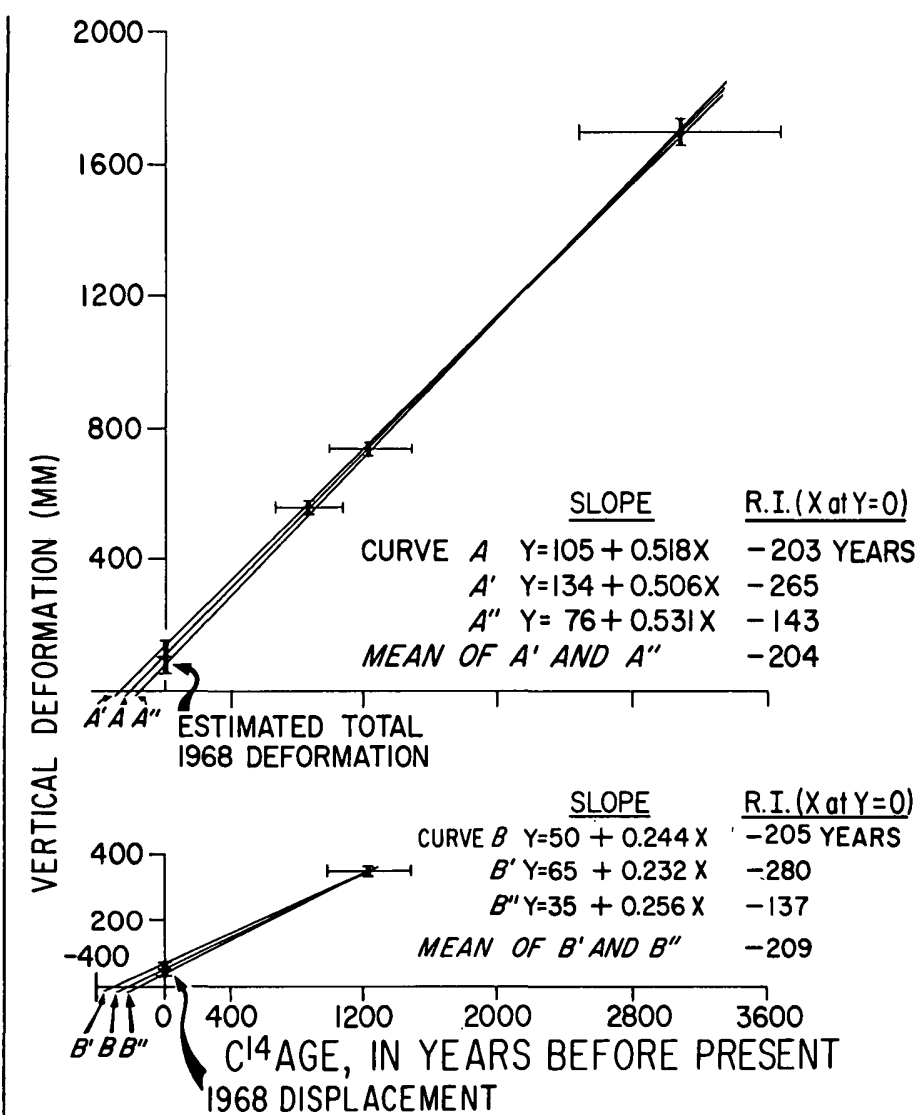

FIGURE 84. - Graphs showing relation of vertical off set to $\mathrm{C}^{14}$ age along a branching break of the 1968 earthquake, determined from late Holocene sediments exposed in trench 1. Data from figure 83 and table 14. Deformation associated with the 1968 earthquake is shown along the vertical axis. Curve $A$ represents the total vertical component of deformation (warping or drag plus slip at the branching break) through mean data values. Curve $B$ represents the vertical component of fault slip only through mean data values. Curves $A^{\prime}, A^{\prime \prime}, B^{\prime}$ and $B^{\prime \prime}$ represent alternate deformation rates based on extreme values for the total and fault-slip-only vertical component of uplift in 1968. Note that the intercepts of these curves with the horizontal axis represent recurrence intervals (R.I.) for vertical deformations on the branching break of the size that occurred in 1968. Curves $A, A^{\prime}$, and $A^{\prime \prime}$ were derived from a least-squares fit of a line through the three $\mathrm{C}^{14}$-controlled data points and vertical deformation, respectively, of $100 \mathrm{~mm}, 150 \mathrm{~mm}$, and $50 \mathrm{~mm}$ at age $=0$. Curves $B, B^{\prime}$ and $B^{\prime \prime}$ drawn, respectively, through vertical deformation of $50 \mathrm{~mm}, 65 \mathrm{~mm}$, and $35 \mathrm{~mm}$ at age $=0$.

of the oldest segment of deformation rate curve $A$ in figure 84 should be greater.

The curves in figure 84 are very sensitive to the values of total vertical deformation and vertical fault slip at the branching break in 1968. Measured fault slip ranged from 0 to $50 \mathrm{~mm}$, but the maximum values (about $50 \mathrm{~mm}$ ) are thought to most accurately represent the true 1968 vertical slip at the fault 


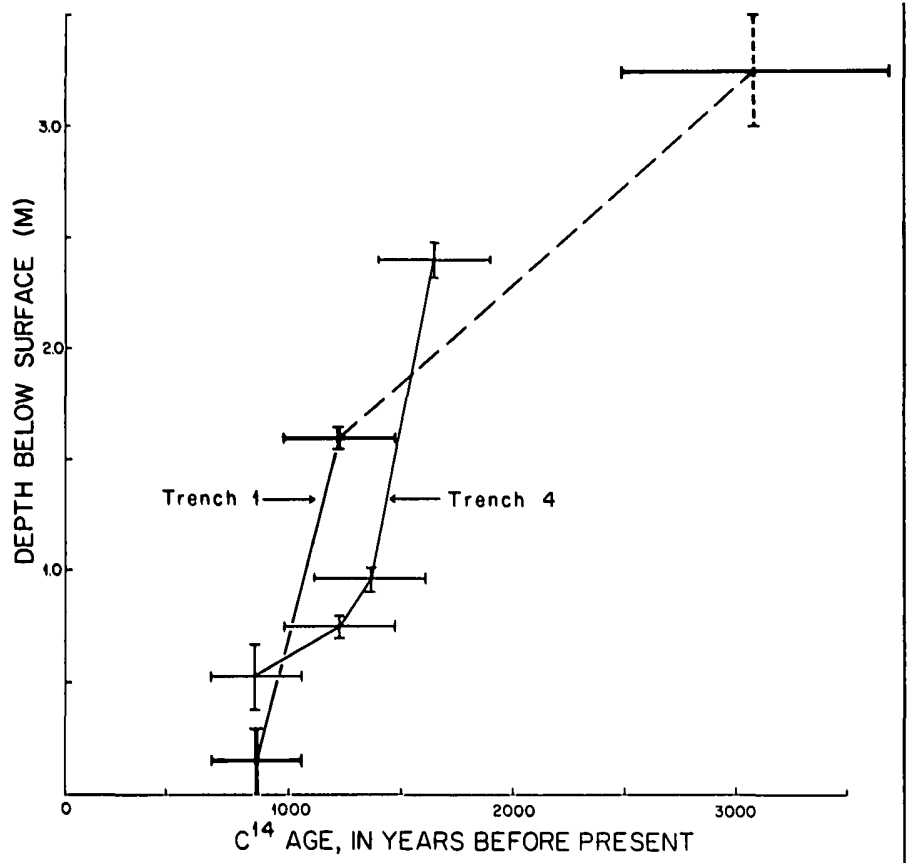

Figure 85. - Deposition rates of sediments southwest of the branching break of 1968 in trenches 1 and 4. Data from figures 83 and 89 and table 14 .

because (1) the break consisted of en echelon fractures along which offsets ranged from 0 to the maximum values observed, (2) measured offsets tend to be minimums owing to the difficulty of accurately measuring and summing all multiple small offsets on partly parallel fractures in a natural land surface, and (3) the branching break was not found until 11 months after the earthquake, when the offsets had been partly obliterated by wind and rain. The precision of this measurement is estimated to be about $15 \mathrm{~mm}$, and accordingly the value of the 1968 vertical slip at the branching break used in figure 84 ranges from about 35 to $65 \mathrm{~mm}$, with an average value of $50 \mathrm{~mm}$. Because the ratio of total vertical deformation to vertical slip for beds in trench 1 is about $2: 1,100 \mathrm{~mm}$ is used for total vertical deformation - twice the most probable value for fault slip alone and the average of the estimated values of total vertical deformation.

Recurrence intervals for 1968-size events on the branching break are represented by the negative intercepts of curves $A$ and $B$ with the axis of abscissas in figure 84 . By using mean values for the 1968 offsets, the recurrence interval based upon both the total vertical deformation (curve $A$ ) and the total vertical offset due to fault slip only (curve $B$ ) is determined to be about 205 years. If the contact $200 \mathrm{~mm}$ above sample $\mathrm{W}-2468$ is significantly younger than 3,080 years, then curve $A$ (taken to be straight) would be steeper, and the recurrence interval derived from it would be shorter than 205 years. If the uncertainties in the amount of 1968 offset are considered, the recurrence interval for total vertical deformation at the branching break would be (without rounding off numbers) $204 \pm 61$ years, and for vertical fault slip alone $209 \pm 72$ years. The fairly close agreement between these numbers and the most likely recurrence intervals (190 yr or perhaps $160 \mathrm{yr}$ ) derived from the structural profiles across the central break lead us to conclude that the recurrence interval for 1968size events on the Coyote Creek fault is about 200 years, with an uncertainty of perhaps 60 or 70 years.

\section{COMPARISON OF RECURRENCE INTERVALS WITH OTHER DATA}

The validity of a 200 -year recurrence interval for 1968-size events on the Coyote Creek fault (table 15) can be evaluated by comparison with other kinds of displacement-rate data. At present, however, only

TABLE 15. - Recurrence intervals for 1968-size events along the Coyote Creek fault based on the vertical component of late Holocene displacements along the central and branching breaks of 1968

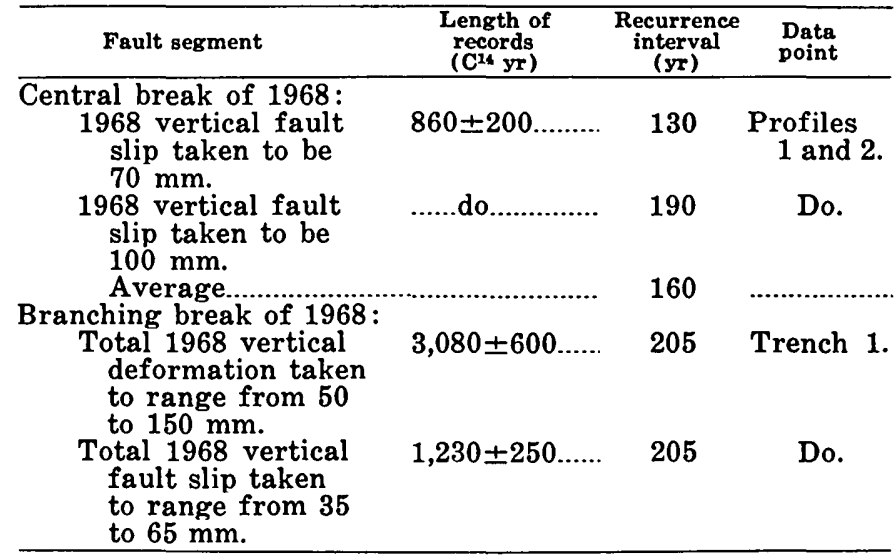

two sets of data seem useful for this purpose, and both are marginally so. One set compares the Coyote Creek and its parent San Jacinto fault with the San Andreas fault with respect to the ratio of recurrence interval to displacement rates. The other comparison in effect checks the 200-year recurrence interval for the Coyote Creek fault against one derived from the length of the 1968 rupture and the 70 -year history of major earthquakes along the San Jacinto fault.

The 200-year recurrence interval derived for 1968size events at a point on the Coyote Creek fault is about seven times longer than the 28-year interval estimated by Wallace $(1970$, figs. 4,8$)$ for events of similar magnitude (6.4) at a given point along the San Andreas fault. Wallace's estimate is based on 
an empirical relation between horizontal offset and Richter magnitude and an assumed long-term horizontal strain accumulation rate of $20 \mathrm{~mm} / \mathrm{yr}$ along the San Andreas fault, whereas the recurrence intervals summarized in table 15 are based on a $\mathrm{C}^{14}$ dependent chronology of late Holocene geologic events at three adjacent points along the Coyote Creek fault.

If the Coyote Creek fault responds to the stresses generated by the northwestward drift of coastal California more or less as does the main San Andreas fault north of the Transverse Ranges, then the recurrence interval for slip events and associated earthquakes of the same magnitude along the two faults should be proportional to their deformation (slip) rates. For the main San Andreas fault north of the Transverse Ranges, $20 \mathrm{~mm} / \mathrm{yr}$ (Wallace, 1970 , p. 2877) seems a reasonable average rate for long-term slip, although modern rates as high as $85 \mathrm{~mm} / \mathrm{yr}$ are suggested in the southern Salton Trough by geodetic data (Scholz and Fitch, 1969, p. 6664) and $60 . \mathrm{mm} / \mathrm{yr}$ is suggested for the Gulf of California region by sea-floor magnetic anomalies (Larson and others, 1968). For the Coyote Creek fault, data on long-term displacement are limited to a record of $5.1+\mathrm{km}$ of right-lateral offset of the early Irvingtonian (early Pleistocene) Bautista Beds of Frick (1921, p. 283-288) along the middle of the parent San Jacinto fault zone (Sharp, 1967, p. 714, 727). This amounts to a rate of $>2.5 \mathrm{~mm} / \mathrm{yr}$, because these beds are probably no more than 2 m.y. old. The long-term slip rate for the San Andreas fault is about eight times as great, which is in general agreement with the $7: 1$ ratio between the recurrence intervals of magnitude 6.4 earthquakes along the two faults. Adjustments in these ratios to accommodate possibly larger post-Irvingtonian displacement on the San Jacinto and higher displacement rates on the San Andreas would tend to increase both ratios. Comparison of ratios involving the main San Jacinto fault zone on one hand (longterm slip) and the Coyote Creek fault on the other (late Holocene recurrence intervals) may be justified because the Coyote Creek fault bears clear evidence of late Holocene activity, whereas definite evidence of such activity has not been discovered on other strands of the San Jacinto fault zone east of Borrego Badlands. The fact that the long-term slip rate and the recurrence interval for magnitude 6.4 events on the San Andreas fault are, respectively, several times larger and smaller than on the San Jacinto-Coyote Creek fault lends some support to our estimated recurrence intervals for the Coyote Creek fault. The large ratios also follow from the fact that the slip rates and recurrence intervals for the San Andreas north of the Transverse Ranges probably reflect most of the relative motion between the Pacific and North American plates, whereas the Coyote Creek fault probably shares this motion with at least three other active fault systems south of the Transverse Ranges (the Banning-Mission Creek segment of the San Andreas, the Elsinore-Agua Caliente, and the Newport-Inglewood fault zones).

Our 200-year recurrence interval for the 1968 break is also compatible with recurrence intervals estimated from historic seismicity of the entire San Jacinto fault zone. Seven earthquakes of magnitude 6.0 or greater have occurred in the zone since 1899 , at an average interval of 11.4 years. (See fig. 86; table 16.) Assuming (1) a length of $215 \mathrm{~km}$ for the San Jacinto fault zone, (2) a constant crustal strain rate along the entire zone, and (3) that ground ruptures are associated only with earthquakes of magnitude 6.0 or greater and release all the strain accumulated along the ruptured length of the zone, a simple relation, derived from the concepts of Wallace (1970), exists:

$$
R_{x}=\frac{R_{t} \times L_{t}}{L_{x}}
$$

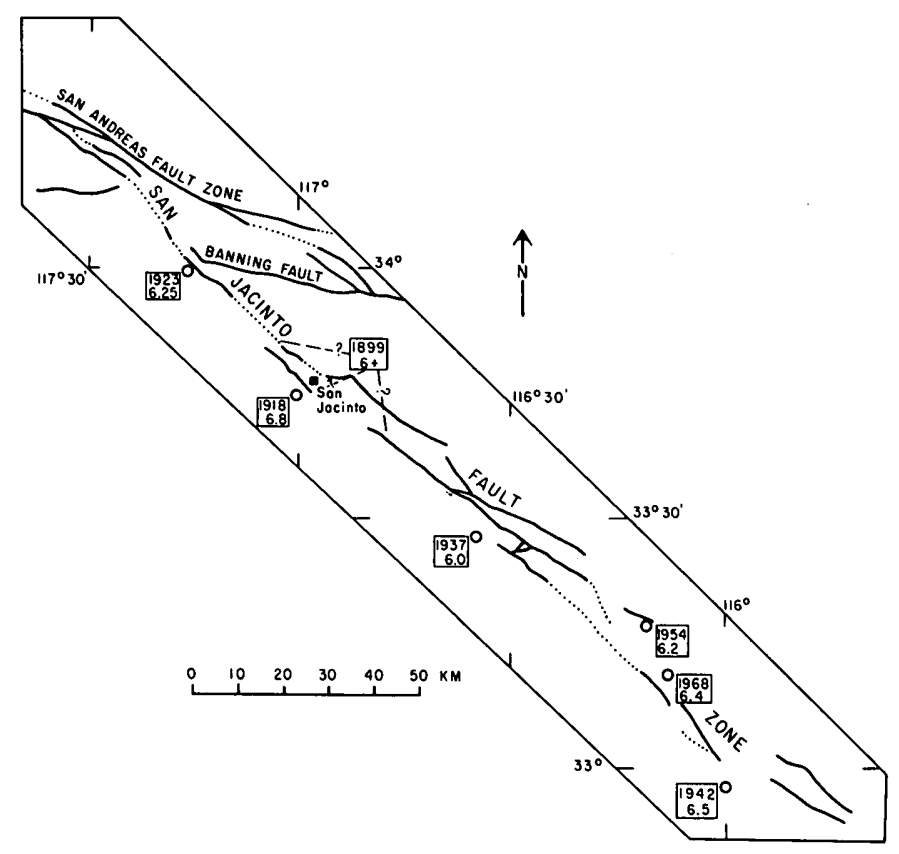

Figure 86. - Historic earthquakes of magnitude 6 and greater along the San Jacinto fault zone (after Allen and others, 1965, fig. 5, table 1). The epicenter and magnitude of the 1899 earthquake are unknown, but descriptions by Danes (1907), Townley (1918), and Rolfe and Strong (1918) indicate that its epicenter was near that of the 1918 earthquake and that its magnitude was comparable to or greater than that of the 1918 earthquake. Fault traces after Sharp (1972). 
TABLE 16. - Earthquakes of magnitude 6.0 and greater along the San Jacinto fault zone since 1899

[After data in Allen, St. Amand, Richter, and Nordquist (1965, table 1, fig. 5)]

\begin{tabular}{|c|c|c|c|c|}
\hline Date & Lat (N.) & Long (W.) & Magnitude & $\begin{array}{c}\text { Interval } \\
\text { since } \\
\text { previous } \\
\text { event (yr) }\end{array}$ \\
\hline $\begin{array}{l}\text { Dec. } 25,1899 \ldots \ldots \\
\text { Apr. } 21,1918 \ldots \ldots . \\
\text { July } 23,1923 \ldots \ldots . \\
\text { Mar. } 25,1937 \ldots \ldots \\
\text { Oct. } 21,1942 \ldots \ldots \ldots \\
\text { Mar. } 19,1954 \ldots \\
\text { Apr. } 9,1968 \ldots \ldots .\end{array}$ & $\begin{array}{l}33 \%^{\circ} \\
34^{\circ} \\
33^{\circ} 28^{\prime} \\
32^{\circ} 58^{\prime} \\
33^{\circ} 17^{\prime} \\
33^{\circ} 12^{\prime}\end{array}$ & $\begin{array}{l}(1) \\
117^{\circ} \\
11714^{\circ} \\
116^{\circ} 35^{\prime} \\
116^{\circ} 00^{\prime} \\
116^{\circ} 11^{\prime} \\
116^{\circ} 08^{\prime}\end{array}$ & $\begin{aligned} 2>6.0 \\
6.8 \\
61 / 4 \\
6.0 \\
6.5 \\
6.2 \\
6.4\end{aligned}$ & $\begin{array}{r}18.3 \\
5.3 \\
13.7 \\
5.6 \\
11.4 \\
14.0\end{array}$ \\
\hline
\end{tabular}

Near San Jacinto.

where

$R_{x}=$ recurrence interval at a point in the zone for earthquakes that produce ruptures,

$R_{t}=$ recurrence interval for all earthquakes along the zone that produce ruptures,

$L_{t}=$ total length of the fault zone, and

$L_{x}=$ length of rupture for a given earthquake. Equation 1 simply states that the number of events, $L_{t} / L_{x}$, required to break the entire length of the zone multiplied by the average time interval between these events, $R_{t}$, equals the interval between successive events at the same point in the zone, $R_{x}$.

For a point in the San Jacinto fault zone $\left(R_{t}=11.4\right.$ years, $L_{t}=215 \mathrm{~km}$ ),

$$
R_{x}=\frac{2450}{L_{x}} \text {. }
$$

Because $L_{x}$ is not known for the six earthquakes that happened before 1968, ${ }^{1}$ equation 2 is plotted as curve $A$ in figure 87 to show better the range of possible values of $L_{x}$ and $R_{x}$.

The recurrence interval of 200 years reported here and the length of the 1968 rupture of $31 \mathrm{~km}$ (pl. 1) are within a factor of about three of values derived from the historic record in equation 2 (fig. 87 , curve $A$ ). A reasonable value for average length of ground rupture, $L_{x}$, on curve $A$ of figure 87, namely $12 \mathrm{~km}$, corresponds to our recurrence interval, $R_{x}$, of 200 years, and this value also falls within the range (roughly 5-70 km for magnitude 6-7 earthquakes) given by Wallace's (1970, fig. 2) leastsquares curve for data showing the relation between rupture length and magnitude of earthquakes in western North America. The 1968 rupture is considerably longer and gives a value for $R_{x}$ of about 80

'No systematic, detailed investigations of the San Jacinto fault zone were reported after the earlier carthquakes. Although Danes (1907) described a 8-km surface rupture resulting from the 1899 earthquake, Sharp (1972) indicated that the known surficial effects probably are entirely of landslide origin. After the 1918 earthquake, Townley (1918) and Rolfe and Strong (1918) found no evidence for surface ruptures at the places they checked. Laughlin, Arnold, and Kew (1923) evidently did not inspect traces of the San Jacinto fault after the 1923 earthquake, and we are not aware of any field investigations of the effects of the earthquakes of 1937,1942 , or 1954 .

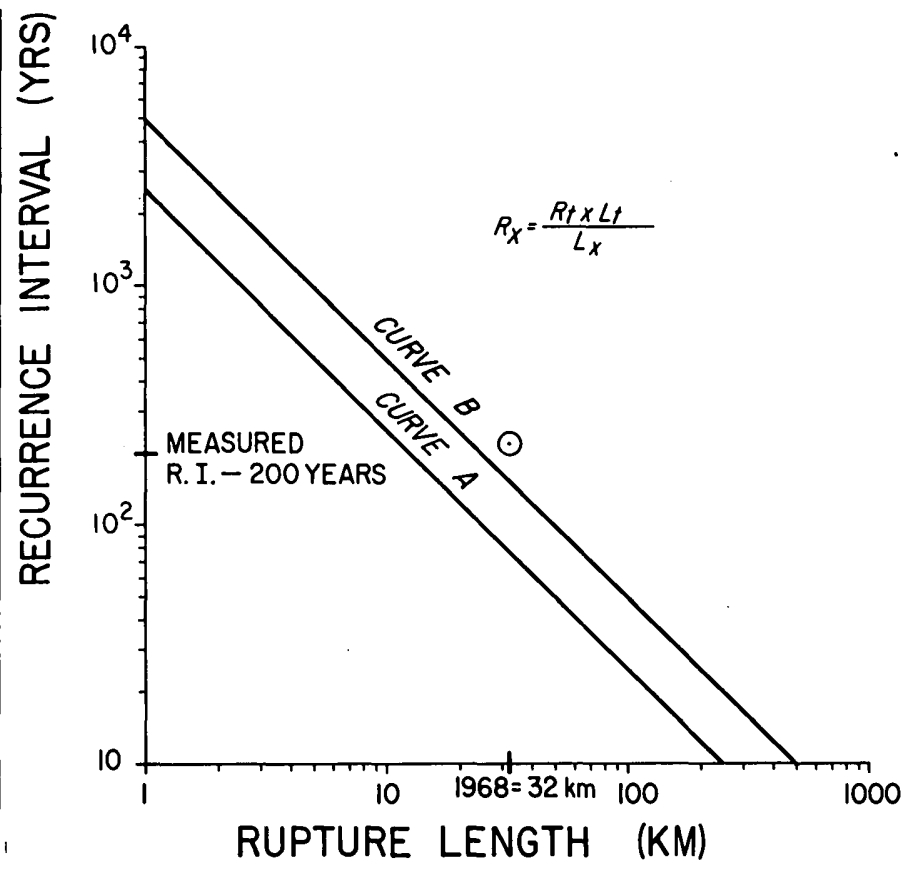

FIGURE 87 . - Recurrence intervals, $R_{x}$, at a point in the San Jacinto fault zone for different values of rupture length, $L_{x}$. Curve $A$, all historic earthquakes of magnitude 6 and greater; curve $B$, earthquakes of $1899,1918,1942$, and 1968 only (magnitude 6.4 and greater). Based on equation 1 and data in table 16, with a total length of fault zone, $L_{t}$, of $215 \mathrm{~km}$. For curve $A$, average interval between earthquakes, $R_{t}$, is 11.4 years. For curve $B, R_{t}$ is 22.8 years.

instead of 200 years on curve $A$ of figure 87 . Considering the broad nature of the assumptions underlying equation 1 , these values represent fair agreement between the two independent estimates of recurrence intervals.

Two additional assumptions about the historic earthquakes bring much closer agreement between the recurrence intervals estimated by the two different methods. Of the historic earthquakes in table 16 , the ones most likely to have been associated with significant ground ruptures are those larger than the 1968 earthquake. Assuming that the earthquake of 1899 was about equal to that of 1918 (magnitude 6.8, fig. 86) and that the earthquakes of 1923, 1937, and 1954 (magnitude less than 6.4) were not associated with significant faulting, the remaining four earthquakes (magnitude 6.4 or greater) have an average interval, $R_{t}$, of 22.8 years. (Notice that the intervals between these four earthquakes, 18.3, 24.6, and 25.4 years, differ less than the intervals between all seven earthquakes given in table 16.) By using the value of $R_{t}(22.8 \mathrm{yr})$ for these four largest earthquakes, equation 1 then becomes

$$
R_{x}=\frac{4900}{L_{x}},
$$


which is plotted as curve $B$ in figure 87 . With curve $B$, our recurrence interval of 200 years corresponds to a rupture length of $25 \mathrm{~km}$; conversely, the 1968 rupture length, $31 \mathrm{~km}$, corresponds to a recurrence interval of about 160 years. These values represent very close agreement between the two estimates of recurrence intervals, particularly if the ends of the 1968 rupture, which had very small displacements (pl. 1), overlap by several kilometers into adjoining segments of the fault broken by other earthquakes. Such overlap would shorten the effective length of the rupture used in this analysis. In view of the uncertainties, however, both in the recurrence intervals estimated from field data and in the assumptions underlying equation 1 , a conclusion that significant faulting and regularity of occurrence attend only those earthquakes of roughly magnitude 6.4 and greater along the San Jacinto fault is highly speculative. The record of historic seismicity, however, definitely does not conflict with a recurrence interval of 200 years; indeed, historic seismicity appears to support it.

RATE OF LATE HOLOCENE STRIKE-SLIP DISPLACEMENT

The rate of late Holocene right-lateral strike-slip along the Coyote Creek fault can be estimated from our measurements in two ways : by applying the 200 year recurrence interval for 1968-size events to the right-lateral strike-slip displacements observed in 1968 or by multiplying the vertical deformation rates by the ratio of the horizontal to the vertical components of slip measured along the relevant part of the central break after the 1968 event. Both estimates rest on a number of assumptions and other estimates, and as with the recurrence intervals, they can be tested only by comparison with other kinds of displacement-rate data. The assumptions are that (1) late Holocene vertical displacement rates across the fault at the structural profiles and trench 1 are proportional to horizontal displacement, rates on the central break; (2) the late Holocene recurrence intervals we derive from the rate of vertical deformation at three points on the central and branching breaks can be extrapolated to the entire Coyote Creek fault; and (3) the total fault slip in 1968, both sudden offset at the time of the earthquake and subsequent creep, is represented in the fault offsets recorded on plate 1 . The chief estimates are of (1) the horizontal and vertical components of slip in 1968 (and the ratio between these components) along the part of the central break that was most strongly uplifted on the southwest side in 1968 and for which we estimated recurrence intervals and (2) the ratio of the horizontal component of drag to strike slip along the central break. Data summarized in the next section suggest that drag at least commonly accompanies slip along faults where they traverse unconsolidated or poorly consolidated sediments near the earth's surface.

Each of the two estimates of late Holocene strike slip rests on the stratigraphically based $\mathrm{C}^{14}$-dated vertical-deformation rates derived from structural data in profiles 1 and 2 . The first estimate, inasmuch as it uses the 200-year recurrence interval, is in addition based on the $\mathrm{C}^{14}$-dated vertical deformation rates derived from structural data in trench 1 . This estimate applies the 200-year recurrence interval to the horizontal-slip components of the 1968 event. The estimate relies heavily on the extent to which the vertical components of displacement near the profiles and at the trench are representative of both the 1968 and earlier events. Comparison with other data in the preceding section suggests that they are, in general, representative.

The results of the first approach are presented in table 17 . The horizontal deformation rates are de-

TABLE 17. - Rate of late Holocene strike-slip deformation on the Coyote Creek fault based on 200-year recurrence interval for 1968-size events

\begin{tabular}{|c|c|c|c|c|}
\hline \multirow[t]{2}{*}{ Break } & \multirow{2}{*}{$\begin{array}{c}\text { Maximum } 1968 \\
\text { horizontal } \\
\text { displacement } \\
(\mathrm{mm})\end{array}$} & \multicolumn{3}{|c|}{$\begin{array}{c}\text { Total horizontal deformation rates based } \\
\text { on various assumed ratios of horizontal } \\
\text { drag to fault slip (mm/yr) }\end{array}$} \\
\hline & & $0: 1$ & $1: 1$ & $2: 1$ \\
\hline North.. & 380 & 1.9 & 3.8 & 5.7 \\
\hline Central. & 300 . & 1.5 & 3.0 & 4.5 \\
\hline 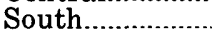 & $140^{\circ}$ & .70 & 1.4 & 2.1 \\
\hline
\end{tabular}

rived by first converting the maximum horizontal fault displacements (slip) observed in 1968 (recorded on pl. 1) to total horizontal deformation by multiplying them by the factors 1,2 , and 3 , corresponding to three alternatives: no horizontal drag, drag equal to fault slip, and drag twice as large as fault slip. The maximum 1968 slip was chosen because we believe it probably most closely approaches "deep" or bedrock displacement. Horizontal drag is probably a significant factor. Allen, Wyss, Brune, Grantz, and Wallace (this volume) reported that such deformation accompanies displacement along the nearby Superstition Hills, Imperial, and San Andreas (Banning-Mission Creek) faults, which also traverse areas of late Cenozoic sedimentary deposits. (See also the following section of this report.) However, they did not report the proportion of horizontal drag to fault slip, so we arbitrarily use the ratios of vertical drag to fault slip 
found in trench 1 and profiles 1 and 2 (1:1 and $2: 1)$ and also the ratio for no drag $(0: 1)$. The ranges in rates so obtained for the more strongly displaced northern and central breaks are, respectively, $1.9-5.7 \mathrm{~mm} / \mathrm{yr}$ and $1.5-4.5 \mathrm{~mm} / \mathrm{yr}$. Within these ranges, the rates corresponding to a drag-toslip ratio of $1: 1$, namely 3.8 and $3.0 \mathrm{~mm} / \mathrm{yr}$, may possibly be best, because this ratio characterized vertical drag in two of the three structural sections measured in profiles 1 and 2 and trench 1 .

A similar analysis, presented in table 18, estimates vertical deformation rates along representative parts of the north, central, and south breaks of 1968. The vertical drag coefficients used, $1: 1$ and $2: 1$, are based on those found in trench 1 and profiles 1 and 2. Such estimates may be useful, for example, in geomorphic, structural, or engineering studies of the hills or scarps that are being tectonically uplifted now in the region of the Coyote Creek fault.

TABLE 18. - Rate of late Holocene vertical deformation at selected points along the Coyote Creek fault based on 200year recurrence interval for 1968-size events

\begin{tabular}{|c|c|c|c|c|}
\hline \multirow[t]{2}{*}{ Break } & \multirow[t]{2}{*}{$\begin{array}{l}\text { Sample } 1968 \\
\text { vertical } \\
\text { fault slip } \\
\text { (mm) }\end{array}$} & \multirow[t]{2}{*}{$\begin{array}{l}\text { Locality } \\
\text { (see pl. 1) }\end{array}$} & \multicolumn{2}{|c|}{$\begin{array}{c}\text { Total vertical deformation } \\
\text { rates based on assumed } \\
\text { ratios of vertical drag } \\
\text { to fault slip } \\
(\mathrm{mm} / \mathrm{yr})\end{array}$} \\
\hline & & & $1: 1$ & $2: 1$ \\
\hline 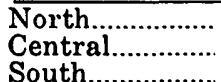 & $\begin{array}{r}200 \\
100 \\
40\end{array}$ & $\begin{array}{r}8.1 \\
19.1 \\
30.2\end{array}$ & $\begin{array}{l}2 \\
1 \\
4\end{array}$ & $\begin{array}{l}3 \\
1.5 \\
.6\end{array}$ \\
\hline
\end{tabular}

The results of the second approach to estimating the rate of late Holocene strike slip along the fault are presented in table 19 . They were derived by multiplying (1) the stratigraphically based late Holocene vertical deformation rates in profiles 1 and 2 and (2) the ratio of the horizontal to the vertical component of slip along the part of the central break showing the greatest and most uniform uplift in 1968. This ratio is the cotangent of the angle between the net slip and the horizontal component of slip if the Coyote Creek fault is indeed vertical, as suggested by field mapping.

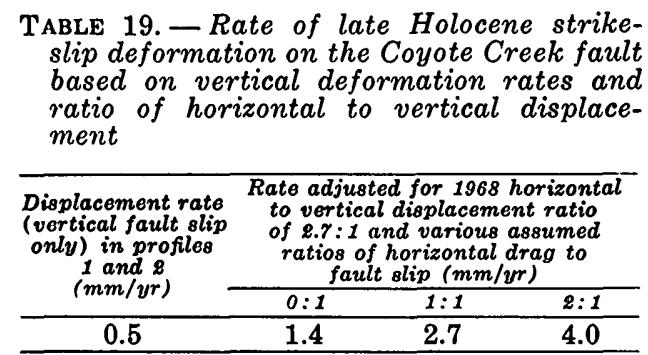

The deformation rates in table 19 are based on an average ratio of horizontal to vertical slip derived from ratios measured at eight places showing large right slip along the most uplifted part of the central break of 1968. These places lie between localities 16.1 and 21.9 on plate 1 and encompass profiles 1 and 2 . The ratios at the eight places range from 1.3 to 4.6 and average 2.7. Although only measurements with more than $100 \mathrm{~mm}$ of right slip in 1968 were used, the average value of the ratio would not differ greatly if all the ratios recorded in plate 1 from the most uplifted area were used. The product of the vertical deformation rate and the average ratio was modified by factors for no horizontal drag, drag equal to, and drag twice as large as horizontal slip. The strike-slip displacement rates that result from this analysis, $1.4-4.0 \mathrm{~mm} / \mathrm{yr}$, are similar to those obtained by the first. This approach avoids using the recurrence intervals, which depend heavily on the representativeness of the 1968 deformation at trench 1 and near profiles 1 and 2 , and it has the advantage of using the average of eight ratios of horizontal to vertical slip from points evenly spaced along $5.8 \mathrm{~km}$ of the most uplifted part of the central break. The considerable variation in this ratio along even this most uplifted part of the central break, however, causes a large uncertainty in the calculated strike-slip deformation rate.

The rates of late Holocene strike slip estimated for the Coyote Creek fault in tables 17 and 19 are comparable to Sharp's (1967) estimate of the rate of Pleistocene slip, $>2.5 \mathrm{~mm} / \mathrm{yr}$, for the main San Jacinto fault. Sharp's rate falls within the range of strike-slip rates estimated by both approaches and lies closest to the displacement rates estimated for the central break with a drag-to-slip ratio of $1: 1$. For the north break, estimated only by the first method, Sharp's minimum rate lies between the values for drag-to-slip ratios of $0: 1$ and $1: 1$. The fairly close correspondence of Sharp's estimates and ours is encouraging but, because of differences in the age and character of the offset features used in Sharp's estimate and ours, does not constitute conclusive verification of our estimates. Nevertheless, it does support the suggestion, based upon our estimates of vertical displacement rates and recurrence intervals for the central break and the most commonly observed ratio of vertical drag to fault slip in the profiles and trenches, that the rate of late Holocene strike-slip displacement on the Coyote Creek fault is about $3 \mathrm{~mm} / \mathrm{yr}$. 


\section{SIGNIFICANCE OF DRAG ADJACENT TO THE FAULT}

Both horizontal and vertical components of fault drag were found adjacent to the fractures of the Coyote Creek fault, even where it crosses late Holocene sedimentary deposits. This evidence of drag indicates that displacements across fractures alone, at least where measured at the earth's surface, may be significantly less than total permanent deformation in this and other fault zones. The vertical component of drag is clearly demonstrated in the deformation of originally flat-lying Holocene sediments exposed in trench 1 (fig. 83) and in steeply dipping Quaternary sediments adjacent to the fault in Ocotillo Badlands; horizontal drag is shown by a characteristic bending of strike of the steeply dipping strata next to the fault (Sharp and Clark, this volume). Thus, the horizontal and vertical offsets recorded on plate 1 , which represent the cumulative slip (including subsequent creep) along the discrete fractures that constitute the 1968 fault break, are probably not a true measure of the total displacement across the fault zone associated with the 1968 earthquake. The fact that figure 83 shows greater vertical drag in successively deeper Holocene beds indicates that the drag is permanent strain.

Our data do not reveal whether the ratios between vertical slip on macrofractures and drag (roughly $1: 1$ in trench 1 and profile 2 and $1: 2$ in profile 1 ) hold also for horizontal movement at those places or elsewhere along the Coyote Creek fault. Preliminary measurements of horizontal creep from an alinement array on the central break established near profile 2 a few days after the 1968 earthquake suggest that postearthquake displacements across the smaller of two fractures in the fault zone consist of roughly equal amounts of slip on fractures and distortion in the adjacent sediments, although the larger fracture does not show drag (Burford, this volume, fig. 75). Whether this distortion is permanent or is instead elastic and temporary is not yet known. Without repetitive measurements of preearthquake lines across the rupture of 1968 on the Coyote Creek fault, the distribution of total strain across the rupture cannot be determined. Repeated regional triangulation across the northern part of the Coyote Creek fault near the Truckhaven road (Whitten, 1956), however, suggests continuing right-lateral strain across the general zone of the Coyote Creek and San Jacinto faults.
It is of interest here that where preearthquake geodetic lines $100 \mathrm{~m}$ long had been established across the segments of the Imperial, Superstition Hills, and San Andreas (Banning-Mission Creek) faults on which slip was triggered by the 1968 earthquake, drag deformation was found to have occurred in the horizontal plane. (See Allen and others, this volume.) The horizontal offsets determined by remeasuring these geodetic lines after the earthquake were consistently larger than the slips measured at the fault itself, indicating that distributed horizontal deformation (drag) accompanied the fault slip.

It should be emphasized that with the exception of the differentially uplifted and intensely deformed block of Ocotillo Badlands, nonelastic strain (slip plus drag) along the Coyote Creek fault in the area of the 1968 break accumulated only in a narrow zone. This zone is about $4 \mathrm{~m}$ wide on the branching break cut by trench 1 and about $100 \mathrm{~m}$ wide in profiles 1 and 2 and in the zone of vertical dips exposed next to the central break of 1968 in the Ocotillo Badlands. Deflected fold axes in the Ocotillo Badlands indicate that the active fault zone there may, at times, have been as much as a few hundred meters wide. Even the large possibly diapiric structural knot of Ocotillo Badlands, which lies between the central and north breaks of the Coyote Creek fault, is less than $2 \mathrm{~km}$ wide. Compared with the elastically strained zone on both sides of such breaks (the elastic zone of the Imperial fault, for example, is $120 \mathrm{~km}$ wide according to Scholz and Fitch, 1969, p. 6652), these zones of nonelastic deformation have negligible width. For regional studies the slip and drag components, where present, can usually be considered together as displacement at the fault, but for detailed studies along active faults, these two aspects of deformation must be differentiated.

Drag has been reported along other active faults. It was a common feature of displacement along the San Andreas fault in 1906. Lawson and others (1908, p. 148) reported that its effects extended, at one place, almost 1 mile from the fault, and they stated that:

The recognition of the distribution of the movement on auxiliary cracks, some of which may not have appeared at the surface, and the deformation of the ground along the zone of rupture, justifies the conclusion that, except under peculiar conditions $* * *$ the maximum figures obtained for the displacement by the measurement of off sets at the surface must be a minimum expression for the true extent of the movement in the firm rocks below.

Drag also accompanies deformation during earthquakes at thrust faults, as at San Fernando in 1971 (U.S. Geol. Survey, 1971; Kamb and others, 1971), 
and at normal faults, as at Hebgen Lake, Montana, in 1959 (Myers and Hamilton, 1964, p. 81-83). It probably occurs, to some extent, in most episodes of faulting.

Drag associated with slip events along active faults is generally difficult to detect where there are few manmade features of known predeformation shape such as roads, fences, or survey lines. Our observations serve as a reminder that where such features or lines are not present, slip measured across the surface breaks of active faults may not represent total offset and should not be taken as a quantitative measure of total strain. Thus, for example, conclusions drawn from comparisons between local strain at faults and regional strain may be in error, and calculations of seismic moment or stress-drop will be too low, if determined solely from fault slip where drag was a significant component of the deformation.

\section{POSITION OF PRE-1968 FRACTURES}

The trenches and natural exposures of Holocene sediments along the 1968 rupture record important information about the position of past fractures. In some locations fracturing has occurred repeatedly along exactly the same fault plane for as long as 3,000 years, whereas elsewhere sediments as much as 1,650 years old were first ruptured in 1968 .

Trench 1 (fig. 83) reveals the clearest evidence of repeated displacement along a single narrow trace. Differential displacement of the three dated layers cut by the trench demonstrates at least three episodes of faulting in the last 3,080 years. Furthermore, if the total vertical deformation from each episode were the same as that in 1968 (estimated $100 \mathrm{~mm}$ ), then 17 such events would have been required to produce the total offset $(1.7 \mathrm{~m}$, including that of 1968) of the oldest dated layer exposed in that trench. Whatever the actual number of tectonic events, the trench shows that about one-half the total vertical deformation in the last 3,080 years has taken place along a fault plane that is in places no more than a few tens of millimeters wide.

Two trenches excavated across the main scarp of the central break also showed that most past fault displacement has been confined to a narrow zone. Trench 2 was at the site of profile 2 (fig. 80 ), and trench 3 was about $1 \mathrm{~km}$ farther southeast along the same scarp. Although no strata could be correlated across the fault in either trench (layer $\mathrm{A}$ was eroded off the surface at each site), the main rupture zone in each consisted of several discrete fractures a few tens of millimeters wide that occupied a band less than $1 \mathrm{~m}$ wide.
About $10 \mathrm{~km}$ farther southeast, along the south break, natural exposures in the walls of a wash that has developed since Lake Cahuilla withdrew also show a long history of repeated activity along the same fault strands. The wash is $1.5 \mathrm{~m}$ deep and cuts across three old en echelon fault strands, two of which moved either at the time of the earthquake or later, as a result of creep (Clark, "Surface Rupture Along the Coyote Creek Fault," this volume, fig. $49 \mathrm{~h} ; \mathrm{pl} .1$, loc. 30.7 ). Figure 88 shows two episodes of channel cutting along a relict fracture of one of these breaks, as exposed in the walls of the wash. Channeling is interpreted to have been caused by water flowing in the fracture toward nearby areas of active collapse in the same fracture. Such collapse occurs only in open fractures (Clark, "Collapse Fissures Along the Coyote Creek Fault," this volume); hence, these channels were cut soon after the faulting that opened the fractures and during periods when Lake Cahuilla had withdrawn below the elevation of this location (about $5 \mathrm{~m}$ below sea level). $\mathrm{C}^{14}$ dates on shells that fill the lower channel show that it may be as much as 2,450 years old (W-2469), whereas the younger channel is post-Lake Cahuilla (860 years) but pre-1968. The earlier date represents a maximum for the older channel, inasmuch as the shells may have been reworked from older deposits.

In contrast to fault strands on which movement had occurred repeatedly in the past, some branching ruptures of 1968 broke late Holocene strata for the first time. One fracture broke sediments $1,650 \pm 250$ years old (W-2454) for the first time in 1968 (fig. 89), yet it joined two fractures - one only $50 \mathrm{~m}$ distant - that show evidence of repeated movement during the last 1,650 years. Elsewhere, several ruptures branching from the central and south breaks appear to have breached the youngest lake bed for the first time. These new fractures outside the main fracture zone, together with the diffuse fractures shown in the other trenches and cuts (figs. 83, 88, 89), contribute to the drag component of displacement adjacent to the main fault breaks and decrease the slip component. New breaks like these may reflect a temporary or permanent shift in position of a fracture zone, and such shifts in the past would decrease the number of tectonic events apparent in the main fracture zone. Although fault breaks tend to remain in the same places during successive local earthquakes, new breaks do form and must be recognized as a hazard, even near a fault with a demonstrated history of repeated movement along preexisting traces. 


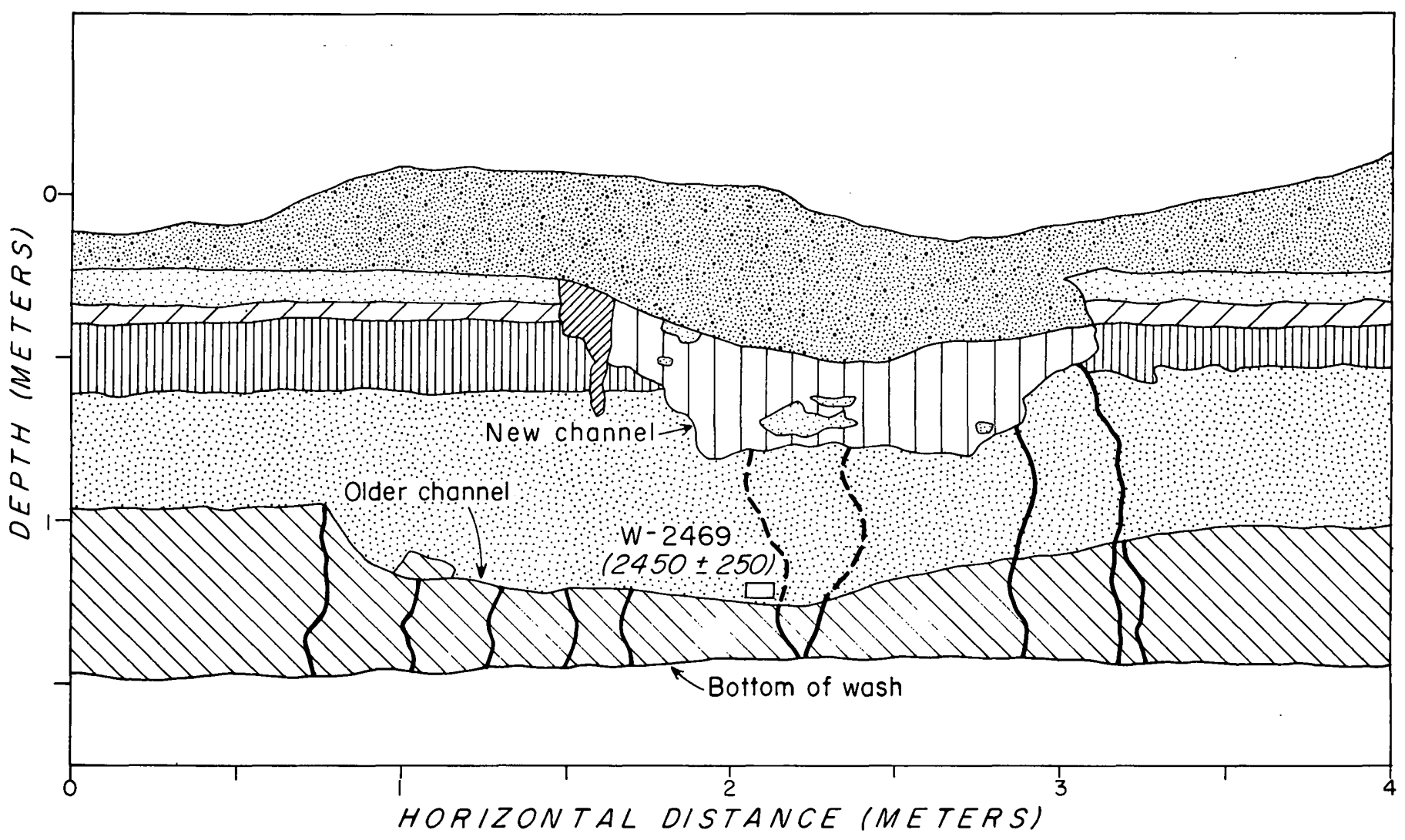

EXPLANATION

?.

Silt and sand

Eolian and fluvial(?)

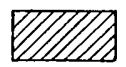

Fill along animal burrow, root, or possible fracture

Consists of material from youngest lake bed and the next older bed

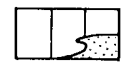

Fill in upper channel Consists of rotated pieces of youngest lake bed in matrix of material from next two older beds

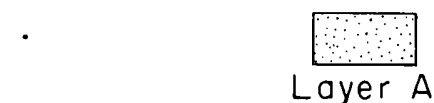

White shelly calcareous silt. Youngest deposit of Lake Cahuilla

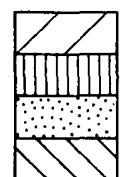

Crossbedded to thinly bedded silt to coarse sand contains some shells; probably lacustrine

\section{Fracture}

Dashed where inferred

$W-2469$

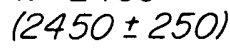

Radiometrically dated sample (See table 14)

Numbers in parentheses indicate $C^{14}$ age, in years before present 


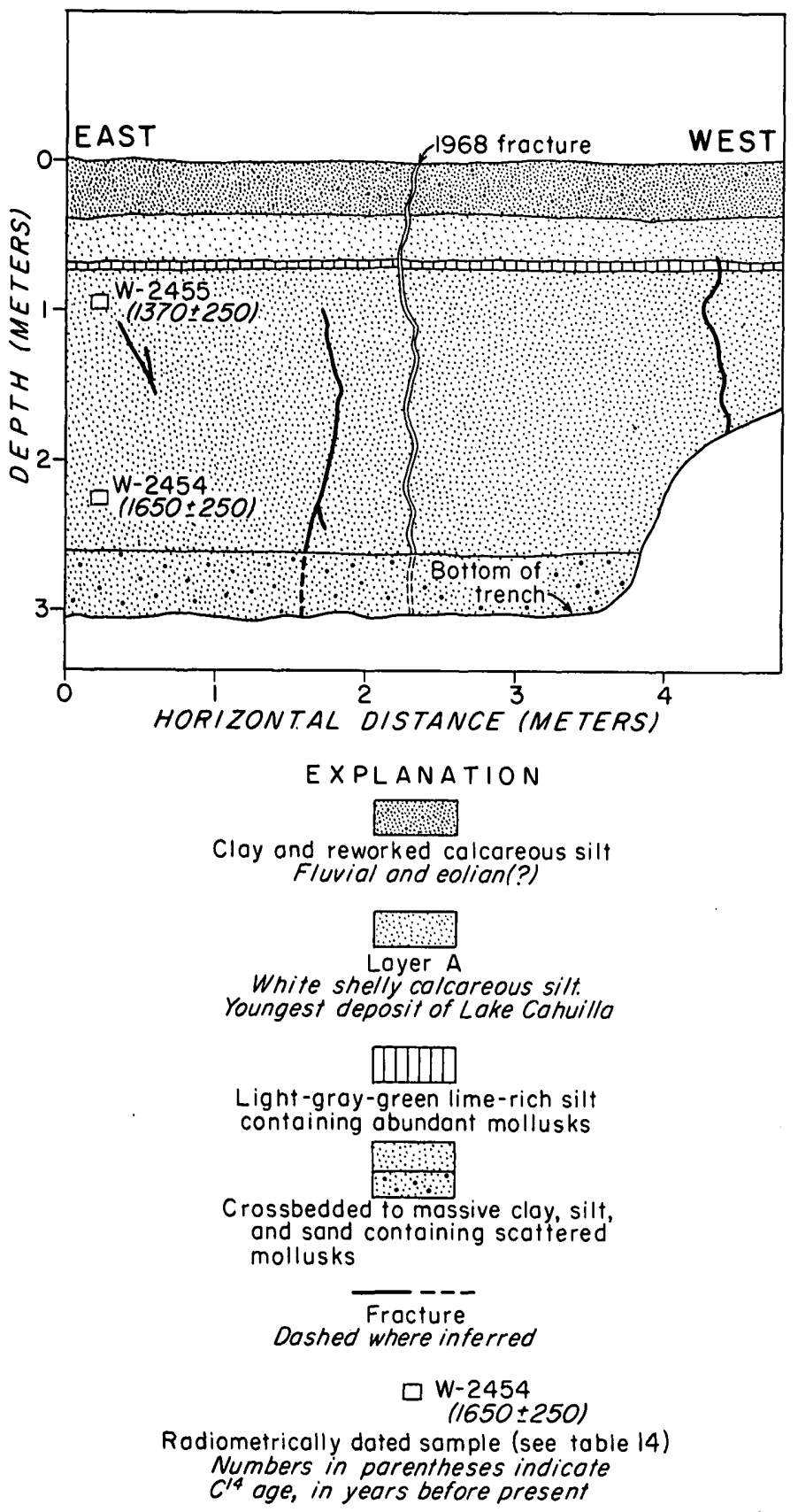

\section{REFERENCES CITED}

Allen, C. R., St. Amand, Pierre, Richter, C. F., and Nordquist, J. M., 1965, Relationship between seismicity and geologic structure in the southern California region: Seismol. Soc. America Bull., v. 55, no. 4, p. 753-797.

Bolton, H. E., 1930, Anza's California expeditions, v. 1, An outpost of empire: Berkeley, Univ. California Press, $529 \mathrm{p}$.

Daneš, J. V., 1907, Das Erdbeben von San Jacinto am 25. December, 1899: Geogr. Gesell. Wien Mitt., nos. 6-7, p. 339-347.

Dibblee, T. W., Jr., 1954, Geology of the Imperial Valley region, in Jahns, R. H., ed., Geology of southern California; chap. 2, Geology of the natural provinces: California Div. Mines and Geology Bull. 170, p. 21-28, pl. 2.

Frick, Childs, 1921, Extinct vertebrate faunas of the badlands of Bautista Creek and San Timoteo Canyon, southern California: California Univ. Pub., Dept. Geol. Sci. Bull., v. 12, p. 277-424.

Hubbs, C. L., and Bien, G. S., 1967, La Jolla natural radiocarbon measurements V: Am. Jour. Sci. Radiocarbon Supp., v. 9, p. 261-294.

Hubbs, C. L., Bien, G. S., and Suess, H. E., 1960, La Jolla natural radiocarbon measurements I: Am. Jour. Sci. Radiocarbon Supp., v. 2, p. 197-223.

1963, La Jolla natural radiocarbon measurements III : Am. Jour. Sci. Radiocarbon Supp., v. 5, p. 254-272. 1965, La Jolla natural radiocarbon measurements IV: Am. Jour. Sci. Radiocarbon Supp., v. 7, p. 66-117.

Jennings, C. W., compiler, 1967, Geologic map of California, Olaf P. Jenkins' edition, Salton Sea sheet: California Div. Mines and Geology, scale 1:250,000.

Kamb, Barclay, Silver, L. T., Abrams, M. J., Carter, B. A., Jordan, T. H., and Minster, J. B., 1971, Pattern of faulting and nature of fault movement in the San Fernando earthquake, in The San Fernando, California, earthquake of February 9, 1971: U.S. Geol. Survey Prof. Paper 733, p. 41-54.

FIGURE 89. - South wall of trench 4 showing a surface fracture associated with the 1968 earthquake that developed where there had never been a break before. Displacement across this fracture was almost entirely pull apart $(30 \mathrm{~mm}$ max.), with virtually no lateral or vertical displacement. None of the older fractures shown is as large as that of 1968. The second and third beds from the surface correlate, respectively, with the first and third beds from the surface in trench 1 (fig. 83). Location of trench 4 is shown in figure 80. (From sketches by M. M. Clark and R. V. Sharp.)
Figure 88. - Pre-1968 fractures and fissure collapse along the Coyote Creek fault as exposed in a natural cut at location 30.7. Although this fracture did not rupture during or after the 1968 earthquake, two nearby strands did. This section shows two pre-1968 episodes of erosion and filling presumably related to collapse along the fracture zone. In both episodes the channels were evidently eroded by water running in or along the fractures toward deeper openings. The younger episode is
post-Lake Cahuilla, as shown by rotated blocks of the youngest lake bed (layer A) in the fill of the upper channel. The older episode is represented by a shallower channel cut into the lowest deposit evidently during a period when Lake Cahuilla had withdrawn from this area. The fill of the lower channel contains a slump block of the lowest deposit. Eolian and fluvial (?) mud, silt, and sand have covered the youngest lake deposit at this location since Lake Cahuilla withdrew. 
Larson, R. L., Menard, H. W., and Smith, S. M., 1968, Gulf of California - A result of ocean floor spreading and transform faulting: Science, v. 161, no. 3843, p. 781-784.

Laughlin, Homer, Arnold, Ralph, and Kew, W. S. W., 1923, Southern California earthquake of July 22, 1923: Seismol. Soc. America Bull., v. 13, p. 105-106.

Lawson, A. C., and others, 1908, The California earthquake of April 18, 1906, Report of the State Earthquake Investigation Commission: Carnegie Inst. Washington Pub. 87 , v. $1,451 \mathrm{p}$.

Mendenhall, W. C., 1909, Ground waters of the Indio region, California, with a sketch of the Colorado Desert: U.S. Geol. Survey Water-Supply Paper 225, 53 p.

Myers, W. B., and Hamilton, Warren, 1964, Deformation accompanying the Hebgen Lake earthquake of August 17, 1959: U.S. Geol. Survey Prof. Paper 435-I, p. 55-98.

Rogers, T. H., compiler, 1965, Geologic map of California, Olaf P. Jenkins edition, Santa Ana sheet: California Div. Mines and Geology, scale 1:250,000.

Rolfe, F., and Strong, A. M., 1918, The earthquake of April 21, 1918, in the San Jacinto Mountains: Seismol. Soc. America Bull., v. 25, p. 63-67.

Scholz, C. H., and Fitch, T. J., 1969, Strain accumulation along the San Andreas fault: Jour. Geophys. Research, v. 74 , no. 27, p. $6649-6666$.
Sharp, R. V., 1967, San Jacinto fault zone in the Peninsular Ranges of southern California: Geol. Soc. America Bull., v. 78, p. $705-730$

1972, Map showing recently active breaks along the San Jacinto fault zone between the San Bernardino area and Borrego Valley, California: U.S. Geol. Survey Misc. Geol. Inv. Map I-675.

Stanley, G. M., 1963, Prehistoric lakes in Salton Sea basin [abs.] : Geol. Soc. America Spec. Paper 73, p. 249-250.

1966, Deformation of Pleistocene Lake Cahuilla shoreline, Salton Sea basin, California [abs.]: Geol. Soc. America Spec. Paper 87, p. 165.

Strand, R. G., compiler, 1962, Geologic map of California, Olaf P. Jenkins edition, San Diego-El Centro sheet: California Div. Mines and Geology, scale 1:250,000.

Townley, S. D., 1918, The San Jacinto earthquake of April 21, 1918: Seismol. Soc. America Bull., v. 8, p. 45-62.

U.S. Geological Survey, 1971, Surface faulting, in The San Fernando, California, earthquake of February 9, 1971: U.S. Geol. Survey Prof. Paper 733, p. 55-76.

Wallace, R. E., 1970, Earthquake recurrence intervals on the San Andreas fault: Geol. Soc. America Bull., v. 81, p. 2875-2890.

Whitten, C. A., 1956, Crustal movement in California and Nevada: Am. Geophys. Union Trans., v. 37, no. 4, p. 393-398. 


\title{
GEOLOGIC EVIDENCE OF PREVIOUS FAULTING NEAR THE 1968 RUPTURE ON THE COYOTE CREEK FAULT
}

\author{
By Robert V. Sharp and Malcolm M. Clark \\ U.S. Geological SuRvey
}

\begin{abstract}
Detailed structural mapping along the segment of 1968 rupture on the Coyote Creek fault reveals a complex zone of prior faulting and folding. Past vertical tectonism shown by small upwarps in alluvium and by uplift along the principal strands is consistently in the same sense as that shown by the 1968 movement. Faults of east-west, northsouth, and northwest-southeast trend diverge from the main strand of the Coyote Creek fault near Borrego Mountain, but none apparently moved in 1968. En echelon discontinuity of the Coyote Creek fault trace at Ocotillo Badlands and compressional folding evident there may be surface manifestations of a continuous fault at depth. Structural complexity along the 1968 trace apparently increases with the age of the exposed rocks. Beyond the ends of the 1968 break, the Coyote Creek fault probably connects northwestward through concealed branch faults with the main strand near Coyote Mountain, and to the southeast it possibly extends along the southwest margin of Superstition Mountain or perhaps to fresh surficial fractures east of Plaster City. The great width of the tectonically disturbed zone indicates an engineering hazard for structures near this or similar faults. Studies and measurements of regional horizontal strain must take into account the complex horizontal and vertical movements that apparently characterize past tectonism in this area.
\end{abstract}

\section{INTRODUCTION}

This paper describes the relation of 1968 ruptures to older fault strands in the region of the Coyote Creek fault. ${ }^{1}$ (See fig. 90.) At several locations along or near the 1968 rupture, excellent exposures reveal clear evidence of earlier movements on the same or other fault strands within the zone and show that complex folding in young sediments is closely associated with some of the faults. Some fault strands can be seen at the surface, but others, in areas where exposures are poorer, are inferred from stratigraphic and structural relations. Our detailed mapping shows that the Coyote Creek fault along the segment of the 1968 rupture is at many places a complex zone consisting of multiple strands that are either widely

"Physiographic evidence of earlier faulting is discussed in greater detail by Clark in "Surface Rupture Along the Coyote Creek Fault," (this volume). divergent from or nearly parallel to the regional trend. Several strands have not been previously recognized.

Holocene deformation along the Coyote Creek fault is expressed at the surface by folding and doming of Quaternary strata, as well as by strike-slip faulting with local uplift shown by scarps. This deformation is locally distributed across a relatively broad zone, and the width of the disturbed belt implies an engineering hazard for structures built across this or similar fault zones. Although there was no movement on many strands near the main Coyote Creek fault during the 1968 earthquake, they should not be considered inactive.

\section{NORTH BREAK ${ }^{2}$}

The northern segment of the 1968 break along the Coyote Creek fault marks the structural boundary between the southern Borrego Badlands to the northeast and the Borrego Mountain area to the southwest (pl. 3). The southern Borrego Badlands are eroded in Pleistocene terrestrial deposits that are downdropped and displaced in a right-lateral sense relative to the granitic basement rocks exposed across the fault at Borrego Mountain. The depth to granitic rock on the northeast side of the fault probably ranges from very shallow opposite East Butte to a few kilometers beyond the north end of the 1968 break. (See pl. 3.) South of State Highway 78 , the sense of net vertical throw on the fault is reversed from that to the north, as shown by the structural and topographic elevation of Pleistocene and Holocene strata in Ocotillo Badlands northeast of the break. Geomorphic and geologic evidence for the exact position of the trace along the north break prior to 1968 is sparse because of a widespread cover of very recent alluvium, particularly along the $10-\mathrm{km}$

2The designation of fault strands in this discussion is the same as that used by Clark ("Surface Rupture Along the Coyote Creek Fault," this volume). They are termed the north, central, and south breaks. 


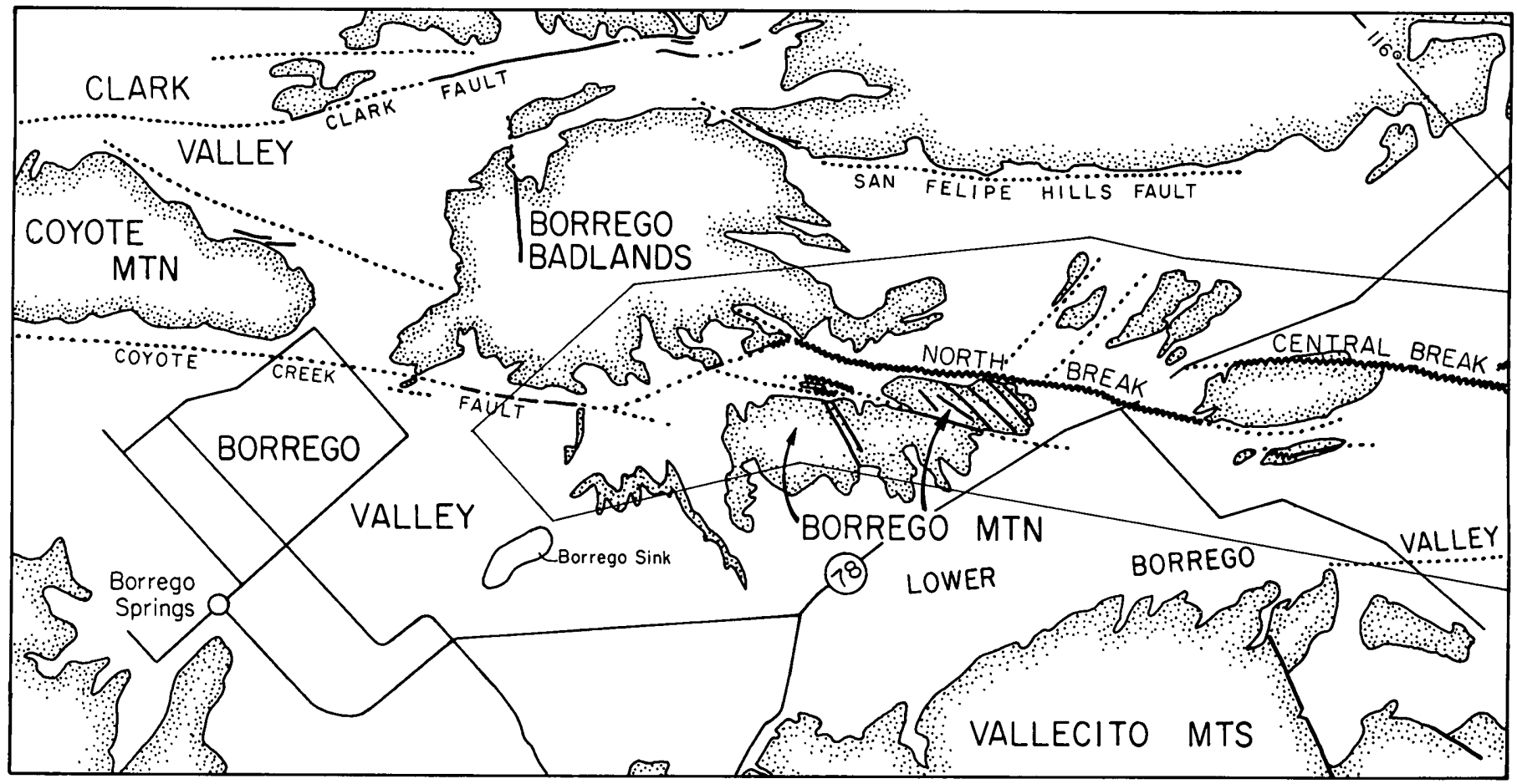

FIGURE 90. - Regional map of the Coyote Creek fault showing its relation to faults outside the 1968 rupture zone. pre-Cenozoic

segment where the fault is followed by the wash of San Felipe Creek.

As indicated by the position of the 1968 break northwest of the north end of East Butte of Borrego Mountain, the Coyote Creek fault traverses the nearly featureless bottom of San Felipe Wash for about $2 \mathrm{~km}$ and then follows the west side of a low, flat bench formed by Holocene terrace gravels for about $1 \mathrm{~km}$. In the next kilometer northwest, the fault again crosses alluvium of San Felipe Creek and projects directly into a small hill at the edge of Borrego Badlands halfway between Big Wash and Third Wash (pl. 3). This is one of the very few places along the entire length of the 1968 break where Cenozoic deposits were apparently newly broken. A few additional minor cracks occurred along the southwest-facing scarp marking a boundary fault at the edge of the badlands for about $2 \mathrm{~km}$ north-northwest of this hill.

The 1968 rupture at the hill between Big Wash and Third Wash apparently did not coincide with the traces of older faulting at that location. Detailed mapping shows that a fault continues for probably at least $2 \mathrm{~km}$ north-northwest into the Borrego Badlands, nearly on the projected trend of the 1968 break south of Third Wash. (See pl. 3; fig. 91.) The fault that borders the southwest side of Borrego Badlands converges east-southeastward with this fault near the hill just mentioned. Apparently, the 1968 rupture at the hill is a new break that approximately bisects the angle between the previously established branch faults. No evidence of earlier movement on projection from the 1968 rupture was found either on the hill or farther north.

The positions of similar fold axes on opposing sides of the fault extending into Borrego Badlands suggest a small right-lateral offset (fig. 91). Although the gross structure throughout the southern Borrego Badlands is a north-dipping homocline, small superimposed folds define unique domains of anomalous southward dip on each side of the fault in southeastern sec. 13, T. 11 S., R. 7 E. The traces of bedding in the fault surface as projected from each side of the fault are nearly identical, supporting the interpretation that the folds are correlative and older than the offset. The amount of horizontal shift is about one-third kilometer; the amount and sense of vertical separation are unknown.

Exactly how far this fault extends northwestward into the Borrego Badlands is uncertain, but it does not simply cross the badlands and join one of the main fractures of the San Jacinto zone near Coyote Mountain (fig. 90), as might be expected from the distribution of aftershocks of the 1968 earthquake in this area. (See Hamilton, this volume.) Exposures of sheared sediments are visible where the fault intersects Big Wash, but the fault is covered by terrace deposits for at least the next kilometer to the 


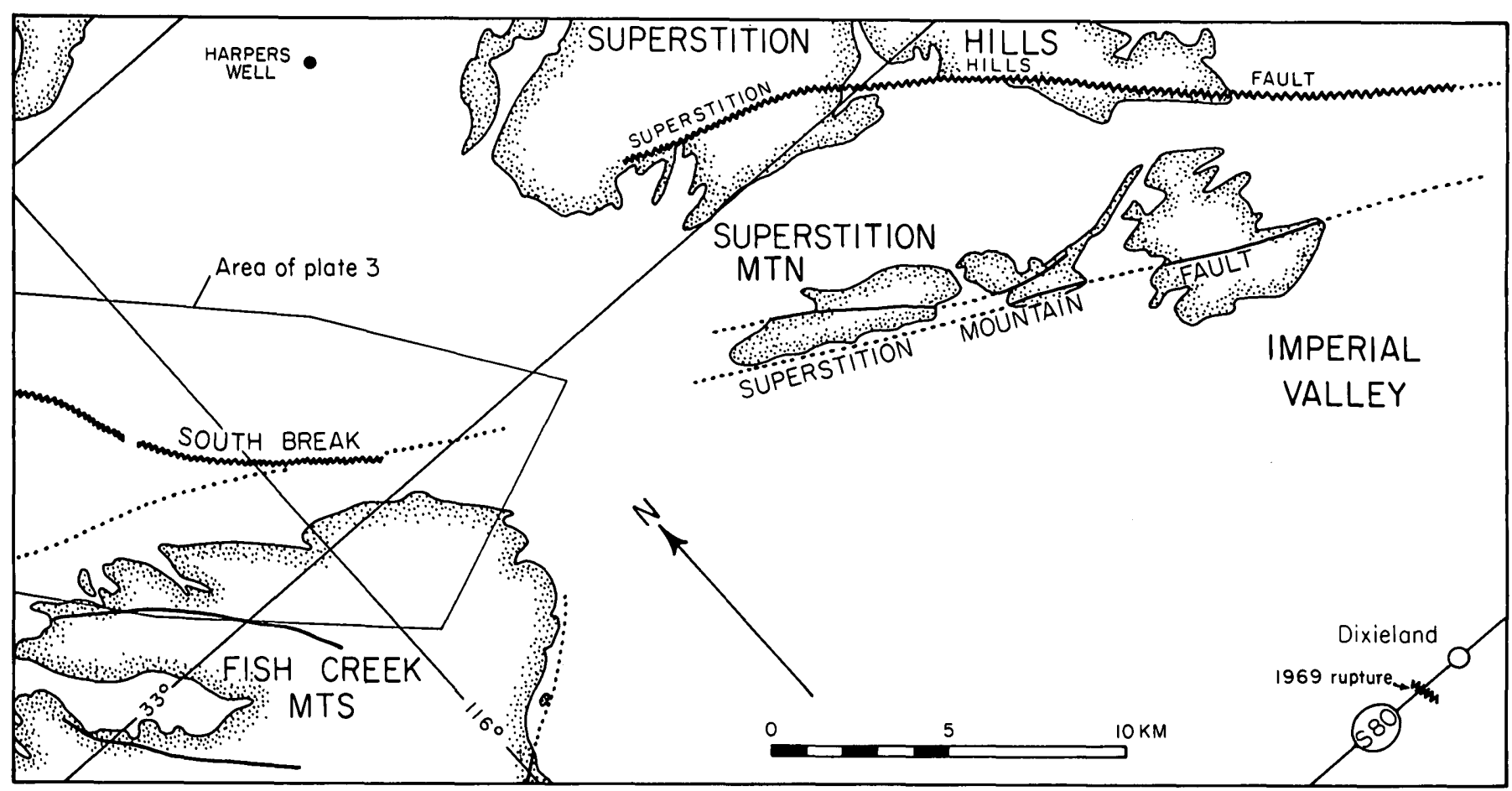

Unstippled areas show Holocene alluvium; stippled areas show Pleistocene and older Cenozoic deposits and crystalline rocks.

northwest. Continuous badland exposures of the Palm Spring Formation (Sharp, "Tectonic Setting of the Salton Trough," this volume) even farther northwest appear to be unfaulted on large-scale aerial photographs, implying either that there is no displacement or possibly that the trace has swung to a slightly more westerly trend and transects the section parallel to the strike of bedding. Although the second possibility has not been investigated fully in the field, such an extension of the fault could at best tie irregularly into the main Coyote Creek fault strand south of Coyote Mountain (fig. 90). Moreover, because intervening parts of the Borrego Badlands are definitely unfaulted, this fault does not connect northward at the surface with the fault bounding the east side of Coyote Mountain.

Although the pre-1968 branch lying within the Borrego Badlands may be an active fault, several lines of evidence suggest that the most active strand of the Coyote Creek fault is now the branch fault lying along the southwest boundary of the badlands. This segment of the fault ruptured at three places in 1968 , and for $1 \mathrm{~km}$ west of the branching point, relatively fresh-looking southwest-facing scarps lie along this strand. In contrast, no geomorphic features of faulting are preserved along the branch extending into the badlands, although a crush zone in the Pliocene and Pleistocene Palm Spring Formation is exposed at Big Wash. Furthermore, the branch along the badlands' boundary lies nearly along the most direct route between the 1968 break and the strand of the Coyote Creek fault that shows evidence of Holocene movement west of Borrego Badlands. (See fig. 90, and Sharp, 1972.)

The Coyote Creek fault along the west side of Borrego Badlands is expressed at the surface only by two rounded northeast-facing linear scarps in alluvium about $2.5 \mathrm{~km}$ north of Borrego Sink Wash (pl. 3). Northwest from these scarps as far as Coyote Mountain (fig. 90), the trace is not visible, but the fault is marked by a very steep gravity gradient reflecting a concealed step in the basement rocks (Biehler and others, 1964, chart 1). Although its trace is concealed by apparently unfaulted alluvium southeast from the same scarps, the fault defines a structural boundary between nearly homoclinally dipping sediments in Borrego Badlands and more intricately folded but otherwise similar beds lying southwest of the fault. Because this strand of the Coyote Creek fault apparently does not directly cross the wash of San Felipe Creek, continuity of faulting to the southeast probably is achieved by an eastward bend or branch of the fault that connects with the northernmost 1968 ruptures.

Southeast from the north end of East Butte to the settlement of Ocotillo Wells, the precise location of the currently active strand of the Coyote Creek fault is known only from the trace of rupturing in 


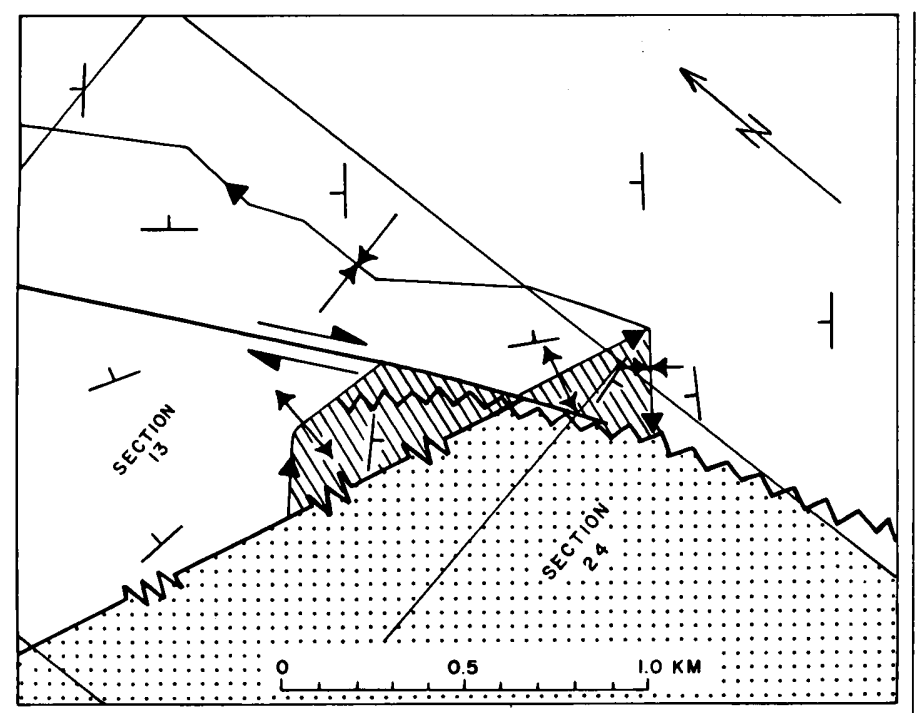

EXPLANATION

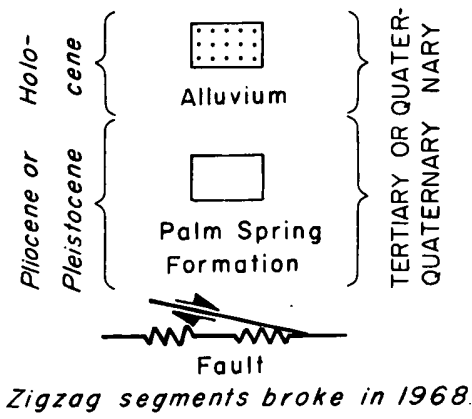

Arrows indicate relative horizontal movement

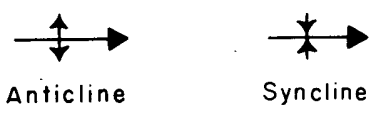

Fold axes, showing plunge

Generalized strike and dip of beds

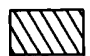

Domain in which dips are in southeast quadrant

In other areas dips are in northeast or northwest quadrants

FiguRE 91. - Simplified geologic map of the area at the north end of 1968 Coyote Creek fault ruptures. Area in this figure is shown on plate 3 .

1968. Extensive alluvial cover and the absence of surficial features of Holocene faulting in the alluvium prevent close delineation of the fault by geologic or physiographic means.

Geologic evidence at Borrego Mountain and in the hills east and northeast of Benson Dry Lake demonstrates that several widely divergent branch faults exist along the north break of the Coyote Creek fault. Granitic rocks and overlying beds referred to the Palm Spring Formation (Sharp, "Tectonic Setting of the Salton Trough," fig. 2, this volume) exposed at East Butte and West Butte of Borrego Mountain (Dibblee, 1954, pl. 2) are broken by several southtrending faults, some of which display small vertical components of offset. There may have been lateral components of displacement on these fractures, but no clear evidence supports strike slip. In contrast, on the east side of the Coyote Creek fault, locally tightly folded and faulted strata of the Imperial Formation, Palm Spring Formation, and Ocotillo Conglomerate of Dibblee (1954) (Sharp, "Tectonic Setting of the Salton Trough," fig. 2, this volume) define a large compressional zone with east-west structural grain. At least two major east-trending faults, one bounding Squaw Peak on the south and the other underlying San Felipe Wash where it turns abruptly eastward, are required to explain convincingly the distribution of the basement rocks and distinctive Cenozoic stratal units. (See fig. 92.) The presence of a fault at Squaw Peak is indicated by the abutment of south-dipping beds of the Palm Spring Formation against the crystalline basement along the south edge of the hills; this relation could be explained by a depositional contact on the south limb of an anticline centered on the axis of the hills, but uniformity of gneissic structure in the basement rocks indicates that such anticlinal arching has not occurred. The southern east-west fracture is shown by stratigraphic relations - in particular, by the omission of the Ocotillo Conglomerate from the section exposed on the north side of San Felipe Creek. The post-Pleistocene compression indicated by the folding subparallel to the traces of both these faults suggests that they may be reverse or thrust faults. The regional gravity map of Biehler, Kovach, and Allen (1964) depicts east-west alinement of gradients in this area and establishes that the structural trends evident at the surface are reflected in the configuration of the basement at depth.

\section{OCOTILLO BADLANDS SECTION OF THE COYOTE CREEK FAULT}

The north and central breaks of the 1968 rupture are disposed in a left-stepping en echelon arrangement along the margins of Ocotillo Badlands (pl. 1; fig. 93). Tarbet and Holman's (1944) Borrego Formation and Dibblee's (1954) Ocotillo Conglomerate exposed in the badlands almost certainly were folded complexly and bulged upward in response to the same tectonic forces that produced the boundary breaks, and the deformation along the faults and on the folds dispersed between them is considered in this report to be interrelated. 


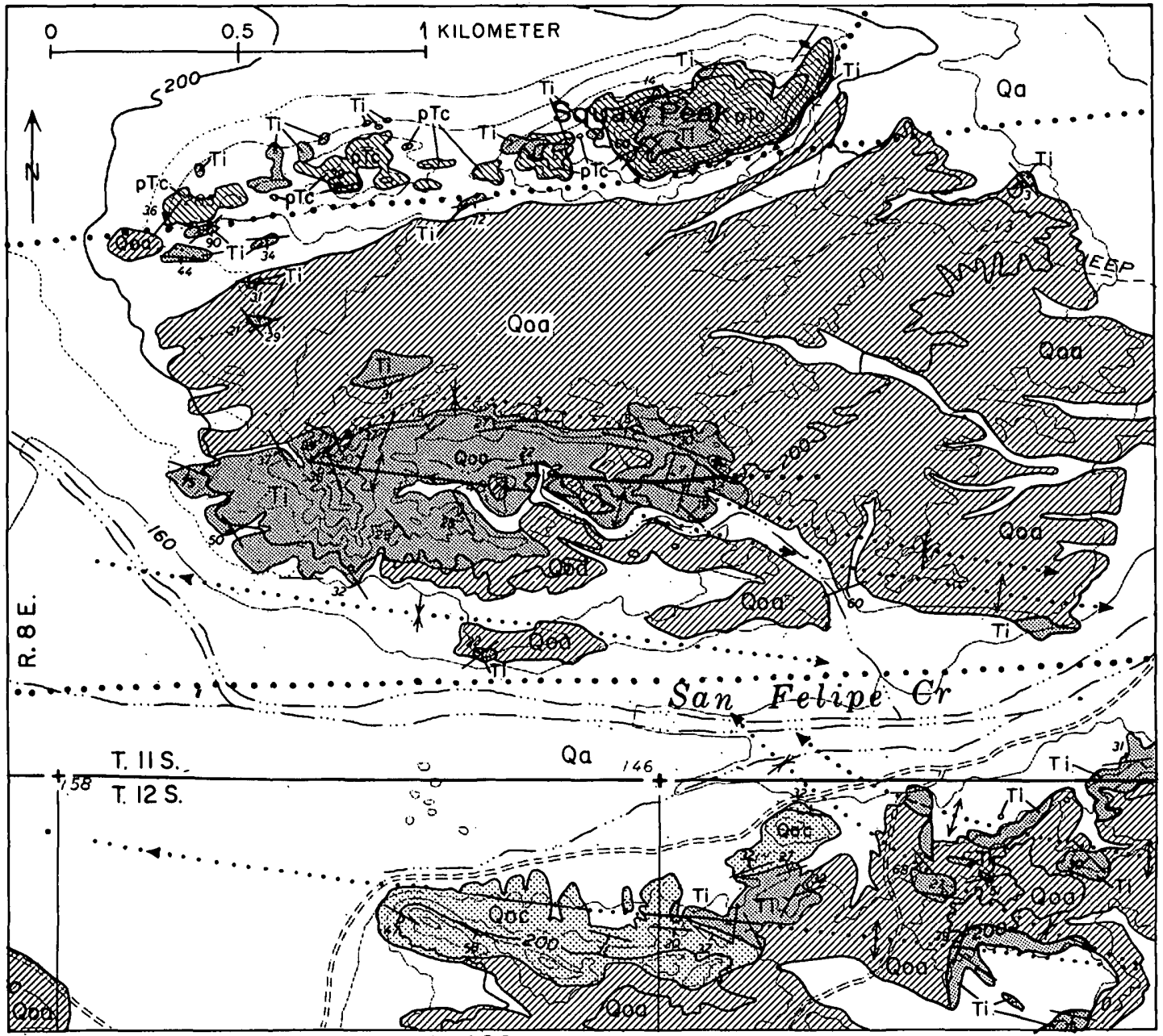

Bose from U.S. Geological Survey 1:24,000

Shell Reef, Borrego Mountain, 1960

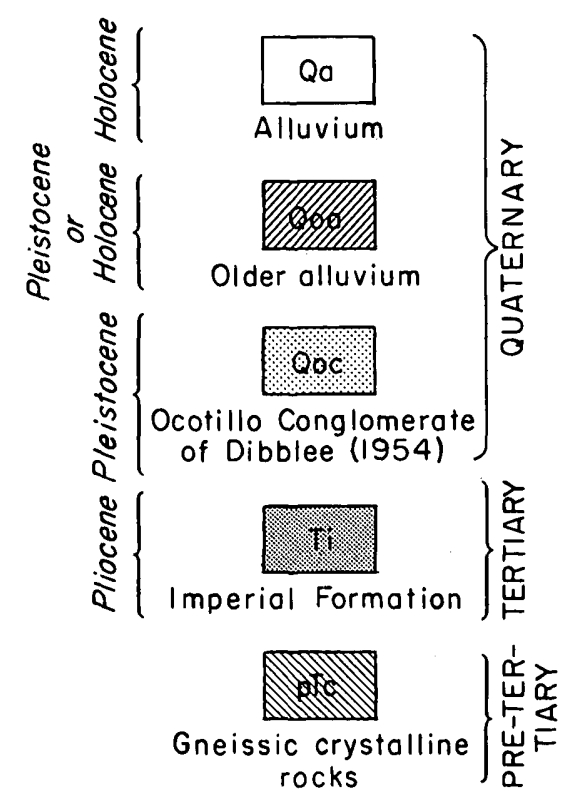

EXPLANATION

Geology by R. V. Sharp, 1969

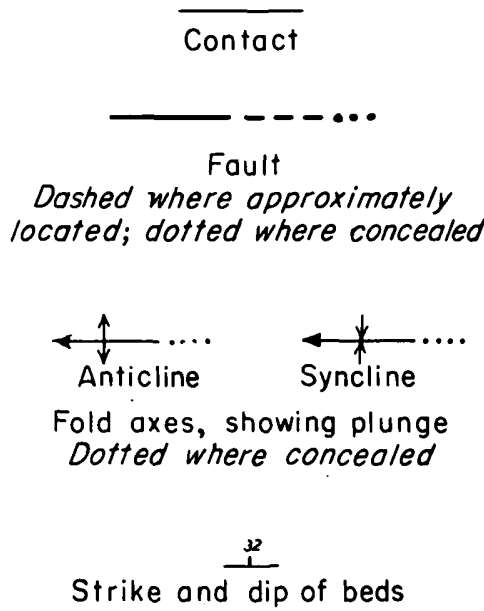

Figure 92. - Geologic map of the area near Squaw Peak. (See pl. 3 for location of this map.) 


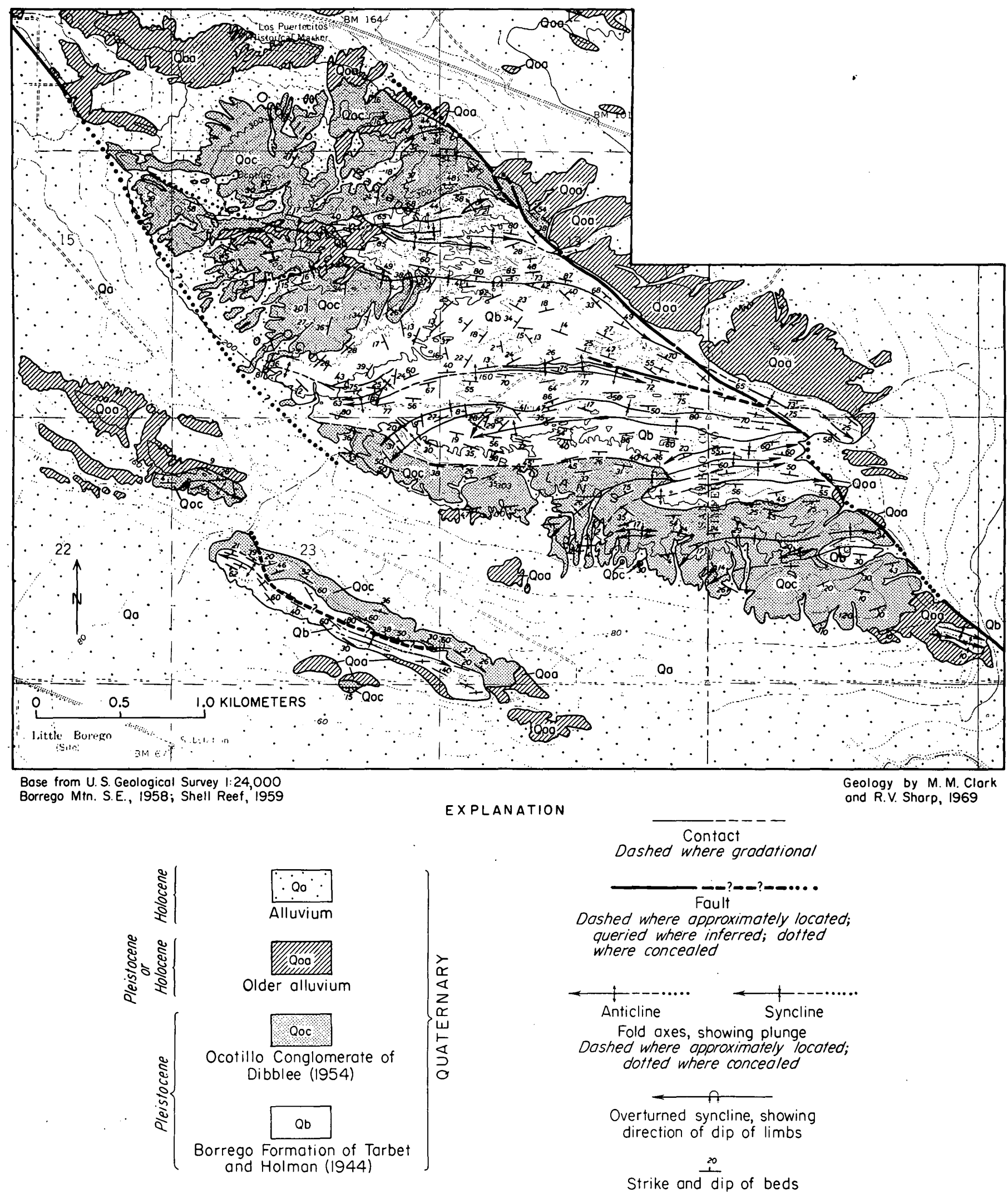

Figure 93. - Geologic map of the Ocotillo Badlands. (See pl. 3 for location of this map.) 
Whether the faults bounding the Ocotillo Badlands project beyond those segments that broke at the time of the 1968 earthquake (the north and central breaks) is unknown. Although the north break terminated as a coherent line of displacement near the northwest corner of the badlands, the linear form of the west margin of the hills suggests that the fault may continue southeastward and may intersect the central break. Abundant geologic evidence demonstrates the continuity of the central break along nearly all the northeast boundary of the hilis. The possibility that the central break extends beyond State Highway 78 at least to Benson Lake is suggested by the alinement of alluvium-filled passes between low hills covered with terrace gravels, but no other evidence for a continuation of the fault is known.

Geologic structure in the Pleistocene Borrego Formation and Ocotillo Conglomerate exposed in the Ocotillo Badlands signifies compressional tectonics (fig. 93). Because bedding in these formations and in older alluvium dips away from the hills around the periphery of the badlands, the gross geologic structure is a closed anticlinal upwarp. However, the detailed structure is extremely complex, involving a large number of tightly folded anticlines and synclines that have essentially an east-west orientation. The folds are relatively open and widely spaced near the west boundary of the hills, where they are exposed in the Ocotillo Conglomerate, but they become tightly appressed and closely spaced eastward in the underlying Borrego Formation. In the central part of the badlands, fold axes are generally straight, but many folds are abruptly terminated either by convergence and cancellation of anticlines by synclines, by offset along minor faults, or by a combination of both mechanisms. In addition, extreme attenuation in the limbs of nearly isoclinal folds locally has destroyed bedding and reduced originally competent sand beds to scattered phacoidal bodies in clay or silt matrix. Near their eastern extremities, the fold axes curve and become parallel with the trace of the central break, suggesting right-lateral drag along the fault. In addition, dips increase to nearly vertical within a few tens of meters of the break, perhaps as a result of drag from the vertical component of displacement.

The geometry of the folds on the southwest side of the badlands is more varied than to the east, but the nonuniformity probably is partly related to the age of the exposed beds. Fold axes in the Borrego Formation trend southwest before dying out or turning northwest near the southwest margin of the bad- lands. Although the folds are not nearly as tight or abundant on the southwest as they are on the northeast, where present their relation to the probable southwestern boundary fault is similar to that of the northeastern folds to the northeastern boundary fault. In contrast, fold axes in the Ocotillo Conglomerate overlying the Borrego Formation at the south end of the badlands bend southwestward and die out in southwest-dipping homoclinal beds. Moreover, at some places in the badlands, bedding in the Ocotillo Conglomerate is strongly discordant to structure in the underlying Borrego Formation. (See fig. 93.) Thus, the structural simplicity apparent along parts of the southwest edge of the badlands may belie more pronounced but concealed folding at depth that may closely resemble the complex structural effects near the northeastern boundary fault. The tightness of folding probably reflects principally the age of the beds involved, but it is also possible that depth of burial and lithology affected the structural development to some degree.

Another structural complexity at the central break in Ocotillo Badlands, in addition to the apparent drag effects, is the presence of several small closed anticlines and synclines elongate parallel to the fault. These closed structures are less than several hundred meters long and lie next to the fault. The fact that they represent both doming and subsidence of strata implies that they resulted from differential vertical movements of material below the surface adjacent to the fault. Restriction of the closed anticlines and synclines to the region of apparent drag effects suggests that they were created by flowage of buried strata of clay and silt adjacent to the fault in reaction to local drag effects. As already noted, tight folds exposed in the center of Ocotillo Badlands reveal widespread flowage of such strata. ${ }^{3}$

Deformation in the Ocotillo Badlands is almost certainly a manifestation of continuity of distributed right-lateral displacement transferred from the north to the central break. Surficial material that is deformed in a right-lateral sense across two breaks with these orientations must theoretically be shortened in the north-south dimension and extended

${ }^{3}$ Closed anticlines and synclines are even more striking $25 \mathrm{~km}$ to the southeast in the uplifted block adjacent to the Superstition Hills fault. Inspection of aerial photographs and brief field investigation show that the upthrown block (the Superstition Hills) consists of a complex sequence of closed, open, and faulted anticlines and synclines in poorly consolidated sediments similar to those of Ocotillo Badlands. The number and complexity of these folded structures increase toward the fault, although the Superstition Hills fault does not everywhere follow a course that is completely obvious from local structure. Future detailed mapping and analysis of the well-exposed structure of Superstition Hills may lead to answers concerning the origin of such small closed folds and also to a better understanding of their relation to the faults associated with them. 
either vertically or in the east-west direction, or both. In this area, there is obvious vertical extension. At great depth, however, it is probable that both surficial breaks converge into a single continuous shear zone, perhaps in the manner shown in figure 94. At depth, such a continuous fault surface would not require the type of strong deformation in the adjoining materials that is now present at the surface in Ocotillo Badlands.

\section{CENTRAL BREAK SOUTHEAST OF OCOTILLO BADLANDS}

Structural expression of the central break southeast of Ocotillo Badlands is simple and is entirely lacking in the southeasternmost part. (See Clark, "Surface Rupture Along the Coyote Creek Fault," this volume.) Briefly, a low scarp (southwest side up) extends along the central break for nearly $4 \mathrm{~km}$ southeast from Ocotillo Badlands. This part of the central break also acts as a barrier to ground water, limiting abundant growth of mesquite (Prosopis juliflora) to the southwest (upthrown) side of the fault. North of Old Kane Spring Road, a secondary break diverges eastward from the scarp, creating a graben between the two breaks. Near the southeast end of this scarp, the sense of vertical uplift is locally reversed, and domed strata are exposed for about

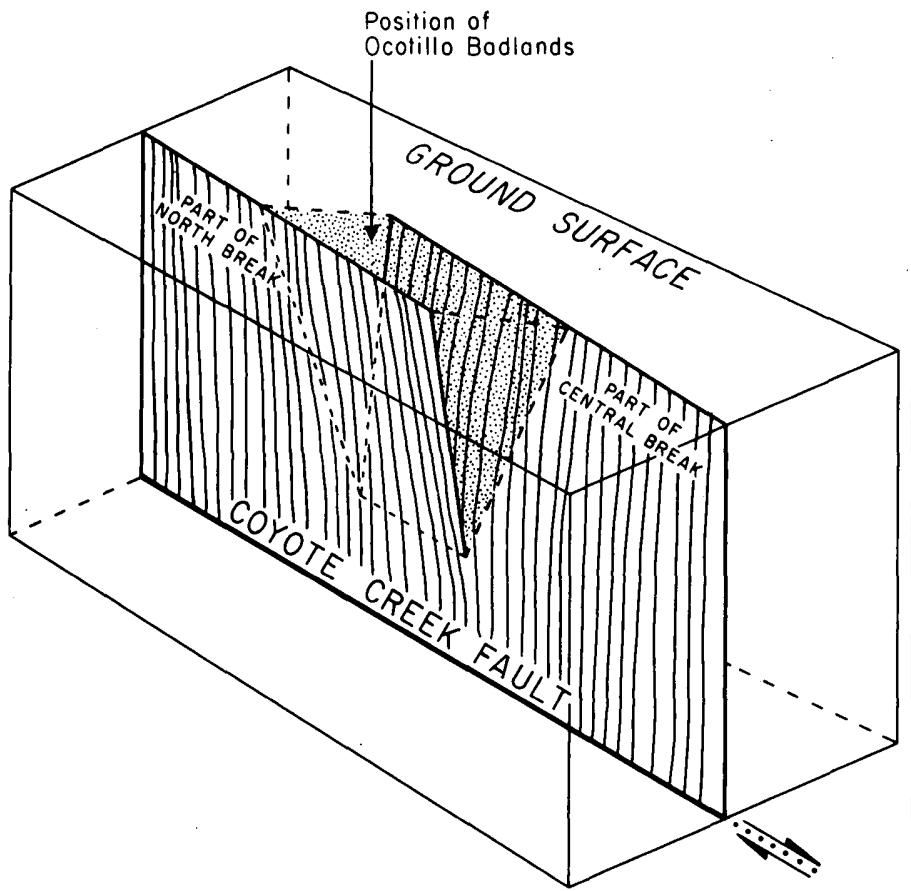

Figure 94. - Three-dimensional view of possible configuration for Coyote Creek fault beneath Ocotillo Badlands. Distal ends of volume of strongly compressed sediments (stippled) shown by dashed and dotted lines.
$100 \mathrm{~m}$ along the northeast side of the break. This small closed anticline resembles those in Ocotillo Badlands described previously but is unusual in its isolated setting and small size. Southeast of the low scarp the central break has no structural expression at the surface.

The scarp, graben, and localized doming resulted from a consistent but small vertical component of displacement associated with the dominant strike slip. These surficial manifestations of faulting are visible today only because the rate of deposition on this alluvial plain (and former lakebed) is less than the rate of tectonic uplift.

\section{AREA EAST OF THE CENTRAL BREAK}

Several of the 1968 breaks extend along isolated low hills east of the central break. The largest of these hills are as much as $1 \mathrm{~km}$ long and consist of upwarped and eroded sediments, commonly in the form of closed anticlines with moderately dipping strata that are easily recognized on vertical aerial photographs; others are covered by unusually large mesquite-stabilized dunes. None is more than $10 \mathrm{~m}$ high.

Although all such hills east of the central break as far as a closed anticline $2 \mathrm{~km}$ south of Harpers Well (fig. 90) were checked in June 1968, fractures were associated with only two: the large upwarp $1 \mathrm{~km}$ east of the central break and immediately south of Old Kane Spring Road, and a prominent hill $5 \mathrm{~km}$ farther southeast and about $3 \mathrm{~km}$ east of the main break. The fracture along the first hill was clearly associated with renewed uplift in 1968, as shown by the sense of vertical displacement (pl. 1). Unfortunately, blown sand obliterated most of the fractures and all evidence of relative displacement along the second hill. However, the surviving fractures lie along the base of the scarp that bounds one side, suggesting renewed uplift of this hill also in 1968 . Because the fractures were probably more extensive originally, it is possible that fracturing occurred during the earthquake or later on other hills within a few kilometers to the northwest and southeast.

The conclusion seems inescapable that these low hills of upwarped sediments are growing as a result of present tectonic activity in this area and that those crossed or bounded by fresh breaks were given additional uplift in 1968. Furthermore, the hills along Old Kane Spring Road and those to the southwest lie approximately on the projection of the Superstition Hills fault, and they may mark a connection between the fracture branching from the central break at Old Kane Spring Road and the Superstition Hills fault. 
HILLS SOUTHWEST OF OCOTILLO BADLANDS

In contrast to the upwarped and gently folded strata that lie to the east of the central break, tightly folded and locally vertical strata are exposed in the linear hills that lie about $1 \mathrm{~km}$ southwest of Ocotillo Badlands. Small ruptures were found in the central part of these hills after the earthquake. Those fractures that traversed exposed strata followed older structural trends, such as offset strata or nearly vertical bedding. However, no new fractures extended to the southeast through the steeply dipping strata of the southern part of the hills, although this area appears to be an anticline with an axial fault. Strata on the flanks of these elongate hills dip gently to moderately away from the axis but become vertical at the axis. By analogy with structure along the northeast margin of Ocotillo Badlands where the 1968 rupture followed a narrow zone of vertically dipping beds, this zone of vertical strata is thought to contain a fault, although its exact position is uncertain.

\section{SOUTH BREAK}

In contrast to the north and central breaks, almost all the south break lacks obvious geologic clues at the surface that indicate the presence of the underlying fault. Vegetation, however, locally defines the position of the active trace, and both the geology and the distribution of vegetation to the southeast suggest connections with other traces. The south break lacks significant vertical displacement and occupies an area of active deposition; as a result, no scarps are visible, and recurrent burial by sediment conceals structural complexity caused by faulting. A channel, however, that crosses the break about $0.7 \mathrm{~km}$ east of R. 9 E. reveals the fault and evidence of repeated activity during the past 2,400 years. (See Clark and others, this volume.) In addition, lines of creosote bushes and shallow sinks along the south break mark several relict tectonic fractures that formed perhaps 20 years before the earthquake. (See Clark, "Surface Rupture Along the Coyote Creek Fault," this volume.) Like segments of the central break, parts of the south break act as a barrier to ground water, encouraging abundant growth of mesquite to the southwest of the fault. (See Clark, preceding reference.) Alined mesquitecovered dunes, which may mark the course of previous surface fractures, and low hills of upwarped alluvium occur along the southeastward projection of the south break and imply a connection between the 1968 break and the colinear fault that forms the southwest boundary of Superstition Mountain. Part of this projected segment lies exactly over a steep gravity gradient interpreted by Biehler, Kovach, and
Allen (1964) to represent a concealed fault. The faults of Superstition Mountain and the low hills between Superstition Mountain and the south break were inspected after the earthquake, but no indication of recent breakage was evident.

The south break and its projection along the southwest boundary of Superstition Mountain trend nearly $20^{\circ} \mathrm{E}$. of the S. $40^{\circ} \mathrm{E}$. average trend of the Coyote Creek fault to the north. According to interpretation of gravity data by Biehler, Kovach, and Allen (1964), faulting along this S. $60^{\circ}$ E. trend probably also continues in the opposite direction (northwestward) west of the 1968 rupture toward the front of the Vallecito Mountains. Alternatively, the principal strand of the Coyote Creek fault may not continue southeastward to Superstition Mountain but instead extends S. $40^{\circ}$ E. from the south break across the nearly featureless alluvial flats that lie to the west of Superstition Mountain. A reconnaissance of this projected trend in December 1970 revealed no evidence of present or past fracturing for a distance of nearly $30 \mathrm{~km}$ from the south break; however, an extensive zone of fresh fractures of possible tectonic origin crosses Highway S80 along the projection about $1.5 \mathrm{~km}$ west of Dixieland (fig. 90).

\section{SUMMARY}

Geologic mapping along the Coyote Creek fault demonstrates various relations of Quaternary structure to the 1968 surface rupture:

1. Bending of fold axes, increase of dips to nearly vertical, and local doming or downwarping of Quaternary strata in the immediate vicinity of the 1968 break give strong evidence of drag effects. These structural clues to tectonic activity and other geologic evidence revealed additional recently active branches and nearby faults in Quaternary deposits along the 1968 break, many of which were inactive during the Borrego Mountain earthquake.

2. Tectonically upwarped hills of alluvial sediment as much as $3 \mathrm{~km}$ from the main 1968 break were apparently uplifted slightly in 1968 .

3. The sense of the vertical component of very recent offset changes at several locations along the fault yet is always consistent with all evidence that shows the sense of earlier movement.

4. Structural complexity along the Coyote Creek fault is greatest in the regions of the north and central breaks, where the oldest sediments and rocks are exposed. Young alluvium and lake deposits may mask complex branch faulting and folding that affect the underlying but 
possibly only slightly older sediments at many locations.

5. The principal fracture of the Coyote Creek fault in the area of 1968 breakage is probably connected with the unbroken main strand farther northwest by a fracture that is mostly concealed beneath the alluvium of San Felipe Creek. Subsidiary faults along the north break diverge from the main fault strand along nearly east-west lines northeast of the fault and along north-south lines on the southwest side.

6. The Coyote Creek fault possibly extends beyond the 1968 breakage east-southeast along isolated uplifted mounds of sediments to the fault that bounds Superstition Mountain on the southwest, although it may also extend southeast to connect with fresh fractures near Highway S80, nearly $30 \mathrm{~km}$ distant.

7. Structural features of the strongly folded and uplifted Cenozoic sediments of Ocotillo Badlands indicate a zone of compression between the en echelon overlap of the north and central breaks. The en echelon pattern, however, may be the surficial expression of a continuous fault at depth.

The pattern of fault strands established by our structural mapping, together with the ruptures formed by the 1968 earthquake, probably only partly reveal the complexity of the Coyote Creek fault. Although many recognizable branch faults diverge from the main zone at small and large angles near Borrego Mountain, other undetected strands and branches of the Coyote Creek fault may also exist, particularly along the south break and parts of the central break that traverse very young Holocene deposits. The definite nature and extent of complex branch faults even in some areas of well-exposed Cenozoic beds may not be revealed until movement occurs again or until extensive exploration is undertaken. The recently detected aseismic ground fracturing near Dixieland (mentioned previously) may prove to be widespread and prevalent; its documentation will certainly supplement and will perhaps be a critical part of the information about geologic structure in this region.

Our investigation emphasizes that the area of potential damage to structures extends far beyond the narrow zone of the main rupture of 1968 and includes outlying low hills of upwarped sediment, splaying faults, and the southeastward and northwestward projection of 1968 ruptures. Any proposed site for a major building in the region of the Coyote Creek fault should be examined carefully below the surface for evidence of Holocene or Pleistocene displacement. The clear evidence of repeated displacement in the past along the 1968 rupture (Clark and others, this volume) suggests that movement can occur again on any trace of the Coyote Creek fault with a record of late Pleistocene or Holocene offset.

This investigation also demonstrates that studies of strain in this region must consider the complex nature of outlying fractures and the widespread occurrence of significant vertical components of movement in the region of the main fault. Attempts to measure regional strain for a simple strike-slip model could fail if stations move vertically in reaction to regional horizontal strain (for example, in Ocotillo Badlands) or if stations undergo complex movement because they are placed in blocks bounded by parallel or splaying faults.

\section{REFERENCES CITED}

Biehler, Shawn, Kovach, R. L., and Allen, C. R., 1964, Geophysical framework of northern end of Gulf of California structural province, in van Andel, T. H., and Shor, G. G., Jr., eds., Marine geology of the Gulf of California: Am. Assoc. Petroleum Geologists Mem. 3, p. 126-143.

Dibblee, T. W., Jr., 1954, Geology of the Imperial Valley region, California, in Jahns, R. H., ed., Geology of southern California; chap. 2, Geology of the natural provinces: California Div. Mines Bull. 170, p. 21-28.

Sharp, R. V., 1972, Map showing recently active breaks along the San Jacinto fault zone between the San Bernardino area and Borrego Valley, California: U.S. Geol. Survey Misc. Geol. Inv. Map I-675.

Tarbet, L. A., and Holman, W. H., 1944, Stratigraphy and micropaleontology of the west side of Imperial Valley, California [abs.]: Am. Assoc. Petroleum Geologists Bull., v. 28 , p. $1781-1782$. 


\title{
INTENSITY DISTRIBUTION AND FIELD EFFECTS, STRONG-MOTION SEISMOGRAPH RECORDS, AND RESPONSE SPECTRA
}

\author{
By Seismological Field Survey, National Oceanic and Atmospheric Administration
}

\begin{abstract}
The Borrego Mountain earthquake was widely felt throughout southern California and neighboring parts of Arizona and Nevada, but damage was generally light owing to the sparse population and lack of structural development. A maximum intensity of VII was assigned to a small area coinciding with the zone of surface breaks along the Coyote Creek fault in the vicinity of Ocotillo Wells.

Strong-motion seismograph records obtained between 41 and 240 miles of the epicenter reveal the following: (1) The peak values for acceleration, $0.14 \mathrm{~g}$, and displacement, $7.25 \mathrm{~cm}$, were recorded at $\mathrm{El}$ Centro. (2) Accelerations recorded at sites on unconsolidated materials were higher than at sites on or near bedrock. (3) Displacement for the first 200 miles was approximately defined by the equation $\log A=$ 2.32-0.011 d ( $A=$ maximum single displacement; $d=$ epicentral distance). (4) Large seismoscope amplitudes oriented at a considerable angle to the trend of faulting were recorded at El Centro. (5) Seismoscope records at earthfill dams indicated an amplification factor for the response of the dams compared to nearby bedrock. (6) A better distribution of strong-motion seismographs would be desirable, particularly along the active fault zones and at free-field sites in the Los Angeles area. (7) Relative velocity response spectra for the El Centro, San Diego, and San Onofre records show the usual rapid decay with distance from the epicenter. With no accelerometer closer than 40 miles from the epicenter, the vertical components recorded are not as important as the horizontal components.
\end{abstract}

\section{INTRODUCTION}

The magnitude 6.4 Borrego Mountain earthquake of April 9, 1968 (G.m.t.) was generally felt over an epicentral radius of 150 miles, and although significant damage was largely restricted to 900 square miles surrounding the region between Ocotillo Wells and Borrego Springs, minor damage occurred in the more populous sections of the Imperial Valley and in the San Diego, Los Angeles, and San BernardinoRiverside areas. Reports from the Borrego SpringsOcotillo Wells area show that broken pipes, minor building cracking, and glass breakage comprised the bulk of vibration-related damage effects, apart from the actual faulting that disrupted State Highway 78 and part of the local airstrip.

Strong-motion seismograph records were obtained from 114 accelerographs operated by the Seismological Field Survey ${ }^{1}$ in California and Nevada, and although relative accelerations were high in only one location, El Centro, the results are particularly valuable for several reasons: (1) The distribution of a large number of records may suggest local and regional geologic influences on strong ground shaking. (2) The first significant earthquake motion to be measured at a nuclear generating plant was recorded at the San Onofre reactor site. (3) The geographic pattern of instrument location and operation could provide a basis for future planning of the strong-motion network. (4) A large number of recordings were obtained in the upper stories of buildings in Los Angeles. These reflect the periods of the structures as excited by seismic forces.

In addition to the acceleration data, records were also obtained from 17 displacement meters and at least nine seismoscopes. Although more than 130 seismoscopes were located in the felt area, recorded amplitudes were generally too small to definitely assign them to the Borrego Mountain earthquake rather than to previous local tremors that had gone unnoticed.

\section{ACKNOWLEDGMENTS}

The authors thank M. Engle, J. Martine, G. Murray, and E. Etheredge, who retrieved and processed the strong-motion records, and also B. J. Morrill, who carried out the field investigation after the earthquake. Thanks are also extended to C. F. Knudson and V. Perez, who reviewed the section on strong-motion seismograph records.

Formerly a unit of the U.S. Coast and Geodetic Survey. 


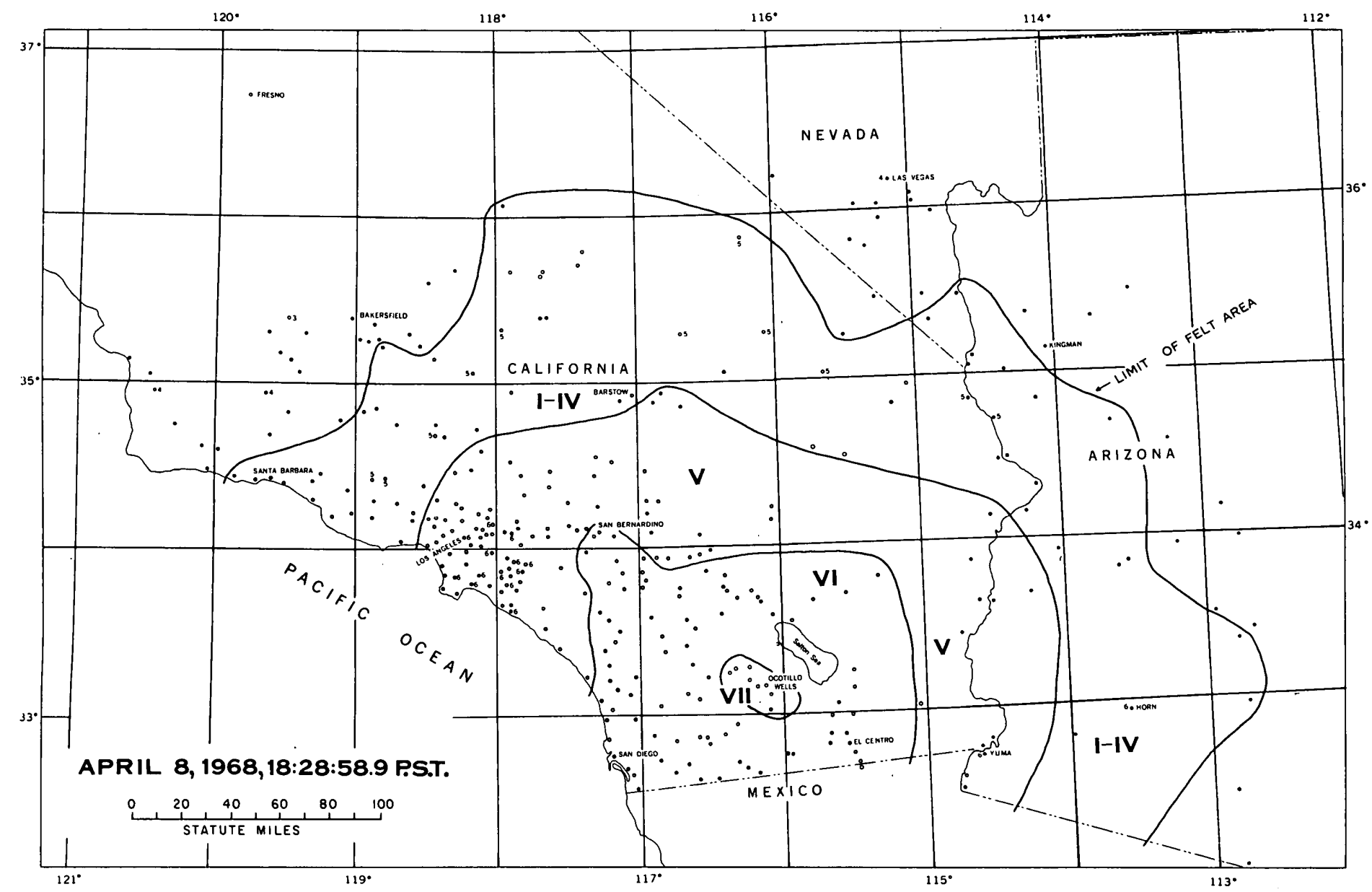

FIGURE 95. - Isoseismal map of the Borrego Mountain earthquake, showing earthquake intensity as measured on the Modified Mercalli scale.

\section{INTENSITY DISTRIBUTION AND FIELD EFFECTS \\ By William K. Cloud Seismograph Station, University of California \\ NinA H. SCOTT}

Seismological Field Survey, National Oceanic and Atmospheric ADMINISTRATION

A review of reports from an extensive questionnaire canvass by the Seismological Field Survey indicates that the generally felt area of the Borrego Mountain earthquake in the United States was approximately 60,000 square miles (fig. 95). Limits of the generally felt area were from Santa Barbara, northeast to Tehachapi, China Lake, Trona, and Tecopa ; in Arizona, southeast to Wikieup, and south to Wenden, Horn, and Dateland. Outside the generally felt area, the shock was reported to be felt slightly at Santa Maria, Fresno, Yosemite Valley, and at Las Vegas. Intensity VII was assigned to a small area in northeastern San Diego County, principally in the Borrego Mountain-Ocotillo Wells area (Coffman and Cloud, 1970). By contrast, Youd and Castle (1970) and Castle and Youd (this volume) report maximum intensities as high as IX, although these were based on ground effects only.

Within the intensity VII area, minor right-lateral displacement on the Coyote Creek fault was observed, and Highway 78 was cracked adjacent to Ocotillo Wells. Park rangers reported that rockslides occurred in Palm Canyon, at Split Mountain, and at Font's Point. Huge boulders blocked the MontezumaBorrego Highway, from the head of Culp Canyon (about 3 miles southwest of Borrego Springs) to near the San Diego County Sheriff's office. Press reports relating to effects at Ocotillo Wells stated that at the Walter Morton home, walls split over doorways and at room corners, a bedroom separated from the rest of the house, dishes and glassware flew out of cupboards, and 3,600 gallons of water gushed out of a storage tank. At the Desert Ironwood Motel, about 3 miles west of Ocotillo Wells, the manager reported loud earth noises, tile block cracked, water pipes in building broke, water from swimming pool flooded the motel, and groundwaves moved toward the north. The manager also reported 
feeling a light tremor about 45 seconds before the main shock. At the M. A. Smith residence, across Highway 78 from the Desert Ironwood Motel, a schoolbus moved 3 inches, furniture moved 4 inches, and the pumice-block building was slightly damaged. At the Borrego Air Ranch, about 10 miles northwest of Ocotillo Wells and west of Borrego Mountain, well water became dark, a pipeline cracked, and a swimming pool lost $11 / 2$ feet of water, but there was no damage to the concrete-block and steel Class "A" building. At the San Felipe Substation, about 3.2 miles south-southeast of Ocotillo Wells, large transformers were shifted, shearing anchor bolts and breaking X-bracing.

Reported effects of the earthquake at sample locations outside the intensity VII area were:

Brea (Orange County). Canned goods fell from shelves. Hanging objects swung violently northwestsoutheast.

Chula Vista (San Diego County). Strong rolling motion. Power out in 4-square-mile area. Many grocery shelves emptied.

Desert Center ( 50 miles northeast of the epicenter; eastern Riverside County). "Waves 6 inches high on swimming pool and about 100 gallons of water splashed out on northwest-southeast sides. Movement of earth was a slow roll and lasted about 30 seconds."

Hemet (Riverside County). "Impossible to walk in trailer during shock. Trailer movement was violent. Water in swimming pool was sloshing over the sides 10 minutes after the shock was apparently over."

Coachella (Riverside County). "Most damage was to grocery and liquor store stocks in this area; a great deal of breakage."

Imperial County :

Power disruptions occurred in some sections of Imperial Valley.

Brawley ( 8 miles west of Wieman Ranch). "I have experienced all shocks here in the last 45 years. This one was the most violent of all but its vibrations were mostly vertical and therefore it did not topple bookcases, china closets, etc., as in previous shocks. Damage was negligible."

Calexico. (Press) Part of the ceiling fell in Safeway store.

El Centro. (Press) Part of a ceiling fell at the J. C. Penney store in Valley Plaza. The Balboa Hotel, which was battered by the 1940 earthquake, was damaged again. Plaster fell from walls and ceilings on the hotel's second floor. Merchandise fell in stores.

Imperial. Damage at Imperial was reported as generally light. About 7,500 books fell from shelves at the public library. About 6 miles west of Imperial, on the Worthington Road grade leading down to New River, there was a crack in the road about 200 feet long and 2 inches wide. "Shock lasted nearly a minute. Sensation of standing in small boat in choppy water. Walking would have been difficult. Feeling of nausea persisted for 15-20 minutes following the shock. Lights were off for 10-15 minutes after the shock."

Westmorland. (Press) Top part of brick wall collapsed at a laundromat. Walls cracked in other buildings.

Long Beach (Los Angeles County). Press reported the passenger liner Queen Mary, in drydock at the Long Beach Naval Shipyard, rocked back and forth on its keel blocks for 5 minutes following the shock. Few windows cracked. People ran outdoors at the U.S. Naval Base.

Los Angeles. (Press) Los Angeles area residents reported instances of chairs sliding across floors, cracks in plaster, and water sloshing out of swimming pools. The following report was received from a structural engineer:

I inspected two downtown buildings on April 16, 1968, for an insurance company. One of the buildings is 13 stories, steel frame, brick filler walls. Both are of about the same construction and were constructed prior to any earthquake code design criteria. Interior partitions are mostly plastered hollow tile. Damage in both buildings was limited almost entirely to plaster cracks where old cracks from the 1933 and 1952 earthquakes were reopened or were slightly enlarged. There was a slight "banging" crumbling where one building is built tight against the other to the north.

Palm Springs (Riverside County). "Light fixture swayed violently 2 or more feet in north-south direction. Dinner guests became partially ill. Neighbors ran outside, some screaming."

Salton Sea. (Press) Residents on the north shore of the Salton Sea reported a 6 -inch tide was measured on concrete retaining wall.

San Diego (San Diego County). Press reported hundreds of broken windows and severed power lines in beach communities of San Diego County. In the south San Diego Bay area, high-tension wires swung and arced over. People seated found it difficult to stand erect.

West Covina (Los Angeles County). Strong rolling northwest-southeast motion, lasted 1 minute. Difficult to walk.

Whittier (Los Angeles County). "Water splashed out of pool and ran into northeast drains, uphill, and back onto deck from northwest drains. Moved filter off of underground filter in northeast direction. Ground visibly wavy." 


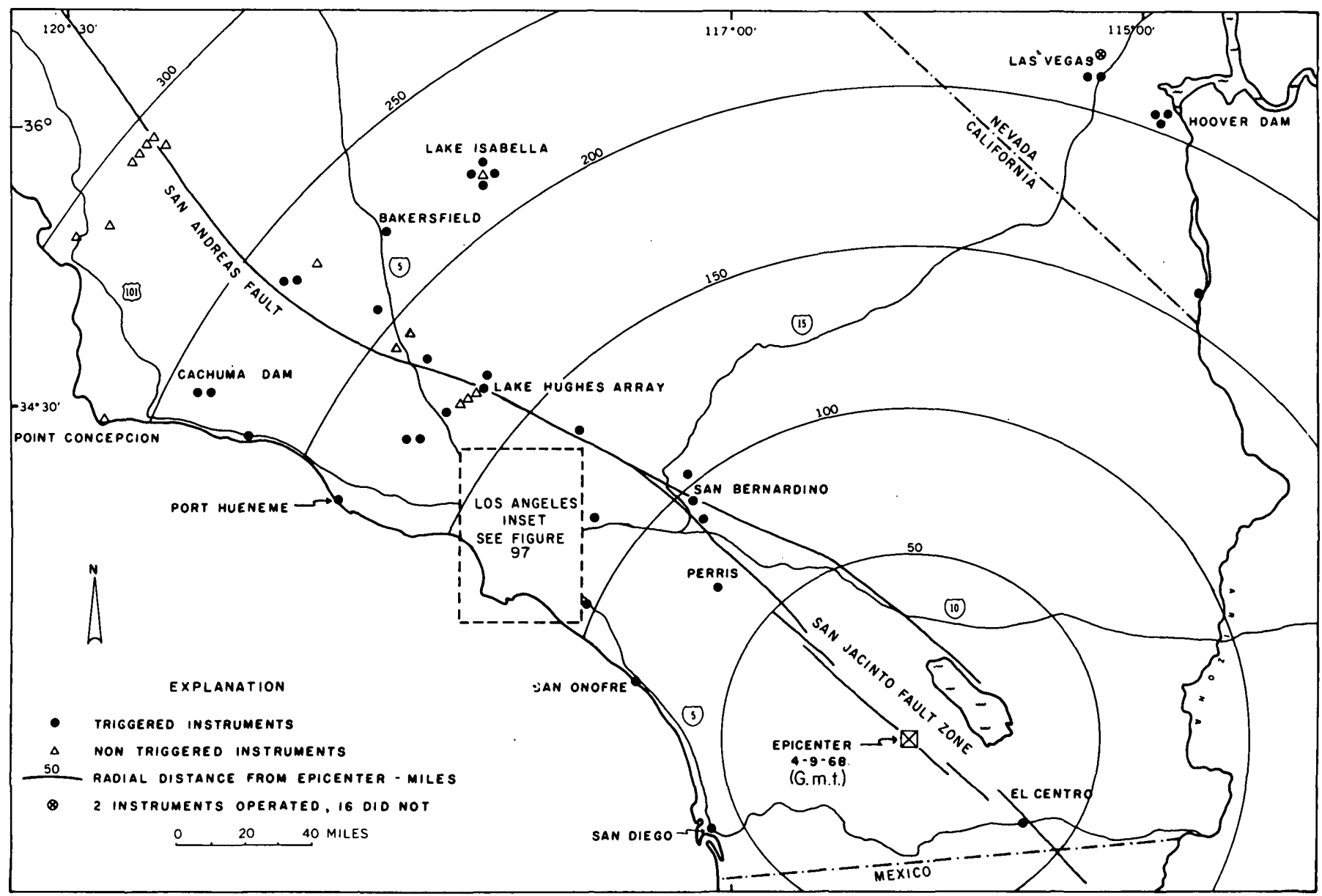

FIGURE 96. - Location of strong-motion seismographs that were triggered by the Borrego Mountain earthquake.

Cantil (Eastern Kern County). Felt by all. Vehicles rocked.

Fillmore (Ventura Couniy). "Water in swimming pools splashed by rocking motion."

Fort Irwin (about 25 miles northeast of Barstow; San Bernardino County). Dizzy sensation experienced.

Blythe (Lost Lake Resort, about 31.5 mi-3s north of Blythe, Colorado River area of eastern Riverside County). "I was sitting on couch in trailer. The trailer shook violently for a few seconds. The effects were very noticeable."

Coso Junction (southern Inyo County). "I was leaning on desk and thought I was having a dizzy spell. Also felt nauseated."

Tecopa (southeast Inyo County). Telephone poles and lines shook.

\section{Arizona :}

Horn (about 150 miles east of the epicenter and just north of Dateland). "One 1,800-foot well pumped red clay. Production of the well dropped off 900 gallons per minute. Desk shifted 1 inch."
Yuma. "Reports of very few cracks in concrete walks and driveways. Noticeable movement of parked vehicles. Water splashed out of pools."

Quartzsite. Water disturbed.

Topock. Small objects and furniture shifted.

Wenden. All persons in home felt a sensation of seasickness.

\section{STRONG-MOTION SEISMOGRAPH RECORDS}

By Richard P. Maley

Seismological Field SURVey, National. Oceanic and Atmospheric ADMinistration

\section{GEOGRAPHIC DISTRIBUTION}

Examination of the geographic distribution of the strong-motion seismographs triggered by the Borrego Mountain earthquake (figs. 96, 97) shows that within the first 170 miles, all 91 accelerographs were triggered, including every Los Angeles area station, whereas from 170 to 240 miles, 21 of 28 operated. In addition, two of the 18 installations maintained by the Environmental Research Laboratories Special Projects Party in Las Vegas, Nev., 215 miles from the epicenter, were also actuated. Most of the 16 


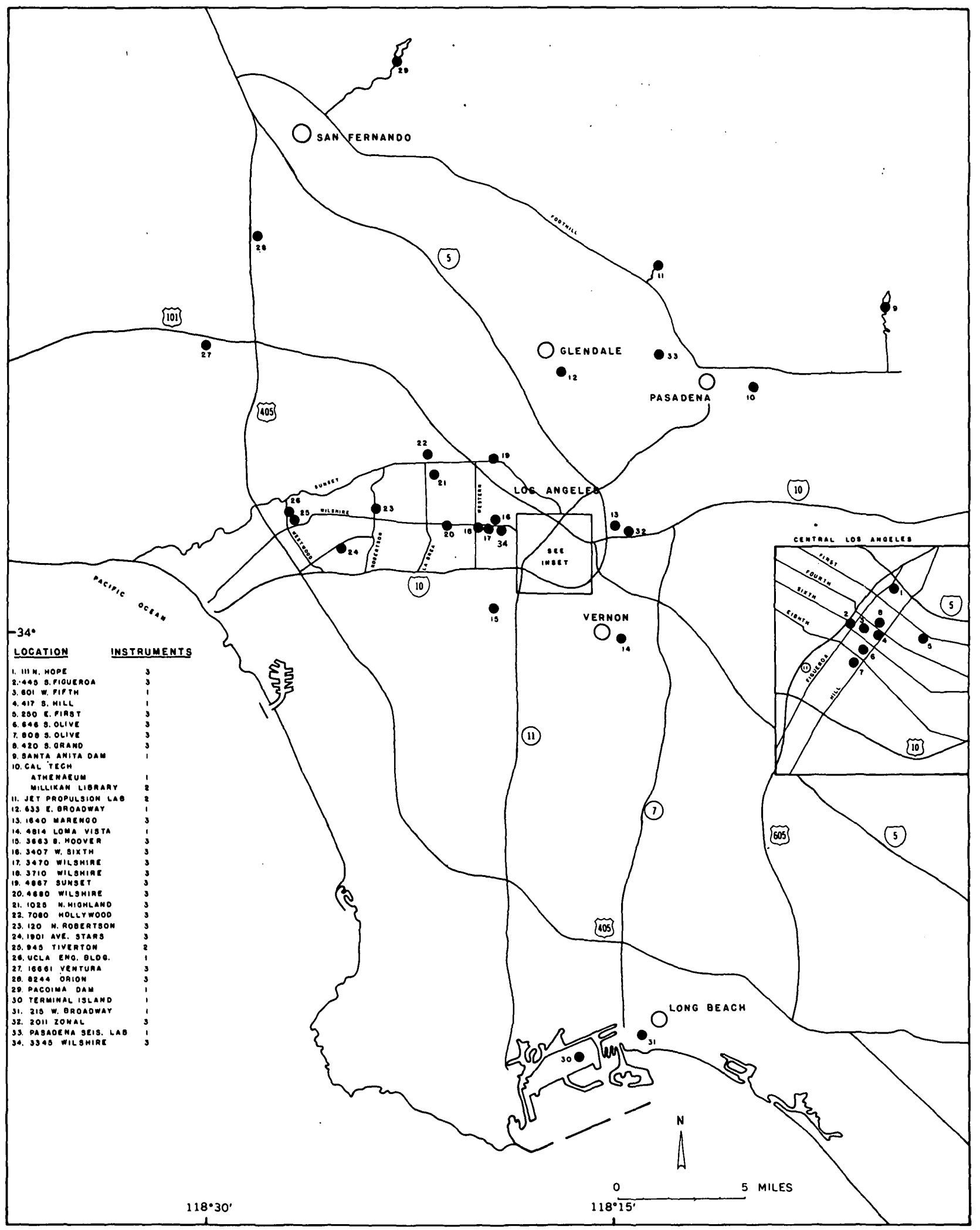

Figure 97. - Location of strong-motion seismographs in the Los Angeles area that were triggered by the Borrego Mountain earthquake. 
that did not operate are in upper levels of taller buildings. It is not known how many of these were in standby condition, since they are generally used to record the effects of nearby nuclear detonations rather than kept in ready status for earthquakes. Table 20 gives the strong-motion stations at increas-

TABLE 20. - Strong-motion seismograph records from the Borrego Mountain earthquake

\begin{tabular}{|c|c|c|c|c|}
\hline \multirow{2}{*}{ Stations } & \multicolumn{3}{|c|}{ Instruments } & \multirow{2}{*}{$\begin{array}{c}\text { Distance } \\
\text { from } \\
\text { epicenter } \\
\text { (miles) }\end{array}$} \\
\hline & C \& GS & $\mathrm{AR}-\mathbf{2 4 0}$ & RFT-250 & \\
\hline 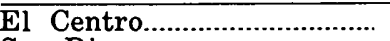 & 1 & $\cdots$ & $\cdots$ & 41 \\
\hline San Diego.......... & 1 & & .... & 67 \\
\hline Perris Dam site... & $\cdots$ & 1 & .... & 78 \\
\hline 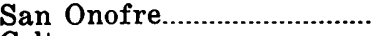 & 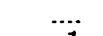 & 1 & .... & 83 \\
\hline Colton & 1 & .... & & 93 \\
\hline San Bernardino.. & 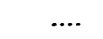 & & 1 & 98 \\
\hline Devil Canyon.......... & .... & 1 & .... & 102 \\
\hline Cedar Springs Dam site.... & $\cdots$ & $\overline{1}$ & .... & 104 \\
\hline Santa Ana & 1 & & .... & 109 \\
\hline Puddingstone Dam & & 1 & & 117 \\
\hline Los Angeles area.................. & 10 & 65 & 4 & $125-145$ \\
\hline Pearblossom ............................. & $\cdots$ & 1 & & 142 \\
\hline 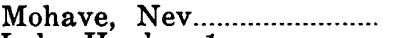 & .... & & 1 & 170 \\
\hline Lake Hughes 1 .. & & 1 & .... & 171 \\
\hline Fairmont Dam... & 1 & & .... & 172 \\
\hline Castaic Dam site.. & .... & 1 & .... & 173 \\
\hline Santa Felicia Dam.................. & .... & 2 & .... & 177 \\
\hline Gorman... & & 1 & .... & 189 \\
\hline Port Hueneme..... & 1 & & .... & 190 \\
\hline Wheeler Ridge... & & 1 & .... & 210 \\
\hline Las Vegas, Nev.. & 2 & .... & .... & 210 \\
\hline Hoover Dam, Nev................... & 3 & .... & & 212 \\
\hline Lake Isabella Dam.............. & & & 14 & 220 \\
\hline Santa Barbara & 1 & .... & .... & 222 \\
\hline 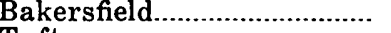 & 1 & $\dddot{m}$ & $\cdots$ & 225 \\
\hline Taft... & 1 & 1 & .... & 237 \\
\hline Cachuma Dam...... & 2 & $\ldots$ & $\ldots$. & 240 \\
\hline Totals & 26 & 78 & 10 & \\
\hline Grand total.................. & 114 & & & \\
\hline
\end{tabular}

${ }^{1}$ Not simultaneously connected. Four operated, one did not.

ing distance from the epicenter, with the type of instrumentation indicated for each site.

The closest nonactuated instruments (table 21)

TABLE 21. - Strong-motion seismographs not triggered by the Borrego Mountain earthquake but within potential operable range

\begin{tabular}{|c|c|c|}
\hline Station & $\begin{array}{r}\begin{array}{r}\text { Number of } \\
\text { Instruments }\end{array} \\
\end{array}$ & $\begin{array}{c}\text { Nominal distance } \\
\text { to epicenter } \\
\text { (miles) }\end{array}$ \\
\hline 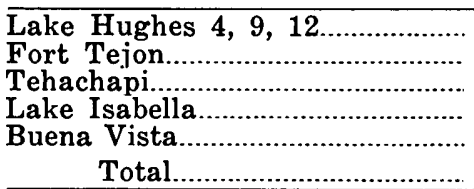 & $\begin{array}{l}3 \\
1 \\
1 \\
1 \\
1 \\
7\end{array}$ & $\begin{array}{l}170 \\
190 \\
195 \\
220 \\
225\end{array}$ \\
\hline
\end{tabular}

were part of the Lake Hughes array, 171 miles from the epicenter. The number 1 station there, a few hundred feet northeast of the San Andreas fault, was triggered, but the three others, 3-9 miles southwest of the fault, were not. Since the maximum accelerations recorded at station 1 were near the instrument's operating threshold, the fact that the three nearby stations were not actuated may reflect minor variations in trigger sensitivities and subtle differences in geology rather than their greater distance from the San Andreas fault. Beyond the 170mile epicentral radius, all accelerographs more than 45 miles inland were not actuated, whereas all those within 30 miles of the coastline and out to the 240mile operable limit were. Preliminary inspection suggested that this pattern was related to the regional variation of geologic structure introduced by the San Andreas fault system and the existence of different tectonic provinces. The seven accelerographs near the coastline are located well west of the San Andreas fault in the Transverse Ranges, while 11 of the 18 inland accelerographs that operated (excluding those in Nevada) are near to and east of the San Andreas fault in the central Transverse Ranges and the southern regions of the San Joaquin Valley and Sierra Nevada. Closer investigation suggests the possibility that this pattern of operation was due to local geologic conditions, rather than any regional structure. Six of the eight nonoperating instruments are on a basement complex or other highly competent rock, while those beyond 170 miles and west of the San Andreas fault are on considerably poorer foundation materials, including earthfill dams and alluvium. None of the near-coastline instruments are at basement-rock sites, and only one is on well-consolidated sediment.

A review of the regional distribution of strongmotion seismograph records show that only six of the 114 records were obtained within 100 miles of the Borrego Mountain earthquake epicenter. The primary reasons for the lack of close-in stations are twofold: (1) A majority of the accelerographs are owned by various organizations and agencies other than the Seismological Field Survey and therefore are operated at the engineering structures of particular interest to those groups. (2) The largest cluster of instrumentation is located in Los Angeles because of the building code provision requiring accelerographs in most taller buildings.

\section{ENGINEERING-TYPE LOCATIONS}

A breakdown of instrument locations according to "structure type" is shown in table 22. Twenty of the larger buildings had simultaneously connected accelerographs at the lowest, intermediate, and highest levels, with identical timing signals and a common starting capability (that is, when any one of three instruments is triggered, the remaining two are immediately actuated, thereby providing a uniform zero time on all records). Of those 20 buildings, 19 have been equipped under the Los Angeles building code that requires three acclerographs in most new structures taller than six stories. Three other buildings have two accelerographs per structure, again 


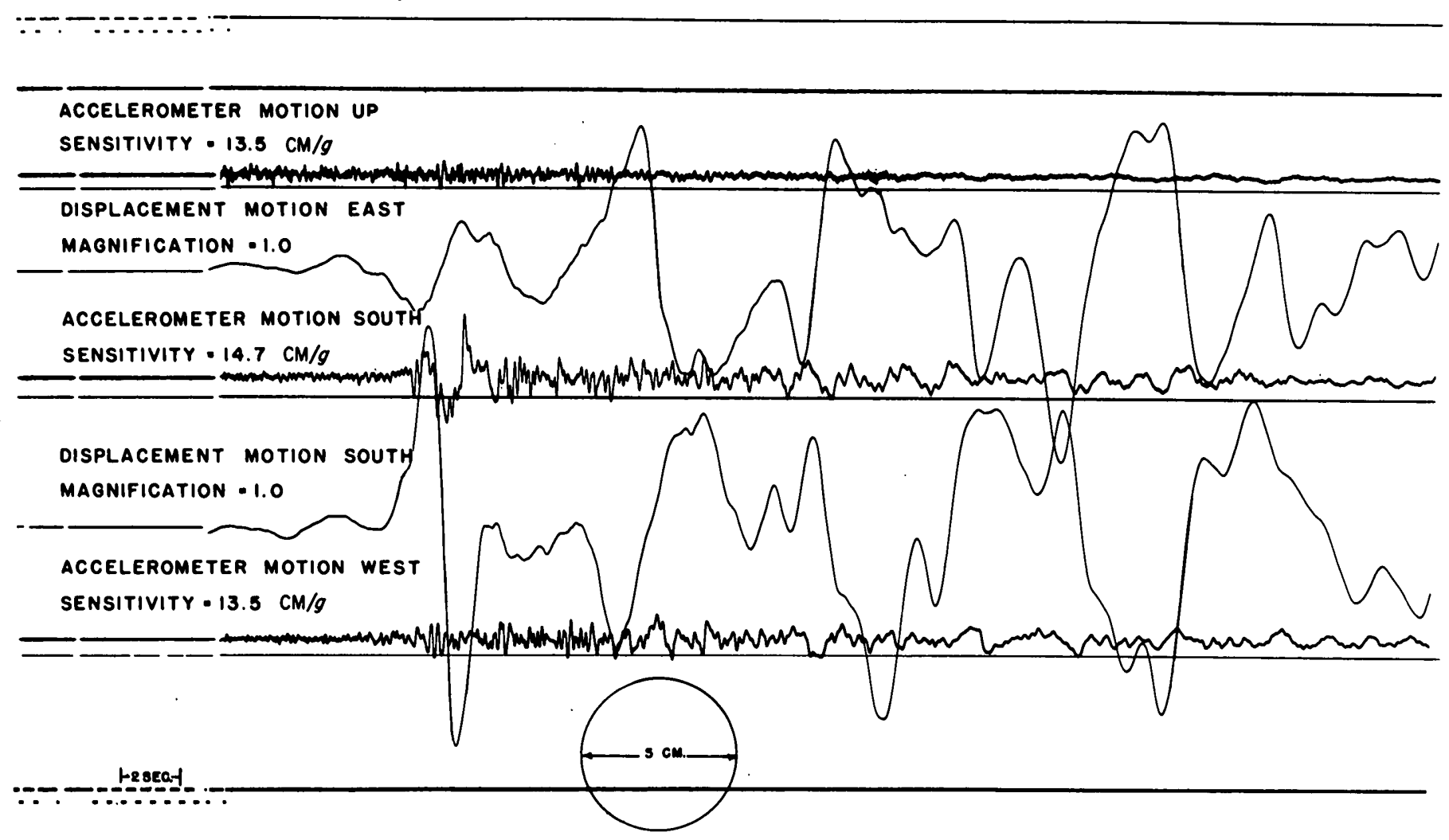

Figure 98. - El Centro accelerograph record from the Borrego Mountain earthquake.

similarly interconnected, and the remaining seven single instruments in the taller buildings are located at the lowest level except the two in Las Vegas that are on the roof. Thirteen accelerographs were in buildings of less than six stories, five of these in the Los Angeles area and the rest in smaller cities throughout central and southern California. Strongmotion seismographs in small buildings or sheds are those related to facilities other than dams in the California Department of Water Resources Feather River project. The 15 records obtained at existing dams are from eight different structures where the instruments have been installed in various configurations of one to five accelerographs per location. The last three stations are at future Department of Water Resources dam sites.

A casual inspection of table 22 reveals that most of the accelerographs were in locations where the records to a considerable extent reflected the characteristic response of a structure rather than pure ground motion. In fact only 20 of the 114 accelerograms were obtained at sites with free-field conditions, and in Los Angeles just one of these was within 15 miles of the central complex of 20 multiple-instrumented buildings. Among the 16 ground stations outside the Los Angeles area, 14 were near existing or planned hydraulic facilities.

\section{ACCELEROGRAPH RECORDS}

Acceleration records were written by three different instruments: the Coast and Geodetic Survey standard strong-motion seismograph and the AR-240 and RFT-250 strong-motion recorders; the latter two are manufactured by Earth Science Division of Teledyne Industries, now Teledyne-Geotech.

Since the three instruments supply similar data (that is, a continuous three-component record of acceleration throughout the duration of a potentially damaging earthquake), it follows that their functional descriptions are closely analogous, although engineering characteristics vary considerably from instrument to instrument. The accelerographs are triggered by a horizontal 1-second pendulum during the early phase of an earthquake, thus actuating the "operate" cycle at an acceleration level of approximately 1 percent of gravity. Three unifilar torsiontype accelerometers detect motion along mutually perpendicular axes, two horizontal and one vertical, and through a system of mirrors deflect a beam of light focused upon translating photographic paper or film behind a collimating lens. Included on the record are 1-second time marks supplied by an internal clock system and a series of reference lines adjacent to each of the three data traces (figs. 98, 


\section{A San Onofre}

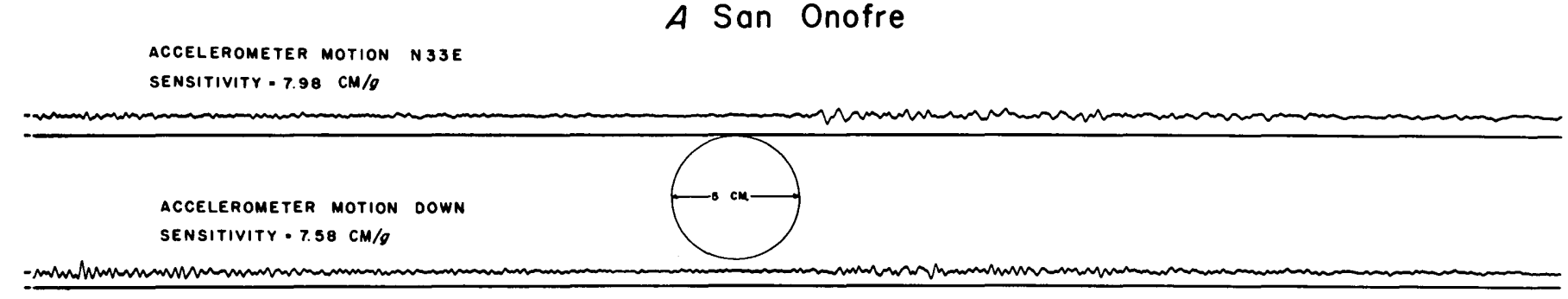

ACCELEROMETER MOTION NSTW

SENSITIVITY $\cdot 7.62 \mathrm{CM} / \mathrm{g}$

(n)

-

\section{$B$ San Diego}

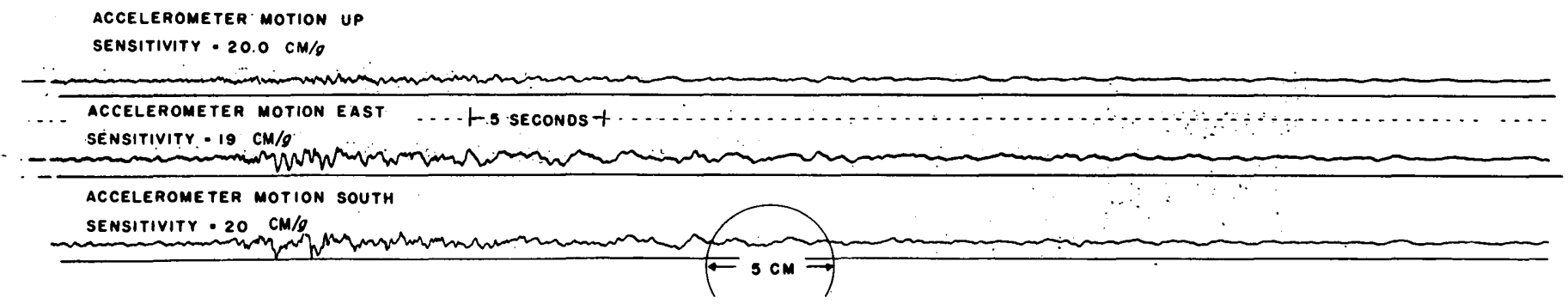

FIGURE 99. - Accelerograph records. $A$, San Onofre accelerograph record from the Borrego Mountain earthquake. Note that the sensitivity of this instrument was about one-third of that at San Diego. B, San Diego accelerograph record from the Borrego Mountain earthquake.

TABLE 22. - Locations of strong-motion seismographs triggered by the Borrego Mountain earthquake

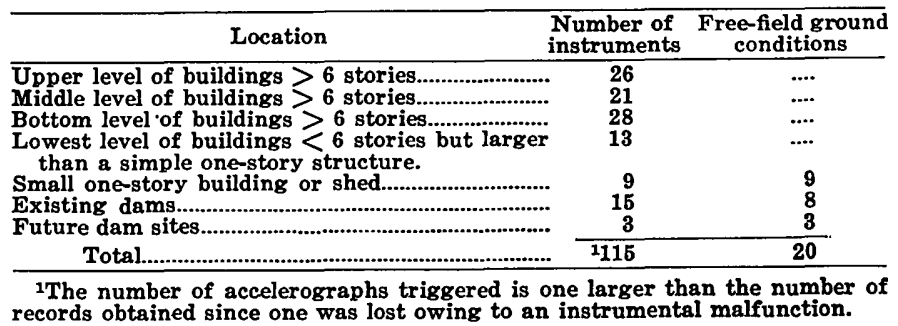

99). Upon cessation of significant shaking, the instrument automatically shuts off but remains in an operable mode in case there are subsequent earthquakes. The nominal physical characteristics of the different accelerographs are given in table 23. (For more detailed descriptions see Halverson, 1969.) Displacement meters that are an integral part of many strong-motion seismographs will be discussed later.

TABLE 23. - General characteristics of strong-motion seismographs

\begin{tabular}{|c|c|c|c|c|c|}
\hline Model & $\begin{array}{l}\text { Period } \\
(\mathrm{sec})\end{array}$ & $\begin{array}{c}\text { Sensitivity } \\
(\mathrm{cm} / g)\end{array}$ & $\begin{array}{l}\text { Damping } \\
\text { (percent of } \\
\text { critical) }\end{array}$ & $\begin{array}{c}\text { Record } \\
\text { size }\end{array}$ & $\begin{array}{l}\text { Recording } \\
\text { speed } \\
(\mathrm{cm} / \mathrm{sec}) \\
\end{array}$ \\
\hline $\begin{array}{l}\text { U.S.C. \& G.S.......... } \\
\text { Teledyne AR-240...... }\end{array}$ & $\begin{array}{r}.045-0.084 \\
.052\end{array}$ & $7-20$ & $\begin{array}{l}60 \\
60 \\
60\end{array}$ & $\begin{array}{l}6 \text { or } 12 \text { in. } \\
12 \text { in. }\end{array}$ & $\frac{1 \text { or } 2}{2}$ \\
\hline Teledyne RFT-250...... & $\begin{array}{l}.062 \\
.048 \\
.070\end{array}$ & $\begin{array}{r}16.2 \\
1.9 \\
13.8\end{array}$ & $\begin{array}{l}60 \\
60 \\
60\end{array}$ & $\begin{array}{l}70 \mathrm{~mm} \\
70 \mathrm{~mm}\end{array}$ & $\begin{array}{ll}1 \\
1 \\
1\end{array}$ \\
\hline
\end{tabular}

'Only four of these models were in use, all in the Los Angeles area.

A maximum acceleration of $0.14 \mathrm{~g}$ was recorded in a north-south direction at El Centro, the station nearest the epicenter, which was 41 miles to the southeast. As previously pointed out by Cloud and Scott (1968), this large pulse had an apparent sinelike character and was superimposed upon a smaller background motion approximately 7 seconds after the recording began (fig. 98). The acceleration 
levels were no higher than $0.06 \mathrm{~g}$ before or after this major pulse in other directions at El Centro, nor for that matter at any other sites. Two other important records shown in figure 99 were obtained from San Onofre and San Diego, respectively 67 and 83 miles from the epicenter. The highest accelerations at San Diego, occurring between 9 and 13 seconds after the instrument was triggered, were about $0.03 \mathrm{~g}$ on both horizontal components. Vertical motions, considerably smaller, had maximums near $0.01 \mathrm{~g}$. The accelerogram from San Onofre, the first significant strong-motion record from a nuclear generating site, shows two separate trains of relatively high amplitude motion: one at the beginning, and the other 16 seconds later. The peak horizontal acceleration was $0.04 \mathrm{~g}$, larger than at San Diego and Perris, two stations nearer the earthquake. Perhaps the most notable feature of the San Onofre record was the vertical acceleration of slightly more than $0.04 \mathrm{~g}$; this acceleration is higher than that recorded at any other site, including El Centro at one-half the epicentral distance. Table 24 lists the

TABLE 24. - Maximum accelerations recorded at stations less than 120 miles from the epicenter

\begin{tabular}{|c|c|c|c|c|}
\hline Station & $\begin{array}{l}\text { Distance } \\
\text { from } \\
\text { epicenter } \\
\text { (miles) }\end{array}$ & $\begin{array}{c}\text { Component } \\
\text { direction }\end{array}$ & $\begin{array}{l}\text { Maximum } \\
\text { accelera- } \\
\text { tion } \\
\left(\mathrm{cm} / \mathrm{sec}^{2}\right)\end{array}$ & Geology \\
\hline 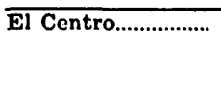 & 41 & $\begin{array}{l}\text { South............ } \\
\text { West.......... } \\
\text { Vertical....... }\end{array}$ & $\begin{array}{r}140 \\
47 \\
33\end{array}$ & $\begin{array}{l}\text { Alluvium, 2,000 ft over } \\
\text { late Tertiary sediments. } \\
\text { Basement at } \\
18,000-20,000 \mathrm{ft} .\end{array}$ \\
\hline San Diego..................... & 67 & $\begin{array}{l}\text { South............ } \\
\text { West.......... } \\
\text { Vertical............. }\end{array}$ & $\begin{array}{l}29 \\
25 \\
10\end{array}$ & $\begin{array}{l}\text { Alluvium, } 50-100 \mathrm{ft} \text { over } \\
\text { Pliocene sediments. } \\
\text { Granite at } \\
4,000-5,000 \mathrm{ft} \text {. }\end{array}$ \\
\hline Perris............................ & 78 & $\begin{array}{l}\text { South............. } \\
\text { West........... } \\
\text { Vertical....... }\end{array}$ & $\begin{array}{r}12 \\
18 \\
6\end{array}$ & $\begin{array}{l}\text { Alluvium, thin veneer } \\
\text { over granitic rocks } \\
\text { (estimated less than } \\
50 \mathrm{ft} \text { thick). }\end{array}$ \\
\hline San Onofre................... & 83 & $\begin{array}{l}\text { N. } 33^{\circ} \mathrm{E} \text {..... } \\
\text { N. } 57^{\circ} \mathbf{W} \\
\text { Vertical......... }\end{array}$ & $\begin{array}{l}40 \\
27 \\
45\end{array}$ & $\begin{array}{l}\text { Slightly cemented Pliocene } \\
\text { sandstone. Estimated } \\
\text { depth to basement rock, } \\
11,000 \mathrm{ft} \text {. }\end{array}$ \\
\hline Colton........................ & 93 & $\begin{array}{l}\text { South............. } \\
\text { West......... } \\
\text { Vertical....... }\end{array}$ & $\begin{array}{l}23 \\
18 \\
19\end{array}$ & $\begin{array}{l}\text { Alluvium, more than } 500 \\
\text { ft. Basement estimated } \\
\text { at } 1,000 \mathrm{ft} \text {. }\end{array}$ \\
\hline $\begin{array}{l}\text { San Bernardino } \\
\text { (Hall of } \\
\text { Records). }\end{array}$ & 98 & $\begin{array}{l}\text { South............ } \\
\text { West.......... } \\
\text { Vertical....... }\end{array}$ & $\begin{array}{r}18 \\
4 \\
3\end{array}$ & $\begin{array}{l}\text { Alluvium, approximately } \\
1,000 \mathrm{ft} \text {. Basement } \\
\text { estimated at } 2,000 \mathrm{ft} \text {. }\end{array}$ \\
\hline $\begin{array}{l}\text { Devill Canyon } \\
\text { (San } \\
\text { Bernardino). }\end{array}$ & 102 & $\begin{array}{l}\text { South............. } \\
\text { West.......... } \\
\text { Vertica1........ }\end{array}$ & $\begin{array}{r}140 \\
47 \\
33\end{array}$ & $\begin{array}{c}\text { Fractured limestone and } \\
\text { gneiss. On Santa Ana } \\
\text { fault (strand of the } \\
\text { San Andreas system). }\end{array}$ \\
\hline Cedar Springs.......... & 104 & $\begin{array}{l}\text { S. } 20^{\circ} \mathrm{E} . \ldots . . \\
\text { N. } 70^{\circ} \mathrm{E} . . . . \\
\text { Vertical...... }\end{array}$ & $\begin{array}{l}6 \\
6 \\
7\end{array}$ & Granitic rocks. \\
\hline Santa Ana................. & 109 & $\begin{array}{l}\text { South............ } \\
\text { West......... } \\
\text { Vertical........ }\end{array}$ & $\begin{array}{r}14 \\
14 \\
5\end{array}$ & $\begin{array}{l}\text { Alluvium, } 350 \mathrm{ft} \text { over } \\
\text { Pliocene sediments. } \\
\text { Basement rock at } \\
\text { approximately } 10,000 \mathrm{ft} .\end{array}$ \\
\hline Puddingstone........... & 117 & $\begin{array}{l}\text { N. } 25^{\circ} \mathrm{E} \ldots \ldots \\
\text { N. } 65^{\circ} \mathrm{W} \\
\text { Vertical....... }\end{array}$ & $\begin{array}{r}16 \\
17 \\
4\end{array}$ & $\begin{array}{l}\text { Volcanic agglomerate over } \\
\text { Miocene sediments. } \\
\text { Basement estimated } \\
\text { at } 500 \text { ft. }\end{array}$ \\
\hline
\end{tabular}

maximum accelerations for the three components at all stations up to 120 miles from the epicenter, and briefly describes the geology of each site. (For a complete tabulation of strong-motion seismograph results, see U.S. Coast and Geodetic Survey and California Institute of Technology, 1968.)
Figure 100 shows the variation of maximum acceleration with epicentral distance for the horizontal components of all strong-motion seismographs that operated during the earthquake. Comparison of the soil conditions at sites less than 120 miles from the epicenter (table 24) indicates that sites on or near bedrock - Perris, Devil Canyon, and Cedar Springs - fall on the lower area of the curve envelope, whereas those sites on alluvium or other less-consolidated materials tend to be on the upper parts of the graph. At Perris there is only a thin veneer of alluvium overlying the basement complex (John Wolfe, oral commun.), and the short seismogram $(6 \mathrm{sec})$ obtained there is more typical of that recorded directly on basement rock (that is, low acceleration level, less than $0.02 \mathrm{~g}$, but relatively high frequency content near $10 \mathrm{~Hz}$ ) than that recorded on alluvium.

Data were also plotted for the maximum vertical accelerations at sites less than 100 miles from the epicenter, thus showing the relatively higher values recorded at San Onofre. The. vertical maximums observed at El Centro, San Diego, and Perris were respectively 75,22 , and 13 percent of that at the San Onofre site.

The square area outlined on figure 100 encloses measurements from 35 ground-level instruments in the Los Angeles area at a nominal epicentral distance of 125-145 miles. Peak accelerations ranged from near zero to slightly greater than $0.01 g$; there was no apparent relationship of attenuation to distance. Attempts to correlate the same information with the local geology were unsuccessful; however, the amplitudes were generally quite small, and the potential scaling error involved is of itself rather large compared with the amplitudes themselves.

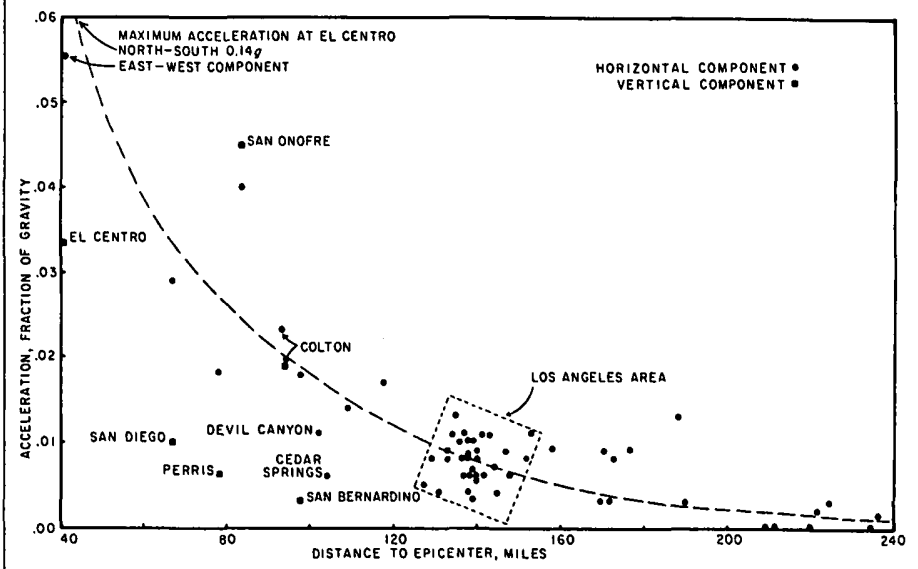

FIGURE 100. - Maximum horizontal accelerations plotted against epicentral distance for the Borrego Mountain earthquake. Vertical accelerations are also included for records obtained at less than 100 miles. 


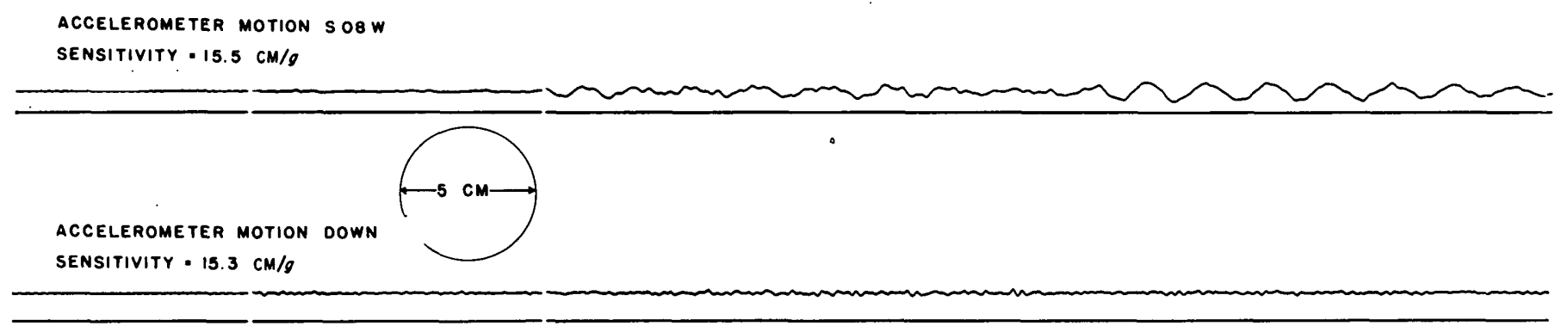

ACCELEROMETER MOTION S B2E

SENSITIVITY - $15.4 \mathrm{CM} / \mathrm{g}$

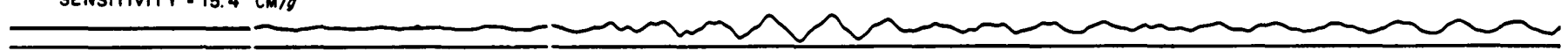

5 SECONDS

FIGURE 101. - Accelerograph record from the Central Engineering building at the Jet Propulsion Laboratory in Pasadena, ninth floor.

Possibly, as suggested by Cloud and Hudson (in U.S. Coast and Geodetic Survey and California Institute of Technology, 1968), "significant studies of the distribution of ground motion can be made" through the use of the entire strong-motion record with complete soil profiles and more sophisticated analytical techniques.

In addition to the 35 ground-level records, 44 accelerograms were written in the middle and upper level of the taller buildings in Los Angeles and Pasadena. The maximum accelerations and the character of these recordings reflect the structural characteristics of individual buildings, as shown by the record in figure 101 which is from the ninth floor of the Central Engineering building at the Jet Propulsion Laboratory in Pasadena. Maximum accelerations on the top floor were $0.03 \mathrm{~g}$, six times those recorded in the basement; the predominant periods were 1.09-1.12 and 1.06-1.14 seconds in north-south and east-west directions, respectively, compared with values of 0.92 and 0.93 second measured during an independent observation of ambient building vibrations. The difference may be attributed to the "softening" effect in the structure under higher horizontal force loads. A peak acceleration of slightly over $0.07 \mathrm{~g}$ at a period of 0.60 second was recorded on the top floor of one structure in Los Angeles, although in most instances the maximums were $0.04 g$ or less, frequently considerably less.

\section{DISPLACEMENT RECORDS}

Displacement measurements were obtained from two different instruments, the Coast and Geodetic Survey displacement meter and the Carder displacement meter, both optional features installed at 11 accelerograph stations where ground-level records were obtained during the earthquake. The Carder displacement meter, operated as an integral component of the accelerograph, is an inverted pendulum suspended by a cross hinge and stabilized by a torsional restraint wire. The seismogram is produced on photographic paper through a system of mirrors that transmit a reflected light ray from the pendulum to the recording surface (Carder, 1964). The Coast and Geodetic Survey displacement meter, although triggered and controlled by the accelerograph, is housed separately from it and records independently. It consists of two horizontal pendulums set at right angles to each other and held level by angular support wires. The axes of the pendulums are free to pivot in a needle-in-jewel arrangement. The record is made by an optical system similar to the one in the Carder displacement meter. Both instruments are normally adjusted for a magnification of 1 . (See table 25 for typical physical characteristics.) 
TABLE 25. - General characteristics of displacement meters

\begin{tabular}{|c|c|c|c|c|c|}
\hline Model & $\begin{array}{l}\text { Period } \\
\text { (sec) }\end{array}$ & $\begin{array}{l}\text { Magni- } \\
\text { fication }\end{array}$ & $\begin{array}{c}\text { Damp- } \\
\text { ing } \\
\text { (percent } \\
\text { of critical) }\end{array}$ & $\begin{array}{c}\text { Record } \\
\text { size } \\
\text { (in.) }\end{array}$ & $\begin{array}{c}\text { Paper } \\
\text { speed } \\
(\mathrm{cm} / \mathrm{sec})\end{array}$ \\
\hline $\begin{array}{l}\text { Carder meter........................ } \\
\text { U.S.C. \& G.S. mete......... }\end{array}$ & $\begin{array}{r}2.0-6.0 \\
10.0 \\
\end{array}$ & $\begin{array}{l}1 \\
1 \\
\end{array}$ & $\begin{array}{l}60 \\
60 \\
\end{array}$ & $\begin{array}{l}6 \text { or } 12 \\
6 \\
\end{array}$ & $\begin{array}{r}1 \text { or } 2 \\
.5\end{array}$ \\
\hline
\end{tabular}

Figure 102 shows the locations of displacement meters at ground-level stations in California and Nevada between 41 and 240 miles of the earthquake epicenter. The largest displacement occurred at El Centro, where a recorded double amplitude of 13.9 $\mathrm{cm}$ (maximum single amplitude of $7.2 \mathrm{~cm}$ ) was correlated in time of occurrence and duration with the sinelike acceleration pulse of $0.14 \mathrm{~g}$ previously referred to in figure 98. (A double amplitude is the total peak-to-peak measurement of successive trace deflections in opposite directions, whereas a single amplitude is a measurement from the null, or zero, line to the deflection in one direction.) As noted by Cloud and Scott (1968), the displacements at El Centro were large compared to the small accelerations, and in fact continuous single amplitudes greater than $4.0 \mathrm{~cm}$ were recorded for more than 90 seconds.

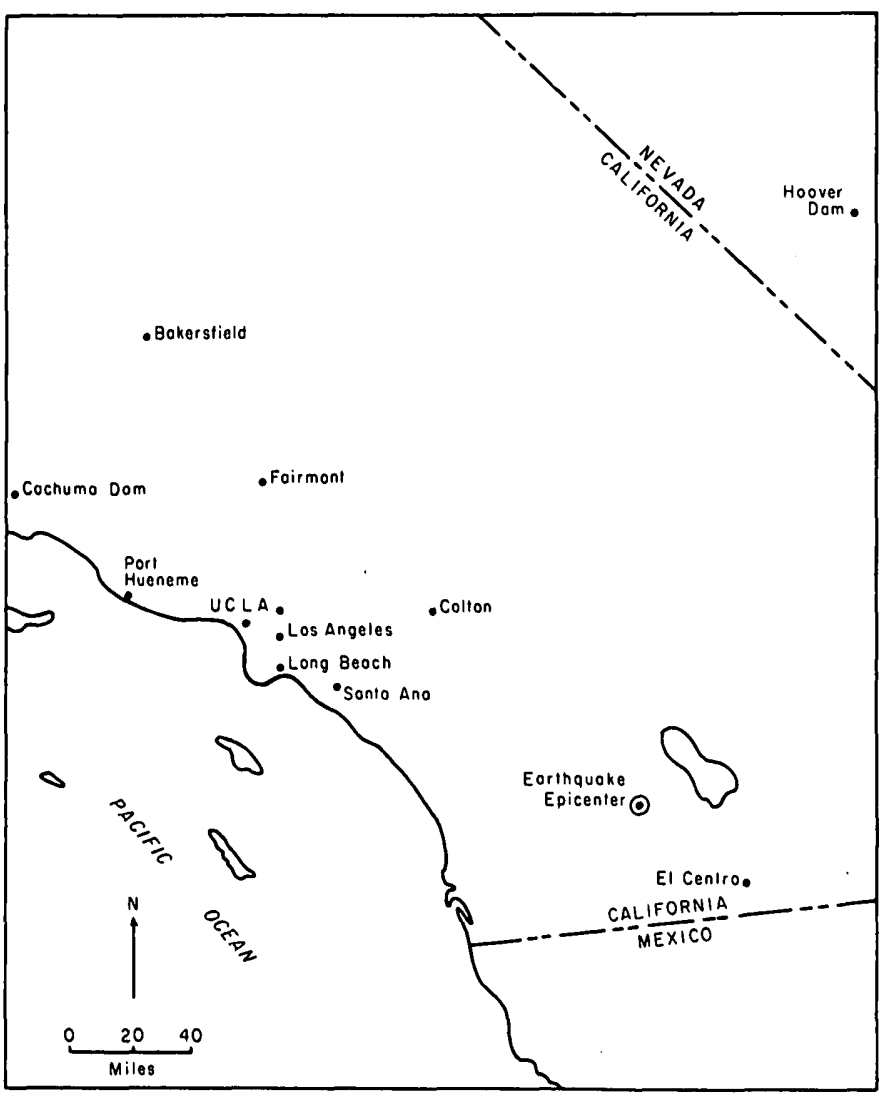

Figure 102. - Location of the ground-level displacement meters actuated by the Borrego Mountain earthquake.
TABLE 26. - Maximum amplitudes recorded by ground-level displacement meters

\begin{tabular}{|c|c|c|c|c|}
\hline \multirow[t]{2}{*}{ Station } & \multirow{2}{*}{$\begin{array}{l}\text { Distance to } \\
\text { epicenter } \\
\text { (miles) }\end{array}$} & \multirow{2}{*}{$\begin{array}{l}\text { Component } \\
\text { direction }\end{array}$} & \multicolumn{2}{|c|}{$\begin{array}{c}\text { Maximum } \\
\text { amplitude }(\mathrm{cm})\end{array}$} \\
\hline & & & Double & Single \\
\hline \multicolumn{5}{|c|}{ Carder displacement meters } \\
\hline 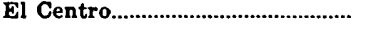 & 41 & South.................... & 13.95 & $\begin{array}{l}7.25 \\
6.80\end{array}$ \\
\hline 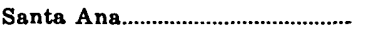 & 109 & S. $4^{\circ} \mathrm{E} \ldots \ldots$ & 2.40 & 1.40 \\
\hline Long Beach... & 127 & 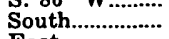 & 1.15 & $\begin{array}{r}1.10 \\
.60\end{array}$ \\
\hline 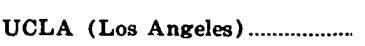 & 148 & 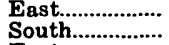 & $\begin{array}{r}1.25 \\
.85\end{array}$ & .70 \\
\hline Fairmont. & 172 & $\begin{array}{l}\text { East............. } \\
\text { N. } 34^{\circ} \text { W......... }\end{array}$ & $\begin{array}{l}.75 \\
.21\end{array}$ & .40 \\
\hline Port Hueneme........... & 190 & 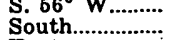 & .20 & .11 \\
\hline Hoover Dam.... & 210 & $\begin{array}{l}\text { East............... } \\
\text { S. } 45^{\circ} \text { W.......... }\end{array}$ & $\begin{array}{l}.70 \\
.45\end{array}$ & $\begin{array}{l}.40 \\
.26\end{array}$ \\
\hline Cachuma Dam...... & 240 & 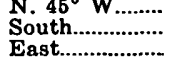 & $\begin{array}{l}.57 \\
.35 \\
.42\end{array}$ & $\begin{array}{l}.35 \\
.20 \\
.31\end{array}$ \\
\hline \multicolumn{5}{|c|}{ U.S. Coast and Geodetic Survey displacement meters } \\
\hline 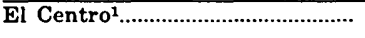 & 41 & South................. & 9.0 & 4.5 \\
\hline Colton..... & 93 & 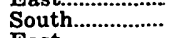 & 3.20 & 1.65 \\
\hline Subway terminal (Los Angeles) & 138 & N. $38^{\circ} \mathrm{E}$ & 3.70 & 2.10 \\
\hline Bakersfield... & 225 & 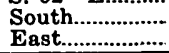 & $\begin{array}{l}3.40 \\
1.30\end{array}$ & $\begin{array}{r}1.95 \\
.65\end{array}$ \\
\hline
\end{tabular}

1Pendulums hit stops.

Peak north-south displacements (table 26) were plotted against epicentral distance in figure 103. Amplitudes measured from the six Carder displacement meter records at distances less than 200 miles fell on a relatively straight line defined by the equation

where

$$
\log A=2.32-0.011 d,
$$

$A=$ maximum single displacement, in millimeters, and

$d=$ epicentral distance, in miles.

Measurements scaled from three of four Coast and Geodetic Survey displacement meters are related to the curve in the following manner: (1) not applicable at El Centro because the pendulums struck the stops at both ends of the swing-arc; (2) reasonably close at Colton, 93 miles from epicenter; and (3) well beyond the arbitrary 200-mile equation limit at Bakersfield. The fourth record, obtained in central Los Angeles, showed abnormally large displacements, the only major deviation from the trend of the curve. Note the comparatively large amplitude also recorded at Bakersfield, 225 miles from the epicenter.

Displacement records were also made by instruments installed on structures at Hoover and Cachuma dams and in the top of two Las Vegas buildings, but these are not considered here.

\section{SEISMOSCOPE RECORDS}

After the earthquake, 131 seismoscope plates were removed from instruments in central and southern California. Since amplitudes were generally quite small, there is no certainty that records thus obtained were from the Borrego Mountain earthquake rather than from minor local earthquakes, except 


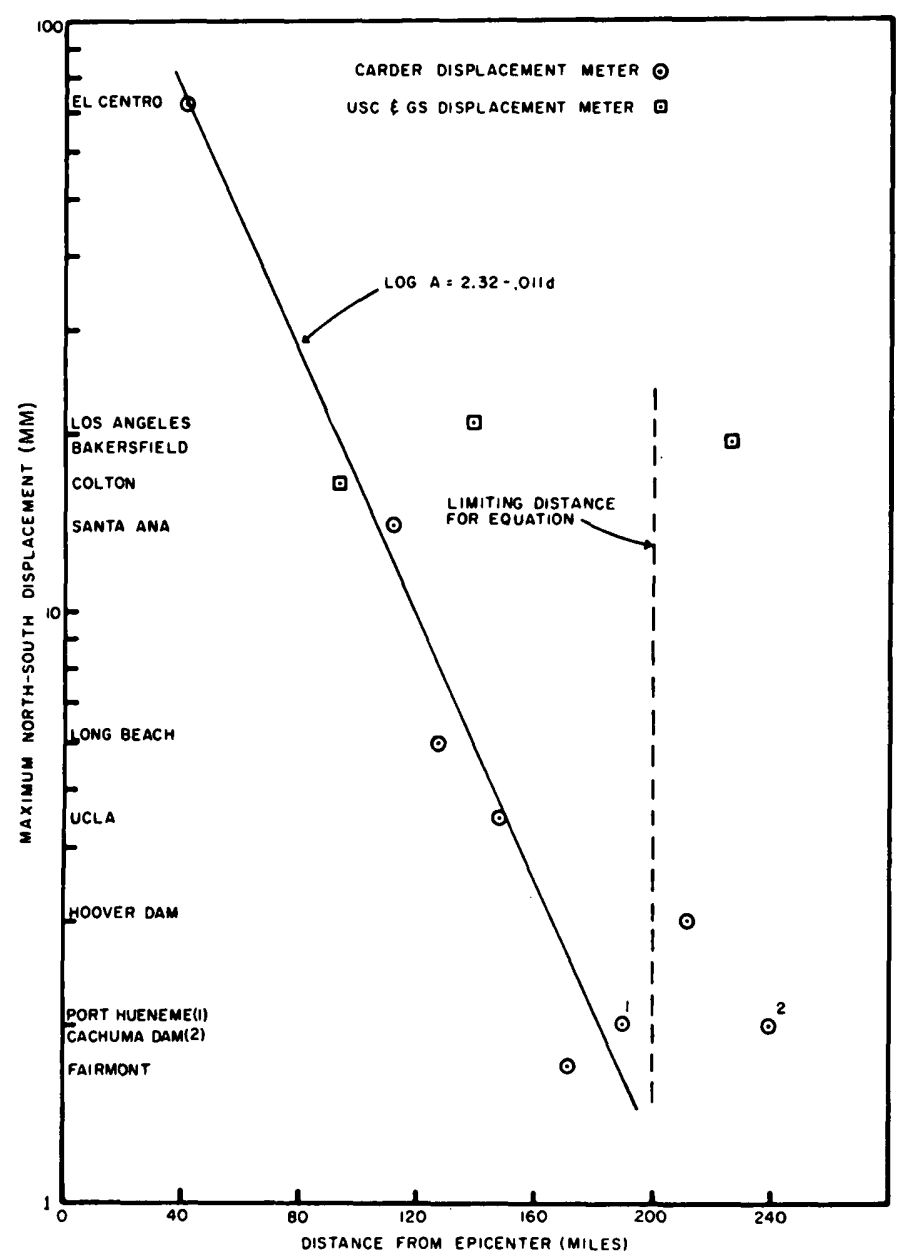

FIGURE 103. - Variation of maximum displacement (northsouth) recorded by ground-level displacement meters with distance from epicenter of Borrego Mountain earthquake.

for El Centro and some dam sites, where instruments are inspected frequently.

The seismoscope consists of a free conical pendulum supported by a single pivot suspension wire providing freedom of movement in all horizontal directions (Cloud and Hudson, 1961). The relative motion between the instrumental base and pendulum is etched on a smoked watchglass by a scriber attached to the frame. As suggested by Hudson and Cloud (1967), the El Centro results are presented in terms of the relative displacement response spectrum that is calculated by the following solution from direct measurements off the watchglass

where,

$$
S_{d}=\frac{g T^{2}}{4 \pi^{2}} \varphi_{\max } \sqrt{\frac{n}{10}}
$$

$S_{d}=$ relative displacement response spectrum, in inches,

$T$ = undamped natural period of the seismoscope,

$$
\varphi_{\max }=\frac{A_{t}}{S}
$$

with,

$A_{t}=$ single amplitude on the record plate,

$S=$ tilt sensitivity of the instrument, approximately 2.15 inches per radian, and

$n$ =fraction of critical damping for the amplitude, $A_{t}$.

Table 27 summarizes the results from four El Centro seismoscopes located at sites $40-42$ miles

TABLE 27.-Relative maximum displacement spectra from the

\begin{tabular}{|c|c|c|c|c|c|c|c|}
\hline Location & $\begin{array}{c}\text { Distance } \\
\text { to } \\
\text { epi- } \\
\text { center } \\
\text { (miles) }\end{array}$ & $\underset{\text { (sec) }}{\text { Period }}$ & $\begin{array}{l}\text { Damp- } \\
\text { ing } \\
\text { (percent } \\
\text { of } \\
\text { critical) }\end{array}$ & $\begin{array}{l}\text { Sensi- } \\
\text { tivity } \\
\text { (in./ } \\
\text { radi- } \\
\text { an) }\end{array}$ & $\begin{array}{c}\text { Trace } \\
\text { Ampli- } \\
\text { tude } \\
\text { (in.) }\end{array}$ & $\begin{array}{c}\text { Maxi- } \\
\text { mum } \\
\text { relative } \\
\text { displace- } \\
\text { ment } \\
\text { (in.) } \\
\end{array}$ & Direction \\
\hline $\begin{array}{l}\text { Central Union } \\
\text { High School........ } \\
\text { Electric substation } \\
\text { Steam plant............ } \\
\text { Waterworks............ }\end{array}$ & $\begin{array}{ll} & \\
\ldots & 40.3 \\
\text { n } & 41.3 \\
. . & 41.5 \\
\ldots & 42.0\end{array}$ & $\begin{array}{r}0.75 \\
.73 \\
.75 \\
.74\end{array}$ & $\begin{array}{l}10.4 \\
11.6 \\
10.0 \\
10.5\end{array}$ & $\begin{array}{l}2.16 \\
2.14 \\
2.19 \\
2.19\end{array}$ & $\begin{array}{r}0.34 \\
.34 \\
.40 \\
.33\end{array}$ & $\begin{array}{r}0.88 \\
.87 \\
1.00 \\
.82\end{array}$ & $\begin{array}{l}\text { N. } 13^{\circ} \mathrm{E} \text {. } \\
\text { S. } 5^{\circ} \mathrm{W} \text {. } \\
\text { S. } 4^{\circ} \mathrm{W} \text {. } \\
\text { S. } 21^{\circ} \mathrm{W} \text {. }\end{array}$ \\
\hline
\end{tabular}
El Centro seismoscope records

from the epicenter. The calculated displacement response spectra ranged from 0.82 to $1.00 \mathrm{inch}$. Superimposed on a map of El Centro at their respective locations (fig. 104), these records indicate that the polarized amplitudes are oriented northsouth, approximately 45 degrees to the trend of ground rupture on the Coyote Creek fault. An angle this large between recorded amplitudes and the trace of nearby fault movement had been observed at Hollister in 1959 and 1960 (Cloud, 1967) and at Parkfield in 1966 (Hudson and Cloud, 1967). Unfortunately, there were no other seismoscopes less than 78 miles from the epicenter, so the angle cannot be measured elsewhere.

At many dams in southern California there are two seismoscopes, one on the crest and the other nearby on rock typical of that underlying the dam. Table 28 gives the unreduced maximum amplitudes scaled directly from the records obtained at five earthfill dams at epicentral distances of 117-166 miles. Although there is no absolute certainty that these records were from the Borrego Mountain earthquake, record size and frequency of inspections suggest that at least those recorded at the Puddingstone, San Gabriel, and San Fernando Dams are from that earthquake. Table 28 also gives records from an earthquake in Borrego Valley on April 28, 1969, and from a shock of unknown origin.

The largest amplitudes from the crest records were essentially normal to the axis of the dam, and when these values were compared with the peak measurements obtained from nearby bedrock regardless of direction, maximum crest to abutment 


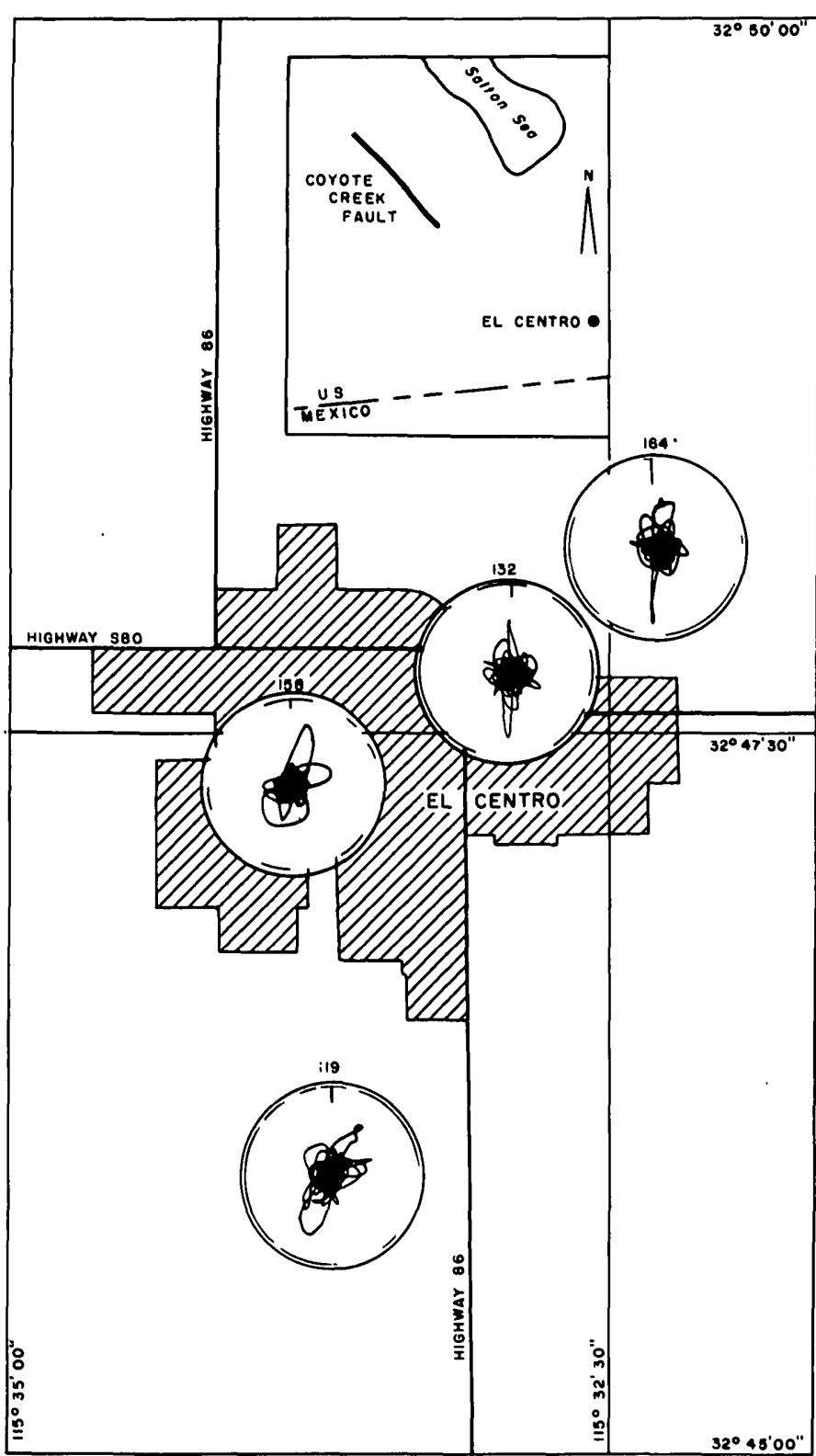

FIgURe 104. - El Centro seismoscope records superimposed on a map of the city.

amplitude ratios ranged from $2: 1$ to $16: 1$. While four of the sites showed ratios between $2: 1$ and $6: 1$, it is interesting that San Gabriel Dam with a crest height of 320 feet, more than twice that of the next largest structure on the list, had ratios of approximately $16: 1$ for two different earthquakes. The rock underlying San Gabriel Dam is a crystalline basement complex, whereas at the other sites the rocks are volcanic agglomerates, Tertiary shale, and alluvium (table 28); consequently, on strictly geologic grounds, a larger range in relative response between the crest and abutment seismoscope may be anticipated at San Gabriel Dam.

One should exercise considerable caution in interpreting these results for two reasons. (1) The values in table 28 are raw measurements scaled directly off the seismoscope plates. (2) The amplitudes represent only one point on the relative displacement response spectrum curve corresponding to the physical characteristics of the instrument. In calculating the relative displacement, it would be necessary to consider the damping factor for very small records (as at the abutments), and since calibration of the seismoscope does not permit sufficiently accurate determination of this variable at such small amplitudes, the inherent error in the displacement response could be quite large. The data presented here merely indicate that there were significant increases of ground shaking on fill-type dams compared to that of the bedrock foundation within the limits of the seismoscope's response range.

Figure 105 shows the comparative records from San Fernando Dam in the San Fernando Valley of Los Angeles, San Gabriel Dam in the foothills above Azusa, and the single record from Puddingstone Dam at San Dimas. The contrast in amplitudes is strongly evident in the first two sets of records, and although there was no abutment seismoscope to compare with the Puddingstone crest, the large amplitude is of itself quite meaningful, considering the relatively large epicentral distance of 117 miles.

\section{RESPONSE SPECTRA \\ By A. Gerald Brady \\ Division of EngineERING AND APPLIED SCIENCr, California Institute of Technology}

The three acceleration records containing the strongest ground motion from the Borrego Mountain earthquake were the El Centro, San Diego, and San Onofre records. Parts of these appear in figures 98 and 99 ; the complete records were published in another report (U.S. Coast and Geodetic Survey and California Institute of Technology, 1968). These three records have been incorporated in a current digitization project under way at the California Institute of Technology. One volume has been published thus far (Earthquake Eng. Research Lab., 1969). Part A of that volume includes two records, IA19 El Centro and IA20 San Diego Light and Power Co., and part B includes the IB40 San Onofre, Southern California Edison record (labeled 68-8T here, the original Caltech reference number). The project report includes digitized accelerograms (v. 1), corrected accelerograms (v. 2), and response spectra (v. 3). 
TABLE 28. - Maximum seismoscope amplitudes from selected dams in southern California

\begin{tabular}{|c|c|c|c|c|c|c|c|c|c|}
\hline Location & $\begin{array}{l}\text { Distance } \\
\text { from } \\
\text { epicenter } \\
\text { (miles) }\end{array}$ & $\begin{array}{l}\text { Orientation } \\
\text { of the } \\
\text { dam crest }\end{array}$ & $\begin{array}{l}\text { Maximum } \\
\text { amplitude }\end{array}$ & $\begin{array}{c}\text { Direction } \\
\text { from } \\
\text { epicenter }\end{array}$ & $\begin{array}{c}\text { Amplitude } \\
\text { ratio, } \\
\text { crest to } \\
\text { abutment }\end{array}$ & Dam type & $\begin{array}{c}\text { Crest } \\
\text { length } \\
\text { (in.) }\end{array}$ & $\begin{array}{c}\text { Height } \\
\text { (ft) }\end{array}$ & $\begin{array}{c}\text { Geologic } \\
\text { foundation }\end{array}$ \\
\hline \multicolumn{10}{|c|}{ Borrego Mountain earthquake of April 9, 1968} \\
\hline Puddingstone crest...... & 117 & N. $62^{\circ}$ E. & 0.36 & N. $30^{\circ}$ W. & (......... & $\begin{array}{l}\text { Earthfill, } \\
\text { curved } \\
\text { crest. }\end{array}$ & 1.080 & 147 & $\begin{array}{l}\text { Volcanic } \\
\text { agglomerate. }\end{array}$ \\
\hline $\begin{array}{l}\text { San Gabriel : } \\
\text { Crest.......................... } \\
\text { Abutment.................. }\end{array}$ & 124 & N. $60^{\circ} \mathrm{E}$. & $\begin{array}{l}.230 \\
.014\end{array}$ & N. $30^{\circ} \mathrm{W}$. & $16: 1$ & $\begin{array}{l}\text { Earthfill } \\
\text { and rock, } \\
\text { straight } \\
\text { crest. }\end{array}$ & $\mathbf{1 , 5 2 0}$ & 320 & $\begin{array}{l}\text { Weathered } \\
\text { gneissoid } \\
\text { granite. }\end{array}$ \\
\hline 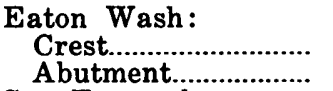 & 134 & $\begin{array}{l}\text { N. } 60^{\circ} \mathbf{E} . \\
\text { to E. }-\mathrm{W} .\end{array}$ & $\begin{array}{l}.06 \\
.03\end{array}$ & S. $20^{\circ}$ E. & $2: 1$ & $\begin{array}{l}\text { Earthfill, } \\
\text { curved } \\
\text { crest. }\end{array}$ & 1,545 & 63 & Alluvium \\
\hline 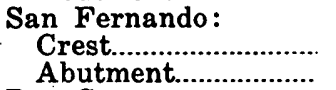 & 157 & N. $73^{\circ} \mathrm{W}$ & $\begin{array}{l}.24 \\
.04\end{array}$ & $\begin{array}{l}\text { N. } 15^{\circ} \text { W. } \\
\text { N. } 70^{\circ} \text { E. }\end{array}$ & $6: 1$ & $\begin{array}{l}\text { Earthfill, } \\
\text { straight } \\
\text { crest. }\end{array}$ & 2,080 & 142 & $\begin{array}{c}\text { Tertiary } \\
\text { shale. }\end{array}$ \\
\hline $\begin{array}{l}\text { Dry Canyon : } \\
\text { Crest........................... } \\
\text { Abutment.................... }\end{array}$ & 166 & N. $80^{\circ} \mathbf{E}$. & $\begin{array}{r}.15 \\
.06 \\
\end{array}$ & $\begin{array}{l}\text { N. } 17^{\circ} \mathrm{E} . \\
\text { N. } 67^{\circ} \mathrm{E} .\end{array}$ & $21 / 2: 1$ & .....do.................... & 510 & 67 & Do. \\
\hline \multicolumn{10}{|c|}{ Borrego Valley earthquake of April 28, 1969, magnitude 5.8 (Pasadena) } \\
\hline $\begin{array}{l}\text { Puddingstone : } \\
\text { Crest......................... } \\
\text { Abutment }\end{array}$ & 117 & N. $62^{\circ}$ E. & $\begin{array}{r}0.09 \\
.04\end{array}$ & N. $56^{\circ} \mathrm{W}$. & $2+: 1$ & \multirow{2}{*}{\multicolumn{4}{|c|}{ See data above. }} \\
\hline $\begin{array}{l}\text { San Gabriel : } \\
\text { Crest.......................... } \\
\text { Abutment.................... }\end{array}$ & 124 & N. $60^{\circ}$ E. & $\begin{array}{l}.063 \\
.004 \\
\end{array}$ & N. $54^{\circ} \mathrm{W}$. & $16: 1$ & & & & \\
\hline \multicolumn{10}{|c|}{ Earthquake of unknown origin between June 1968 and March 1969} \\
\hline $\begin{array}{l}\text { Dry Canyon : } \\
\text { Crest.......................... } \\
\text { Abutment................... } \\
\end{array}$ & 166 & N. $80^{\circ} \mathrm{E}$. & $\begin{array}{r}0.180 \\
.035 \\
\end{array}$ & N. $56^{\circ}$ W. & …...... & & See & a above & \\
\hline
\end{tabular}

The response spectra described herein have been obtained from the uncorrected accelerograms of volume 1 . They will be similar to those forthcoming in volume 3 , since the particular baseline correction techniques are not critical in response-spectra calculations (Amin and Ang, 1966).

The response spectra in figures 106 and 107 have been calculated from the total length of the available records, that is, approximately 87,79 , and 45 seconds. The period ranges from 0 to 10 seconds, and the five damping values are $0,2,5,10$ and 20 percent of critical. All three components of acceleration are analyzed. The computer programs used are those described by Nigam and Jennings (1968); changes were made on subroutines PCN05, PCN06 and PCN07 so that they could be run on the IBM 360 at present at Caltech. In addition, a few of the digitized time coordinates had to be further separated so that the rounding to 0.005 second, performed in subroutine PCN05, resulted in nonequal increasing times in every record. These changes were of the same magnitude as the rounding off changes which are shown to affect response spectra calculations by less than 2 percent.

Figure 106 shows the relative velocity response spectra for the El Centro record in the south, west, and vertical directions. The station at El Centro is approximately 41 miles southeast of the epicenter in the general direction of the San Jacinto and
Imperial faults. (The focal depth, for comparison, was $11 \mathrm{~km}$.)

The two horizontal components here are at the same angle, 45 degrees, to the radial motion from the epicenter, and therefore both components are affected by ground motion both longitudinal and transverse to the fault. Peaks in the spectra are well distributed over the longer periods, those greater than 1 second. Of particular note are peaks at 2 seconds in the south component and at $51 / 2$ seconds in the west component. Vertical motion shows considerably lower spectral values with no predominant peak.

Figures $107 A, B, C$ are the relative velocity response spectra for the south, east, and vertical components of the record of the San Diego Light and Power Co., 67 miles west-southwest of the epicenter. This direction is nearly perpendicular to the San Jacinto fault.

Again the horizontal component directions of the record do not coincide with longitudinal or transverse motion, and therefore both motion components are present in both record components. Peaks are apparent at periods of 2 and $31 / 2$ seconds in both these components.

Figures $107 D, E, F$ are the spectra for the record from the San Onofre Southern California Edison Power Plant, 83 miles west-northwest of the epicenter. The component directions are N. $33^{\circ}$ E., N. 


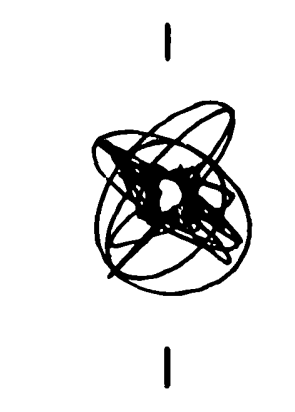

San Fernando Crest
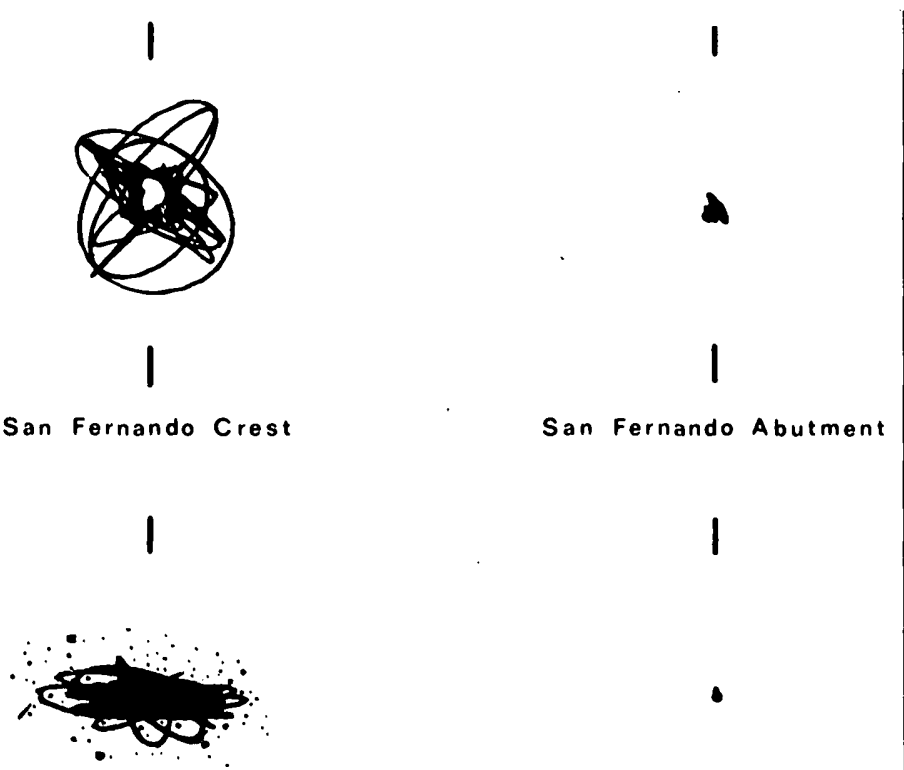

San Gabriel Crest

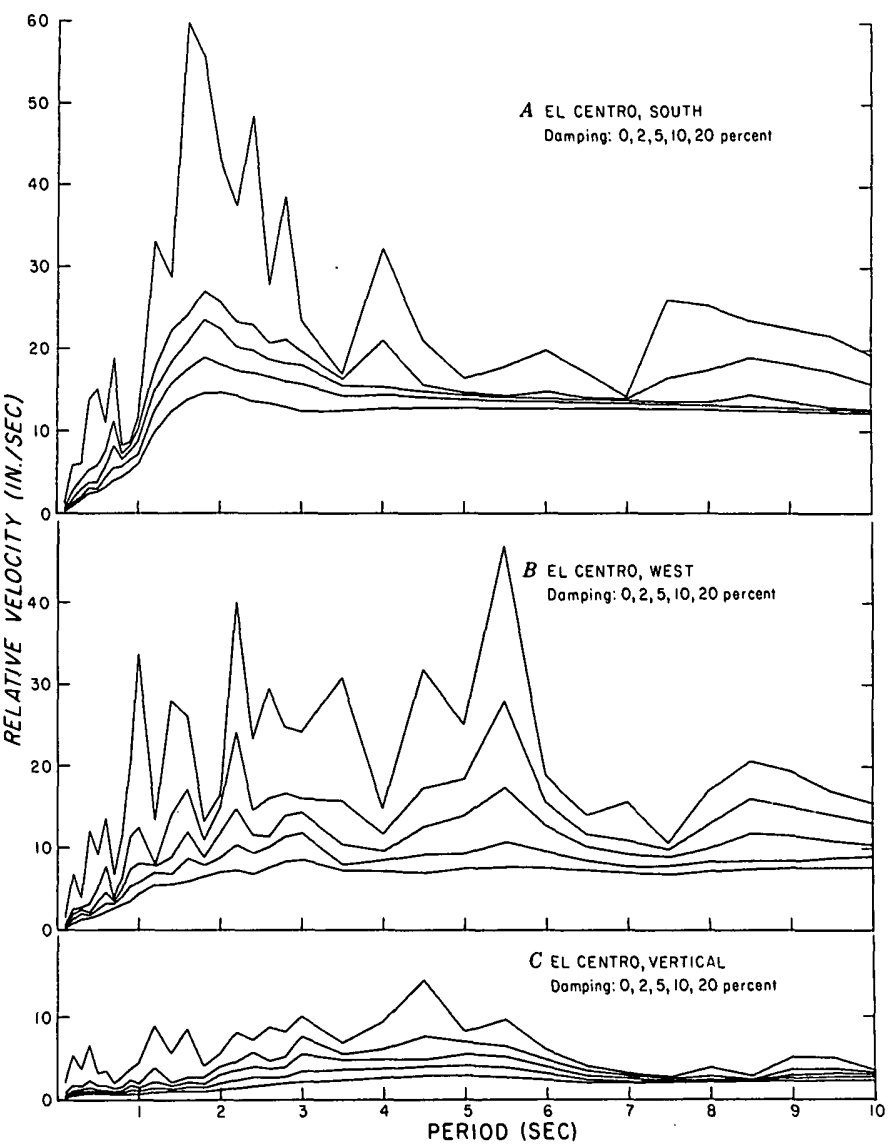

FIgURE 106. - Response spectra from El Centro. $A$, South; $B$, West; $C$, Vertical.

influence (Hudson and others, 1969) on the undamped spectra, particularly for the long-period components, which tend to be amplified by energy buildup during long excitations. The short-period components decay with distance more rapidly than longer period components; the El Centro spectra, obtained 41 miles from the epicenter, indicate this clearly. The predominant 2-second component appearing at El Centro and San Diego has decayed to an insignificant amount at San Onofre.

With no accelerometer closer than 40 miles from the epicenter, the vertical components recorded are not as important as the two horizontal components. In particular, the short-period vertical motion that is usually displayed only close to the epicenter was not recorded.

\section{SUMMARY AND CONCLUSIONS}

The Borrego Mountain earthquake was felt over 60,000 square miles of California, Nevada, and Arizona, but damages were generally minor owing to the sparsity of population in the immediate epicentral region. A maximum intensity of VII, based upon the routine questionnaire canvass, was as- 

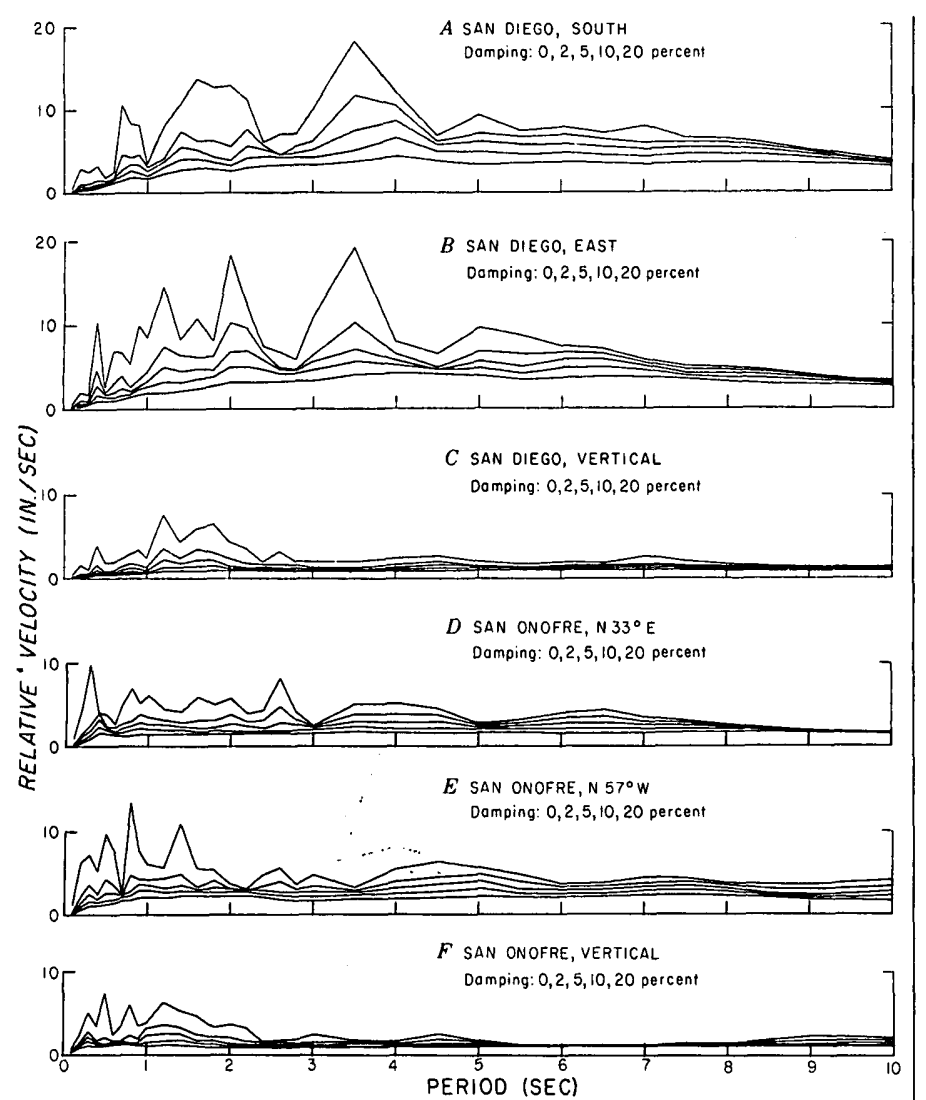

Figure 107. - Response spectra from San Diego and San Onofre. $A$, San Diego, south; $B$, San Diego, east; $C$, San Diego, vertical; $D$, San Onofre, N. $33^{\circ}$ E.; $E$, San Onofre, N. $57^{\circ}$ W.; $F$, San Onofre, vertical.

signed to an area near the Coyote Creek fault in the Borrego Springs-Ocotillo Wells region.

The earthquake actuated 115 strong-motion seismographs operated by the Seismological Field Survey; these instruments are located between 41 and 240 miles from the epicenter. The maximum acceleration and displacement occurred at the nearest station, El Centro, where values of $0.14 \mathrm{~g}$ and $7.25 \mathrm{~cm}$ were measured. Seismoscope plates were collected from 131 instruments, but owing to the small amplitudes, only five records from the Los Angeles area dams and four from El Centro can definitely be considered to have been written by this particular earthquake. The largest relative displacement response spectra that were measured at $\mathrm{El}$ Centro ranged from 0.82 to 1.00 inch.

After examining the results of this earthquake, several conclusions may be drawn concerning the pattern of strong-motion seismograph operations and the evaluation of their records.

(1) Since only 6 of 114 records were obtained less than 100 miles from the earthquake epicenter, it is evident that a greater geographical distribution of strong-motion seismographs would be desirable in southern California, particularly near the very active San Jacinto fault zone, where there have been eight shocks of magnitude 6 or greater between 1915 and 1968-an average of one every 7 years (Allen and others, 1965). As previously stated, most of the existing accelerographs are located at structures of particular interest to the organizations that own the equipment; therefore, a cooperative program was instituted between the Seismological Field Survey and the California Institute of Technology to select and instrument various new locations to fill in the existing geographic gaps in the network. By 1970, eight accelerographs had been located along the San Jacinto and Imperial fault zones, and additional sites were selected for a number of future installations.

(2) At the time of the earthquake, there were only four free-field stations in the Los Angeles area, and only one was near the 20 buildings with multiple accelerograph installations. Because more groundlevel stations would be advantageous, four sites were selected for future instrumentation to provide control stations that would be on basement rock as well as being near the centers of present high-rise construction. Three of these sites were equipped with instruments by mid-1970.

(3) The pattern of strong-motion seismograph triggering between 170 and 240 miles from the epicenter was correlated with the geologic foundation; that is, most instruments on basement rock failed to operate, but nearly all of them on relatively poor soils were actuated by the earthquake.

(4) Geologic conditions seemed to exert a general influence on the level of acceleration at stations less than 120 miles from the epicenter; that is, those instruments located on or near basement rock recorded lower values than those on alluvium or relatively less consolidated materials. The level of acceleration in the Los Angeles area, about $0.01 \mathrm{~g}$, was too small to show any effective relationship between soil conditions and seismic response, at least not without more comprehensive evaluation.

(5) The displacement attenuation decrement up to a 200-mile epicentral distance was shown to be logarithmic and may be defined by the equation

$$
\log A=2.32-0.011 d \text {. }
$$

Beyond the 200-mile limit there was no discernible pattern to the recorded data.

(6) Strong polarized amplitudes at a large angle to the trend of faulting were observed on seismoscope records from El Centro. Perhaps the installation of paired seismocopes on opposite sides of active fault zones, a program currently in progress, may help to shed more light on this phenomenon. 
(7) Seismoscope records from several earthfill dams show maximum amplitude ratios, crest to abutment, ranging from $2: 1$ to $16: 1$. Although these records were quite small and the ratios were obtained from unreduced scalings, it is sufficiently evident that future investigation of this apparent amplification in terms of the dams' structural behavior under applied earthquake stresses would be of considerable value, especially if more complete instrumentation were made available.

(8) For the first time in the history of strongmotion seismology, a large number of instruments in California were triggered by a single event, a possibility that could become routine for even moderate-sized earthquakes, provided they occurred near an appropriate concentration of accelerographs. At the time of the Borrego Mountain earthquake, there were 108 accelerographs in southern California, defined as the area south of a line connecting Cachuma Dam and the Lake Hughes array (fig. $100)$. By the end of 1969 there were 200 instruments in this area, 150 of these in Los Angeles alone. It is quite clear that future shocks on or near the San Andreas fault system that are equivalent in magnitude to or stronger than the Borrego Mountain earthquake will trigger a considerable part of the network, in any event probably more than 100 instruments.

(9) The three acceleration records showing strongest ground motion are included in a Caltech digitization project, and the relative velocity response spectra have been calculated. The distances from the epicenter are such that short-period components have been considerably attenuated at two of the sites, but not at San Onofre; here peaks in the spectra are well distributed over the whole period range.

\section{REFERENCES CITED}

Allen, C. R., St. Amand, Pierre, Richter, C. F., and Nordquist, J. M., 1965, Relationship between seismicity and geologic structure in the southern California region: Seismol. Soc. America Bull., v. 55, p. 753-797.

Amin, Mohammad, and Ang, A. H.-S., 1966, A nonstationary stochastic model for strong-motion earthquakes: Illinois Univ. Civil Eng. Studies, Structural Research Ser. No. 306, $115 \mathrm{p}$.

Carder, D. S., ed., 1964, Earthquake investigations in the western United States, 1931-64: U.S. Coast and Geodetic Survey Pub. 41-2, $264 \mathrm{p}$.

Cloud, W. K., 1967, Seismoscope results from three California earthquakes in the Hollister, California area: Seismol. Soc. America Bull., v. 57, p. 1445-1448.

Cloud, W. K., and Hudson, D. E., 1961, A simplified instrument for recording strong-motion earthquakes: Seismol. Soc. America Bull., v. 51, p. 159-174.

Cloud, W. K., and Scott, N. H., 1968, The Borrego Mountain, California, earthquake of 9 April 1968: A preliminary engineering seismology report:' Seismol. Soc. America Bull., v. 58 , p. 1187-1191.

Coffman, J. L., and Cloud, W. K., 1970, United States earthquakes 1968: U.S. Coast and Geodetic Survey, 111 p.

Earthquake Engineering Research Laboratory, California Institute of Technology, 1969, Strong-motion earthquake accelerograms, digitized and plotted data: v. 1, pt. A, $164 \mathrm{p}$.

Halverson, H. T., 1969, Some recent developments in strongmotion seismographs: Teledyne-Geotech Pub., Monrovia, Calif., $32 \mathrm{p}$.

Hudson, D. E., and Cloud, W. K., 1967, An analysis of seismoscope data from the Parkfield earthquake of June 27, 1966: Seismol. Soc. America Bull., v. 57, p. 1143-1159.

Hudson, D. E., Nigam, N. C., and Trifunac, M. D., 1969, Analysis of strong-motion accelerograph records: 4th World Conf. of Earthquake Eng., Santiago, Chile, 1969, Proc., Sec. A-2, 17 p.

Nigam, N. C., and Jennings, P. C., 1968, Digital calculation of response spectra from strong-motion earthquake records: Earthquake Eng. Research Lab., California Inst. Technology, $65 \mathrm{p}$.

U.S. Coast and Geodetic Survey, Seismological Field Survey, and California Institute of Technology, Earthquake Engineering Research Laboratory, 1968, Strong-motion instrumental data on the Borrego Mountain earthquake of 9 April 1968: San Francisco, Calif., 119 p.

Youd, L. T., and Castle, R. O., 1970, Borrego Mountain earthquake of April 8, 1968: Am. Soc. Civil Engineers Proc., Jour. Soil Mechanics and Found. Div., v. 96, no. SM4, p. 1201-1219. 


\title{
ENGINEERING GEOLOGY
}

\author{
By R. O. Gastle and T. L. Youd \\ U.S. Geological SurveY
}

\begin{abstract}
Effects of the magnitude 6.4 Borrego Mountain earthquake that relate to enginering geology were surveyed between Coronado and Glamis, Calif. Of particular significance were the complexity and $0.15-1.0-\mathrm{km}$ maximum half width of the main tectonic fracture zone along the Coyote Creek fault and the secondary (or spatially separated) faulting at least $2.4 \mathrm{~km}$ from the main trace. This pattern and distribution of tectonic ruptures, particularly where associated with predominantly transcurrent movement, generally have been identified with shocks of magnitude $8+$. Of possibly equal engineering significance was the surficial rupturing along the Banning-Mission Creek, Imperial, and Superstition Hills faults, which suggests that fault displacements may be seismically triggered along any discontinuity associated with accumulated elastic strain. Modified Mercalli intensities of VIII-IX were indicated for the epicentral region, in good agreement with those predicted for a magnitude 6.4 shock.

Terrain effects attributable to seismic shaking included rockfalls and soil falls, slumps and incipient slumps or ground cracking, dislodged pebbles and cobbles, shattered crusts overlying poorly indurated dry deposits, seismic compaction ruptures, and sand boils. Most of these features were located in the epicentral area, east of the Coyote Creek fault.

Structural damage, which was confined chiefly to the epicentral and Salton City areas, was remarkably light for an earthquake of this magnitude, even when consideration is given to the very limited development of this region. Cracked and spalled concrete bridge piers constituted the most serious damage to a modern structure in the epicentral region. Broken and cracked concrete-block walls were observed locally, and unreinforced masonry walls were toppled as far away as $65 \mathrm{~km}$ from the epicenter. Damage to wood-frame structures was generally minor, expressed chiefly as cracked plaster and stucco walls. Projected and displaced objects were reported from Coronado to about $8 \mathrm{~km}$ east of Brawley. The most severe damage beyond the immediate area of the epicenter occurred over the late Cenozoic lake deposits underlying most of the Imperial Valley.
\end{abstract}

\section{INTRODUCTION}

The magnitude 6.4 Borrego Mountain earthquake of April 9, 1968, was felt over approximately 155,000 sq $\mathrm{km}$ of the southwestern United States (Cloud and Scott, 1968a, p. 1187; Seismological Field Survey, National Oceanic and Atmospheric Administration, this volume) and an undetermined part of north- western Mexico. Perceptible shaking extended from Dateland, Wenden, and Horn, Ariz., westward to the Pacific Ocean and from Yosemite Valley on the north (Cloud and Scott, 1968a, p. 1187; 1968b, p. 19) to at least as far south as Ensenada, Baja California, Mexico (M. G. Bonilla, oral commun., 1968). Permanent effects, although generally minor outside the epicentral region (pl. 4), were reported from as far away as Los Angeles, about $220 \mathrm{~km}$ from the epicenter.

Although the main shock was clearly associated with the release of considerable seismic energy, its location within the sparsely settled and generally undeveloped western Colorado Desert precluded extensive damage. In spite of the generally light damage, however, this earthquake is considered significant to engineering geology in at least four respects :

1. The main shock was associated with the formation of two partly overlapping or en echelon zones of tectonic surface ruptures along a 32$\mathrm{km}$ reach of the Coyote Creek fault. (See pl. 5; Clark, "Surface Rupture along the Coyote Creek Fault," this volume). This breakage was locally complex, particularly within the area of overlap where the zone of fracturing was as much as $3 \mathrm{~km}$ wide. Although shocks of magnitude 6 and greater have commonly produced surface ruptures (Brown and others, 1967, p. 22), the complexity and width of the zone of tectonic rupturing generated during the Borrego Mountain event have not generally been reported for earthquakes of this or lesser magnitude, particularly along strike-slip faults. The complexity was such, moreover, that although the approximate locations of the rupture zones were generally predictable, the precise locations of the individual fractures were only locally predictable.

2. Temporally associated fault displacements occurred on the Banning-Mission Creek, Impe- 
rial, and Superstition Hills faults at distances of as much as $50 \mathrm{~km}$ or more from the epicenter. (See Allen and others, this volume). This phenomenon suggests that we should not, as a matter of course, restrict our predictions of tectonic surface rupturing to the fault (or fault zone) associated with the main shock, even when dealing with relatively small earthquakes.

3. Secondary terrain effects (those derived from shaking) were generated in profusion in the epicentral area. These effects, including both free-face movements and various liquefaction phenomena, identify the epicentral area with Modified Mercalli intensities of VIII or more. Similar terrain effects suggest that intensities were as high as VII over several thousand square kilometers of the western Imperial Valley.

4. Even when consideration is given to the low population density of the epicentral region, structural damage was remarkably light for an earthquake of magnitude 6.4 and Modified Mercalli intensities of $\mathrm{V}$ or greater over at least $65,000 \mathrm{sq} \mathrm{km}$. (See Cloud and Scott, 1968b, p. 26.) Local terrain effects and transient effects consistent with intensity VIII or greater suggest that structural damage should have been much more severe, far more pervasive, and experienced over a much wider area than it actually was.

\section{NATURE AND PURPOSE OF THE INVESTIGATION}

This report describes the effects of the Borrego Mountain earthquake of significance to engineering geology and attempts to relate these effects to the local and regional geology.

The field study focused on a relatively thorough, systematic investigation of the effects (particularly terrain effects) identified along a belt arbitrarily restricted to $8 \mathrm{~km}$, extending from Coronado on the Pacific coast eastward to Glamis, Calif. (pl. 4) ; a few excursions were made outside this belt, both north and south of the primary route. The main traverse was coincident with State Highway 78 over much of its extent. This route was designed to pass through the epicentral region at a high angle to the Coyote Creek fault and thus to provide a selective and representative sample of effects over contrasting geologic terranes at steadily increasing distances from the epicenter. In order to establish some sort of standardized scale of effects, special attention was directed toward several guide features, particularly bridges and post-World War II supermarkets. Spot checks were made for evidence of damage to other types of structures. Our direct observations were supplemented by eyewitness reports, both written and oral, press accounts, and information collected in a questionnaire canvass conducted by the Coast and Geodetic Survey. (See Cloud and Scott, 1968a, p. 1187; 1968b, p. 19; Seismological Field Survey, National Oceanic and Atmospheric Administration, this volume.)

Fieldwork began the day after the earthquake with a brief reconnaissance of effects between Banning and Ocotillo Wells. Systematic field investigations were carried out during the periods April 11-17, April 24-27, and May 9-11, 1968. About twothirds of the fieldwork was concentrated in the epicentral region - that is, within an area extending 6-8 $\mathrm{km}$ to either side of the main tectonic trace.

\section{ACKNOWLEDGMENTS}

The cooperation of many individuals and a number of public agencies contributed immeasurably to the preparation of this report. We wish to thank in particular the staff members of the Engineering and Utilities Department of the City of San Diego, the County Engineer and Road Department of the County of San Diego, the El Centro Office of the California Division of Highways, and the Public Works Departments of Imperial County; several significant effects would have gone unrecognized had we not been alerted to their existence by these groups. K. C. Crowther and D. R. Nichols of the Geological Survey ably assisted in mapping a variety of terrain effects. Partial support was provided by the Division of Reactor Development and Technology, Atomic Energy Commission.

\section{REGIONAL GEOLOGIC SETTING}

The epicentral area of the Borrego Mountain earthquake is located along the east margin of the Peninsular Range province of southern California (Jahns, 1954b). This province occupies the southwest corner of California and extends southward into Baja California; it is bounded on the west by the Pacific Ocean and abuts the Coachella and Imperial Valleys of the Salton Trough on the east (Jahns, 1954a).

Jahns (1954b) has described the geology of the Peninsular Range province, and Dibblee (1954) has described the geology of the Imperial Valley region. The geology along the San Jacinto fault, the dominant structural feature of the eastern Peninsular Range province, was described by Sharp (1967), whose summary of the tectonic setting of the Salton Trough appears elsewhere in this volume.

The inland parts of the Peninsular Ranges (pl. 4) are underlain chiefly by igneous and metamorphic 
rocks of Paleozoic and Mesozoic age. These rocks are in turn overlain with profound unconformity by irregular patches of volcanic rocks and nonmarine sedimentary deposits of Late Cretaceous to late Cenozoic age and, in the coastal areas, by Pleistocene marine terrace deposits. Along the east margin of the province, and within the Salton Trough, the older crystalline rocks are mantled by locally thick chiefly nonmarine upper Tertiary and Quaternary deposits. These rocks consist largely of fanglomerate and conglomerate, lacustrine clay and sand, and various volcanic assemblages. The upper part of this section, the Pliocene or Pleistocene Ocotillo Conglomerate of Dibblee $(1954$, p. 24), grades eastward into the Pleistocene lacustrine Brawley Formation of Dibblee (1954, p. 24), consisting of claystone and thin interbeds of sandstone. The upper Pliocene and Pleistocene deposits of lacustrine origin that can be conveniently separated from other types of Cenozoic deposits are shown on plate 4 as chiefly Quaternary lake deposits; elsewhere these deposits are included with the Pleistocene to Upper Cretaceous volcanic and nonmarine sedimentary deposits. The lake deposits probably thicken to at least 600 or $800 \mathrm{~m}$ and perhaps $1,500-3,000 \mathrm{~m}$ beneath the Salton Trough (Dibblee, 1954, p. 24, pl. 2). Poorly indurated Quaternary dune sand, alluvium, and nonmarine deposits are grouped together as " $Q$ " on plate 4. The thicknesses of these surficial deposits probably vary widely, but they are almost certainly very thin compared with the underlying lake deposits or Upper Cretaceous to Pleistocene sedimentary rocks. (See Dibblee, 1954, pl. 2.)

The Peninsular Ranges are traversed by a conspicuous series of steeply dipping northwest-trending faults, many of which project into or beneath the Salton Trough (Jahns, 1954a, p. 19; 1954b, pl. 3 ; Dibblee, 1954, pl. 2). These faults are most numerous in the area eastward from Santa Ysabel and Julian, and most, if not all, have been intermittently active during Cenozoic time (Jahns, 1954a, p. 19; 1954b, pl. 3; Dibblee, 1954, pl. 2). Movement on one such fault - identified as the San Jacinto fault by Dibblee (1954, pl. 2) and Jahns (1954b, pl. 3) but distinguished from the San Jacinto and mapped as the Coyote Creek fault by Sharp $(1967$, pl. 1) - apparently generated the Borrego Mountain earthquake of 1968.

\section{TRANSIENT EFFECTS}

Transient effects of the Borrego Mountain earthquake are described and discussed in detail elsewhere in this volume (Seismological Field Survey, National Oceanic and Atmospheric Administration; Waananen and Moyle); those reported from along the route of our reconnaissance are indicated on plates 4 and 5. Selected aspects of engineering significance are summarized here.

The most significant and most generally observed transient effect was the ground motion generated in response to the passage of seismic waves. Clearly perceptible shaking occurred over at least 155,000 sq km (Cloud and Scott, 1968a, 1968b; Seismological Field Survey, National Oceanic and Atmospheric Administration, this volume), and two reports of felt ground motion came from Yosemite Valley, about $650 \mathrm{~km}$ from the epicenter and roughly 300 $\mathrm{km}$ beyond the generally felt area.

Estimates of the duration of felt motion ranged from $1 / 2$ to 1 minute in the epicentral region, from $1 / 4$, to 1 minute in the metropolitan San Diego area, and from a few seconds to about 1 minute in the metropolitan Los Angeles area. Strong-motion records were not obtained within the epicentral area of this earthquake as they were, for example, for the Parkfield-Cholame earthquake of June 27, 1966 (Brown and others, 1967, p. 51). Accelerations (as measured for a single horizontal component) of $0.03 \mathrm{~g}$ or more, however, persisted for at least 26 $\mathrm{sec}, 65 \mathrm{~km}$ from the epicenter at El Centro (Cloud and Scott, 1968a, p. 1189; 1968b, p. 104). Because the attenuation of acceleration between the epicenter and a point $65 \mathrm{~km}$ distant has been given as about one-third (Cloud, 1968), strong motion (accelerations of $0.10 \mathrm{~g}$ or greater) may have persisted for as much as 26 seconds in the epicentral region.

\section{PERMANENT EFFECTS}

Permanent effects associated with the Borrego Mountain earthquake are classified (pl. 4) much as were those associated with the Parkfield-Cholame earthquakes of 1966 (Brown and others, 1967, p. 41-43). The basic classification is adapted from Richter (1958, p. 81) and designates as direct or primary those effects due directly to differential movement along fault surfaces and as indirect or secondary those effects due to shaking generated by the passage of seismic waves. The permanent effects are further classified according to object affected and as effects on terrain or manmade structures. Insofar as is now known, all the permanent effects described were associated with the main shock.

\section{PRIMARY EFFECTS ON TERRAIN TECTONIC FRACTURES ALONG COYOTE CREEK FAULT}

Tectonic surface ruptures, produced chiefly along a 33-km segment of the Coyote Creek fault, formed the most dramatic and probably the most significant terrain effect associated with this earthquake. (See 
pls. 4, 5.) Measurable slip along individual fractures was generally right lateral and was as much as about $38 \mathrm{~cm}$. (See Clark, "Surface Rupture along the Coyote Creek Fault," this volume.) Near the Ocotillo Badlands, the breaks were distributed mainly along two narrow en echelon zones along the margins of the hills, but an especially complex pattern of surficial fractures also formed within the badlands. (See pl. 5.)

Allen, Grantz, Brune, Clark, Sharp, Theodore, Wolfe, and Wyss (1968, p. 1185) observed that “*** In every area where a pre-existing fault was indicated by a scarp or ground-water barrier, the rupture took place almost exactly along the line of earlier breaks." However, because clear evidence of earlier faulting was absent along perhaps half the ruptured trace of the Coyote Creek fault and because the ruptured zones were locally as much as several hundred meters wide, indications of earlier faulting constituted imperfect guides to the location of these breaks.

The width of the main zone of surficial faulting and the distances of the secondary tectonic fractures from the main trace are greater than would have been anticipated for a shock of magnitude 6.4, particularly one associated with predominantly strike-slip movement. Thus, Bonilla (1967, p. 29, table 1) observed that the half widths of the main zone of tectonic fracturing associated with earthquakes of up to magnitude 8+ (accompanying transcurrent faulting) have been as much as about $0.09 \mathrm{~km}$ and that secondary, or spatially separated, tectonic fracturing is not known to have occurred more than $2.9 \mathrm{~km}$ from the main trace. The main zone of surficial faulting produced during the Borrego Mountain event was characterized by maximum half widths of about $1 \mathrm{~km}$ in the area of overlap near Ocotillo Badlands and as much as $0.15 \mathrm{~km}$ elsewhere; secondary tectonic fracturing (excluding that associated with the Banning-Mission Creek, Imperial, and Superstition Hills faults) occurred at distances of at least $2.5 \mathrm{~km}$ and perhaps $3 \mathrm{~km}$ or more from the main trace. (See Clark, "Surface Rupture along the Coyote Creek Fault," this volume.)

The character and distribution of the Coyote Creek tectonic fractures, with respect to both their geographic occurrence and various significant geologic features, are described in detail elsewhere in this volume by Clark ("Surface Rupture along the Coyote Creek Fault") and by Sharp and Clark.

TEMPORALLY ASSOCIATED TECTONIC FRACTURES

The most surprising of the major terrain effects associated with the Borrego Mountain earthquake consisted of minor horizontal displacements of up to about $2.2 \mathrm{~cm}$ on the Superstition Hills, Imperial, and Banning-Mission Creek (San Andreas) faults (see pl. 4) at distances of about 45,70 , and $50 \mathrm{~km}$, respectively, from the epicenter (Allen and others, 1968, p. 1185). Although these effects were probably triggered by the April 9 shock on the Coyote Creek fault and are thus indirect or secondary (in the sense that their existence could be attributed to the passage of seismic waves), they are nonetheless primary in the sense that the breaks are certainly due directly to fault movement.

If these displacements can be considered secondary tectonic fractures (tectonic fractures spatially separated from the main fault zone - see classification of Bonilla, 1967, p. 5), they are spectacularly unique among recognized examples of this phenomenon. Secondary faulting was previously reported at maximum distances of $13 \mathrm{~km}$ (associated with an estimated magnitude 8.3 shock), $13.7 \mathrm{~km}$ (associated with a magnitude 7.1 shock), and, possibly, $34 \mathrm{~km}$ (associated with a magnitude 8.5-8.6 shock) from the main fault (Bonilla, 1967, table 1). These extreme examples were characterized by dip-slip or oblique-slip movement on the main fault; secondary faulting associated with predominantly strike-slip movement had never before been recognized at distances greater than $2.9 \mathrm{~km}$ from the main fault (Bonilla, 1967, table 1).

\section{SECONDARY EFFECTS ON TERRAIN}

Terrain effects attributable to seismic shaking consisted chiefly of slope failures of several types and various compaction or liquefaction phenomena. Slope failures, including rockfalls, soil falls, rockslides, slumps; and block glides (classification after Varnes, 1958), formed the largest group of secondary terrain effects. They were widely distributed within the almost universally dry surficial deposits from San Diego to at least as far east as the central Imperial Valley. Compaction effects occurred chiefly in the epicentral region and scattered areas east of the epicenter. The largest slope failure observed during our reconnaissance was a reactivated slump or block glide near Seely in the west-central Imperial Valley (see pl. 4); its significance lies less in its size, perhaps, than in its distance $(65 \mathrm{~km})$ from the epicenter. Soil falls apparently were generated over hundreds of square kilometers; the distribution of the resulting dust clouds clasely matched the courses of many of the major arroyos over the area extending eastward from Borrego Springs to the Salton Sea. 


\section{ROCKFALLS AND SOIL FALLS}

Rockfalls were unrecognized over the main route of our reconnaissance. They were dramatically developed elsewhere, however, particularly in Split Mountain Canyon (about $15 \mathrm{~km}$ south of Ocotillo Wells), where several automobiles and camper vehicles were seriously damaged. Rockfalls and (or) soil falls attributable to the earthquake were also reported from the Sunset Cliffs district of San Diego; these falls, however, involved materials being actively undercut by wave erosion.

Soil falls (falls composed of materials too poorly lithified to be identified as rock) were generated within many of the bedded alluvial deposits along the generally shallow but almost universally steepsided arroyos that characterize this part of the southwest. They occurred at least as far west as Yaqui Well, about $25 \mathrm{~km}$ west of the epicenter, but they were only sparsely developed east of there to within $3 \mathrm{~km}$ of the main trace of the Coyote Creek fault. They persisted in abundance eastward from the fault for many $\mathrm{km}$ and were observed as far east as the south tip of the Salton Sea. The relatively few soil falls west of the Coyote Creek fault, as contrasted with their abundance east of this break, may correlate with an apparently abrupt increase in the thickness of the poorly consolidated sedimentary cover. (See Hamilton, 1970, p. 378.)

Soil falls were particularly common along major washes and arroyos and in the badlands sections of the epicentral area. The most impressive soil falls or rockfalls were seen in the Fonts Point region of the Clay Hills, north of Borrego Mountain. Incipient failures of this type are shown in figure 108, where a series of blocks has been partly detached from the rim of this steep badlands slope. These particular failures are difficult to classify and could just as easily have been designated as slumps, for they clearly have been rotated or displaced laterally.

One of the most intense concentrations of soil falls was along Fish Creek Wash (fig. 109), south of Ocotillo Wells. These falls were distributed westward along the banks of the wash almost without interruption for at least $2-3 \mathrm{~km}$ beyond the ruptured trace of the Coyote Creek fault. They presumably continued eastward for an equal distance; we did not, however, examine this terrain. The material in which Fish Creek Wash is incised consists of generally poorly sorted and poorly indurated but well-jointed sandy alluvium. (See fig. 109.)

\section{ROCKSLIDES AND DEBRIS SLIDES}

Rockslides occurred along a number of cuts in the strongly foliated and highly fractured crystalline rocks cropping out along the Montezuma-Bor-

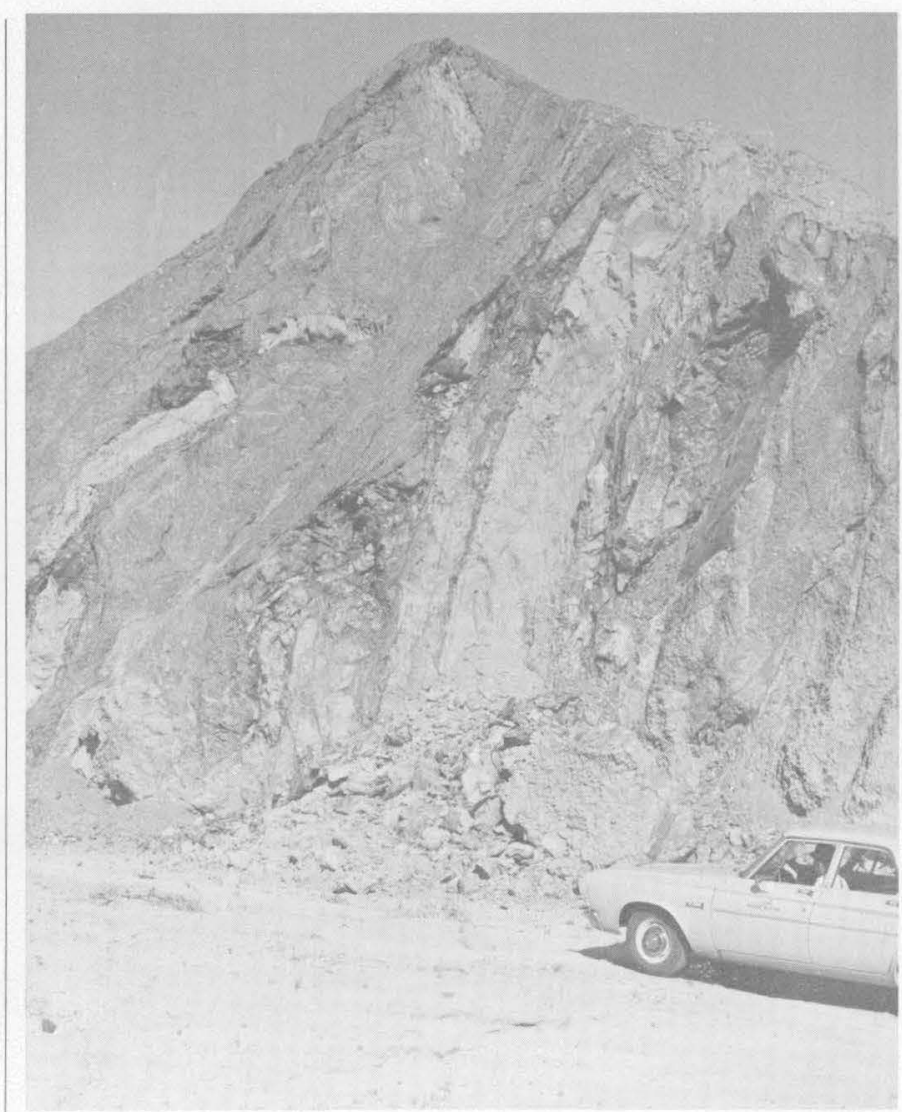

$A$

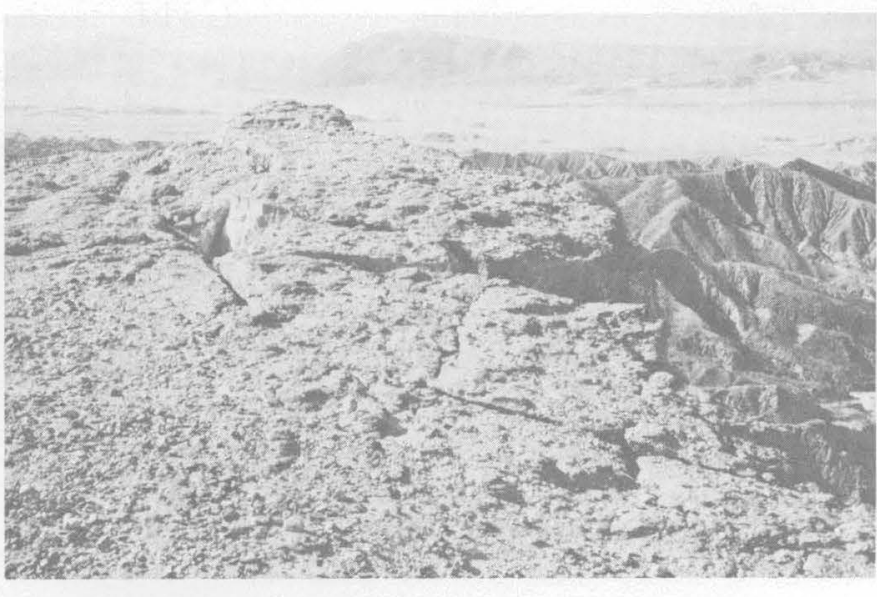

$B$

Figure 108. - Rockfalls and soil falls in lacustrine silts and sands of probable Pliocene age. $A$, Complex rockfall and debris slide in the central Ocotillo Badlands. This failure is typical of many of those found in the badlands. $B$, Incipient rockfalls or soil falls in the Clay Hills, near Fonts Point, about $3 \mathrm{~km}$ north of the north end of the ruptured fault trace.

rego highway, leading west from Borrego Springs. Many of the slides were along dip slopes, but the highly fractured character of the rock, which surely was aggravated by blasting during construction, 


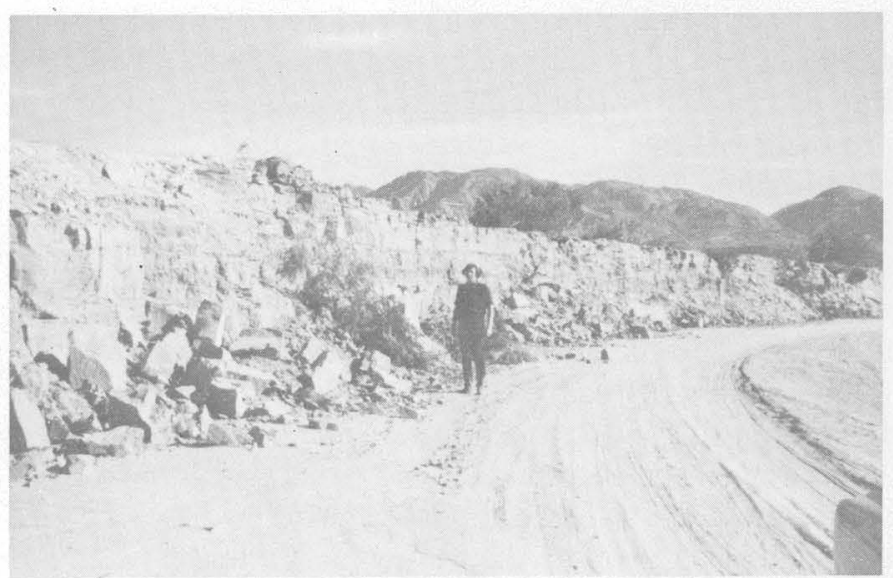

Figure 109, - Soil falls produced in sandy alluvium along the near-vertical walls of Fish Creek Wash, immediately west of the main trace of the Coyote Creek fault.

must have contributed substantially to the failures. (Rockslides closed this route to traffic for a number of hours after the main shock; this was the only known example of a road closure attributable to the earthquake.)

Rockslides or debris slides (together with associated soil falls) also occurred locally on the oversteepened natural slopes of the Ocotillo Badlands underlain by the folded Borrego Formation of Tarbet and Holman (1944) and Ocotillo Conglomerate of Dibblee (1954), both of Quaternary age. (See pl. 1.) These failures, an example of which is shown in figure $108 \mathrm{~A}$, were generally too complex to be classified simply as falls, slumps, or slides.

\section{SLUMPS}

Slumps and incipient slumps of various types were the most prevalent slope failures recognized in the epicentral region, if not within the entire area we examined. Most were incipient failures, in the sense that they were generally characterized by open cracks or fissures of the sort shown in figure 110 rather than by well-developed headwall scarps or clearly defined toes. Although slumps attributable to the earthquake were identified many kilometers from the epicenter, they were most abundant on the gentle to moderate slopes underlain by the Ocotillo Conglomerate of Dibblee (1954), the Borrego Formation of Tarbet and Holman (1944), and the Palm Spring Formation (Sharp, "Tectonic Setting of the Salton Trough," this volume) within and immediately north of the Ocotillo Badlands (fig. 110). The density of slump or incipient slump features was generally very low more than $3-5 \mathrm{~km}$ from the trace of the Coyote Creek fault. We were unable to identify any good correlation between the distribution of tectonic ruptures and slumps within the

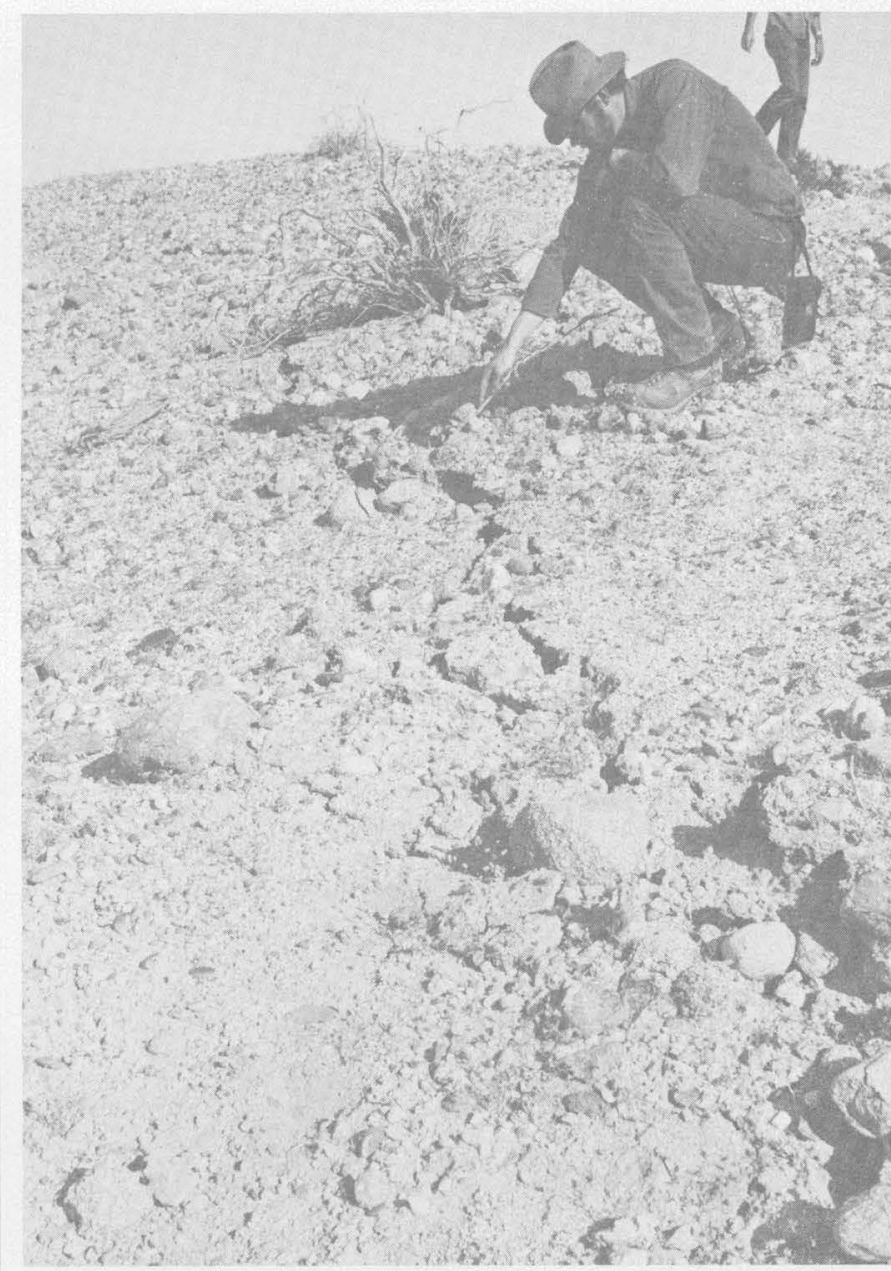

FIGURE 110. - Incipient slump in the low-relief hills north of the Ocotillo Badlands.

3- to 5-km-wide zone in which both were well developed. What was evident, however, was an almost complete restriction of the slumps to the area east of the mapped tectonic ruptures. (See pl. 5.) Older landslides within the zone of intensive slumping were nonexistent or at least inconspicuous. The general configuration of the hills, however, suggests that slumping may have been locally operative for a long time. The boundary between the hills and the surrounding alluvial lowlands forms a convoluted pattern in plan view that, together with the prominently steepened ridge crests toward the ends of their respective spurs, suggest great viscous flows edging out upon the adjacent plain.

The best expressed and generally largest slumps were produced in the Ocotillo Badlands; a representative example of this terrain is shown in figure 111. The scarp shown in figure 112 could be traced for nearly $0.5 \mathrm{~km}$; smaller, arcuate scarps of the sort shown in figure $112 B$ were the most commonly recognized slump features in both the Ocotillo Badlands and the hills immediately to the north. 


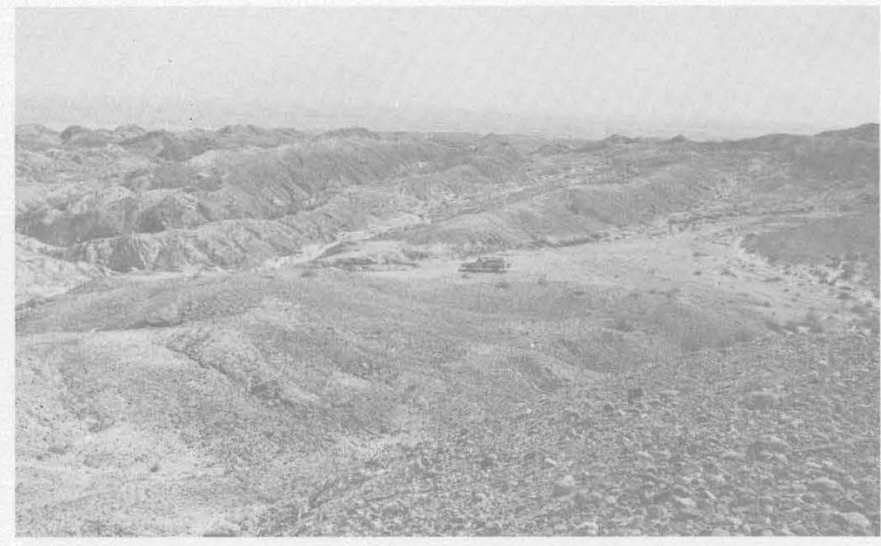

FIgURE 111. - Gently rolling hills and steep-sided washes of the central Ocotillo Badlands characterized by abundant slope failures. View northeast.

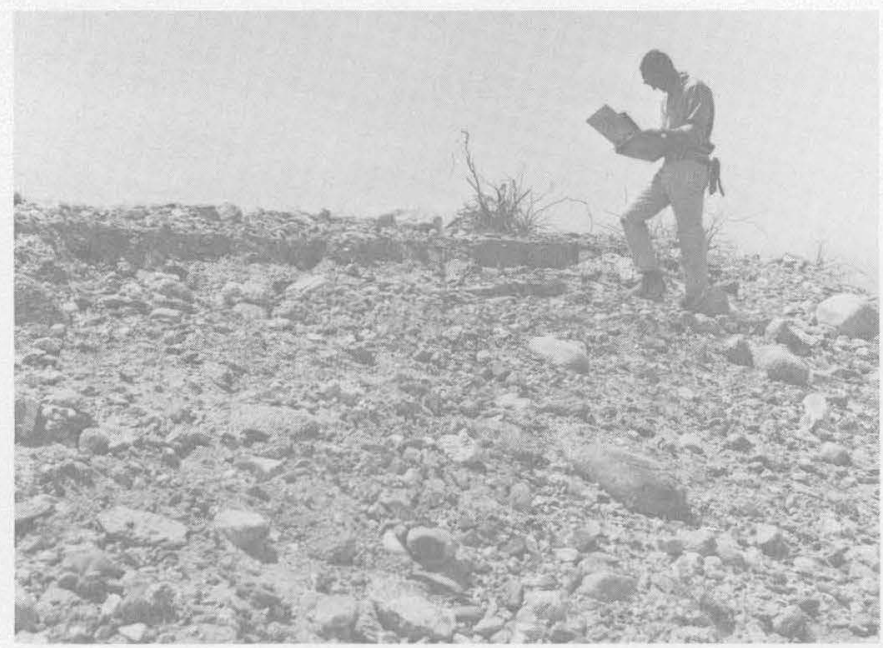

A

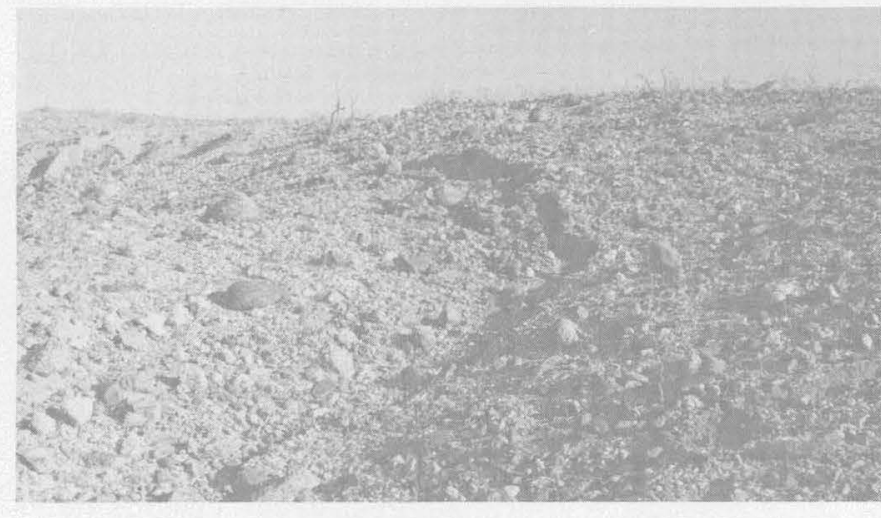

$B$

Frgure 112. - Slump scarps in the gently to steeply folded Pleistocene Ocotillo Conglomerate of Dibblee (1954) along or near the crests of hills in the epicentral area. $A$, Failure along east-west-trending ridge in the Ocotillo Badlands, about $2 \mathrm{~km}$ southeast of Ocotillo Wells. This scarp extended for more than $0.5 \mathrm{~km}$ along the ridge crest. Photograph by E. W. Wolfe. $B$, Typical scarp produced in the gently sloping hills about $3 \mathrm{~km}$ east of Ocotillo Wells.
A reactivated slump or block glide within the generally silty Quaternary lacustrine deposits, about 65 $\mathrm{km}$ southeast of the epicenter at the Highway 80 crossing of the New River, constituted the largest single slope failure identified during the postearthquake investigation. This failure was expressed as a simple break (fig. 113), about $125 \mathrm{~m}$ east of and roughly parallel to the river; vertical separations along this break of as much as $5 \mathrm{~cm}$ formed a subdued headwall scarp that extended south from Highway 80 for more than $0.4 \mathrm{~km}$.

Slumps were recognized in artificial fills near the south end of the Santa Rosa Mountains and in several areas around the Imperial Valley. The most significant of these consisted of small- to moderatesized slumps in engineered fills forming parts of building pads in the Salton City area and along a recently completed highway between Borrego Valley

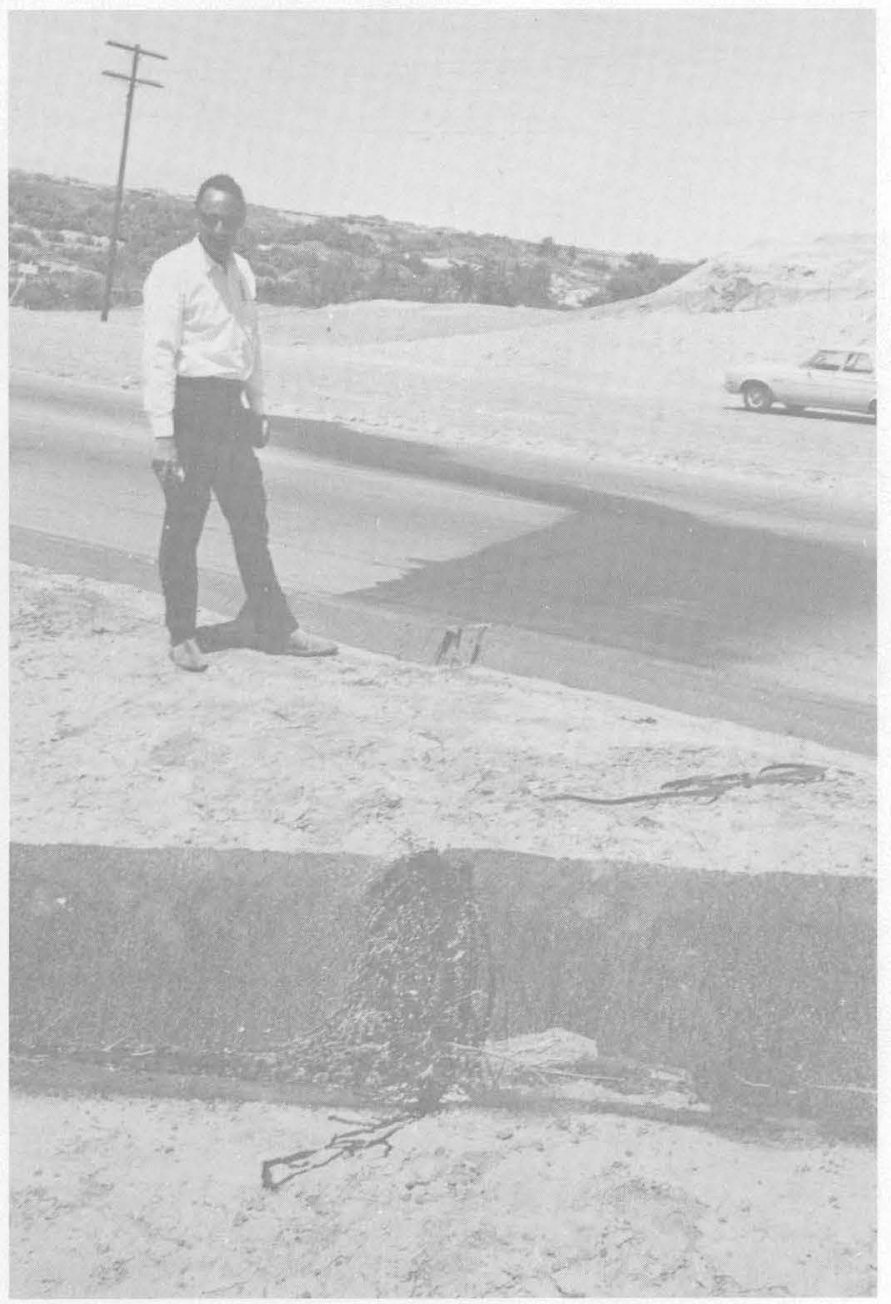

Figure 113. - Headwall scarp of slump or block glide immediately east of Highway 80 crossing of the New River. Vertical separations of as much as $5 \mathrm{~cm}$ (see displaced curbs) occurred along a $125-\mathrm{m}$ segment of this break. 


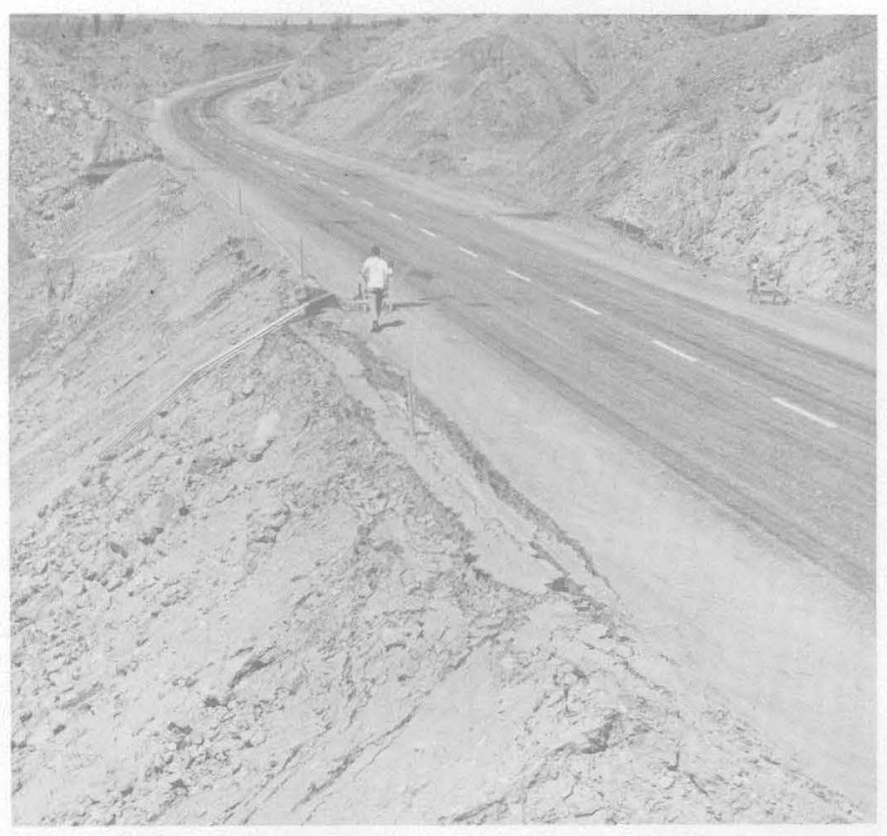

FIGURE 114. - Slump in artificial fill along a recently completed highway north of the Borrego Badlands.

and Salton City (fig. 114). Slumps were also generated in wet and possibly saturated soils forming fills or fill base along the edge of the Salton Sea and along river and canal embankments in the Imperial Valley bottomlands.

\section{COMPACTION AND LIQUEFACTION EFFECTS}

Seismic compaction and liquefaction effects attributable to the earthquake were discovered at a number of widely separated points. Perhaps the most intriguing of these were the linear cracks and differential displacements produced chiefly along the margins of several recently irrigated fields. These ruptures were particularly conspicuous in the sandy alluvial deposits of the epicentral region, where they occurred in some measure within or around every well-wetted field we examined (figs. 115 and 116). Probably the least expected example of compactioninduced rupturing was found near the center of a thoroughly soaked alfalfa field in the Imperial Valley, about $15 \mathrm{~km}$ west-northwest of Westmorland, or more than $30 \mathrm{~km}$ east of the epicenter. Shaking of the silty Quaternary lake beds on which the field was established seemingly generated a small graben about $3 \mathrm{~m}$ wide, $150 \mathrm{~m}$ long, and characterized by vertical displacements of as much as $15 \mathrm{~cm}$ (fig. 117).

Sand boils, the only direct evidence of seismically elevated pore-water pressures and soil liquefaction (or near liquefaction) that we discovered, were seen in only two areas: within a concentric array of compaction fractures in the epicentral area and landward of slumps along the New River northwest

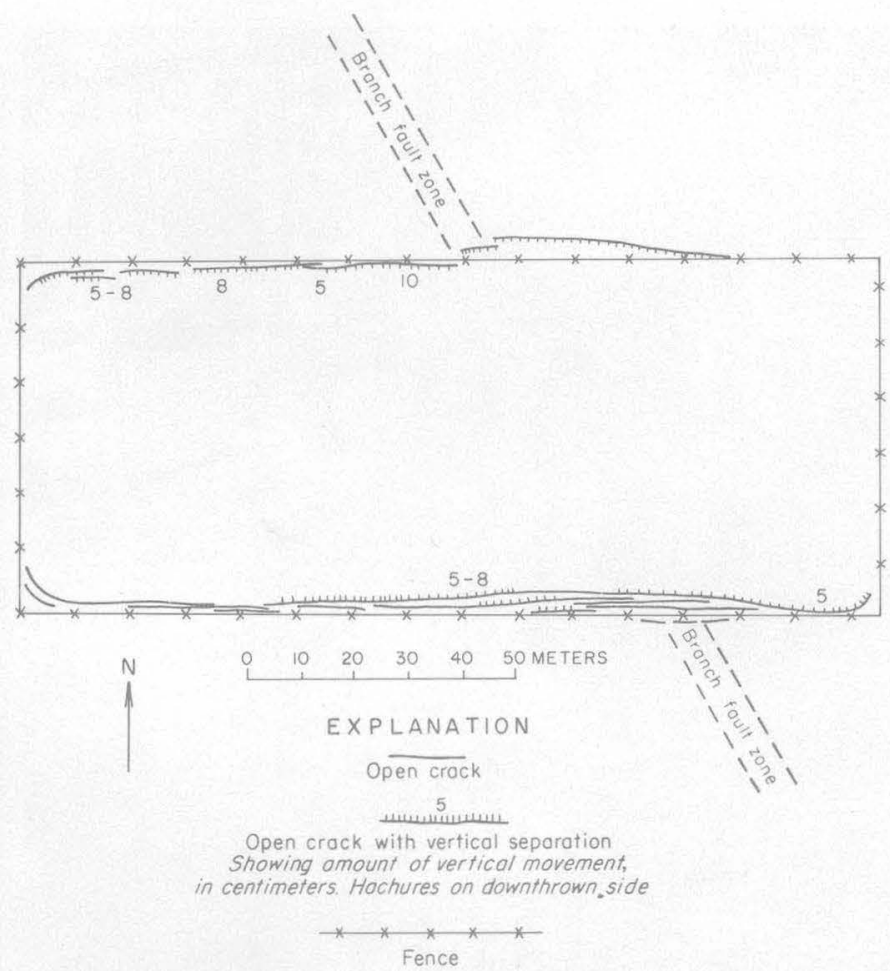

FIGURE 115. - Sketch map showing distribution of seismiccompaction ruptures (see fig. 116) around irrigated field adjacent to main trace of the Coyote Creek fault, about $1.5 \mathrm{~km}$ southeast of Ocotillo Wells. Note that the cracking associated with branch fault zone does not project through field.

of El Centro (fig. 118). The second association suggests that this slumping, at least, was attributable in whole or in part to liquefaction of the underlying materials.

\section{MISCELLANEOUS TERRAIN EFFECTS}

Many of the poorly indurated surficial deposits, such as dune sands, alluvium, and artificial fill, found within the western Imperial Valley are veneered with thin lightly cemented brittle crusts. Not surprisingly, these crusts were locally shattered by seismic shaking (fig. 119). The distribution of this effect, however, was seemingly random and largely unpredictable. The occurrence of shattered dunes south of the Ocotillo Badlands (fig. 119), for example, was apparently unrelated to their location with respect to the ruptured trace of the Coyote Creek fault. The most puzzling expression of crustal shattering discovered during our reconnaissance occurred about $13 \mathrm{~km}$ east of Ocotillo Wells (and about $6 \mathrm{~km}$ from the nearest strand of the Coyote Creek fault). The shattering there was confined to a linear zone that was as much as $100 \mathrm{~m}$ wide and extended for about $0.4 \mathrm{~km}$ (fig. 120). This peculiarly restricted zone, which was produced on a nearly featureless and gently sloping surface overlying ancient lake deposits, led eastward from the base of 


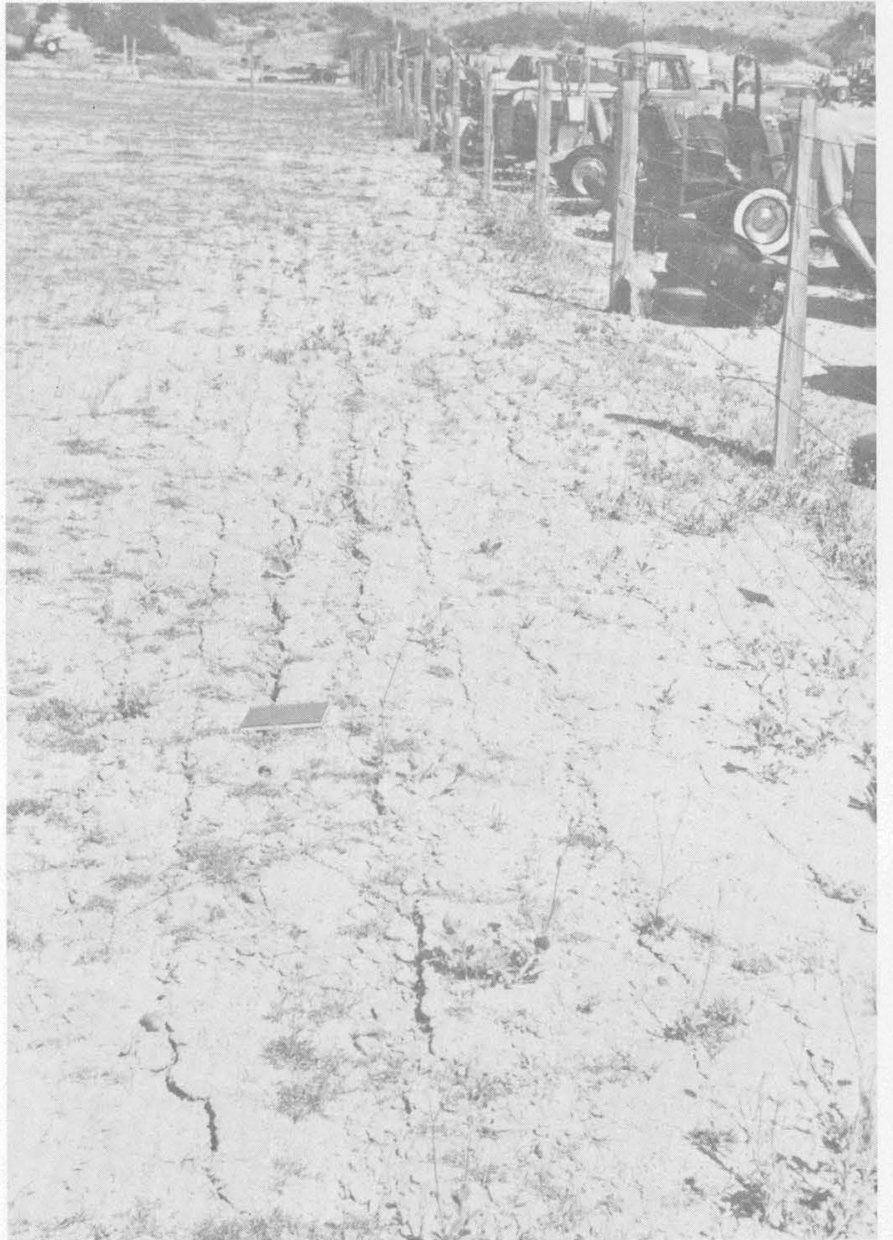

A

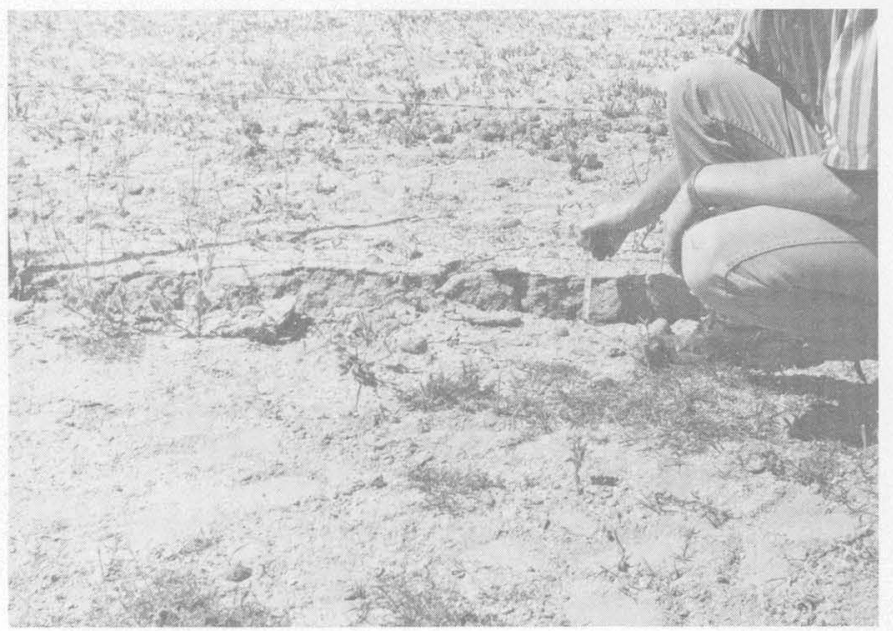

$B$

FIGURE 116. - Seismic-compaction ruptures along the periphery of an irrigated field adjacent to the main trace of the Coyote Creek fault. (See fig. 115.) Soil sample from this field had a mean particle size of $0.26 \mathrm{~mm}$ and a uniformity coefficient of 6 . $A$, Parallel array along south side of field. $B$, Rupture along the north side of the field on which maximum vertical separation was about $10 \mathrm{~cm}$.

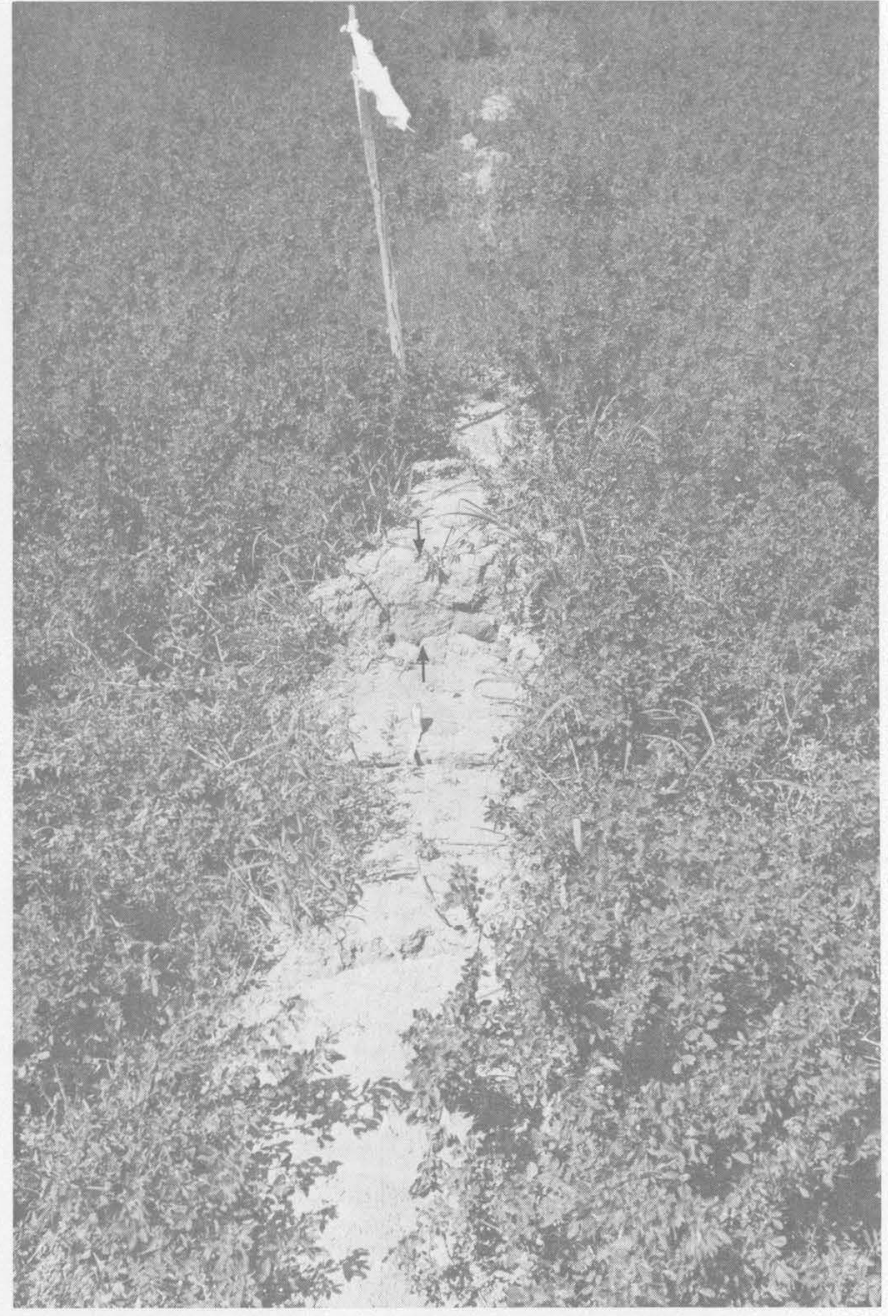

FIGURE 117. - Fifteen-cm scarp (at arrow) along the edge of a compaction-produced graben in the center of an alfalfa field on the Elmore Ranch, $22 \mathrm{~km}$ west-northwest of Westmorland (pl. 4).

a shattered sand dune and veered northward across Highway 78 near its east terminus. Differential displacements were unrecognized along the shattered zone, even where it was faintly expressed as fresh cracks in the asphalt pavement of Highway 78 . Furthermore, we could in no way define the zone other than through the occurrence of the shattered crust itself. Thus, because this effect seems to be only very generally related to any evident geologic feature, its localization may be due to a peculiar focusing of seismic energy.

Dislodged or displaced pebbles and cobbles (fig. 121) were found throughout much of the epicentral region. Their relative abundance showed a good correlation with that of the slumps and incipient slumps in the low hills of the epicentral region. 


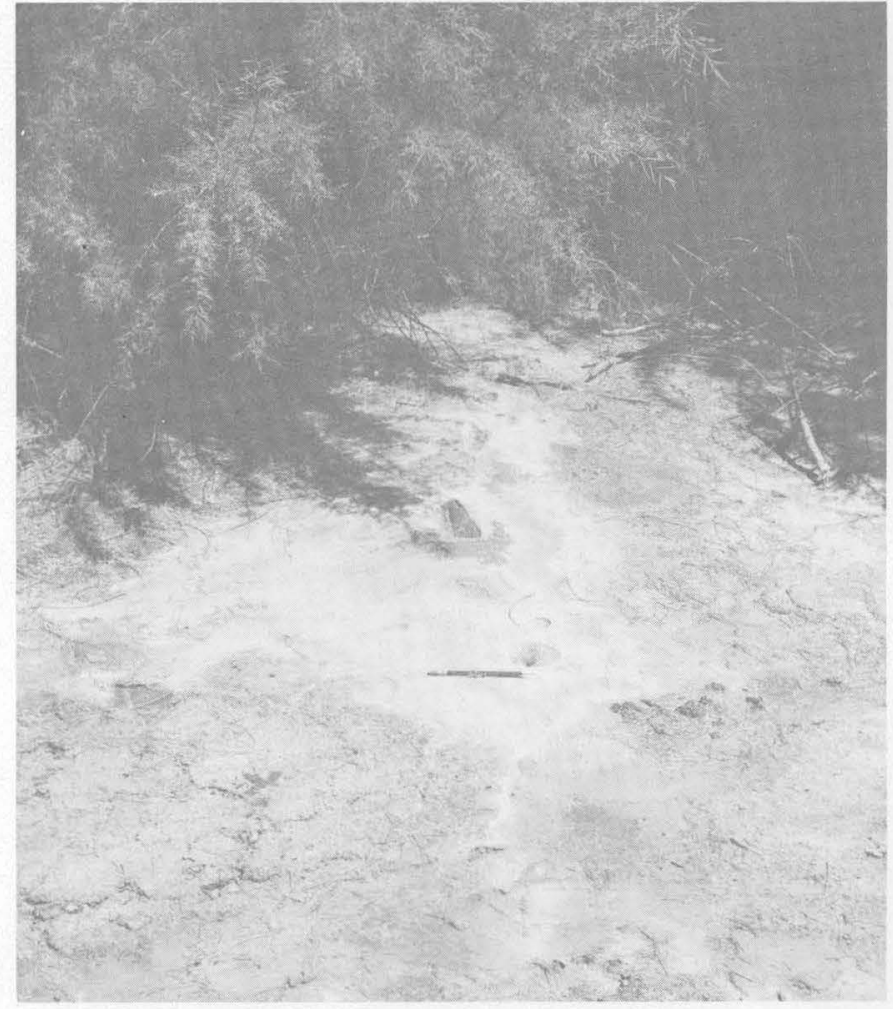

FIGURE 118. - Sand boils in late Quaternary lake-bottom deposits along the New River, about $55 \mathrm{~km}$ southeast of the epicenter.

\section{PRIMARY EFFECTS ON STRUCTURES}

Significant primary effects on manmade structures were limited almost entirely to rending or cracking of roads and highways. Chief among these was the cracking, displacement, and partial rotation of the pavement at the intersection of Highway 78 with the ruptured trace of the Coyote Creek fault. Minor cracking was also detected at a number of road intersections with the trace of the Imperial fault (Allen and others, this volume).

Additional minor primary effects on structures included the rupture and offset of a $2.5-\mathrm{cm}$ water pipeline that intersected the Coyote Creek fault about $1.5 \mathrm{~km}$ southeast of Ocotillo Wells and the extension and bending of an airplane tiedown cable at the Ocotillo Wells county airport on Benson Dry Lake. (See pl. 5.)

\section{SECONDARY EFFECTS ON STRUCTURES}

Secondary effects on manmade structures, although widespread, were generally minor. They were concentrated in two general areas: the epicentral region around Ocotillo Wells and the Salton City area, about $22 \mathrm{~km}$ northeast of the epicenter. Although our sample was poor owing to the sparse construction in this area, it is clear that the most

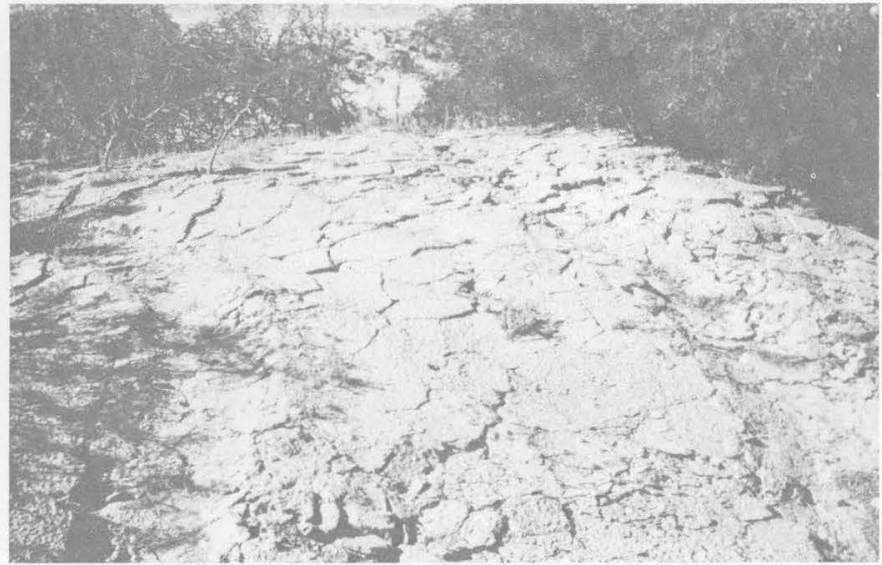

A

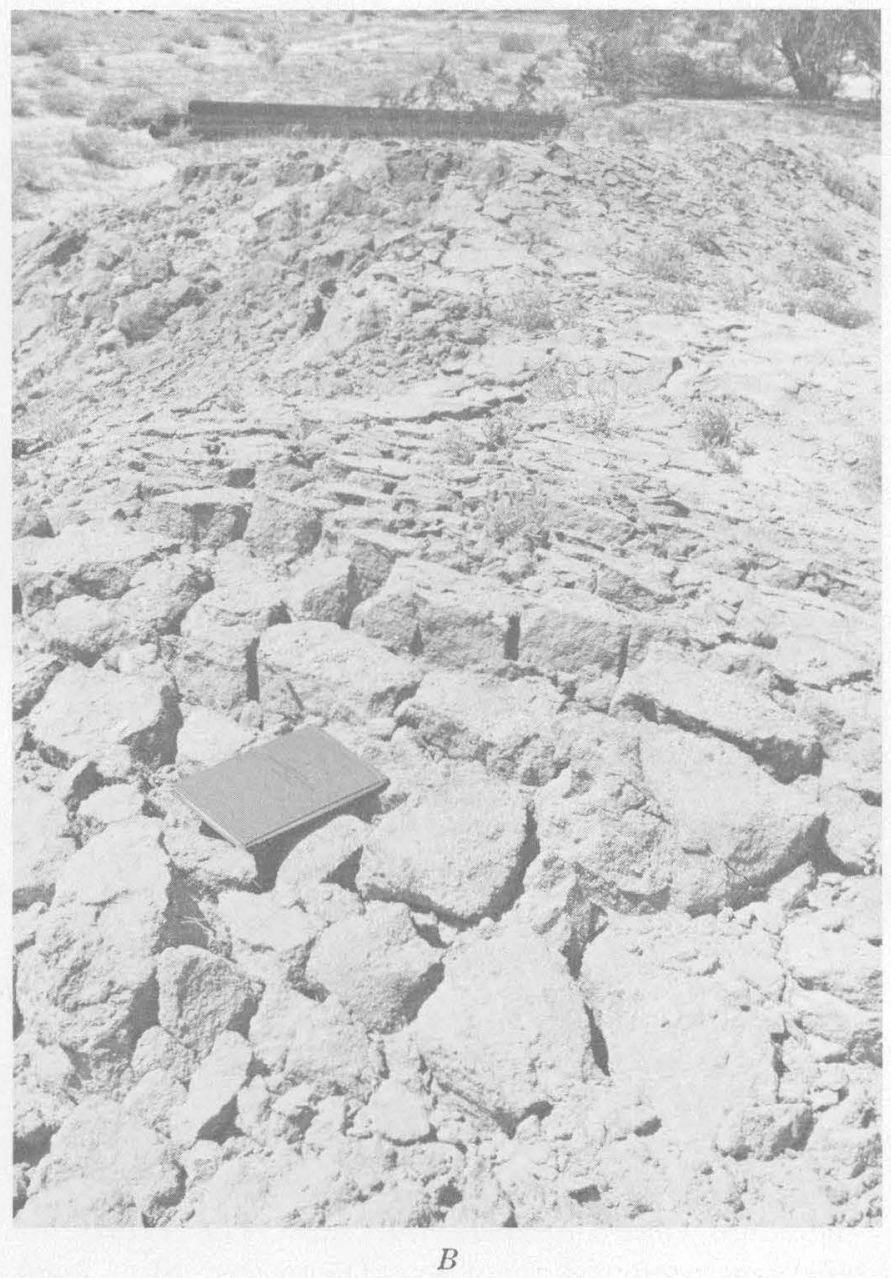

FIGURE 119. - Shattered lightly cemented crust overlying poorly indurated materials in the epicentral area. $A$, Sand dune south of the Ocotillo Badlands. $B$, Artificial fill about $1.5 \mathrm{~km}$ west of Ocotillo Wells.

severe damage away from the immediate area of the epicenter occurred on the old, chiefly Quaternary lacustrine deposits that underlie most of the Imperial Valley. Damage west of the Coyote Creek fault, 


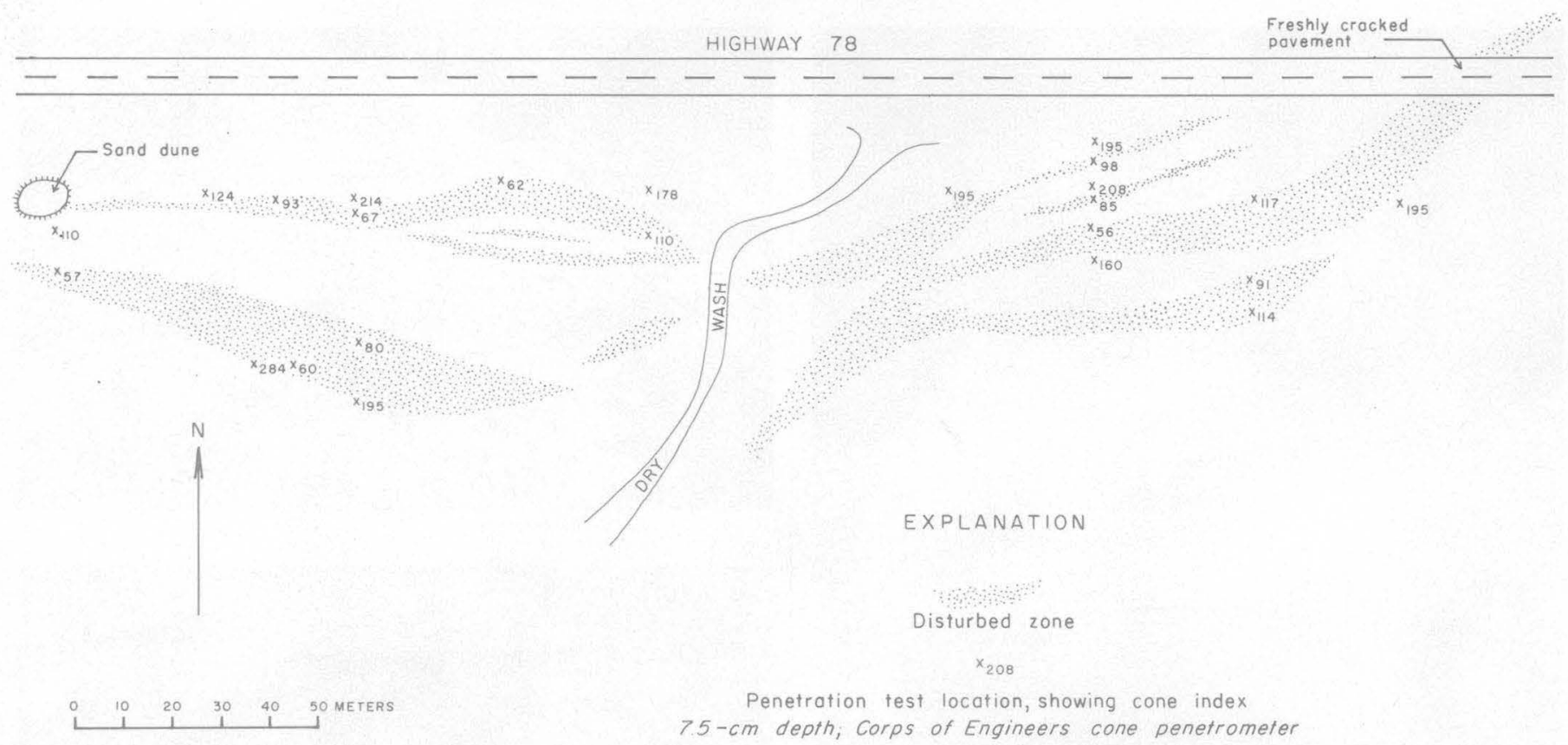

FIGURE 120. - Map showing distribution of shattered brittle crust overlying generally sandy lake-bottom(?) deposits about $13 \mathrm{~km}$ east of Ocotillo Wells. Relative differences in penetration resistance indicated by Corps of Engineers cone penetrometer measurements along and across zone.

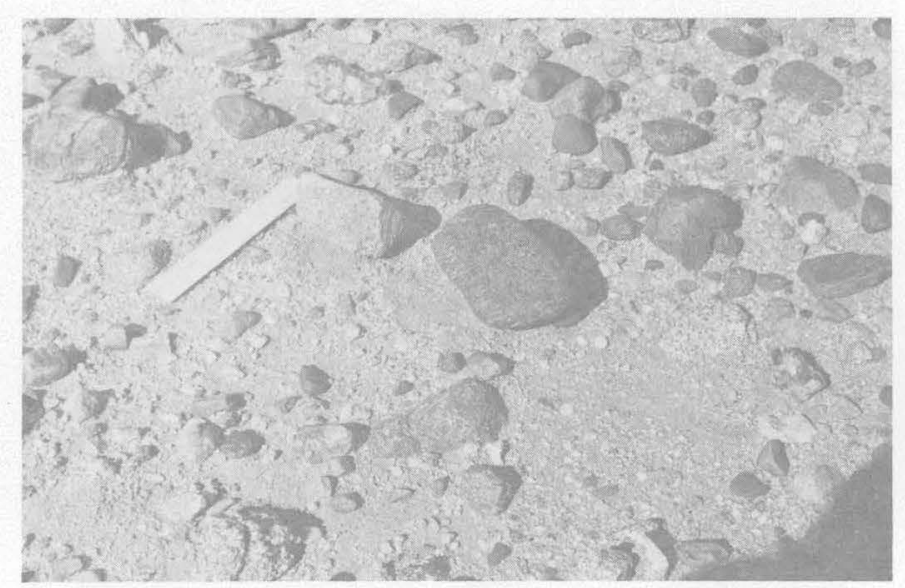

Figure 121. - Cobbles dislodged or displaced by seismic shaking, about $2.5 \mathrm{~km}$ east of Ocotillo Wells.

particularly among the crystalline rocks of the Peninsular Ranges, was generally very light (pl. 4).

Damage that impaired the safety or utility of structures occurred from San Diego east to at least Brawley and Calexico. (See Cloud and Scott, 1968a, p. 1190.) Frame structures, other than those that were poorly built or were particularly old, sustained very little damage. Unreinforced masonry structures were more severely affected, but we saw only two masonry failures that could be described as "impressive," and both of these were well removed from the epicenter.
Toppled or otherwise damaged chimneys were reported from as far west as Ramona (unconfirmed by our observations). We have no reports, however, of damaged chimneys east of the epicentral area or of damage to modern reinforced chimneys from any locality. The partly collapsed fieldstone chimney in Ocotillo Wells, shown in figure 122, was the most severely affected of any we observed.

Masonry walls sustained damage over an area ranging from Borrego Springs to at least as far east as Brawley and as far south as El Centro. The most spectacular failures occurred in pre-1940 unreinforced brick and adobe walls in Westmorland (fig. 123) and Seeley, 45 and $65 \mathrm{~km}$ east and southeast, respectively, of the epicenter. Unreinforced fieldstone walls, which formed parts of two abandoned structures near Ocotillo Wells, collapsed almost completely during the earthquake. One of these structures (fig. 124), an abandoned cellar, was overlain by a concrete slab roof, the inertial effect of which may have contributed to the failure. Lightly damaged reinforced concrete-block walls were observed from Borrego Springs east and southeast as far as Salton City and Brawley, respectively. Because nearly all the supermarkets in this part of California are built of concrete blocks and because we examined a very large percentage of these, our knowledge of the distribution of this type of damage probably is good. Breakage in the block walls generally consisted of cracking along construction 


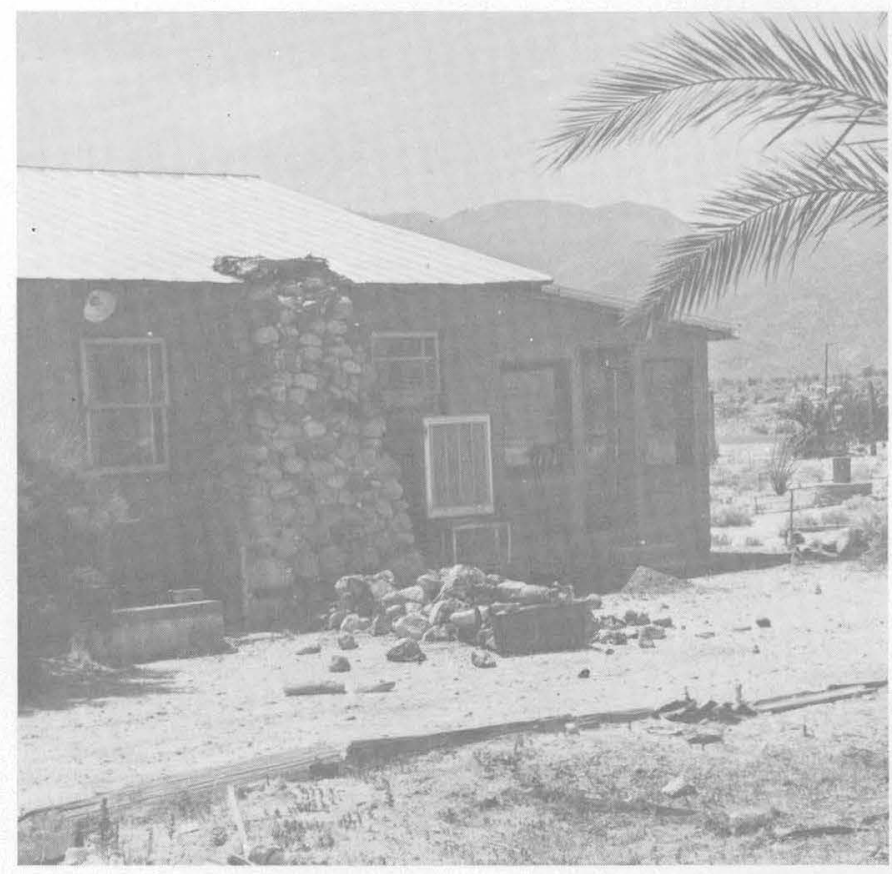

Figure 122. - Partly toppled fieldstone chimney in community of Ocotillo Wells.

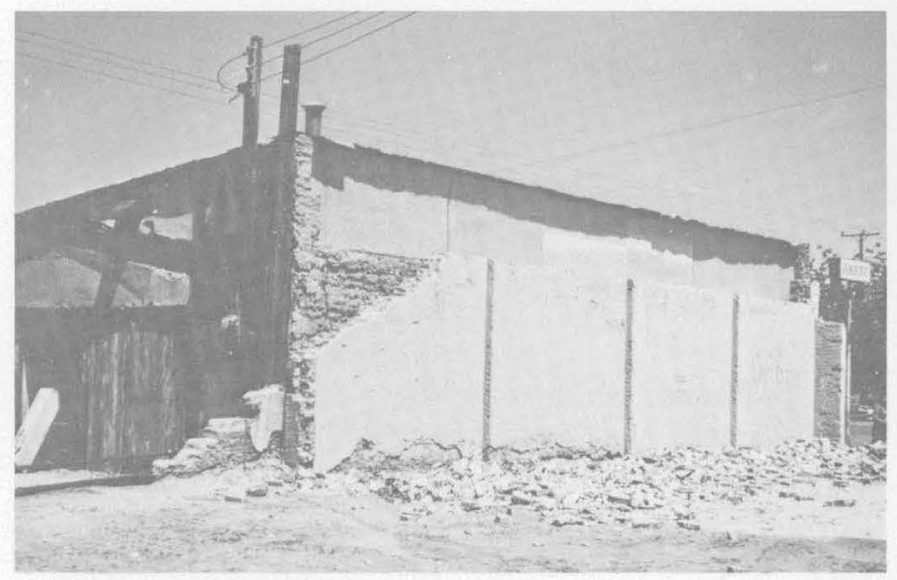

FIGURE 123. - Partly collapsed unreinforced brick wall in Westmorland.

joints, but individual blocks were rent locally (fig. 125). We have no substantiated reports of even partly collapsed reinforced block walls.

Cracked plaster or stucco walls were the most common type of damage incurred by frame structures. The westernmost examples of cracked plaster walls (other than hairline breaks associated with little if any debris) were seen in a pre-World War II Lakeside drugstore, just northeast of San Diego. Both interior plaster and exterior stucco walls were damaged enough to require repair. Plaster and stucco walls of frame structures in the Salton City area, none of which is probably more than 10 years old, seemed particularly susceptible to cracking and

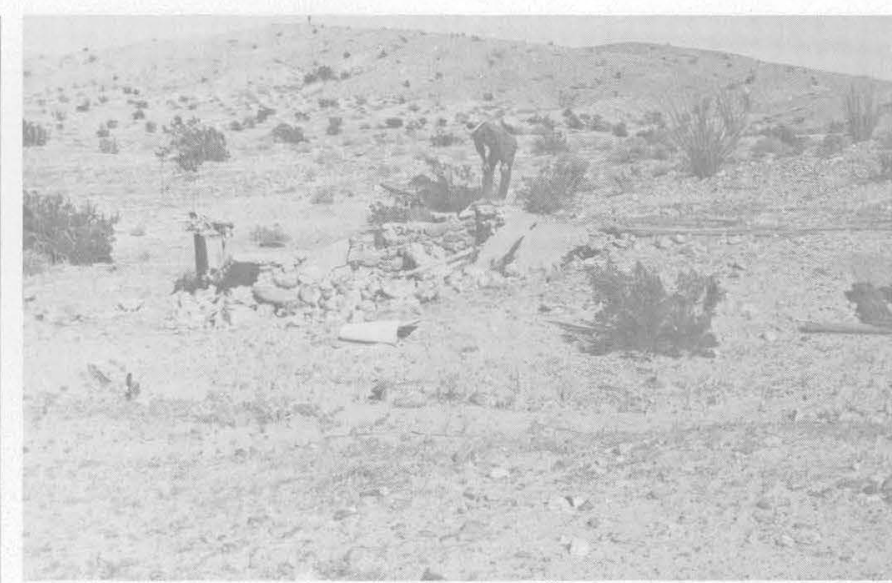

Figure 124.- Collapsed concrete-roofed fieldstone cellar about $1 \mathrm{~km}$ east of Ocotillo Wells.

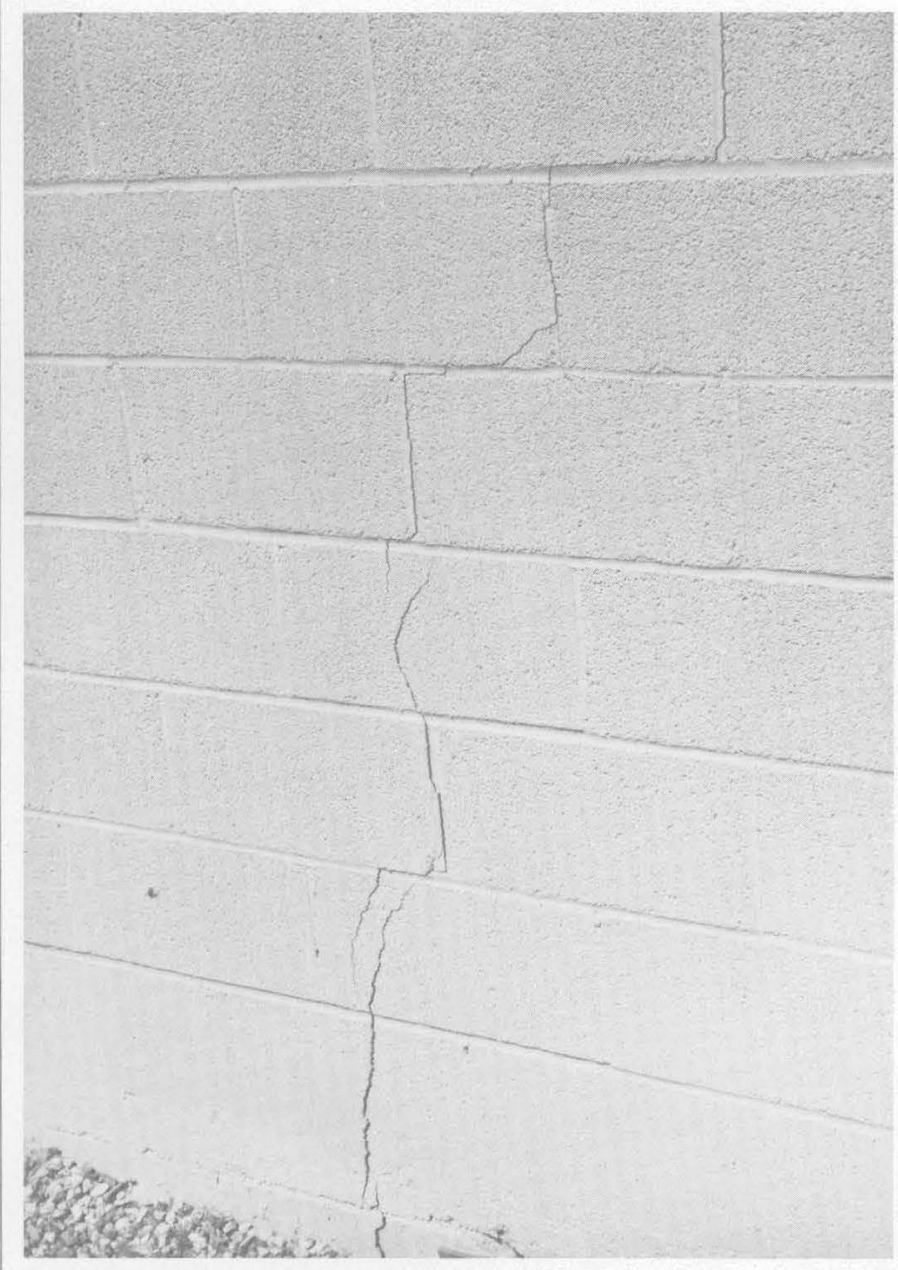

FIGURE 125. - Cracked reinforced concrete-block wall in Salton City building.

spalling. Most of the cracking in the Salton City area was minor, but the walls of a two-story woodframe motel in the center of town were extensively damaged (fig. 126). The cracks in the motel general- 


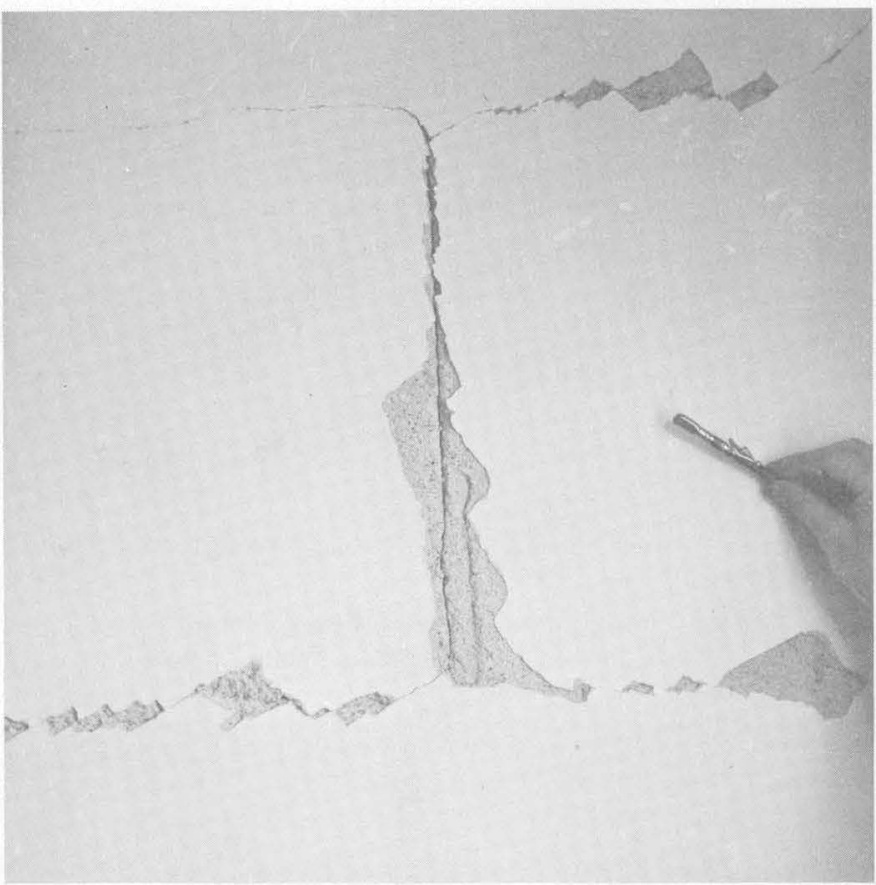

$A$
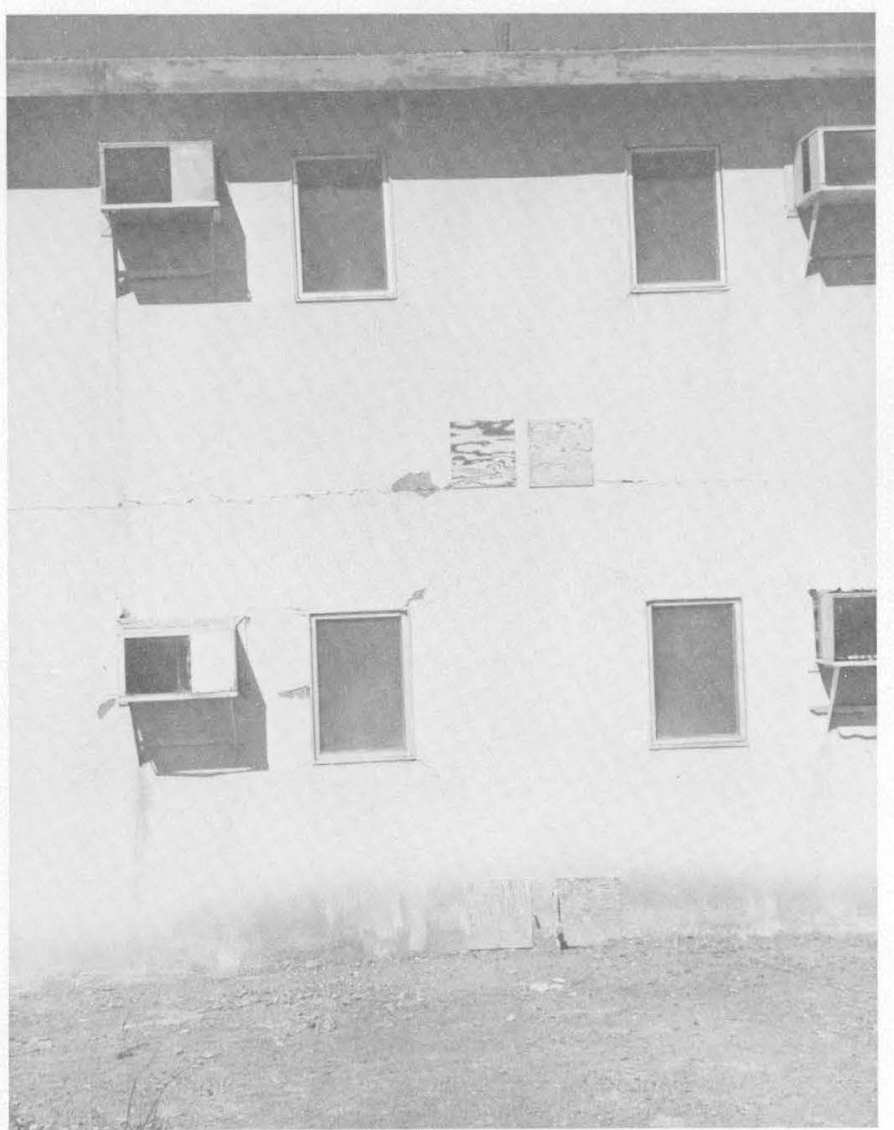

$B$

FIGURE 126. - Damage to plaster and stucco walls of a two-story motel in Salton City. A, Cracked interior wall. $B$, Cracked stucco wall. Note cracks radiating away from corners of sashwork and between floors. ly followed the studs, jumped from stud to stud along straight lines (fig. 126A), or radiated away from sashwork corners (fig. 126B) ; the most conspicuous damage occurred on the first floor, particularly at the interior corners between wings.

Separated or rent frame walls, partitions, or other structural units were seen in Salton City, Ocotillo Wells, and Borrego Springs. One very poorly built frame house in Ocotillo Wells was characterized by shifted, rocked, or possibly vertically lifted and projected walls. The displacement of a wooden column at its junction with an overhead beam in this same house very nearly led to the collapse of a section of roof. Nonetheless, damage of this sort was very light, and the only examples of separated or rent frame units that we saw were ones in which the unit was poorly bonded to the adjacent member.

Broken windows, which were observed locally over nearly the entire extent of our traverse, were, nevertheless, not generally evident. Press accounts of "hundreds of broken windows" in the San Diego area (Cloud and Scott, 1968a, p. 1190) may have been exaggerated, for a single cracked plate-glass window in an office building at $2 \mathrm{~d}$ and Broadway was the only example of a broken window that we saw or were reliably informed of in the metropolitan San Diego area.

Nearly all the highway bridges between San Diego and Glamis are of modern design and construction; most are supported by heavy wooden piles or concrete piers. Of the tens of bridges we examined, only the monolithic reinforced concrete bridge at the Highway 78 crossing of San Felipe Creek sustained damage that could be fairly definitely associated with the earthquake. The concrete piers supporting this bridge were circumferentially cracked and slightly spalled at their junctions with the overlying pier caps (fig. 127); although the cracks seemed to be equally distributed about the piers, the spalling was most conspicuous on the northwest sides of the piers. This particular damage constituted the only evident deterioration of a large, massive, modern structure discovered in our investigation. The only other shaking effects associated with the highway bridges along routes 78 and 99 were slumps or cracks within or along the margins of the abutments and aprons and cracking of the soil around the piers. The stream-bottom deposits within the arroyos intersected by Highway 78 about $10 \mathrm{~km}$ east of the San Felipe bridge, for example, displayed concentric and radiating arrays of cracks around the supporting bridge piers. It was not always possible, however, to be certain that these cracks and slumps were derived entirely from seis- 


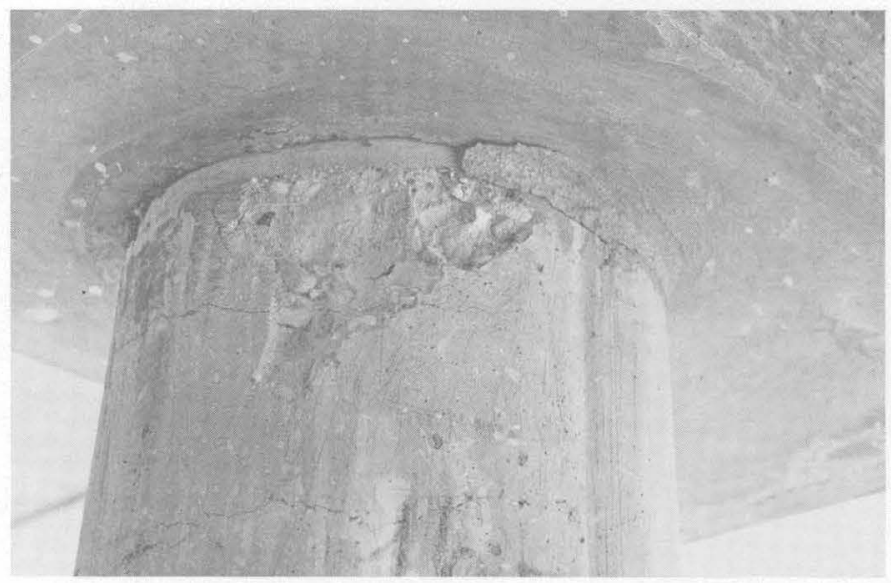

Figure 127. - Cracked and spalled 40-cm-diameter concrete pier supporting the Highway 78 bridge over San Felipe Creek, about $8 \mathrm{~km}$ east of Ocotillo Wells.

mic shaking rather than from heavy vehicular pounding.

A railroad bridge was damaged several hundred yards southeast of Glamis along the main line of the Southern Pacific Railroad (pl. 4). The concrete-abutment retaining walls supporting this bridge reportedly displayed $1 / 2$ - to 1 -cm-wide cracks prior to April 9; these expanded at the time of the earthquake into the 8 - to 10 -cm-wide cracks shown in figure 128.

Ruptured buried concrete pipes on Jacobs Ranch east of Ocotillo Wells and cracked concrete canal linings on the Elmore Desert Ranch west of Westmorland (see pl. 4) constituted the only other type of secondary structural damage of which we are aware. The cracks in the canal linings paralleled both the contour of the lining slope and fresh cracks in the adjacent soil; both sets of cracks probably were generated by free-face movements of the canal embankments.

\section{EFFECTS ON LOOSE OBJECTS}

Loose objects (those unrestrained or only partly restrained against free movement) are classified as heavy (for example, furniture, safes, automobiles) or light (for example, shelf goods, lamps, whisky bottles). Although heavy objects within the epicentral region commonly were displaced or shifted, we have few reports of this sort of movement more than 4 or $5 \mathrm{~km}$ from the epicenter. Shifting or projection of light objects, on the other hand, occurred over almost all of our traverse.

An abandoned kitchen stove in Ocotillo Wells that had been flipped upside-down (R. V. Sharp, written commun., 1970) and toppled bales of hay, about $3.5 \mathrm{~km}$ south of Ocotillo Wells, were the only reported examples of overturned heavy objects.

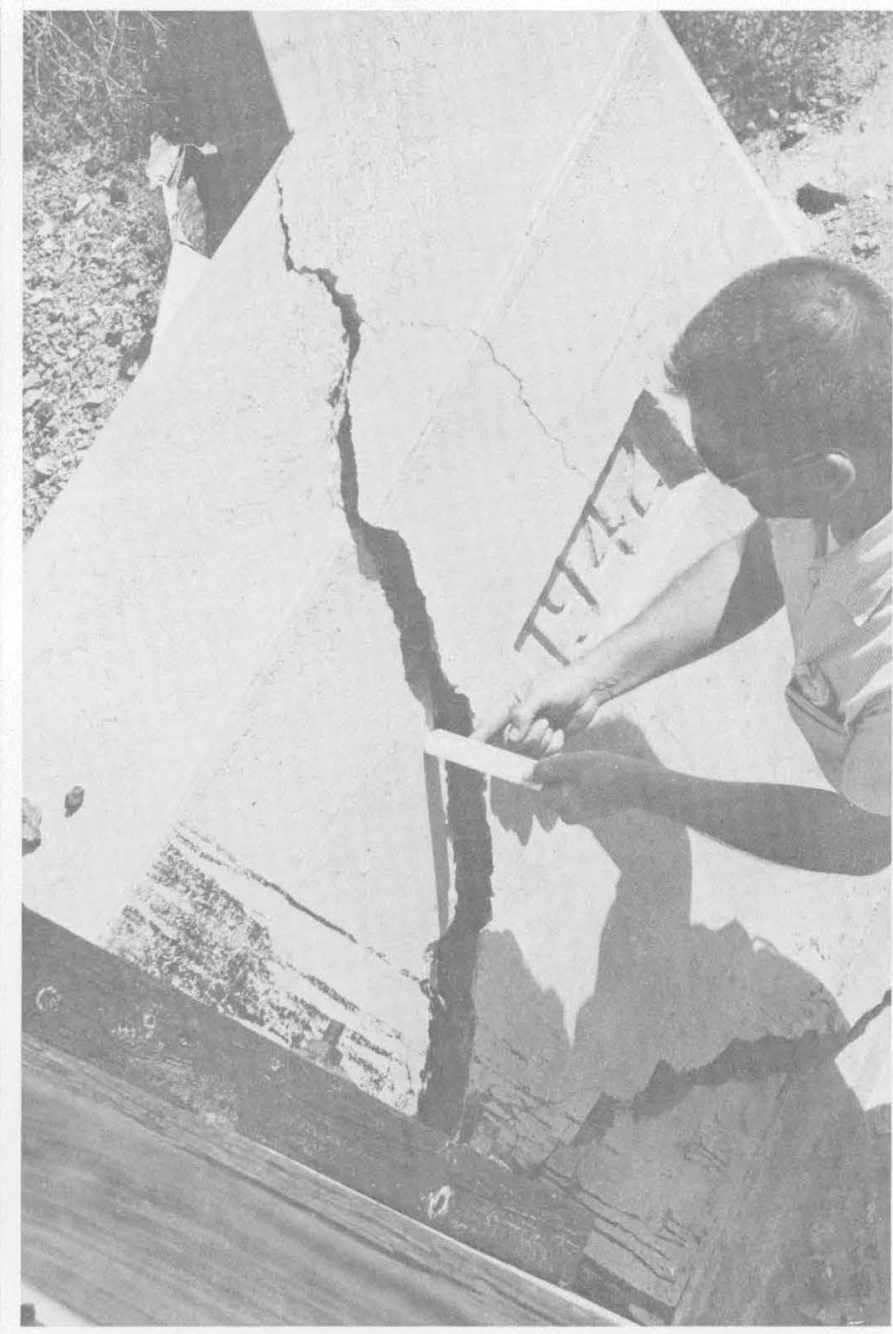

FIGURE 128. - Cracked concrete railroad bridge abutment about $0.3 \mathrm{~km}$ southeast of Glamis. Gaping cracks shown here reportedly widened at the time of the earthquake from $1 / 2$ - to $1-\mathrm{cm}$ openings to $8-10 \mathrm{~cm}$.

Shifted or displaced heavy objects, however, abounded within the epicentral region. Automobiles commonly were shifted about, and a number of house trailers parked in the Ocotillo Wells area were knocked off their parking jacks or other light foundations. Large transformers at the San Felipe substation, about $5 \mathrm{~km}$ south-southeast of Ocotillo Wells, were shifted with such force as to shear their anchor bolts (Cloud and Scott, 1968a, p. 1189). Stoves, refrigerators, and various types of furniture slid around within the stores and dwellings of Ocotillo Wells; interviews with local witnesses indicate that these objects were displaced preferentially toward the northeast (that is, toward the trace of the Coyote Creek fault). Away from the epicenter, a large high-pressure fuel tank inside a restaurant about $5 \mathrm{~km}$ east of Borrego Springs shifted or pivoted about $8 \mathrm{~cm}$ to the south-southeast, 
and heavy roller-mounted vaults in a Salton City bank moved about $25 \mathrm{~cm}$ west-southwest.

Vibrational penetration of automobiles into loosely compacted sands occurred near the main trace of the Coyote Creek fault. About a dozen heavy vehicles parked near the Morton house, about $1.5 \mathrm{~km}$ southeast of Ocotillo Wells, were shaken into the ground to depths of 8-10 cm (fig. 129).

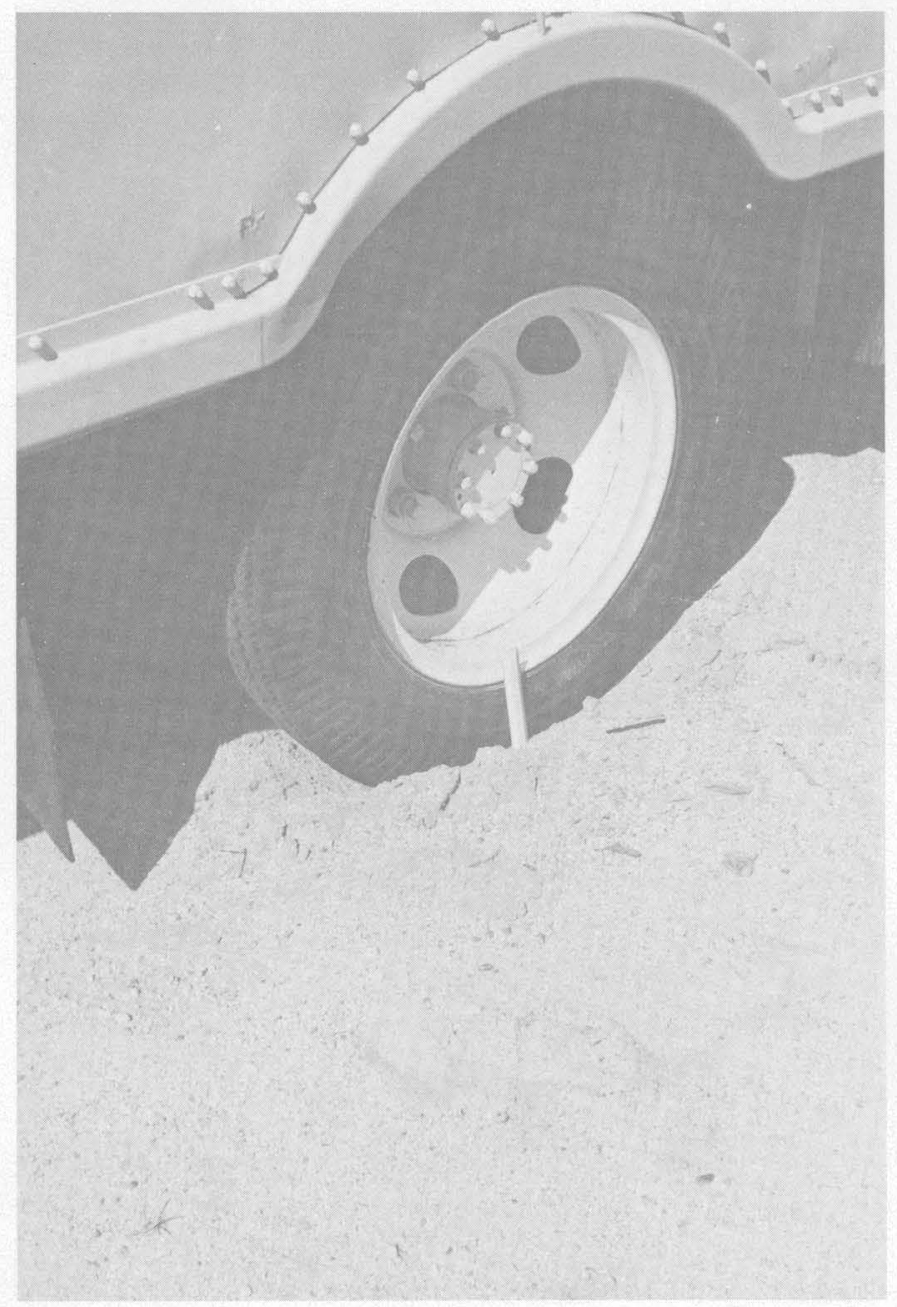

Figure 129. - Light truck that sank several centimeters into the dry desert sand, owing to vibratory ground motion, about $1.5 \mathrm{~km}$ southeast of Ocotillo Wells.

Projected or displaced light objects were reported from Coronado to about $8 \mathrm{~km}$ east of Brawley; they were particularly conspicuous near the epicenter (fig. 130). Interviews with three residents of Glamis (virtually the entire population) failed to elicit a single report of displaced objects at this most easterly point of our reconnaissance. Goods shaken from shelves were both the commonest and most widespread permanent effect discovered. Two thousand dollars worth of breakage in a Salton City liquor

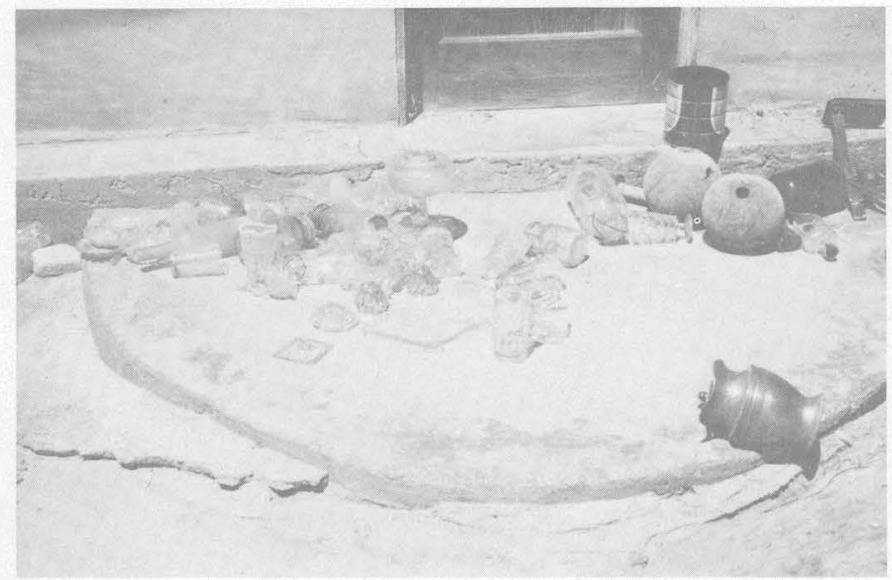

Figure 130. - Projected and displaced objects about 1.5 $\mathrm{km}$ southeast of Ocotillo Wells. All those objects for which the height of the center of gravity was equal to or greater than the diameter of the base were knocked over. Glass tumbler nearest the camera was placed in upright position before the picture was taken.

store was, in fact, the single most distressing effect of the Borrego Mountain earthquake.

The distribution of displaced and projected light objects is of interest. We have reports of extensive breakage in the metropolitan San Diego area from Chula Vista north to Mission Bay and reports of limited breakage in supermarkets in central El Cajon and various stores in Lakeside and Ramona. (See pl. 4.) All these areas are underlain by Quaternary terrace deposits, beach or lagoonal deposits, or alluvium that range from a few to perhaps hundreds of meters in thickness. There were, by contrast, startlingly few reports of fallen objects from Ramona eastward to and including the west edge of the Borrego Valley; this region is underlain almost entirely by crystalline rocks. The occurrence of fallen objects diminished generally eastward from the epicenter, but local pockets of breakage were recognized in Salton City, Westmorland, Imperial, and El Centro, all of which are underlain by late Cenozoic lacustrine deposits several thousand meters thick.

Several small-scale associations between the distribution of fallen objects and certain physiographic features attracted our attention. A relatively new supermarket about $0.3 \mathrm{~km}$ west of the center of Lakeside had no more than about $\$ 10$ worth of breakage, whereas a similar supermarket near the center of town apparently sustained extensive breakage (exact amount unknown). The only apparent difference between the two situations was the location of the second market on what appeared to be a slightly lower (by 3-4 m) river terrace. The distribution of breakage in Brawley was particularly 
interesting. The modern concrete-block Mayfair Market here sustained about $\$ 1,000$ worth of breakage, yet according to the manager, breakage was minor in similar stores elsewhere around Brawley. The Mayfair Market may be unique because it is both the westernmost of the Brawley supermarkets and only about $0.6 \mathrm{~km}$ east of the high (approximately $12 \mathrm{~m}$ ) steep-banked New River flood plain.

\section{INFERRED INTENSITY}

Maximum intensities of at least VIII and possibly IX (Modified Mercalli) were attained during the earthquake. The highest intensities occurred within a 3-6-km-wide band along the ruptured trace of the Coyote Creek fault and along the west edge of the Salton Sea near Salton City. Intensities of VIII are indicated by partial loss of control of moving automobiles, minor damage to reinforced masonry, collapsed chimneys, cracked stucco walls, local changes in well flow, and cracks on steep slopes. Intensities of IX may be indicated for the epicentral region by the destruction of poorly constructed masonry walls, separated or rent frames, broken underground pipes, local ground cracking, sand boils, and landslides in dry soils. (See Richter, 1958, p. 136-138.) Although some effects, such as large slumps in the Ocotillo Badlands, suggest intensities even greater than IX, the general survival of frame structures and the absence of serious bridge damage argue that intensities of $\mathrm{X}$ probably were not attained anywhere within the area we investigated.

Intensities midway between VIII and IX were correlated with magnitude 6.4 shocks by Gutenberg and Richter (1956, p. 132), a correlation in agreement with what has been observed for this earthquake. Gutenberg and Richter $(1956$, p. 131) also correlated intensities of VIII and IX with ground accelerations of $0.20 \mathrm{~g}$ and $0.30 \mathrm{~g}$, respectively. These correlations, in turn, are in good agreement with epicentral accelerations of about $0.22 \mathrm{~g}$ correlated with magnitude 6.4 shocks (Lockheed Aircraft Corp. and Holmes and Narver, Inc., 1963, p. 14). Epicentral accelerations of $0.35 \mathrm{~g}$ to $0.40 \mathrm{~g}$ may be deduced from accelerations measured at El Centro and attenuation curves presented by Cloud (1968); however, we have assumed in using these attenuation curves that differing initial accelerations decline proportionately with distance, and this procedure may have led us to overestimate epicentral accelerations. Clark ("Intensity of Shaking Estimated from Displaced Stones," this volume), nevertheless, concluded from observations of displaced pebbles that horizontal accelerations approaching $1 g$ may have been attained locally within the epicentral region.
Our field investigation indicates that intensities of VIII or more were attained over at least 500-600 $\mathrm{sq} \mathrm{km}$ and that intensities of VII or greater were attained over several thousand square kilometers. Isoseismal maps are presented elsewhere in this report. (See Seismological Field Survey, National Oceanic and Atmospheric Administration, this volume.)

\section{The occurrence of the Borrego Mountain earth-} quake in one of the least developed sections of southern California scarcely minimizes the significance of this event to engineering geology and foundation engineering. The earthquake effects of greatest importance to these two disciplines are as follows:

1. The earthquake was felt over at least $155,000 \mathrm{sq}$ $\mathrm{km}$ and was characterized by maximum intensities of VIII-IX within a narrow band extending $32 \mathrm{~km}$ or more along the Coyote Creek fault. The inferred epicentral intensities are in close agreement with those predicted for a magnitude 6.4 shock.

2. The main shock was accompanied by the formation of a locally complex zone of tectonic surface ruptures that extended about $32 \mathrm{~km}$ along the Coyote Creek fault. Zones of this complexity have not generally been reported for earthquakes of magnitude 6.4 or less. The precise locations of the ruptures, moreover, could have been predicted on the basis of ordinary field criteria over no more than about one-half their extent.

3. Temporally associated surficial fault displacements were generated along the Superstition Hills, Imperial, and Banning-Mission Creek faults. Although very slight, they could have damaged sensitive structures situated on the fault trace. It now seems likely that even relatively distant shocks may trigger displacements along any discontinuities identified with accumulated elastic strain of whatever source.

4. The epicentral region was characterized by a variety of dry-soil slope failures and compaction effects that could be reasonably expected to accompany a magnitude 6.4 shock. Direct and indirect effects of soil consolidation generated at distances of $30-60 \mathrm{~km}$ from the epicenter were less expected; these distant features were all underlain by probably thick upper Cenozoic lacustrine sediments.

5. Structural damage was generally minor for an earthquake of this size and was concentrated in the epicentral region and over the lacustrine deposits in and around Salton City. It seems 
unlikely that this light damage can be due entirely to the limited development of the region; it may be equally attributable to a relatively short duration of strong shaking or to generally good natural foundations beneath those structures close to the epicenter.

The fortunate location of this shock should not, as we have observed, minimize its significance to those concerned with most aspects of earthquake engineering. Had the locally complex pattern of tectonic surface ruptures and the various shaking effects associated with this shock occurred in a densely populated region (especially one characterized by water-saturated ground), the damage would certainly have been much greater and locally severe.

\section{REFERENCES CITED}

Allen, C. R., Grantz, Arthur, Brune, J. N., Clark, M. M., Sharp, R. V., Theodore, T. G., Wolfe, E. W., and Wyss, Max, 1968, The Borrego Mountain, California, earthquake of 9 April 1968 - A preliminary report: Seismol. Soc. America Bull., v. 58, no. 3, p. 1183-1186.

Bonilla, M. G., 1967, Historic surface faulting in continental United States and adjacent parts of Mexico: U.S. Geol. Survey open-file report, 36 p. (also U.S. Atomic Energy Comm. Rept. TID-24124, 36 p.).

Brown, R. D., Vedder, J. G., Wallace, R. E., Roth, E. F., Yerkes, R. F., Castle, R. O., Waananen, A. O., Page, R. W., and Eaton, J. P., 1967, The Parkfield-Cholame, California earthquakes of June-August 1966 - Surface geologic effects, water-resources aspects, and preliminary seismic data: U.S. Geol. Survey Prof. Paper 579, 66 p.

Cloud, W. K., 1968, Strong-motion seismograph records, in State-of-the-art symposium, earthquake engineering of buildings: Earthquake Eng. Research Inst. Mtg., San Francisco, Feb. 5-6, 1968, Program.

Cloud, W. K., and Scott, N. H., 1968a, The Borrego Mountain, California, earthquake of 9 April 1968 - A preliminary engineering seismology report: Seismol. Soc. America Bull., v. 58 , no. 3 , p. $1187-1191$.

$1968 \mathrm{~b}$, Field reports and isoseismal map, in Strongmotion instrumental data on the Borrego Mountain earth- quake of 9 April 1968: Seismological Field Survey of U.S. Coast and Geodetic Survey and Earthquake Eng. Lab. of California Inst. Technology, p. 19-119.

Dibblee, T. W., Jr., 1954, Geology of the Imperial Valley region, California, in Jahns, R. H., ed., Geology of southern California; chap. 2, Geology of the natural provinces: California Div. Mines Bull. 170, p. 21-28.

Gutenberg, Beno, and Richter, C. F., 1956, Earthquake magnitude, intensity, energy, and acceleration, $2 \mathrm{~d}$ paper: Seismol. Soc. America Bull., v. 46, no. 2, p. 105-145.

Hamilton, R. M., 1970, Time-term analysis of explosion data from the vicinity of the Borrego Mountain, California, earthquake of 19 April 1968: Seismol. Soc. America Bull., v. 60 , p. $367-381$.

Jahns, R. H., 1954a, Investigations and problems of southern California geology, in Jahns, R. H., ed., Geology of southern California; chap. 1, General features: California Div. Mines Bull. 170, p. 5-29.

1954b, Geology of the Peninsular Range province, southern California and Baja California, in Jahns, R. H., ed., Geology of southern California; chap. 2, Geology of the natural provinces: California Div. Mines Bull. 170, p. 29-52.

Jennings, C. W., compiler, 1967, Geologic map of California, Olaf P. Jenkins edition, Salton Sea sheet: California Div. Mines and Geology, scale 1:250,000.

Lockheed Aircraft Corp. and Holmes and Narver, Inc., 1963, Nuclear reactors and earthquakes: Div. Reactor Development, U.S. Atomic Energy Comm. Rept. TID-7024, $411 \mathrm{p}$.

Richter, C. F., 1958, Elementary seismology: San Francisco, Calif., W. H. Freeman and Co., 768 p.

Rogers, T. H., compiler, 1965, Geologic map of California, Olaf P. Jenkins edition, Santa Ana sheet: California Div. Mines and Geology, scale $1: 250,000$.

Sharp, R. V., 1967, San Jacinto fault zone in the Peninsular Ranges of southern California: Geol. Soc. America Bull., v. 78 , no. 6 , p. $705-730$.

Strand, R. G., compiler, 1962, Geologic map of California, Olaf P. Jenkins edition, San Diego-El Centro sheet: California Div. Mines and Geology, scale 1:250,000.

Varnes, D. J., 1958, Landslide types and processes, chap. 3, in Eckel, E. B., ed., Landslides and engineering practice: Natl. Research Council, Highway Research Board Spec. Rept. 29, NAS-NRC Pub. 544, p. 20-47. 


\title{
INTENSITY OF SHAKING ESTIMATED FROM DISPLACED STONES
}

\author{
By Malcolm M. Clark
}

U.S. Geological Survey

\begin{abstract}
Characteristics of stones displaced during the Borrego Mountain earthquake of April 9, 1968, allow rough estimates of the accelerations responsible for displacement. Areas of displaced surface stones were generally restricted to certain high ridges in Ocotillo Badlands, indicating that topography magnified the intensity of shaking. Nearly every unrestrained stone on the surface of one such ridge was either tipped, slid, or flung as much as $0,5 \mathrm{~m}$ in one direction, whereas only cobbles and boulders taller than about $50 \mathrm{~mm}$ moved on an adjacent ridge, sliding a maximum of $0.1 \mathrm{~m}$ in the opposite direction. These directions of displacement indicate that the horizontal shaking responsible was oriented $70^{\circ}-80^{\circ}$ from the general trend of surface breakage; however, the influence exerted on these directions by the position, shape, or material of the ridges is unknown.

Theoretical considerations indicate that in the absence of adhesion, simple sliding of a rectangular block depends only on the values of coefficient of friction, slope, and acceleration and is independent of size and shape. Tipping depends only on slope, shape, and acceleration. Size of the block affects sliding or tipping only if adhesion or other surface forces are present or if rocking has begun.

Estimates of coefficients of friction, combined with characteristics of size, shape and movement, suggest that horizontal accelerations on the first ridge were greater than $0.5 g$ and possibly as high as $1 g$, whereas vertical accelerations were definitely less than $1 \mathrm{~g}$. Stones of the second ridge experienced smaller accelerations, with the horizontal component apparently in the range of 0.4-0.9 g. Adhesion between stones and the surface of the second ridge prevented stones shorter than $50 \mathrm{~mm}$ from moving.
\end{abstract}

\section{INTRODUCTION}

This chapter describes and evaluates the implications of stones near the surface rupture of the Coyote Creek fault that were displaced by shaking during the Borrego Mountain earthquake of April 9, 1968. Such stones offer a crude measure of intensity of shaking (as in Oldham, 1897, p. 129-133), and an analysis of the stones and their displacements permits some general statements about the accelerations responsible for displacements. The chapter presents observations of such stones in Ocotillo Badlands, an analysis of how stones may react to shaking, and estimates of local accelerations in the badlands.
T. L. Youd, R. V. Sharp, and R. O. Castle pointed out flaws and omissions in the analysis and made many suggestions for improvement of the manuscript.

\section{LOCATION OF DISPLACED STONES}

In general, surface gravels are not abundant along the 1968 break; however, a lag of granitic and metamorphic gravel covers most of the low hills in the vicinity of the break and much of the ridges and lower flanks of Ocotillo Badlands. Scattered patches of gravels left by modern debris flows also occur locally near washes elsewhere along the break.

Few of these surface gravels anywhere in the region of the break were moved by shaking of the earthquake, except on several high parts of ridges of Ocotillo Badlands (for example fig. $131 A, B$, and $C$ ), where a majority of the pebbles, cobbles, and boulders resting on the surface were rolled, slid, or flung from their preearthquake positions. In contrast, the gravels that cover most of the lower slopes of the Badlands did not move, except for those at location $E$, figure 131. According to R. V. Sharp (oral commun., 1969), about 20 percent of the surface stones at location $E$ moved down the $10^{\circ}$ slope on which they rested. Also, Castle and Youd (this volume) report displaced stones near slump fractures elsewhere in Ocotillo Badlands and in the low hills to the northeast.

The ridges with displaced stones in Ocotillo Badlands evidently experienced significantly higher accelerations than elsewhere. As noted by Clark ("Surface Rupture Along the Coyote Creek Fault," this volume), these same hills were literally shattered with small landslides and faults. Although the abundance of landslides and slips is probably a manifestation of magnified accelerations, the moved stones do not appear to have been directly affected by such landsliding. Several slides originated on ridges containing the moved stones described in this study, but none of these stones rested on such slides. 


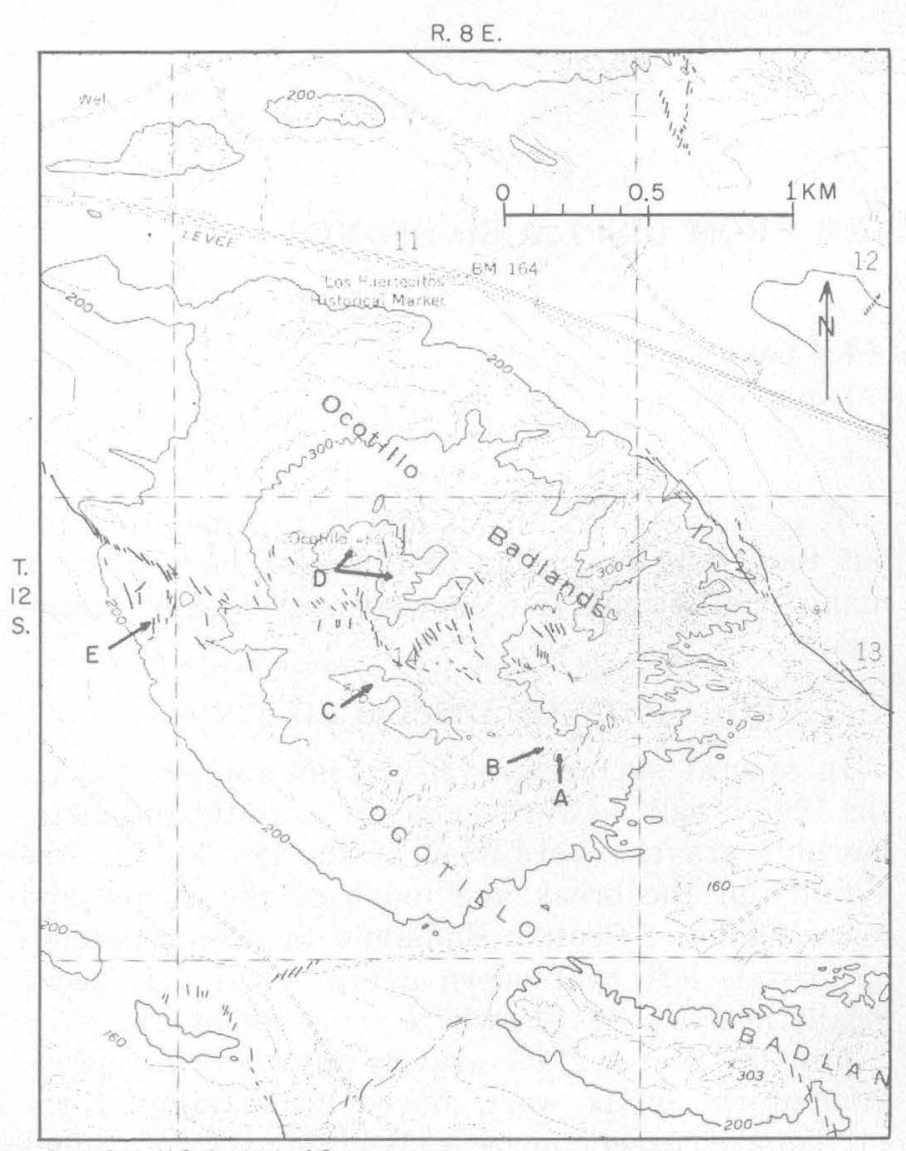

Base from U.S. Geological Survey
$1: 24,000$ Borrego Mountain SE, 1958 and Shell Reef, 1959

Figure 131. - Area of moved surface gravels. $A$ and $B$, Location of ridges on which many stones were displaced by shaking. $C$, Ridge containing many displaced stones but which was not investigated. $D$, High ridges on which virtually no stones were displaced. $E$, Only area where many boulders were moved that was not on a ridgetop. Lines show surface ruptures associated with the earthquake of April 9, 1968.

\section{DISPLACED STONES OF OCOTILLO BADLANDS}

The distribution of the areas of abundant displaced stones in Ocotillo Badlands appears to be irregular. Stones possibly shifted on high ridges not visited, in addition to those that moved on ridges at $A, B$, and $C$ (fig. 131). No stones, however, moved on the high ridges at $D$, suggesting that other factors such as orientation of the ridges, position with respect to breaks, or local topography, lithology, and structure might also determine locations of magnified shaking.

Stones displaced by shaking were easily identified. Differences in tone and color marked the seats from which cobbles and boulders had moved and identified formerly buried parts of stones. Furthermore, many stones moved from seats embedded as much as 100 $\mathrm{mm}$ into the surface, revealing original position and orientation exactly (figs. 133, 134).

The observations of moved stones that form the basis for this report were made on two parallel ridges (locs. $A, B$, fig. 131). Only enough measurements were made to characterize broadly the movement of the stones; hence, the data lead simply to general statements about shaking and displacements and to estimates of the probable ranges of acceleration.

\section{RIDGE A}

Ridge $\mathrm{A}$ lies about $100 \mathrm{~m}$ from ridge $\mathrm{B}$, and both trend about $\mathrm{N} .45^{\circ} \mathrm{E}$. Ridge $\mathrm{A}$ is covered by predominantly granitic gravel that ranges from small pebbles to subrounded and rounded blocks with minimum dimensions greater than $0.5 \mathrm{~m}$ (fig. 132). The gravels examined occupy the crest and west and northwest slopes on surfaces inclined from $0^{\circ}$ to about $20^{\circ}$. The earthquake either tipped, slid, or

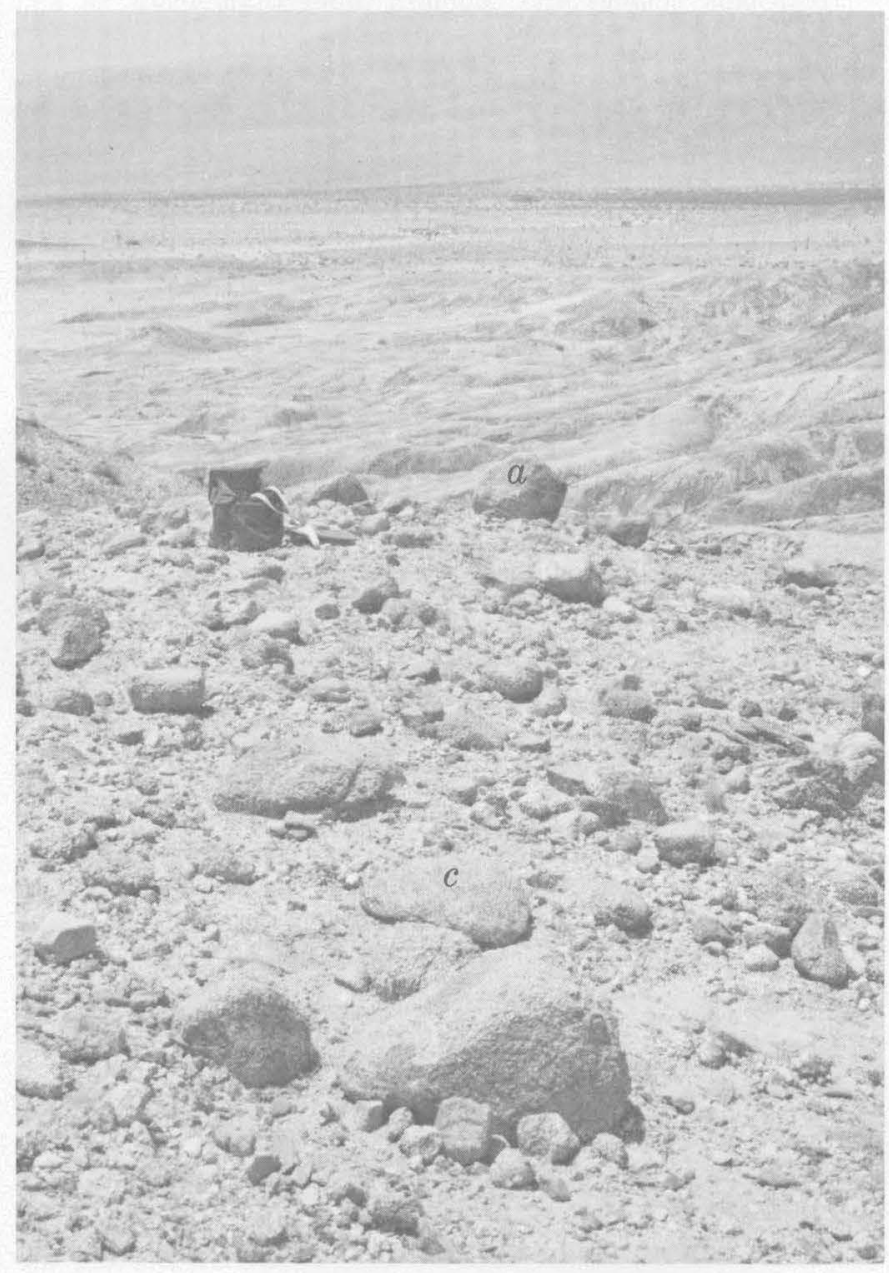

Figure 132. - Gravel on top of ridge A. Shaking displaced most of the stones in view in a direction away from the camera and slightly to the right. Detail of boulder $a$ is in figure 133, and detail of boulder $c$ in figure 134 . 


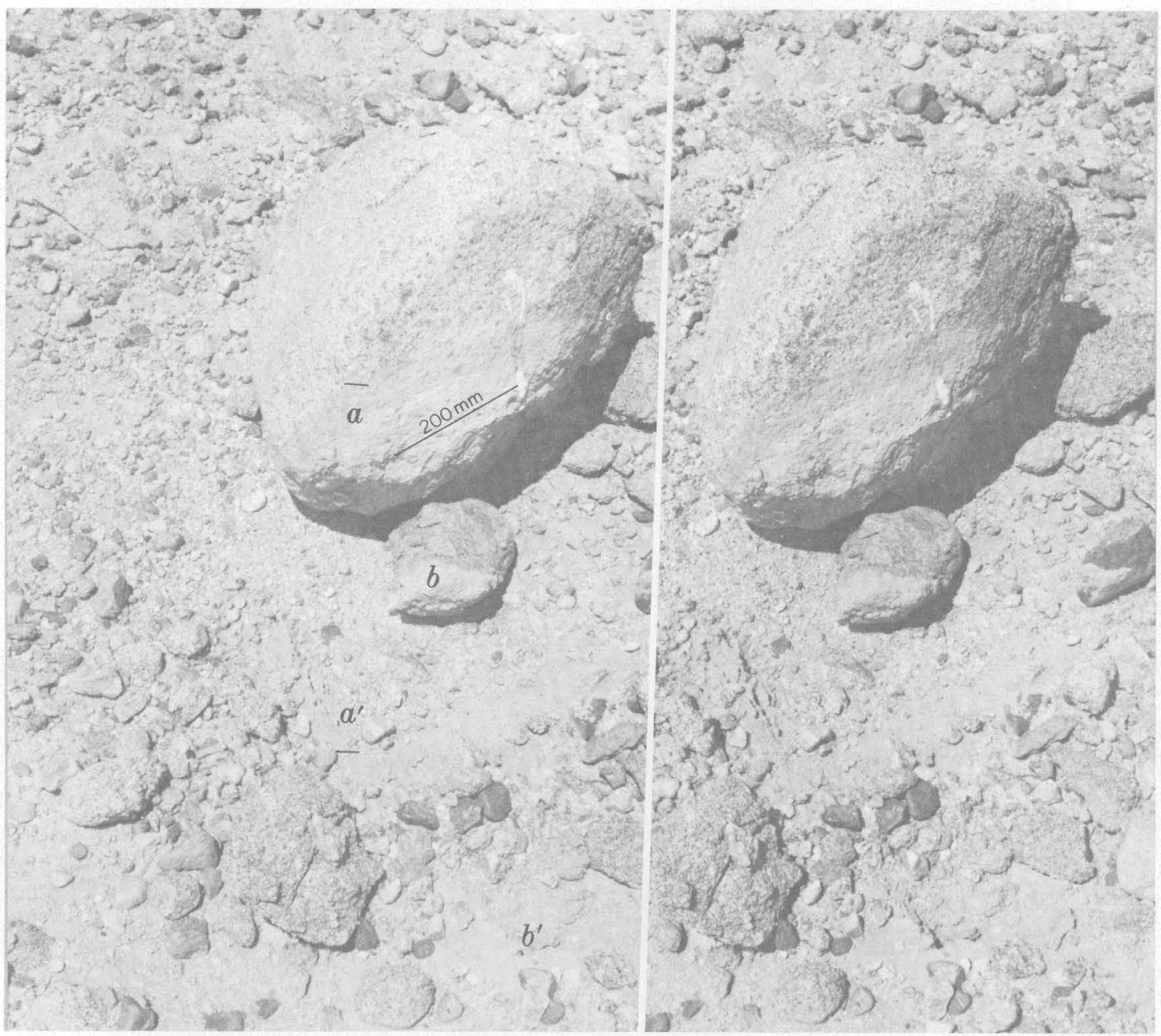

Figure 133. - Stereo view of a 0.6-m-high tetrahedron tipped over by shaking. $a$ marks matching points on the boulder and its seat. Cobble $b$ moved more than $0.5 \mathrm{~m}$ across pebbles from its seat $\left(b^{\prime}\right)$ into that vacated by the tetrahedron. Most stones in this picture have moved, including all that occupy the vacated seat of the tetrahedron. View is downhill; average slope is about $10^{\circ}$.

flung virtually every unrestrained stone on ridge A in one direction, about S. $70^{\circ}$ W. Although this direction includes a component of downslope movement for most stones on this ridge, many others that moved either rested on the flat ridge crest, had level bases, or were originally nested as much as $100 \mathrm{~mm}$ below the surface. Boulders and cobbles commonly moved an amount at least equal to their own diameters, and many pebbles and cobbles moved into the seats of other displaced clasts (figs. 133, 134). Moving cobbles and boulders also struck and displaced numerous pebbles, and one elongate boulder rotated $180^{\circ}$ when it struck a buried boulder (fig. 134). The evidence of large and apparently rapid displacements shown in figures 133 and 134 suggests large local accelerations, and possibly displacements, during shaking of the ground at ridge $\mathrm{A}$.

\section{RIDGE B}

Surface gravel on ridge $\mathrm{B}$ is not so abundant as on ridge $\mathrm{A}$ and includes many tabular schistose cobbles, which were scarce on ridge A. Furthermore, all the displaced stones examined here occupy the nearly flat crest of the ridge. As on ridge A, displaced stones were easy to identify because of the strong color contrast between newly exposed seats and the surrounding surface. In addition, many of the large cobbles and small boulders that did move (and many smaller cobbles that did not) rested on low sand-silt pedestals that not only clearly defined preearthquake position of their covering stones but also offered no material barrier to sliding.

In contrast to ridge $\mathrm{A}$, the displaced stones of ridge $B$ were neither flung nor tipped but slid uniformly in one direction, about N. $65^{\circ}$ E., nearly 


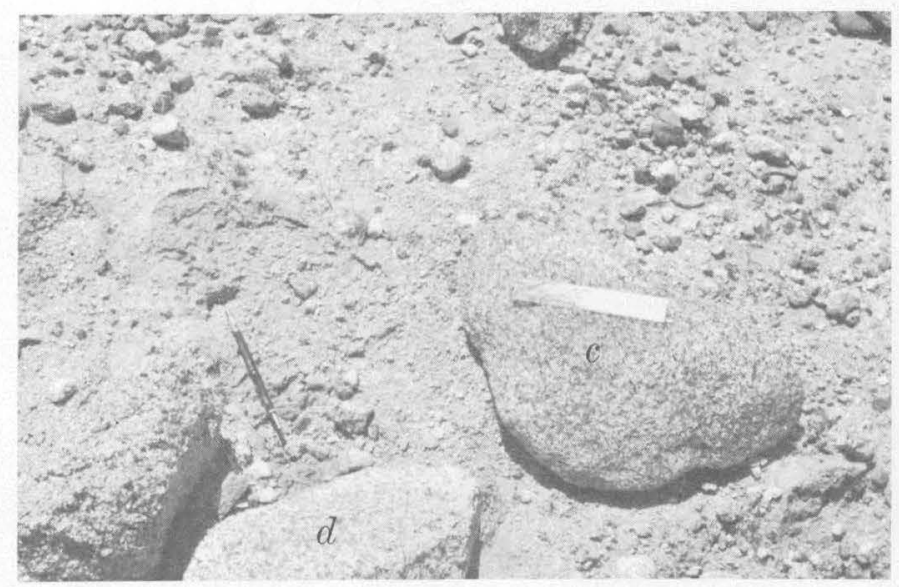

Figure 134. - Boulder $c$, located on ridge A, was propelled from its nearly horizontal seat (pencil) and rotated $180^{\circ}$ after striking the partly buried boulder $d$. Pebbles moved into the seat of boulder $c$ after it moved out. The scale is $150 \mathrm{~mm}$ (6 in.) long.

opposite to those of ridge A. No displacements exceeded $0.1 \mathrm{~m}$. A striking characteristic of the stones of ridge $B$ was a well-defined size difference between clasts that moved and those that did not. Most stones less than about $50 \mathrm{~mm}$ high, which included many tabular schistose cobbles, did not move, regardless of shape.

A 500-g laboratory spring scale was used to obtain crude measurements of coefficient of sliding friction and adhesion between stones and the surface of ridge $\mathrm{B}$. Four determinations of coefficient of friction yielded values between 0.4 and 0.7 . The adhesion of 13 quasi-rectangular, tabular cobbles lying on the flat surface of the ridge was measured by lifting one end only of each with the hook on the end of the spring scale. Each of the 13 cobbles required a force greater than half its weight to break it free from the surface when lifted in this manner. Half the weight of each cobble was determined from the scale reading after the surface adhesion was broken. Theoretically the net force required to break adhesion, divided by one-half the contact area of each cobble, equals the adhesive strength between each cobble and the surface (table 29). The measurements in table 29 show that the adhesive force may reach a large fraction of the total weight of small cobbles. If the two extreme values of table 29 are excluded, the average of the remaining 11 values of adhesive strength is $270 \mathrm{~N} / \mathrm{m}^{2}$ (roughly $0.04 \mathrm{psi}$ ). The tensile adhesive strength measured this way is assumed to be roughly the same as the shear adhesive strength; measurement of the shear adhesive strength of one flat cobble on ridge $B$ gave a value of $400 \mathrm{~N} / \mathrm{m}^{2}$, well within the range of values in table 29 .
TABLE 29. - Adhesion of cobbles to the surface of ridge $B$

\begin{tabular}{|c|c|c|c|c|c|}
\hline $\begin{array}{l}\text { Force used to } \\
\text { break cobble } \\
\text { free from } \\
\text { surface } \\
(\mathrm{g})\end{array}$ & $\begin{array}{c}\text { Weight of } \\
\text { one end of } \\
\text { cobble } \\
(\mathrm{g})\end{array}$ & $\begin{array}{l}\text { Net force } \\
\text { used to } \\
\text { overcome } \\
\text { adhesion } \\
(\mathrm{g})\end{array}$ & $\begin{array}{c}\text { Dimensions } \\
\text { of cobble } \\
(\mathrm{mm})\end{array}$ & $\begin{array}{l}\text { Contact } \\
\text { area of } \\
\text { cobble } \\
\left(\mathrm{mm}^{2}\right)\end{array}$ & $\begin{array}{l}\text { Adhesive } \\
\text { strength } \\
\left(\mathrm{N} / \mathrm{m}^{2}\right)\end{array}$ \\
\hline $\begin{array}{l}120 \\
170 \\
120 \\
120 \\
320 \\
100 \\
160 \\
140 \\
140 \\
160 \\
240 \\
320 \\
400\end{array}$ & $\begin{array}{r}110 \\
150 \\
100 \\
100 \\
280 \\
80 \\
110 \\
100 \\
70 \\
100 \\
170 \\
140 \\
200\end{array}$ & $\begin{array}{r}10 \\
20 \\
20 \\
20 \\
40 \\
20 \\
50 \\
40 \\
70 \\
60 \\
70 \\
180 \\
200\end{array}$ & $\begin{array}{r}90 \times 50 \\
80 \times 60 \\
90 \times 50 \\
70 \times 50 \\
70 \times 90 \\
50 \times 50 \\
80 \times 50 \\
70 \times 40 \\
70 \times 60 \\
80 \times 40 \\
90 \times 30 \\
80 \times 70 \\
100 \times 50\end{array}$ & $\begin{array}{l}4,500 \\
4,800 \\
4,500 \\
3,500 \\
6,300 \\
2,500 \\
4,000 \\
2,800 \\
4,200 \\
3,200 \\
2,700 \\
5,600 \\
5,000\end{array}$ & \begin{tabular}{r|}
40 \\
80 \\
90 \\
110 \\
130 \\
160 \\
250 \\
290 \\
330 \\
360 \\
500 \\
640 \\
800
\end{tabular} \\
\hline
\end{tabular}

${ }^{1}$ Lifting one end only of each cobble with a 500-g spring scale (see text). For these measurements, $100 \mathrm{~g}$ is assumed to equal $1 \mathrm{~N}$ (newton).

${ }^{2}$ Measured after cobble broken free from surface.

${ }^{3}$ Rounded to the nearest $10 \mathrm{~N} / \mathrm{m}^{2}$.

On ridge $\mathrm{A}$, most of the unrestrained material on the surface was either tipped, slid, or flung as much as $0.5 \mathrm{~m}$ either horizontally or partly downslope in a west-southwest direction. In contrast, on adjacent ridge $\mathrm{B}$ only boulders and cobbles taller than about $50 \mathrm{~mm}$ moved, and they did so by sliding as much as $100 \mathrm{~mm}$ in a direction almost opposite to that of the displaced gravel on ridge A.

These differences were observed on ridges less than $100 \mathrm{~m}$ apart. The parallel directions of displacement on the two ridges (although opposite in sense) indicate that the direction of strongest horizontal shaking here was roughly N. $70^{\circ}$ E. to S. $70^{\circ}$ W., which is $70^{\circ}-80^{\circ}$ from the general trend of the surface rupture along the Coyote Creek fault and about $25^{\circ}$ from the trend of the ridges. Whether the direction of most intense shaking and the sense of displacement are controlled primarily by the position, geometry, and composition of the ridges or by the regional orientation of seismic waves cannot be determined from these observations. Slope may have determined the sense of movement on ridge $\mathrm{A}$, but differences in the position and shape of the two ridges or differences, if any, in the material underlying them could also have been important. The available data, however, may help to reveal the magnitude of the accelerations on these ridges and to explain why the shorter clasts of ridge $B$ did not move.

\section{MECHANICS OF DISPLACEMENT INDUCED BY SHAKING}

Before atte ${ }_{11}$ pting to explain the displacements on ridges $\mathrm{A}$ and $\mathrm{B}$, we should consider the different ways in which a stone may react to the shaking of an earthquake. For simplicity, we will consider the forces acting between a rigid rectangular block and the inclined surface upon which it rests 


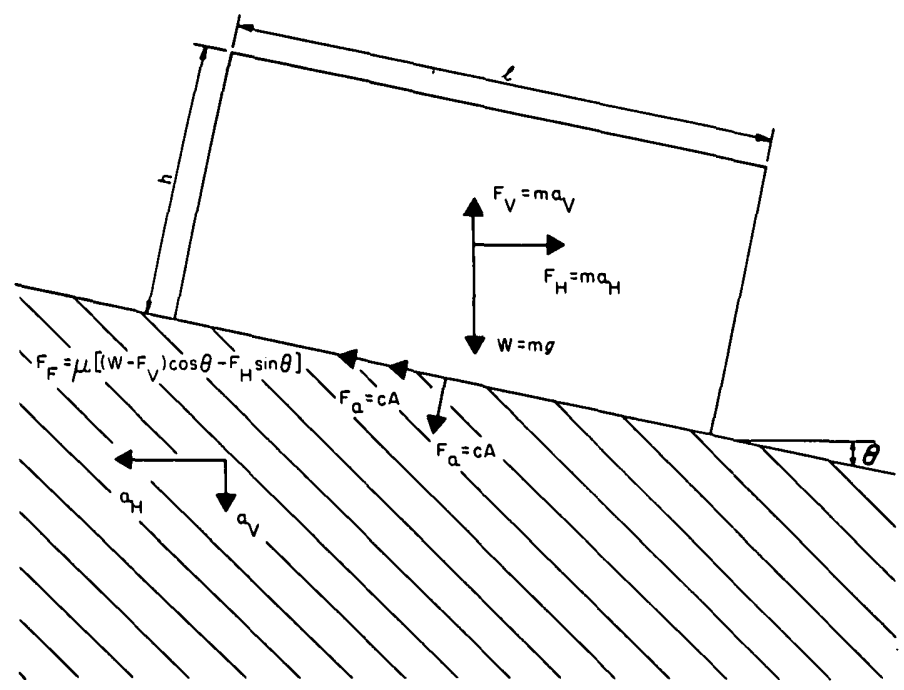

Figure 135. - Forces, $F$, acting between a block of mass, $m$, density, $\rho$, and basal area, $A$, and the inclined surface on which it rests, as a result of friction, adhesion, and shaking of the surface. $a_{1}$ and $a_{\| r}$, vertical and horizontal accelerations of shaking; $g$, gravity; $\mu$, coefficient of friction; $h$, height; $I$, length; $\theta$, inclination of surface; $c$, adhesive strength between the surface and block (assumed here to be the same for shear and tension, to be independent of weight, and to embrace all surface forces, including nonideal components of friction); $F_{1}$ and $F_{11}$, vertical and horizontal inertial reactions to shaking; $W$, weight; $F_{r}$, frictional resistance to sliding; $F_{n}$, adhesion.

(shown in fig. 135). The block will slide during shaking when the downslope components of both weight and horizontal and vertical inertial reactions to shaking equal or exceed the combined frictional and adhesive resistance to sliding:

$W \sin \theta-F_{V} \sin \theta+F_{H} \cos \theta \geqslant F_{F}+F_{a}$.

(Symbols are defined in fig. 135.) Substituting the definitions of these forces given in figure 135 gives $m\left(g-a_{v}\right) \sin \theta+m a_{1 \prime} \cos \theta \geqslant \mu\left[m\left(g-a_{v}\right) \cos \theta\right.$

$$
\left.-m a_{H} \sin \theta\right]+c A,
$$

which reduces to

$a_{I \prime} \cos \theta+\left(g-a_{v}\right) \sin \theta \geqslant \mu\left[\left(g-a_{v}\right) \cos \theta\right.$

$$
\left.-a_{H} \sin \theta\right]+\frac{c}{\rho h} .
$$

The critical horizontal acceleration, $a_{H c}$, necessary for sliding is then

$$
a_{H c} \geqslant \frac{\left(g-a_{v}\right)(\mu-\tan \theta)+c / h \rho \cos \theta}{1+\mu \tan \theta} .
$$

When vertical acceleration is upward rather than downward or when horizontal acceleration acts out from rather than into the slope, sliding will be more difficult. It is assumed for this simple analysis that tipping moments do not change overall frictional resistance of the block.

In the absence of adhesion $(c=0)$, the acceleration that initiates sliding, $a_{H c}$, depends only on $g$, $\mu$, $\theta$, and $a_{V}$ and is in no way affected by shape or size of the block, unless the block tips or is rocking. (See succeeding paragraphs.) Thus rectangular blocks of any size or shape on a slope will start moving at the same time when the critical horizontal acceleration is reached. It is important to remember that like inertial forces, frictional resistance of a block to sliding is proportional to the weight of the block rather than to its contact area, as is sometimes implied (for example, Richter, 1968, p. 56). Vertical accelerations are not likely to change drastically the values of $a_{\| l c}$. If maximum vertical accelerations range from about one-third to two-thirds as large as the values of associated horizontal accelerations (Housner, 1970, p. 79), $a_{H c}$ decreases little with increasing $a_{v}$, particularly with lower values of $\mu$ (fig. 136). Even the extreme example of $a_{V}=a_{H}$ reduces $a_{H c}$ by only 35 percent, if $\mu=0.5$. However, an increase in the slope, $\theta$, of the surface may greatly reduce the value of $a_{H c}$ (fig. 137). For $\mu=0.5$, an increase in slope from $0^{\circ}-10^{\circ}$ lowers $a_{H c}$ more than does an increase of $a_{v}$ from 0 to $2 / 3 a_{t}$ (fig. 136).

It is also important to note that rocking probably allows sliding in some situations in which sliding would otherwise not occur. Variations in the period,

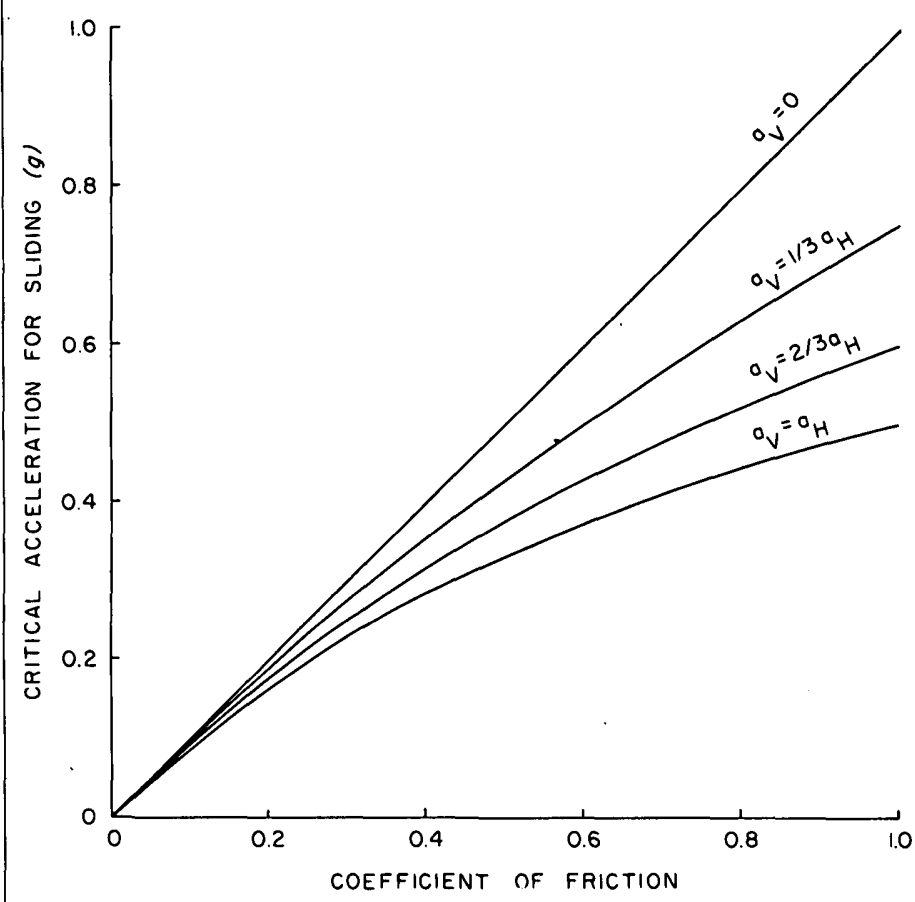

FIGURE 136. - Relation between coefficient of friction, $\mu$, and minimum horizontal acceleration, $a_{t r}$, necessary to slide a block that rests on a horizontal plane, for different values of associated upward vertical acceleration, $a_{1}$. From equation 4 , with $\theta=0$ and $c=0$. Adhesion between the block and the surface on which it rests will always raise the value of the horizontal acceleration required for sliding. 


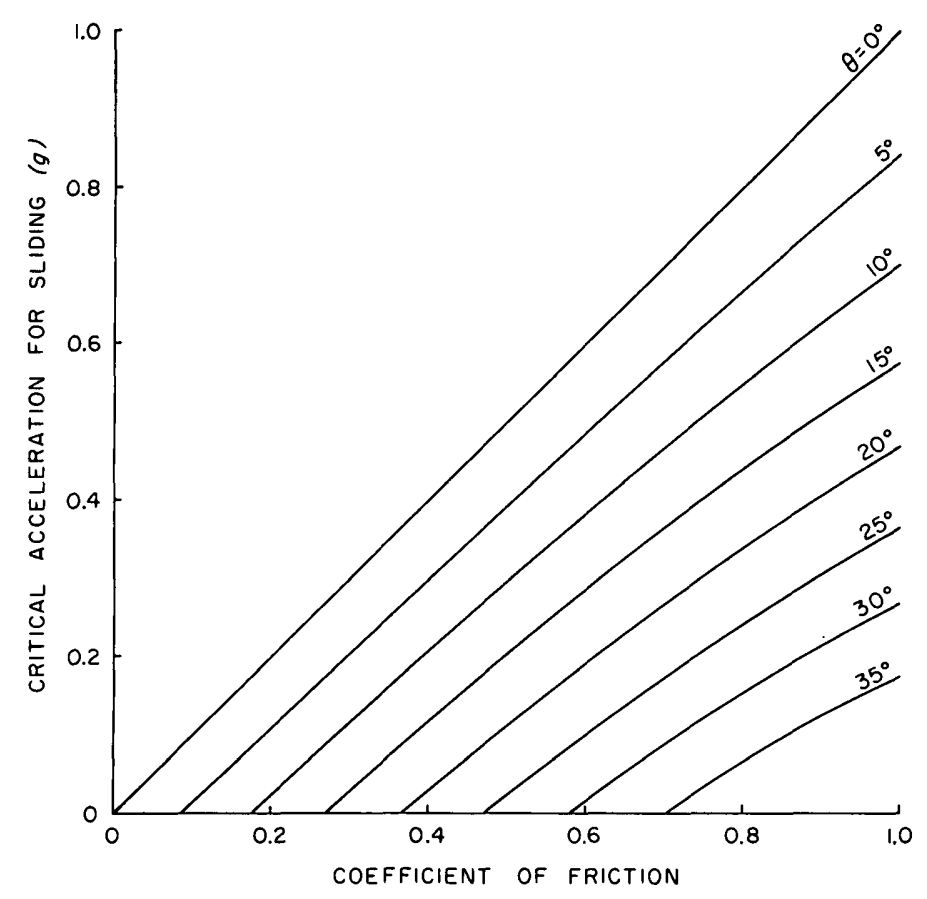

FIGURE 137.- Relation between coefficient of friction, $\mu$, and the minimum horizontal acceleration, $a_{H c}$, necessary to slide a block that rests on an inclined plane, for different values of inclination, $\theta$. From equation 4 , with vertical acceleration of shaking and adhesion assumed to be zero. Inclusion of positive values for $a_{V}$ will depress the righthand part of each curve an amount proportional to and smaller than the depression of the corresponding curves in figure 136.

magnitude, and direction of shaking are likely to yield ground movements that can slide a rocking block at vulnerable positions in its cycle of movement.

If either $a_{H}$ or $a_{V}$ exceeds $1 g$, the block will probably be propelled from its seat. Oldham, however, pointed out $(1897$, p. 133$)$ that there must be an upper limit to the size of boulders that can be lifted by the vertical component of shaking, which is determined by the strength of the surface on which they rest. Because the ratio of mass to contact area increases as size increases, boulders larger than some critical size will simply deform the surface beneath them as it shakes.

Adhesion may drastically change the behavior of shorter blocks. If adhesion is present, $a_{H c}$ increases, and the size term, $h$, becomes important in equation 4. Thus a lower limit, $h$, for the height of rectangular blocks that will slide exists for every value of $a_{H o}$ greater than the value in the absence of adhesion. This limit exists because adhesive resistance to sliding varies with contact area rather than with mass and is proportionately larger for shorter blocks than are inertial forces. Thus when $c>0$, the ratio of surface forces, $F_{a}$, to inertial forces increases for progressively shorter rectangular blocks until surface forces become dominant. As $a_{H c}$ increases in equation 4 , the critical height, $h$, that separates blocks that move from those that do not, decreases.

The block may also respond to shaking by tipping or rocking. For tipping or rocking to occur, the block must be tall enough that the overturning moment about an edge of the block exceeds the resisting moment (fig. 135), or

$$
\begin{aligned}
\frac{h}{2}-\left[F_{H} \cos \theta+\left(W-F_{V}\right) \sin \theta\right] & +\frac{l}{2} F_{H} \sin \theta \\
& \geq \frac{l}{2}\left(W-F_{V}\right) \cos \theta+c A .
\end{aligned}
$$

The critical horizontal acceleration for tipping, then, is

$$
a_{H c} \geqslant \frac{\left(g-a_{V}\right)[(l / h)-\tan \theta]+(l / h)(c / h \rho \cos \theta)}{1-(l / h) \tan \theta} \text {. }
$$

Generally, stones tip if they are partly buried in the surface or if they are somewhat taller than they are wide, so that the critical acceleration for tipping is less than that for sliding.

In the absence of adhesion $(c=0)$, initial tipping of stones is not controlled by size but only by shape $(l / h)$, slope, and the accelerations due to shaking. That is, all objects of a certain shape on a given slope will tip at some critical acceleration, regardless of their size. Rocking of an object during shaking, however, is controlled by size. The larger the object of a given shape the longer is its natural period of rocking. Hence, those objects whose natural rocking period is close to the period of strong shaking may be overturned or translated, while others of similar shape but different size may not be so affected. This size-controlled response manifests itself only after tipping starts; it has no effect if the acceleration is too low to initiate tipping or rocking.

If the block adheres to the surface, however, a larger horizontal acceleration is required to tip it, and size again becomes important. Depending on the magnitude of adhesion, all blocks of a given shape that are taller than some critical height, $h$, will tip at some level of acceleration greater than that necessary to tip the blocks in the absence of adhesion. Thus, if rocking occurs or if adhesion is present, a size effect, in addition to a shape effect $(h / l)$, will control which blocks tip and which do not.

Thus in the absence of adhesion, simple sliding of a rectangular block depends only on slope, coefficient of friction, and acceleration and is independent of size and shape. Tipping depends only on slope, shape, and acceleration. Size of the block affects sliding or tipping only if adhesion or other surface 
forces are present. Once rocking begins, size also controls its period.

\section{MECHANICS OF SHAKING APPLIED TO OBSERVATIONS AT OCOTILLO BADLANDS}

The preceding analysis treats very simple situations. The stones observed at Ocotillo Badlands, however, show great differences in shape and size; few are rectangular blocks, and some are embedded in the surface. Although coefficients of friction probably are fairly uniform, adhesion shows greater variation. Richter $(1968$, p. 140$)$ pointed out that even arrays of columns of different sizes and shapes designed to determine earthquake motion commonly yield ambiguous information, presumably because of rocking and complex shaking. Nevertheless, the observations of Ocotillo Badlands do permit estimates of the probable ranges of acceleration.

The evidence of flinging and large displacements of stones of all sizes of ridge $\mathrm{A}$, including many resting on slopes of less than $5^{\circ}$, almost certainly indicates either that horizontal accelerations exceeded $0.5 \mathrm{~g}$ and perhaps approached $1 \mathrm{~g}$ or that significant vertical accelerations accompanied somewhat smaller horizontal accelerations, or both. However, because many boulders that were partly buried in the unconsolidated surface material showed no evidence of vertical displacement, vertical accelerations were definitely less than $1 \mathrm{~g}$. Slope may have strongly influenced the sense, if not direction, of displacement and probably contributed to some of the larger downslope displacements. Probably some of the boulders tipped over or slid only because they rested on slopes or because their bases sloped. However, the fact that virtually every unrestrained stone on the surface moved, including many whose bases were originally horizontal, strongly suggests that large accelerations, rather than slope, were primarily responsible for movement.

On ridge $B$, smaller displacements and the fact that small stones did not move indicate that accelerations were smaller and that size was important. Either shaking produced rocking on ridge $\mathrm{B}$ or adhesion prevented the smaller stones from moving.

Rocking of stones on ridge $B$ is almost certainly ruled out, because clasts that were displaced have the same shapes as clasts that remained stationary. Furthermore, on ridge $\mathrm{B}$, a block about $0.25 \mathrm{~m}$ high by $0.25 \mathrm{~m}$ wide by $0.4 \mathrm{~m}$ long slid horizontally $50 \mathrm{~mm}$ parallel to the $0.4 \mathrm{~m}$ length. The most reasonable combinations of horizontal and vertical accelerations sufficient to rock this relatively low block parallel to its long dimension and thus to permit it to slide would have been large enough to move virtually every stone on this ridge. Because many did not move, including cobbles with more favorable ratios of height to length, it seems very unlikely that differences in the natural period of rocking caused the size difference observed between those stones that were and were not displaced.

Adhesion to the underlying surface very probably prevented the shorter stones on ridge $B$ from sliding. This conclusion is supported both by the observed height rather than shape difference between stones that did and did not slide and by the values of adhesion measured on ridge $B$ (table 29). Figure 138 shows that these values are compatible with reasonable levels of excess acceleration over that necessary in the absence of adhesion. For example, if $\mu=0.5$ and $a_{v}=0$ in the absence of adhesion, $a_{H c}=0.5^{\prime} g$ for all stones on a flat surface (fig. 136). If adhesion, however, is $270 \mathrm{~N} / \mathrm{m}^{2}$ (the average of the measured values), an excess acceleration of $0.2 \mathrm{~g}\left(2 \mathrm{~m} / \mathrm{sec}^{2}\right.$; see fig. 138), or $a_{H c}=0.7 \mathrm{~g}$, will be required to move all stones taller than $50 \mathrm{~mm}$ (fig. 138). In other words, with this value of adhesion, only stones taller than $50 \mathrm{~mm}$ will be sliding when $a_{H}$ reaches $0.7 \mathrm{~g}$. These numbers indicate that reasonable values of acceleration can account for the observations on

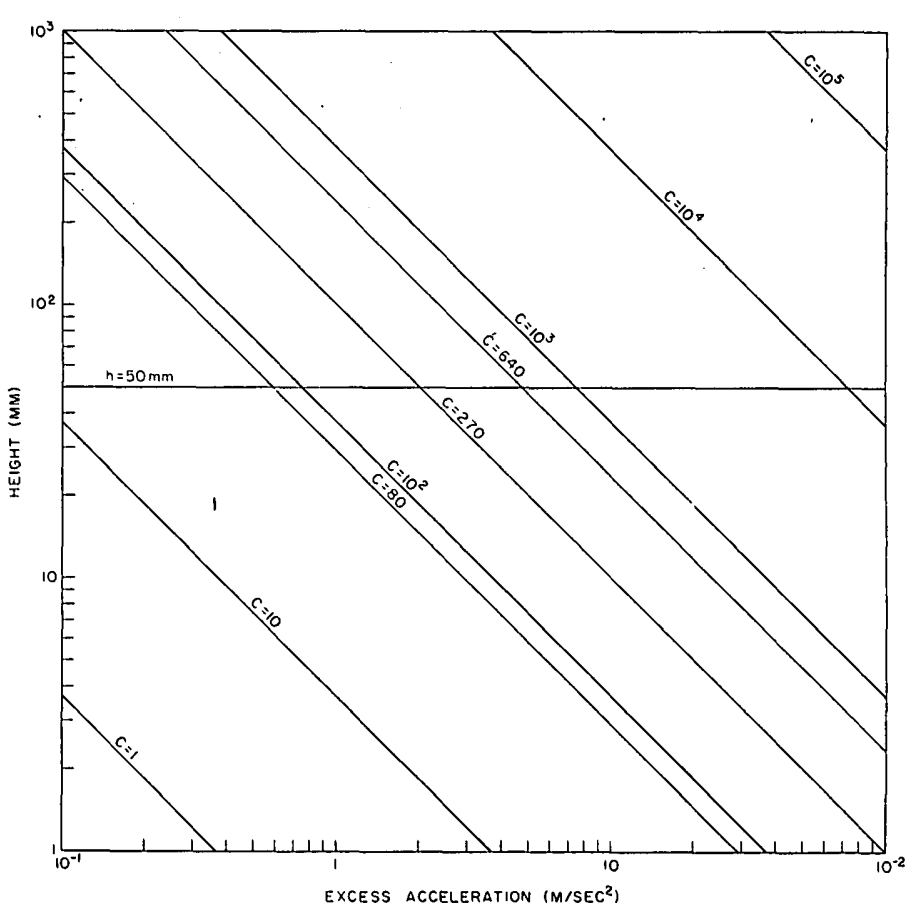

FIGURE 138. - Relation between height, $h$ (separating blocks that slide on a horizontal surface from those that do not), and excess acceleration, $a_{H c}-\mu\left(g-a_{v}\right)$ (excess over that necessary to slide blocks in the absence of adhesion), between blocks and the surface on which they rest for different values of adhesive strength, $c,\left(\mathrm{~N} / \mathrm{m}^{2}\right)$. From equation 4 , with $\theta=0$ and $\rho=2.7 \times 10^{3} \mathrm{~kg} / \mathrm{m}^{3}$; other symbols as defined in figure 135. 
ridge $\mathrm{B}$. Moreover, figure 138 shows that reasonable local variations in $\mu, c, a_{H}$, and $a_{V}$ do not drastically change the value of $h$ that separates stones that will and will not move.

A determination of the likely values of acceleration on ridge $B$ requires an estimate of both the value of coefficient of friction and the magnitude of the vertical component of acceleration, $a_{v}$, in addition to the value of adhesion. By using the range of measured coefficients of friction, 0.4-0.7, and by assuming that maximum vertical accelerations were one-third to two-thirds the magnitude of horizontal accelerations, figure 136 yields values of $a_{H}$ ranging from about 0.3 to $0.7 \mathrm{~g}$. If, in addition, we include the excess acceleration of about $0.2 \mathrm{~g}$, indicated by the preceding consideration of adhesion, the final estimate of horizontal acceleration on ridge $B$ lies between 0.5 and $0.9 \mathrm{~g}$.

It is worth noting that if values of adhesion on ridge $A$ were similar to those measured on ridge $B$, the fact that virtually all stones moved increases the likelihood that $a_{H}$ was close to $1 g$ on ridge $\mathrm{A}$.

In summary, the observations of displaced stones of ridges $\mathrm{A}$ and $\mathrm{B}$ indicate that on ridge $\mathrm{A}$ horizontal accelerations were probably greater than $0.5 g$ and perhaps as high as $1 g$, whereas vertical accelerations were definitely less than $1 \mathrm{~g}$. Ridge B was subjected to somewhat lower horizontal accelerations, probably in the range of $0.5-0.9 \mathrm{~g}$. Stones were displaced in the same direction, $70^{\circ}-80^{\circ}$ to the trend of the main surface rupture zone, although in opposite sense, on these two ridges.

The study also demonstrates that higher parts of the ridges experienced greater shaking than the lower slopes of the same ridges, other ridges, low hills, and flat alluvium. Studies elsewhere indicate that surface shaking generally increases with greater depth of unconsolidated material (for example, Borcherdt, 1970; McCulloch and Bonilla, 1970, p. 69-72). Observations at Ocotillo Badlands, supported by more recent findings at San Fernando (Nason, 1971), indicate that $100 \mathrm{~m}$ of topographic relief in poorly consolidated sediments may also amplify shaking.

\section{REFERENCES CITED}

Borcherdt, R. D., 1970, Effects of local geology on ground motion near San Francisco Bay: Seismol. Soc. America Bull., v. 60 , p. 29-61.

Housner, G. W., 1970, Strong ground motion, chap. 4, in Wiegel, R. L., ed., Earthquake engineering: PrenticeHall, p. 75-91.

McCulloch, D. S., and Bonilla, M. G., 1970, Effects of the earthquake of March 27, 1964, on The Alaska Railroad: U.S. Geol. Survey Prof. Paper 545-D, p. D1-D161.

Nason, R. D., 1971, Shattered earth at Wallaby Street, Sylmar, in The San Fernando, California, earthquake of February 9, 1971: U.S. Geol. Survey Prof. Paper 733, p. 97-98.

Oldham, R. D., 1897, Report on the great earthquake of 12 th June, 1897: Geol. Survey India Mem., v. 29, 379 p.

Richter, C. F., 1958, Elementary seismology: San Francisco, W. H. Freeman and Co., $768 \mathrm{p}$. 


\title{
WATER-RESOURCE EFFECTS
}

\author{
By A. O. Waananen and W. R. Moyle, JR. \\ U.S. Geological SURveY
}

\begin{abstract}
The principal effect of the Borrego Mountain earthquake on water resources was seismic in nature and almost entirely transient, as shown by the charts from recorders on groundwater observation wells in California, Arizona, and Nevada, and at gaging stations in California. Hydroseisms were recorded at several sites nearly $500 \mathrm{~km}$ from the epicenter. At most sites, the effects were limited to water-level or water-surface fluctuations, with some minor and insignificant net changes in water level at many wells; changes in streamflow were observed at only two sites. Hydroseisms were observed also in many swimming pools near the epicenter, in San Diego County, and near Los Angeles. The effects on water quality were minor, and no significant problems were reported.
\end{abstract}

\section{INTRODUCTION}

Seismic shocks associated with the Borrego Mountain earthquake of April 9, 1968, ${ }^{1}$ caused noticeable fluctuations in ground-water levels, and in the stages of streams, canals, and lakes, over a wide area extending nearly $500 \mathrm{~km}$ from the epicenter near Borrego Mountain, about $27 \mathrm{~km}$ southwest of the Salton Sea. Fluctuations were caused also by a few of the aftershocks that were associated with the Borrego Mountain earthquake (centered near the town of Ocotillo Wells). Effects from the M (magnitude) 6.4 principal shock occurring at $6: 29$ p.m. (P.s.t.) April $8(02: 28: 59.1$ G.m.t. April 9) were noted on the records from more than 120 water-stage recorders; the effects of two aftershocks (M 5.2 and M 4.7) were recorded at a few stations.

The effects of the earthquake on water resources can be classified in four main groups: (1) Waterlevel fluctuations in wells, (2) temporary changes in ground-water levels, (3) changes in spring flow or in the base flow of streams, and (4) variations in the magnitude of surface-water fluctuations along channels caused by variations in channel geometry. Some of these effects occurred immediately after the three principal shocks, but others, such as changes in the

${ }^{1}$ Greenwich mean time (G.m.t.). Local time was April 8, 1968. Pacific standard time (P.s.t.) used for time reference in this discussion. flow of streams, developed slowly over a period of days and were sustained for several days or weeks. No permanent changes in water level or discharge are known. A noticeable but temporary increase in sediment content of the water was observed in one well as a result of the Borrego Mountain earthquake.

Selected terms will be used in the subsequent discussions, as follows:

"Hydroseism" refers to all seismically induced waterlevel fluctuations other than tsunamis (tidal waves). The term, as defined by Vorhis (1967, p. C2), pertains to fluctuations of water levels in wells, streams, lakes, ponds, and reservoirs.

"Hydroseismic data" refers to both the charts that record hydroseisms and the information taken from the charts.

"Seiche" is a term applied to standing waves set up on lakes and similar closed bodies of water by wind, change in barometric pressure, and other factors. Seiches caused by earthquakes are termed "seismic seiches." A special form of hydroseism, they are characterized by the symmetrical fluctuations (equal water-level rises and declines) set up on lakes, reservoirs, and ponds at times corresponding to the passage of seismic waves from an earthquake.

\section{FLUCTUATIONS IN GROUND-WATER LEVELS}

The principal transient effect of the April 8, 1968, earthquakes on the water resources was the abrupt and pronounced fluctuation of water level in response to the seismic shock, as shown by the records from many water-stage recorders. These hydroseisms commonly are shown by analog-type recorders in areas affected by earthquakes. At times of large shocks, the recorder traces show vertical lines caused by movement of the recorder float as a result of wave action in the gage well or observation well induced by ground motion associated with the seismic shock. There is a rough relation of the amplitude of the fluctuations to the severity of the shock, the distance 


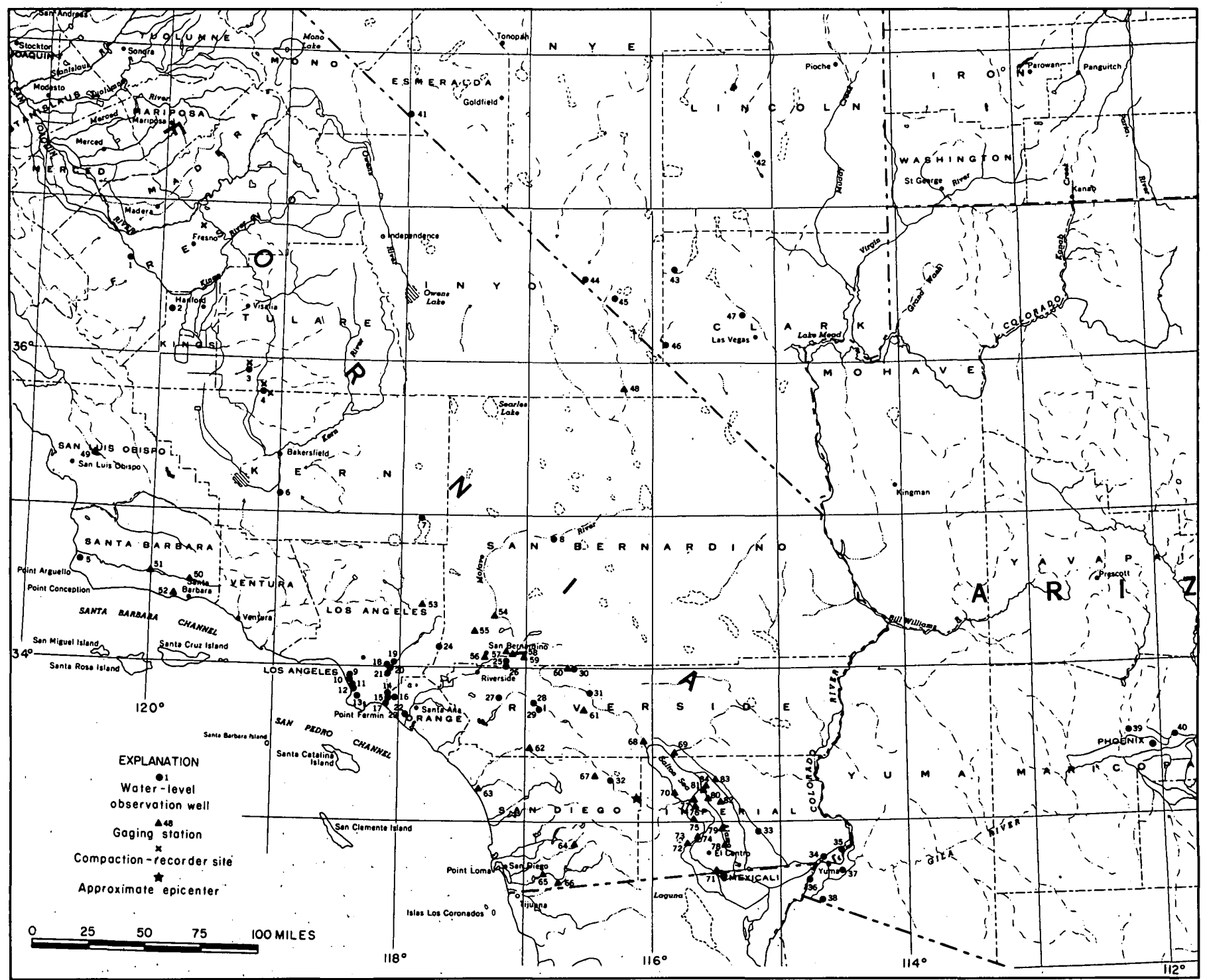

FIgURE 139. - Selected observation wells, gaging stations, and compaction-recorder sites that showed effects of the Borrego Mountain earthquake. Observation wells and gaging stations are given in tables 30 and 31, respectively.

from the epicenter, and the kind of underlying soil and rock formations. The records for several observation wells also demonstrate some postearthquake short-term changes in water levels as a result of the shocks.

The greatest areal extent of the hydroseismic effects was shown by water-level recorders on ground-water : observation wells in California, Arizona, and Nevada, and by compaction recorders in California. These recorders were operated by the Geological Survey and by state, county, and local agencies. The location of the recorders that showed effects of the Borrego Mountain earthquake is shown in figure 139. Three sites in Fresno and Kings Counties in California (sites 1,2, and the compaction- recorder site near Fresno) and one site in Esmeralda County in Nevada ( site 41 near the California border $84 \mathrm{~km}$ southwest of Tonopah) are nearly $500 \mathrm{~km}$ northwest of the epicenter.

Hydroseismic data for the selected wells shown in figure 139 (sites 1-47) are given in table 30 . The wells listed are identified by both a site number and a well number. The well numbers indicate the location of wells according to the rectangular system for the subdivision of public lands. The site numbers identify the township, range, section, location within the section, serial number of the well in the section, and the baseline and meridian in accord with the standard practices in California, Arizona, and $\mathrm{Ne}$ vada. 
TABLE 30.-Fluctuations of water level in observation wells, April 8, 1968

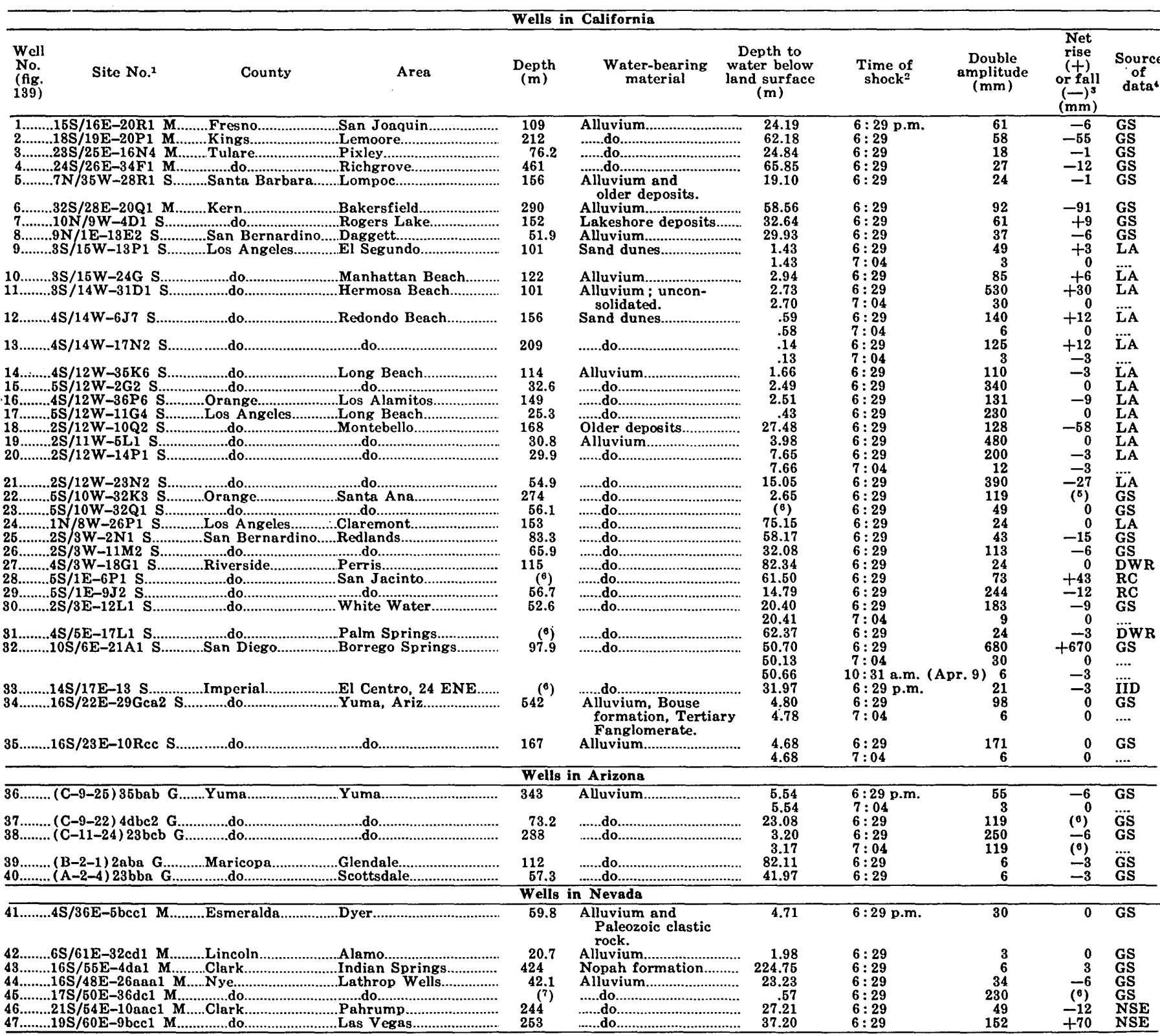

'Location referred to baseline and meridian as follows: M, Mount Diablo:

S, San Bernardino; G. Gila and Salt River.
Time of shock in Pacific standard time (P.s.t.) ; add 8 hours for Greenwich mean time (G.m.t.).

A Approximate water-level offset before and after shock.

Agencies as follows: GS, U.S. Geological Survey ; LA, Los Angeles County Flood Control District; DWR, California Department of Water Resources ; District; NSE, Nevada State Engineer.

A water-level rise of $0.67 \mathrm{~m}$ in well $10 \mathrm{~S} / 6 \mathrm{E}-21 \mathrm{~A} 1$ $S$ near Borrego Springs (site 32) was the greatest change observed; most water-level changes did not exceed $0.09 \mathrm{~m}$. The fluctuations reflected principally the response to the main Borrego Mountain earthquake shock at $6: 29$ p.m. (P.s.t.) April 8. The records for several observation wells indicated movement in response to the $M 5.2$ shock that occurred about 35 minutes after the principal shock. The well

'Not recorded ; pen off chart.

Not known. 'Recorder in Devil's Hole, a spring in cavernous limestone; exploration by
scuba divers indicates depths greater than $60 \mathrm{~m}$ and diameter more than
$2 \mathrm{~m}$.

near Borrego Springs (site 32) responded also to the M 4.7 shock at 10:31 a.m. (P.s.t.) April 9.

Examples of direct responses to the seismic shocks are illustrated in figures 140 and 141. The hydroseisms shown are representative of the more pronounced effects observed, including the range in the fluctuations and the changes in water levels. Most of the records available, however, indicate only shortterm fluctuations at the time of the principal shock, 


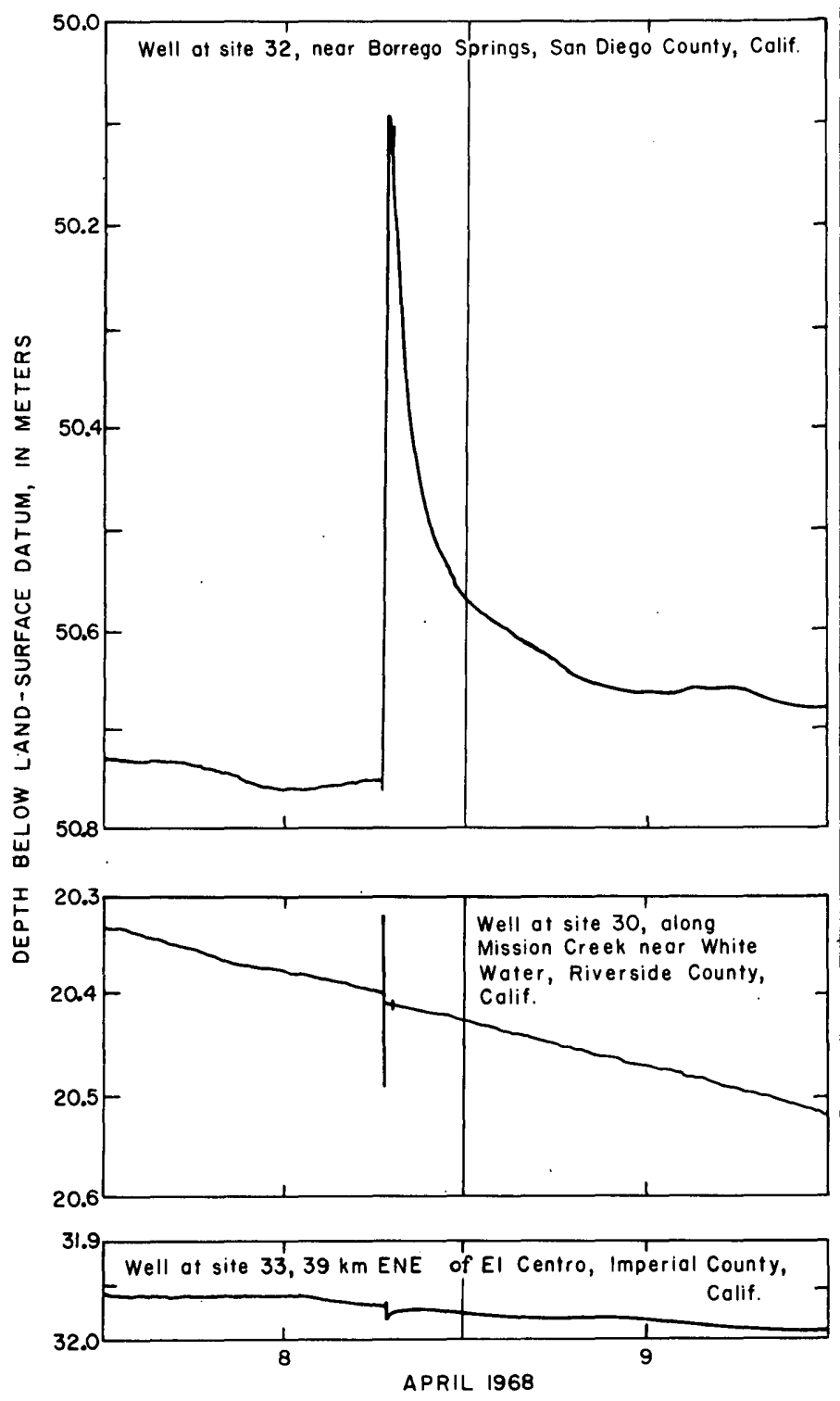

FIgURE 140. - Fluctuations recorded at observation wells in the Salton Sea basin during and after the Borrego Mountain earthquake. Time is Pacific standard time.

and a few demonstrate the response to one or more aftershocks.

Parts of the recorder charts for three observation wells in the Salton Sea basin (sites 32, 30, and 33) are shown in figure 140, and those for three observation wells in central and southern California outside of the Salton Sea basin (sites 21,.11, and 6) are shown in figure 141. The well at site 32 near Borrego Springs, $23 \mathrm{~km}$ northwest of the epicenter of the principal shock, was the nearest well observed. For several hours prior to the earthquake, the water level in the well showed a small decline and a slight rise. Immediately after the main shock, the water level started to rise rapidly; it rose $0.67 \mathrm{~m}$ in $15 \mathrm{~min}$ -
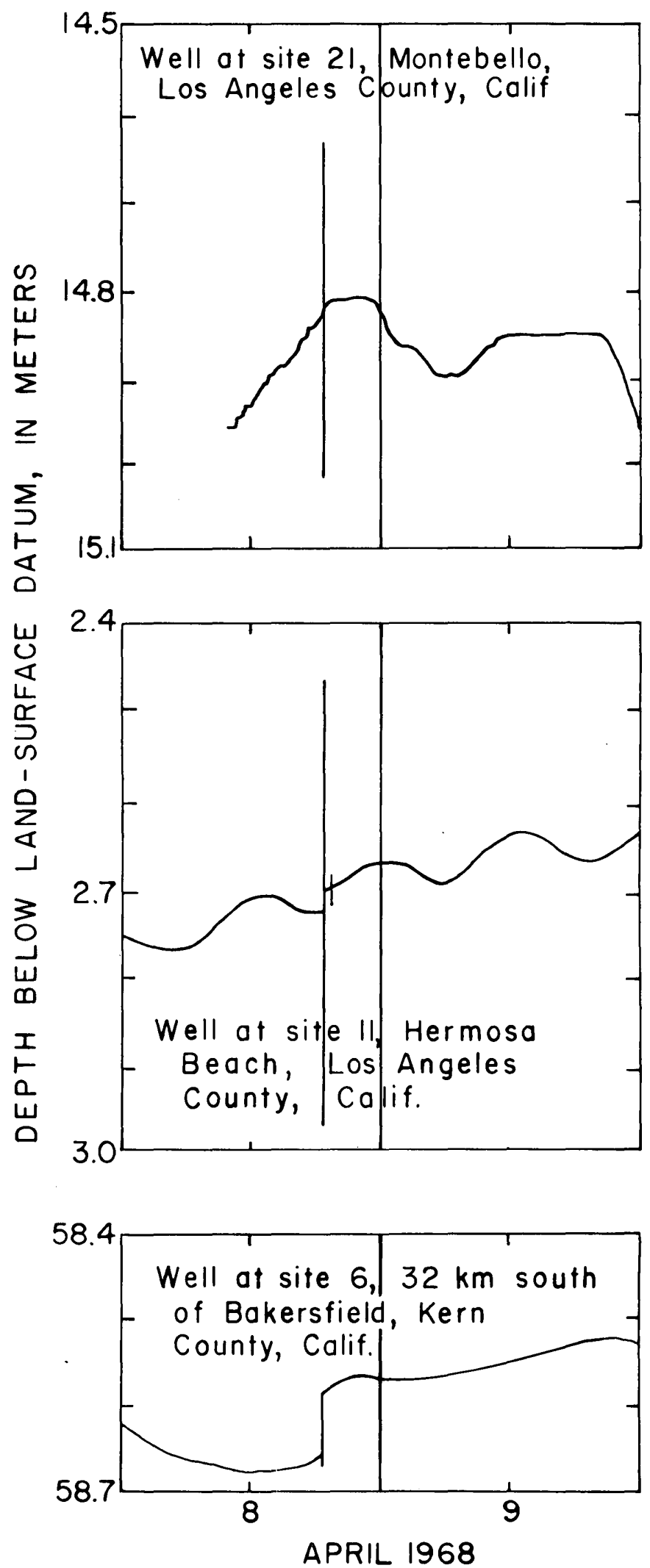

FIGURE 141. - Fluctuations recorded at observation wells in central and southern California during and after the Borrego Mountain earthquake. Time is Pacific standard time. 
utes and then declined in 18 hours to a stable level about $0.09 \mathrm{~m}$ higher than that before the shock. The rapid water-level rise of $0.67 \mathrm{~m}$ may have been caused by compaction or compression of the aquifer, which resulted in the upward movement of water. As the aquifer pressures returned to equilibrium, the water level declined very gradually for several days after April 9, and the $0.09-\mathrm{m}$ residual change subsequently disappeared. The record for this well also showed a minor response to the seismic shock at 10:31 a.m. (P.s.t.) April 9 (fig. 140).

The record for the Mission Creek well (site 30, fig. 140) indicates multiple water-level fluctuations at the time of the principal shock at 6:29 p.m. (P.s.t.) April 8 and for a short period after this shock. This multiple response possibly is the result of the aftershocks at $6: 33$ and $6: 37$ p.m. April 8 . This well also responded to the $M 5.2$ shock at 7:04 p.m.

Compaction recorders in Tulare and Kern Counties $50-60 \mathrm{~km}$ north of Bakersfield and one in Fresno County $16 \mathrm{~km}$ northeast of Fresno (fig. 139) showed movement resulting from ground motion at the time of the principal seismic shock on April 8. Compaction recorders, as described by Lofgren and Klausing (1969, p. B69), are special installations of recorders connected to heavy weights emplaced in the underlying formations. These instruments record changes in thickness of particular sequences in water-bearing deposits as the water table declines. The amplitude of the fluctuations recorded on April 8 was small, but the time of the movement indicated that they resulted from the earthquake.

The magnitude of the hydroseisms, as shown in table 30 , demonstrates local differences in the responses of water levels to the effects of seismic shocks. These differences reflect variations in well construction, distances from the epicenter, and geological influences. Deep wells that have adequate perforations, for example, may have fluctuations of greater magnitude than shallow wells. The 274-m deep well at site 22 in Orange County $8 \mathrm{~km}$ southwest of Santa Ana had a fluctuation of $119 \mathrm{~mm}$, while an adjacent well (site 23), $56 \mathrm{~m}$ deep, had a fluctuation of $49 \mathrm{~mm}$. The water level in this area was less than $3 \mathrm{~m}$ below the land surface. Wells in Los Angeles County in the Montebello area, along the coast near Santa Monica Bay southwest of Los Angeles, and in the Long Beach area south of Los Angeles had fluctuations in water level with amplitudes as great as $530 \mathrm{~mm}$ (site 11, at Hermosa Beach); the net changes, however, were small, less than $60 \mathrm{~mm}$.

In an earlier study of the effects of earthquakes on water levels, La Rocque (1941) reported that during the Long Beach, Calif., earthquake of March 10, 1933, the water-level fluctuation in one well (4S/ $12 \mathrm{~W}-28 \mathrm{H} 2 \mathrm{~S}$ ) reached an amplitude of $3.23 \mathrm{~m}$ with a net rise of $2.51 \mathrm{~m}$. This well is located in Long Beach just east of Signal Hill; the epicenter of the earthquake was only $27 \mathrm{~km}$ southeast of Signal Hill. La Rocque reported also that lines of equal surge, sketched on the basis of recorded data, indicated the occurrence of surges greater than $3 \mathrm{~m}$ along a northwest-southeast trending band about $8 \mathrm{~km}$ long just east of Signal Hill. This area is closed to the so-called Inglewood-Newport fault zone. The underlying unconsolidated deposits contain thick impervious beds, and water in the deepest aquifers is confined under a hydrostatic head as great as $460 \mathrm{~m}$. The pattern of the lines of water-level surge suggested, as noted by La Rocque, that the magnitude of the surge may have been caused to a considerable degree by the thickness, perviousness, and elasticity of the waterbearing zones and by the effectiveness of the confining beds.

\section{SURFACE-WATER FLUCTUATIONS AND TURBIDITY}

Seismic effects were shown by water-stage recorders at many gaging stations on streams in the Salton Sea basin and in basins in central and southern California. The effects, which were generated largely by wave motion induced in the stilling wells by ground motion associated with the seismic shock, caused movement of the recorder floats and consequent vertical tracings on the recorder graphs. This recorded rise and fall of the water surface constitutes an approximate measure of the severity of the earth shock.

Two distinct events were recorded at some gaging stations on April 8, one corresponding to the principal shock at $6: 29$ p.m., and the second about 35 minutes later. The second event was also recorded in many ground-water observation wells.

The location of selected gaging stations that showed the shock effects is shown in figure 139 (sites 48-84). Pertinent data on the range of the fluctuations and the net change in stage are given in table 31. These data demonstrate the area affected by the earthquake. Most of the recorder traces showed only a direct response to the shock or aftershocks, with no sustained changes in stage or flow.

Parts of the recorder charts from five selected stations that show the fluctuations in response to the principal shock and the secondary event are reproduced in figure 142. The record for Salt Creek near Mecca (site 69) consists of water-surface oscillations that continued for several minutes; this response is 
TABLE 31.-Fluctuations and net changes in water level recorded at gaging stations in California, April 8, 1968

\begin{tabular}{|c|c|c|c|}
\hline \multirow{2}{*}{$\begin{array}{l}\text { Stream, reservoir, or } \\
\text { canal, and station }\end{array}$} & \multicolumn{2}{|c|}{$\begin{array}{c}\text { Fluctuations in } \\
\text { gage well (double } \\
\text { amplitude, in mm) }\end{array}$} & \multirow{2}{*}{$\begin{array}{c}\text { Net } \\
\text { changes } \\
\text { rise }(+) \\
\text { or } \\
\text { fall }(-) \\
(\mathrm{mm})\end{array}$} \\
\hline & $\begin{array}{l}6: 29 \\
\text { p.m. }\end{array}$ & $\begin{array}{l}7: 04 \\
\text { p.m. }\end{array}$ & \\
\hline 48.......Amargosa River at Tecopa...... & 3 & ......... & 0 \\
\hline 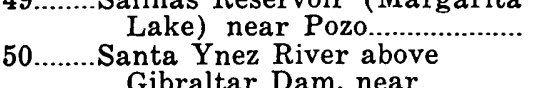 & 121 & ...... & 0 \\
\hline 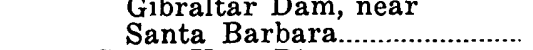 & 12 & ......... & 0 \\
\hline 51........Santa Ynez River near & • & & \\
\hline 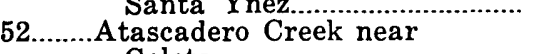 & 6 & 然. & 0 \\
\hline Goleta.......................... & 6 & .......... & 0 \\
\hline $\begin{array}{l}\text { Valyermo } \\
\text { 54.... West Fork Mojave River }\end{array}$ & 12 & $\ldots$ & 0 \\
\hline $\begin{array}{l}\text { near Hesperia...................... } \\
55 \text {. Pine Creek near }\end{array}$ & 6 & ..... & 0 \\
\hline 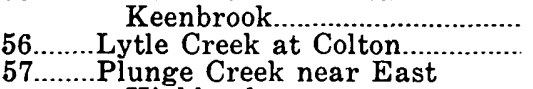 & $\begin{array}{l}30 \\
70\end{array}$ & (n...... & $\begin{array}{r}0 \\
-3\end{array}$ \\
\hline $\begin{array}{l}\text { Highlands........................... } \\
\text { 58..... }\end{array}$ & 37 & ........ & 0 \\
\hline grounds near Mentone & 58 & ......... & 0 \\
\hline $\begin{array}{l}\text { Mill Creek power c } \\
\text { near Yucaipa }{ }^{2} \ldots\end{array}$ & 18 & ... & 0 \\
\hline $\begin{array}{l}\text { 60........Mission Creek near Desert } \\
\text { Hot Springs. }\end{array}$ & 15 & & -3 \\
\hline 61......Palm Canyon Creek near & & & \\
\hline $\begin{array}{l}\text { 62......Temecula Creek at Vail Dam. } \\
63 \ldots . . . \text { San Luis Rey River at }\end{array}$ & 27 & (........ & 0 \\
\hline 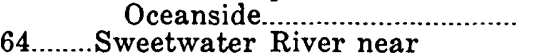 & 6 & ......... & 0 \\
\hline Descanso............ & 6 & … & 0 \\
\hline $\begin{array}{l}65 \ldots . . . \text { Jamul Creek near Jamul........ } \\
66 \ldots \ldots . . \text { Cottonwood Creek above }\end{array}$ & 30 & .......... & 0 \\
\hline $\begin{array}{l}\text { Tecate Creek, near Delzura. } \\
\text { 67....... Borrego Palm Creek near }\end{array}$ & 21 & ......... & 0 \\
\hline Borrego Springs............ & 6 & ․ & $\left({ }^{3}\right)$ \\
\hline 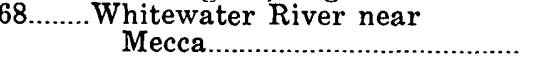 & 214 & & $4-82$ \\
\hline 69........Salt Creek near Mecca.. & 70 & 9 & +3 \\
\hline $\begin{array}{l}\text { 70........ Salton Sea near } \\
\text { Westmorland... }\end{array}$ & 250 & & -9 \\
\hline 71...... New River at $\mathrm{Me}$ & & 21 & 0 \\
\hline Boundary ${ }^{5}$ & 95 & & \\
\hline 72........West Side Main canal at & 17 & 12 & \\
\hline 73....... Dixie Pond automatic spill & & & \\
\hline gate 5 & 6610 & ......... & +6 \\
\hline
\end{tabular}

representative of gaging stations in the Salton Sea basin.

Change in streamflow, a significant and common response of the water regime to earthquakes, is indicated by the records for Borrego Palm Creek near Borrego Springs (site 67) and Whitewater River near Mecca (site 68). Prior to the earthquake, the flow in Borrego Palm Creek, after a rise April 2 to $0.04 \mathrm{~m}^{3} / \mathrm{sec}$ (cubic meters per second) in response to $21 \mathrm{~mm}$ of precipitation, had declined by April 8 to $0.006 \mathrm{~m}^{3} / \mathrm{sec}$. The shock at $6: 29$ p.m. April 8 caused only minor wave motion in the gage well. About 8 hours later, however, without any rain, the flow at the gaging station increased suddenly and was $0.03 \mathrm{~m}^{3} / \mathrm{sec} 16$ hours after the shock. This
TABLE 31. - Fluctuations and net changes in water level recorded at gaging stations in California, April 8, $1968-$ Continued

\begin{tabular}{|c|c|c|c|}
\hline \multirow{2}{*}{$\begin{array}{l}\text { No. } \\
\text { (fig. } \\
139 \text { ) }\end{array}$} & \multicolumn{2}{|c|}{$\begin{array}{l}\text { Fluctuations in } \\
\text { gage well (double } \\
\text { amplitude, in } \mathrm{mm} \text { ) }\end{array}$} & \multirow{2}{*}{$\begin{array}{l}\text { Net } \\
\text { changes, } \\
\text { rise (+) } \\
\text { or }(-) \\
\text { fall (mm) }\end{array}$} \\
\hline & $\begin{array}{l}6: 29 \\
\text { p.m. }\end{array}$ & $\begin{array}{l}7: 04 \\
\text { p.m. }\end{array}$ & \\
\hline $\begin{array}{l}74 \ldots \ldots \text { West Side Main canal at } \\
\text { automatic gate of No. } 8 \\
\text { pond } 5\end{array}$ & 6300 & 6300 & +82 \\
\hline 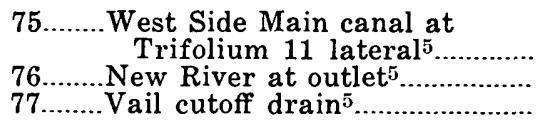 & $\begin{array}{r}70 \\
183 \\
158\end{array}$ & …....... & $\begin{array}{r}0 \\
-24 \\
0\end{array}$ \\
\hline $\begin{array}{c}\text { 78........ Rositas Pond at Rose } \\
\text { heading }\end{array}$ & 265 & 21 & 0 \\
\hline 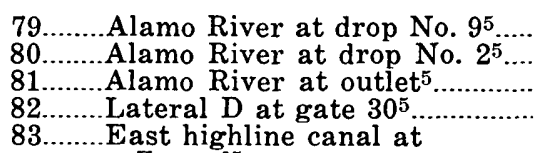 & $\begin{array}{r}37 \\
280 \\
43 \\
195\end{array}$ & $\begin{array}{l}21 \\
\cdots \cdots \cdots \\
\cdots \cdots \cdots \\
\cdots \cdots \cdots\end{array}$ & $\begin{array}{r}+6 \\
-3 \\
-21 \\
+3\end{array}$ \\
\hline 84 pond $\quad$ T drain ${ }^{5}$ & $\begin{array}{r}92 \\
150\end{array}$ & $\cdots \cdots$ & $\begin{array}{r}+30 \\
-3 \\
0\end{array}$ \\
\hline $\begin{array}{l}\text { 2Data from Southern California Edison Co. } \\
\text { 3Delay in response : rise in stage and increa } \\
8 \text { hours after principal shock. } \\
\text { 4Flow decreased } 0.57 \mathrm{~m}^{3} / \mathrm{sec} \text {. } \\
\text { } 5 \text { Data from Imperial Irrigation District. } \\
\text { }{ }^{\circ} \text { Complete rotation of recorder drum; : exact }\end{array}$ & ni & & \\
\hline
\end{tabular}

increase may have been the result of increased spring discharge at some point upstream from the gaging station. The increase in flow seems to have been sustained for more than a month. Flow at the gaging station ceased seasonally June 16 .

Substantial water-surface fluctuations were recorded at the gaging station on Whitewater River near Mecca (site 68) at the time of the principal shock, and the stage declined suddenly (fig. 142); a secondary fluctuation occurred about 35 minutes later. The flow declined from $4.0 \mathrm{~m}^{3} / \mathrm{sec}$ before the principal shock to $3.4 \mathrm{~m}^{3} / \mathrm{sec}$ after the event, and it continued at a reduced level for more than a week. Subsequently, the flow increased gradually to the preearthquake magnitude. The decline may be attributable to short-term changes in the storage and drainage capabilities of the irrigated lands adjacent to the river.

The record for the Salinas Reservoir (Margarita Lake) near Pozo, Calif. (site 49) shows a seismic seiche that had an initial range of $21 \mathrm{~mm}$ and continued for about 2 hours. The reservoir is $470 \mathrm{~km}$ northwest from the epicenter. The Alaskan earthquake of March 27, 1964, caused a seiche in this reservoir that had an initial range of $128 \mathrm{~mm}$ and continued for nearly 6 hours. In contrast, the Parkfield-Cholame earthquake of June 27, 1966, caused only a short-term hydroseism with an amplitude of $55 \mathrm{~mm}$ (Waananen and Page, 1967), although the distance from the epicenter was less than $80 \mathrm{~km}$. The Salton Sea, however, as shown by the record 


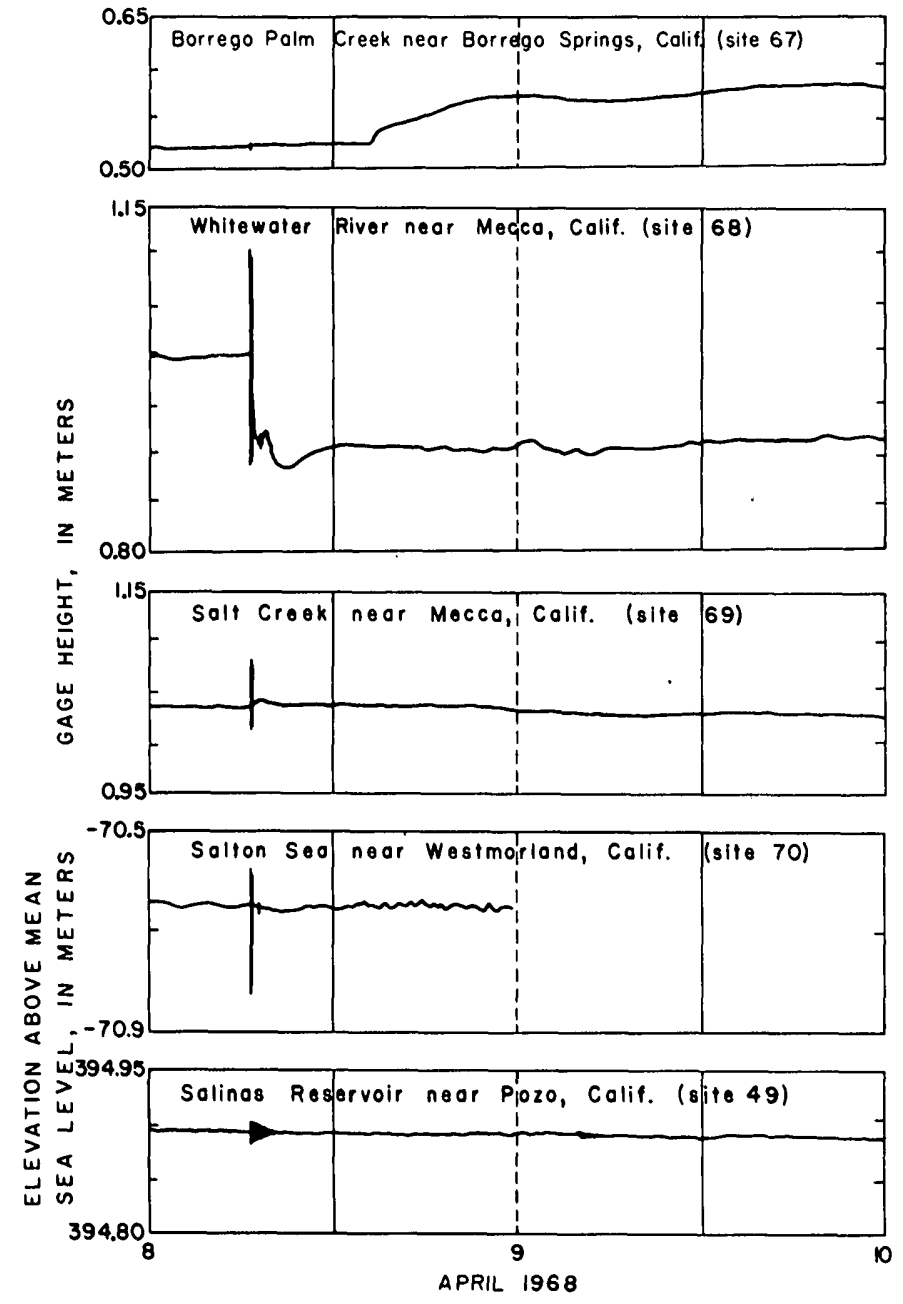

Figure 142. - Fluctuations recorded at gaging stations in response to the Borrego Mountain earthquake. Time is Pacific standard time.

near Westmorland (site 70, fig. 142), evidently did not develop any seiche, although the water-surface fluctuations in the gage well had an amplitude of $250 \mathrm{~mm}$ and the oscillations continued for several minutes; one aftershock was also recorded.

Many water-stage recorders on streams, canals, ponds, and drains operated by the Imperial Irrigation District south of the Salton Sea showed large hydroseisms at the time of the principal shock and the secondary event April 8. Data for 14 of the stations (sites 71-84, fig. 139) are given in table 31 . The amplitude of the fluctuations exceeded $150 \mathrm{~mm}$ at many sites and varied widely between adjacent sites, owing to differences in the width and depth of channels and the effects of canal junctions, automatic spill gates at ponds, and other manmade features. The fluctuations resulting from the principal shock continued for several minutes. The records for the stations at the automatic gates show extreme fluctuations that may have resulted from secondary wave motion caused by operation of the gates in response to water-surface oscillation in the ponds. The available records did not indicate any significant changes in flows that could be attributed to the shocks.

Seiches, especially in small pools and ponds, may have been the most common surface-water effect attributable to the Borrego Mountain earthquakes. Countless swimming pools in San Diego County and over widespread adjacent areas in southern California were subjected to seiches and sloshing water. The effects ranged from minor oscillations to severe spill. The Ironwood Motel at Ocotillo Wells, about $6 \mathrm{~km}$ south of the epicenter, was flooded by water sloshed out of an adjacent pool (Cloud and Scott, 1968 a, p. $1189 ; 1968$ b, p. 20). At Cypress, in Orange County, $13 \mathrm{~km}$ west of Anaheim and $32 \mathrm{~km}$ southeast of the Los Angeles Civic Center, the hydroseism in a pool was sufficient to cause a splash of more than $150 \mathrm{~mm}$ spill from the pool. A lesser motion, without spill, was observed in a motel pool near the community of Borrego Springs in Borrego Valley, about 40 $\mathrm{km}$ west of Salton Sea and less than $22 \mathrm{~km}$ from the epicenter. The full extent of this effect has not been determined.

The earthquake may have caused the roiling or muddying of water in some wells and springs, a common effect with respect to water quality. A noticeable temporary increase in well-water turbidity was observed by the owner of a well along State Highway 67, about $8 \mathrm{~km}$ west of Ramona, San Diego County, and about $40 \mathrm{~km}$ northeast of San Diego. The effects of the Borrego Mountain earthquake in most wells may have been so slight or of such short duration that they either were not visible or were not observed.

\section{REFERENCES CITED}

Cloud, W. K., and Scott, N. H., 1968a, The Borrego Mountain, California, earthquake of 9 April 1968 - a preliminary engineering seismology report: Seismol. Soc. America Bull., v. 58, no. 3, p. 1187-1191.

$1968 \mathrm{~b}$, Field reports and isoseismal map, in Strongmotion instrumental data on the Borrego Mountain earthquake of 9 April 1968: Seismological Field Survey, U.S. Coast and Geodetic Survey and Earthquake Engineering Lab., California Inst. Technology, p. 19-119.

La Rocque, G. A., Jr., 1941, Fluctuations of water level in wells in the Los Angeles basin, California during five strong earthquakes 1933-40: Am. Geophys. Union Trans., pt. II, p. 374-386.

Lofgren, B. E., and Klausing, R. L., 1969, Land subsidence due to ground-water withdrawal, Tulare-Wasco area, California: U.S. Geol. Survey Prof. Paper 437-B, 101 p.

Vorhis, R. C., 1967, Hydrologic effects of the earthquakes of March 27, 1964, outside Alaska: U.S. Geol. Survey Prof. Paper 544-C, $54 \mathrm{p}$

Waananen, A. O., and Page, R. W., 1967, Water-resources aspects, in Brown, R. D., and others, The ParkfieldCholame, California, earthquakes of June-August 1966 : U.S. Geol. Survey Prof. Paper 579, p. 53-56. 


\title{
COLLAPSE FISSURES ALONG THE COYOTE CREEK FAULT
}

\author{
By Malcolm M. Clark \\ U.S. Geological Survey
}

\begin{abstract}
Gaping collapse fissures that are locally as much as $4 \mathrm{~m}$ deep, $3 \mathrm{~m}$ wide, and $100 \mathrm{~m}$ long have developed along the Coyote Creek fault on many tectonic fractures associated with the Borrego Mountain earthquake of Ápril 9, 1968. Most of these collapse fissures developed only in zones of active creep during rainfall sufficiently intense to cause surface runoff. Surface water, pouring into new tectonic fractures or into those kept open by creep, tunnels horizontally and cascades downward at many levels within the fractures, eroding sediment from the walls and from foundered blocks and transporting it deeper into the fractures. This removal of material leads to foundering and slumping of additional blocks from the walls of the fractures until flow of water ceases or until the fissures become clogged or filled at depth. Many fissures that remain open after flow of water ceases become clogged by slumping or are filled with windborne debris within weeks or months, unless additional tectonic creep keeps them open. Renewed creep may also reopen fissures that have been clogged for several months or years, thus permitting additional collapse. After a fissure becomes clogged, it slowly fills with windborne and waterborne material, a process that may take years in some locations. Concentration of surface runoff in fissures leads to vigorous growth of vegetation along them that may persist long after the fissures fill. Because collapse depends on fortuitous occurrence of runoff soon after tectonic movement, it may be a relatively unusual fate of tectonic fractures in this arid area.
\end{abstract}

Assuming that tectonic fractures in the weak sediments broken by the 1968 rupture remain open to a depth at least as great as that of the water table (about $5-30 \mathrm{~m}$ ), in most tectonic fractures the open space above that level is large enough to accommodate the material removed from the collapsed parts at the surface.

Four groups of collapse fissures at the south end of the 1968 surface rupture predate the 1968 earthquake. The two oldest groups probably opened 15 years or more before the earthquake. Another group became active after 1953 to 1956 but apparently ceased enlarging several years before 1968 . The latest collapse on the youngest group occurred less than a year and perhaps merely a few months before the earthquake.

Collapse fissures have developed elsewhere, usually in arid areas. In a few places they start from tectonic fracturing, but generally they start from fractures caused by other agents such as desiccation, soil piping, and subsidence. Nevertheless, the development of collapse along fractures of any origin, which evidently requires conditions that are met in

arid regions, is probably similar to the development of collapse observed along the tectonic fractures of the 1968 earthquake.

By far the most prominent surface features to develop in the Coyote Creek fault zone after the Borrego Mountain earthquake of April 9, 1968 have been large gaping fissures along tectonic fractures (figs. 143-150; see also fig. 42). Many of these fissures are as much as $4 \mathrm{~m}$ deep and $3 \mathrm{~m}$ wide, and some are hundreds of meters long. They form when runoff from infrequent heavy rain pours into open (typically 20-100 $\mathrm{mm}$ wide) tectonic fractures and carries slumped and foundered material deep into the fractures; removing this material causes additional slumping and collapse of the walls of the fractures. The development of these collapse fissures, as they are called in this report, is one of the most obvious signs of active or very recent creep along the 1968 rupture. Collapse has also revealed the presence of many otherwise inconspicuous tectonic fractures that were overlooked during early phases of the postearthquake investigation because they were not along the main rupture. Because collapse increases the time required for sediment to fill tectonic fractures, it extends the period during which these fractures are evident. Collapse fissures collect surface runoff, encouraging unusually abundant and vigorous vegetation to grow along them and to mark the location of some original fractures for years or decades. Fissures constitute a hazard to the increasing numbers of high-speed dune buggies, jeeps, and motorcycles that frequent the flat terrain of this generally undeveloped region. Development of collapse fissures might also endanger structures that happen to be built upon tectonic fractures.

One of the most important characteristics of collapse fissures is that their extent and time of development correspond closely to those of creep. Because slumping or blown sand and silt filled or closed fractures rapidly along the 1968 rupture, the only fractures open at the surface during periods of heavy 


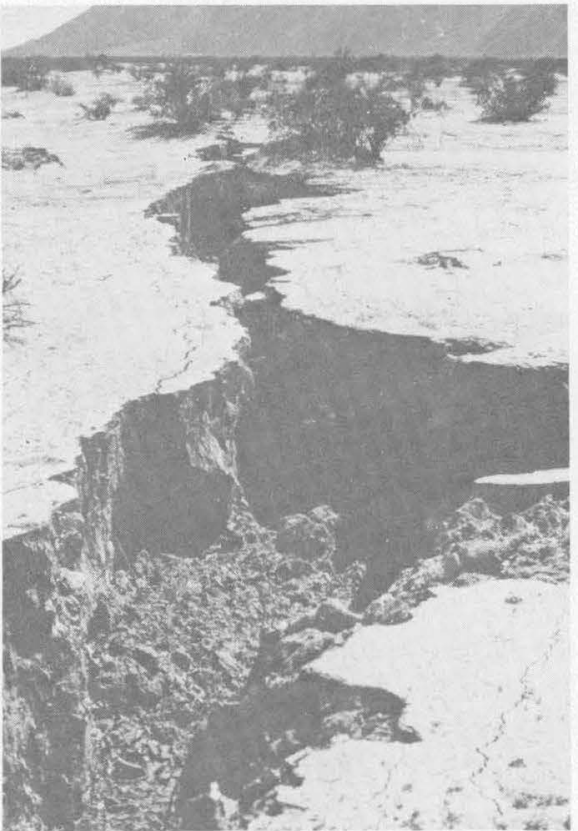

A

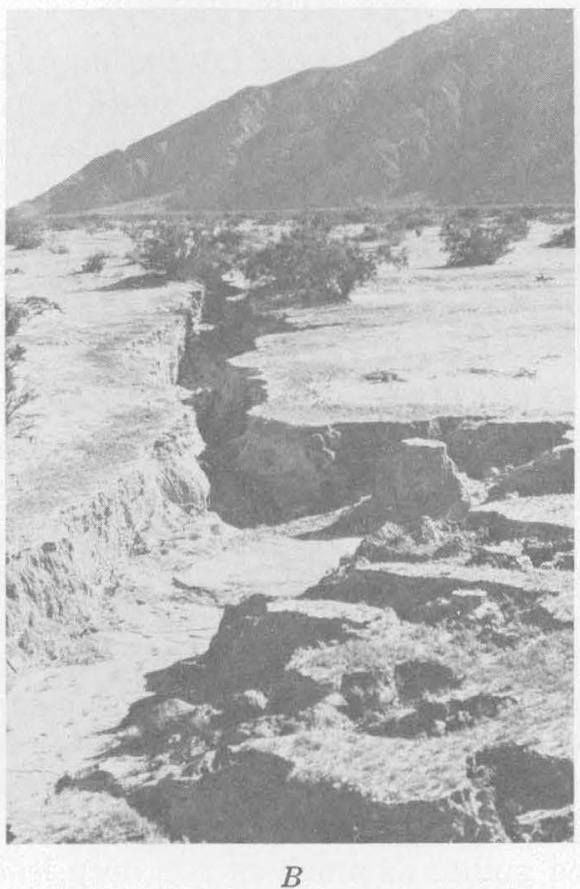

rain, and hence susceptible to collapse, were those that were either actively creeping or had been creeping no more than a few months before the rain. (Heavy rain falls in the area of the 1968 rupture only a few times each year; see fig. 40.) The first major collapse fissures to form along the 1968 rupture after the earthquake were caused by heavy rains in July 1968, the first to fall after the earthquake. Collapse fissures developed then only along fractures, all part of the central break, that were actively creeping during or before the rains. Subse-

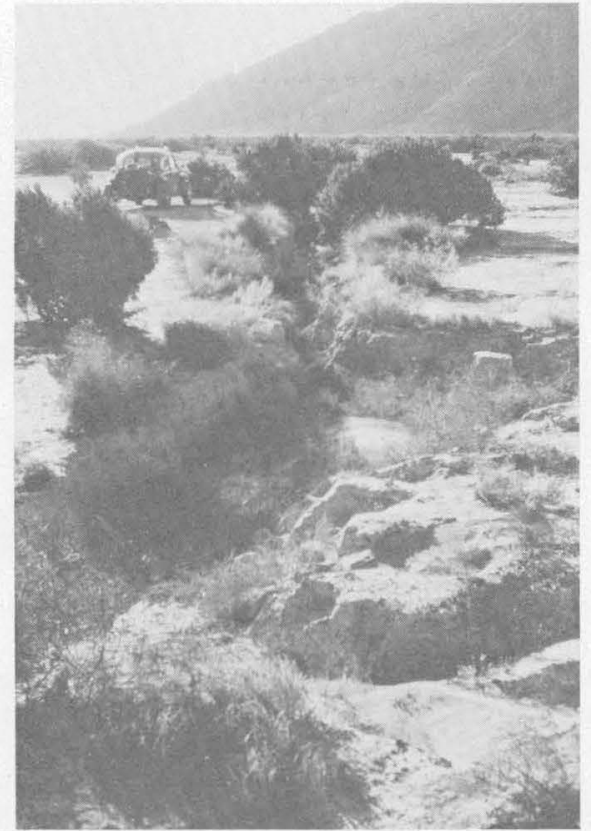

C

Figure 143. - Collapse fissure on the south break (loc. 30.3) that developed on a tectonic fracture after the rains of September 1969. A, October 1969. This recently collapsed fissure shows steep to vertical wall and peripheral cracks that bound the blocks that will collapse next. Small desiccation cracks break the mud locally in the bottom of the fissure, but collapse of the walls continued along most of the fissure long after any ponded water had dried up after the September runoff. $B$, January 1970. Water inflow during rains early in November 1969 widened the fissure by additional collapse, but material accumulated faster than the water conducted it down into the fissure, thus causing the fissure to fill. Little collapse occurred after water ceased flowing. A grassy band is evident along the sides of the fracture and along the channel that conducted water into it from the right. $C$, December 1970. Runoff and wind during 1970 have added a small amount of material to the fissure, but the most obvious change is in vegetation. The grass grew taller than $0.1 \mathrm{~m}$ and died, and many shrubs have appeared in the grassy band. Creosote bushes along the fissure (to the right of the car) have increased markedly in size and vigor, whereas those distant from the fissure are little changed.

quent collapse on the central break occurred only in areas where creep opened fractures immediately before or during episodes of runoff. No collapse fissures developed either in July 1968 or later on the north break, because blown silt and sand rapidly filled fractures created at the time of the earthquake, and no creep occurred to reopen them. No collapse fissures developed in July 1968 along the south break for the same reason, and none formed until nearly $11 / 2$ years after the earthquake because of a delay in the onset of creep along that break. Thereafter 


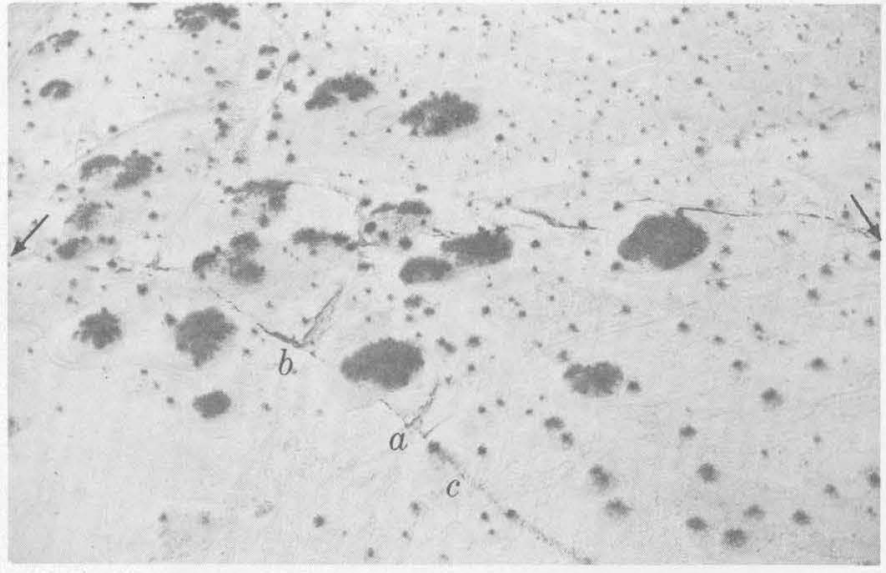

FIgURe 144. - Aerial view southwestward of collapse fissures and channels leading into them on south break (arrows) at location 31.4, January 1970. Vehicle tracks give scale. Branching fracture in lower half of view formed after January 1969 but before September 1969, and it continued to open and extend after September. Collapse shown here occurred in September and November 1969. The largest parts of the fissure are $a$ and $b$, located where wide shallow channels cross the fracture. Collapse developed on either side of the channels along the fracture, indicating subsurface flow of intercepted surface water in the fracture itself. Figures 145, 146, and 150 show details at $a, b$, and c, respectively. The large plants are mesquite (Prosopis); most of the small ones are creosote bush (Larrea).

collapse occurred on the south break only in areas of continued or renewed creep.

This paper describes the collapse fissures that formed along the 1968 rupture and offers some explanations of how they form. They represent a potential, if not common, feature of tectonic fractures in this region, so they can be expected to form after future surface faulting here and perhaps elsewhere in desert regions. An understanding of these fissures, especially their connection with creep, can be important in future investigations of surface faulting in desert regions. Indeed, the presence of new collapse fissures in a fault zone constitutes presumptive evidence of new fracturing. Furthermore, if the new fractures are caused by creep, subsequent collapse may be the first obvious sign of the presence of fractures and hence of the presence of creep.

This report draws heavily from information in a preceding paper in this volume (Clark, "Surface Rupture Along the Coyote Creek Fault"), which describes the fractures that formed along the Coyote Creek fault during and after the earthquake. The observations supporting the explanation of collapse fissures reported here were made during periodic visits to monitor creep along the 1968 rupture. As will be explained below, development of collapse fissures along tectonic fractures is closely related to creep, hence the reader should look at the sections of the preceding paper that deal with the postearthquake investigation and with creep. This paper will use the names north, central, and south breaks for the three main sections of the 1968 rupture, and locations will be given in the coordinate system of plate 1.

\section{ACKNOWLEDGMENTS}

Because the investigation of collapse fissures grew out of the study of the surface rupture of the 1968 earthquake along the Coyote Creek fault, most of the people acknowledged in that report (see Clark, "Surface Rupture Along the Coyote Creek Fault," this volume) also helped in this investigation. In addition, I have had helpful discussions with Arthur Grantz and E. A. Jenne of the U.S. Geological Survey. M. G. Bonilla, Arthur Grantz, D. H. Radbruch, and R. V. Sharp made many suggestions to improve the manuscript.

\section{OBSERVATIONS OF THE DEVELOPMENT OF COLLAPSE FISSURES ALONG THE 1968 RUPTURE}

The most complete record of the development of collapse fissures comes from the south break, which not only has collapse fissures that predate the earthquake, but also afforded the best observation of the evolution of new collapse fissures because of a fortunate timing of creep, rainfall, and field visits (fig. 40). Although less complete, observations of collapse fissures along the central break support the findings from the south break.

\section{EFFECTS OF THE RAINS OF SEPTEMBER 1969}

I first saw the collapse fissures of the south break in October 1969, about one month after the runoff that had created most of them. (See figs. 143A, 144, $145 \mathrm{~A}$, and $146 \mathrm{~A}$.) Most of the prominent fissures had developed on new and reopened long single fractures rather than on complex or short en echelon breaks. The roots of creosote bushes were stretched tautly across some fractures, indicating tectonic movement (fig. 145). Runoff from adjacent nearly flat terrain had eroded gullies as much as $2 \mathrm{~m}$ deep leading into the developing fissures. The water also eroded subhorizontal tunnels along the plane of the fractures at various levels in the exposed upper $3 \mathrm{~m}$. These tunnels evidently induced collapse well beyond the point of entry of the runoff. (Fig. 83 shows a trench excavated across a fracture of the central break about $20 \mathrm{~m}$ from the nearest collapsed section and $40 \mathrm{~m}$ from the nearest major entry point for surface runoff; this trench revealed a tunnel along the fracture $2 \mathrm{~m}$ below the surface; see also figs. 144 and 147.) The freshly exposed vertical walls and 


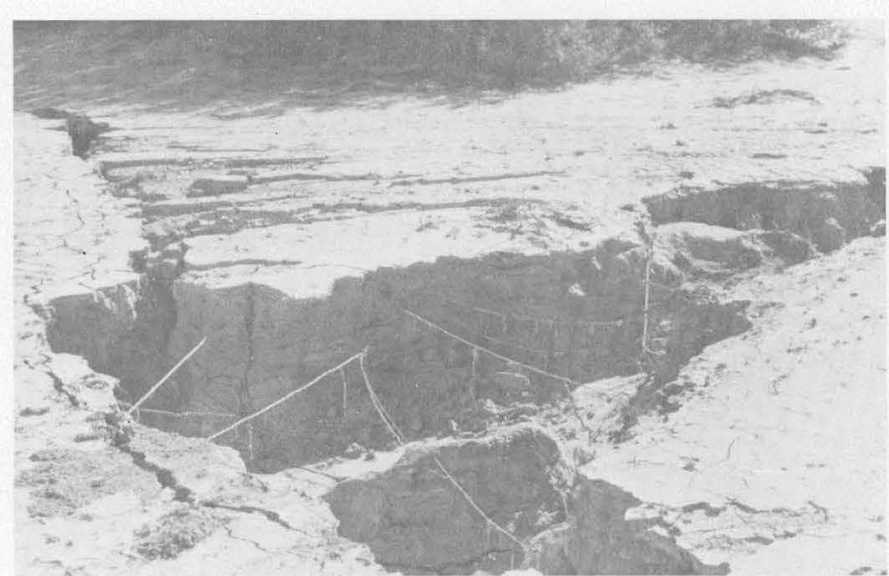

$A$

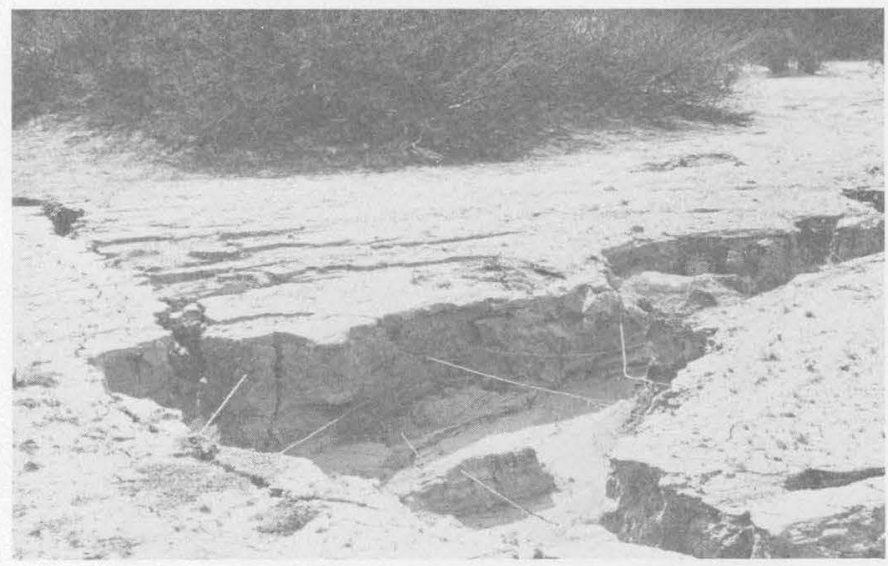

$B$

FIgUre 145. - Detail of channel and collapse fissure shown at $a$ in figure 144. $A$, October 1969. The tectonic fracture extends from upper left to lower right. Water entered the fracture from the right and eroded channels into the fracture. A taut root spans the open fracture at the left, whereas limp roots hang across the eroded channel to the right. Original tectonic opening of this fracture was 20 $30 \mathrm{~mm}$. Peripheral cracks flank both the fracture and the channel. Desiccation cracks in the bottom indicate some filling of the fissure during the first episode of runoff and collapse in September 1969. Additional creep of several millimeters during 1970 and 1971 was not sufficient to lead to further collapse. $B$, January 1970. Runoff from the rains of November 1969 has added $0.1-0.2 \mathrm{~m}$ of sediment to the fissure, but almost no further collapse has occurred.

jumbled debris in the bottoms of the fissures on the south break indicated that collapse had continued after rain and inflow of the surface water had ceased, although how long after cessation is unknown (figs. $143 A, 145 A, 146 A$, and $148 A$ ). Some of the tectonic fractures (for example, the one in fig. 144) continued to extend after the rain and thus were prepared for further collapse during the next heavy rain.

\section{EFFECTS OF THE RAINS OF NOVEMBER 1969}

Heavy rains fell on the collapse fissures on the south break November 9 to 10,1969 , again causing surface runoff. Inspection in January 1970 revealed

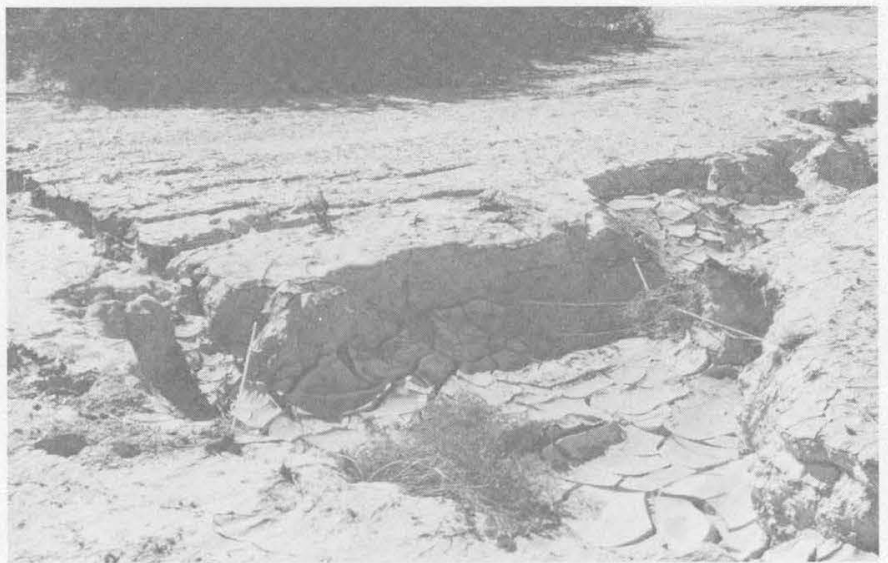

C

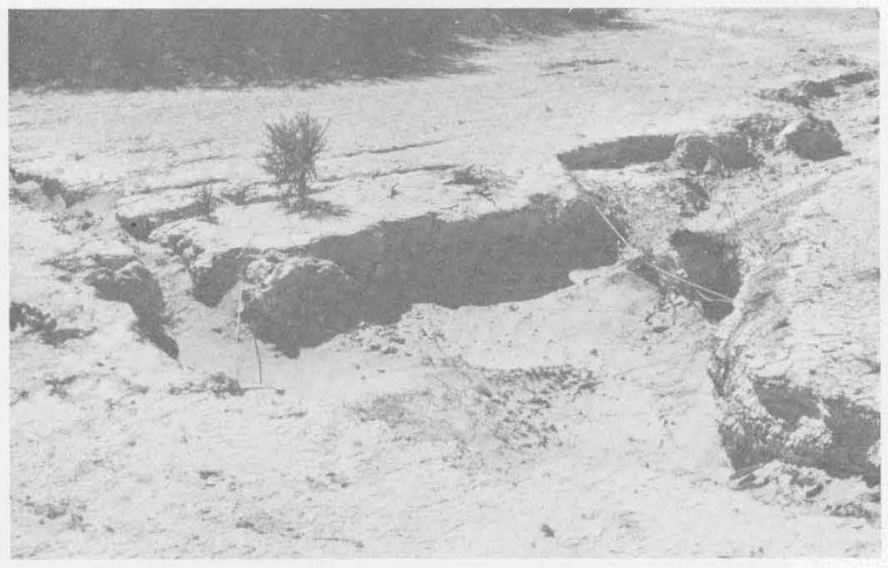

$D$

Rainfall was not intense enough to cause general sheet flow on the immediately surrounding surface. $C$, December 1970. Runoff during one or more storms in 1970 filled and overflowed the sealed fissure but added no more than 200 $\mathrm{mm}$ of sediment to the bottom. Debris plastered on weeds growing in the channel immediately downstream from the fissure indicates a maximum water depth there of only about $70 \mathrm{~mm}$; this depth implies a small sediment-carrying capacity for the channel. New desiccation polygons have formed in the fresh layer of silt and mud 2-10 $\mathrm{mm}$ thick on the surrounding surface. $D$, November 1971. Runoff from one or more storms since December 1970 has overflowed the fissure again but has added little to the sediment in the bottom.

that rainfall had caused major changes in the fissures that formed in September 1969, most of which experienced little further creep after September, and in the few new fractures formed between September and November 1969 (figs. $143 B, 145 B, 146 B, 148 B$, and 149).

Most open collapse fissures that developed in September were partly filled with waterborne and slumped mud and silt by January 1970 ; this filling created shallower rounded and polygonally cracked bottoms that contrasted with earlier steep walls and deep irregular bottoms (figs. 143 and 146). Many 

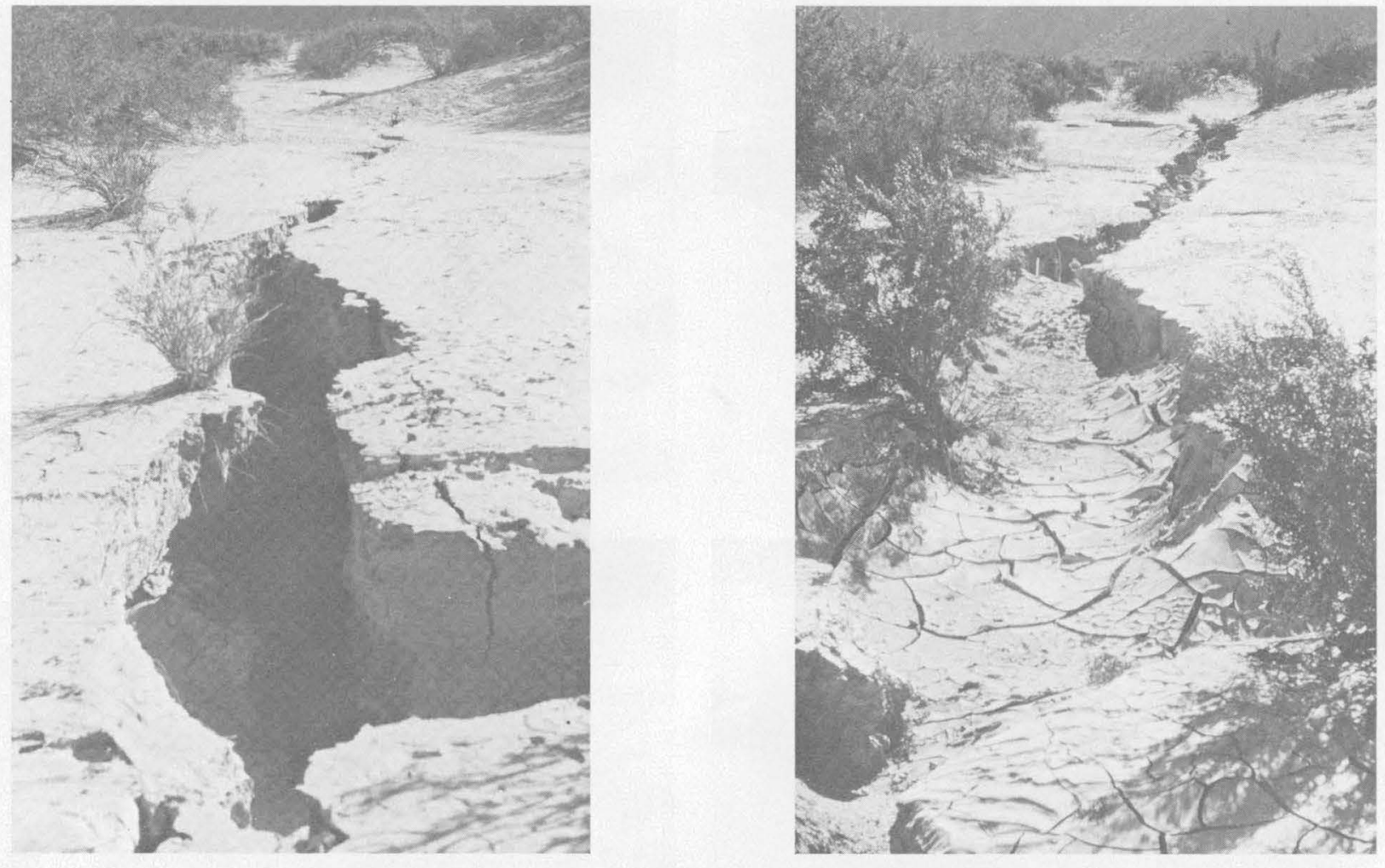

$A$
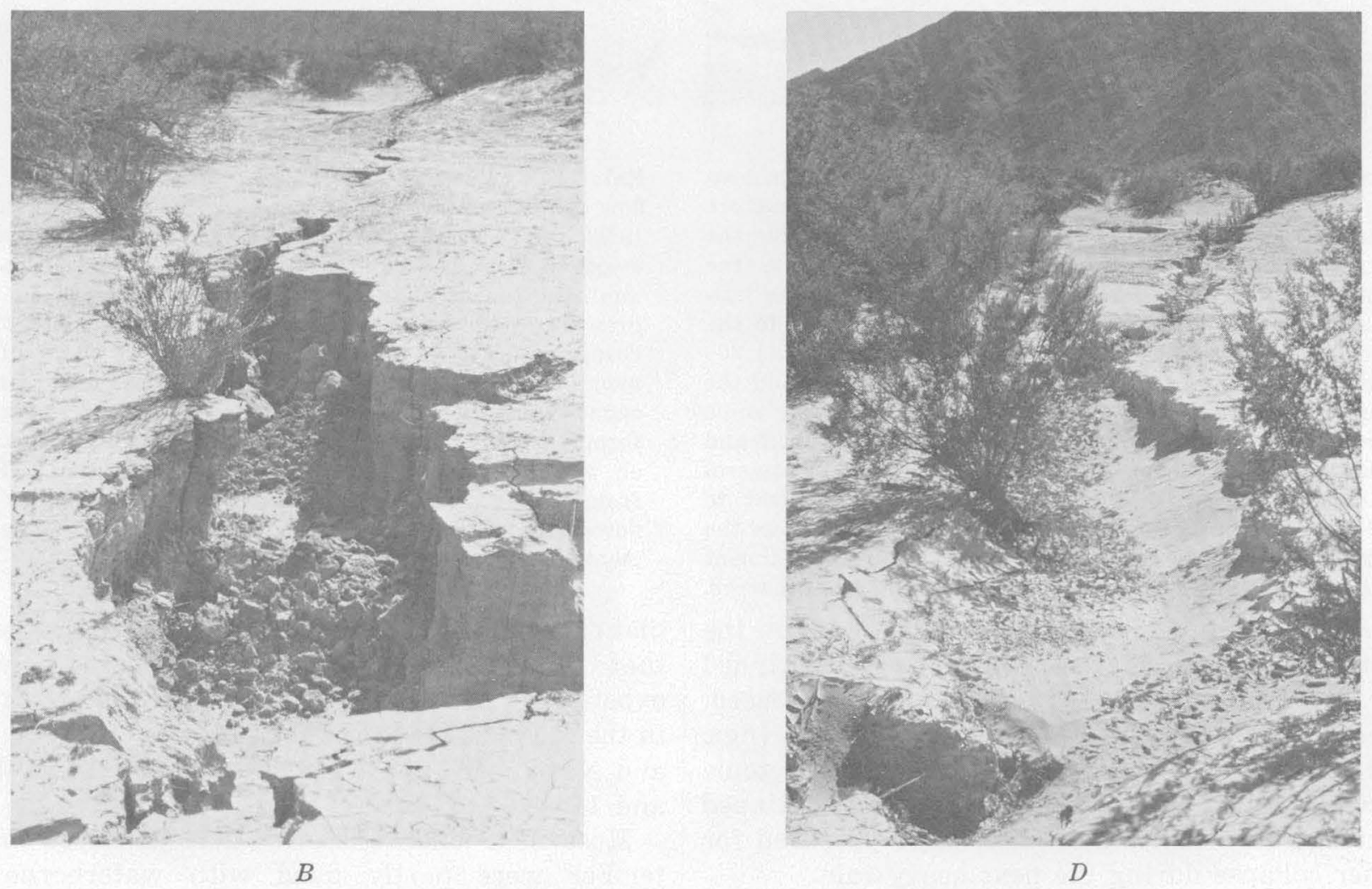

Figure 146. - Explanation on opposite page. 
had been widened by additional collapse, and some of the gullies leading into collapse fissures had enlarged by both headward erosion and widening, although they were now also shallower because they and the fissures had been partly filled (figs. 143 and 148). The U-shaped cross sections and cracked mud of the bottoms of the fissures indicated that draining or drying of pools of muddy water were the last major events to occur, rather than foundering and slumping of walls as in September.

In contrast, many of the fissures which had just begun to collapse in October, plus the tectonic fractures that developed or opened further after the September rain, had now collapsed more as a result of the November rains. Collapse, rather than ponding of water, was the last event to occur in these new fissures after the November rains, as it was for the older fissures after the September rains.

A significant clue to the collapse mechanism, noted in October 1969, was the development of fractures parallel to the main fractures or fissures and usually within $1 \mathrm{~m}$ on both sides (figs. 143, 145, and 150). These cracks initially bounded small graben along the fracture and represented the first step of collapse visible at the surface. After the central blocks foundered, the side walls progressively caved in along similar cracks that developed farther away from the fracture (figs. 143 and 146). Although a small number of peripheral cracks accompanied new tectonic fractures, most developed during ensuing weeks or months (fig. 150), presumably either by delayed collapse toward the free faces presented by

FIGURE 146. - Detail of the collapse fissure at $b$, figure 144 . The fracture runs directly away from the camera, near the center of the photograph. Water entered the fissure via channels from the right. $A$, October 1969. Collapse resulting from the rains of September 1969. Walls are nearly vertical in the 3 -m-deep fissure. Peripheral cracks define blocks that may collapse. $B$, January 1970. More water entered the fissure during the rain of early November and caused additional collapse before the fissure started to fill and flow ceased. The fissure evidently contained standing water at the end of the runoff episode, although some blocks collapsed after the bottom dried. C, December 1970 . Heavy rains of 1970 caused some additional collapse in the foreground of this photograph and extensive additional collapse in the background. Runoff in the foreground overflowed the fissure. The high level of water in the fissure probably caused much of the new collapse. $D$, November 1971. Water overflowed the fissure again after December 1970 but did not deposit large amounts of sediment. Blown debris is accumulating along one wall of the slowly filling fissure. Vegetation alongside has grown rapidly since the fissure developed.

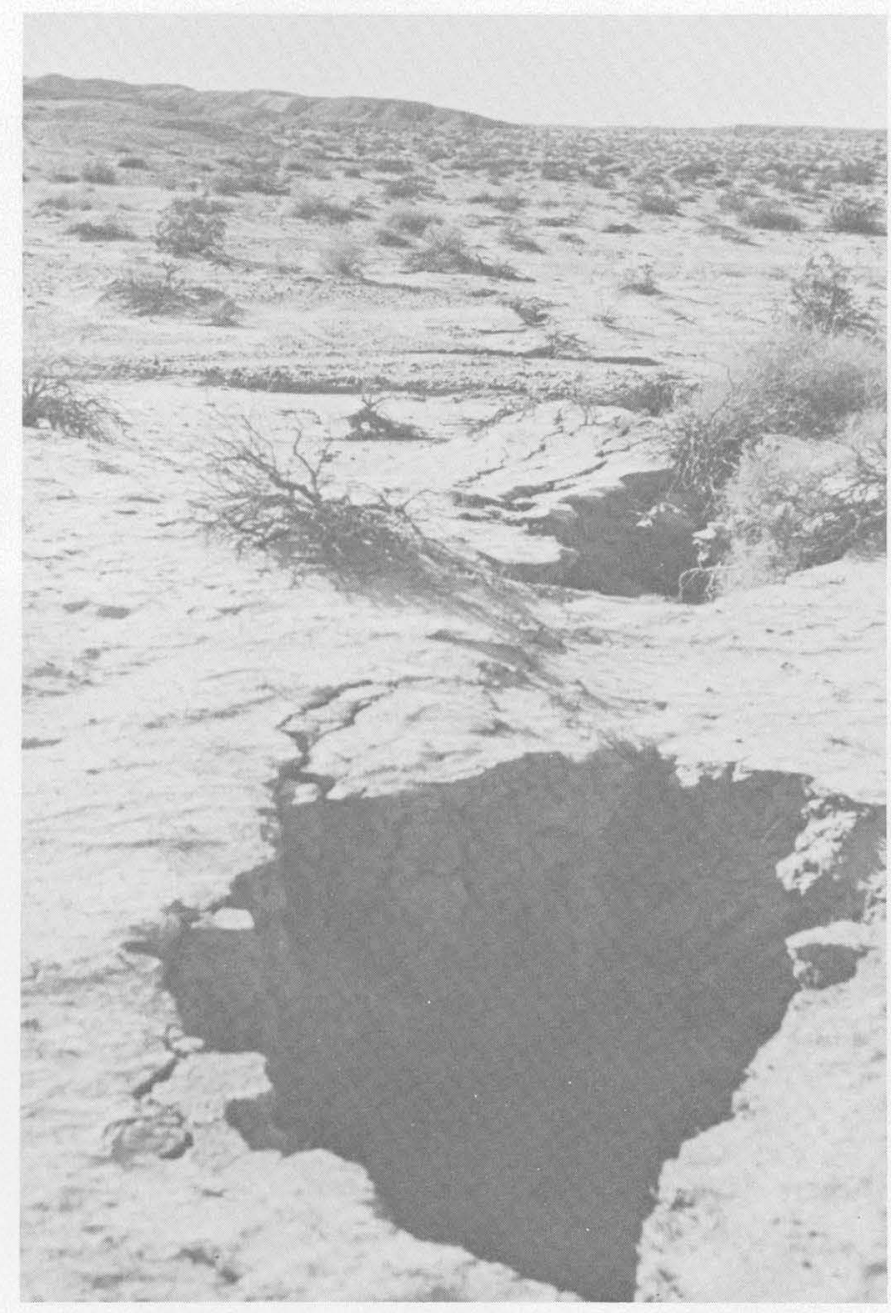

Figure 147. - Collapse across a silty mound demonstrates subsurface flow of water in the fissure from the channel extending from left to right beyond the mound. Almost no runoff poured into the parts of the fissure visible in this photograph. The tectonic fracture extends from the bottom of the photograph to the right middle distance. Location 19.2 .

the tectonic fractures or by removal of support by running water during subsequent rainstorms. These peripheral cracks bounded nearly all material that fell into the fissures, and their development appears to be a necessary step in the formation of collapse fissures.

A noteworthy event after the heavy rain of November 10 was the appearance of bands of short thick green grass in the zone of peripheral cracks along many collapse fissures and the gullies leading into them (figs. $143 B, 148 B$, and 149). The grass greatly aided the search for small collapse fissures in January 1970 and made them particularly obvious from the air. At those places where peripheral 


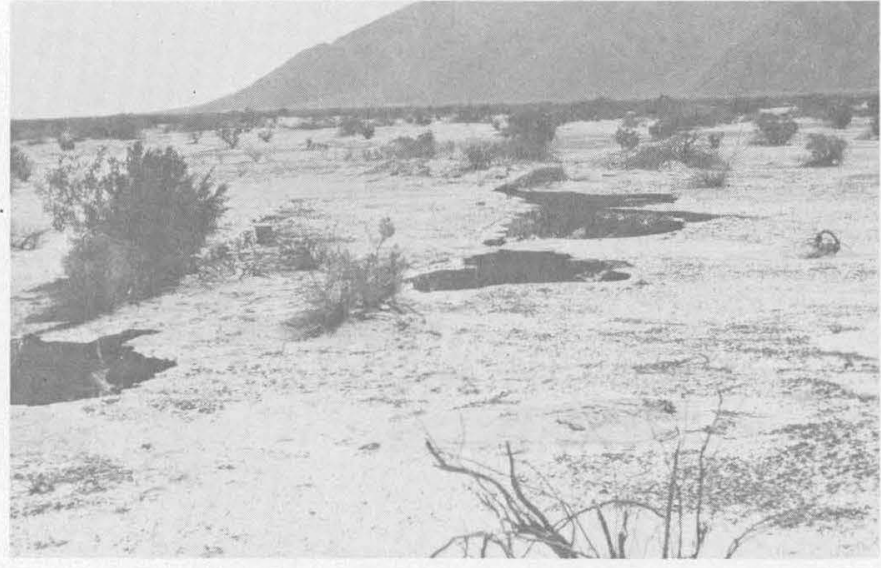

A

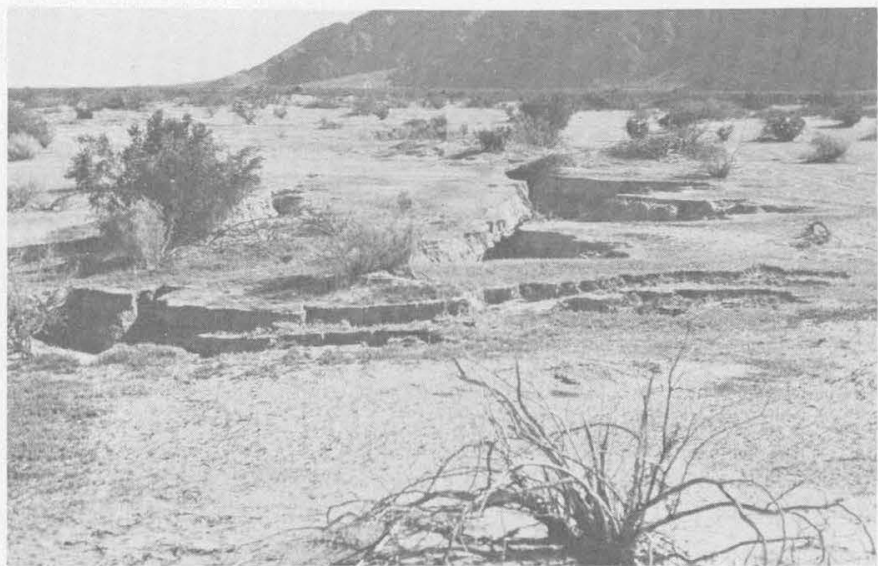

$B$

Figure 148. - Collapse fissures on the south break (loc. $30.5)$. Fractures extend from lower left to right center. Water entered the fractures from the right. $A$, October 1969. September rains created deep, steep-walled fissures; a peripheral crack parallels the left side of the large fissure near the center of the photograph. $B$, January 1970. November rains have caused headward erosion of the channels leading to the fissures. A block bounded by the peripheral fracture visible in $A$ has collapsed. Short grass surrounds the fissures and the gullies leading into them. $C$, December 1970. Rain and wind have further modified and filled the fissure, but the dramatic increase in vitality

cracks were present, full-sized grassy bands even flanked some tectonic fractures that had not yet collapsed. In contrast, grassy bands were missing from the few uncollapsed tectonic cracks that lacked peripheral cracks. The grass almost certainly grew in response to the increased penetration of rain and surface water afforded by the peripheral cracks. (The low permeability of undisturbed surface silts in the vicinity is indicated by large areas that consist of small desiccation polygons that are typically 20 $50 \mathrm{~mm}$ wide and $2-6 \mathrm{~mm}$ thick; see fig. $145 \mathrm{C}$.) Except for these $1 / 2$ - to 1 -m-wide bands of grass along collapse fissures and newly opened tectonic fractures, grass grew elsewhere after this November rain only

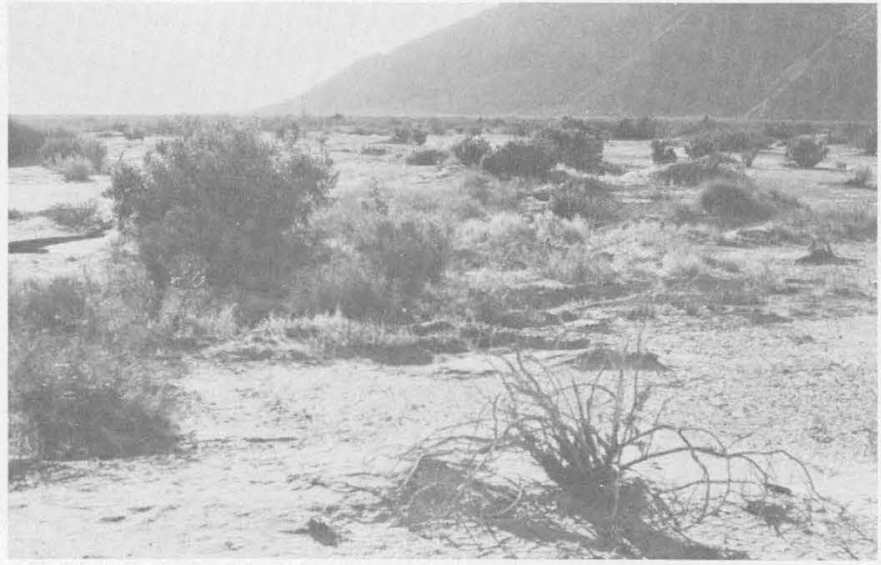

C

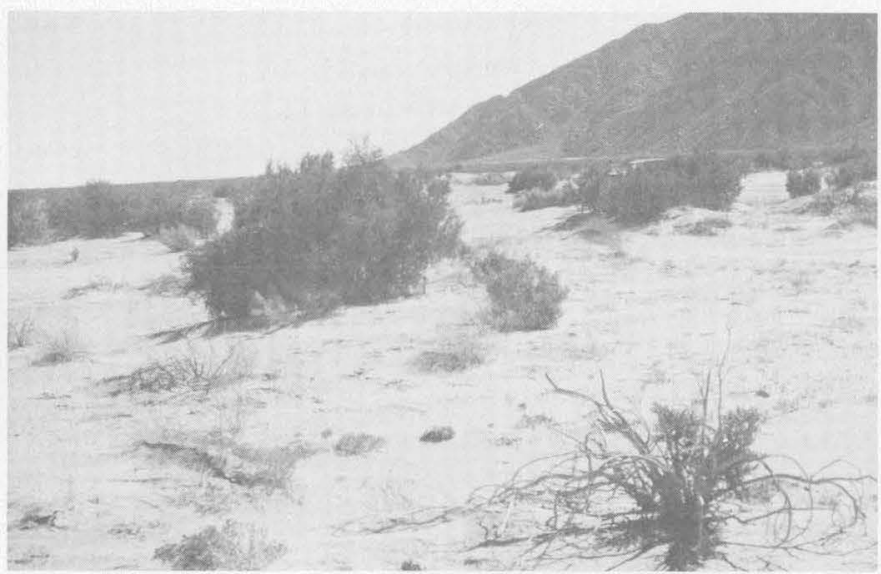

D

and amount of vegetation next to the fissure is the most obvious change since January 1970. The same storms that caused overflow of the fissure shown in figures 145 and $146,1 \mathrm{~km}$ distant, did not fill this fissure nor those shown in figure 149 with water and added very little sediment to the fissures at this location. D, November 1971. Unusually large amounts of blown silt and sand during 1971 filled this and many nearby fissures. Although the grass and most of the small bushes have died, the large creosote bushes along the former fissure have grown noticeably since December 1970; they are far larger and greener than other bushes nearby.

in widely scattered small surface depressions of the otherwise nearly featureless surrounding terrain.

The width of the bands of grass showed no relation to the greatly varied depth of the fissures - further evidence that the grass resulted from increased penetration of surface water into the cracked zone flanking the fissures rather than from a high level of water in the fissures. Many parts of fissures more than $2 \mathrm{~m}$ deep showed no evidence of having been filled with water, yet the grass was just as well developed on the surface next to these fissures as it was on the surface next to shallow fissures that showed evidence of ponded water less than $0.5 \mathrm{~m}$ from the top. 


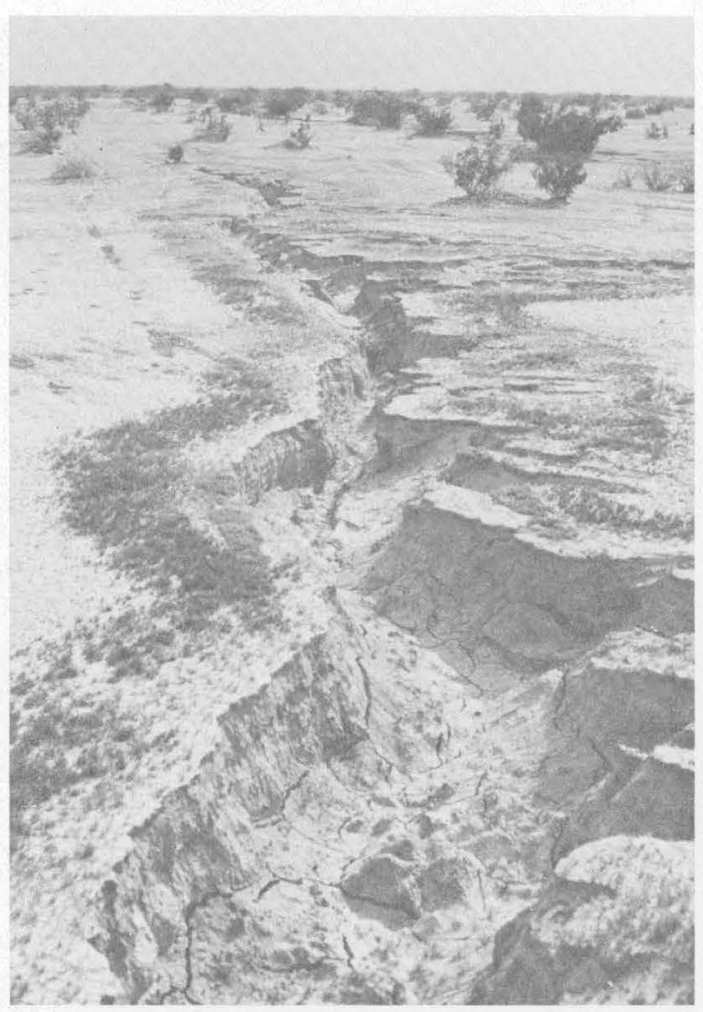

$A$

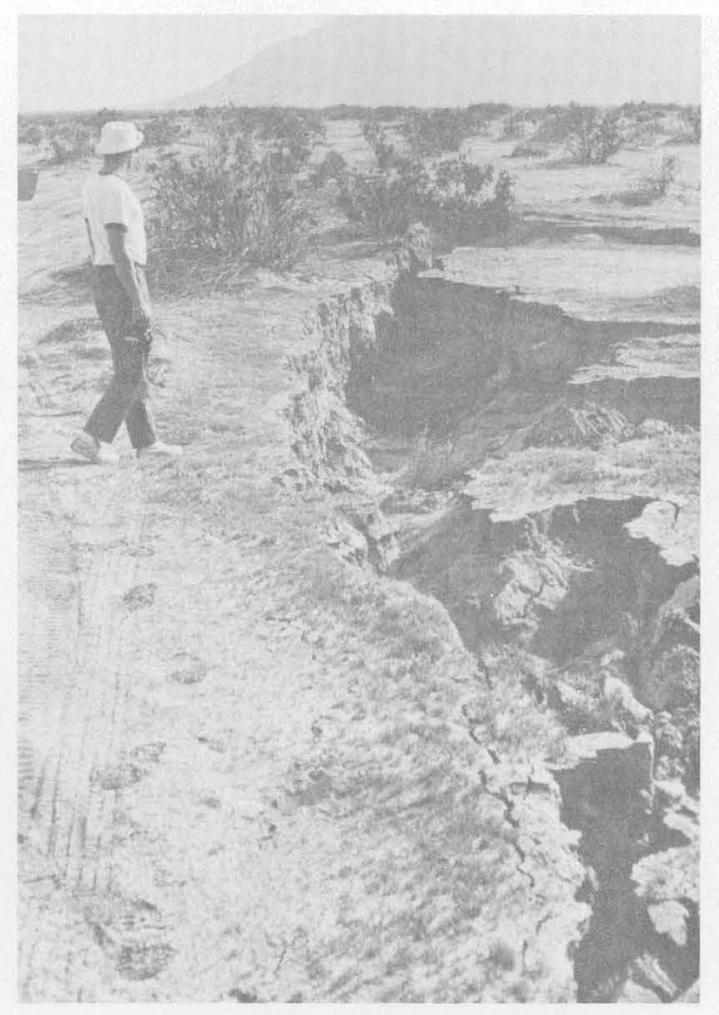

$B$

FIgURE 149. - January 1970. Collapse fissures on the south break formed by runoff in September 1969 (loc. 30.2). Rain in November 1969 led to growth of grass in the zone of peripheral cracks flanking the fissures and the gullies leading into them. The rounded cracked bottom of the fissure shown in $A$ is typical of fissures in the early stages of filling. The November rain caused little additional collapse except that shown in the lower right in $B$, where peripheral cracks have opened further and some blocks with grassy tops have collapsed after the grass reached its present size (see text).

The grass also demonstrated that some fissure walls collapsed long after flow of water ceased. Figure $149 B$ shows grass-topped segments of slumped walls in a fissure. The segments were much too small to have supported the root structure of such thick grass. The blades of grass were perpendicular to the sloping tops of the segments, hence the slumping happened after the grass had reached its present size. Because the grass beside the fissure continued to grow after the photograph (fig. $149 B$ ) was taken (for example, fig. 148C), slumping must have occurred shortly before the photograph was taken in January 1970, 5-8 weeks after water last entered the fissure in early November.

\section{CHANGES DURING 1970-71}

Except for minor collapse along a few new fractures formed after November 1969, most collapse fissures gradually filled during 1970-71 (figs. $143 C$, $145 C, 146 C$, and $148 C$ ). The small amount of creep during this period on both the central and south breaks has not lead to significant further collapse.
Strong winds during 1971 filled many collapse fissures along the central and south breaks that were scarcely affected by blown debris during 1969 or 1970 (see fig. $148 D$ ).

\section{GENERAL OBSERVATIONS ON COLLAPSE FISSURES}

The history of development of collapse fissures shows that at some time after the onset of wateraggravated collapse, either the fissures became clogged with sediment and could no longer conduct water, or they filled with water and detritus. At many of the fractures described above, this clogging or filling evidently occurred during the rain of November 1969 , which generally caused some further collapse before the fissures started to pond water.

The low gradients (about 0.003 ) and relief of the areas surrounding the south break apparently do not enable runoff to carry large loads of sediment to the fissures. Most channels that lead into fissures on the south break are merely wide swales, some less than $0.1 \mathrm{~m}$ deep. They drain small areas, typically thousands to tens of thousands of square meters of nearly 


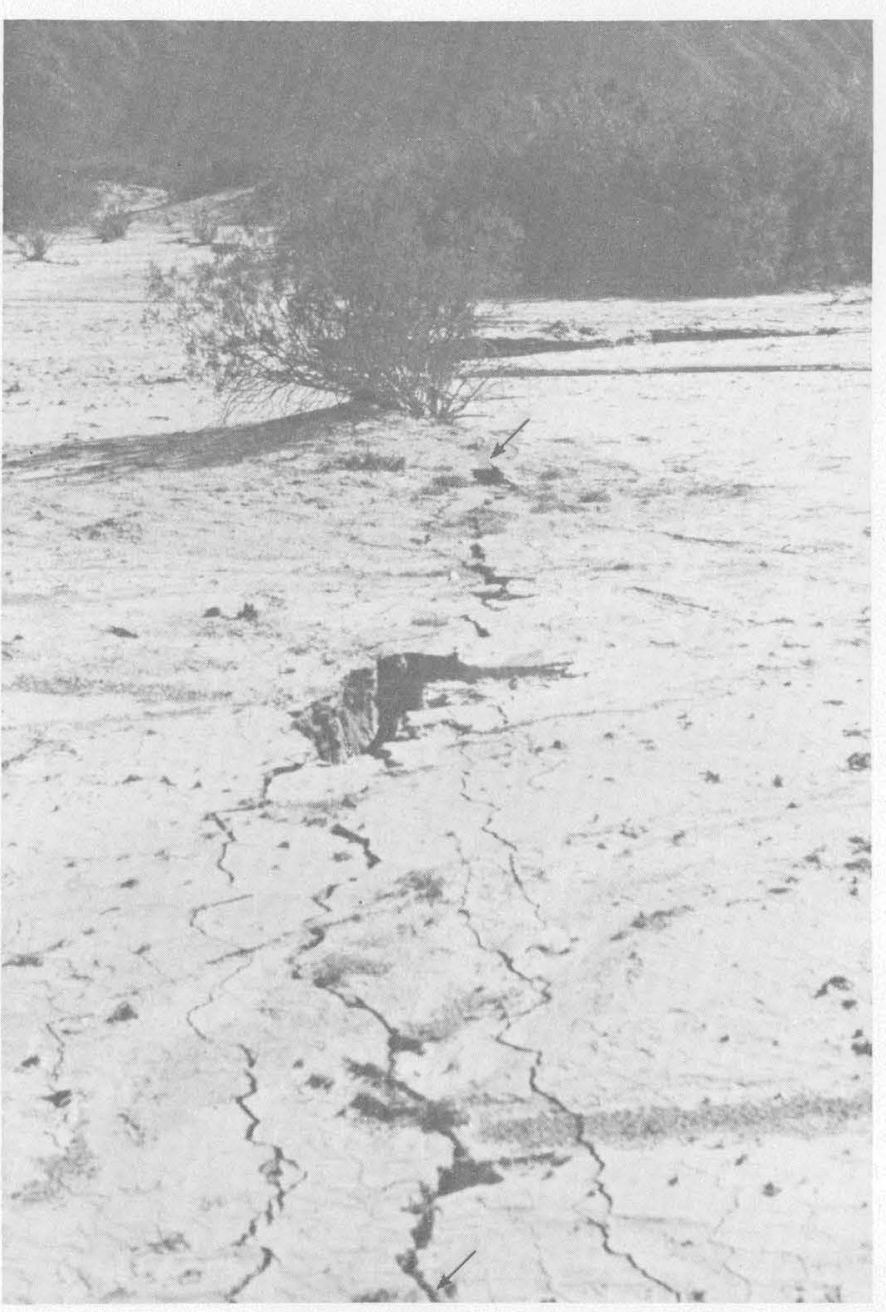

Figure 150. - October 1969. Detail at $c$, figure 144. Blocks are foundering between the tectonic (center) fracture (arrows) and the peripheral crack to the left. In the foreground the block next to the open fissure has dropped about $20 \mathrm{~mm}$. The tectonic fracture is prerunoff (September 1969), whereas peripheral cracks are postrunoff; these facts indicate that some peripheral cracks form slowly after subsurface excavation by intercepted water flowing in the fracture.

flat terrain, and transport relatively little sediment into the fissures (see figs. 145 and $146 B, C$, and $D$ ). Each of the increments of waterborne sediment shown in figures 145 and 146 is a small fraction of the total amount of sediment that has collapsed from the walls of these fissures. These observations indicate that most fissures on the south break do not act as receptacles for large amounts of waterborne sediment from the surrounding surface. Indeed, the observed persistence of shallow sinks and depressions for years in areas free of large amounts of wind-transported material along apparently inactive fractures of the south break implies locally low rates of water erosion and transport.
In contrast, parts of the few fractures and fissures along both the central and south breaks that developed across major channels apparently filled with sediment during the initial or subsequent episodes of collapse. Evidently, the relatively large sediment load in these larger channels either rapidly clogged or filled nearby parts of these fractures and fissures. As a consequence, little or no collapse occurred on parts of many fractures that cross larger channels.

The final stage of the existence of these collapse fissures, in the absence of renewed opening by creep or rapid filling by blown debris, appears to be gradual filling, with the persistence of alined shallow depressions or sinks for perhaps years or decades. The sinks may represent either residual unfilled parts of fissures, minor renewed opening and collapse, or later subsidence from compaction or gradual collapse of cavities in the fissure. Because an unfilled fissure collects surface water, small plants and the larger creosote bush and mesquite tend to concentrate and flourish along them (figs. 143 and 148).

The sequence of formation, growth, and filling of collapse fissures, as determined from the foregoing observations, is summarized in figure 151. This sequence should apply to the development of collapse regardless of whether the initial open fracture is caused by tectonism, desiccation, subsidence, or in

Frgure 151. - Hypothetical development of a collapse fissure. $A$, A new tectonic fracture may develop small peripheral cracks after the earthquake; slumping then takes place along these cracks. Without subsequent surface runoff, windblown sand and silt will eventually fill or seal the fracture. $B$, The first episode of surface runoff occurs before the fracture is clogged with debris. Water entering a nearby part of the fracture erodes a tunnel above a level that became clogged before or during runoff. The tunnel, by removing support from the overlying material, promotes the formation of peripheral failure planes. Blocks slump along the planes into the tunnel. $C$, End of the first episode of surface runoff. Water running in the fissure has removed most of the slumped material to other parts of the fissure and fracture, but after the water ceased flowing more material slumped in. After runoff ceases, peripheral cracks continue to form back from the walls of the fissure. $D$, The second episode of surface runoff causes more slumping; the fissure, which became clogged after the first runoff ceased or during the second runoff, begins to fill. Standing water at the end of the second episode of runoff leaves a rounded muddy surface in the bottom of the fissure. Concentration of water during the first and later episodes of runoff causes pronounced increase in the vitality of bushes growing next to the fissure. $E$, The collapse fissure gradually fills with windborne and waterborne debris and leaves a line of shallow sinks and prominent bushes that may persist for years or decades. 


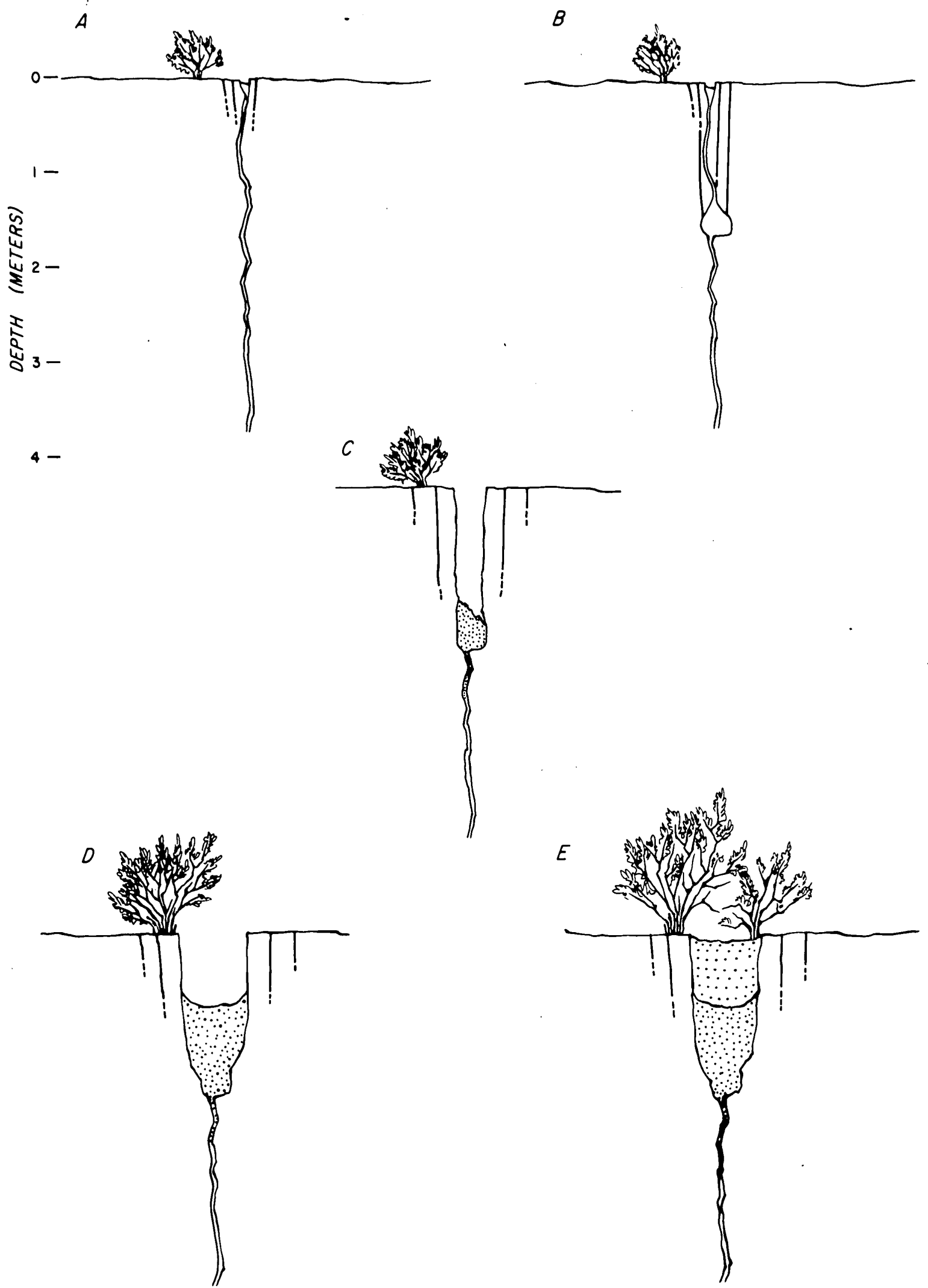

Figure 151. - Explanation on opposite page. 
some situations, piping. In photographs, these other features appear indistinguishable from the collapse fissures described here. A brief description of the development of desiccation fissures in Texas and Chihuahua by Underwood and DeFord (1969) accords with the development observed in Lower Borrego Valley.

\section{MECHANISM OF COLLAPSE THE PROCESS OF COLLAPSE}

For foundering of blocks and collapse to occur at all requires that the walls of fractures remain relatively coherent and that they not fail by crumbling or general slumping soon after opening. These conditions prevail in much of the unconsolidated Pleistocene and Holocene alluvial and lacustrine sediments along the 1968 rupture. Vertical and overhanging walls are common along freshly cut margins of nearby channels, and some of these exceed $6 \mathrm{~m}$ in height. These observations indicate that tectonic fractures should also remain intact and open to a depth of at least $6 \mathrm{~m}$ below the surface.

Collapse begins when blocks 0.5 to at least $4 \mathrm{~m}$ tall founder into tectonic fractures along nearly vertical surfaces (the peripheral cracks described above) that form as much as $1 \mathrm{~m}$ back from the fractures or from the walls of the subsequent fissures. Water flowing in fractures or fissures removes the material of the foundered blocks; removal of this material leads to additional foundering. This removal of the foundered and slumped blocks is a necessary part of collapse, for, in general, the major tectonic fractures along the 1968 break opened less than about $100 \mathrm{~mm}$. This amount of opening permitted only a comparable amount of settling of foundering blocks before they contacted the other side of the irregular surface of the tectonic fractures and stopped.

A few small collapse fissures (less than about 0.3 $\mathrm{m}$ wide and $2 \mathrm{~m}$ deep) did develop without the help of any water. Some formed along the main breaks during the days immediately after the earthquake. Before any rain fell, some blocks dropped 10-50 $\mathrm{mm}$ between bounding tectonic fractures less than $1 / 2 \mathrm{~m}$ apart. Smaller graben along some new fractures completely crumbled out of sight. Such collapse must have occurred when blocks foundered and slumped a short distance and then disintegrated into the space created below in the tectonic fractures. However, the amount of collapse caused in this fashion was very minor compared to that caused by water flowing into open fractures.

Clearly, water removes to deeper levels the material that slumps into a growing fissure, as is shown by postrunoff fissures $1 \mathrm{~m}$ wide and 3-4 $\mathrm{m}$ deep that develop from cracks that exhibit as little as 20$30 \mathrm{~mm}$ of tectonic opening. The subhorizontal tunnels described above and the extension of collapse beyond the immediate points of entry of runoff indicate that some water flows in channels along the plane of the fracture near the base of the collapsing zone. Observations of collapse fissures in different stages of evolution show that water flowing within fractures enlarges fissures in two ways. It removes material that slumps into and would otherwise fill and support the walls of the fissures, and it also undercuts the walls, inducing additional foundering and slumping.

The evidence for extensive subsurface flow of water along low gradients within collapsing fissures shows that local clogging commonly impedes the downward movement of water in fissures. Such lateral flow may occur at many levels in the fractures and may lead to collapse between levels. Certainly, during collapse, large volumes of water must be continually eroding and widening conduits, whereas falling and collapsing walls of the enlarging fissures must be clogging them. The arrangement of passageways probably changes rapidly in the fissures as erosion and removal of material by water causes, but in turn is affected by, collapse.

Collapse fissures cease enlarging when water can no longer leave the upper collapsed zone of the fissures as fast as it enters. This may happen during or immediately after episodes of runoff if waterborne sediment or the collapsing material clogs or fills the conduits and channels by which surface water descends the fractures, or it may happen if windborne material clogs or fills the fissures between runoff events. Both types of blockage occurred along the collapse fissures associated with the 1968 break. Of course, it is important to remember that continued or renewed tectonic displacement (creep) can keep fissures open or reopen clogged ones, allowing additional collapse.

\section{SPACE AVAILABLE IN TECTONIC FRACTURES}

Probably the most intriguing aspect of the collapse fissures is the great volume of both water and debris (mud, silt, and sand) that disappears into them. The volume of sediment eroded from the fissures near the surface alone indicates large storage capacity at depth. Although direct observation from the surface was limited to the upper $4 \mathrm{~m}$ of the fissures, indirect evidence reveals many of the conditions that probably exist at greater depths.

It seems very unlikely that the soupy mix cascading into the depths of a tectonic fracture penetrates very far into porous strata that may exist below. Porous strata next to recharge wells that inject water containing suspended sediments rapidly be- 
come clogged (Sniegocki, 1962, p. F14-F15). This type of clogging constitutes a major problem with injection wells (for example, Meinzer, 1946, p. 197; Todd, 1959, p. 262; Sniegocki, 1962, p. F12). Perhaps a small amount of sediment penetrates strata in the lower parts of new fractures, but because of the tendency for the same fractures to break repeatedly (see Clark and others, this volume), strata surrounding reopened fractures are likely to have been clogged during earlier episodes of infiltration. Therefore, it seems unlikely that much solid material removed by collapse and erosion from above goes anywhere except into lower parts of the fracture system itself, although the water may eventually penetrate through the clogged areas into porous strata.

The existence in this area of cavernous voids underground or interconnected conduits emerging at the surface some distance away at lower elevation can be neither established nor disproved with available observations. If underground conduits to remote surface openings or large underground storage places do exist, it would seem probable that at least some collapse fissures would enlarge without limit, but none have. All preearthquake and postearthquake fissures along the 1968 rupture have stopped growing after reaching a maximum width of about $3 \mathrm{~m}$ and depth of $4 \mathrm{~m}$. No other evidence so far even suggests that cavernous voids underground or interconnected conduits may be principal methods of removing collapsed solids. ${ }^{1}$

The fact that collapse fissures along tectonic fractures have not increased beyond a certain maximum size implies that each tectonic event creates a finite storage volume. The most reasonable storage volume is that of the newly opened fractures themselves.

In order to estimate the volume available in a tectonic fracture, we must determine the depths to which fractures can remain open. If water-saturated clay strata are present at depth, fractures in them could probably not remain open long because extrusion of these moist clays would close the fractures. Clays make up perhaps 30-40 percent of the upper 200-300 feet of strata underlying the central break, according to drilling records of wells 5-10 miles - north and northwest (Moyle, 1968). Water is 15$20 \mathrm{~m}$ below the surface in wells $3-4 \mathrm{~km}$ east and west of the collapse fissures near Old Kane Spring Road

\footnotetext{
A minor example of piping occurred on the south break at location 30.6 at the intersection of a channel 1-2 $\mathrm{m}$ deep with a fracture that reopened nfter January 1970. Two subhorizontal tunnels formed in the fracture at the level of the bottom of the channel and conducted runoff into the channel. Nearby fractures, however, collapsed to levels deeper than the bottom of this channel, so this piping could not have been the principal mechanism for removal of water and debris.
}

(Moyle, 1968). In 1969, water was $27 \mathrm{~m}$ below the surface in an abandoned well about $11 / 2 \mathrm{~km}$ southeast of the collapse fissures on the south break. These depths represent minimum distances to saturated sediments, because the water in the wells may have risen from deeper strata under artesian pressure. Thus, $15-30 \mathrm{~m}$ seems to be a maximum depth that fractures might remain open along the central and south breaks, if extrusion of water-saturated clay limits the maximum depth of open fractures.

Whether the clay, silt, sand, and gravel strata above the level of water saturation are able to maintain an open fracture remains a problem. This problem cannot be completely solved without knowledge of the physical properties of the material at depth. T. L. Youd (written commun., 1970) estimates that the material would require a cohesive strength of roughly $70,000 \mathrm{~N} / \mathrm{m}^{2}$ (newtons per square meter) to maintain an open crack $30 \mathrm{~m}$ below the surface and half that at $15 \mathrm{~m}$. These strengths fall within the $35,000-100,000 \mathrm{~N} / \mathrm{m}^{2}$ range (about 5-15 psi) determined for dry loess (Gibbs and others, 1960, p. 126-127) and seem at least plausible for the alluvial and lacustrine sediments underlying the 1968 break. If this estimate of strength is correct, the fractures probably remain open to a depth of at least $15 \mathrm{~m}$ and possibly as much as $30 \mathrm{~m}$ below the surface.

The space available in such fractures to receive material carried down from the surface can be calculated. The net volume in a fracture that is $30 \mathrm{~mm}$ wide and $15 \mathrm{~m}$ deep is $0.45 \mathrm{~m}^{3}$ for each meter of length of the fault. If the fracture is open to a depth of $27 \mathrm{~m}$, then the volume is about $0.8 \mathrm{~m}^{3}$ for each meter of length of the fault. If the fracture opens $60 \mathrm{~mm}$ wide, the volumes double, and so on.

Considering only the volume of sediment derived from collapsing walls, the space in a fracture $30 \mathrm{~mm}$ wide and $27 \mathrm{~m}$ deep could accept enough material to create a surface fissure about $0.9 \mathrm{~m}$ wide and $0.9 \mathrm{~m}$ deep or $0.4 \mathrm{~m}$ wide and $2 \mathrm{~m}$ deep if all the water entered surrounding strata and if solids filled the fracture at the same density they had before collapse (for this approximate calculation, we ignore the fact that the collapsed part of the original fracture at the surface is not available for storage).

These collapsed dimensions are compatible with those of the most continuous collapse fissure near the south break ( $c$ in fig. 152; see below); this collapse fissure opened probably no more than about $30 \mathrm{~mm}$ and formed where the water table is probably at least $27 \mathrm{~m}$ deep. The cross-sectional area of this fissure ranges from about $0.1 \mathrm{~m}^{2}$ near the north end to perhaps $0.5-1 \mathrm{~m}^{2}$ locally elsewhere. The average collapsed volume along the entire length of the fissure 
is probably between 0.2 and $0.8 \mathrm{~m}^{3}$ per meter of length. Assuming a tectonic opening of $30 \mathrm{~mm}$, the fracture can supply the necessary storage volume for this observed range of average collapsed volume if it remains open to depths of $7 \mathrm{~m}$ and $27 \mathrm{~m}$, respectively.

However, fissures 1-3 $\mathrm{m}$ wide and 3-4 $\mathrm{m}$ deep that developed on fractures $20-30 \mathrm{~mm}$ wide have already been described, and the volumes of these fissures cannot be accommodated in the fracture below, even using the $27 \mathrm{~m}$ depth. A solution to this problem is that these large collapsed sections are seldom more than 3-5 m long, and they generally occur on fractures that have many uncollapsed or only slightly collapsed parts (fig. 144). Indeed, most fractures that have collapsed since 1968 near the south break - where the deepest and widest fissures occur have about as many uncollapsed as collapsed parts. Water flowing within the fissures must commonly remove material from one part of the fissure and deposit it elsewhere in the lower parts of the same fracture beneath sections that did not collapse. Hence, to an initial approximation, each collapse fissure studied along the 1968 break appears to have enough volume at depth to receive the amount of material that has been carried below plus minor amounts of sediment brought in by runoff.

\section{CONDITIONS CONTROLLING THE DEVELOPMENT OF COLLAPSE FISSURES}

If the preceding explanation for the development of large collapse fissures from tectonic fractures is correct, then the conditions necessary for their creation are fairly restrictive. The primary requirements are simple: The fractures must remain open to a depth of at least $10 \mathrm{~m}$ or so, and water sufficient to erode or to undermine material from the walls, but not so loaded with sediment as to clog or fill the fractures, must enter before other agents, such as wind or slumping, close them. Many factors may affect these primary requirements.

Certainly, the physical properties and conditions of the material in which fractures form are critical. Such properties as cohesion and shear strength and such conditions as moisture content and depth to the zone of water saturation not only affect the depth to which the crack will remain open but also probably influence the nature of the fracture itself whether it will be a single fracture or a narrow band of multiple, interconnected, or en echelon fractures, which may not be so likely as a single break to offer an open path to lower levels.

Delivery of a relatively large volume of water with a low suspended particle load to many places along tectonic fractures requires some minimum in- tensity and quantity of rain and is facilitated by surfaces with low slope, relief, and permeability. If the surface is also largely free of vegetation, a lower intensity or total amount of rainfall will supply the necessary amount of runoff, although lack of vegetation may also increase the sediment load. Thus collapse fissures presumably will not form in soil too weak to maintain open cracks or in places where surface runoff is either small or quickly localized into narrow channels.

Indeed, collapse fissures may not be a certain consequence of each episode of surface faulting in Lower Borrego Valley. The relations observed between creep and collapse imply that at least some collapse fissures depend on a delicately timed sequence of tectonic opening and heavy rain. For large collapse fissures to occur at all, heavy rain must fall before tectonic fractures are sealed near the surface. Widespread development of collapse fissures, as in 196869 , may be a relatively unusual part of the tectonic activity of this part of the Coyote Creek fault.

\section{OTHER COLLAPSE FISSURES IN LOWER BORREGO VALLEY}

\section{PREEARTHQUAKE AND RELICT COLLAPSE FISSURES ALONG THE COYOTE CREEK FAULT}

Four groups of preearthquake collapse fissures are recognizable along the south break (fig. 152). The

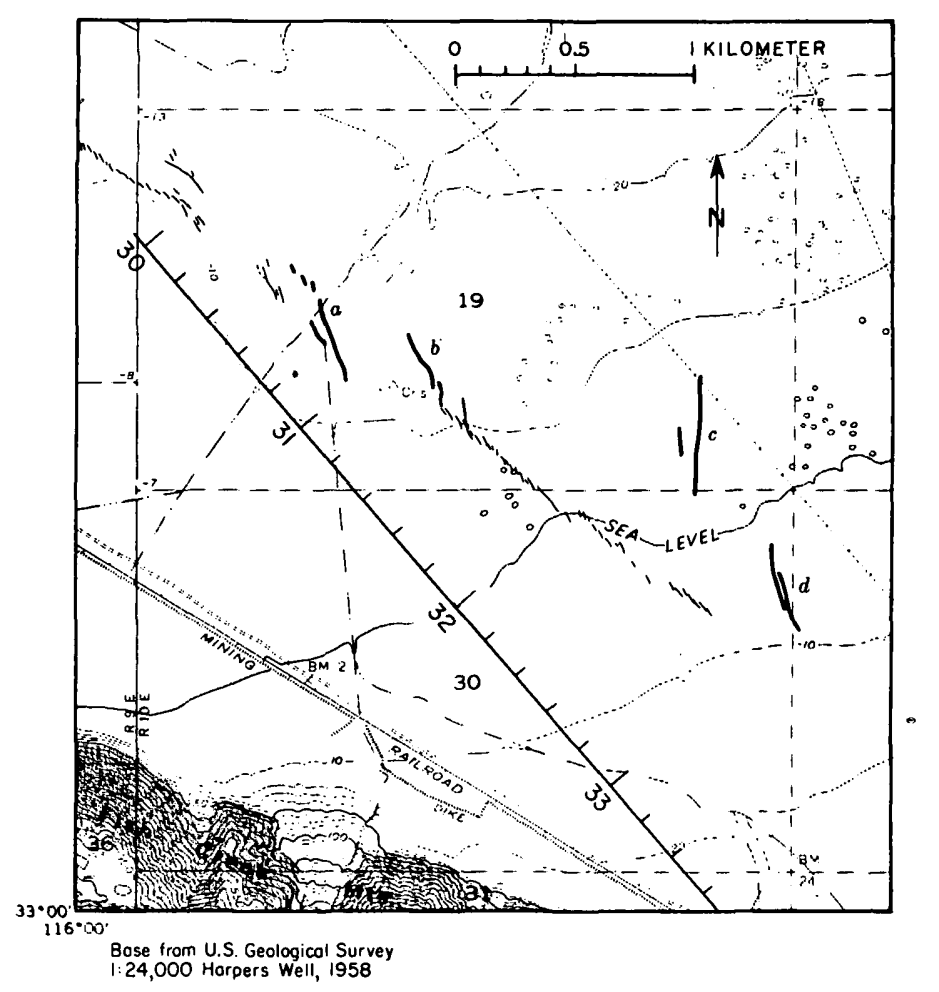

Figure 152. - Relict (preearthquake) collapse fissures $(a-d)$ near the south break of the 1968 rupture along the Coyote Creek fault. 
oldest developed before 1953; the youngest opened shortly before the earthquake, and abundant vegetation has not yet grown. Alined vegetation, with or without shallow sinks, marks the three older groups. These older linear features can be identified as relict tectonic collapse fissures because (1) they are parallel to major en echelon fractures of 1968 , (2) they are located next to the 1968 break, and (3) they are the older members of a gradational series that ends with nearby younger fissures created since the earthquake.

The oldest two groups of fissures (fig. 152, $a$ and $d$ ) are now mostly filled and are represented by alined creosote bushes, small brush-stabilized dunes, and linear sinks less than $0.5 \mathrm{~m}$ deep and $2 \mathrm{~m}$ long. These oldest relict fissures are distinctly more conspicuous on 1953 aerial photos than they are on 1968 and 1969 photographs; indeed, on the 1953 photos, one at $d$ (fig. 152) appears to have an open collapse fissure, although the scale does not permit positive identification.

The relict fissure at $b$ (fig. 152) consists of alined bushes, dunes, and sinks like those at $a$ and $d$ but is evidently younger. Unlike the older fissures, most of it is not visible on the 1953 photos, and in 1969, the alined sinks of the southern part were nearly continuous and deeper than those of the fissures at $a$ and $d$. Furthermore, the alined bushes of this fissure have become increasingly prominent since 1953, when only a few grew near the northern end. More bushes appear on a 1956 aerial photograph (fig. 38), and by 1968 , large and prominent bushes marked the entire length shown in figure 152. The discontinuous nature of this relict fissure and the rounded shallow sinks along it indicate that the fissure stopped growing several years ago. Moreover, it showed no evidence of fresh cracking or opening at the surface either shortly before or since the earthquake, even though it nearly joins one of the two new fractures formed by postearthquake creep in September 1969 (e, fig. 49).

By far the most prominent collapse fissure - preearthquake or postearthquake - along the entire fault is at $c$ (fig. 152). When first seen on April 10, 1968 , it was a straight, almost continuously open fissure nearly $500 \mathrm{~m}$ long and as much as $2 \mathrm{~m}$ wide and deep (figs. 153-155). Moist polygonally cracked mud in the bottom, presumably created by drying after inflow of water during heavy preearthquake rains of April or March 1968, did not crack further during the earthquake. This fissure is not visible on the aerial photographs of 1953 and 1956, and thus was almost certainly not in existence then because it is very conspicuous on 1968 aerial photographs of similar scale.

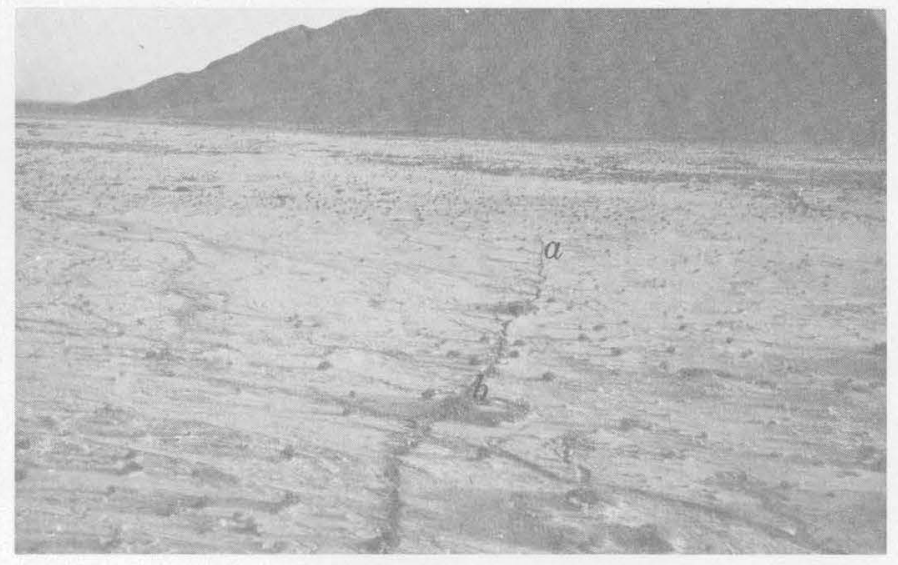

Figure 153. - Aerial view southward over part of the most prominent preearthquake fissure (fig. $152, c$ ). This photograph shows a $400-\mathrm{m}$ length of the fissure as it appeared on April 10, 1968. The southeast part of the south break passes immediately in front of the dense vegetation in the middle distance (figs. 154 and 155 show details at $a$ and b). Photograph by J. P. Lockwood, U.S. Geological Survey.

At least some of the collapse took place less than a year before the earthquake, although parts of the fissure could have been older. The southernmost section of fissure $c$ had the steep walls and deep narrow bottom typical of initial collapse of a new fracture. Moreover, a relatively fresh set of tire tracks crossed the fissure at a place that had subsequently collapsed. As seen a few days after the earthquake, the tracks were obvious and appeared fresher than many 1-year-old tracks studied along the break after the earthquake. Other parts of fissure $c$ looked as though they had been subjected to two or more episodes of surface runoff, because they had the U-shaped cross section and mud polygons of a fissure that is filling. The abundance of small bushes in and along the northernmost part of the fissure suggested that that section was more than several months old. In 1968 much of this fissure had not yet stimulated a vigorous growth of creosote bushes typical of the older collapse fissures nearby; thus, it was at most only a few years old. Two large mesquite-stabilized dunes athwart the fissure imply the existence of an earlier fracture in the same location, but the position of these dunes could be fortuitous. Preearthquake rainfall as late as March and April of 1968 (fig. 40) might have caused most of the latest collapse, although it seems more likely that rainfall in 1967 or earlier caused some of the initial collapse. Nevertheless, the condition of the fissure, the tire tracks, and the pattern of rainfall in early 1968 indicate at least some collapse shortly before the earthquake.

Although this fissure is longer, is farther from the fault, and has a slightly different trend (fig. 152) 


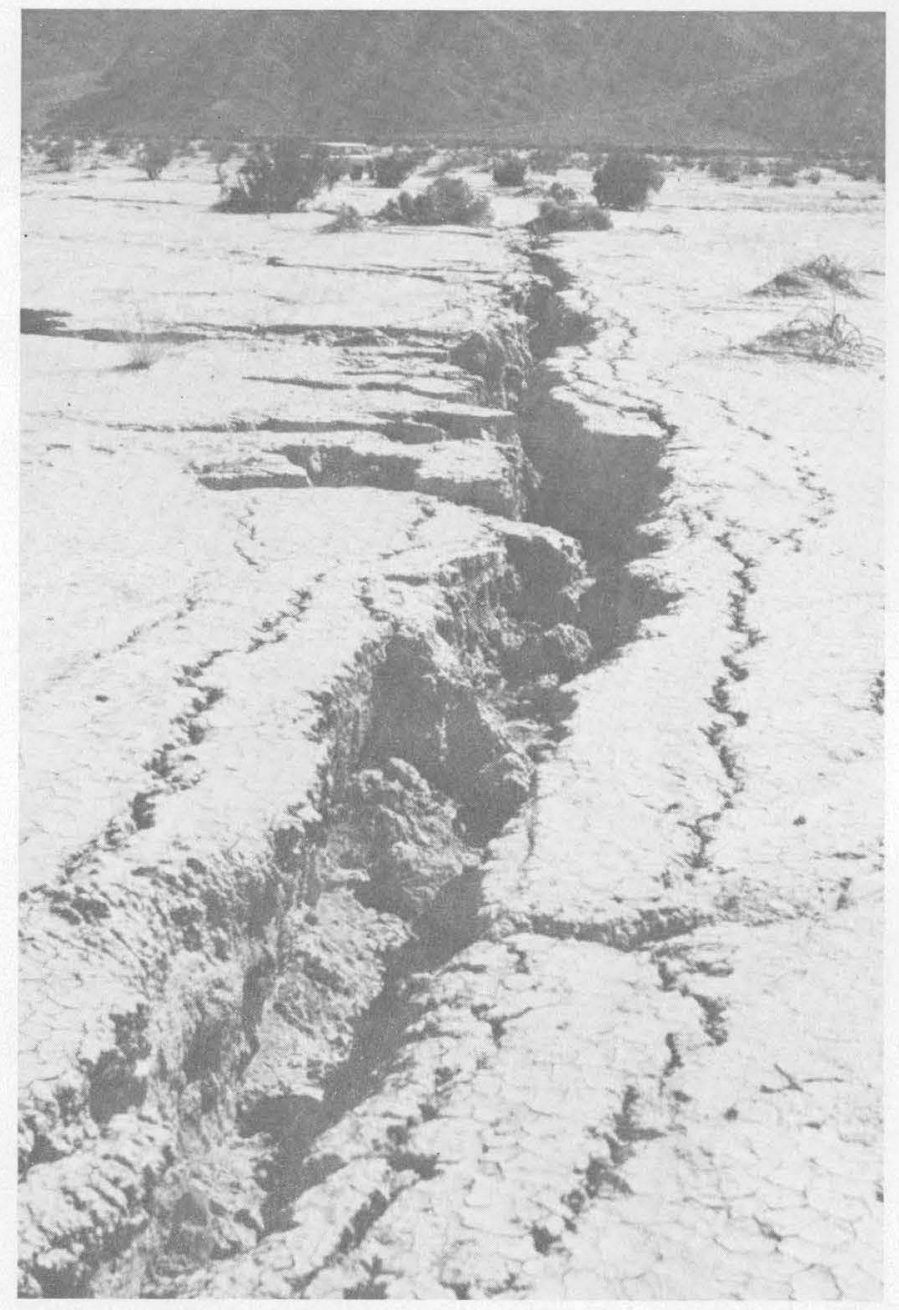

FIgURE 154. - South end of the relict collapse fissure shown at $a$ in figure 153. Water entering from the left has eroded deep gullies into the fissure. Peripheral cracks bound the blocks that would slump next or that would founder into the fissure if it were growing. However, the fissure has been slowly filling with waterborne material since it was first observed in April 1968, although it opened slightly and some minor collapse oceurred during 1970.

than the other relict and younger fissures nearby, it appears to have had the same tectonic origin. Fractures and fissures somewhat similar in appearance to these relict fissures have been described, but they result from other causes, such as desiccation (for example, Lang, 1943; Knechtel, 1952; Willden and Mabey, 1961; Neal and others, 1968; Underwood and DeFord, 1969), piping (for example, Carroll, 1949; Fletcher and others, 1954), broad subsidence, possibly related to lowering of artesian head (Robinson and Peterson, 1962; Kam, 1965), or near-surface compaction (hydrocompaction) at the time of initial wetting of alluvial deposits (Bull, 1964, 1970; Lofgren, 1969).

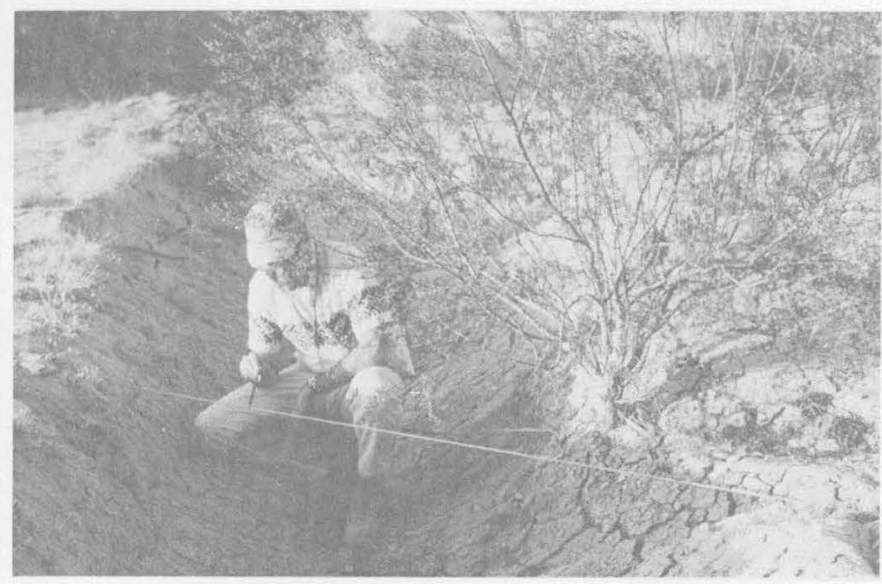

Figure 155. - A taut root across the relict collapse fissure at $b$ in figure 153 may have been stretched by tectonic opening of the original fracture. The bottom of the fissure shows the shrinkage polygons and rounded cross section typical of a collapse fissure that is filling rather than deepening.

The preearthquake fissures of figure 152 along the south break lack important characteristics of each of these other types. The fissures of the south break have neither the polygonal arrangement nor playa setting of desiccation cracks. They do not trend toward the walls of Carrizo Wash (pl. 1), the only nearby free face sufficiently high to account for depths observed in the fissures (about $4 \mathrm{~m}$ ), if they have been caused by collapse of subsurface pipes. These fissures bound no apparent subsiding area; the closest pumping of ground water since before the earthquake is at a ranch nearly $10 \mathrm{~km}$ to the north, and the older fissures predate these wells. Finally, these fissures show neither the proximity nor orientation parallel to channels that might be causing hydrocompaction of adjacent sediment (Bull, 1970 , p. 55, 152). The straight channels that cross the south break at location 30.6 (across the relict fissure at $a$, fig. 152) are actively deepening their channels, which evidently follow old roads or manmade courses. These channels receive runoff concentrated by culverts under the nearby railroad. If this augmented flow across the fault were causing hydrocompaction, the channels should subside and fractures should form parallel to them, but neither is apparent. Thus there is no convincing evidence that these relict fissures have developed from anything except tectonic fractures associated with the Coyote Creek fault zone. Moreover, groups of small alined preearthquake sinks along the south break at locations 28.4 and 32.0 provide further evidence of tectonic activity shortly before the earthquake. These sinks represent partly filled collapse fissures and 


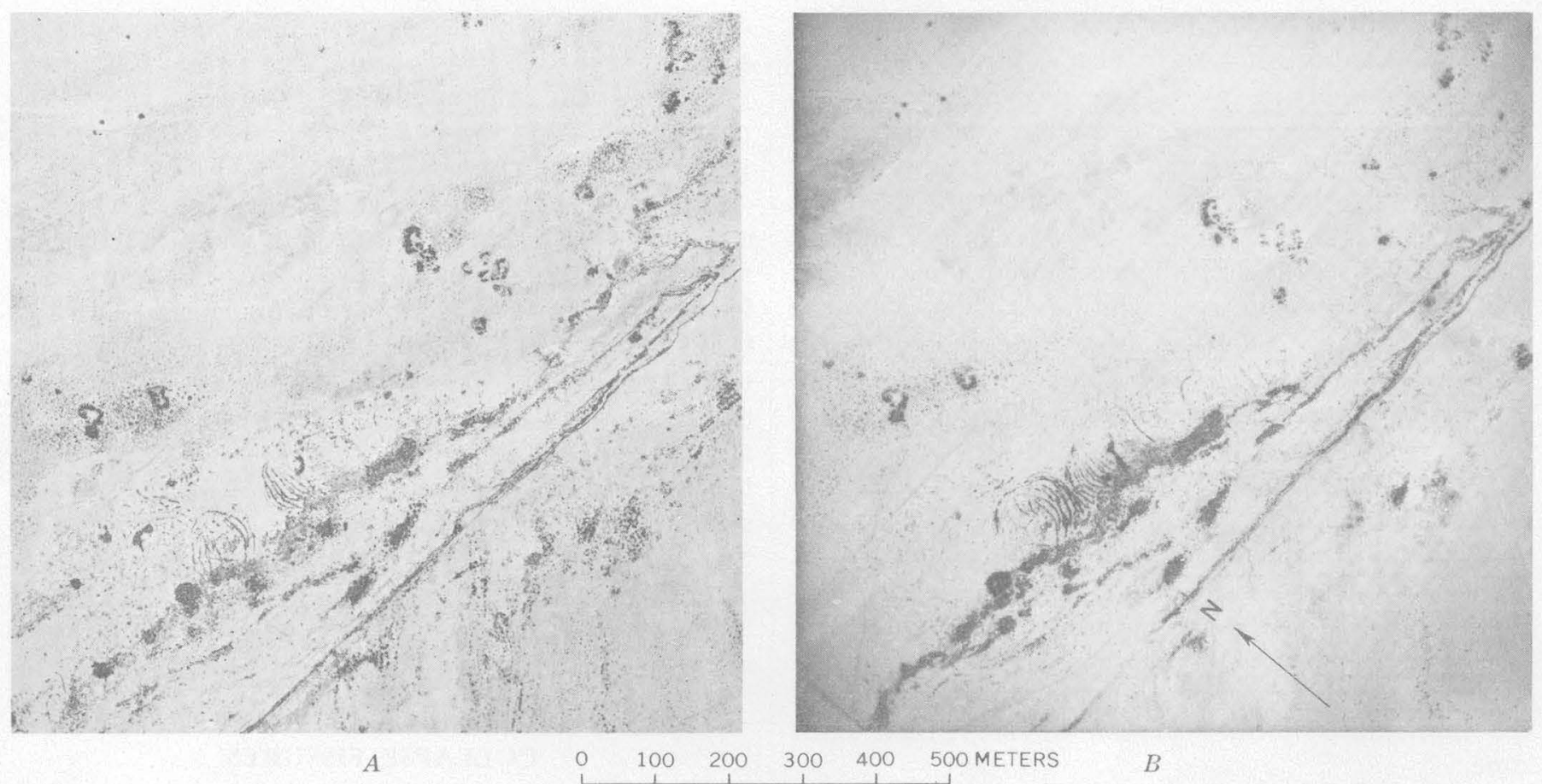

Figure 156. - Vertical aerial photographs of concentric collapse fissures at location 18. A, April 1953. Circles (apparently older) are faintly visible north and northwest of the prominent circles. $B$, April 1969. Although many new concentric fissures have formed between the two prominent sets, many fissures visible in $A$ are much less prominent or no longer evident. The distinct E-W lineament is Old Kane Spring Road.

appear to be comparable in age to the young preearthquake fissure at $c$ (fig. 152).

\section{OTHER FISSURES IN LOWER BORREGO VALLEY}

Two groups of fissures and relict fissures that are not obviously related to the fault occur near the 1968 rupture. The larger group lies $1-2 \mathrm{~km}$ southwest of the south break alongside and within one-half kilometer of the base of the Fish Creek Mountains between locations 28 and 32 . The smaller group is about $10 \mathrm{~km}$ to the northwest near uplifted sediments at location 18 , about $3 \mathrm{~km}$ west of the central break (fig. 35 ). For the most part, these fissures consist of continuous and discontinuous shallow sinks in circular, straight, or irregular alinements. Bushes are concentrated along all the fissures, which range in length from tens to hundreds of meters. A few sinks are as much as $1 \mathrm{~m}$ wide and $2 \mathrm{~m}$ deep and are nearly continuous for tens of meters. Some fissures are merely conspicuous lines of bushes that connect widely spaced small (less than $0.5 \mathrm{~m}$ wide) sinks. Many of the fissures are apparent on 1953 aerial photographs.

Most of the fissures fall into two classes. All those in the smaller northwestern group and many in the larger southern group form clusters of as many as seven or eight concentric rings within a diameter of $30-150 \mathrm{~m}$ (fig. 156). The other class, entirely in the southern group, comprises many nearly straight fissures that roughly parallel the adjacent front of the Fish Creek Mountains.

Although these features were not systematically mapped, aerial photographs taken in 1969 recorded some of them and revealed definite changes from 1953. The concentric fissures in particular show obvious evolution between 1953 and 1969. In the southern group, several circular fissures formed in new locations after 1953, whereas in the group to the northwest, many new concentric fissures developed around those present in 1953. In contrast, many of the less prominent fissures of 1953 (presumably shallow sinks) had almost entirely disappeared by 1969 (fig. 156). The nearly straight fissures along the Fish Creek Mountains show similar changes, and partial aerial photographic coverage of the southern group since the earthquake suggests that new fractures are appearing more rapidly than old ones are disappearing.

There seems little reason to doubt that these fissures developed by collapse from open fractures, but the origin of the fractures remains uncertain. Except for some of those in the northwestern group, none of the fissures showed signs of active enlargement at the time of field observations in December 1970, nor were any uncollapsed fractures found. It is difficult to imagine that concentric fractures $150 \mathrm{~m}$ or less in diameter could have originated at great depth or been formed by tectonic forces. The circular 
fissures closely resemble "ring fissures" described by Neal, Langer, and Kerr (1968, p. 86). Ring fissures supposedly develop from desiccation contraction of soil around a phreatophyte as the plant lowers the water table and capillary fringe directly beneath it. Although no single central phreatophyte (mesquite, in this area) is obvious in the rings in figure 156, several of the concentric fissures in the southern group encircle individual large mesquite bushes. In the absence of any direct evidence for some other form of local compaction or subsidence, the phreatophyte explanation seems the most reasonable for the circular fissures. The position of the straight fissures of the southern group alongside and subparallel to the mountain front suggests that more widespread subsidence of the sediments might result from either tectonic movement or, possibly, compaction. Regardless of their origin, these unusual outlying fissures demonstrate the potential for collapse in the sediments of Lower Borrego Valley beyond the immediate vicinity of the Coyote Creek fault zone.

\section{DEVELOPMENT OF COLLAPSE FISSURES ALONG OTHER ACTIVE FAULTS}

Few investigators have reported collapse of tectonic fractures elsewhere. Neither the detailed descriptions nor abundant illustrations of the report on the 1906 California earthquake include collapse fissures (Lawson and others, 1908): None were mentioned in the brief report on the Imperial earthquake of 1940 (Ulrich, 1941). The intensive investigation of the San Fernando earthquake of February 9, 1971, revealed no collapse fissures (U.S. Geological Survey, 1971) despite intense rain February 17th, 8 days later (Environmental Data Service, 1971, p. 47). However, in 1907, W. D. Johnson photographed and described sinks as much as $1 \mathrm{~m}$ deep that had developed and persisted on the breaks of the 1872 earthquake in Owens Valley, California (unpub. data, U.S. Geological Survey library, Denver). Many of these sinks were little changed in 1971. Some of the sinks developed, however, in sandy alluvium within $100 \mathrm{~m}$ upstream of a scarp 6-7 m high, and piping could have caused these, rather than the mechanism proposed for the fissures in Lower Borrego Valley. Warne (1955) described fractures caused by the Arvin-Tehachapi earthquake of 1952 that intercepted irrigation water, and he included a photograph of what appears to be subsequent collapse of such a fracture (his fig. 20). Although no collapse fissures were reported from the ground breaks that formed at the time of the Parkfield-Cholame earthquake of 1966 on the San Andreas fault (Brown and others, 1967), R. E. Wallace has photographs of some fissures (written commun., 1971) that were present 1 year later along the fractures. What connection, if any, these fissures have to creep and rainfall is unknown.

The fact that a few collapse fissures have been associated with other earthquakes, plus the development of similar fissures elsewhere from piping, desiccation, subsidence, or unknown causes, indicates that the conditions for their formation are not unique to Lower Borrego Valley. Fissures will develop along earth fractures whenever all the necessary conditions are present, whatever those may be. That most of the reports of fissures in the United States are restricted to arid or semiarid areas strongly suggests that in desert areas soil and sediment, surface characteristics, rainfall patterns, ground-water conditions, or combinations of these or other factors are most favorable for the development of fissures.

\section{ENGINEERING SIGNIFICANCE OF COLLAPSE FISSURES}

Collapse fissures of the size that developed along the 1968 rupture present a distinct but manageable hazard to manmade structures. Moreover, if the mechanism of collapse proposed here is correct, collapse can be prevented by diverting runoff away from new fractures. Once collapse has started, it can be stopped by dumping sand and dirt into the growing fissures. Fissures that have stopped growing, of course, can easily be filled.

Structures threatened by collapse of fractures are also threatened by the generally greater hazard of ground displacement. Tectonic opening or continuing creep sufficient to permit collapse is likely to do more damage to most structures built across fractures than will subsequent collapse. Moreover, collapse can be controlled; tectonic movement cannot.

\section{SIGNIFICANCE OF COLLAPSE FISSURES IN FAULT STUDIES}

Probably the most important characteristic of collapse fissures in fault studies is that their presence in a fault zone is one of the most obvious signs of recent fracturing. Along the 1968 rupture, new fractures formed by creep were discovered only by investigators who crossed them on foot, but collapse fissures that developed from these fractures were easily visible from cars and airplanes or on aerial photographs. Although collapse is never a necessary consequence of fracturing, new collapse fissures in a fault zone are an almost certain indication that new fractures have formed, either during an earthquake or, afterward, from creep. 


\section{REFERENCES CITED}

Brown, R. D., Jr., Vedder, J. G., Wallace, R. E., Roth, E. F., Yerkes, R. F., Castle, R. O., Waananen, A. O., Page, R. W., and Eaton, J. P., 1967, The Parkfield-Cholame California, earthquakes of June-A ugust 1966 - surface geologic effects, water resources aspects, and preliminary seismic data: U.S. Geol. Survey Prof. Paper 579, 66 p.

Bull, W. B., 1964, Alluvial fans and near-surface subsidence in western Fresno County, California: U.S. Geol. Survey Prof. Paper 437-A, $71 \mathrm{p}$.

1970, Prehistoric near-surface subsidence cracks in western Fresno County, California: U.S. Geol. Survey open-file report, $256 \mathrm{p}$.

Carroll, P. H., 1949, Soil piping in southeast Arizona: U.S. Dept. Agriculture Soil Conservation Service Regional Bull. 110, Soil Series 13, 21 p.

Environmental Data Service, 1971, Climatological data, California: Natl. Oceanog. and Atmospheric Adm., v. 75.

Fletcher, J. E., Harris, K., Peterson, H. B., and Chandler, V. N., 1954, Piping: Am. Geophys. Union Trans., v. 35, p. 258-263.

Gibbs, H. J., Hills, J. W., Holtz, W. G., and Walker, F. C., 1960 , Shear strength of cohesive soils, in Research conference on shear strength of cohesive soils, Boulder, Colo., 1960: Am. Soc. Civil Engineers, Soil Mechanics and Found. Div., p. 33-162.

Kam, William, 1965, Earth cracks - a cause of gullying, in Geological Survey research 1965: U.S. Geol. Survey Prof. Paper 525-B, p. B122-B125.

Knechtel, M. M., 1952, Pimpled plains of eastern Oklahoma: Geol. Soc. America Bull., v. 63, p. 689-700.

Lang, W. B., 1943, Gigantic drying cracks in Animas Valley, New Mexico: Science, v. 98, p. 583-584.

Lawson, A. C., and others, 1908, The California earthquake of April 18, 1906, Report of the state earthquake investigation commission: Carnegie Inst. of Washington, v. 1, $451 \mathrm{p}$.
Lofgren, B. E., 1969, Land subsidence due to application of water, in Varnes, D. J.., and Kiersch, G., eds., Reviews in engineering geology, v. 2: Geol. Soc. America, 350 p.

Meinzer, O. E., 1946, General principles of ground water recharge: Econ. Geology, v. 41, no. 3, p. 191-201.

Moyle, W. R., Jr., 1968, Water wells and springs in Borrego, Carrizo, and San Felipe Valley areas, San Diego and Imperial Counties, California: California Dept. Water Resources Bull. 91-15, 16 p.

Neal, J. T., Langer, A. M., and Kerr, P. F., 1968, Giant desiccation polygons of Great Basin playas: Geol. Soc. America Bull., v. 79, p. 69-90.

Robinson, G. M., and Peterson, D. E., 1962, Notes on earth fissures in southern Arizona: U.S. Geol. Survey Circ. $466,7 \mathrm{p}$.

Sniegocki, R. T., 1962, Problems in artificial recharge through wells in the Grand Prairie region, Arkansas: U.S. Geol. Survey Water-Supply Paper 1615-F, 25 p.

Todd, D. K., 1959, Ground water hydrology: New York, John Wiley \& Sons, Inc., $336 \mathrm{p}$.

Ulrich, F. P., 1941, The Imperial Valley earthquakes of 1940: Seismol. Soc. America Bull., v. 31, p. 13-31.

Underwood, J. R., Jr., and DeFord, R. K., 1969, Large-scale desiccation fissures in alluvium, Trans-Pecos Texas and northern Chihuahua [Abs.]: Geol. Soc. America Abs. with Programs, v. 1, pt. 2, p. 31 .

U.S. Geological Survey, 1971, Surface faulting, in The San Fernando, California earthquake of February 9, 1971: U.S. Geol. Survey Prof. Paper 733, p. 55-76.

Warne, A. H., 1955, Ground fracture patterns in the southern San Joaquin Valley resulting from the ArvinTehachapi earthquake, in Oakeshott, G. B., ed., Earthquakes in Kern County, California during 1952: California Div. Mines Bull. 171, p. 57-66.

Willden, Ronald and Mabey, D. R., 1961, Giant desiccation fissures on the Black Rock and Smoke Creek Deserts, Nevada: Science, v. 133, p. 1359-1360. 